$$
\text { DVE/ER/75879-T2: }
$$

\title{
Mixed Field Dosimetry Using Focused and Unfocused Laser Heating of Thermoluminescent Materials
}

\author{
A Thesis \\ Presented to \\ The Academic Faculty \\ by \\ Seungjae Han
}

DISCLAIMER

This report was prepared as an account of work sponsored by an agency of the United States Government. Neither the United States Government nor any agency thereof, nor any of their employees, makes any warranty, express or implied, or assumes any legal liability or responsibility for the accuracy, completeness, or usefulness of any information, apparatus, product, or process disclosed, or represents that its use would not infringe privately owned rights. Reference herein to any specific commercial product, process, or service by trade name, trademark, manufacturer, or otherwise does not necessarily constitute or imply its endorsement, recommendation, or favoring by the United States Government or any agency thereof. The views and opinions of authors expressed herein do not necessarily state or reflect those of the United States Government or any agency thereof.

In Partial Fulfillment of the Requirements for the Degree Doctor of Philosophy

Georgia Institute of Technology
March 1994 
This thesis is dedicated to my loving wife, Youngrae, whose assistance, encouragement, and patience made this work possible; and to my wonderful children, Zeesoo and Zeeyeon, who have been a constant source of inspiration and joy. 


\section{ACKNOWLEDGEMENTS}

I would like to express my sincere gratitude to Professor Kimberlee J. Kearfott for her support, guidance and encouragement during this work. I am grateful to Professor CK. Chris Wang for his continual assistance and for his valuable suggestions without which this work could not be completed. I am also thankful to the following members of my thesis committee for their suggestions and help during my graduate study: Dr. Bernd Kahn, Dr. Mostafa Ghiaasiaan, Dr. Ahmet Erbil, and Dr. William R. Callen.

I am especially grateful to Dr. Jefferey A. Donnell for his review and valuable comments on writing of this dissertation. I am deeply indebted to Moonkyu Hwang for his assistance and encouragement.

Thanks to Professor William J. Wepfer and Ms Claudette Noel, the staff at the Woodruff School of Mechanical Engineering.

This work is supported by U.S. Department of Energy contract No. DE-FG0292ER 75703, that is hereby gratefully acknowledged.

Finally, I would like to thank my and my wife's parents and express my love and appreciation for their love and support of my studies; and especially to my wife Youngrae and my daughters Zeesoo and Zeeyeon for their love, patience, understanding, and continuous support and encouragement. 


\section{TABLE OF CONTENTS}

ACKNOWLEDGEMENTS iii

TABLE OF CONTENTS iv

LIST OF TABLES vi

LIST OF FIGURES

NOMENCLATURE XXiv

GLOSSARY $\quad$ Xxv

SUMMARY XXvi

I. INTRODUCTION

1.1 Motivation 1

1.2 Theory of Thermoluminescence 4

1.3 Recent Developments in TLD 8

1.3.1 Multi-element, Filtered Thermoluminescence Dosimeters $\quad 8$

1.3.2 Thin-element Beta Detectors 9

1.3.3 New Heating Techniques 10

$\begin{array}{ll}1.4 \text { Thesis Outline } & 12\end{array}$

II. COMPUTATIONAL METHODS 15

2.1 Laser Beam Heating 15

2.1.1 Gaussian Laser Beam $\quad 15$

2.1.2 Uniform Square Beam 20

2.2 Heat Conduction with Temperature-Dependent Thermal conductivity 23

2.3 Calculation of Thermoluminescence Glow Curve 25

2.4 Unfolding of Depth-Dose 26

III. DEVELOPMENT AND IMPLEMENTATION OF COMPUTER CODES 28

3.1 Description of Computer Codes 28

3.1.1 Codes for Heat Conduction and Glow Curve Calculation 28

3.1.2 Code for Electron and Photon Transport Calculations 29

3.1.3 Code for Unfolding Depth-Dose Distributions 30

3.2 Description of Calculations 31

3.2.1 Benchmark Calculations 31

3.2.1.1 Heat Conduction Calculations 31

3.2.1.2 TL Glow Curve Calculations 33 
3.2.2 Design-Basis Heat Conduction Calculations 34

3.2.2.1 Single Pulse Heating 34

3.2.2.2 Multiple Pulse Heating 37

3.2.3 Design-Basis TL Glow Curve Calculations 40

3.2.4 Design-Basis Depth-Dose Calculations Using EGS4 41

3.2.5 Response Function Calculations 43

3.2.6 Unfolding of Depth-Dose Distribution and Optimization of Pulsed Heating

3.2.6.1 Unfolding of Depth-Dose Distribution

3.2.6.2 Approach of Optimum Pulsed Heating Scheme 44

3.2.6.3 Determination of Shallow and Deep Doses 46

3.2.7 Sensitivity and Uncertainty Analysis 47

3.2.7.1 Stability of Computer Code 47

3.2.7.2 Noise Analysis of Glow Curves and Depth-Dose $\quad 47$

3.2.7.3 Uncertainties of EGS4 Depth-Dose 48

IV.RESULTS AND DISCUSSIONS $\quad 50$

4.1 Temperature Profiles 50

4.1.1 Temperature Profiles for Benchmark Calculations $\quad 50$

4.1.2 Temperature Profiles of Thick Laser TLD 51

4.2 TL Glow Curves 57

4.2.1 TL Glow Curves for Benchmark Calculations 57

4.2.2 TL Glow Curves in Thick Laser TLD 59

4.3 Design-Basis Depth-Dose Distribution 61

4.3.1 Depth-Dose Distributions Obtained from EGS4 61

4.3.2 Uncertainties of Depth-Dose Distributions 61

4.4 Pulsed Heating and Response Functions 62

4.4.1 Pulsed Heating and Response Functions for Focused Beam $\quad 62$

4.4.2 Pulsed Heating and Response Functions for Uniform Beam $\quad 62$

4.5 Unfolding of Depth-Dose Distribution and Optimization of Heating

Scheme

4.5.1 Unfolding of Depth-Dose Distribution with a Two-Pulse Heating Scheme

4.5.2 Optimum Two-Pulse Heating

4.5.3 Unfolding of Depth-Dose Distribution with a Three-Pulse Heating Scheme

4.5.4 Optimum Three-Pulse Heating 67

4.6 Deep and Shallow Doses $\quad 70$

4.6.1 Deep and Shallow Doses for Focused Beam $\quad 70$

4.6.2 Deep and Shallow Doses for Uniform Beam $\quad 70$

4.7 Noise Analysis Results 72

4.7.1 Noise of Depth-Dose by Focused Beam 72

4.7.2 Noise of Depth-Dose by Uniform Beam

4.8 Results of Energy Response Calculation 75

4.9 Dosimetric Evaluation of Thick Laser TLD 77

4.9.1 Characteristics of Depth-Dose within TL Dosimeter 77

4.9.2 Temperature and TL Light Gradient 77

4.9.3 TL Light Attenuation and Energy Response $\quad 79$

$\begin{array}{ll}\text { V. CONCLUSIONS } & 80\end{array}$

VI. SUGGESTIONS FOR FUTURE WORK

6.1 Construction of the Thick TLD Heating System 83 
6.2 Practical Application of the TL Dosimeters

84

6.3 Uncertainty Study of Thermal Parameters

84

REFERENCES

APPENDIX 


\section{LIST OF TABLES}

Table

1. Various heat conduction and glow curve parameters. The symbol $(\dagger)$ marked parameters were used through the mathematical models in this study.

page

2. Summary of input data to run EGS4 electron/photon transport code.

3. Summary of benchmark heat conduction and TL glow curve calculations of laser beam heating on LiF TL materials.

4. Specifications of focused and unfocused laser system for a Laakmann Electro-Optics RF-445 laser [Abtahi, 1985; Grupen, 1989].

5. Summary of mixed field dosimetry calculations using laser beam on a thick laser TLD.

6. Two-pulse heating approaches of focused laser beam.

7. DOELAP radiation fields for dosimeter calibration [DOE/EH-0026, 1986]. These radiation fields were used to calculate the actual depth-doses within the thick TL dosimeter by EGS4 code. 50,000 history Monte Carlo simulation was performed for every single and mixed sources by $1: 1$ mixing ratio.

8. The surface to surface temperature difference for different thick TLD.

9. Surface temperature variations by temperature dependence of thermal conductivity coefficient for $0.09 \mathrm{~cm}$ LiF TLD by $10 \mathrm{~W}$ uniform laser beam heating.

10. Surface temperature variations by temperature dependence of thermal conductivity coefficient for $0.38 \mathrm{~cm}$ LiF TLD by $10 \mathrm{~W}$ uniform laser beam heating.

11. Comparison of numerical glow curve with experimental glow curve. Numerical glow curve is the result from HANG1 and experimental glow curve is the result from Abtahi [Abtahi et al., 1985] following heating with a $0.084 \mathrm{~cm}$ diameter focused $4.93 \mathrm{~W}$ laser beam.

12. Comparison of numerical glow curve with experimental glow curve. Numerical glow curve is the result from HANG1 and experimental glow curve is the result from Grupen [Grupen, 1989] following heating with a $0.170 \mathrm{~cm}$ diameter unfocused $4 \mathrm{~W}$ laser beam. 
13. Comparison of numerical glow curve with experimental glow curve. Numerical glow curve is the result from HANG2 and experimental glow curve is the result from Braunlich [Braunlich, 1990] following heating with a with a $0.3 \mathrm{~cm}$ square uniform laser beam.

14. Least-squares fitting values of EGS4 depth-dose from Sr/Y-90 beta-particles in shallow and deep dose region and related errors.

15. Least-squares fitting values of EGS4 depth-dose from Sr/Y-90 beta-particles in shallow and deep dose region and related errors.

16. Least-squares fitting values of EGS4 depth-dose from Cs-137 gamma-rays in shallow and deep dose region and related errors.

17. Unfolded results of shallow doses from pulsed heating scheme of focused laser beam on the thick laser TLD exposed by single or mixed beta/gamma radiation. The radiation exposure was simulated by the EGS4 Monte Carlo code with 50,000 histories for each radiation field.

18. Unfolded results of deep doses from pulsed heating scheme of focused laser beam on the thick laser TLD exposed by single or mixed beta/gamma radiation. The radiation exposure was simulated by the EGS4 Monte Carlo code with 50,000 histories for each radiation field.

19. Unfolded results of shallow doses from pulsed heating scheme of uniform laser beam on the thick laser TLD exposed by single or mixed beta/gamma radiation. The radiation exposure was simulated by the EGS4 Monte Carlo code with 50,000 histories for each radiation field.

20. Unfolded results of deep doses from pulsed heating scheme of uniform laser beam on the thick laser TLD exposed by single or mixed beta/gamma radiation. The radiation exposure was simulated by the EGS4 Monte Carlo code with 50,000 histories for each radiation field.

21. Variations of TL glow curves for a thick laser TLD with systematic noises of various thermal parameters following pulsed heating with a $0.032 \mathrm{~cm}$ diameter focused $4 \mathrm{~W}$ laser beam. TL dosimeter exposed to TI-204 beta particles was used for this calculations.

22. Variations of deep and shallow doses for a thick laser TLD with systematic noises of various thermal parameters following pulsed heating with a $0.032 \mathrm{~cm}$ diameter focused $4 \mathrm{~W}$ laser beam. TL dosimeter exposed to T1-204 beta particles was used for this calculations.

23. Variations of TL glow curves for a thick laser TLD with systematic noises of various thermal parameters following pulsed heating with a $0.3 \mathrm{~cm}$ square uniform laser beam. TL dosimeter exposed to $20 \mathrm{keV}$ X-rays was used for this calculations.

24. Variations of shallow and deep doses for a thick laser TLD with systematic noises of various thermal parameters following pulsed heating with $a .3 \mathrm{~cm}$ square uniform laser beam. TL dosimeter exposed to $20 \mathrm{keV}$ x-rays was used for this calculations. 
25. Thermoluminescence light attenuation data for TLD dosimeters manufactured by Harshaw/Filtrol*. 


\section{LIST OF FIGURES}

Eigure

1. Schematic diagram of thick LiF laser TL dosimeter. Actual size is $0.3 \times 0.3 \mathrm{x}$

page

$0.38 \mathrm{~cm}^{3}$. The shallow dose and deep doses are determined at $0.0027 \mathrm{~cm}(7$ $\left.\mathrm{mg} / \mathrm{cm}^{2}\right)$ and $0.38 \mathrm{~cm}\left(1000 \mathrm{mg} / \mathrm{cm}^{2}\right)$, respectively. LiF is semi-opaque with the absorption coefficient of $40 \mathrm{~cm}^{-1}$ for $10.6 \mu \mathrm{m}$ IR laser photons.

2. Schematic diagram of the logistics of the computational study.

3. A schematic diagram of unfocused and focused laser heating of a TLD sample; (a) In unfocused laser heating of a TL dosimeter of thickness $\mathrm{L}$, the laser waist, or $2 \omega_{0}$, is positioned at the surface of the TL dosimeter; (b) In focused laser heating of a TL dosimeter of thickness $L$, the focused waist, or $2 \omega_{\mathrm{f}}$, is positioned at the surface of the TL dosimeter.

4. Schematic diagram of subroutines (user codes) of EGS4 code.

5. Cross-sectional views of three different laser beams: (a) Unfocused Gaussian beam (0.170 cm diameter); (b) Focused Gaussian beam $(0.032 \mathrm{~cm}$ diameter); and (c) Uniform square beam ( $0.3 \times 0.3 \mathrm{~cm}^{2}$ in size), which is exactly the same as the dosimeter cross-section. Focused and unfocused beam originate from $4 \mathrm{~W}$ continuous wave (CW) laser beam. Uniform beam originates from $10 \mathrm{~W}$ continuous wave $(\mathrm{CW})$ laser beam by beam shaping optics

6. A schematic diagram of uniform laser beam heating of a sample; This uses the IST (International Sensor Technology, Inc.) TLD reader [Braunlich, 1990] which features an RF-excited $\mathrm{CO}_{2}$ waveguide laser capable of delivering a uniform beam of around $0.3 \times 0.3 \mathrm{~cm}^{2}$ spot size (adjustable) to discrete a TL sample of thickness $L$.

7. Thermoluminescence TL glow curves from LiF (TLD-100) analyzed by McKeever [McKeever, 1980]. These are the numerical fitting parameters obtained by experimental fits of LiF glow peaks (peaks 2-5), notably the trap depths of $1.13,1.23,1.54$, and $2.17 \mathrm{eV}$ and frequency factors of $6.1 \times 10^{13}$, $4.0 \times 10^{13}, 7.3 \times 10^{15}$, and $4.0 \times 10^{21} / \mathrm{sec}$.

8. Schematic diagram of nodal points and meshes for TL glow curve calculation. TL intensity was generated at every nodal point (closed circles), then integrated with the surrounding cylindrical volume (annular ring) elements; For nodes inside and at the center: volume element, $\Delta V=\pi(\Delta \mathrm{r} / 2)^{2} \Delta \mathrm{z}$ and $\Delta V=\pi / 2(\Delta r / 2)^{2}(\Delta z / 2)$, respectively; For nodes at edge: $\Delta V=2 \pi r(\Delta r / 2)^{2} \Delta z$ and For other nodes: $\Delta \mathrm{V}=2 \pi \mathrm{r} \Delta \mathrm{r} \Delta \mathrm{z}$. 
9. Schematic representation of computer code structure for heat conduction and TL glow curve calculation for laser heating system.

10. A schematic diagram of geometry of focused laser heating of a TLD sample; (a) Long focal length, and (b) Short focal length. These view the dependence of beam diameter varying with the depth within the thick TL dosimeter. The focused beam with a typical focal length $10 \mathrm{~cm}$ lens was used in this study.

11. Calculation method of depth-dose distribution within TL dosimeter. The depth-doses were calculated as total energy deposited in a bin, $\mathrm{dz}$ at $\mathrm{z}$ and distribution for all 380 bins.

12. Beta-ray spectra from $\mathrm{Pm}-147\left(\mathrm{E}_{\max }=0.224 \mathrm{MeV}\right)$ source [Cross, 1983]. The beta particles from this source were used as input data to EGS4 [Nelson, 1985] Monte Carlo simulation. The continuous slowing down approximation (CSDA) range corresponding to the maximum beta energy within LiF TL dosimeter is $0.025 \mathrm{~cm}$ [NBSIR 82-2550, 1982].

13. Beta-ray spectra from $\mathrm{Tl}-204\left(\mathrm{E}_{\max }=0.766 \mathrm{MeV}\right)$ source [Cross, 1983]. The beta particles from this source were used as input data to EGS4 [Nelson, 1985] Monte Carlo simulation. The continuous slowing down approximation (CSDA) range corresponding to the maximum beta energy within LiF TL dosimeter is $0.147 \mathrm{~cm}$ [NBSIR 82-2550, 1982].

14. Beta-ray spectra from $\mathrm{Sr} / \mathrm{Y}-90\left(\mathrm{E}_{\max }=2.28 \mathrm{MeV}\right)$ source [Cross, 1983]. The beta particles from this source were used as input data to EGS4 [Nelson, 1985] Monte Carlo simulation. The continuous slowing down approximation (CSDA) range corresponding to the maximum beta energy within LiF TL dosimeter is $0.53 \mathrm{~cm}$ [NBSIR 82-2550, 1982].

15. Optimization procedure of pulsed heating scheme for pure and mixed radiation fields. The optimization was performed to determine a unique top-and-bottom pulsed heating scheme by doing depth-dose unfolding and comparison with actual dose for 23 DOELAP radiation fields.

16. Descriptions of the data points averaged to determine the shallow and deep doses following the focused laser heating: (a) data points at the shallow dose region (above) and (b) data points at deep dose region.

17. Descriptions of the data points averaged to determine the shallow and deep doses following the uniform laser heating: (a) data points in the box at the shallow dose region (above) and (b) data points in the box at deep dose region.

18. Temperature distribution in radial and axial directions in a $0.09 \mathrm{~cm}$ thick $\mathrm{LiF}$ TLD, predicted using an analytical solution, following heating with a $0.084 \mathrm{~cm}$ diameter focused $4.93 \mathrm{~W}$ continuous wave (CW) laser beam for $100 \mathrm{msec}$. Axial and radial distances were normalized to maximum dimensions of $\mathrm{L}=0.09 \mathrm{~cm}$ and $\mathrm{R}=0.15 \mathrm{~cm}$, respectively. 
19. Temperature distribution in radial and axial directions in a $0.09 \mathrm{~cm}$ thick $\mathrm{LiF}$ TLD, predicted using an analytical solution, following heating with a $0.084 \mathrm{~cm}$ diameter focused $4.93 \mathrm{~W}$ continuous wave (CW) laser beam for $200 \mathrm{msec}$. Axial and radial distances were normalized to maximum dimensions of $\mathrm{L}=0.09 \mathrm{~cm}$ and $\mathrm{R}=0.15 \mathrm{~cm}$, respectively.

20. Temperature distribution in radial and axial directions in a $0.09 \mathrm{~cm}$ thick $\mathrm{LiF}$ TLD, predicted using an analytical solution, following heating with a $0.084 \mathrm{~cm}$ diameter focused $4.93 \mathrm{~W}$ continuous wave (CW) laser beam for $300 \mathrm{msec}$. Axial and radial distances were normalized to maximum dimensions of $\mathrm{L}=0.09 \mathrm{~cm}$ and $\mathrm{R}=0.15 \mathrm{~cm}$, respectively. The dotted lines superimposed are the simulated data published by Abtahi [Abtahi et. al, 1986].

21. Temperature distribution in radial and axial directions in a $0.09 \mathrm{~cm}$ thick $\mathrm{LiF}$ TLD, predicted using an numerical solution, following heating with a $0.084 \mathrm{~cm}$ diameter focused $4.93 \mathrm{~W}$ continuous wave (CW) laser beam for $300 \mathrm{msec}$. Axial and radial distances were normalized to maximum dimensions of $\mathrm{L}=0.09 \mathrm{~cm}$ and $\mathrm{R}=0.15 \mathrm{~cm}$, respectively. The dotted lines superimposed are the simulated data published by Abtahi [Abtahi et. al, 1986].

22. Temperature profiles in axial direction in a $0.038 \mathrm{~cm}$ thick LiF TLD, predicted using analytical solution (solid line) and numerical solution (dotted line), following heating with a $0.3 \times 0.3 \mathrm{~cm}$ square $10 \mathrm{~W}$ uniform laser beam for $0.5 \mathrm{sec}$ and $0.8 \mathrm{sec}$, respectively.

23. Temperature profiles in axial direction in a $0.09 \mathrm{~cm}$ thick LiF TLD, predicted using analytical solution (solid line) and numerical solution (dotted line), following heating with a $0.3 \times 0.3 \mathrm{~cm}$ square $10 \mathrm{~W}$ uniform laser beam for $0.5 \mathrm{sec}$ and $0.8 \mathrm{sec}$, respectively.

24. Temperature profiles in a thick laser TL dosimeter after $0.5 \mathrm{sec}$ of heating by a $0.170 \mathrm{~cm}, 4 \mathrm{~W}$ unfocused laser.

25. Temperature profiles of a $0.09 \mathrm{~cm}$ thick laser $\mathrm{TL}$ dosimeter after $0.5 \mathrm{sec}$ of heating by a $0.170 \mathrm{~cm}, 4 \mathrm{~W}$ unfocused laser.

26. Variations of heating depths corresponding to the peak temperatures of peaks 2-5 with various unfocused beam heating durations. These simulated results were obtained for a thick laser TL dosimeter heated with $0.170 \mathrm{~cm}$ diameter $4 \mathrm{~W}$ unfocused laser beam.

27. Radial temperature profiles at the surface of a thick laser TL dosimeter. The TLD is heated with a $0.170 \mathrm{~cm}$ diameter, $4 \mathrm{~W}$ unfocused laser beam for $0.4 \mathrm{sec}, 1.0 \mathrm{sec}, 2.0 \mathrm{sec}$, and $3.0 \mathrm{sec}$.

28. Axial temperature profiles at the center line of a thick laser TL dosimeter. The TLD is heated with a $0.170 \mathrm{~cm}$ diameter, $4 \mathrm{~W}$ unfocused laser beam for $0.4 \mathrm{sec}, 1.0 \mathrm{sec}, 2.0 \mathrm{sec}$, and $3.0 \mathrm{sec}$.

29. Axial temperature gradients at the center of a thick laser TL dosimeter with various unfocused laser heating times. The data were obtained for a $0.170 \mathrm{~cm}$ diameter, $4 \mathrm{~W}$ unfocused Gaussian laser beam. 
30. Radial temperature gradients at the surface of a thick laser TL dosimeter with various unfocused laser heating times. The data were obtained for a $0.170 \mathrm{~cm}$ diameter, $4 \mathrm{~W}$ unfocused Gaussian laser beam.

31. Calculated heating rate at center of the surface of a thick laser TL dosimeter by unfocused laser beam heating with a $0.170 \mathrm{~cm}$ diameter, $4 \mathrm{~W}$ unfocused laser beam.

32. Two-dimensional temperature distributions in a thick laser TL dosimeter following heating with a $0.032 \mathrm{~cm}$ diameter focused $4 \mathrm{~W}$ continuous wave (CW) laser beam for $18 \mathrm{msec}$.

33. Temperature profiles in a $0.09 \mathrm{~cm}$ thick LiF TL dosimeter after $18 \mathrm{msec}$ of heating by a $0.032 \mathrm{~cm}, 4 \mathrm{~W}$ focused laser.

34. Variations of heating depths corresponding to the peak temperatures of peaks $2-5(383 \mathrm{~K}, 421 \mathrm{~K}, 457 \mathrm{~K}$, and $483 \mathrm{~K})$ with various focused beam heating duration. These results were obtained for a $0.09 \mathrm{~cm}$ TL dosimeter heated with a $0.032 \mathrm{~cm}$ diameter, $4 \mathrm{~W}$ focused laser beam.

35. Variations of heating depths corresponding to the peak temperatures of peaks $2-5(383 \mathrm{~K}, 421 \mathrm{~K}, 457 \mathrm{~K}$, and $483 \mathrm{~K})$ with various focused beam heating duration. These results were obtained for a thick laser TL dosimeter heated with a $0.032 \mathrm{~cm}$ diameter, $4 \mathrm{~W}$ focused laser beam.

36. Surface temperatures as a function of heat duration for a $0.09 \mathrm{~cm}$ thick TLD and a thick laser TL dosimeter. These results were obtained with a 0.032 cm diameter, $4 \mathrm{~W}$ focused Gaussian laser beam.

37. Axial temperature profiles at the center line of a thick laser TL dosimeter heated with various focused laser beam heating durations: $5 \mathrm{~ms}, 10 \mathrm{~ms}, 15 \mathrm{~ms}$, and $18 \mathrm{~ms}$. The focused beam is $0.032 \mathrm{~cm}$ in diameter and $4 \mathrm{~W}$ in power.

38. Radial temperature profiles at the surface of a thick laser TL dosimeter arising from various focused laser beam durations: $5 \mathrm{~ms}, 10 \mathrm{~ms}, 15 \mathrm{~ms}$, and $18 \mathrm{~ms}$. The focused beam is $0.032 \mathrm{~cm}$ in diameter and $4 \mathrm{~W}$ in power.

39. Axial temperature profiles at the center line of a thick laser TL dosimeter with various focused laser heating durations. The focused beam is $0.032 \mathrm{~cm}$ in diameter and $4 \mathrm{~W}$ in power.

40. Radial temperature profiles at the surface of a thick laser TL dosimeter with various focused laser heating durations. The focused beam is $0.032 \mathrm{~cm}$ in diameter and $4 \mathrm{~W}$ in power.

41. Calculated heating rates at the center of surface of a thick laser TL dosimeter. The results were obtained for a focused laser beam heating with a $0.032 \mathrm{~cm}$ diameter and $4 \mathrm{~W}$ in power.

42. The variations of focused beam diameter as a function of depth in a thick TL dosimeter. These results were obtained with various focal length, $f$ for $4 \mathrm{~W}$ focused laser beam heating. 
43. Relative differences of temperatures calculated with $\omega_{0}$ and $\omega(z)$, respectively, at the center $(r=0)$ of a thick laser TL dosimeter with various heating durations. These results were obtained for $0.032 \mathrm{~cm}$ diameter $4 \mathrm{~W}$ focused laser beam and heating durations of $10 \mathrm{~ms}, 15 \mathrm{~ms}$, and $18 \mathrm{~ms}$.

44. Relative differences of temperatures calculated with $\omega_{0}$ and $\omega(z)$, respectively, at the surface $(z=0)$ of a thick laser TL dosimeter with various heating durations. These results were obtained for $0.032 \mathrm{~cm}$ diameter $4 \mathrm{~W}$ focused laser beam and heating durations for $10 \mathrm{~ms}, 15 \mathrm{~ms}$, and $18 \mathrm{~ms}$.

45. Temperature distributions in axial direction of a thick LiF TLD, following heating with a $0.3 \mathrm{~cm} \times 0.3 \mathrm{~cm} 10 \mathrm{~W}$ uniform laser beam for $0.5 \mathrm{sec}$ and $0.8 \mathrm{sec}$, respectively. The results were obtained using the numerical solution assuming that no heat transfer is allowed on the TLD boundaries.

46. Temperature profiles in $0.09 \mathrm{~cm}$ thick TL dosimeter using an average thermal conductivity $\mathrm{k}_{\mathrm{o}}$ (solid line) and a temperature-dependent thermal conductivity $\mathrm{k}(\mathrm{T})$ (dashed line) for a $0.3 \mathrm{~cm} \times 0.3 \mathrm{~cm} 10 \mathrm{~W}$ uniform laser beam.

47. Temperature profiles at $0.8 \mathrm{sec}$ in a thick laser TL dosimeter using an average thermal conductivity, $\mathrm{k}_{\mathrm{o}}$ (solid line) and a temperature-dependent thermal conductivity, $\mathrm{k}(\mathrm{T})$ (dashed line) for a $0.3 \mathrm{~cm} \mathrm{x} 0.3 \mathrm{~cm} 10 \mathrm{~W}$ uniform laser beam.

48. Variations of TL dosimeter depths corresponding to peak 5 temperature $(483 \mathrm{~K})$ as a function of heating duration. These results were obtained for a $0.09 \mathrm{~cm}$ thick TLD and a thick laser TLD heated with $0.3 \mathrm{~cm} \times 0.3 \mathrm{~cm}$, $10 \mathrm{~W}$ uniform beam.

49. Surface temperature as a function of heating duration for a $0.09 \mathrm{~cm}$ thick TLD and a thick laser TL dosimeters. The results were obtained for a $0.3 \mathrm{~cm} \times 0.3 \mathrm{~cm} 4 \mathrm{~W}$ uniform laser beam.

50. Surface temperature as a function of heating duration for a $0.09 \mathrm{~cm}$ thick TLD and a thick laser TL dosimeters. The results were obtained for a $0.3 \mathrm{~cm} \times 0.3 \mathrm{~cm} 10 \mathrm{~W}$ uniform laser beam.

51. Calculated heating rates at the surface of a thick laser TL dosimeter by $0.3 \times 0.3 \mathrm{~cm}$ square laser beam with various powers:(a) $2 \mathrm{~W}$; (b) $3 \mathrm{~W}$; (c) $4 \mathrm{~W}$; (d) $10 \mathrm{~W}$.

52. Glow curves for a $0.09 \mathrm{~cm}$ thick LiF TLD, predicted both by analytical solution (solid line) and by numerical solution (dotted line). The TLD was heated with a $0.084 \mathrm{~cm}$ diameter focused $4.93 \mathrm{~W}$ continuous wave (CW) laser beam. The published results [Abtahi et al., 1986] is also included (in dots) for comparison.

53. Glow curves calculated using a numerical solution (dotted line) for a $0.09 \mathrm{~cm}$ thick LiF TLD, following heating with a $0.084 \mathrm{~cm}$ diameter focused $4.93 \mathrm{~W}$ continuous wave (CW) laser beam. The experimental result (dots) published by Abtahi [Abtahi et al., 1986] is also shown for comparison. 
54. Theoretical TL glow curves obtained from a $7 \mathrm{mg} / \mathrm{cm}^{2}(0.0027 \mathrm{~cm}) \mathrm{LiF}$ (TLD100) with a focused Gaussian laser beam of fixed $0.059 \mathrm{~cm}$ diameter and various laser powers.

55. Experimental TL glow curves for a $0.0027 \mathrm{~cm}\left(7 \mathrm{mg} / \mathrm{cm}^{2}\right)$ LiF TLD (TLD-100) with a focused Gaussian laser beam of fixed $0.059 \mathrm{~cm}$ diameter and various laser powers [Braunlich, 1990].

56. Theoretical and experimental glow curves for a $0.038 \mathrm{~cm}$ thick LiF TLD following heating with a $0.170 \mathrm{~cm}$ diameter unfocused $4 \mathrm{~W}$ continuous wave (CW) laser beam; (a) glow curve calculated with frequency factors reduced by two orders of magnitude and (b) the experimental glow curve published by Grupen [Grupen, 1989]. Both curves were normalized to the peak values.

57. TL glow curves obtained from a $0.038 \mathrm{~cm}$ thick LiF (TLD-100) Harshaw chip with a uniform laser beam heating; (a) Experimental glow curve (dotted line); (b) Theoretical glow curve (solid line). These data were obtained for $0.3 \mathrm{~cm} \times 0.3 \mathrm{~cm}, 10 \mathrm{~W}$ uniform laser beam. Both curves were normalized to their major peak values.

58. Glow curve for a thick laser TL dosimeter, predicted using numerical solution with assumed uniform dose, following heating with a $0.170 \mathrm{~cm}$ diameter, $4 \mathrm{~W}$ unfocused laser beam for 1.2 sec.

59. Variations of glow curve response for focused laser beam heating for time mesh sizes of (a) $1.0 \times 10^{-5} \mathrm{sec}$ (Solid line), (b) $1.0 \times 10^{-4} \mathrm{sec}$ (Dotted line), and (c) $1.0 \times 10^{-3} \mathrm{sec}$ (Dashed line). Radial mesh size of $7.5 \times 10^{-4} \mathrm{~cm}(\mathrm{R} / 200)$ and axial mesh size of $9.0 \times 10^{-4} \mathrm{~cm}(\mathrm{~L} / 422)$ were used. Calculation were performed for a $0.032 \mathrm{~cm}, 4 \mathrm{~W}$ focused Gaussian laser beam.

60. Variations of glow curve response for focused laser beam heating for time mesh sizes of (a) R/200; L/422 (Solid line), (b) R/200; L/211 (Dotted line), and (c) $\mathrm{R} / 100 ; \mathrm{L} / 106$ (Dashed line). Time mesh size of $1.0 \times 10^{-4} \mathrm{sec}$ was used. Calculation were performed for a $0.032 \mathrm{~cm}, 4 \mathrm{~W}$ focused Gaussian laser beam.

61. Theoretical glow curves of a thick laser TL dosimeter exposed to various beta sources. The depth-dose profile used for glow curve calculation were obtained from EGS4 Monte Carlo calculations with 50,000 electron histories for each beta source. TL light intensity were calculated for the TLD heated with a $0.032 \mathrm{~cm}$ diameter, $4 \mathrm{~W}$ focused Gaussian laser beam.

62. Glow curves based on a numerical solution for a $0.038 \mathrm{~cm}$ thick and a $0.09 \mathrm{~cm}$ thick LiF TL dosimeters, following heating with $0.3 \mathrm{~cm} \times 0.3 \mathrm{~cm}$ $10 \mathrm{~W}$ uniform laser beam for $0.8 \mathrm{sec}$.

63. Variations of glow curves of a $0.09 \mathrm{~cm}$ thick TL dosimeter using temperaturedependent thermal conductivity, $\mathrm{k}(\mathrm{T})$ for a $0.3 \mathrm{~cm}$ x $0.3 \mathrm{~cm} 10 \mathrm{~W}$ uniform laser beam. 
64. Variations of glow curves for uniform laser beam heating for time mesh sizes: $1.0 \times 10^{-3}$ (dashed line), $1.0 \times 10^{-4}$ (dotted line), and $1.0 \times 10^{-5}$ dashed line). Calculations were performed for a $0.3 \times 0.3 \mathrm{~cm} 10 \mathrm{~W}$ uniform laser beam, and the axial mesh size used was $1.8 \times 10^{-3} \mathrm{~cm}$.

65. Variations of glow curves for uniform laser beam heating with axial mesh sizes: $7.2 \times 10^{-3} \mathrm{~cm}$ (dotted line), $3.6 \times 10^{-3} \mathrm{~cm}$ (broken line), and $1.8 \times 10^{-3}$ (dashed line). Time mesh size of $1.0 \times 10^{-4} \mathrm{sec}$ was used. Calculations were performed for a $0.3 \mathrm{~cm} \mathrm{x} 0.3 \mathrm{~cm} 10 \mathrm{~W}$ uniform laser beam, and the time mesh size used was $1.0 \times 10^{-4} \mathrm{sec}$.

66. Variations of glows of a thick laser TL dosimeter using a temperaturedependent thermal conductivity, $\mathrm{k}(\mathrm{T})$ for $\mathrm{a} 0.3 \mathrm{~cm} \times 0.3 \mathrm{~cm} 10 \mathrm{~W}$ uniform laser heating.

67. Numerically calculated TL light gradients as function of dosimeter depth for various TL dosimeters following heating with a $0.3 \mathrm{~cm} \times 0.3 \mathrm{~cm} 10$ W laser beam for $0.8 \mathrm{sec}$ ( (a) $0.038 \mathrm{~cm}$ TLD (dashed line); (b) $0.09 \mathrm{~cm}$ TLD (solid line); (c) $0.38 \mathrm{~cm}$ TLD (dotted line).

68. Calculated depth-dose curves of a thick laser TL dosimeter with Pm-147 source. These data were obtained from 50,000 history particle transport simulation by the EGS4 Monte Carlo code.

69. Calculated depth-dose curves of a thick laser TL dosimeter with T1-204 source. These data were obtained from 50,000 history particle transport simulation by the EGS4 Monte Carlo code.

70. Calculated depth-dose curves of a thick laser TL dosimeter with $\mathrm{Sr} / \mathrm{Y}-90$ source. These data were obtained from 50,000 history particle transport simulation by the EGS4 Monte Carlo code.

71. Calculated depth-dose curves of a thick laser TL dosimeter with $20 \mathrm{keV}$ NBS filtered X-ray source. These data were obtained from 50,000 history particle transport simulation by the EGS4 Monte Carlo code.

72. Calculated depth-dose curves of a thick laser TL dosimeter with Cs-137 $(\mathrm{E}=662 \mathrm{keV})$ gamma-ray source. These data were obtained from 50,000 history particle transport simulation by the EGS4 Monte Carlo code.

73. Surface temperature of a thick laser TL dosimeter as a function of time for the following heating scheme: $10 \mathrm{~ms}$ heating (first pulse), $40 \mathrm{~ms}$ cooling, and $15 \mathrm{~ms}$ heating (second pulse). The data were obtained for a $0.032 \mathrm{~cm}$ diameter, $4 \mathrm{~W}$ focused Gaussian laser beam.

74. Surface temperature of a thick laser TL dosimeter as a function of time for the following heating scheme: $10 \mathrm{~ms}$ heating (first pulse), $40 \mathrm{~ms}$ cooling, and $20 \mathrm{~ms}$ heating (second pulse). The data were obtained for a $0.032 \mathrm{~cm}$ diameter, $4 \mathrm{~W}$ focused Gaussian laser beam. 
75. TL light vs. depth of a thick laser TL dosimeter heated with the following pulsed heating scheme: $10 \mathrm{msec}$ of first pulse, and $40 \mathrm{msec}$ of cooling, and then followed by $15 \mathrm{msec}$ of second pulse. The data were obtained for $0.032 \mathrm{~cm}$ diameter, $4 \mathrm{~W}$ focused Gaussian laser beam.

76. TL light vs. depth of a thick laser TL dosimeter heated with the following pulsed heating scheme: $10 \mathrm{msec}$ of first pulse, and $40 \mathrm{msec}$ of cooling, and then followed by $20 \mathrm{msec}$ of second pulse. The data were obtained for $0.032 \mathrm{~cm}$ diameter, $4 \mathrm{~W}$ focused Gaussian laser beam.

77. Temperature vs. depth of a thick laser TL dosimeter for various combinations of laser powers and heating durations: $10 \mathrm{~W}, 0.3 \mathrm{sec}$; $4 \mathrm{~W}, 1.2 \mathrm{sec} ; 3 \mathrm{~W}, 2.0 \mathrm{sec} ; 2 \mathrm{~W}, 4.0$ sec.

78. Variations of temperature-depth distributions of a thick laser TL dosimeter during various cooling periods (a time duration after laser is turned off): $0.424 \mathrm{sec}, 0.848 \mathrm{sec}, 2.12 \mathrm{sec}, 8.48 \mathrm{sec}$, and $12.72 \mathrm{sec}$. The data were obtained for a $0.3 \times 0.3 \mathrm{~cm}$ square $10 \mathrm{~W}$ uniform laser beam.

79. Pulsed heating schemes selected for use in this study. (a) Single pulse of $0.6 \mathrm{sec}$ duration; (b) two $0.6 \mathrm{sec}$ pulses separated with $0.6 \mathrm{sec}$ of cooling, and (c) three $0.6 \mathrm{sec}$ pulses with $0.6 \mathrm{sec}$ of cooling between pulses. All three schemes employ the same laser power of $10 \mathrm{~W}$.

80. (a) Temperature profiles of $10 \mathrm{~W} 0.6 \mathrm{sec}$ heating and $0.6 \mathrm{sec}$ cooling and (b) the corresponding TL response of a thick laser TL dosimeter for $0.3 \mathrm{~cm} \times 0.3 \mathrm{~cm}$ uniform laser beam.

81. (a) Calculated temperature profiles for a thick laser TL dosimeter following two $10 \mathrm{~W} 0.6 \mathrm{sec}$ pulses separated with $0.6 \mathrm{sec}$ of cooling. (b) The corresponding TL response. The pulses heating were based on a $0.3 \mathrm{~cm} \times 0.3 \mathrm{~cm}$ uniform laser beam

82. (a) Calculated temperature profiles for a thick laser TL dosimeter following three $10 \mathrm{~W}, 0.6 \mathrm{sec}$ pulses with $0.6 \mathrm{sec}$ of cooling between pulses. (b) The corresponding TL responses. The pulsed heating was based on a $0.3 \mathrm{~cm} \times 0.3 \mathrm{~cm}$ uniform laser beam.

83. The calculated TL light intensity vs. depth for a thick laser TL dosimeter following three $10 \mathrm{~W}, 0.6 \mathrm{sec}$ pulses with $0.6 \mathrm{sec}$ of cooling between pulses. Three curves correspond to the TL light generated in the three durations: (a) beginning of the first pulse to $0.6 \mathrm{sec}$ of cooling after the first pulse, (b) beginning of the second pulse to $0.6 \mathrm{sec}$ of cooling after the second pulse, and (c) beginning of the third pulse to $0.6 \mathrm{sec}$ of cooling after the third pulse. The pulsed heating was based on a $0.3 \mathrm{~cm} \times 0.3 \mathrm{~cm}$ uniform laser beam.

84. The heating schemes based on three consecutive pulses with varied powers, duration, and cooling time between pulses: (a) $10 \mathrm{~W}-10 \mathrm{~W}-4 \mathrm{~W}$ heating scheme: first pulse (10 W $0.7 \mathrm{sec}$ ) and cooling $(1.4 \mathrm{sec})$; (b) 10W-4W-2W heating scheme: first pulse $(10 \mathrm{~W} 0.7 \mathrm{sec})$ and cooling (1.2 sec); second pulse (4 W $1.6 \mathrm{sec})$ and cooling $(2.0 \mathrm{sec})$; third pulse $(3.5 \mathrm{sec})$. 
85. The calculated temperature profiles of a thick laser TL dosimeter for two pulsed heating based on three consecutive pulses with various powers, durations and cooling time between pulses: (a) $10 \mathrm{~W}-10 \mathrm{~W}-4 \mathrm{~W}$ heating scheme: $0.7 \mathrm{sec} 10 \mathrm{~W}$ first pulse, followed by $1.4 \mathrm{sec}$ of cooling time, which is then followed by a $1.0 \mathrm{sec} 10 \mathrm{~W}$ second pulse with $3.5 \mathrm{sec}$ cooling, and then another $2.0 \sec 4 \mathrm{~W}$ third pulse; (b) $10 \mathrm{~W}-4 \mathrm{~W}-2 \mathrm{~W}$ heating scheme: $0.7 \mathrm{sec} 10 \mathrm{~W}$ first pulse, followed by $1.2 \mathrm{sec}$ of cooling time, which is then followed by a $1.6 \mathrm{sec} 4 \mathrm{~W}$ second pulse with $2.0 \mathrm{sec}$ of cooling, and then another $3.5 \sec 2 \mathrm{~W}$ third pulse. The three curves (i.e., 1, 2 and 3) of each figure correspond to temperatures at the end of cooling period following each pulse.

86. The calculated TL light intensity vs. TLD depth of three pulsed heating schemes based on three consecutive pulses with various powers, duration and cooling time between powers: (a) 10W-10W-4W heating scheme: $0.7 \mathrm{sec} 10 \mathrm{~W}$ first pulse, followed by $1.4 \mathrm{sec}$ of cooling, which is then followed by a $1.0 \mathrm{sec} 10 \mathrm{~W}$ second pulse with $3.5 \mathrm{sec}$ cooling, and then another 2.0 sec $4 \mathrm{~W}$ third pulse; (b) $10 \mathrm{~W}-4 \mathrm{~W}-2 \mathrm{~W}$ heating scheme: $0.7 \mathrm{sec}$ $10 \mathrm{~W}$ first pulse, followed by $1.2 \mathrm{sec}$ of cooling time, which is then followed by a $1.6 \mathrm{sec} 4 \mathrm{~W}$ second pulse with $2.0 \mathrm{sec}$ of cooling, and then another $3.5 \sec 2 \mathrm{~W}$ third pulse. The three curves (i.e., 1,2 and 3 ) of each figure correspond to temperatures at the end of cooling period following each pulse.

87. Calculated glow curves produced by pulsed heating using a $0.032 \mathrm{~cm}$ diameter $4 \mathrm{~W}$ focused laser beam for a thick laser TL dosimeter exposed to a broad perpendicular beam of Sr/Y-90 beta-particles. The pulsed heating scheme includes a $10 \mathrm{msec}$ first pulse followed with a $40 \mathrm{msec}$ of cooling, and then a $15 \mathrm{msec}$ second pulse.

88. Unfolded depth-dose for a thick laser TL dosimeter exposed to $\mathrm{Sr} / \mathrm{Y}-90$ beta-particles. The pulsed heating scheme includes a $10 \mathrm{msec}$ first pulse followed with a $40 \mathrm{msec}$ of cooling, and then a $15 \mathrm{msec}$ second pulse.

89. Calculated glow curves produced by pulsed heating using a $0.032 \mathrm{~cm}$ diameter $4 \mathrm{~W}$ focused laser beam for a thick laser TL dosimeter exposed to a broad perpendicular beam of Sr/Y-90 beta-particles. The pulsed heating scheme includes a $10 \mathrm{msec}$ first pulse followed with a $40 \mathrm{msec}$ of cooling, and then a $20 \mathrm{msec}$ second pulse.

90. Unfolded depth-dose for a thick laser TL dosimeter exposed to St/Y-90 beta-particles. The pulsed heating scheme includes a $10 \mathrm{msec}$ first pulse followed with a $40 \mathrm{msec}$ of cooling, and then a $20 \mathrm{msec}$ second pulse.

91. Unfolded depth-dose for a thick laser TL dosimeter exposed Sr/Y-90 beta-particles following pulsed heating using a $0.032 \mathrm{~cm}$ diameter $4 \mathrm{~W}$ laser beam: first heating ( $20 \mathrm{msec}$ ) and cooling ( $40 \mathrm{msec}$ ), and second heating $(72 \mathrm{msec})$ 
92. Calculated glow curves of a thick laser TL dosimeter exposed to $\mathrm{Sr} / \mathrm{Y}-90$ beta-particles using pulsed heating of uniform laser beam: (a) $10 \mathrm{~W}-10 \mathrm{~W}-4 \mathrm{~W}$ heating approach: first heating (10 W $0.7 \mathrm{sec})$ and cooling $(1.4 \mathrm{sec})$; second heating (10 W $1.0 \mathrm{sec}$ ) and cooling ( $3.5 \mathrm{sec})$; third heating $(2.0 \mathrm{sec})$ (above); (b) $10 \mathrm{~W}-4 \mathrm{~W}-2 \mathrm{~W}$ heating approach: first heating (10 W $0.7 \mathrm{sec})$ and cooling $(1.2 \mathrm{sec})$; second heating ( 4 W $1.6 \mathrm{sec})$ and cooling ( $2.0 \mathrm{sec})$; third heating (3.5 sec)(below).

93. Unfolded depth-dose of a thick laser TL dosimeter exposed to $\mathrm{Sr} / \mathrm{Y}-90$ beta-particles using pulsed heating of uniform laser beam ( 3 pulsed heating on the top); first heating (10 W, $0.7 \mathrm{sec})$ and cooling (1.4 sec); second heating $(10 \mathrm{~W}, 1.0 \mathrm{sec})$ and cooling $(3.5 \mathrm{sec})$; third heating $(2 \mathrm{~W}, 2.0 \mathrm{sec})$.

94. Unfolded depth-dose of a thick laser TL dosimeter exposed by $\mathrm{Sr} / \mathrm{Y}-90$ beta-particles. $0.7 \mathrm{sec} 10 \mathrm{~W}$ first pulse, followed by $1.2 \mathrm{sec}$ of cooling, which is then followed by a $1.6 \mathrm{sec} 4 \mathrm{~W}$ second pulse with $2.0 \mathrm{sec}$ of cooling, and then another $3.5 \sec 2 \mathrm{~W}$ third pulse.

95. The calculated temperature profiles of a thick laser TL dosimeter for pulsed heating of uniform laser beam; $0.6 \mathrm{sec} 10 \mathrm{~W}$ first pulse, followed by $1.3 \mathrm{sec}$ of cooling time, which is then followed by a $1.6 \mathrm{sec} 4 \mathrm{~W}$ second pulse with $2.0 \mathrm{sec}$ of cooling, and then another $3.5 \mathrm{sec} 2 \mathrm{~W}$ third pulse.

96. The calculated TL light intensity vs. TLD depth of three-pulse heating schemes based on three consecutive pulses with various powers, duration and cooling time between powers: $0.6 \mathrm{sec} 10 \mathrm{~W}$ first pulse, followed by $1.3 \mathrm{sec}$ of cooling, which is then followed by a $1.6 \mathrm{sec} 4 \mathrm{~W}$ second pulse with $2.0 \mathrm{sec}$ of cooling, and then another $3.5 \sec 2 \mathrm{~W}$ third pulse.

97. Calculated glow curves produced by pulsed heating using uniform laser beam for a thick laser TL dosimeter exposed to a broad perpendicular beam of Sr/Y-90 beta-particles. The pulsed heating scheme includes a $0.6 \mathrm{sec}$ $10 \mathrm{~W}$ first pulse, followed by $1.3 \mathrm{sec}$ of cooling, which is then followed by a $1.6 \mathrm{sec} 4 \mathrm{~W}$ second pulse with $2.0 \mathrm{sec}$ of cooling, and then another $3.5 \mathrm{sec}$ $2 \mathrm{~W}$ third pulse.

98. Unfolded depth-dose of a thick laser TL dosimeter exposed to Sr/Y-90 beta-particles. The pulsed heating scheme includes a $0.6 \mathrm{sec} 10 \mathrm{~W}$ first pulse, followed by $1.3 \mathrm{sec}$ of cooling, which is then followed by a 1.6 $\sec 4 \mathrm{~W}$ second pulse with $2.0 \mathrm{sec}$ of cooling, and then another $3.5 \mathrm{sec}$ $2 \mathrm{~W}$ third pulse.

99. Unfolded depth-dose for a thick laser TL dosimeter exposed to Cs-137 gamma-rays. The pulsed heating scheme includes a $0.6 \mathrm{sec} 10 \mathrm{~W}$ first pulse, followed by $1.3 \mathrm{sec}$ of cooling, which is then followed by a 1.6 $\sec 4 \mathrm{~W}$ second pulse with $2.0 \mathrm{sec}$ of cooling, and then another $3.5 \mathrm{sec}$ $2 \mathrm{~W}$ third pulse. 
100. Optimum pulsed heating schemes for uniform laser beam on the top and bottom surface of the thick laser TL dosimeter The pulsed heating scheme includes (a) Top heating (above): a $0.2 \mathrm{sec} 10 \mathrm{~W}$ first pulse, followed by $1.7 \mathrm{sec}$ of cooling, which is then followed by a $1.8 \mathrm{sec}$ $4 \mathrm{~W}$ second pulse with $2.0 \mathrm{sec}$ of cooling, and then another $3.5 \mathrm{sec} 2 \mathrm{~W}$ third pulse; (a) Bottom heating (below): a $0.6 \mathrm{sec} 10 \mathrm{~W}$ first pulse, followed by $1.3 \mathrm{sec}$ of cooling, which is then followed by a $1.6 \sec 4 \mathrm{~W}$ second pulse with $2.0 \mathrm{sec}$ of cooling, and then another $3.5 \mathrm{sec} 2 \mathrm{~W}$ third pulse.

101. The calculated temperature profiles of a thick laser TL dosimeter for pulsed heating of uniform laser beam. The pulsed heating scheme includes

(a) Top heating (above): a $0.2 \mathrm{sec} 10 \mathrm{~W}$ first pulse, followed by $1.7 \mathrm{sec}$ of cooling, which is then followed by a $1.8 \sec 4 \mathrm{~W}$ second pulse with $2.0 \mathrm{sec}$ of cooling, and then another $3.5 \mathrm{sec} 2 \mathrm{~W}$ third pulse; (a) Bottom heating (below): a $0.6 \mathrm{sec} 10 \mathrm{~W}$ first pulse, followed by $1.3 \mathrm{sec}$ of cooling, which is then followed by a $1.6 \sec 4 \mathrm{~W}$ second pulse with $2.0 \mathrm{sec}$ of cooling, and then another $3.5 \sec 2 \mathrm{~W}$ third pulse.

102. Calculated glow curves of a thick TL dosimeter exposed by St/Y-90 beta-particles using pulsed heating of uniform laser beam. The pulsed heating scheme includes (a) Top heating (above): a $0.2 \mathrm{sec} 10 \mathrm{~W}$ first pulse, followed by $1.7 \mathrm{sec}$ of cooling, which is then followed by a $1.8 \mathrm{sec}$ $4 \mathrm{~W}$ second pulse with $2.0 \mathrm{sec}$ of cooling, and then another $3.5 \mathrm{sec} 2 \mathrm{~W}$ third pulse; (a) Bottom heating (below): a $0.6 \mathrm{sec} 10 \mathrm{~W}$ first pulse, followed by $1.3 \mathrm{sec}$ of cooling, which is then followed by a $1.6 \sec 4 \mathrm{~W}$ second pulse with $2.0 \mathrm{sec}$ of cooling, and then another $3.5 \mathrm{sec} 2 \mathrm{~W}$ third pulse.

103. Unfolded depth-dose of a thick laser TL dosimeter exposed to $\mathrm{Sr} / \mathrm{Y}-90$ beta-particles using pulsed heating of uniform laser beam. The pulsed heating scheme includes (a) Top heating (above): a $0.2 \mathrm{sec} 10 \mathrm{~W}$ first pulse, followed by $1.7 \mathrm{sec}$ of cooling, which is then followed by a $1.8 \mathrm{sec}$ $4 \mathrm{~W}$ second pulse with $2.0 \mathrm{sec}$ of cooling, and then another $3.5 \mathrm{sec} 2 \mathrm{~W}$ third pulse; (a) Bottom heating (below): a $0.6 \mathrm{sec} 10 \mathrm{~W}$ first pulse, followed by $1.3 \mathrm{sec}$ of cooling, which is then followed by a $1.6 \sec 4 \mathrm{~W}$ second pulse with $2.0 \mathrm{sec}$ of cooling, and then another $3.5 \sec 2 \mathrm{~W}$ third pulse.

104. Unfolded depth-dose for a thick laser TL dosimeter exposed to Sr/Y-90 beta-particles using pulsed heating of uniform laser beam.(3 pulsed heating on the top and 1 pulsed heating on the bottom); (a) Top; first heating ( $10 \mathrm{~W}, 0.2$ sec) and cooling (1.7 sec); second heating ( $4 \mathrm{~W}, 1.8 \mathrm{sec})$ and cooling (2.0 sec); third heating ( $2 \mathrm{~W}, 3.5 \mathrm{sec})$; (b) Bottom; first heating (10 W, $0.6 \mathrm{sec})$.

105. Unfolded depth-dose of a thick laser TL dosimeter exposed to $\mathrm{Sr} / \mathrm{Y}-90$ beta-particles using pulsed heating of uniform laser beam ( 3 pulsed heating on the top and 2 pulsed heating on the bottom); (a) Top; first heating (10 W, $0.2 \mathrm{sec})$ and cooling $(1.7 \mathrm{sec}) ;$ second heating $(4 \mathrm{~W}, 1.8 \mathrm{sec})$ and cooling $(2.0 \mathrm{sec})$; third heating $(2 \mathrm{~W}, 3.5 \mathrm{sec})$; (b) Bottom; first heating $(10 \mathrm{~W}, 0.6 \mathrm{sec})$ and cooling $(1.3 \mathrm{sec})$; second heating $(4 \mathrm{~W}, 1.6 \mathrm{sec})$.

106. Unfolded depth-dose of a thick laser TL dosimeter exposed to $\mathrm{Sr} / \mathrm{Y}-90$ beta-particles using pulsed heating of uniform laser beam ( 2 pulsed heating on the top and 2 pulsed heating on the bottom); (a) Top; first heating ( $10 \mathrm{~W}, 0.2$ sec) and cooling (1.7 sec); second heating ( $4 \mathrm{~W}, 1.8 \mathrm{sec})$; (b) Bottom; first heating ( $10 \mathrm{~W}, 0.6 \mathrm{sec})$ and cooling $(1.3 \mathrm{sec})$; second heating $(4 \mathrm{~W}, 1.6 \mathrm{sec}) .215$ 
107. Unfolded shallow depth-dose for a thick laser TL dosimeter exposed to $\mathrm{Pm}-147$ beta-particles following pulsed heating of $0.032 \mathrm{~cm}$ diameter $4 \mathrm{~W}$ laser beam.

108. Unfolded shallow depth-dose for a thick laser TL dosimeter exposed to Tl-204 beta-particles following pulsed heating of $0.032 \mathrm{~cm}$ diameter $4 \mathrm{~W}$ laser beam.

109. Unfolded shallow depth-dose for a thick laser TL dosimeter exposed to $\mathrm{Sr} / \mathrm{Y}-90$ beta-particles following pulsed heating of $0.032 \mathrm{~cm}$ diameter $4 \mathrm{~W}$ laser beam.

110. Unfolded shallow depth-dose for a thick laser TL dosimeter exposed to $20 \mathrm{keV} x$-rays following pulsed heating of $0.032 \mathrm{~cm}$ diameter $4 \mathrm{~W}$ laser beam.

111. Unfolded shallow depth-dose for a thick laser TL dosimeter exposed to $59 \mathrm{keV} x$-rays following pulsed heating of $0.032 \mathrm{~cm}$ diameter $4 \mathrm{~W}$ laser beam.

112. Unfolded shallow depth-dose for a thick laser TL dosimeter exposed to $120 \mathrm{keV}$ x-rays following pulsed heating of $0.032 \mathrm{~cm}$ diameter $4 \mathrm{~W}$ laser beam.

113. Unfolded shallow depth-dose for a thick laser TL dosimeter exposed to Cs-137 gamma-rays following pulsed heating of $0.032 \mathrm{~cm}$ diameter $4 \mathrm{~W}$ laser beam.

114. Unfolded deep depth-dose for a thick laser TL dosimeter exposed to $\mathrm{Sr} / \mathrm{Y}-90$ beta-particles following pulsed heating of $0.032 \mathrm{~cm}$ diameter $4 \mathrm{~W}$ laser beam.

115. Unfolded deep depth-dose for a thick laser TL dosimeter exposed to 20 $\mathrm{keV} \mathrm{x}$-rays following pulsed heating of $0.032 \mathrm{~cm}$ diameter $4 \mathrm{~W}$ laser beam.

116. Unfolded deep depth-dose for a thick laser TL dosimeter exposed to 59 $\mathrm{keV} \mathrm{x}$-rays following pulsed heating of $0.032 \mathrm{~cm}$ diameter $4 \mathrm{~W}$ laser beam.

117. Unfolded deep depth-dose for a thick laser TL dosimeter exposed to $120 \mathrm{keV} \mathrm{x}$-rays following pulsed heating of $0.032 \mathrm{~cm}$ diameter $4 \mathrm{~W}$ laser beam.

118. Unfolded deep depth-dose for a thick laser TL dosimeter exposed to Cs137 gamma-rays following pulsed heating of $0.032 \mathrm{~cm}$ diameter $4 \mathrm{~W}$ laser beam.

119. Unfolded shallow depth-dose for a thick laser TL dosimeter exposed to $\mathrm{Sr} / \mathrm{Y}-90$ beta-particles following pulsed heating of uniform laser beam (3 pulsed heating on the top and 3 pulsed heating on the bottom).

120. Unfolded shallow depth-dose for a thick laser TL dosimeter exposed to $20 \mathrm{keV}$ x-rays following pulsed heating of uniform laser beam ( 3 pulsed heating on the top and 3 pulsed heating on the bottom). 
121. Unfolded shallow depth-dose for a thick laser TL dosimeter exposed to $59 \mathrm{keV}$ x-rays following pulsed heating of uniform laser beam ( 3 pulsed heating on the top and 3 pulsed heating on the bottom).

122. Unfolded shallow depth-dose for a thick laser TL dosimeter exposed to $120 \mathrm{keV} \mathrm{x}$-rays following pulsed heating of uniform laser beam ( 3 pulsed heating on the top and 3 pulsed heating on the bottom).

123. Unfolded shallow depth-dose for thick laser TL dosimeter exposed to Cs-137 gamma-rays following pulsed heating of uniform laser beam (3 pulsed heating on the top and 3 pulsed heating on the bottom).

124. Unfolded deep depth-dose for a thick laser TL dosimeter exposed to $\mathrm{Sr} / \mathrm{Y}-90$ beta-particles following pulsed heating of uniform laser beam (3 pulsed heating on the top and 3 pulsed heating on the bottom).

125. Unfolded deep depth-dose for a thick laser TL dosimeter exposed to $20 \mathrm{keV}$ x-rays following pulsed heating of uniform laser beam ( 3 pulsed heating on the top and 3 pulsed heating on the bottom).

126. Unfolded deep depth-dose for a thick laser TL dosimeter exposed to $59 \mathrm{keV}$ x-rays following pulsed heating of uniform laser beam ( 3 pulsed heating on the top and 3 pulsed heating on the bottom).

127. Unfolded deep depth-dose for a thick laser TL dosimeter exposed to $120 \mathrm{keV} \mathrm{x}$-rays following pulsed heating of uniform laser beam ( 3 pulsed heating on the top and 3 pulsed heating on the bottom).

128. Unfolded deep depth-dose for thick laser TL dosimeter exposed to Cs-137 gamma-rays following pulsed heating of uniform laser beam ( 3 pulsed heating on the top and 3 pulsed heating on the bottom).

129. (1) Variations of TL glow curve with (a) $\pm 2 \%$ changes of beam density (above) and (2) $\pm 2 \%$ changes of absorption coefficient (below) of a thick laser TL dosimeter exposed to T1-204 beta particles following pulsed heating of focused laser beam.

130. Variations of glow curve with (1) $\pm 2 \%$ changes of thermal conductivity (above) and (2) $\pm 2 \%$ changes of specific heat (below) of a thick laser TL dosimeter exposed to Tl-204 beta particles following pulsed heating of focused laser beam.

131. Variations of TL glow curves with (a) $\pm 2 \%$ changes of beam density (above) and (b) $\pm 2 \%$ changes of absorption coefficient (below) of a thick laser TL dosimeter exposed to Tl-204 beta particles following pulsed heating of focused laser beam.

132. Variations of $T L$ glow curves with (a) $\pm 2 \%$ changes of thermal conductivity (above) and (b) $\pm 2 \%$ changes of specific heat (below) of a thick laser TL dosimeter exposed to Tl-204 beta particles following pulsed heating of focused laser beam. 
133. Variations of the depth-doses with (1) $\pm 2 \%$ changes of beam density (above) and (2) $\pm 2 \%$ changes of absorption coefficient (below) for a thick laser TL dosimeter exposed to Tl-204 beta particles following pulsed heating of focused laser beam.

134. Variations of the depth-doses with (1) $\pm 2 \%$ changes of thermal conductivity (above) and (2) $\pm 2 \%$ changes of specific heat (below) of a thick laser TL dosimeter exposed to Tl-204 beta particles following pulsed heating of focused laser beam.

135. Variations of TL glow curves with $\pm 5 \%$ changes of beam density of a thick laser TL dosimeter exposed to $20 \mathrm{keV}$ x-rays following pulsed heating of uniform laser beam.

136. Variations of glow curve with $\pm 5 \%$ changes of absorption coefficient of a thick laser TL dosimeter exposed to $20 \mathrm{keV}$ x-rays following pulsed heating of uniform laser beam.

137. Variations of glow curve with $\pm 5 \%$ changes of thermal conductivity of a thick laser TL dosimeter exposed to $20 \mathrm{keV}$ x-rays following pulsed heating of uniform laser beam.

138. Variations of glow curve with $\pm 5 \%$ changes of specific heat of a thick laser TL dosimeter exposed to $20 \mathrm{keV}$ x-rays following pulsed heating of uniform laser beam.

139. Variations of TL glow curves with (a) $\pm 2 \%$ changes of beam density (above) and (b) $\pm 2 \%$ changes of absorption coefficient (below) of a thick laser TL dosimeter exposed to $20 \mathrm{keV} x$-rays following pulsed heating of uniform laser beam.

140. Variations of $T L$ glow curves with (a) $\pm 2 \%$ changes of thermal conductivity (above) and (b) $\pm 2 \%$ changes of specific heat (below) of a thick laser TL dosimeter exposed to $20 \mathrm{keV}$ x-rays following pulsed heating of uniform laser beam.

141. Variations of the depth-doses with (1) $\pm 5 \%$ changes of beam density (above) and (2) $\pm 5 \%$ changes of absorption coefficient (below) of a thick laser TL dosimeter exposed to $20 \mathrm{keV}$ x-rays following pulsed heating of uniform laser beam.

142. Variations of the depth-doses with (1) $\pm 5 \%$ changes of thermal conductivity (above) and (2) with $\pm 5 \%$ changes of specific heat (below) of a thick laser TL dosimeter exposed to $20 \mathrm{keV}$ x-rays following pulsed heating of uniform laser beam.

143. Theoretical beta-ray energy responses at the depth of $7 \mathrm{mg} / \mathrm{cm}^{2}$ of various LiF TL dosimeters as a function of mean beta energy. The data were obtained for conventional heating (planchet heating) at heating rate of $4.3 \mathrm{~K} / \mathrm{s}$ during $60 \mathrm{sec}$. The depth-doses from each beta source were calculated by EGS4 Monte Carlo transport code and based on the same dose rates. The symbols $(\mathrm{x})$ superimposed are the experimental data for a $0.09 \mathrm{~cm}$ thick LiF TLD published by Gupta [Gupta, 1983]. 
144. Theoretical beta-ray energy responses of various thick LiF TL dosimeters as a function of maximum beta energy. The data were obtained for conventional heating (planchet heating) on TLDs at heating rate of $4.3 \mathrm{~K} / \mathrm{s}$ during $60 \mathrm{sec}$. The depth-doses from each beta source were calculated by EGS4 Monte Carlo transport code and based on the same dose rates.

145. Theoretical beta-ray energy responses of $\mathrm{LiF} \mathrm{TL}$ dosimeter. Heating times correspond to a given depth of maximum thermoluminescence emission; $6 \mathrm{~ms}$ $(0.0025 \mathrm{~cm}), 8 \mathrm{~ms}(0.01 \mathrm{~cm}), 12 \mathrm{~ms}(0.018 \mathrm{~cm})$, and $16 \mathrm{~ms}(0.024 \mathrm{~cm})$. The data were obtained for $0.032 \mathrm{~cm}$ diameter, $4 \mathrm{~W}$ focused laser beam heating on $0.09 \mathrm{~cm}$ thick LiF TLD. The depth-doses from each beta source were calculated by EGS4 Monte Carlo transport code and based on the same dose rates.

146. Theoretical beta-ray energy responses of LiF TL dosimeter. Heating times correspond to a given depth of maximum thermoluminescence emission; $0.1 \mathrm{~s}$ $(0.0025 \mathrm{~cm}), 0.5 \mathrm{~s}(0.0050 \mathrm{~cm}), 0.6 \mathrm{~s}(0.024 \mathrm{~cm}), 0.7 \mathrm{~s}(0.035 \mathrm{~cm})$, and $0.8 \mathrm{~s}(0.045 \mathrm{~cm})$. The data were obtained for $0.3 \mathrm{~cm} \mathrm{x} 0.3 \mathrm{~cm}, 10 \mathrm{~W}$ uniform laser beam heating on $0.09 \mathrm{~cm}$ thick LiF TLD. The depth-doses from each beta source were calculated by EGS4 Monte Carlo transport code and based on the same dose rates.

147. A semilog scale plot of per cent transmitted intensity LiF absorber thickness $(\mathrm{mm})$ for pure beta emitters. This result was obtained from 50,000 history particle transport simulation by the EGS4 Monte Carlo code (lines). Experimental values superimposed: Pm-147 (open square), T1-204 (closed square), and Sr/Y-90 (open hole)[Paliwal et al., 1976].

148. Conceptual diagram of automatic TL reading system. Laser heating is performed in chambers 1 and 2 . In differential heating mode, laser 1 is used heat top of TLD, collecting TL light by PM tube 1; laser 2 is used to heat bottom of TLD collecting TL light by PM tube2. Focusing lenses 1 and 2 are used to focused the beam while Ge lenses 1 and 2 to prevent the laser beam from passing to the PM tube. 


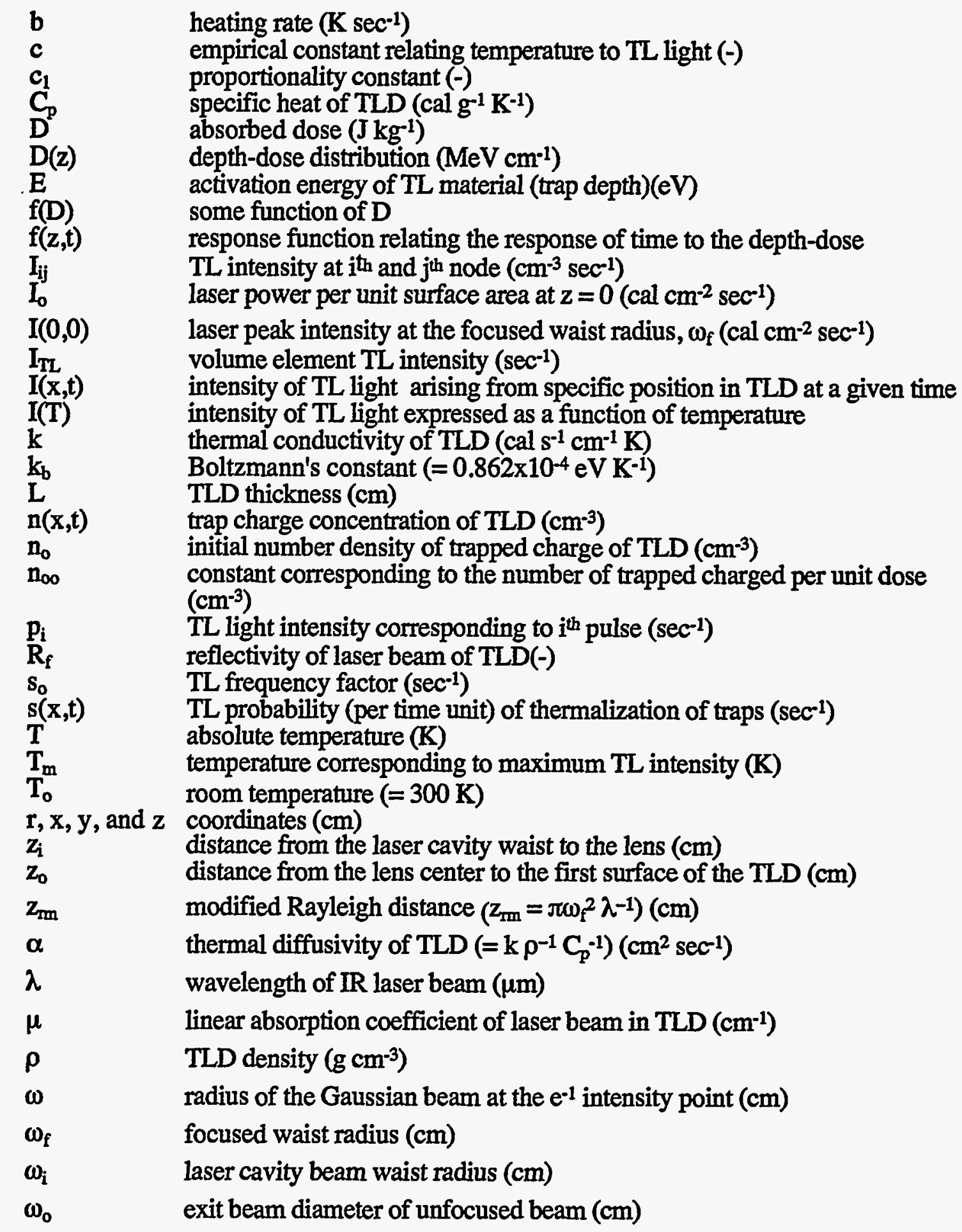




\section{GLOSSARY}

Thick laser TLD: A hypothetical LiF TLD having dimension of $0.3 \mathrm{~cm} \times 0.3 \mathrm{~cm} \times 0.38$ cm.

Focused beam: A Gaussian laser beam with a $0.032 \mathrm{~cm}$ focused diameter and $4 \mathrm{~W}$ power.

Unfocused beam: A Gaussian laser beam with a $0.17 \mathrm{~cm}$ unfocused diameter and $4 \mathrm{~W}$ power.

Uniform beam: A uniform square laser beam with dimensions of $0.3 \mathrm{~cm} \times 0.3 \mathrm{~cm}$ and $10 \mathrm{~W}$ power.

Cooling: A temperature redistribution in the TLD after the laser power is turned off.

Unfolding: A computer technique which converts glow curve data to depth-dose distribution.

Response function: A set of theoretical glow curve data in the form of TL light intensity versus depth of the TLD. The data were calculated by the first-order kinetic model by assumption of uniform dose distribution in the TLD. 


\section{SUMMARY}

The incidents at the Three Mile Island and Chernobyl have triggered the need for better personnel dosimetry methods in mixed radiation fields. In mixed radiation field dosimetry, both shallow and deep doses are important. Thermoluminescent (TL) materials have been used as personnel dosimeters in mixed radiation fields. The conventional methods to extract shallow and deep doses in such mixed fields have been to use multielement TL dosimeters with contact heating schemes (i.e., conduction or convection).

This thesis presents a detailed computational study of a new method for mixed radiation field dosimetry using single-element TL dosimeters with pulsed laser heating schemes. The main objective of this study was to obtain an optimum heating scheme so that the depth-dose distribution in a thick TL dosimeter could be accurately determined. The major parts of the study include: (a) heat conduction calculations for TL dosimeters with various heating schemes, (b) glow curve calculations for TL dosimeters based on a firstorder kinetic model, (c) unfolding of the depth-dose distribution based on the glow curve data, and (d) estimation of shallow and deep doses from the unfolded depth-dose distribution.

Two optimum heating schemes were obtained in this study. The first one was obtained for a focused laser beam, and the second one was obtained for a uniform laser beam. Both heating schemes consist of two processes: top surface heating and bottom surface heating, and each process in turn consists of a sequence of laser pulses with various heating durations and power levels. Compared to the "true" depth-dose distribution obtained using Monte Carlo transport code EGS4, relative errors associated with the shallow and deep doses obtained from the unfolded depth-dose distributions are 5\% and 
Xxviii

$25 \%$, respectively, for the focused laser beam, and $15 \%$ in both doses for the uniform laser beam. 


\section{CHAPTER I}

\section{INTRODUCTION}

\subsection{Motivation}

Mixed beta/gamma dosimetry typically involves determining of the shallow (skin) dose and deep dose for a human body exposed to mixed beta/gamma radiation fields. The International Commission on Radiological Protection (ICRP) [ICRP, 1977] has recommended that an appropriate measurement of skin dose is that integrated between tissue depths of 5 and $10 \mathrm{mg} / \mathrm{cm}^{2}(50-100 \mu \mathrm{m})$ which corresponds to the depth of cells in the basal layer of the body. Recently, the International Commission on Radiological Units and Measurements (ICRU) [ICRU, 1985] prescribed two new operational quantities intended for application to individual monitoring: the Individual Dose Equivalent Penetrating, $H_{p}(d)$, and Individual Dose Equivalent Superficial, $H_{s}(d)$. They are defined as the dose equivalent in soft tissue below a specified point on the body at depths of 10.0 and $0.07 \mathrm{~mm}$, respectively.

The control of skin dose becomes an important matter when high-intensity beta or low-energy gamma- or X-ray environments are present. High-intensity beta radiation fields are frequently encountered in the nuclear industry [NCRP, 1971; CFR, 1992]. Some examples of these beta fields include those that exist in maintenance of steam generators at pressurized water reactors (PWRs), turbine repair at boiling water reactors (BWRs) and cleanup of contaminated experimental areas. The Three Mile Island (TMI) [Hildebrand et al., 1983] and Chernobyl accidents [IAEA, 1986] increased interest in beta dosimetry. As a 
result of high-level beta fields at the TMI nuclear generating station following the 1979 accident, attention was focused on errors and improvements with respect to beta and mixed beta/gamma dosimetry, especially the problems with TLDs. Alexander describes the dosimetric problems following the TMI accident:

..Workers were handling samples of cooling water in which the beta contamination caused overexposure to the hands. These overexposures were calculated afterwards rather than measured because the beta survey instruments and procedures available to the worker apparently were inadequate. Following the first worker entry into the building, dosimeter readouts and algorithm results were anomalous because part of the energetic beta contribution registered on their dosimeter as a deep dose rather than as a skin dose. The higher energy beta contribution to the deep element resulted in too large a subtracted correction from the shallow element readout. Therefore, due to the hitherto unrecognized shortcomings of the dosimeter design and algorithm, deep doses were probably overestimated and shallow doses were probably underestimated [Alexander, 1983].

The problem was more seriously recognized when the Chernobyl nuclear power accident occurred in 1986. The IAEA report on Chernobyl accident, [IAEA 1986] described the personnel exposure to radiation as follows:

.....(Health Effect).. The early effects were limited to the power plant personnel and firemen exposed shortly after the reactor disruption. Of about 300 persons admitted to hospitals, 203 had symptoms of acute radiation syndrome which resulted from whole body gamma radiation doses from about $2 \mathrm{~Gy}$ up to $16 \mathrm{~Gy}$. In addition to being irradiated by penetrating gamma-rays, a number of those irradiated with high doses were exposed to beta emitting radionuclides. In some cases, this beta exposure caused very extensive radiation burns of the skin that were extremely difficult to treat and contributed substantially to the deaths of 29 victims. Technical measures should be taken to prevent the occurrence of extensive skin burns should an accident of a similar nature occur in the future...

Currently, the shallow and deep dose quantities are determined for personnel dosimetry monitoring by using multiple TLD chips of different types with appropriate physical filters [Sherbini et al., 1983; Sherbini et al., 1985; Moscovitch et al., 1988; Devine et al., 1990; Stanford et al., 1990; Rayes et al., 1990]. A computational algorithm is applied to derive the equivalent deep and shallow doses from the individual TLD readings [Sherbini et al., 1983; Sherbini et al., 1985; Moscovitch et al., 1988; Devine et 
al., 1990; Stanford et al., 1990; Rayes et al., 1990]. Although the dosimetric approach often provides reasonable results, uncertainties may be magnified in the current TL dosimeter systems due to the random errors associated with the characteristics of radiation fields.

The conventional method of heating TLDs to extract their signal has involved the use of a thermal source which elevates the temperature of the entire chip. The heating was carried out by bringing the sample into mechanical contact with a heated metal support or by immersing it in a heating gas or fluid. Useful heating rates in this way were limited to little more than about $10 \mathrm{~K} / \mathrm{s}$. In recent years the use of laser beams to heat TLDs has been studied as a direct, rapid and noncontact heating method providing a high heating rate of about $10^{4} \mathrm{~K} / \mathrm{s}$. Laser heating has been shown to be a promising technique to increase the signal-to-noise ratio of TL emission signals because the dark current background signal of the detector reduces proportionally to the reduction in time achieved over slow heating. This thesis studies the use of pulsed laser heating schemes to extract depth-dose information from a thick TLD chip. 


\subsection{Theory of Thermoluminescence}

Thermoluminescence is an atomic electron phenomenon where a material absorbs energy from some form of electromagnetic or ionizing irradiation. The absorbed energy is stored as trapped charge, and part of it is released in the form of light when the material is thermally stimulated. The trapped charge release mechanism can be described by a firstorder kinetic expression (or Randall-Wilkins model) [Randall and Wilkins, 1945] given as

$$
\frac{d n(x, t)}{d t}=-s(x, t) n(x, t) \quad n(x, 0)=n_{0}
$$

where $n(x, t)$ is the trap charge concentration as a function of time $t$ and position $x, s(x, t)$ is the probability (per time unit) of thermalization of traps, and $n_{0}$ is the concentration of initially trapped charges. The term, $s(x, t)$ can be expressed as

$$
s(x, t)=s_{0} \exp \left[-\frac{E}{k_{b} T(x, t)}\right]
$$

where $k_{b}$ is Boltzmann's constant, $T$ is the absolute temperature, $s_{o}$ is the frequency factor, and $E$ is the activation energy (trap depth). Substitution of Eq (1.2) into Eq (1.1) yields

$$
\frac{d n(x, t)}{d t}=-n(x, t) s_{o} \exp \left[-\frac{E}{k_{b} T(x, t)}\right]
$$

The physical meaning of Eq (1.3) is that the $n(x, t)$ trap charges vibrate back and forth in the potential well with frequency $s_{0}$, so that the rate of escape is $n(x, t) \cdot s_{0}$ multiplied by the exponential term. The exponential term is the well known Boltzmann factor expressing the probability that the trap charge will escape each time it approaches the potential barrier. Eq (1.3) can be solved by rearrangement, substitution of the definition for 
the heating rate $b, b=d T(x, t) / d t$ (linear heating rate) and application of initial conditions (at $t=0, T(x, t=0)=T_{0}$ or room temperature and $\left.n(x, 0)=n_{0}\right)$. The result is

$$
n(x, t)=n_{0} \exp \left\{-\left(\frac{S_{0}}{b}\right) \int_{T_{0}}^{T} \exp \left[-\frac{E}{k_{b} T(x, t)}\right] d T\right\}
$$

The intensity, $I(x, t)$ of released trapped charges and recombining of electrons with holes as a function of time can be written as:

$$
I(x, t)=-c\left(\frac{\operatorname{dn}(x, t)}{d t}\right)
$$

where $\mathrm{c}$ is an empirical constant for each solid (less than 1.0 since each detrapping event may not lead to light emission) corresponding to the efficiency by which the release of trap charges is converted into light. Substitution of Eq (1.3) into Eq (1.5), integration of the resulting equation, and application of initial condition yields an expression for the intensity of light produced in terms of the temperature $T$,

$$
I(T)=c n_{0} S_{o} \exp \left[-\frac{E}{k_{b} T}\right] \exp \left\{-\left(\frac{S_{0}}{b}\right) \int_{T_{0}}^{T} \exp \left[-\frac{E}{k_{b} T}\right] d T\right\}
$$

For simplicity the notation substitution $T=T(x, t)$ has been made in the above equation.

Plotting this total intensity versus temperature (or time) yields the glow curve. Typically glow curves represent the signal integrated over the entire TLD, whereas the above equations include potential dependencies. The temperatures of maximum luminescent intensity shift to higher temperatures as $E$ or $b$ increases or as $s_{o}$ decreases. The area under the glow curve is proportional to $\mathrm{n}_{0}$ and hence is proportional to the absorbed dose. In $\mathrm{Eq}$ 
(1.6), if the maximum $\mathrm{TL}$ intensity occurs at temperature $\mathrm{T}_{\mathrm{m}}$, differentiation of $\mathrm{Eq}$ (1.6) with respect to temperature and equating it with zero yields

$$
\frac{T_{m}^{2}}{b}=\frac{E \exp \left[E / k_{b} T_{m}\right]}{k_{b} s_{o}}
$$

Substitution of $\mathrm{Eq}$ (1.7) in Eq (1.6) yields

$$
I\left(T_{m}\right)=\frac{c n_{0} b E}{k_{b} T_{m}^{2}} \exp \left\{-\left(\frac{S_{o}}{b}\right) \int_{T_{0}}^{T_{m}} \exp \left[-\frac{E}{k_{b} T}\right] d T\right\}
$$

Assuming that the radiation dose dependence of $n_{0}$ is given by

$$
\mathrm{n}_{\mathrm{o}}=\mathrm{n}_{00} \mathrm{f}(\mathrm{D})
$$

where $D$ is the dose, $f(D)$ is some function of $D$, and $n_{00}$ is a constant corresponding to the number of trapped charge produced per unit dose. Substituting Eq (1.9) into Eq (1.8) then yields

$$
\frac{I\left(T_{m}, D\right)}{D}=\frac{c n_{0 o} f(D) b E}{D k_{b} T_{m}{ }^{2}} \exp \left\{-\left(\frac{S_{o}}{b}\right) \int_{T_{o}}^{T_{m}} \exp \left[-\frac{E}{k_{b} T}\right] d T\right\}
$$

The quantity, $I\left(T_{m}, D\right) / D$ is linearly proportional to the sensitivity of the detector at dose $D$, and depends on the product of the terms in $\mathrm{Eq}(1.10)$. The right hand side of $\mathrm{Eq}(1.10)$ is proportional to: 


$$
p=\frac{b}{T_{m}^{2}} \exp \left\{-\left(\frac{S_{o}}{b}\right) \int_{T_{0}}^{T_{m}} \exp \left[-\frac{E}{k_{b} T}\right] d T\right\}
$$

Typically, $\mathrm{E}=1 \mathrm{eV}$ and $\mathrm{s}_{\mathrm{o}}$ is $10^{12} \mathrm{~s}^{-1}$. As a result, $\mathrm{p}$ values are $1.198 \times 10^{-5}$ and $3.349 \times 10^{-5}$ $\mathrm{K}^{-1} \mathrm{~s}^{-1}$ respectively for the two heating rates $5 \mathrm{Ks}^{-1}$ and $10 \mathrm{Ks}^{-1}$. This analysis predicts that the peak temperature of the glow curve shifts to a higher value with increasing heating rate approximately according to $\mathrm{Eq}(1.7)$, and it predicts that the glow peak height is dependent on the heating rate according to $\mathrm{Eq}$ (1.11). Note that in $\mathrm{Eq}(1.11), \mathrm{b}=\mathrm{dT} / \mathrm{dt}$ is assumed to be independent on time. Gasiot has shown that the shifts might reach $250 \mathrm{~K}$ for increases of $b$ from several $\mathrm{Ks}^{-1}$ to $10^{4} \mathrm{Ks}^{-1}$ [Gasiot et al., 1982]. This points to one aspect of TLD laser heating that has to be considered carefully in experiments and applications. The glow peak can easily be shifted into a temperature range in which incandescent background radiation is not negligible. Proper selection of filters and photocathodes of the photomultiplier tube may, therefore, be essential in certain cases. However, in the experiment by Gasiot [Gasiot et al., 1982] the problem did not appear to be insurmountable although no special filters were used even when extreme heating rates up to $10^{4} \mathrm{Ks}^{-1}$ were employed. 


\subsection{Recent Developments in TLD}

\subsubsection{Multi-element. Filtered Thermoluminescence Dosimeters}

Practical personnel dosimetry for mixed radiation fields typically utilizes two or more TLD elements. Each has a different filter of thickness to selectively remove portions of the field prior to reaching the detector. The elements are usually incorporated into a personnel badge. This approach has been adopted for film [Landauer; Siemens] and modified to include detectors having different energy sensitivities for TLDs [Panasonic; Harshaw]. Algorithms, or empirical computational approaches, are then devised to resolve the shallow and deep dose components of the field [Gesell et al., 1979; Heizelmann et al., 1982; Scherpelz et al., 1983; Sherbini et al., 1983; Sherbini et al., 1985].

An immediate limitation of the multi-element dosimeter approach arises from difficulties related to the detectors themselves. Dosimetry film is plagued by a high lower limit of detectability [Brodsky, 1963; Becker, 1966; Kathren, 1966; Kearfott et al., 1991 a]. Dosimeter-to-dosimeter variations in TLD response, as indicated by the requirement of individual calibration of each element [McKeever, 1985; Kearfott et al., 1990 a], combined with the history-dependence of TLD response [Kartha, 1971] complicates mixed field approaches. Both film and TLDs are subject to serious angular dependencies, which introduce additional inaccuracies, particularly when these are to be used with filters [Ishiguro at al., 1980; Sherbini et al., 1983]. The multi-element method has the advantage of using existing TLD systems. However, the dose estimate with that method is encumbered with high random errors at low beta energies, and dosimeter design may become prohibitively expensive [Christensen, 1986; Uchrin, 1986; Kathren, 1987].

The difficulties associated with detector response are compounded by the limitations of the algorithms, which are typically discontinuous and are derived for very specific radiation fields. These algorithms tend to produce anomalous results for particular combinations of detector readings. Although this problem has drawn recent attention 
[Stanford et al., 1990], the algorithm remains complex. Furthermore, the overall multielement system has difficulty in discriminating between low energy photons and high energy betas [Kearfott et al., 1991 b]. In conclusion, the multi-element, filtered approach remains, at best, a purely empirical mixed field dosimetry method which is subject to potentially serious errors, especially in certain mixed radiation fields.

\subsection{Thin-element Beta Detectors}

It is desirable to resolve personnel dose into the skin or shallow dose component and the deep dose component, defined as the integrated dose deposited below the skin. Often unknown, quantitative knowledge of the beta energy spectrum is critical for accurate measurement of skin dose using thick TLDs [Driscoll et al., 1984]. Thus, thin-element beta detectors are designed to avoid dependence upon the incident beta spectra.

One unique method of differential beta dosimetry involves the use of a theoretical computation of the dose deposition for some experimental spectrum to derive the TLD sensitivity for a specific field [Shen et al., 1987]. Another approach to this problem, which avoids radiation field distortions caused by the presence of the detector, is to use ultra-thin TLDs or detection layers which do not appreciably attenuate beta radiation [Marshall and Docherty, 1971; Charles, 1977]. Unfortunately these thin TLDs are relatively insensitive, fragile, inconvenient to use, and have substantial detector-to-detector sensitivity variation [Gotlib et al., 1988]. Another method for satisfying conflicting requirements for thin detectors is to use a thick dosimeter made effectively thin by incorporating an opaque material such as carbon. This has the effect of making the detector partially opaque thus facilitating discrimination between luminescence emission from the surface and from deep layers. The experimental results indicate that whereas their relative sensitivity to low energy beta radiation has improved, the absolute sensitivity has diminished, resulting in high detection thresholds [Francis et al., 1986; Francis et al., 1989]. 


\subsubsection{New Heating Techniques}

Surface-heating approaches to mixed field and beta dosimetry have been reported recently. The beta contribution in a mixed beta-gamma field has been isolated using rapid contact heating of low heat conductivity teflon-LiF TLDs [Sahre, 1983; Gotlib et al., 1988]. However, this approach has not received widespread attention, possibly because of the low sensitivity of the TLDs, associated with their low heat conductivity. Possible practical difficulties may also exist related to the reproducibility and speed of planchet heating. This suggests that an alternate, more rapid heat transfer method is needed.

Use of laser beams to heat TLDs has the advantages of rapid, reproducible, positional heating. Numerous studies have discussed The heating and glow curve characteristics obtained when laser heat sources are used for local and positional heating of TLDs for both unfocused [Grupen-Shemansky, 1989] and focused lasers [Braunlich et al., 1984; Abtahi et al., 1985; Moreau et al., 1986; Abtahi et al., 1986 a; Abtahi et al., 1986 b; Kelly et al., 1987; Grupen-Shemansky, 1989; Young et al., 1989; Zangaro et al., 1990]. Several applications and practical systems have used lasers to heat TLDs [Braunlich et al., 1986; Kearfott et al., 1990 b; Kearfott et al., 1990 c]. Abtahi [Abtahi et al., 1986 b; Kelly et al., 1987] recommends heating the TLD with a laser beam of uniform intensity profile for accurate and sensitive personnel and environmental dosimetry. The energy transfer from a uniform square beam to a layered structure (weakly absorbing layer of TLD-100 on a thin substrate) has been studied in detail by Braunlich [Braunlich et al, 1986]. He developed the IST (International Sensor Technology, Incorporated) TLD reader, which features an RF-excited $\mathrm{CO}_{2}$ waveguide capable of delivering an uniform beam of around $0.3 \times 0.3 \mathrm{~cm}^{2}$ spot size (adjustable) to discrete thin dosimeter spots of the same area. TLDs are affixed to substrates of thicknesses between $125 \mu \mathrm{m}$ and $180 \mu \mathrm{m}$. The resulting temperature distribution was uniform over a large portion of the exposed region on the layer and, except for an initial steep rise, the heating rate remains constant. Rapid heating of 
TLDs has also permitted further investigation into the basic properties of TL materials [Abtahi et al., 1987]. Although the previous studies suggested that lasers be used to heat selectively various depths in a TLD and to extract mixed field information, such an approach has not been thoroughly studied for practical mixed-field dosimetry [Quam, 1983 a; Quam, 1983 b; Sahre, 1983; Braunlich et al., 1984; Grupen-Shemansky, 1989]. This thesis is primarily a detailed computational study of the use of lasers for selective heating. 


\subsection{Thesis Outline}

The novel approach presented in this dissertation enables the direct measurement of deep and shallow doses using a single TLD chip, and it could permit more accurate personnel dosimetry measurements. The proposed dosimetry approach consists of heating a thick TLD (herein denoted thick laser TL dosimeter or thick laser TLD) with a laser beam. The thick laser TLD is a square of LiF TL material, measuring $0.3 \mathrm{~cm}$ on its sides and 0.38 cm thickness. It is conceptually designed as described in Figure 1. The main feature of this dosimeter is that with an appropriate heating scheme the shallow dose can be determined directly at $0.0027 \mathrm{~cm}\left(7 \mathrm{mg} / \mathrm{cm}^{2}\right)$ and the deep dose can be determined at $0.38 \mathrm{~cm}(1000$ $\left.\mathrm{mg} / \mathrm{cm}^{2}\right)$.

The original hypothesis of this work was that a thick TL dosimeter $(>0.1 \mathrm{~cm})$ may be used to determine shallow and deep doses in mixed field radiation dosimetry using a differential heating technique. This technique employs a focused and uniform laser beam to selectively heat a thick TLD. First it is heated superficially releasing shallow dose information. Superficial heating is then followed by more thorough heating of the detector to extract depth-dose information. This approach is then expanded to the technique of pulsed laser heating, i.e., sequential pulses with varying power and duration, which will result in a temporal output of TL signal, the shape and magnitude of which is a function of both depth-dose and the heating sequence which was applied. The TL signal can then be utilized to extract depth-dose information using deconvolution techniques.

This dissertation is a computational study which includes various numerical and analytical methods used to simulate and optimize the performance of the proposed thick laser TLD. Figure 2 provides the logistics of the computational study. The idea is based on the fact that the depth-dose distribution in a TLD may be unfolded from a collection of TL light emissions following a sequence of laser heating. The unfolding is possible because 
one may select a particular heating scheme, i.e., sequence of laser pulses of given power and duration, so that each pulse preferentially extracts TL light from a certain depth in a TLD. The main objective of this work is to obtain the optimum heating scheme which allows successful unfolding of depth-dose distribution. This objective is accomplished through a series of steps illustrated in Figure 2. As indicated in Figure 2, the temperature profile of a TLD is first obtained by solving the heat conduction equation with a specific heating scheme. The TL light emitted as a function of time, or glow curve, is then calculated using a first-order kinetic model and a depth-dose distribution, which is calculated using electron/photon Monte Carlo code, EGS4 [Nelson et al., 1985]. The TL light thus obtained is then used together with the response function of the TLD and heating system to unfold the depth-dose distribution. The response function matrix is a twodimensional matrix, which consists of TL light intensity for each depth and for each pulse included in a heating scheme under the condition that the depth-dose distribution is uniform in the TLD. Finally, the unfolded depth-dose information is compared with the original, with good agreement indicating an appropriate heating scheme. Several depth-dose distributions are studied to test system behavior for different radiation fields. As indicated in Figure 2, the search for the optimum heating scheme is an iterative process. In this study, the search is started with an arbitrary heating scheme which is then modified many times using trial and error until the depth-dose distributions which result from the unfolding EGS4 agree with the original EGS4 distributions.

The computational methods used for heat conduction, glow curve generation, and depth-dose unfolding are described in detail in Chapter 2. Several computer codes were developed based on the computational methods, and they must be run in sequence in order to simulate the performance of the proposed thick laser TLD system. Chapter 3 describes in detail the computer codes and their implementations in the course of this study. The results of the TLD system simulation and optimization are presented and discussed in Chapter 4. The conclusions drawn from the results are reemphasized in Chapter 5 , and the suggestions 
for future work are summarized in Chapter 6. Finally, the actual raw data for depth-dose distributions in thick TL dosimeter are presented in Appendix A. The computer codes, HANG1 and HANG2 are presented in Appendix B. 


\section{CHAPTER II}

\section{COMPUTATIONAL METHODS}

This chapter discusses various computational methods that were used in this study. These methods were developed to: (1) calculate temperature profile in a TLD, (2) calculate thermoluminescence glow curve, and (3) unfold the depth-dose distribution.

\subsection{Laser Beam Heating}

This section presents a two-dimensional, time-dependent numerical model for heating a TLD slab with a continuous carbon dioxide Gaussian laser beam $\left(\mathrm{TEM}_{00}\right)$ and uniform laser beam. The model is developed for use with any TLD material, shape, and thickness provided that its thermal and physical properties are known.

\subsubsection{Gaussian Laser Beam}

As a first step in the theory of TL emission we calculate $T(\vec{r}, t)$ by solving the heat conduction equation

$$
\rho C_{p} \frac{\partial T}{\partial t}=k \nabla^{2} T+S
$$

where $\nabla^{2}$ is, in the cylindrical coordinate system

$$
\nabla^{2}=\frac{1}{r} \frac{\partial}{\partial r} r \frac{\partial}{\partial r}+\frac{\partial^{2}}{\partial z^{2}}
$$


and, in the rectangular coordinate system, is

$$
\nabla^{2}=\frac{\partial^{2}}{\partial x^{2}}+\frac{\partial^{2}}{\partial y^{2}}+\frac{\partial^{2}}{\partial z^{2}}
$$

where $\rho$ is the density, $C_{p}$ is the specific heat capacity, $k$ is the thermal conductivity, which is illustrated in Table 1 , and $S$ is the laser power per unit volume present at position $(r, z)$ or $(x, y, z)$ during laser exposure (heat source function). Starting at time $t=0$ a narrow, circularly symmetric, Gaussian beam of light propagating in the $\mathrm{z}$ direction illuminates the surface. Correcting for reflective loss on the front surface at $z=0$, the heat source function or incident intensity distribution becomes

$$
S(r, z, t)=\mu\left(1-R_{f}\right) I_{0} \exp \left(-\frac{r^{2}}{\omega^{2}}\right) \exp (-\mu z)
$$

where $\omega$ is the radius of the Gaussian beam at the $e^{-1}$ intensity point (i.e., $2 \omega$ is the full width at $1 / \mathrm{e}$ power of the laser beam), $R_{f}$ is the reflectivity, $\mu$ is the absorption coefficient of the laser beam, and $I_{0}$ is the laser power per unit surface area at $z=0$, which is called laser beam power density. Eq (2.1) has the following boundary conditions:

a) No heat loss occurs at the boundaries $z=0$ and $z=L, i . e ., d T / d z=0$. This assumption is approximately valid in vacuum in the absences of radiative heat loss.

b) The TLD is infinite in the radial direction and has a thickness $L$.

The initial condition is $T(r, z, 0)=T_{0}$, i.e., room temperature $(300 \mathrm{~K})$, and the laser beam is turned on at time $t=0$. The temperature obtained from $\mathrm{Eq}(2.1)$ is the actual temperature increase above $\mathrm{T}_{0}$. An analytical solution to the problem of Eq (2.1) with $\mathrm{Eq}(2.4)$ is possible [Braunlich, 1984]. The solution is 


$$
\begin{aligned}
T(r, z, t) & =\frac{\left(1-R_{f}\right) \alpha \omega^{2} I_{0}}{k L} \int_{0}^{t} d \tau\left[\frac{e^{-r^{2}}\left[4 \alpha(t-\tau)+\omega^{2}\right]}{4 \alpha(t-\tau)+\omega^{2}}\right] \\
& {\left[\left(1-e^{-\mu L}\right)+2 \mu^{2} \sum_{m=1}^{\infty} \frac{e^{-\alpha m^{2}(t-\tau) / \pi^{2} L^{2}}}{\mu^{2}+\left(\frac{m \pi}{L}\right)^{2}} \times\left(1-e^{-\mu L} \cos m \pi\right)(\cos m \pi z / L)\right] }
\end{aligned}
$$

where $\alpha$ is thermal diffusivity $\left(=\mathrm{k} / \rho \mathrm{C}_{\mathrm{p}}\right), \mathrm{L}$ is TLD thickness, and $\mathrm{m}=1,2,3, \cdots \cdot$

Solving Eq (2.1) with the cylindrical coordinate system numerically, the implicit technique [Ozisik, 1980] cannot be directly applied, because there are two spatial coordinates rather than one. Nevertheless, it is still possible to obtain stable solutions by separately applying the 1-D technique to each dimension. In the so-called alternating direction-implicit (ADI) technique [Mansuripur, 1982], one divides the time step $\Delta t$ into two equal half steps, $\Delta t / 2$ each. In going from $k \Delta t$ to $(k+1 / 2) \Delta t$, one treats $r$ explicitly and $z$ implicitly; in going from $(k+1 / 2) \Delta t$ to $(k+1) \Delta t$, one treats $z$ explicitly and $r$ implicitly (The order of $\mathrm{r}$ and $\mathrm{z}$ is unimportant). The basic equations for the TL slab are represented by the following:

First half-step:

$$
\begin{aligned}
& \frac{T_{i, j}^{*}-T_{i, j}^{k}}{\Delta t / 2}=\frac{\alpha}{r} \frac{T_{i+1, j}^{*}-T_{i-1, j}^{*}}{2 \Delta r}+\alpha \frac{T_{i-1, j}^{*}-2 T_{i, j}^{*}+T_{i+1, j}^{*}}{(\Delta r)^{2}}+\alpha \frac{T_{i, j-1}^{k}-2 T_{i, j}^{k}+T_{i, j+1}^{k}}{(\Delta z)^{2}}+\frac{S}{\rho C_{p}} \\
& T_{i r, j}^{*}=0 \\
& T_{1, j}^{*}=\frac{4}{3} T_{2, j}^{*}-\frac{1}{3} T_{3, j}^{*}
\end{aligned}
$$

Second half-step: 


$$
\begin{aligned}
& \frac{T_{i, j}^{k+1}-T_{i, j}^{*}}{\Delta t / 2}=\frac{\alpha}{r} \frac{T_{i+1, j}^{*}-T_{i-1, j}^{*}}{2 \Delta r}+\alpha \frac{T_{i-1, j}^{*}-2 T_{i, j}^{*}+T_{i+1, j}^{*}}{(\Delta r)^{2}}+\alpha \frac{T_{i, j-1}^{k+1}-2 T_{i, j}^{k+1}+T_{i, j+1}^{k+1}}{(\Delta z)^{2}}+\frac{S}{\rho C_{p}} \\
& T_{i, j+1}^{k+1}=T_{i, j r}^{k+1} \\
& T_{i, 1}^{k+1}=T_{i, 2}^{k+1}
\end{aligned}
$$

where $\alpha$ is thermal diffusivity, $i$ is the radial nodal index, and $j$ is the axial (z) nodal index, and $k$ is the time steps. In the equations $T_{i, j}^{k}$ stands for the approximation to temperature at $r$ $=i \Delta r, z=j \Delta z$, and $t=k \Delta t$. The $T_{i, j}^{k}$ is calculated by the iteration procedure. The symbol (*) means the temperature at the intermediate time step. The ADI method [Birkhoff et al., 1962; Mansuripur et al., 1982] has the advantages of being very stable and of saving computing time. Such a numerical solution allows the flexibility for easily studying focused laser beams and considering more realistic geometry and boundary conditions. This approach was therefore implemented for use as the main tool for studying the extraction of depth-dose information using the Gaussian laser heating of TLDs.

Laser heating is achieved by transferring radiant energy from an infrared (IR) laser beam to the absorbing medium or TLD in this case. Figure 3 displays a schematic diagram of unfocused and focused laser beam heating. Figure 4 represents a cross sectional view of each beam incident perpendicularly on the TLD. In unfocused beam heating, the exit beam diameter, $2 \omega_{0}$, after it is corrected with a beam divergence from a laser cavity beam waist, $2 \omega_{i}$, is used on the front surface of the TLD. In focused laser heating of a TL layer of thickness, $\mathrm{L}$, the focused waist diameter, $2 \omega_{\mathrm{f}}$ is used on the front surface of the TLD. The intensity distribution of the focused beam is

$$
\frac{I(\sigma, \beta)}{I(0,0)}=\frac{1}{1+\beta^{2}} \exp \left[\frac{-2 \sigma^{2}}{\left(1+\beta^{2}\right)}\right], \quad \text { [Grupen-Shemansky, 1989] }
$$


where $\sigma=\mathrm{r} / \omega_{\mathrm{f}}, \beta=\left(\mathrm{z}-\mathrm{z}_{\mathrm{o}} / \mathrm{z}_{\mathrm{i}}\right) / \Gamma \mu_{\mathrm{o}}{ }^{2}, \Gamma=\pi \omega_{\mathrm{i}}^{2} / \mathrm{z}_{\mathrm{i}} \lambda, \mu_{\mathrm{o}}=\omega_{\mathrm{f}} / \omega_{\mathrm{i}}, \omega_{\mathrm{f}}$ is the output or focused waist radius at the $1 / \mathrm{e}$ intensity point, $\omega_{\mathrm{i}}$ is the $1 / \mathrm{e}$ input or laser cavity waist radius, $z_{\mathrm{o}}$ is the distance from the lens center to the first surface of the TLD, $z_{i}$ is the distance from the laser cavity waist to the lens, $\mathrm{r}$ is the radial distance measured from the beam axis, $\mathrm{z}$ is the axial distance from the center of the lens, and $\lambda$ is the wavelength $\left(10.6 \mu \mathrm{m}\right.$ for $\mathrm{CW} \mathrm{CO}_{2}$ laser beam). Eq (2.8) may be rearranged in the dimensional form as follows:

$$
\frac{I(r, z)}{I(0,0)}=\frac{z_{r m}^{2}}{z_{r m}^{2}+\left(z-z_{0}\right)^{2}} \exp \left[-\frac{r^{2}}{\omega^{2}} \frac{z_{r_{m}}^{2}}{z_{r m}^{2}+\left(z-z_{0}\right)^{2}}\right]
$$

where $I(0,0)$ is the peak intensity at the focused waist radius, $\omega_{\mathrm{f}}$, and $z_{\mathrm{rm}}=\pi \omega_{\mathrm{f}}^{2} / \lambda$. The modified Rayleigh distance, or $z_{\mathrm{rm}}$, is the distance from the focused waist to where the peak intensity is one-half that at $\omega_{\mathrm{f}}$.

$\mathrm{Eq}$ (2.9) represents the unattenuated laser beam intensity. To convert Eq (2.9) into a heat source function for a TL laser, an absorption coefficient $\mu$ for t0s follows:

$$
-\frac{\partial \mathrm{I}}{\partial \mathrm{z}}=\mu \mathrm{I}(\mathrm{z})
$$

where $I(z)$ is the intensity of the monochromatic beam as a function of $z$ within the absorbing medium. Assuming that a uniformly attenuated Gaussian beam remains Gaussian with no change in the radial beam profile, the $\mathrm{I}(\mathrm{r}, \mathrm{z})$ expression in $\mathrm{Eq}(2.9)$ may replace I(z) in Eq (2.10) after correcting for surface reflection losses and axial absorption within the TL layer. The $\mathrm{z}$ dependent heat source function of the focused beam is derived as follows:

$$
-\frac{\partial I}{\partial z}=\mu\left(1-R_{f}\right) I_{0} \frac{z_{r m}^{2}}{z_{r m}^{2}+\left(z-z_{0}\right)^{2}} \exp \left[-\frac{r^{2}}{\omega^{2}} \frac{z_{r m}^{2}}{z_{r m}^{2}+\left(z-z_{0}\right)^{2}}\right] \exp (-\mu z)
$$


where $R_{f}$ is the reflectivity and $\mathrm{z}$ is the depth within the TLD from the laser-heated surface as displayed in Figure 3.

\subsubsection{Uniform Square Beam}

For accurate and sensitive personnel and environmental dosimetry, heating with a laser beam of uniform intensity profile is highly desirable. By a special feature of the laser beam optics, it is possible to convert a Gaussian $\mathrm{CO}_{2}$ beam profile to a square, uniform one [Braunlich, 1990]. Figure 5 shows a schematic diagram of a uniform square laser beam as it heats a sample. Figure $4($ c) represents a cross sectional view of a uniform (square) beam incident perpendicularly on the TLD. The heating system uses the IST (International Sensor Technologies) TLD reader [Braunlich, 1990] which features an RFexcited $\mathrm{CO}_{2}$ waveguide laser capable of delivering a uniform beam of around $0.3 \times 0.3 \mathrm{~cm}^{2}$ spot size (adjustable) to discrete a TL sample of thickness L. The transient temperature response of the TLD slab irradiated by a uniform beam can be estimated by a model given by

$$
\rho C_{p} \frac{\partial T}{\partial t}=k\left(\frac{\partial^{2} T}{\partial x^{2}}+\frac{\partial^{2} T}{\partial y^{2}}+\frac{\partial^{2} T}{\partial z^{2}}\right)+S
$$

where $\rho$ is the density, $C_{p}$ is the specific heat capacity, $k$ is the thermal conductivity, and $S$ is the laser power per unit volume present at position $(x, y, z)$ during laser exposure (heat source function). Correcting for reflective loss on the front surface at $z=0$, the heat source function becomes

$$
S(z)=\mu\left(1-R_{f}\right) I_{0} \exp (-\mu z)
$$


where $\mathrm{z}$ is the depth in TLD, $R_{f}$ is the reflectivity, $\mu$ is the absorption coefficient of laser beam, and $\mathrm{I}_{0}$ is the beam power density. $\mathrm{Eq}(2.12)$ has the following boundary conditions:

a) No heat loss occurs at the boundaries, i.e., $d T / d x=d T / d y=d T / d z=0$.

b) An appropriate initial condition is $T(x, y, z, 0)=T_{0}$, i.e., room temperature, and the laser beam is turned on at $t=0$.

Eq (2.12) can be solved analytically by the Green's function technique [Ozisik, 1980]. The general solution for the TLD having dimension, $x=a, y=b$ and $z=c$ is

$$
T(x, y, z, t)=\frac{\alpha}{k} \int_{z=0}^{t} d \tau \int_{x=0}^{z} d x^{\prime} \int_{y=0}^{b} d y^{\prime} \int_{z=0}^{c} d z^{\prime} G\left(x, y, z, t I x^{\prime}, y^{\prime}, z^{\prime}, \tau\right) g(x, y, z, t)
$$

where $\mathbf{G}$ is Green's function derived as:

$$
\begin{aligned}
& G\left(x, y, z, t \mid x^{\prime}, y^{\prime}, z^{\prime}, t\right)=\frac{1}{a b c}+\left(\frac{2}{a b c}\right)\left(\sum_{m=1}^{\infty} e^{-\alpha \beta_{m}^{2} t} \cos \beta_{m} x \cos \beta_{m} x^{\prime}+\sum_{n=1}^{\infty} e^{-\alpha \gamma_{n}{ }^{2} t} \cos \gamma_{n} y \cos \gamma_{n} y^{\prime}\right. \\
& \left.+\sum_{p=1}^{\infty} e^{-\alpha \eta_{p}^{2} t} \cos \eta_{p} z \cos \eta_{p^{\prime}} z^{\prime}\right)+\left(\frac{4}{a b c}\right)\left(\sum_{m=1}^{\infty} \sum_{n=1}^{\infty} e^{-\alpha\left(\beta_{m}^{2}+\gamma_{n}^{2}\right) t} \cos \beta_{m} x \cos \beta_{m} x^{\prime}\right. \\
& \cos \gamma_{n} y \cos \gamma_{n} y^{\prime}+\sum_{n=1}^{\infty} \sum_{p=1}^{\infty} e^{-a\left(\gamma_{n}^{2}+\eta_{p}{ }^{2}\right) t} \cos \gamma_{n} y \cos \gamma_{n} y^{\prime} \cos \eta_{p} z \cos \eta_{p} z^{\prime} \\
& \left.+\sum_{m=1}^{\infty} \sum_{\mathrm{p}=1}^{\infty} \mathrm{e}^{-\alpha\left(\beta_{\mathrm{m}}{ }^{2}+\eta_{\mathrm{p}}{ }^{2}\right) t} \cos \beta_{\mathrm{m}} \mathrm{x} \cos \beta_{\mathrm{m}} \mathrm{x}^{\prime} \cos \eta_{\mathrm{p}} \mathrm{z} \cos \eta_{\mathrm{p}} \mathrm{z}^{\prime}\right)+\left(\frac{8}{\mathrm{abc}}\right) \\
& \sum_{m=1}^{\infty} \sum_{n=1}^{\infty} \sum_{p=1}^{\infty} e^{-\alpha\left(\beta_{m}{ }^{2}+\gamma_{n}{ }^{2}+\eta_{p}{ }^{2}\right) t} \cos \beta_{m} x \cos \beta_{m} x^{\prime} \cos \gamma_{n} y \cos \gamma_{n} y^{\prime} \cos \eta_{p} z \cos \eta_{p} z^{\prime}
\end{aligned}
$$

where $\beta_{m}=m \pi / a$ for $m=1,2,3, \cdots, \gamma_{m}=n \pi / b$ for $n=1,2,3, \cdots$, and $\eta_{m}=p \pi / c$ for $p=$ $1,2,3, \cdots \cdot$. The time-dependent temperature distribution for a TLD with thickness, $L$ is: 


$$
\begin{gathered}
T(z, t)=\frac{\alpha\left(1-R_{f}\right) I_{0}}{k L}\left(1-e^{-\mu L}\right) t+\frac{\alpha}{k} \sum_{m=1}^{\infty} \int_{\tau=0}^{t} e^{-\alpha\left(\frac{m \pi}{L}\right)^{2} e^{-\alpha\left(\frac{m \pi}{L}\right)^{2}} \cos \left(\frac{m \pi}{L}\right) z} \\
{\left[\frac{2 \mu^{2}\left(1-R_{f}\right) I_{0}}{\mu^{2} L+m^{2} L^{2}}\right]\left(1-e^{-\mu L} \cos m \pi\right) d \tau}
\end{gathered}
$$

Eq (2.12) is also solved numerically using the explicit technique [Ozisik, 1980]. A numerical solution to $\mathrm{Eq}(2.12)$ requires the replacement of the continuous space-time domain with a discrete set of points. Defined as the approximation to the temperature at $(x, y, z, t)=(i \Delta t, j \Delta y, k \Delta z, n \Delta t)$, where $n>0$ and $0<i<i_{\max }=a / \Delta x, 0<j<j_{\max }=b / \Delta y$, and $0<\mathrm{k}<\mathrm{k}_{\max }=\mathrm{L} / \Delta \mathrm{z}, \mathrm{Eq}(2.12)$ is discretized with finite-difference equations as follows:

$$
\begin{gathered}
\frac{T_{i, j, k}^{n+1}-T_{i, j, k}^{n}}{\Delta t}=\alpha\left[\frac{T_{i+1, j, k}^{n}-2 T_{i, j, k}^{n}+T_{i-1, j, k}^{n}}{(\Delta x)^{2}}+\frac{T_{i, j+1, k}^{n}-2 T_{i, j, k}^{n}+T_{i, j-1, k}^{n}}{(\Delta y)^{2}}+\right. \\
\left.\frac{T_{i, j, k+1}^{n}-2 T_{i, j, k}^{n}+T_{i, j, k-1}^{n}}{(\Delta z)^{2}}\right]+\frac{S_{i, j, k}^{n}}{\rho C_{p}}
\end{gathered}
$$

The following rearrangement of Eq (2.17) shows how the solution at time $n+1$ can be directly derived from the solution at time $\mathrm{n}$ :

$$
\begin{aligned}
T_{i, j, k}^{n+1}= & T_{i, j, k}^{n}+\alpha \Delta t\left\{\left[\frac{T_{i+1, j, k}^{n}-2 T_{i, j, k}^{n}+T_{i-1, j, k}^{n}}{(\Delta x)^{2}}+\frac{T_{i, j+1, k}^{n}-2 T_{i, j, k}^{n}+T_{i, j-1, k}^{n}}{(\Delta y)^{2}}+\right.\right. \\
& \left.\frac{T_{i, j, k+1}^{n}-2 T_{i, j, k}^{n}+T_{i, j, k-1}^{n}}{(\Delta z)^{2}}\right]+\frac{\left.S_{i, j, k}^{n}\right\}}{\rho C_{p}}
\end{aligned}
$$


2.2 Heat Conduction with Temperature-Dependent Thermal Conductivity

From the heat conduction equation, Eq (2.1), the parameters, $\rho$ (density), $C_{p}$ (specific heat) and $\mathrm{k}$ (thermal conductivity) are related to the material properties, and the source term, $S$, is related to laser beam properties. The variables $k$ and $C_{p}$ are temperature sensitive parameters. Table 1 summarizes the values used by different investigators for these two parameters. The thermal conductivity, $\mathrm{k}=0.00658 \mathrm{cal} \mathrm{s}^{-1} \mathrm{~cm}^{-1} \mathrm{~K}^{-1}$ is approximately one third of the value measured for a single crystal at room temperature [Abtahi et al., 1985]. The value, $\mathrm{k}=0.0282 \mathrm{cal} \mathrm{s}^{-1} \mathrm{~cm}^{-1} \mathrm{~K}^{-1}$ was the averaged value within a temperature range $0-500{ }^{\circ} \mathrm{C}$ [Gotlib et al., 1984]. The thermal conductivity and the specific heat values from Braunlich are used throughout this study. However, the temperature dependence of $\mathrm{k}$ is studied using a heating model as described in $\mathrm{Eq}$ (2.12) assuming $\mathrm{k}$ decreases with increasing temperature approximately as $\mathbf{T}^{-1}$ [Petrov et al., 1976].

With temperature dependent thermal conductivity, $\mathrm{k}(\mathrm{T}) \mathrm{Eq}(2.12)$ can be modified:

$$
\rho C_{p} \frac{\partial T}{\partial t}=\nabla \cdot(k(T) \nabla T)+S
$$

where $\nabla \cdot(k(T) \nabla T)$ is equivalent to $\nabla k(T) \cdot \nabla T+k(T) \nabla^{2} T$. By vector product

$$
\nabla k(T) \cdot \nabla T=\frac{\partial k}{\partial T}\left[\left(\frac{\partial T}{\partial x}\right)^{2}+\left(\frac{\partial T}{\partial y}\right)^{2}+\left(\frac{\partial T}{\partial z}\right)^{2}\right]
$$

Substituting Eq (2.20) into Eq (2.19) yields

$$
\rho C_{p} \frac{\partial T}{\partial t}=k(T) \nabla^{2} T+\frac{\partial k}{\partial T}\left[\left(\frac{\partial T}{\partial x}\right)^{2}+\left(\frac{\partial T}{\partial y}\right)^{2}+\left(\frac{\partial T}{\partial z}\right)^{2}\right]+S
$$


Now, $\mathrm{Eq}(2.21)$ is discretized and rearranged

$$
\begin{aligned}
T_{i, j, k}^{n+1}=T_{i, j, k}^{n} & +\frac{k(T) \Delta T}{\rho C_{p}}\left[\frac{T_{i+1, j, k}^{n}-2 T_{i, j, k}^{n}+T_{i-1, j, k}^{n}}{(\Delta x)^{2}}+\frac{T_{i, j+1, k}^{n}-2 T_{i, j, k}^{n}+T_{i, j-1, k}^{n}}{(\Delta y)^{2}}+\right. \\
& \left.\frac{T_{i, j, k+1}^{n}-2 T_{i, j, k}^{n}+T_{i, j, k-1}^{n}}{(\Delta z)^{2}}\right]+\left(\frac{1}{\rho C_{p}}\right)\left(\frac{\partial k}{\partial T}\right)\left[\left(\frac{T_{i+1, j, k}^{n}+T_{i-1, j, k}^{n}}{2 \Delta x}\right)^{2}\right. \\
& \left.\left(\frac{T_{i, j+1, k}^{n}+T_{i, j-1, k}^{n}}{2 \Delta y}\right)^{2}+\left(\frac{T_{i, j+1, k}^{n}+T_{i, j-1, k}^{n}}{2 \Delta y}\right)^{2}\right]+\frac{S_{i, j, k}^{n}}{\rho C_{p}}
\end{aligned}
$$

where $\mathrm{k}(\mathrm{T})=\mathrm{k}_{\mathrm{o}} \mathrm{T}_{\mathrm{o}} / \mathrm{T}, \mathrm{dk} / \mathrm{dT}=-\mathrm{k}_{\mathrm{o}} \mathrm{T}_{\mathrm{o}} / \mathrm{T}^{2}, \mathrm{k}_{\mathrm{o}}=0.0275 \mathrm{~W} / \mathrm{cmK}, \mathrm{T}_{\mathrm{o}}=300 \mathrm{~K}, \mathrm{C}_{\mathrm{p}}=1.56$ $W s / g K$ and $\rho=2.635 \mathrm{~g} / \mathrm{cm}^{3}$ for LiF TLD. 


\subsection{Calculation of Thermoluminescence Glow Curve}

Glow curves obtained from a TLD following laser beam heating can only be calculated numerically by integrating the TL light generated in each discretized volume. The thermoluminescence glow curve peaks considered in this study are commonly called peaks $2,3,4$, and 5 (Figure 6), corresponding to the trap depths of $1.13,1.23,1.54$ and $2.17 \mathrm{eV}$ and frequency factors of $6.1 \times 10^{13}, 4.0 \times 10^{13}, 7.3 \times 10^{15}$, and $4.0 \times 10^{21} \mathrm{sec}^{-1}$ [McKeever, 1980] via a fit of experimental curves to first-order kinetics. Laser heating generates a timedependent transient spatial temperature distribution which is cylindrically symmetric for a given depth $z$. The TLD volume is divided in elements of size of $2 \pi \mathrm{rdrd} z$ around the axis as shown in Figure 7. The TL intensity $\mathrm{I}_{\mathrm{TL}}$ for each volume element at a given time is calculated by $\mathrm{Eq}$ (1.6) and these contributions over the total volume are integrated to obtain the total thermoluminescence emission at this time, i.e.,

$$
I_{\text {II }}(t)=\iint 2 \pi r d r d z I(r, z, t)
$$

where $I(r, z, t)$ is the TL emission intensity per unit volume. In the actual computer simulation, TL intensity is

$$
I_{I L}(t)=\sum_{i=1}^{i r} \sum_{j=1}^{j r} 2 \pi r d r d z I_{i j}
$$

where $I_{i j}$ is the TL intensity at ith and jth node calculated using Eq (1.6). The volumes at the center, corner and edge are described as shown in Figure 7. Plotting this total emission intensity vs time yields the glow curve. 


\subsection{Unfolding of Depth-Dose}

Consider a depth-dose distribution $\mathrm{D}(\mathrm{z})$, the resulting TL light intensity corresponding to each pulse of a heating scheme can be expressed as

$$
p_{i}=c_{1} \int_{u_{1}} \int_{0}^{L} D(z) f(z, t) d z d t
$$

where $t_{i}$ is the duration of the $i^{\text {ith }}$ pulse, $f(z, t)$ is the response function relating the production of TL light at any given pulse to the depth-dose, $L$ is the TLD thickness, and $c_{1}$ is the proportionality constant. In order to carry out the unfolding scheme to obtain the depthdose distribution, $\mathrm{Eq}(2.25)$ is converted to matrix form:

$$
\mathrm{p}_{\mathrm{i}}=\sum_{\mathrm{j}} \mathrm{D}_{\mathrm{j}} \mathrm{f}_{\mathrm{ij}}
$$

where $f_{i j}$ is the response function matrix described in Section 1.4, which is the TL light intensity for $j^{\text {th }}$ depth and $i^{\text {th }}$ pulse, $D_{j}$ is a vector of dimension $M$, corresponding to measurements of total TL intensity during some interval. $f_{i j}$ is a matrix of dimension $\mathrm{Nx}$ $\mathrm{M}$, characteristic of the TL dosimeter and heating scheme including total pulses of $N$. Eq (2.26) is the basic function describing the unfolding problem.

Unfolding procedure The depth-dose unfolding technique used in this study is based on the unfolding algorithm used for unfolding of neutron spectrum by Wang [Wang et al., 1990] and an iterative algorithm developed by Doroshenko [Doroshenko et al., 1977]. The iteration process is started with uniform dose distribution by the following procedure: 
(1) Determine $f_{i j}$ from known experiments.

(2) Give an initial guess of $D_{j}$ (for example, uniform depth-dose distribution).

(3) Calculate $\mathrm{p}_{\mathrm{i}, \text { computed, }}$ the calculated TL light for the $\mathrm{i}^{\text {ith }}$ pulse from

$$
p_{i, \text { computed }}=\sum_{j} D_{j} f_{i j}
$$

(4) Update $D_{j}$, the unfolded depth-dose distribution using:

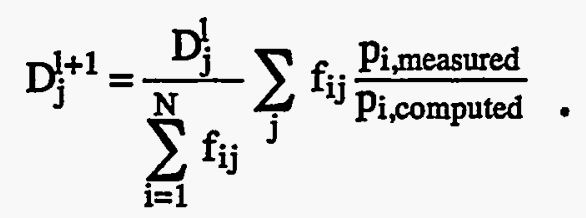

where $p_{i, \text { measured }}$ is the measured TL light intensity for the $i^{\text {th }}$ pulse and $D_{j}^{l}$ is the depth-dose distribution during the $1^{\text {th }}$ iteration.

(5) As is shown in Eq (2.28), the depth-dose distribution for each iteration is calculated using the results of a previous iteration and the ratio of the measured and calculated TL light intensity. The iteration is continued until either a specified number of iterations is exceeded or a specified convergence criterion is met. The convergence criterion is based on the deviation of the calculated TL light intensity from the input TL light intensity. The deviation is described by the equation

$$
\mathrm{e}^{\mathrm{l}}=\frac{1}{\mathrm{~N}} \sum_{\mathrm{k}=1}^{\mathrm{N}}\left[\left(\frac{\mathrm{p}_{\mathrm{k}, \text { computed }}^{\mathrm{l}}-\mathrm{p}_{\mathrm{k}, \text { measured }}}{\mathrm{p}_{\mathrm{i} \text {,measured }}}\right)^{2}\right]^{\frac{1}{2}}
$$




\section{CHAPTER III}

\section{DEVELOPMENT AND IMPLEMENTATION OF COMPUTER CODES}

As was described in Chapter 1, the simulation and performance optimization of the thick laser TLD system is an iterative process. The parameter which is most relevant to the system's performance is the depth-dose information. Several computer codes were developed based on the computational methods discussed in Chapter 2, and some were adapted to simulate the performance of the TLD system. The computer codes and the various calculations associated with the system optimization process are discussed in detail in this chapter.

\subsection{Description of Computer Codes}

\subsubsection{Codes for Heat Conduction and Glow Curve Calculation}

A computer code, called HANG (Heat Conduction ANd Glow curve calculations), was developed to implement heat conduction and glow curve calculations. The code was written to model the two-dimensional, non-steady state production of thermoluminescence (TL) following laser heating. A schematic diagram for the structure and data flow of the computer code is displayed in Figure 8. As shown in the figure, each code consists of a heat conduction model and a TL glow curve calculation model. The input data include TLD type, TLD dimension, laser power, laser beam size, and heating duration. The output data include transient spatial temperature profiles, heating rate, spatial TL light distribution and transient TL light distribution. Because different mathematical approaches are required for Gaussian beam and uniform beam cases, two versions of code, HANG1 and HANG2 
were written. HANG1 employs ADI technique [Mansuripur et al., 1982] to solve the transient, 2-dimensional heat conduction equation for focused and unfocused beam heating. HANG2 employs the explicit technique [Ozisik, 1980] to solve the transient, 1-dimensional heat conduction equation for uniform beam heating. Boundary conditions for the heat conduction calculation include convective, adiabatic, and infinite boundaries. The heat source produced by a laser beam is spatially dependent, and it was calculated based on the laser beam diameter, laser absorption coefficient and reflectivity. As shown in Figure 7, a TLD chip was subdivided into a large number of spatial meshes based on the cylindrical coordinate system. Temperature was calculated for each spatial mesh. Both versions of HANG employ the first-order kinetic model of thermoluminescence described by Eq (1.6) and the trapping parameters for LiF [McKeever, 1980] to calculate TL light emission. TL light intensity was calculated for each spatial mesh, and then integrated over the entire volume of the TLD to obtain the glow curve. The codes were written in FORTRAN, and run on SUN workstations [SUN].

\subsubsection{Code for Electron and Photon Transport Calculations}

A recently released version of Monte Carlo electron/photon transport code, EGS4 was used in this study for calculating depth-dose distributions for various beta and gamma sources. The EGS (Electron-Gamma Shower) system of computer codes is a general purpose package. It is used for the Monte Carlo simulation of the coupled transport of electrons and photons in an arbitrary geometry, and it is useful for particles with energies from a few $\mathrm{keV}$ up to several TeV. The current version is the EGS4 Code System by Nelson [Nelson et al., 1985] and it is more commonly referred to as EGS4.

For an EGS4 run, the main program and two subroutine programs, HOWFAR and AUSGAB must be provided by users. HOWFAR is used to specify the geometry, while AUSGAB is used to score the energy deposited in depth bins. The relationship of these subroutine programs with other EGS4 programs is displayed in Figure 9. Table 2 provides 
the input data for material and radiation which are required by the main program. The electron cross section data for $\mathrm{LiF}$ TL were generated using the cross section generation code, PEGS4. The cutoff energy is $1 \mathrm{keV}$ for both electrons and photons. For beta-particle transport calculations, a beta spectrum was converted to a cumulative density function, using a computer code, called ENSRC.

\section{1,3 Code for Unfolding Depth-Dose Distributions}

The unfolding code is based on the iterative algorithm described in Section 2.4. The major input data of the code includes the response function matrix $\mathrm{f}_{\mathrm{ij}}$ (which is described in Section 2.4) and a set of integrated TL intensities corresponding to pulsed heating. The output is the absorbed dose versus depth distribution data. From the input data, the code sets up two matrices: a one-dimensional matrix for the set of integrated TL intensities, and a two-dimensional matrix for the response function. To unfold the depth-dose distribution, an initial guess was first given. The guessed solution was then tested and modified using the iterative algorithm until the solution converge to a preset accuracy. As discussed in Section 2.4, each element in a response function matrix corresponds to the integrated TL light intensity generated in each depth for each laser pulse included in a heating scheme. In general, the success of unfolding is very much dependent on the unique characteristic behavior of the response function matrix. For an ideal response function matrix, which gives a response which is a strong function of depth, the unfolding process may only take very few iterations. In the case of non-characteristic response function, however, the solution may never converge to the preset accuracy regarding the number of iterations. 


\subsection{Description of Calculations}

This section describes various calculations included in this study. These calculations were performed for a thick laser TL dosimeter heated with multiple pulses of a laser beam. Three types of laser beams were included: focused, unfocused, and uniform as shown in Figure 4, which represents a cross sectional view of each beam incident perpendicularly on TLD. These calculations include: (a) heat conduction calculation, (b) TL glow curve calculation, (c) response function calculation, (d) depth-dose calculation, (e) unfolding of depth-dose spectrum, and ( $f$ ) data analysis.

To make sure that the computational results are credible, several benchmark heat conduction and TL glow curve calculations, summarized in Table 3, were performed using HANG, and their results are shown in Chapter 4. Code input parameters were chosen so that the benchmark calculation results are in good agreement with previously published theoretical or experimental data or analytical results. Although these benchmark calculations only involve single laser beam heating, there is no reason to doubt that the method should also work for heating with multiple laser pulses.

\subsubsection{Benchmark Calculations}

\subsection{1:1 Heat Conduction Calculations}

Benchmark heat calculations include those with focused, unfocused, and uniform laser beams. For the purpose of the calculations for focused and unfocused heating, the manufacturer's specifications for a Laakmann Electro-Optics RF-44 laser [Laakmann; Grupen-Shemansky, 1989; Abtahi et al., 1985] were chosen. These specification data were illustrated in Table 4.

Focused laser beam A LiF TL dosimeter of $0.3 \times 0.3 \mathrm{~cm}^{2}$ and thickness of $0.09 \mathrm{~cm}$ was chosen. It was heated with a $0.084 \mathrm{~cm}$ diameter, $4.93 \mathrm{~W}$ continuous wave (CW) 
focused laser beam. This LiF TLD was assumed to have a uniform dose deposition (which is defined as a flat dose distribution or one in which the dose is unity within a given TL layer) throughout the TL dosimeter. Figure 3(b) provides the geometric configuration. As shown in figure, a Gaussian laser beam of $4.93 \mathrm{~W}$ power and $0.084 \mathrm{~cm}$ full width impinges on the center of the TL slab at $z / L=0$. The energy of the laser beam (wavelength of $10.6 \mu \mathrm{m}$ for $\mathrm{CW} \mathrm{CO}_{2}$ laser) is transferred to the TL dosimeter with absorption coefficient of $40 \mathrm{~cm}^{-1}$, thus raising the temperature within the TLD. The calculations were performed with heat source function of $\mathrm{Eq}(2.4)$ and parameters illustrated in Table 1.

An analytical solution model described in $\mathrm{Eq}$ (2.5) was implemented for use in benchmarking comparison for temperature distributions within the TL dosimeter. The computer simulation required that TLD thickness, laser beam power and size, and heating time be entered upon execution as shown in Figure 8. A numerical solution model described in Eq (2.6) and Eq (2.7) was run assuming boundary conditions of the room temperature.

In both the analytical and numerical calculations, the TLD was assumed to be 0.3 $\mathrm{cm}$ in diameter, which accounts for finite radial dimensions. The test cases were run with heating times of $100 \mathrm{msec}, 200 \mathrm{msec}$, and $300 \mathrm{msec}$, since longer heating times would result in maximum local TLD temperatures exceeding $700-750 \mathrm{~K}$, a temperature that would (a) violate the boundary conditions due to temperature pileup in the TLD [Braunlich et al., 1984; Abtahi et al., 1985] and (b) avoid incandescent light emission to the TLD.

Unfocused Laser Beam Two LiF TL dosimeters of $0.3 \times 0.3 \mathrm{~cm}^{2}$ and thickness of $0.038 \mathrm{~cm}$ and $0.09 \mathrm{~cm}$ were chosen to investigate the thermal and TL parameters with an unfocused laser beam heating. Each dosimeter was assumed to have a uniform depth-dose distribution. A Gaussian laser beam of $4 \mathrm{~W}$ power and $0.170 \mathrm{~cm}$ full width impinges on the center of the TL slab at $z / L=0$ as described in Figure 3(a). The calculations were performed with heat source function of Eq (2.4) using numerical solution model, Eq (2.6) 
and $\mathrm{Eq}$ (2.7) assuming the boundary condition of a nitrogen atmosphere at the TLD surface and parameters illustrated in Table 1.

Uniform Laser Beam In order to demonstrate the applicability of uniform heating on the thick laser TL dosimeter, two types of TL dosimeters widely used in personnel dosimetry were chosen and simulated for heat conduction. As in the unfocused experiment, these were LiF dosimeters measuring $0.3 \times 0.3 \times 0.038 \mathrm{~cm}^{3}$ and $0.3 \times 0.3 \times 0.09 \mathrm{~cm}^{3}$. Temperature profiles of each TL dosimeter following laser heating were calculated by both analytical and numerical models as described in $\mathrm{Eq}$ (2.16) and Eq (2.18) for comparison. In all cases, TL dosimeters were heated by a $10 \mathrm{~W}, 0.3 \mathrm{~cm} \times 0.3 \mathrm{~cm}$ uniform laser beam, with the heating geometry shown in Figures $4(\mathrm{c})$ and 5 . Heating times of $0.5 \mathrm{sec}$ and 0.8 sec were studied, and the same thermal and glow curve parameters used for focused and unfocused beam heating were used.

\subsubsection{TL Glow Curve Calculations}

TL glow curves of TLD are calculated by Eq (2.24) using the data for the trap depths, frequency factors and relative number of traps (Figure 6) obtained by McKeever [McKeever, 1980]. Figure 7 describes the meshes and nodes used for heat conduction calculation and TL light integration during heating with the Gaussian laser beam. The time transient theoretical TL light intensity is calculated at every node with the peak temperatures $(373,421,456$, and $483 \mathrm{~K}$ ) of the peaks $2-5$ and the individual $\mathrm{TL}$ intensities are integrated for volume element, $2 \pi \mathrm{rdrdz}$ containing the node. The $T L$ intensities per small volume are then summed for total dosimeter volume for a given time. This calculation results in TL light intensity versus time by simulating the photomultiplier tube. Otherwise, the TL light gradient with depth including the characteristic feature of TL response, can be calculated using $\mathrm{Eq}$ (2.24). It is important to evaluate the variation of the TL light gradient since it implies the depth-dose distribution within TLD. Therefore, the TL light gradient was used as the key factor to determine a pulsed laser heating scheme effectively. The glow curves of 
LiF TLDs were obtained for use in benchmarking comparison with those previously reported. These are: (a) a LiF TL dosimeter of $0.3 \times 0.3 \mathrm{~cm}^{2}$ and thickness of $0.09 \mathrm{~cm}$ for a $0.084 \mathrm{~cm}$ diameter focused beam from a $4.93 \mathrm{~W}$ CW laser, (b) a LiF TL dosimeter of 0.3 $\mathrm{x} 0.3 \mathrm{~cm}^{2}$ and thickness of $0.038 \mathrm{~cm}$ for $0.17 \mathrm{~cm}$ diameter from a $4 \mathrm{~W} \mathrm{CW}$ laser, and (c) a $0.3 \times 0.3 \times 0.0027 \mathrm{~cm}^{3} \mathrm{LiF}$ TL dosimeter for $0.059 \mathrm{~cm}$ diameter beam and powers of 1.8 , 2.4 , and $2.7 \mathrm{~W}$, respectively. The $0.3 \times 0.3 \times 0.0027 \mathrm{~cm}^{3} \mathrm{LiF}$ TL dosimeter was utilized to study the theoretical analysis of the TL intensity distributions and the onset of incandescent light emission.

The theoretical calculations were compared to experimental results both qualitatively and quantitatively. Experimental results for TL dosimeters with $0.09 \mathrm{~cm}\left(230 \mathrm{mg} / \mathrm{cm}^{2}\right)$ [Abtahi et al., 1985], $0.038 \mathrm{~cm}\left(1000 \mathrm{mg} / \mathrm{cm}^{2}\right)$ [Grupen-Shemansky, 1989], and 0.0027 $\mathrm{cm}\left(7 \mathrm{mg} / \mathrm{cm}^{2}\right)$ [Braunlich, 1990] thicknesses were available from the previous investigators.

\section{2,2 Design-Basis Heat Conduction Calculations}

\section{3,2,2,1 Single Pulse Heating}

In order to control and design the laser heating system, it was necessary to study a variety of thermal response parameters such as the surface temperature spreading (i.e., surface temperature profile), the propagation of heat in the axial direction, the heating rate, the distribution of glow curve peak temperature, the dependence of beam diameter on the axial distance. For the purpose of these studies, a number of simulations were performed with a thick laser TL dosimeter using focused and unfocused laser beam, and uniform laser heating. TLDs are heated by a single laser pulse (i.e., single-pulsed heating). Table 5 summarizes of those heating simulations. In addition, the simulations with a $0.09 \mathrm{~cm}$ thick TLD were also included for comparison.

Temperature Profiles at Center and Surface (Heat Spreading) Heat conduction simulations were performed to obtain the temperatures as a function of TLD depth at $r=0$, 
and as a function of radial distance at TL surface $z=0$. These calculations were to analyze the spreading of surface and axial temperatures for the entire LiF thickness during different heating durations.

Gaussian beam profiles, which are not uniform outward from the beam center, can heat the TLD locally. Incandescent emission temperatures (approximately $750 \mathrm{~K}$ ) then may be reached at the location leading to intolerable background emission as well as loss of glow curve resolution [Gasiot et al., 1982]. Therefore, it is important to study the TLD surface temperature. The temperature profiles at the surface of a thick laser TL dosimeter were calculated with various focused laser beam heating duration; (a) $5 \mathrm{msec}$, (b) $10 \mathrm{msec}$, (c) $15 \mathrm{msec}$, and (c) $18 \mathrm{msec}$. These simulations were performed by heating the TLD with a focused laser beam of $0.032 \mathrm{~cm}$ diameter, and $4 \mathrm{~W}$ power (hereafter denoted focused beam ). In addition, the temperature gradients of a thick laser TL dosimeter were calculated with various focused laser heating durations.

Surface and axial temperature profiles of a thick laser TL dosimeter were calculated with various unfocused laser beam heating durations; (a) $0.4 \mathrm{sec}$, (b) $1.0 \mathrm{sec}$, (c) $2.0 \mathrm{sec}$, and (c) $3.0 \mathrm{sec}$. These simulations were obtained by heating the TLD with an unfocused laser beam of $0.170 \mathrm{~cm}$ diameter, and $4 \mathrm{~W}$ (hereafter denoted unfocused beam ).

Heating Rate Numerically the heating rate was calculated as the temperature rise per heating time interval. The heating rate at the surface of a thick laser TL dosimeter by focused laser beam heating was calculated through simulating heating by focused beam with $18 \mathrm{~ms}$ pulse duration.

The heating rate at the surface of a thick laser TL dosimeter by unfocused laser beam heating was also calculated. The simulation was performed using heating by unfocused beam with $1.2 \mathrm{sec}$ pulse duration.

The heating rate at the surface of a thick laser TL dosimeter due to uniform laser beam heating was calculated based on the use of $10 \mathrm{~W}$ power, $0.3 \mathrm{~cm} \times 0.3 \mathrm{~cm}$ uniform beam (hereafter denoted uniform beam ) with $0.8 \mathrm{sec}$ pulse duration. 
Distribution of Glow Curve Temperature Variations of heating depths corresponding to the peak temperatures of peaks $2-5(383 \mathrm{~K}, 421 \mathrm{~K}, 457 \mathrm{~K}$, and $483 \mathrm{~K}$ ) were calculated with various focused, unfocused, and uniform beam heating duration. In order to investigate the temperature rise at the surface of thick laser TL dosimeters, maximum surface temperatures were calculated with increasing heating time using the focused beam. The simulated data were compared with those for the $0.09 \mathrm{~cm}$ TL dosimeter.

Dependence of Beam Diameter on the Axial Distance It is necessary to consider that $1 /$ diameter, $2 \omega$ of a focused laser beam incident on the thick laser dosimeter depends on the axial distance, $z$, within the dosimeter as shown in Figure 10. This effect can cause an increase of uncertainties between theoretical and practical performances. In this study the variation of laser beam diameter was tested with various focal lengths. Calculations based on equations, Eq (2.4) and Eq (2.11) were performed to observe the change of temperatures in both the axial and the radial directions. In order to observe z-dependence of focused beam diameter (radius) within the thick TL dosimeter, the spread beam diameter, $\omega(\mathrm{z})$ within the dosimeter and the beam diameter, $\omega_{0}$ at the dosimeter surface were calculated with focal lengths $f=10,12,14$, and $17 \mathrm{~cm}$. The $\%$ differences of temperatures due to $\omega_{0}$ and $\omega(z)$, respectively, at the center $(r=0)$ and at the surface $(z=0)$ of a thick laser TL dosimeter were calculated with heating times for $10 \mathrm{~ms}, 15 \mathrm{~ms}$, and $18 \mathrm{~ms}$. The calculations were performed simulating heating by focused beam.

Temperature Dependence of Thermal Conductivity The thermal conductivity, $\mathrm{k}=$ $0.00658 \mathrm{cal} \mathrm{s}^{-1} \mathrm{~cm}^{-1} \mathrm{~K}^{-1}$, which was used throughout this study is approximately one third of the value measured for a single crystal at room temperature [Braunlich et al., 1984]. In order to investigate the significance of the effect of temperature-dependent thermal conductivity, the temperature dependence of $\mathrm{k}$ was studied using a heating model as described in $\mathrm{Eq}$ (2.22), assuming that $\mathrm{k}$ decreases with increasing temperature approximately as $\mathrm{T}^{-1}$ [Petrov et al., 1976]. Calculations of temperature profiles in $0.09 \mathrm{~cm}$ 
thick TL dosimeter were performed with two uniform laser heating durations of 0.5 and $0.8 \mathrm{sec}$.

\subsubsection{Multiple Pulse Heating}

Differential Heating In order to investigate the heating technique that gives a temperature gradient within the thick laser TL dosimeter, several heating techniques as a function of different combinations of laser power and heating time (i.e., pulse duration), or W-sec were examined. For example, the combination of a high power with short pulse duration (e.g., $3 \mathrm{~W}$-sec) was intended to obtain a high temperature gradient with depth while primarily heating the superficial layer. A combination of low power and slow heating (i.e., long pulse duration)(e.g., $8 \mathrm{~W}$-sec) was intended for heating the deep region of the TLD (i.e., differential heating). In addition to heating, cooling was allowed. Cooling, which is a temperature redistribution in the TLD after the laser power turns off, was required not only to keep cooling down the surface temperature to a desired value below that which produces thermal damage, but also to increase temperature in a deeper area prior to another heating. In order to study the effect of cooling, time-dependent isotherms were calculated at the various times, $0.424,0.828,2.12,8.48$ and $12.72 \mathrm{sec}$ following after a $0.424 \mathrm{sec}$ heating by uniform beam.

Related to the cooling a heating technique employing a uniform beam was also studied to determine the TL glow curve response to various heating approaches of pulsed heating schemes. Temperature profiles and TL light generation (TL glow curve) were studied for the thick laser TL dosimeter with (a) $0.6 \mathrm{sec} 10 \mathrm{~W}$ uniform beam heating followed by a $0.6 \mathrm{sec}$ cooling period (one heating and one cooling scheme), (b) two sequential $0.6 \mathrm{sec}$ heating and $0.6 \mathrm{sec}$ cooling periods, and (c) three sequential $0.6 \mathrm{sec}$ heating and $0.6 \mathrm{sec}$ cooling periods.

Two-Pulse Heating Approach A pulsed, focused beam using two different laser pulses was chosen and used to perform the differential heating of the thick laser TLDs 
(hereafter denoted two-pulse heating scheme). That is, a two-pulsed heating scheme was intended and studied to perform the differential heating for the layers of the TLD by focused beam and then to unfold the depth-dose distribution. In this scheme one pulsed heating was designed to obtain the TL glow curve by heating of the very thin superficial layer. Some amount of cooling was then allowed until the second heating began. Another heating was performed to obtain the TL glow curve from a slightly deeper layer than the first heated layer. Table 6 illustrates two-pulse heating scheme.

The two-pulse heating scheme was used to obtain depth-dose distribution in the shallow region obtained by heating on the top (or front) surface of the TLD. The two-pulse heating scheme was also used to obtain depth-dose distribution in the deep region obtained by heating on the bottom (or back) surface of the TLD.

For this study, the TLD was exposed to the beta-particles (50,000 histories) from the $\mathrm{Sr} / \mathrm{Y}-90$ source. In each heating scheme, laser pulses were performed sequentially. Surface temperature profiles and glow curves were calculated following heating and cooling.

Three-Pulse Heating Approach In order to obtain the differential heating layers such as superficial, middle, and deep layers using uniform laser pulses, an appropriate combination of laser pulses with different duration was designed, tested using the software tools, and modified on a trial and error basis. The combination of laser pulses was based on two ideas: 1) high power and short duration to extract TL light in the superficial layer and 2) low power and longer duration to allow the heating of deep layer. Cooling between two successive heatings was required to control surface temperature and to raise the temperature in a deep region of the TLD. For the purpose of heating design, the thick laser TLDs were heated with a pulsed, uniform beam using three laser pulses (hereafter denoted three-pulse heating scheme). The heating scheme was designed to obtain TL glow curves corresponding to heating of the shallow, middle and deep parts of TLDs. Selection of laser power and heating time was based on the results of the experiments by individual heating 
with various parameters. Some examples of the three-pulsed heating scheme are 10W$10 \mathrm{~W}-4 \mathrm{~W}$ and $10 \mathrm{~W}-4 \mathrm{~W}-2 \mathrm{~W}$. The $10 \mathrm{~W}-10 \mathrm{~W}-4 \mathrm{~W}$ heating scheme consists of a $0.7 \mathrm{sec}, 10$ W first pulse followed by $1.4 \mathrm{sec}$ cooling, which is then followed by a $1.0 \mathrm{sec}, 10 \mathrm{~W}$ second pulse with $3.5 \mathrm{sec}$ cooling, and then another $2.0 \sec 4 \mathrm{~W}$ heating (third pulse). The heating scheme of $10 \mathrm{~W}-4 \mathrm{~W}-2 \mathrm{~W}$ is a similar example to that of $10 \mathrm{~W}-10 \mathrm{~W}-4 \mathrm{~W}$, but is designed for heating of much deeper part of the TLD. That is, it consists of a $0.7 \mathrm{sec}, 10 \mathrm{~W}$ first pulse followed by $1.2 \mathrm{sec}$ of cooling, which is then followed by a $1.6 \mathrm{sec}, 4 \mathrm{~W}$ second pulse with $2.0 \mathrm{sec}$ of cooling, and then another $3.5 \mathrm{sec}, 2 \mathrm{~W}$ third pulse. In both heating schemes, the third pulses were designed to heat a deeper part (i.e., bottom) of the TLD using a combination of lower power and longer heating. Spatial temperature distributions and corresponding TL light distributions were calculated.

Due to the advantage of relatively slow heating (i.e., deep heating), the three-pulsed heating scheme can make possible the unfolding of the entire depth-dose distribution in the thick laser TLD. That is, it is the unfolding of the entire depth-dose distribution based on the glow curves produced by both three-pulsed heating schemes on the top and bottom of the TLD. Therefore, a total of 6 response functions are produced by three-pulsed heatings on the top and bottom of the TLD. Unfolding of the entire depth-dose distribution is performed by the response functions and the glow curve produced by the thick laser TLD irradiated with the radiation. 


\subsubsection{Design-Basis TL Glow Curve Calculations}

Glow Curves Responses A simple modification of the glow curve model was all that was required to account for non-uniform distribution of absorbed dose in the thick laser TL dosimeter. First-order kinetic model was solved using the numerical method, involving multiplication of the intensity by the depth-dose information and some sensitivity constant. Test cases were run using depth-dose information which has been computed for the radioactive sources of interest by EGS4 Monte Carlo code. A group of calculations was performed to simulate theoretical glow curve responses of a thick laser TL dosimeter exposed to T1-204, Sr/Y-90, and Pm-147 beta sources with the same Monte Carlo histories $(50,000)$, respectively. The depth-doses from the sources were used to calculate the corresponding TL light intensity. All single-pulsed heating calculations were simulated with focused beam of heating.

For the two-pulse heating schemes as illustrated in Table 6, the TL glow curves were calculated using the method described in Eq (2.24). During the heating and cooling periods the TL glow curves as a function of time and position were computed, and each light pulse was integrated in time to provide a response function, i.e., TL light as a function of depth.

The TL glow curves were also calculated following three-pulse heating schemes, e.g., 10W-10W-4W and 10W-4W-2W.

Energy Response Calculations The proposed detector system may reduce energy dependence, since it employs a differential heating technique, which selects thin TL layers by different heating duration. This problem even may be ignored since the new detector system is based on TL glow curve due to the uniform dose. Energy response calculation was, however, intended to demonstrate the improvement of energy dependence for thick TLDs using laser beam heating, demonstrating in particular, the advantage of laser beam heating employing differential heating technique. 
The energy response of LiF TL dosimeter at $7 \mathrm{mg} / \mathrm{cm}^{2}$ as a function of mean beta energy was obtained by simulation of the TL glow curve response from conventional (planchet) heating. For this calculation the 1-D heat conduction model using ADI technique, which was modified from Eq (2.6) and Eq (2.7) was utilized. The experimental results for the $0.09 \mathrm{~cm}$ thick TLD (with $7 \mathrm{mg} / \mathrm{cm}^{2}$ filter) [Gupta, 1983] was used for comparison to those calculated energy responses. It should be noted that the calculation has been based upon a TL layer without any filter on it. Calculations were performed on TL dosimeters irradiated by 50,000 history beta particles from Pm-147 $\left(E_{\mathrm{av}}=0.062 \mathrm{MeV}\right), \mathrm{Tl}-204\left(\mathrm{E}_{\mathrm{av}}=\right.$ $0.238 \mathrm{MeV})$, and $\mathrm{Y}-90\left(\mathrm{E}_{\mathrm{av}}=0.934 \mathrm{MeV}\right)$, respectively. The different kinds of energy responses of the $\mathrm{LiF} \mathrm{TL}$ dosimeter, which are the energy responses for the entire TLD depth (i.e., dosimeter responses), as a function of maximum beta energy were calculated by simulating conventional (planchet) heating. Calculations were performed on TL dosimeters irradiated by 50,000 history beta particles from $P m-147\left(E_{\max }=0.224 \mathrm{MeV}\right)$, Tl-204 $\left(E_{\max }=0.766 \mathrm{MeV}\right)$, and Y-90 $\left(E_{\max }=2.28 \mathrm{MeV}\right)$, respectively. All energy response calculations were performed by simulating focused and uniform laser beam heating with a $0.09 \mathrm{~cm}$ thick TL dosimeter.

\subsubsection{Design-Basis Depth-Dose Calculations Using EGS4}

In order to evaluate shallow and deep doses within the TL dosimeter, its dose response for radiation over the expected exposure range for both mixed pure and mixed radiation fields needs to be known in advance. This may be determined experimentally by exposing the dosimeters on proper phantoms to photons and beta particles of different energies. However, such experiments would not provide information about the actual dose distribution as a function of depth within a single TLD. In this work, the dose responses of a dosimeter were determined by performing a Monte Carlo simulation of the conceptual TL dosimeter described in Figure 11. 
The EGS4 code calculates the energy deposited by electrons/photons in a LiF layer as a function of depth as shown in Figure 11. The purpose of the code in beta radiation field is to calculate $D(x, E)$ for $E$ corresponding to various incident electron fluences, $N(E)$ and finally to calculate depth dose distribution, $D(x)$. Figures 12 through 14 show the beta spectra used to calculate $\mathrm{N}(\mathrm{E})$, for $\mathrm{Pm}-147\left(\mathrm{E}_{\max }=0.224 \mathrm{MeV}\right)$, Tl-204 $\left(\mathrm{E}_{\max }=0.766\right.$ $\mathrm{MeV})$, and $\mathrm{Sr} / \mathrm{Y}-90\left(\mathrm{E}_{\max }=2.28 \mathrm{MeV}\right) . \mathrm{D}(\mathrm{x}, \mathrm{E})$ is called an energy deposition function. $D(x, E)$ is the energy deposited in a semi-infinite LiF slab at depth $x$ by a unit fluence of monoenergetic electrons incident normal to the slab.

Table 7 includes a description of the 23 pure or mixed beta and gamma radiation fields which are the DOELAP radiation fields for dosimeter calibration [DOE/EH-0026, 1986]. In Table $7 \mathrm{NBS}$ filtered $\mathrm{x}$-rays $(20 \mathrm{keV}, 36 \mathrm{keV}, 70 \mathrm{keV}$ and $120 \mathrm{keV} \mathrm{x}$-rays) were simulated as monoenrgetic $\mathrm{x}$-rays because of limit of availability of energy spectra. The actual depth-dose response of the TLD to these radiation sources was calculated by EGS4 code. 50,000 histories were used for every single source and were mixed by 1:1 ratio for mixed sources. The slab source was assumed for the source-to-target geometry, so that all radiation was incident perpendicularly to the $\mathrm{TL}$ dosimeter. The code transports individual electrons or photons one by one, selecting from statistical distributions of inelastic and elastic events until an electron's or photon's energy drops below an assumed threshold energy of $1 \mathrm{keV}$, respectively. Electron energy depositions were then determined in every $0.001 \mathrm{~cm}$ interval, and for a total of 380 depth bins.

The resulting depth-dose distribution data resulting from the EGS4 runs for the 23 types of pure or mixed beta-gamma radiation fields and $\mathrm{Pm}-147$ beta source were written as data files which could be read by HANG1 or HANG2 heat conduction and glow curve calculation code. 


\subsubsection{Response Function Calculations}

As described in Section 2.4, the response function is defined as a function relating the TL light obtained at a given time as a function of depth for a given heating scheme. The response function is a two-dimensional matrix, which consists of TL light intensity for each depth and for each pulse included in a heating scheme. This function is obtained from the TL glow curve response for TLD depth due to uniform dose. In order to obtain the response function, the TL light intensities for predetermined depth intervals are calculated for a TL dosimeter exposed to an assumed uniform dose. The TL intensity distribution is characteristic of the heating scheme for a given TL dosimeter. The response function is, therefore, sensitive to the heating scheme.

Response Function for Two-pulse Heating Response functions are generated by simulation for the two-pulsed heating scheme, and an assumed uniform depth-dose distribution. During the entire heating period, laser pulses are applied sequentially with different duration, e.g., $10 \mathrm{msec}$ for first heating with $40 \mathrm{msec}$ for cooling, and $15 \mathrm{msec}$ for second heating using single focused beam.

Response Function for Three-pulse Heating A three-pulse heating scheme, e.g., $10 \mathrm{~W}-4 \mathrm{~W}-2 \mathrm{~W}$ on the top and 10W-4W-2W on the bottom, is applied to the thick laser TLDs to generate the response functions. In this case, thick laser TLDs are irradiated by the uniform dose. During the entire heating period, heat pulses are applied one after another with different duration, giving the 3 heat pulses on the top and then 3 heat pulses on the bottom sequentially. On the top $10 \mathrm{~W}, 0.2 \mathrm{sec}$ for the first heating and $1.7 \mathrm{sec}$ cooling, 4 $\mathrm{W}, 1.8 \mathrm{sec}$ for the second heating and $2.0 \mathrm{sec}$ cooling, and $2 \mathrm{~W}, 3.5 \mathrm{sec}$ for the third heating are performed. On the bottom $10 \mathrm{~W}, 0.6 \mathrm{sec}$ for the first heating and $1.3 \mathrm{sec}$ cooling, $4 \mathrm{~W}, 1.6 \mathrm{sec}$ for the second heating and $2.0 \mathrm{sec}$ cooling, and $2 \mathrm{~W}, 3.5 \mathrm{sec}$ for the third heating are also performed. 


\subsubsection{Unfolding of Depth-Dose Distribution and Optimization of Pulsed Heating}

\subsubsection{Unfolding of Depth-Dose Distribution}

In order to unfold the depth-dose distribution from actual the dose response function, the unfolding technique described in Section 2.4 has been utilized. The unfolding calculation requires that measuring TL light intensity data, which are the integrated TL light intensities corresponding to the number of heating duration of pulsed heating, and measuring the response function, which is TL light distribution versus depth data. TL light intensities would be measured as the signal from photomultiplier (PM) tube. For the focused beam case, two integrated TL light intensities corresponding to two pulses of heating, one for the top and one for the bottom of the dosimeter, are used. The results are then the depth-dose spectra in the top heating region (herein, shallow dose region) and in the bottom heating region (herein, deep dose region), respectively. For the uniform beam case, six integrated TL light intensities corresponding to six heating pulses are used (3 times in top heating and 3 times in bottom heating), together. The result is then a single depth-dose spectrum for the entire TL dosimeter. This spectrum-unfolding technique is based on an iterative algorithm developed by Doroshenko [Doroshenko et al., 1977].

\subsubsection{Approach of Optimum Pulse Heating Scheme}

The optimization process is designed to determine a best, unique combination of heating pulses capable of minimizing errors for shallow and deep doses from any kind of single or mixed-field radiation field. The process is to modify iteratively the heating scheme and the corresponding response function by evaluating unfolded depth-doses by comparison with EGS4 depth-dose as illustrated in Figure 15. It is started with three radiation fields, $\mathrm{Sr} / \mathrm{Y}-90, \mathrm{Cs}-137$, and $20 \mathrm{keV}$ NBS filtered $\mathrm{x}$-rays chosen to evaluate initial heating schemes. These heating schemes are designed based on the results from single beam heating. Heating schemes were iteratively modified for every radiation field until the 
process finally finds a heating scheme giving the best fit between the unfolded data and the EGS4 data.

Performance Test of Optimum Two-pulsed Heating Glow curves were simulated for the pulsed focused beam heating scheme for the 23 types of DOELAP radiation fields in Table 7 and a Pm-147 beta source. In this case, thick laser TLDs were irradiated by the mixed radiation field $(50,000$ histories per each radiation field). During the entire heating period, heat pulses with different durations were applied sequentially, i.e., $10 \mathrm{msec}$ for first heating with $40 \mathrm{msec}$ for cooling, and $15 \mathrm{msec}$ for second heating using a $0.032 \mathrm{~cm}$ diameter, $4 \mathrm{~W}$ focused Gaussian laser beam heating. The pulses were first performed on the top of TL dosimeter, and then on the bottom of the dosimeter. A total of 46 simulated glow curves (23 top and 23 bottom) for the DÓELAP radiation fields, and 2 actual glow curves for Pm-147 beta source were generated for this heating method.

Performance Test of Optimum Three-Pulsed Heating A variable power pulsed heating method of uniform beam, $10 \mathrm{~W}-4 \mathrm{~W}-2 \mathrm{~W}$ on the top and $10 \mathrm{~W}-4 \mathrm{~W}-2 \mathrm{~W}$ on the bottom, was applied to thick laser TLDs to generate the actual glow curve for 23 types of radiation fields. In this case, thick laser TLDs were irradiated by the mixed radiation field $(50,000$ histories per each radiation field). During the entire heating period, heat pulses were applied one after another with different duration, giving the 3 heat pulses on the top and then 3 heat pulses on the bottom sequentially. On the top $10 \mathrm{~W}, 0.2 \mathrm{sec}$ for the first heating and $1.7 \mathrm{sec}$ cooling, $4 \mathrm{~W}, 1.8 \mathrm{sec}$ for the second heating and $2.0 \mathrm{sec}$ cooling, and 2 . $\mathrm{W}, 3.5 \mathrm{sec}$ for the third heating were performed. On the bottom $10 \mathrm{~W}, 0.6 \mathrm{sec}$ for the first heating and $1.3 \mathrm{sec}$ cooling, $4 \mathrm{~W}, 1.6 \mathrm{sec}$ for the second heating and $2.0 \mathrm{sec}$ cooling, and 2 $\mathrm{W}, 3.5 \mathrm{sec}$ for the third heating were also performed. After the top heating, the bottom was assumed to be at room temperature $\left(T_{0}=300 \mathrm{~K}\right)$. In the uniform heating case, the actual dose response function was the response function including TL responses from both the top and bottom heating, simultaneously. Therefore, a total of 23 actual dose response functions for the DOELAP radiation fields were generated from this heating method. 


\section{3,2,6.3 Determination of Shallow and Deep Doses}

By definition the shallow and deep doses should be determined as the doses at $0.0027 \mathrm{~cm}$ in depth $\left(7 \mathrm{mg} / \mathrm{cm}^{2}\right)$ and $0.38 \mathrm{~cm}$ in depth $\left(1000 \mathrm{mg} / \mathrm{cm}^{2}\right)$ with a $\mathrm{LiF} \mathrm{TL}$ dosimeter, respectively. According to this work, the doses can be determined by extracting the corresponding values from the dose distribution established (by unfolding) around both dose regions of interest or around the entire TL dosimeter. This technique may have the advantage of achieving a high level of accuracy because the dose distribution within the thick laser TL dosimeter represents the spectrum of the depth-doses received from radiation fields. In this study the shallow and deep dose were estimated by two methods: 1) direct reading of a single dose at the depths at which shallow and deep dose are defined, and 2) averaging of the dose data around dose regions of interest corresponding to the depths at which shallow and deep dose are defined. Figures 16 and 17 display the data points averaged out to determine the shallow and deep dose with errors in the focused and uniform heating cases. For the shallow and deep doses from focused laser beam heating, the doses with errors were evaluated by averaging out the 5 data points with intervals ( $|\Delta \mathrm{L}|$ $=0.0018 \mathrm{~cm}$ for shallow dose and $|\Delta L|=0.0045 \mathrm{~cm}$ for deep dose). For shallow and deep doses from uniform laser beam heating, the doses with errors were evaluated by averaging out the 2 data points with intervals $(|\Delta L|=0.0009 \mathrm{~cm}$ for shallow dose and $|\Delta L|=0.0036$ cm for deep dose). The shallow and deep dose values associated the errors were then determined, and compared with the actual dose values obtained from the EGS4 calculations. 


\section{3,2.7 Sensitivity and Uncertainty Analysis}

\section{3,2.7.1 Stability of Computer Code}

The stability of the two main computer codes using different numerical methods (ADI technique and Explicit technique) was tested with variation of mesh sizes such as time, axial and radial meshes. Tests were performed in the way of each time run of computer code with changed mesh size and comparison of TL glow curves. From these tests the values to give the glow curve data with minimum noise were found, then chosen to use in this study.

Variations of the low curve response with various time mesh sizes by focused laser beam heating on a thick laser TLD were calculated. Mesh sizes used in this calculation were $1.0 \times 10^{-5} \mathrm{sec}, 1.0 \times 10^{-4} \mathrm{sec}$, and $1.0 \times 10^{-3} \mathrm{sec}$. During the calculation, radial mesh size of $7.5 \times 10^{-4} \mathrm{~cm}(\mathrm{R} / 200)$ and axial mesh size of $9.0 \times 10^{-4} \mathrm{~cm}(\mathrm{~L} / 422)$ were used. Variations of glow curve response with various axial and radial mesh sizes under conditions of focused laser beam heating also were calculated. Mesh sizes used in this calculation were (a) R/200 and $L / 422$, (b) $R / 200$ and $L / 211$, and (c) $R / 100$ and $L / 106$. In all three cases the time mesh size of $1.0 \times 10^{-4} \mathrm{sec}$ was used. Calculations were performed using $0.032 \mathrm{~cm}, 4 \mathrm{~W}$ focused Gaussian laser beam. In the uniform heating case, mesh sizes used in the test calculation were (a) $\mathrm{L} / 211$, (b) $\mathrm{L} / 106$, and $\mathrm{L} / 53$ with a time mesh of $1 \times 10^{-4} \mathrm{sec}$. Other tests with time meshes of $1 \times 10^{-3} \mathrm{sec}$ and $1 \times 10^{-5} \mathrm{sec}$ were performed.

\section{3,2.7.2 Noise Analysis of Glow Curves and Depth-Dose}

From the practical situation, the primary source of systematic error is generally associated with the electronics of the TL reading system. The systematic error of the conventional TL reading system is about $4-10 \%$. These errors were based upon the fluctuation of the light output response between various TL measurement readings. Many other factors can contribute to the systematic error including the stability of the 
electrometer, the variability of the heating cycle parameters (i.e. heating rate and maximum temperature), the fluctuation of heating temperature as a function of time, the response variation of the photomultiplier (PM) tube, the stability of the power supply, and other electronic-related factors.

The following factors affect glow curve unfolding in real situations: (1) laser beam power density $\left(I_{0}\right)$ from the heating system, and (2) laser beam absorption coefficient $(\mu)$ and thermal parameters (i.e., thermal conductivity, $k$ and specific heat, $C_{p}$ ) from TL material. Beam power density $\left(\mathrm{cal} / \mathrm{cm}^{2} / \mathrm{sec}\right)$ is determined by laser power and beam diameter, since it is defined as the power per unit surface area. Therefore, errors in beam power density may be expected due to changed beam spread or "mistakes" in measuring the beam diameter, and due to minute oscillations of laser power, or "blunder" in measuring the power from the power indicator. The laser beam absorption coefficient may be varied with TL material. The value used in calculation of theoretical response function assumed by uniform dose is $40 \mathrm{~cm}^{-1}$, but it may change in real situations since this factor is related to the material. It is possible that the change of this factor will have an impact on the temperature profile within TL material, since it is directly related to laser energy deposition. Thermal conductivity and specific heat may change with TL material in real situations. These two values must be examined statistically for TL material batches when the experimental materials are prepared. Therefore, the error analyses were studied to determine how the glow curve would be changed with (1) the change of beam power density, (2) the change of laser beam absorption coefficient, (3) the change of thermal conductivity, and (4) the change of specific heat capacity.

\section{3,2,7,3 Uncertainties of EGS4 Depth-Dose}

Theoretical depth-dose distribution (actual depth-dose distribution) on a thick laser TL dosimeter by EGS4 beta/gamma Monte Carlo code was calculated to determine total energy deposited in every $0.001 \mathrm{~cm}$ thickness. This small bin of thickness was reasonable 
since the shallow dose is determined at $7 \mathrm{mg} / \mathrm{cm}^{2}$ actually corresponding to $0.0027 \mathrm{~cm}$ within the dosimeter. Thus, depth-dose was given by energy deposited at 380 bins $(0.38$ $\mathrm{cm} / 0.001 \mathrm{~cm}=380$ bins) within the dosimeter. However, depth-dose values calculated by such a small bin of thickness showed lots of fluctuations throughout the dosimeter. In order to estimate the errors, the least-squares fitting for actual depth-dose data were performed and the fitting data obtained. Errors related to actual depth-dose were then calculated by comparing each data point to the fitted data and to the actual dose data. 


\section{CHAPTER IV}

\section{RESULTS AND DISCUSSIONS}

This chapter presents and discusses the results of both the benchmark calculations

and the design-basis calculations described in Chapter 3. Due to the large number of calculations which were performed in this study, the presentation and discussion of calculational results are divided into sections:

(1) Temperature profiles

(2) Glow curves

(3) Design-based depth-dose distributions

(4) Pulsed heating and response functions

(5) Unfolding of depth-dose and optimization of heating scheme

(6) Deep and shallow doses

(7) Noise analysis results

(8) Energy response calculations

(9) Dosimetric evaluation of thick laser TLD

\subsection{Temperature Profiles}

\subsubsection{Temperature Profiles for Benchmark Calculations}

Gaussian Laser Beam Heating. Figures 18 through 20 show the temperature profiles, obtained based on the analytical solution, at times of $100 \mathrm{msec}, 200 \mathrm{msec}$, and $300 \mathrm{msec}$ following the initiation of Gaussian laser beam heating. These temperature 
profiles were obtained for a $0.09 \mathrm{~cm}$ TLD following heating with $0.084 \mathrm{~cm}$ diameter 4.93 W focused laser beam. Each isotherm (i.e., $383 \mathrm{~K}, 421 \mathrm{~K}, 457 \mathrm{~K}$ and $483 \mathrm{~K}$ ) corresponds to one of the glow peaks (i.e., peak numbers $2,3,4$, and 5) for the LiF TLD. In addition, these profiles were computed assuming that no heat transfer is allowed at the surfaces of the TLD (i.e., zero temperature gradient at the boundary) and that the TLD's radial dimension is infinitely large compared to the laser beam diameter. In particular, Figure 20 shows excellent agreement between the temperature profiles obtained from the HANG code and those previously reported [Abtahi et al., 1985], and therefore suggests that the code implementation was correct. The increase in temperature at depth as a function of time is apparent in the figure. Figure 21 shows the temperature profiles obtained based on the numerical solution for a $0.09 \mathrm{~cm}$ TLD following $300 \mathrm{msec}$ of heating with $0.084 \mathrm{~cm}$ diameter $4.93 \mathrm{~W}$ focused laser beam. Comparison of Figure 21 with the results (dotted lines superimposed) obtained by others [Abtahi et al., 1985] reveals a good agreement.

Uniform Laser Beam Heating Figures 22 and 23 display temperature distributions in axial direction in a $0.038 \mathrm{~cm}$ thick LiF TLD and a thick laser TLD, following heating with a square uniform laser beam for $0.5 \mathrm{sec}$ and $0.8 \mathrm{sec}$, respectively. The solid line corresponds to the analytical solution, and the dotted line corresponds to the numerical solution. The $\mathrm{TL}$ dosimeters were heated with a $10 \mathrm{~W} \mathrm{CO}_{2}, 0.3 \mathrm{~cm} \times 0.3 \mathrm{~cm}$ uniform laser beam. The temperature profiles were obtained from both analytical and numerical solutions, in which no heat transfer is allowed on the surfaces of TLD (i.e., zero temperature gradient boundary assumptions). As shown, a good agreement between the temperature profiles was obtained from the two solutions.

\subsubsection{Temperature Profiles of Thick Laser TLD}

Unfocused Beam Figure 24 shows the two-dimensional temperature profiles for a thick laser TL dosimeter heated by unfocused beam. The corresponding heating time is 0.5 sec. 
Figure 25 shows the temperature profiles of a $0.09 \mathrm{~cm}$ thick TL dosimeter following the same conditions as those in Figure 24. As the results, the maximum surface temperature (i.e., temperature at $T(0,0)$ ) of a thick laser TLD was $468.5 \mathrm{~K}$ and that of a $0.09 \mathrm{~cm}$ thick TLD was $485.0 \mathrm{~K}$. The isotherm of $483 \mathrm{~K}$ peak temperature (main peak temperature) of a thick laser TLD was not observed during heating. Figure 26 shows the variations of heating depths corresponding to the peak temperatures for peaks 2-5 (i.e., 383 $\mathrm{K}, 421 \mathrm{~K}, 457 \mathrm{~K}$, and $483 \mathrm{~K}$ ). As illustrated, a heating time of $1 \mathrm{sec}$ is required to obtain the temperature of $483 \mathrm{~K}$ at $0.02 \mathrm{~cm}$ depth and the heating depth saturates after $2 \mathrm{sec}$ of heating.

Figure 27 shows the radial temperature profiles as a function of heating time for a thick TLD. Figure 28 shows the temperature as a function of TL layer depth at a TLD center line. According to Figures 27 and 28, the temperature in a thick TLD saturates after 2 sec of heating.

Figure 29 shows the axial temperature profiles at the center line of a thick laser TL dosimeter with various heating times. Figure 30 shows radial temperature profiles at the surface of a thick laser TL dosimeter with various heating times. According to these two figures the temperature gradients in a thick TLD do not change much with heating times. The result suggests that the unfocused beam is not suitable for differential heating.

Figure 31 shows heating rate versus time at the center of a thick TL dosimeter. As shown, the heating rate follows a very fast decaying curve. The initial heating rate is about $7.0 \times 10^{2} \mathrm{~K} / \mathrm{s}$.

Focused Beam Figure 32 shows the isotherms of peak temperatures for peaks 2-5 (i.e., $383 \mathrm{~K}, 421 \mathrm{~K}, 457 \mathrm{~K}$, and $483 \mathrm{~K}$ ) of a thick LiF TLD heated by focused beam. The temperature profiles were obtained at the end of heating $(18 \mathrm{msec})$. The maximum surface temperature was $619.7 \mathrm{~K}$ at $18 \mathrm{msec}$. The non-uniformity (i.e., large temperature gradients) of the temperature distribution by a Gaussian laser beam heating (non-uniform heating) is clearly shown. 
Figure 33 shows the isotherms of a $0.09 \mathrm{~cm}$ thick TL dosimeter following $18 \mathrm{msec}$ of heating with focused beam. The maximum surface temperature was $670.6 \mathrm{~K}$ at $18 \mathrm{msec}$. From these figures, it is concluded that the focused laser beam results in a much more rapid surface heating of the TLD. This is expected because the focused beam instantaneously heats a skinny hot cylinder with its axis parallel to the beam, and it tends to dissipate heat preferentially in the radial direction. This result is promising, because the use of focused laser beam heating would permit selective extraction of superficial dose from a TLD. That is, the shallow dose could be directly determined by using an appropriate beam power and a specific heating time such that primarily shallow dose information is extracted selectively. Therefore, the focused heating tends to not only result in a much more rapid emission of TL light, but allows the more rapid extraction of dose information from shallower depths. In addition, it showed the possibility of local heating due to its small beam diameter. The result also suggests that a heating scheme based on using a short-duration focused laser pulse, followed with a longer-duration uniform pulse would allow the sequential readout of shallow and deep doses.

Figures 34 and 35 show how the glow peak temperatures propagate in depth as a function of heating time for a $0.09 \mathrm{~cm}$ TLD and a thick laser TLD, respectively. The TLDs were heated by focused beam. These two figures show the heating time required for a differential heating layer. For instance, the heating of $10 \mathrm{~ms}$ on the thick laser TLD may detrap the electrons up to a minimum depth of $0.01 \mathrm{~cm}$, while the heating of $10 \mathrm{~ms}$ on 0.09 $\mathrm{cm}$ thick TLD may detrap the electrons up to the minimum depth of $0.015 \mathrm{~cm}$.

Figure 36 shows the maximum surface temperatures as a function of heat duration for a thick laser TL dosimeter. These results were obtained with heating by focused beam. Since the temperature maximizes at the TLD surface, the temperature at the surface dictates the maximum allowable heating time. The surface temperature of approximately $750 \mathrm{~K}$ was found to cause incandescent emissions [Gasiot et al., 1982], thermal shock or crack on TL layer [Gasiot et al., 1982; Grupen-Shemansky, 1989]. 
Figure 37 displays the axial temperature profiles at the center line of a thick laser TL dosimeter for various heating times and Figure 38 shows radial temperature profiles for the same TL and under the same conditions at the surface of a thick laser TL dosimeter with various heating times. As illustrated, the temperature gradients change with heating times, indicating that a focused beam may be used for differential heating. Figures 39 and 40 describe temperature gradients for the thick laser TL dosimeter. As shown, heat penetrates faster in the axial direction than in the radial direction.

One additional characteristic of focused laser heating is its high heating rates. Figure 41 shows heating rate versus time at the center of the surface of a thick laser TL dosimeter heated with focused beam. The initial heating rate using a $4 \mathrm{~W}$ laser is about $4.4 \times 10^{4} \mathrm{~K} / \mathrm{s}$ for the focused beam (with beam diameter $=0.032 \mathrm{~cm}$ ). According to TL kinetic theory, the peak temperatures of the glow curve shift to higher values when heating rate increases. Therefore, the increased heating rates may shift the glow curve peak temperatures into the incandescent region, and reduce the signal to noise ratio. Figure 41 also shows that the heating rates decay exponentially with heating time.

Figure 42 shows the variations of focused beam diameter as a function of depth in a thick TLD. These results were obtained with various focal lengths, $f$ for a $4 \mathrm{~W}$ focused laser beam. The variations of laser beam diameter, $\omega(\mathrm{z})$ were represented as the percent increase relative to the beam diameter at the dosimeter surface, $\omega_{0}$. As illustrated, $\omega(\mathrm{z})$ with small focal length $(f=10 \mathrm{~cm}$ ) increase more rapidly with the depth in the TLD than that of large focal length $(f=17 \mathrm{~cm})$. Figure 43 displays the relative difference of temperatures calculated with $\omega(z)$ and $\omega_{0}$ at the center line of the TLD. As shown, the difference was not strongly dependent on the heating time. Figure 44 shows the relative difference of temperatures at the surface of a thick TLD. In both figures the relative errors were found to be less than $0.063 \%$.

Uniform Beam Figure 45 shows the temperature profiles, at $0.5 \mathrm{sec}$ and $0.8 \mathrm{sec}$ for a thick laser TL dosimeter. The TL dosimeter was heated by uniform beam. These 
temperature profiles were obtained using the numerical solution, assuming that no heat transfer is allowed on the TLD boundaries.

Table 8 illustrates the temperature difference between the top and bottom surfaces for various thicknesses of TLDs. From this table, one concludes that heating of a thin TLD (i.e., with thickness of $0.038 \mathrm{~cm}$ ) is rather uniform and that the axial temperature gradient of a TLD decreases with increase of heating time. Heat appears to dissipate more rapidly on the surface than in the axial dimension. This provides a differential heating of TL layer by uniform laser beam to allow the more selective extraction of superficial dose from the TL dosimeter.

Figures 46 and 47 show the change of temperature profiles by considering temperature-dependent thermal conductivity (i.e., $\mathrm{k}(\mathrm{T})=\mathrm{k}_{0} * \mathrm{~T}_{0} / \mathrm{T}$ ) for a $0.09 \mathrm{~cm}$ thick $\mathrm{LiF}$ TLD and a thick laser TL dosimeter at $0.8 \mathrm{sec}$ following heating by uniform beam. As shown, the TLD temperature profile is rather sensitive to the change of thermal conductivity. The quantitative results are provided in Tables 9 and 10. These tables show that the surface temperatures of a 0.09 thick LiF TLD may vary by as much as $5.5 \%$ while the surface temperatures of a thick laser LiF TLD may vary by as much as $8 \%$ for the two different thermal conductivities used.

Figure 48 shows how the peak no. 5 temperature propagates in depth as a function of heating time for uniform beam. As shown, for the thick laser TL dosimeter heated with $0.5 \mathrm{sec}$ uniform beam, TL lights are emitted from a superficial layer of approximately $0.005 \mathrm{~cm}$ of depth. Whereas, for a $0.09 \mathrm{~cm}$ thick TL dosimeter heated with $0.5 \mathrm{sec} 10 \mathrm{~W}$, TL lights are emitted from the layer having a depth of $0.015 \mathrm{~cm}$. Therefore, selective heating of various thicknesses of TL layer is possible based on a number of selected heating times.

Figures 49 and 50 illustrate the relationship between heating time and surface temperature for the $0.09 \mathrm{~cm}$ thick LiF TLD and the thick laser TLD, respectively. The TLDs were heated with uniform laser beam. Since a surface temperature above $750 \mathrm{~K}$ is 
known to cause incandescent emission and material damage, the heating time is limited by the TLD surface temperature. The surface temperature of TLDs with various thicknesses and laser powers can be estimated from Figure 49 and 50.

Figure 51 shows the surface heating rate of the thick laser TL dosimeter using a uniform beam with various laser powers. It is shown that the initial heating rates using a 10 $\mathrm{W}$ and a $4 \mathrm{~W}$ laser are about $1,000 \mathrm{~K} / \mathrm{s}$ and $400 \mathrm{~K} / \mathrm{s}$, respectively. These heating rates are moderate compared to the initial heating rate, of $4 \times 10^{4} \mathrm{~K} / \mathrm{s}$ shown in Figure 41 for the focused beam heating. 


\subsection{TL Glow Curves}

\subsubsection{TL Glow Curves for Benchmark Calculations}

Focused Beam Figure 52 shows the TL glow curves predicted by both analytical and numerical solutions for a $0.09 \mathrm{~cm}$ thick LiF TLD heated with a $0.084 \mathrm{~cm}$ diameter, $4.93 \mathrm{~W}$ focused beam. In order to free the results from numerical artifacts (especially, numerical integration) and to achieve good spatial resolution, spatial meshes of 50 in the radial dimension and 50 in the axial dimension were used in the calculation. As shown in Figure 52, the computed curve is in excellent agreement with previously reported theoretical results [Abtahi et al, 1985]. The portion of the glow curve arising from a depth of $0.09 \mathrm{~cm}$ indicates that the shallow portions of the TLD begin to glow very quickly following initiation of heating. Figure 52 shows good agreement among the analytical solution, the numerical solution, and the published results [Abtahi et al., 1985]. The more appropriate boundary conditions used in the numerical solution result in slower heating and release of TL light. Nevertheless, the overall agreement supports to the numerical solution.

Figure 53 shows the TL glow curves calculated using a numerical solution for a $0.09 \mathrm{~cm}$ thick LiF TLD, following heating with a $0.084 \mathrm{~cm}$ diameter focused $4.93 \mathrm{~W}$ continuous wave (CW) laser beam. The TLD was heated for a duration of $0.3 \mathrm{sec}$. The collected data were then normalized to the TL light value at $0.3 \mathrm{sec}$. The experimental result by Abtahi [Abtahi et al., 1985] is also included in the figure for comparison. Table 11 illustrates the quantitative comparison. As shown, the agreement between the result obtained with numerical calculations and that obtained from the experiment is reasonable.

Figure 54 shows the theoretical TL glow curves obtained for a $0.0027 \mathrm{~cm} \mathrm{LiF}$ (TLD-100) with a focused Gaussian laser beam of fixed $0.059 \mathrm{~cm}$ diameter and various laser powers. The calculations were performed with $\mathrm{Eq}(2.6)$ and (2.7) and the first-order 
kinetic model parameters (frequency factor, trap depth) determined by McKeever for peaks 2-5 for TLD. The endpoint of each glow curve represents the onset of incandescence corresponding to a maximum temperature of approximately $750 \mathrm{~K}$. The corresponding TL glow curves obtained experimentally [Braunlich, 1990] are shown in Figure 55. The data obtained by Braunlich was for TL layer on a $125 \mathrm{~mm}$ thick glass slide. The agreement between the two figures is reasonable in light of the uncertainties concerning the exact electron-kinetic model description of the thermoluminescence emission from the material and the fact that the experimental TLD has a glass substrate which was not assumed in the calculations.

Unfocused Beam Figure 56 shows both the theoretical and the experimental glow curves for $0.038 \mathrm{~cm}$ thick TL ribbon simulated with heating by unfocused beam. The theoretical glow curves were calculated with frequency factors reduced by two orders of magnitude. The smaller frequency values are simply McKeever's [McKeever, 1980] values reduced in proportion to the increase in heating rate under laser heating (approximately 100 times) as speculated by Kelly [Kelly et al., 1984]. The experimental glow curve data are based on that published by Grupen-Shemansky [Grupen-Shemansky, 1989]. Both curves shown in Figure 56 were normalized to the peak values. Table 12 illustrates the quantitative comparison. The agreement between the two curves is reasonable except for heating time $1.4 \mathrm{sec}$ beyond which the experimental value is higher.

Uniform Beam Figure 57 shows a comparison between experimental glow curve and theoretical glow curve for a $0.038 \mathrm{~cm}$ thick LiF (TLD-100) Harshaw chip with a uniform laser beam. The theoretical glow curves were calculated for a $0.038 \mathrm{~cm}$ thick LiF TL dosimeter heated with uniform beam. The experimental TL glow curve is based on a $0.038 \mathrm{~cm}$ thick LiF (TLD-100) Harshaw chip mounted on the glass substrate and a $10 \mathrm{~W}$ laser of uniform intensity profile [Braunlich, 1990]. There is a general agreement between the two curves characteristically. Table 13 illustrates the quantitative comparison. The difference between the absolute TL intensities is attributed to the fact that the experimental 
glow curve was obtained based on the TLD mounted on the glass substrate, whereas the theoretical calculation did not include this condition.

\section{2,2 TL Glow Curves in Thick Laser TLD}

Gaussian Laser Beam Figure 58 shows the theoretical glow curve of a thick laser TL dosimeter with assumed uniform dose. The TLD was heated by unfocused beam for $1.2 \mathrm{sec}$.

Figure 59 displays the TL glow curve of a thick laser TL dosimeter heated by focused beam. It also shows the variations of glow curves for various time mesh sizes

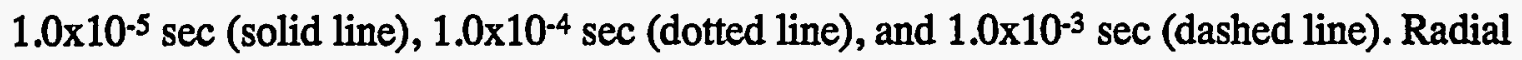
mesh size of $7.5 \times 10^{-4} \mathrm{~cm}$ and axial mesh size of $9.0 \times 10^{-4} \mathrm{~cm}$ were used. In addition, Figure 59 shows that a time mesh size of $10^{-4} \mathrm{sec}$ would produce stable calculational results. Figure 60 displays the variations of glow curve with various axial and radial mesh sizes. The heating conditions for Figure 60 are the same as those for Figure 59. Figure 60 also indicates that an axial mesh size of $1.8 \times 10^{-3} \mathrm{~cm}$ and a radial mesh size of $7.5 \times 10^{-4} \mathrm{~cm}$ would produce stable calculational results. Figure 61 shows the theoretical glow curves of a thick laser TL dosimeter exposed to various beta sources. These glow curves were calculated based on the depth-dose distributions obtained from EGS4 results. The difference in TL responses due to the non-uniformity of depth-dose distribution is clearly illustrated in Figure 61:

Uniform Beam The theoretical glow curves are presented here for the purpose of qualitative comparison of the characteristic feature of TL response obtained by heating TLD with a square laser uniform beam. Figure 62 shows the time transient theoretical glow curves calculated using Eq (2.24) for LiF TLD thicknesses of $0.038 \mathrm{~cm}$ and $0.09 \mathrm{~cm}$ thicknesses. These glow curves were calculated for the TLDs heated by uniform beam for $0.8 \mathrm{sec}$. The calculation utilized the parameters derived by McKeever [McKeever, 1980] with assumed uniform dose. As shown in the figure, the glow curve for the $0.038 \mathrm{~cm}$ TLD 
indicates that the entire TLD could be heated to emit the total thermoluminescent light. Figure 63 shows the theoretical glow curve results corresponding to the temperature profiles of Figure 46. As shown, the TLD glow curve is quite sensitive to the change of thermal conductivity. The effect of glow curve and depth-dose distribution by the change of thermal conductivity will be discussed more in Section 4.8.

Figure 64 displays the TL glow curve of a thick laser TL dosimeter heated by uniform beam. It also shows the variations of glow curves for various time mesh sizes: $1.0 \times 10^{-3} \mathrm{sec}$ (dashed line), $1.0 \times 10^{-4} \mathrm{sec}$ (dotted line), and $1.0 \times 10^{-5} \mathrm{sec}$ (solid line). An axial mesh size of $1.8 \times 10^{-3} \mathrm{~cm}$ was used in these calculations. In addition, Figure 64 shows that the mesh sizes of $10^{-3}$ or $10^{-4}$ sec both produce stable calculational results. Figure 65 displays the variations of glow curve with various axial meshes. The heating conditions for Figure 65 are the same as those for Figure 64. Figure 65 also shows that an axial mesh size of $1.8 \times 10^{-3} \mathrm{~cm}$ would produce stable calculational results. Figure 66 displays the variations of glow curves of a thick laser TL dosimeter with temperature dependence of thermal conductivity, $\mathrm{k}$ for heating by uniform beam. Figure 67 shows TL intensity versus depth of TL dosimeter. obtained from the same TL layers and test conditions as used in Figure 67. 


\subsection{Design-Basis Depth-Dose Distributions}

\subsection{Depth-Dose Distributions Obtained from EGS4}

Figures 68 through 72 present the EGS4 results of depth-dose distribution calculations, the depth-dose distributions in the thick laser TL dosimeter exposed with radiation sources, $\mathrm{Pm}-147, \mathrm{Tl}-204, \mathrm{Sr} / \mathrm{Y}-90,20 \mathrm{keV}$ x-rays and Cs-137. These results were obtained with 50,000 particle histories. Absorbed doses were calculated based on the deposition in small spatial bins which are $0.001 \mathrm{~cm}$ thick. The fluctuations of depth-dose distributions shown in Figures 68 through 72 are caused by the small spatial bins, rendering a small number of events of energy deposition in each bin. The fluctuations are more pronounced near the depth corresponding to the beta range because very few particles actually reach there. According to electron stopping power and range data [Berger et al., 1982], the continuous slowing down approximation (CSDA) ranges for Pm-147, Tl-204 and $\mathrm{Sr} / \mathrm{Y}-90$ are $0.025 \mathrm{~cm}, 0.147 \mathrm{~cm}$, and $0.53 \mathrm{~cm}$, respectively. These depth-dose distributions were used as the design-basis standard in the unfolding procedure, through which the heating schemes were optimized. Sections 4.5 and 4.6 discuss of unfolding results and optimization of heating schemes.

\subsubsection{Uncertainties of Depth-Dose Distributions}

In reality, Figures 68 through 72 shows fluctuations of depth-dose distributions near the depths corresponding to the beta ranges. These fluctuations may be reduced by running more particles in the EGS4 calculations. In this study, however, we determined these fluctuations quantitatively by using the least-squares fit. Numerical comparisons between EGS4 and the fitted data were illustrated in Tables 14 through 16. 


\subsection{Pulsed Heating and Response Functions}

\subsubsection{Pulsed Heating and Response functions for Focused Beam}

Thick laser TLDs were heated with a pulsed, focused beam using two different heating schemes illustrated in Table 6. In each heating scheme, laser pulses were applied sequentially. Figures 73 and 74 show the surface temperature of the dosimeter as a function of time for: (a) $10 \mathrm{msec}$ heating (first pulse), $40 \mathrm{msec}$ cooling, and $15 \mathrm{msec}$ heating (second pulse), and (b) $10 \mathrm{msec}$ heating (first pulse), $40 \mathrm{msec}$ cooling, and $20 \mathrm{msec}$ heating (second pulse). Figures 75 and 76 show the corresponding response functions, the TL light distributions versus the depth of TL dosimeter. The response functions indicate that the first heating duration of $10 \mathrm{msec}$ detraps the electrons within approximately 0.01 $\mathrm{cm}$ depth, and the longer second heating duration gives an additional dose information in the deeper region. These figures clearly demonstrate the advantage of laser beam heating with differential heating technique.

\subsubsection{Pulsed Heating and Response Functions for Uniform Beam}

Figure 77 shows temperature profiles within a thick laser TL dosimeter for various combinations of laser powers and heating duration. It demonstrates that the combination of high power and a short heating duration ( $3 \mathrm{~W}$-sec) results in a high temperature gradient with superficial heating. A combination of low power and long heating duration ( $8 \mathrm{~W}$-sec) results in low temperature gradient and deep heating.

Figure 78 shows a heating scheme which employs a cooling period after the laser is turned off. In Figure 78, the time-dependent temperature profiles within a thick laser TLD were obtained at the various times, $0.424,0.828,2.12,8.48$ and $12.72 \mathrm{sec}$ following a pulse of $0.424 \mathrm{sec}$, uniform beam. The cooling period is required not only to keep the surface temperature of TLD below a set value, but also to increase temperature in the deep 
part of TLD before another laser pulse applied. The temperature near the superficial layer decreases while the temperature at the bottom layer increases during the cooling period.

Figure 79 illustrates the various heating schemes (i.e., single, double, and triple pulsed heatings) selected for use in this study. These heating schemes use the same laser power. Figure 80 illustrates the temperature profiles and TL light intensity calculated for the thick laser TL dosimeter following the single pulse heating scheme (i.e., heating for $0.6 \mathrm{sec}$ $10 \mathrm{~W}$ with uniform beam and cooling of $0.6 \mathrm{sec}$ ). The tail of the glow curve represents the TL light generated during the cooling time. Figure 81 shows the temperature profiles and TL light distribution calculated for the thick laser TLD following the double heating scheme (i.e., two $10 \mathrm{~W}, 0.6 \mathrm{sec}$ pulses separated with $0.6 \mathrm{sec}$ cooling). In Figure 81(b) the glow curve by the second heating is higher than that of the first pulse due to increase of heated area, and TL emission from deeper part of TLD during the second pulse as illustrated in Figure 81(a). Although the TLD surface temperature increases from $500 \mathrm{~K}$ to $600 \mathrm{~K}$ between the end of two laser heating pulses, the surface no longer emits TL light since the trapped electrons were already depleted from this region during the first heating pulse.

Figure 82 shows the temperature profiles and TL light distribution calculated for the thick laser TLD following the triple heating scheme (i.e., three $0.6 \sec 10 \mathrm{~W}$ pulses with $0.6 \mathrm{sec}$ of cooling between pulses). As shown in Figure 82(a), the third pulse does not contribute much to heating the deep part of the TLD. The amount of heating area in deep part of TLD during the third pulse was slightly decreased. This is understood because the penetration of peak temperature increases logarithmically as shown in Figure 48. From the point of view of TL light generation, the glow curve during the third pulse may be smaller than that of the second pulse because a considerable amount of TL light has already been emitted in this region during the cooling period after the second pulse. Figure 83 shows the calculated TL light distribution versus depth (or TL light gradient) for a thick laser TLD following the triple pulse heating scheme. It indicates that the TL light intensities corresponding to the three pulses are characteristic to the depth of the TLD. 
Figures 84 through 86 show another set of pulsed heating schemes, the resulting temperature and TL light distributions. Figure 84(a) displays a 10W-10W-4W heating scheme. That is, it shows a $0.7 \mathrm{sec} 10 \mathrm{~W}$ first pulse followed by $1.4 \mathrm{sec}$ of cooling, which is then followed by a $1.0 \mathrm{sec} 10 \mathrm{~W}$ second pulse with $3.5 \mathrm{sec}$ cooling, and then another 2.0 $\sec 4 \mathrm{~W}$ (third pulse). For this heating scheme, the temperature distributions corresponding to times following the cooling after each pulse are shown in Figure 85(a). The third pulse uses a combination of low power and long duration, and was planned to heat the deep part of the TLD. Figure 84(b) is a similar example to the heating scheme of the Figure 84(a), and is intended for heating much deeper part of the TLD. The heating follows $10 \mathrm{~W}-4 \mathrm{~W}-$ $2 \mathrm{~W}$ scheme. That is, $0.7 \mathrm{sec} 10 \mathrm{~W}$ first pulse followed by $1.2 \mathrm{sec}$ of cooling, which is then followed by a $1.6 \mathrm{sec} 4 \mathrm{~W}$ second pulse with $2.0 \mathrm{sec}$ of cooling, and then another $3.5 \mathrm{sec}$ third pulse. Corresponding temperature distributions at times following the cooling of each pulse are shown in Figure 85(b). The calculated TL intensity versus TLD depth for the two heating schemes is shown in Figure 86. In Figure 86(a) the TL light generated due to the third pulse entirely overlaps with TL light due to the second pulse. Therefore, the TL light generated due to the third pulse does not add the deep dose information. In Figure 86(b), the TL light generated due to the third pulse, however, is contributed from a much deeper part of the TLD than that generated due to the second pulse. Therefore, the third pulse does add to the deep dose information. 
4.5 Unfolding of Depth-Dose Distribution and Optimization of Heating Scheme

\subsubsection{Unfolding of Depth-Dose Distribution with a Two-Pulse Heating Scheme}

Figure 87 shows the calculated glow curves produced by a two-pulse heating scheme applied to a thick laser TL dosimeter irradiated with the beta-particles emitted from Sr/Y-90 source. This two-pulse heating scheme uses a focused beam at $4 \mathrm{~W}$. The first pulse lasts $10 \mathrm{msec}$, it is followed by a $40 \mathrm{msec}$ cooling period, and then a second pulse of 15 msec. The first shorter pulse is intended to extract an accurate superficial dose and the second pulse is intended to extract the deep dose. The integrated TL intensities under the glow curve were used as input data to unfold the depth-dose distribution. Figure 88 shows the unfolded depth-dose distribution and the distribution calculated by the EGS4 (which is considered as the true depth-dose), respectively. In general, the agreement between the unfolded results and the true depth-dose is good for shallow regions of the TLD, but it is not good for deep regions.

Figure 89 shows another example of the calculated glow curves produced by another two-pulse heating scheme which uses an increased duration in the second pulse. This two-pulse heating scheme consists of a $10 \mathrm{msec}$ first pulse followed by a $40 \mathrm{msec}$ cooling period, and then a $20 \mathrm{msec}$ second pulse. Figure 90 shows the unfolded depthdose distribution and the distribution calculated by the EGS4, respectively. As shown, the unfolded data agree well with the EGS4 results, especially within the depth of $0.02 \mathrm{~cm}$ of the TLD. The results shown in Figures 88 and 90 confirm that a two-pulse heating scheme is good for unfolding the depth-dose distribution in shallow regions in the thick laser TLD, but not good for deep regions. The fact that the second pulse does not help in extracting deep dose information is due to the maximum temperature limit of $750 \mathrm{~K}$ at the TLD surface. The unfolded depth-dose distribution shown in Figure 88 is based on the results of 73 iterations with $10 \%$ convergence criterion using Eq (2.29). 
Successful unfolding of depth-dose distribution by pulsed heating depends on the shape of the response function produced by a designed heating scheme. An ideal set of response functions (i.e., TL light intensity vs. depth) should behave like a set of Gaussian distributions having their peaks located at various depths in the TLD. In practice, however, it is impossible to obtain such an ideal set of responses. With an uniform laser beam, however, it is possible to produce a set of response functions which can be used for depthdose unfolding.

\subsubsection{Optimum Two-Pulse Heating}

As discussed in Section 4.5.1, the unfolding of depth-dose distribution is based on the choice of the pulsed heating scheme. The optimum pulse-heating scheme for the thick laser TLD is based on a large number of trials of combinations of laser pulses and duration. These trials are by no means arbitrary. They were based on the general results (e.g., thermal and glow curve characteristics) obtained by single laser beam heating as described in Section 4.4.

Figure 91 shows the unfolded depth-dose data, which do not agree well with the depth-dose data calculated by EGS4. The two-pulse heating scheme in this case consists of a $20 \mathrm{msec}$ first pulse followed by a $40 \mathrm{msec}$ cooling period, and then a $72 \mathrm{msec}$ second pulse. Compared with the heating schemes in Figures 87 and 89, this heating scheme has long pulse duration in both the first and the second pulses. According to Figure 35, a pulse with $20 \mathrm{msec}$ duration results in temperature rise of $483 \mathrm{~K}$ at $0.25 \mathrm{~cm}$ depth with surface temperature of $630 \mathrm{~K}$ (See Figure 45).

In this study the two-pulse heating scheme shown in Figure 87 is an optimum twopulse heating scheme for the thick laser TLD. This scheme was chosen technically based on the procedure shown in Figures 2 and 15. The two-pulse heating scheme was used to obtain shallow depth-dose distribution, which is an unfolded depth-dose distribution in the shallow region obtained by heating the top (or front) surface of the TLD. The two-pulse 
heating scheme was also used to obtain deep depth-dose distribution, which is an unfolded depth-dose distribution in the deep region obtained by heating the bottom (or back) surface of the TLD.

\subsubsection{Unfolding of Depth-Dose Distribution with Three-Pulse Heating Scheme}

Figure 92(a) shows the calculated glow curve produced by the three-pulse heating scheme for a thick laser TL dosimeter irradiated with the beta-particles emitted from $\mathrm{Sr} / \mathrm{Y}$ 90 source. The three-pulsed heating scheme is a $10 \mathrm{~W}-10 \mathrm{~W}-4 \mathrm{~W}$ heating scheme as described in Figure 84. Figure 93 shows the unfolded depth-dose distribution and the depth-dose distribution calculated by EGS4 (which is considered as the true depth-dose), respectively. As shown, the unfolded data agree with the EGS4 results to a depth of 0.07 $\mathrm{cm}$ of the TLD (as opposed to $0.02 \mathrm{~cm}$ with the two-pulsed heating case).

Figure 92(b) shows another example of the calculated glow curve produced by the three-pulse heating scheme, 10W-4W-2W as described in Figure 84. Figure 94 shows the unfolded depth-dose distribution and the depth-dose distribution calculated by the EGS4, respectively. As shown, the unfolded data agree with the EGS4 results to the depth of 0.2 $\mathrm{cm}$ depth of the TLD. The good agreement is attributed to the good response function produced by the uniform surface heating. Moreover, due to the advantage of relatively slow heating (i.e., deep heating), the three-pulse heating scheme can unfold the entire depth-dose distribution in the thick laser TLD. That is, the entire dèpth-dose distribution may be unfolded by applying both three-pulse heating schemes on both the top and the bottom of the TLD. Therefore, a total of 6 response functions are produced.

\subsubsection{Optimum Three-Pulse Heating}

Figure 94 indicates a better unfolded depth-dose distribution in the superficial region when duration of the first pulse is slightly changed. Figure 95 shows a $10 \mathrm{~W}-4 \mathrm{~W}-$ $2 \mathrm{~W}$ heating scheme, in which the duration of the first pulse is reduced by $0.1 \mathrm{sec}$ and the 
cooling time increased by $0.1 \mathrm{sec}$. Figure 95 also shows the corresponding temperature profiles on the thick laser TLD. Figure 96 shows the corresponding set of response functions. Figure 97 shows the calculated glow curve produced by this heating scheme for the thick laser TLD. Figure 98 shows unfolded depth-dose distribution and the distribution calculated by the EGS4. As shown, the unfolded data agree well with the EGS4 results to a depth of $0.2 \mathrm{~cm}$ of the depth.

Figure 99 shows the unfolded depth-dose distribution obtained for a thick laser TLD irradiated with gamma-rays emitted from the Cs-137 source. As shown, the unfolded data do not agree well with the EGS4 results. In this case, the heating scheme may need a change in the duration of the first pulse because the depth-dose from Cs-137 gamma-rays increases very rapidly in the superficial region. In general, the optimum heating scheme should be one pulsed-heating scheme which is capable of producing the unfolded depthdose distribution to approximate the EGS4 results for all types of depth-dose distributions.

Figure 100 is the optimum three-pulse heating scheme obtained following the procedure of Figures 2 and 15. As shown, the thick laser TLD was heated from the top and bottom surfaces by three-pulse heating schemes to obtain the entire depth-dose distribution. Figures 100 (a) and (b) show the heating scheme used for the top surface heating, and the bottom surface heating. Figure 101 shows corresponding temperature profiles. Figure 102 shows the calculated glow curves produced by each three-pulse heating scheme for a thick laser TLD, respectively. The TLD was irradiated with the beta-particles emitted from Sr/Y90 source. Figure 103 shows the unfolded depth-dose distribution and the depth-dose distribution calculated by EGS4. As shown, the unfolded data agree with the EGS4 result through the TLD. Various unfolded depth-dose distributions from the TLDs irradiated by pure or mixed field sources are shown in Appendix A-1.

Figures 104 through 106 show the variations of the unfolded depth-dose distribution based on various combinations of heating pulses. Figure 104 shows the unfolded depth-dose distribution calculated for a thick laser TLD heated with 3 pulses from 
the top surface and 1 pulse from the bottom surface. Figure 105 shows the unfolded depth-dose distribution for a thick laser TLD heated with 3 pulses from the top surface and 2 pulses from the bottom surface. Figure 106 shows the unfolded depth-dose distribution calculated for a thick laser TLD heated with 2 pulses from the top surface and 1 pulse from the bottom surface. 


\subsection{Deep and shallow Doses}

\subsubsection{Deep and Shallow Doses for Focused Beam}

Figures 107 through 113 show some of results of dose distributions calculated in the shallow dose region ( $0-0.02 \mathrm{~cm}$ depth) of the thick laser TLD exposed to radiation fields. The summary of shallow dose results were tabulated in Table 17. Table 17 illustrates that the pulsed heating of the focused beam works well for estimation of shallow doses within 5\% errors except for the radiation fields of Cs137, $59 \mathrm{keV}$, and $70 \mathrm{keV}$ sources. The estimated shallow dose from these radiation fields, however, have an agreement with the EGS4 doses less than $10 \%$ error.

Figures 114 through 118 show some of results of dose distributions calculated in the deep dose region ( $0-0.01 \mathrm{~cm}$ depth) of the thick laser TLD for radiation fields. The summary of deep dose results were tabulated in Table 18. Table 18 illustrates that the pulsed heating of the focused beam works well for estimation of deep doses within $25 \%$ errors except for the radiation fields of $16 \mathrm{keV}, 20 \mathrm{keV} \mathrm{x}$-rays, $59 \mathrm{keV} \mathrm{x}$-rays, $120 \mathrm{keV} \mathrm{x}$ rays, and $\mathrm{Sr} 90 / \mathrm{Y}$ (pure radiation fields), $20 \mathrm{keV}+\mathrm{Sr} 90 / \mathrm{Y}, 120 \mathrm{keV}+\mathrm{Sr} 90 / \mathrm{Y}$, and Cs137+Sr90/Y (mixed radiation fields). The estimated deep dose from these radiation fields, however, have an agreement with the EGS4 dose less than $30 \%$ error. In general, the high errors for the deep dose estimation were considered since the actual depth-doses calculated by Monte Carlo simulation has considerable fluctuations in the deep dose region. Those errors would be reduced by using the least-squares fitting data for the EGS4 depthdoses.

\subsubsection{Deep and Shallow Doses for Uniform Beam}

Shallow Doses Figures 119 through 123 shows some of results of calculated dose distributions in the shallow dose region from the surface to $0.01 \mathrm{~cm}$ depth. The summary 
of shallow dose results for the various radiation fields was also tabulated in Table 19. In Table 19 the results indicate that the uniform heating scheme works well for estimation of deep doses. Table 19 illustrates that the pulsed heating of the focused beam works well for estimation of shallow doses within around 15\% errors except for the radiation fields of 59 $\mathrm{keV}, 70 \mathrm{keV} x$-rays, Cs137 (pure radiation fields), $36 \mathrm{keV}+\mathrm{Cs} 137,70 \mathrm{keV}+\mathrm{Cs} 137$, and

. $20 \mathrm{keV}+\mathrm{Cs} 137$ (mixed radiation fields).

Deep Doses Figures 124 through 128 shows some of results of calculated deep doses and dose distributions in the deep dose region from $0.37 \mathrm{~cm}$ to the bottom of TL dosimeter with $10 \%$ errors. The summary of deep dose results for the various radiation fields was also tabulated in Table 20 . Table 20 shows that the method performed well overall, within around 15\%, for the deep dose estimation for most of the radiation categories except for the $59 \mathrm{keV}$ and $70 \mathrm{keV}$ x-rays radiation fields, and the 120 $\mathrm{keV}+\mathrm{S} r 90 / \mathrm{Y}$ mixed radiation field. In general, the high errors for the deep dose estimation were considered since the actual depth-doses calculated by Monte Carlo simulation has considerable fluctuations in the deep dose region. Those errors would be reduced by using the least-squares fitting data for the EGS4 depth-doses. 


\subsection{Noise Analysis Results}

\subsubsection{Noise of Depth-Dose for Focused Beam}

Figures 129 and 130 show variations in the TL glow curves corresponding to the thick laser TLD system due to systematic errors of $\pm 2 \%$ independently added to the thermal parameters (i.e., beam density, absorption coefficient, thermal conductivity, and specific heat) following the focused laser beam heating. Figures 131 and 132 show the \% relative errors in $T L$ intensities determined for $\pm 2 \%$ independently added to the thermal parameters without the added errors. Table 21 illustrates the minimum and maximum values of relative errors (\%) of TL intensities corresponding to the thick laser TLD system due to systematic errors of $\pm 1, \pm 2, \pm 3$, and $\pm 5 \%$ independently added to the thermal parameters to TL intensities without the added errors. As an example, from Table 21, adding systematic error of $-1 \%$ beam density, the TL light intensity would have a minimum change of $-22.1 \%$ and maximum change of $-1.9 \%$ relative to TL intensity without any error. The TL intensity responses to beam intensity and absorption coefficient linearly increased with increasing added errors, while response decreased with increasing thermal conductivity and specific heat increased negatively. Generally, TL glow curves were very sensitive to the systematic errors of thermal parameters. The small amount of errors gave the wide range of response errors. In Table 21, the thermal conductivity showed relatively small sensitivity, comparing with other parameters.

Figures 133 and 134 show variations of depth-doses corresponding to the thick laser TLD due to systematic errors of $\pm 2 \%$ independently added to the thermal parameters. Table 22 illustrates the values of relative errors (\%) of shallow and deep doses

corresponding to the thick laser TLD system due to systematic errors of $\pm 1, \pm 2, \pm 3$, and $\pm 5 \%$ independently added to the thermal parameters to TL intensities without the added errors. In Table 22(a), from the systematic error of $-1 \%$ beam density the shallow dose has 
$-10.0 \%$ change relative to the shallow dose without any error, while the deep dose has $-1.9 \%$ change relative to the deep dose without any error. In Table 22(b), adding systematic error of $+1 \%$ beam density, the shallow dose would have a change of $11.1 \%$ relative to the shallow dose without any error, while the deep dose would have a change of $13.0 \%$ relative to the deep dose without any error. According to the calculation, the $\pm 1 \%$ of systematic error may come from either the difference of $40 \mathrm{~mW}$ of laser power level or the difference of $32 \mu \mathrm{m}$ for $0.032 \mathrm{~cm}$ diameter focused laser beam at the TLD surface. In order to obtain the shallow and deep doses within $\pm 15 \%$ accuracy, the allowance of $32 \mu \mathrm{m}$ for focused laser beam diameter or the allowance of $40 \mathrm{~mW}$ of laser power is required.

\subsubsection{Noise of Depth-Dose for Uniform Beam}

Figures 135 and 138 show variations in the TL glow curves corresponding to the thick laser TLD system due to systematic errors of $\pm 5 \%$ independently added to the thermal parameters (i.e., beam density, absorption coefficient, thermal conductivity, and specific heat) following the uniform laser beam heating. Figures 139 and 140 show the \% relative errors in $T L$ intensities determined for $\pm 2 \%$ independently added to the thermal parameters without the added errors. Table 23 illustrates the minimum and minimum values of relative errors (\%) of TL intensities corresponding to the thick laser TLD system due to systematic errors of $\pm 2, \pm 3, \pm 4$, and $\pm 5 \%$ independently added to the thermal parameters to TL intensities without the added errors. As an example, from Table 23(a), adding systematic error of $-2 \%$ to beam density, the TL light intensity would have a maximum change of $-68.5 \%$ and minimum change of $-2.3 \%$ relative to $T L$ intensity without any error. The TL intensity responses to beam intensity and absorption coefficient linearly increased with increasing added errors, while response decreased with increasing thermal conductivity and specific heat increased negatively. Generally, TL glow curves were very sensitive to the systematic errors of thermal parameters. The small amount of errors gave the wide range of 
response errors. In Table 23 , the thermal conductivity showed relatively small sensitivity, comparing with other parameters.

Figures 141 and 142 show variations of depth-doses corresponding to the thick laser TLD due to systematic errors of $\pm 5 \%$ independently added to the thermal parameters. Table 24 illustrates the values of relative errors (\%) of shallow and deep doses corresponding to the thick laser TLD system due to systematic errors of $\pm 2, \pm 3, \pm 4$, and $\pm 5 \%$ independently added to the thermal parameters to TL intensities without the added errors. In Table 24 , from the systematic error of $-2 \%$ beam density the shallow dose has $-19.3 \%$ change relative to the shallow dose without any error, while the deep dose has $-7.7 \%$ change relative to the deep dose without any error. In Table 24 , adding systematic error of $+2 \%$ beam density, the shallow dose would have a change of $22.9 \%$ relative to the shallow dose without any error, while the deep dose would have a change of $7.9 \%$ relative to the deep dose without any error. According to the calculation, the systematic error of $\pm 2 \%$ may come from the differences of $\pm 200, \pm 80$, and $\pm 40 \mathrm{~mW}$ of $10 \mathrm{~W}, 4 \mathrm{~W}$, and $2 \mathrm{~W}$ laser power levels or the difference of $30 \mu \mathrm{m}$ for $0.3 \mathrm{~cm}$ square laser beam at the TLD surface. In order to obtain the shallow and deep doses within $\pm 25 \%$ accuracy, the allowance of $30 \mu \mathrm{m}$ for square laser beam size or the allowance of $\pm 200, \pm 80$, and \pm 40 $\mathrm{mW}$ for $10 \mathrm{~W}, 4 \mathrm{~W}$, and $2 \mathrm{~W}$ laser power levels, respectively, is required. 


\subsection{Results of Energy Response Calculation}

Figure 143 shows the theoretical energy response of $\mathrm{LiF} T L$ dosimeter at $7 \mathrm{mg} / \mathrm{cm}^{2}$ as a function of mean beta energy obtained from conventional heating. The experimental results for $0.09 \mathrm{~cm}$ thick TLD (with $7 \mathrm{mg} / \mathrm{cm}^{2}$ filter) [Gupta, 1983] is also given for comparison to those calculated energy responses. It should be noted that the calculation has been based upon a TL layer without any filter on that. Calculation were performed on TL dosimeters irradiated by 50,000 particle history from Pm-147 $\left(E_{\mathrm{av}}=0.062 \mathrm{MeV}\right), \mathrm{Tl}-204$ $\left(E_{a v}=0.238 \mathrm{MeV}\right)$, and $\mathrm{Y}-90\left(\mathrm{E}_{\mathrm{av}}=0.934 \mathrm{MeV}\right)$, respectively. In comparison, Figure 143 shows a decreasing energy response occurs with low energy betas and energy response is improved by decreasing dosimeter thickness. The difference between data exists since the results from Gupta was based on $7 \mathrm{mg} / \mathrm{cm}^{2}$ filtered TL dosimeter. In addition, Figure 143 illustrates that the theoretical energy responses may be utilized to estimate energy response of TL layers.

Figure 144 shows the theoretical energy response of LiF TL dosimeter with different thickness by conventional heating. Calculation were performed on TL dosimeters irradiated by 50000 history from Pm-147 $\left(E_{\mathrm{av}}=0.224 \mathrm{MeV}\right), \mathrm{Tl}-204\left(\mathrm{E}_{\mathrm{av}}=0.766 \mathrm{MeV}\right)$, and $Y-90\left(E_{a v}=2.28 \mathrm{MeV}\right)$, respectively. In Figure the response of the thin TL dosimeter, $0.005 \mathrm{~cm}\left(\sim 13 \mathrm{mg} / \mathrm{cm}^{2}\right)$ was considerably better than $0.09 \mathrm{~cm}\left(\sim 240 \mathrm{mg} / \mathrm{cm}^{2}\right)$ thick. dosimeter.

The theoretical energy responses of LiF TL dosimeter calculated by focused and uniform laser beam heating are represented in Figures 145 and Figure 146, respectively. Laser beam heating has done on a $0.09 \mathrm{~cm}$ thick TL dosimeter with various heating time. Both Figures show the responses increase as heating time decreases. This is reasonable since differential heating was employed by different heating time as described in Chapter 3. This is encouraging since new TL dosimeter employs not several elements or thin TL layer 
but a single thick TLD. Therefore, the use of differential heating by laser beam on the thick TLD can solve the problems not only on superficial layer heating but also on the energy response, simultaneously. This provides a justification for the possible use of a single thick TLD for mixed beta/gamma radiation dosimetry. 


\subsection{Dosimetric Evaluation of Thick Laser TLD}

\subsubsection{Characteristics of Depth-Dose within TL Dosimeter}

The thick laser TL dosimeter may be used to measure the beta and gamma doses received in mixed beta/ gamma fields. TL dosimeter is exposed to a beta source, the front, can directly receive the incident betas. If a gamma-ray field is also present a uniform distribution of trapped electrons will be superimposed on the beta caused gradient. A depthdose curve may be established within TL dosimeter which may be characteristic of the particular type of mixed radiation field.

Figure 147 shows comparisons between calculated depth-dose and experimental depth-dose curves for the LiF TLD with the beta-particles from Pm-147, T1-204 and Sr/Y90 sources, respectively. The calculated depth-doses were calculated by the EGS4 Monte Carlo code. The experimental depth-doses are based on the results of the attenuation of beta spectra [Paliwal et al., 1976]. The experimental study indicates that the electron intensity measured initially decreases exponentially with the increasing thickness $\mathrm{x}$ of the $\mathrm{LiF}$ absorber: $N=N_{0} \exp (-\mu x / \rho)$ where $\mu / \rho$ is the average mass attenuation coefficient. There are general agreements between the calculated and experimental curves charcteristically. As results, it showed that a thick laser TL dosimeter is able to measure the depth-doses received in mixed beta/gamma fields.

\subsubsection{Temperature and TL Light Gradient}

The advantage inherent in the laser-TLD concept is the ability to obtain a signal which is indicative of the beta dose in mixed beta/gamma fields. Focused and uniform beam heating of a thick TL dosimeter were employed to achieve superficial heating, releasing shallow dose information, which could then be followed by more thorough 
heating of the dosimeter to extract depth-dose information; such an approach had advantages over existing approaches to mixed field dosimetry. The heating approach was enhanced by heating of TL dosimeter with a special pulsed heating scheme, i.e., pulsing of the beam with cooling periods and variable beam intensity, which resulted in a temporal output of signal which enables the extraction of depth-dose information. Two pulse-heating model of focused beam developed to perform the differential heating on the thick TL dosimeter has shown the temperature gradient and TL light gradient. As results of depthdose distributions, this model resulted in immediately that $T L$ material could detect and distinguish mixed field radiation. It was demonstrated that, in this simulations, one pulsed heating could be performed in order to obtain the TL glow curve by heating of the very thin superficial layer and another heating was used to obtain the TL glow curve from a little deeper layer than the first heating. Some amount of cooling was allowed between the two pulses helped control the surface temperature from rising to damaging levels.

Three-pulse heating model of uniform beam developed to perform the differential heating on the thick $T L$ dosimeter has also shown the temperature gradient and TL light gradient. This heating scheme used a different combination of laser power and heating time. A combination of high power and short time heating was used to heat and extract TL light in superficial layer. A combination of low power and slow heating was used to heat and extract TL light in deep layer. The intermediate layer heating was appropriately controlled from high and low power heating. Cooling between pulses was effectively used to control surface temperature and to increase temperature in a deep area.

In summary, the formation of temperature and TL light gradients within a thick laser TL dosimeter by both focused and uniform heating were satisfied with the use as a mixed field radiation dosimeter. The pulsed heating of a thick TLD resulted in release of the signal due to shallow dose, which was then followed by the release of the signal due to the deep dose as the deeper portions of the TLD were heated to TL temperature. 


\subsubsection{TL Light Attenuation and Energy Response}

TL Light Attenuation The sensitivity of TL dosimeter is not only related to TL light output generated per unit dose in dosimeter, but also the attenuation of TL light. Therefore, TL light attenuation should be considered when designing a personnel dosimeter which uses a thick laser TL dosimeter. As shown in Table 25, however, if an appropriate TL material is selected, then TL light attenuation may be neglected. As illustrated in Table 25, the mean free path (or effective thickness) for LiF (TLD-100) was approximately $114 \mathrm{~cm}$. This value indicates that if $\mathrm{LiF}$ is selected for a TL dosimeter, $\mathrm{TL}$ attenuation within the dosimeter may be neglected and it is a good requirement for the personnel dosimeter.

Energy Response The theoretical energy responses of LiF TL dosimeter calculated by focused and uniform laser beam heating showed the energy responses increased as heating time decreased. The improvement of the energy response may be possible only by using the differential heating of focused and uniform beams. This may be possible to mention that a thick laser TLD is satisfied with the use as a mixed field radiation dosimeter. 


\section{CHAPTER V}

\section{CONCLUSIONS}

A unique method of performing mixed-field beta/gamma dosimetry using a thick TLD heated with a pulsed laser beam, thick laser TL dosimeter method, has been studied. This study is entirely computational. The thick TL dosimeter modeled in the study was based on LiF thermoluminescence material, and it is $0.38 \mathrm{~cm}$ in thickness and $0.3 \times 0.3 \mathrm{~cm}$ in cross section. The thick laser TLD system employed a new heating technique, which consists of sequence of pulses, each with different power and duration from focused or uniform laser beam. These heating schemes cause differential heating at various TLD in depth, and result in a temporal output of signal. The resulting glow curves contain information which is characteristic of the depth-dose which produced them, which may be unfolded to extract the depth-dose distribution.

In conclusion, this study resulted in computer tools and their utilizations which include various numerical methods used to simulate laser heating of TLD and especially to maintain the performance of the proposed thick laser TLD.

A two-pulse heating scheme was adapted in this study for a focused laser beam of a $0.032 \mathrm{~cm}$ in diameter and a $4 \mathrm{~W}$ in power. This heating scheme employed pulsed heating, consisting of $10 \mathrm{~ms}$ first heating pulse, a $40 \mathrm{~ms}$ cooling period, and a $15 \mathrm{~ms}$ second heating pulse. This heating scheme was applied to the top surface of the TLD to obtain the shallow dose and to the bottom surface of the TLD to obtain the deep dose, respectively. The resulting glow curves were then used to unfold the depth-doses. The shallow and deep dose were then determined from the depth-dose curves, respectively. The results of the 
thick laser TLD system performance yielded good predictions of the shallow and deep doses in the various pure and mixed radiation fields. The two-pulse heating method using the focused beam showed that the thick laser TL dosimeter could be used as a personnel dosimeter with estimations of the shallow and deep doses within the relative errors of $5 \%$, and $25 \%$, respectively.

A three-pulse heating scheme was also obtained for an uniform laser beam having its power uniformly distributed in the $0.3 \mathrm{~cm} \times 0.3 \mathrm{~cm}$ beam cross section. This heating scheme also employed pulsed heating, consisting of two heating processes: top surface heating and bottom surface heating. The powers and duration of the pulses associated with the top surface heating are: $10 \mathrm{~W}-0.2 \mathrm{sec}, 4 \mathrm{~W}-1.8 \mathrm{sec}$, and $2 \mathrm{~W}-3.5 \mathrm{sec}$. The two cooling periods which separate the three pulses are $1.7 \mathrm{sec}$ and $1.3 \mathrm{sec}$. The powers and duration of the pulses associated with the bottom surface heating are: $10 \mathrm{~W}-0.6 \mathrm{sec}, 4 \mathrm{~W}$ $1.6 \mathrm{sec}$, and $2 \mathrm{~W}-3.5 \mathrm{sec}$. The two cooling periods which separate the three pulses are 1.3 sec and $2.0 \mathrm{sec}$. The top surface heating was applied first then followed with the bottom surface heating. The resulting glow curves were successfully used to unfold the depth-dose distribution in the TL dosimeter. The results of the thick laser TLD system performance yielded good predictions of the shallow and deep doses in the various pure and mixed radiation fields. This three-pulse heating method using the uniform beam showed that a thick laser TL dosimeter could be used as a personnel dosimeter with estimations of the shallow and deep doses within the relative errors of $15 \%$ respectively.

Finally, to help in deciding between the two heating schemes, a list of advantages and disadvatages has been drawn up. These are described by the following:

a) Two-pulse heating:

Advantages

1) Simpler mechanism for using a single laser power (i.e., $4 \mathrm{~W}$ ).

2) Fast heating $\left(\sim 10^{4} \mathrm{~K} / \mathrm{sec}\right)$ and accurate superficial depth-dose $(>10 \mathrm{msec})$. 
Disadvatages

1) Nonuniform heating of Gaussian beam produces high local temperature in the TLD.

2) High heating rate can shift the glow curve peak to incandescent region.

b) Three-pulse heating:

Advantages

1) Relatively low heating $\left(\sim 10^{2} \mathrm{~K} / \mathrm{sec}\right)$ and stable glow peak.

2) Uniform heating may control the thermal shock of the TLD.

3) Unfolding of depth-dose distribution in the TLD is possible.

Disadvatages

1) Relatively complicated mechanism for beam shaping.

2) Changing laser powers may reduce reproducibility. 


\section{CHAPTER VI}

\section{RECOMMENDATIONS FOR FUTURE WORK}

The future work relating to the development of a thick laser thick laser TL dosimeter will be focused on (a) the construction of a prototype thick laser TLD heating system, (b) practical applications to validate the theoretical work (correlation between theoretical and experimental works), and (c) the study of uncertainty due to nonuniformity of parameters.

\subsection{Construction of the Thick TLD Heating System}

A schematic diagram of a thick laser TLD system is presented in Figure 148. In this figure, the TLD is heated automatically in two laser beam heating chambers. One chamber is for the top heating of TLD and the other chamber is for the bottom heating of the TLD. A guiding channel or moving channel can be used to deliver TLDs into the heating chamber automatically. As simulated in the theoretical study, TL dosimeter is heated on the top and the bottom, respectively, using two pulsed beams from the focused laser. In the uniform laser beam case, TLD is heated by three pulsed variable power heating on the top and the bottom, respectively. The heating duration of the laser beam is controlled by the timer,

which has a function of programmed time settings. Heating chambers may be kept in vacuum condition, but convection boundary by nitrogen atmosphere may be possible. TL light is collected by the photomultiplier (PM) tube during each heating duration. The PM tube is then connected to the multichannel analyzer (MCA) or personal computer (PC) for on-line data acquisition and processing. 


\subsection{Practical Application of the TL Dosimeter}

In practical application of a prototype TL dosimeter system, the unfolded dose value should be validated as the actual dose. To do this, the dosimeter will be exposed to the known dose using the standard source. The heating procedure will be applied to collect the TL light to use it for unfolding of the depth-dose. From the known dose and unfolded dose, the shallow dose and deep dose calibration curves will be calculated. Therefore, the unfolded doses (shallow and deep doses) received from the unknown radiation field will be used as input data to the calibration curves to obtain the shallow and deep doses, respectively. In practice, however, the dosimeter calibration will have to be performed using a standard procedure such as ANSI Standard N13.11 [ANSI, 1981] or DOELAP personnel dosimetry performance testing program [DOE/EH-0026, 1986]. These studies include more detailed depth-dose simulations for NBS filtered $\mathrm{x}$-rays illustrated in Table 7.

\subsection{Uncertainty Study of Thermal Parameters}

The thermal parameters such as thermal conductivity, specific heat, and laser beam absorption coefficient are constants related to the material. Variations of these constants may occur due to a nonuniformity of material composition, or a paticular change of temperature. As the simulation study showed that the glow curve has a sensitivity due to those variations, careful work will have to be concentrated on the uncertainty study of thermal parameters. For this kind of work, statistical study of dose calculations for the TL dosimeter batches will be needed as the future work. 
Table 1. Various heat conduction and glow curve parameters for LiF TL material. The symbol $(\dagger)$ marked parameters were used through the mathematical models in this study.

\begin{tabular}{clll}
\hline $\begin{array}{c}\text { Parameter } \\
\text { Symbol }\end{array}$ & Definition & Unit & Values \\
\hline $\mathrm{k}$ & Thermal & & \\
& conductivity & $\mathrm{cal} / \mathrm{s} / \mathrm{cm} / \mathrm{K}$ & $\begin{array}{l}0.00658 \dagger \\
0.007125 \ddagger \\
0.00336 \mathrm{~g}\end{array}$ \\
& & & $0.0282 \S$ \\
$\rho$ & LiF density & $\mathrm{g} / \mathrm{cm}^{3}$ & $2.635 \dagger$ \\
& & & $2.4 \ddagger \mathrm{J}$ \\
$\mathrm{C}_{\mathrm{p}}$ & LiF specific & & $2.7 \S$ \\
& heat & $\mathrm{cal} / \mathrm{g} / \mathrm{K}$ & $0.373 \dagger$ \\
& & & $0.431 \ddagger$ \\
& & & $0.29 \S$ \\
$\mu$ & LiF absorption & $\mathrm{g} / \mathrm{cm}^{3}$ & 40 \\
& coefficient & & \\
$\mathrm{R}_{\mathrm{f}}$ & LiF reflection & & 0.0 \\
$\mathrm{k}_{\mathrm{b}}$ & coefficient & & \\
& Boltzmann & $\mathrm{eV} / \mathrm{K}$ & $0.862 \times 10^{-4}$ \\
\hline
\end{tabular}

$\uparrow$ Approximately one third of the thermal conductivity measured for a single crystal at room temperature [Abtahi et al., 1985]

† [Grupen-Shemansky, 1989]

I [Quam, 1983 a]

$\S$ A value averaged within a temperature range 0 - $500^{\circ} \mathrm{C}$ [Gotlib et al., 1984] 
Table 2. Summary of input data to run EGS4 electron/photon transport code.

\begin{tabular}{lll}
\hline $\begin{array}{l}\text { Variable } \\
\text { name }\end{array}$ & \multicolumn{1}{c}{ Description } & $\begin{array}{l}\text { Numerical } \\
\text { value used }\end{array}$ \\
\hline NMED & Number of media being used & 1 \\
ZBIN & Number of depth bins & $0.001 \mathrm{~cm}$ \\
& Charged particle cutoff energies & $0.001 \mathrm{MeV}$ \\
ECUT & in MeV & \\
PCUT & Photon cutoff energies in MeV & $0.001 \mathrm{MeV}$ \\
ZBOUND & Slab thickness & $0.38 \mathrm{~cm}$ \\
NSPEC & Number of energy bins & 44 \\
NCASE & Number of histories & 50000 \\
IQIN & Incident particle description & $-1($ electron $)$ \\
& & $0($ photon) \\
EIN & Kinetic energy & E +0.511 MeV(electron) \\
& & E MeV(photon) \\
IRIN & Starting region & 2 \\
WTIN & Weight for variance reduction & 1 \\
& & \\
\hline
\end{tabular}


Table 3. Summary of benchmark heat conduction and TL glow curve calculations of laser beam heating on $\mathrm{LiF}$ TL materials.

\begin{tabular}{|c|c|c|c|c|c|}
\hline $\begin{array}{l}\text { Beam } \\
\text { geometry }\end{array}$ & $\begin{array}{l}\text { Beam } \\
\text { diameter } \\
(\mathrm{cm})\end{array}$ & $\begin{array}{l}\text { Laser } \\
\text { power } \\
\text { (Watts) }\end{array}$ & $\begin{array}{l}\text { Material } \\
\text { dimensions } \\
\left(\mathrm{cm}^{3}\right)\end{array}$ & $\begin{array}{l}\text { Simulation } \\
\text { methods }\end{array}$ & $\begin{array}{l}\text { Comparison } \\
\text { with literature }\end{array}$ \\
\hline \multirow[t]{2}{*}{$\begin{array}{l}\text { Focused } \\
\text { (Circular, } \\
\text { Gaussian) }\end{array}$} & 0.084 & 4.93 & $0.3 \times 0.3 \times 0.09$ & $\begin{array}{l}\text { Analytical } \\
\text { Numerical } \dagger\end{array}$ & $\begin{array}{l}\text { Simulation and } \\
\text { Experiment } \\
\text { [Abtahi et al., 1985] }\end{array}$ \\
\hline & 0.059 & $\begin{array}{l}1.8 \\
2.4 \\
2.7\end{array}$ & $0.3 \times 0.3 \times 0.0027$ & Numerical $\dagger^{\dagger}$ & $\begin{array}{l}\text { Experiment } \\
\text { [Braunlich, 1990] }\end{array}$ \\
\hline $\begin{array}{l}\text { Unfocused } \\
\text { (Circular, } \\
\text { Gaussian) }\end{array}$ & 0.170 & 4 & $0.3 \times 0.3 \times 0.038$ & Numerical $\dagger$ & $\begin{array}{l}\text { Experiment } \\
\text { [Grupen-Shemansky } \\
\text { 1989] }\end{array}$ \\
\hline $\begin{array}{l}\text { Square } \\
\text { (Uniform) }\end{array}$ & $\begin{array}{l}0.3 \mathrm{~cm} \\
\text { square }\end{array}$ & 10 & $0.3 \times 0.3 \times 0.038$ & Numericalł & $\begin{array}{l}\text { Experiment } \\
\text { [Braunlich, 1990] }\end{array}$ \\
\hline
\end{tabular}


Table 4. Specifications of focused and unfocused laser system for a Laakmann Electro-Optics RF-445 laser [Laakmann; Abtahi et al., 1985; GrupenShemansky, 1989].

\begin{tabular}{cll}
\hline $\begin{array}{l}\text { Parameter } \\
\text { Symbols }\end{array}$ & \multicolumn{1}{c}{ Definitions } & Values \\
\hline $2 \omega_{\mathrm{i}}$ & Laser cavity beam waist & $1.35 \mathrm{~cm}$ \\
$\mathrm{~d}$ & Beam divergence & $10 \pm 1.5 \mathrm{mrads}$ \\
$\mathrm{z}_{\mathrm{f}}$ & Rayleigh distance & $13.5 \mathrm{~cm}$ \\
$\mathrm{z}_{\mathrm{i}}$ & Laser waist to lens distance & $50.0 \mathrm{~cm}$ \\
$\mathrm{f}$ & Lens focal distance & $10.0 \mathrm{~cm}$ \\
$\omega_{\mathrm{f}}$ & laser beam focal waist radius & $0.016 \mathrm{~cm}$ \\
$\omega_{\mathrm{o}}$ & Laser waist radius & $0.085 \mathrm{~cm}$ \\
\hline
\end{tabular}


Table 5. Summary of mixed field dosimetry calculations using laser beam on a thick laser TLD.

\begin{tabular}{|c|c|c|c|c|}
\hline $\begin{array}{l}\text { Beam } \\
\text { geometry }\end{array}$ & $\begin{array}{l}\text { Beam } \\
\text { diameter } \\
(\mathrm{cm})\end{array}$ & $\begin{array}{l}\text { Laser } \\
\text { power } \\
\text { (Watts) }\end{array}$ & $\begin{array}{l}\text { Computer } \\
\text { model }\end{array}$ & Output \\
\hline $\begin{array}{l}\text { Focused } \\
\text { (Circular, } \\
\text { Gaussian) }\end{array}$ & 0.032 & 4 & $\mathrm{ADI} \uparrow$ & $\begin{array}{l}\text { For single-pulse heating: } \\
\text { - Temperature profiles } \\
\text { - Z-dependence of focused } \\
\text { beam } \\
\text { - Heating rate } \\
\text { - Variations of glow curves } \\
\text { - Surface temperature profile }\end{array}$ \\
\hline $\begin{array}{l}\text { Unfocused } \\
\text { (Circular, } \\
\text { Gaussian) }\end{array}$ & 0.170 & 4 & ADI & $\begin{array}{l}\text { For multiple-pulse heating: } \\
\text { - Pulsed heating } \\
\text { - Response functions } \\
\text { - Depth-doses } \\
\text { - Deep and shallow doses }\end{array}$ \\
\hline $\begin{array}{l}\text { Square } \\
\text { (Uniform) }\end{array}$ & $\begin{array}{l}0.3 \mathrm{~cm} \\
\text { square }\end{array}$ & $\begin{array}{r}10 \\
4 \\
2\end{array}$ & Explicit & \\
\hline
\end{tabular}

$\dagger$ Alternating Direction Implicit (ADI) technique

Explicit technique 
Table 6. Two-pulse heating approaches of focused laser beam.

\begin{tabular}{|c|c|c|c|}
\hline $\begin{array}{l}\text { Heating } \\
\text { scheme }\end{array}$ & $\begin{array}{l}\text { First } \\
\text { heating }\end{array}$ & $\begin{array}{l}\text { Cooling } \\
\text { period }\end{array}$ & $\begin{array}{l}\text { Second } \\
\text { heating }\end{array}$ \\
\hline $\begin{array}{l}1 \\
2\end{array}$ & $\begin{array}{l}10 \mathrm{~ms}(4 \mathrm{~W}) \\
10 \mathrm{~ms}(4 \mathrm{~W})\end{array}$ & $\begin{array}{l}40 \mathrm{~ms} \text { (no power) } \\
40 \mathrm{~ms} \text { (no power) }\end{array}$ & $\begin{array}{l}15 \mathrm{~ms} \mathrm{(4W)} \\
20 \mathrm{~ms}(4 \mathrm{~W})\end{array}$ \\
\hline
\end{tabular}


Table 7. DOELAP radiation fields for dosimeter calibration [DOE/EH-0026, 1986]. These radiation fields were used to calculate the actual depth-doses within the thick TL dosimeter by EGS4 code. 50,000 history Monte Carlo simulation was performed for every single source, and a 1:1 mixing ratio applied to sums of single source results to obtain mixed sources.

\begin{tabular}{|c|c|c|}
\hline $\begin{array}{l}\text { Source } \\
\text { number }\end{array}$ & $\begin{array}{l}\text { Radiation } \\
\text { field }\end{array}$ & Description \\
\hline $\begin{array}{l}1 \\
2 \\
5 \\
8 \\
11 \\
14 \\
15 \\
27 \\
29 \\
3 \\
6 \\
9 \\
12 \\
16 \\
4 \\
7 \\
10 \\
13 \\
18 \\
20 \\
22 \\
24 \\
26\end{array}$ & $\begin{array}{l}\text { K16 } \\
\text { M30 } \\
\text { S60 } \\
\text { M150 } \\
H 150 \\
\text { K59 } \\
\text { Cs137 } \\
\text { Sr90/Y } \\
\text { TI204 } \\
\text { M30+TI204 } \\
\text { S60+TI204 } \\
\text { M150+TI204 } \\
\text { H150+TI204 } \\
\text { Cs137+TI204 } \\
\text { M30+Cs137 } \\
\text { S60+Cs137 } \\
\text { M150+Cs137 } \\
H 150+C s 137 \\
\text { M30+Sr90/Y } \\
\text { S60+Sr90/Y } \\
\text { M150+Sr90/Y } \\
H 150+S r 90 / Y \\
\text { Cs137+Sr9ON }\end{array}$ & 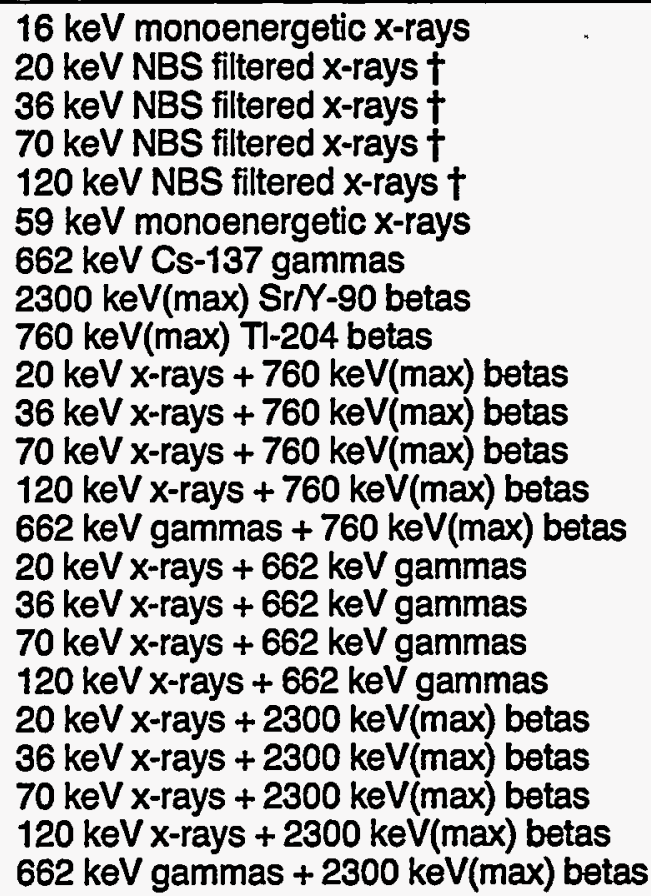 \\
\hline
\end{tabular}

† Monoenergetic x-rays used. 
Table 8. The surface to surface temperature difference for different thick TLDs.

\begin{tabular}{llc}
\hline $\begin{array}{l}\text { TLD } \\
\text { thickness }(\mathrm{cm})\end{array}$ & $\begin{array}{l}\text { Heating } \\
\text { time }(\mathrm{sec})\end{array}$ & $\begin{array}{l}\text { Difference } \\
\mathrm{DT}(\%) \dagger\end{array}$ \\
\hline 0.038 & 0.5 & 2.5 \\
& 0.8 & 1.9 \\
0.09 & 0.5 & 17.6 \\
0.38 & 0.8 & 15.1 \\
& 0.2 & 24.7 \\
& 0.5 & 45.1 \\
& 0.8 & 45 \\
\hline † Relative difference ratio $\left(\mathrm{T}_{\text {surface }}-\mathrm{T}_{\text {botrom }}\right) / \mathrm{T}_{\text {surface }} \times 100(\%)$
\end{tabular}


Table 9. Surface temperature variations by temperature dependence of thermal conductivity coefficient for $0.09 \mathrm{~cm}$ LiF TLD by $10 \mathrm{~W}$ uniform laser beam heating.

\begin{tabular}{cccc}
\hline Heating Time & $\mathrm{k}=\mathrm{k}_{\mathrm{o}} \dagger$ & $\mathrm{k}=\mathrm{k}(\mathrm{T}) \ddagger$ & \% Error I \\
\hline 0.1 & 359.03 & 360.72 & 0.5 \\
0.2 & 398.92 & 404.20 & 1.3 \\
0.3 & 432.76 & 442.47 & 2.2 \\
0.4 & 463.98 & 478.45 & 3.0 \\
0.5 & 494.05 & 513.34 & 4.0 \\
0.6 & 523.61 & 547.70 & 4.4 \\
0.7 & 552.94 & 581.78 & 5.0 \\
0.8 & 582.18 & 615.70 & 5.5 \\
\hline
\end{tabular}

\footnotetext{
$\dagger \mathrm{k}_{0}$ is thermal conductivity coefficient at $293 \mathrm{~K}\left(\mathrm{k}_{0}=0.0658 \mathrm{cal} / \mathrm{s} / \mathrm{cm} / \mathrm{K}\right)$ $\ddagger \mathrm{k}(\mathrm{T})=\mathrm{k}_{\mathrm{O}} * \mathrm{~T}_{0} / \mathrm{T}$, where $\mathrm{T}_{0}=293 \mathrm{~K}$

I $\%$ Error $=\left(\mathrm{k}(\mathrm{T})-\mathrm{k}_{0}\right) / \mathrm{k}_{\mathrm{O}} * 100(\%)$
} 
Table 10. Surface temperature variations by temperature dependence of thermal conductivity coefficient for $0.38 \mathrm{~cm} \mathrm{LiF} \mathrm{TLD} \mathrm{by} 10 \mathrm{~W}$ uniform laser beam heating.

\begin{tabular}{cccc}
\hline Heating Time & $\mathrm{k}=\mathrm{k}_{\mathrm{o}} \dagger$ & $\mathrm{k}=\mathrm{k}(\mathrm{T}) \ddagger$ & \% Error I \\
\hline 0.1 & 359.02 & 360.72 & 0.5 \\
0.2 & 398.63 & 403.97 & 1.3 \\
0.3 & 431.18 & 441.25 & 2.3 \\
0.4 & 459.54 & 475.09 & 3.3 \\
0.5 & 485.04 & 506.62 & 4.3 \\
0.6 & 508.41 & 536.44 & 5.2 \\
0.7 & 530.11 & 564.95 & 6.2 \\
0.8 & 550.84 & 592.40 & 7.0 \\
0.9 & 569.69 & 618.97 & 8.0
\end{tabular}

\footnotetext{
$\dagger \mathrm{ko}$ is thermal conductivity coefficient at $293 \mathrm{~K}(\mathrm{ko}=0.0658 \mathrm{cal} / \mathrm{s} / \mathrm{cm} / \mathrm{K})$

$\neq k(T)=k o *$ To $T$, where $T o=293 \mathrm{~K}$

I $\%$ Error $=(\mathrm{k}(\mathrm{T})-\mathrm{ko}) / \mathrm{ko} * 100(\%)$
} 
Table 11. Comparison of numerical glow curve with experimental glow curve. Numerical glow curve is the result from HANG1 and experimental glow curve is the result from Abtahi [Abtahi et al., 1985] following heating with a $0.084 \mathrm{~cm}$ diameter focused $4.93 \mathrm{~W}$ laser beam.

\begin{tabular}{llll}
\hline $\begin{array}{l}\text { Heating } \\
\text { time (sec) }\end{array}$ & $\begin{array}{l}\text { Experimental } \\
\text { glow curve } \\
\text { [Abtahi et al., 1985] }\end{array}$ & $\begin{array}{l}\text { Numerical } \\
\text { glow curve } \\
\text { (HANG1) }\end{array}$ & $\begin{array}{l}\text { Relative } \\
\text { \% error } \uparrow\end{array}$ \\
\hline 0.013 & 0.000 & 0.000 & 0.0 \\
0.035 & 0.070 & 0.031 & -55.7 \\
0.039 & 0.103 & 0.055 & -46.6 \\
0.043 & 0.137 & 0.088 & -35.8 \\
0.050 & 0.203 & 0.165 & -18.7 \\
0.058 & 0.304 & 0.250 & -17.8 \\
0.070 & 0.405 & 0.395 & -2.5 \\
0.079 & 0.506 & 0.537 & 6.1 \\
0.091 & 0.609 & 0.725 & 19.0 \\
0.105 & 0.711 & 0.814 & 14.5 \\
0.125 & 0.816 & 0.878 & 7.6 \\
0.138 & 0.852 & 0.898 & 5.4 \\
0.155 & 0.887 & 0.915 & 3.2 \\
0.198 & 0.913 & 0.935 & 2.4 \\
0.213 & 0.922 & 0.942 & 2.2 \\
0.246 & 0.937 & 0.959 & 2.3 \\
0.283 & 0.955 & 0.986 & 3.2 \\
0.300 & 1.000 & 1.000 & 0.0 \\
\hline
\end{tabular}

$\dagger$ (Numerical glow curve-Experimental glow curve)/Experimental glow curve x $100(\%)$ 
Table 12. Comparison of numerical glow curve with experimental glow curve. Numerical glow curve is the result from HANG1 and experimental glow curve is the result from Grupen-Shemansky [Grupen-Shemansky, 1989] following heating with a $0.170 \mathrm{~cm}$ diameter unfocused $4 \mathrm{~W}$ laser beam.

\begin{tabular}{llll}
\hline $\begin{array}{l}\text { Heating } \\
\text { time (sec) }\end{array}$ & $\begin{array}{l}\text { Experimental } \\
\text { glow curve }\end{array}$ & $\begin{array}{l}\text { Numerical } \\
\text { glow curve } \\
\text { (HANG1) }\end{array}$ & $\begin{array}{l}\text { Relative } \\
\% \text { error } \dagger\end{array}$ \\
\hline 0.400 & 0.042 & 0.022 & -47.6 \\
0.470 & 0.083 & 0.080 & -3.6 \\
0.530 & 0.137 & 0.179 & 30.6 \\
0.670 & 0.442 & 0.440 & -0.4 \\
0.740 & 0.737 & 0.574 & -22.1 \\
0.800 & 0.895 & 0.765 & -14.5 \\
0.930 & 1.000 & 1.000 & 0.0 \\
1.070 & 0.872 & 0.850 & -2.5 \\
1.200 & 0.716 & 0.684 & -4.5 \\
1.260 & 0.652 & 0.616 & -5.5 \\
1.330 & 0.604 & 0.550 & -8.9 \\
1.470 & 0.546 & 0.426 & -22.0 \\
1.600 & 0.519 & 0.342 & -34.1 \\
1.730 & 0.421 & 0.277 & -34.2 \\
\hline
\end{tabular}

$\dagger$ (Numerical glow curve-Experimental glow curve)/Experimental glow curve x 100 (\%) 
Table 13. Quantitative comparison of numerical glow curve with experimental glow curve obtained from a $0.0027 \mathrm{~cm}$ thick LiF TLD with a $0.3 \mathrm{~cm}$ square uniform $10 \mathrm{~W}$ laser beam.

\begin{tabular}{llll}
\hline $\begin{array}{l}\text { Heating } \\
\text { time (sec) }\end{array}$ & $\begin{array}{l}\text { Experimental } \\
\text { glow curve } \\
\text { [Braunlich, 1990] }\end{array}$ & $\begin{array}{l}\text { Numerical } \\
\text { glow curve } \\
\text { (HANG2) } \text { I }\end{array}$ & $\begin{array}{l}\text { Relative } \\
\text { \% error } \dagger\end{array}$ \\
\hline 0.140 & 0.020 & 0.001 & -95.0 \\
0.160 & 0.020 & 0.003 & -85.0 \\
0.180 & 0.024 & 0.007 & -70.8 \\
0.200 & 0.040 & 0.016 & -60.0 \\
0.220 & 0.066 & 0.036 & -45.4 \\
0.240 & 0.167 & 0.075 & -55.1 \\
0.260 & 0.318 & 0.138 & -56.6 \\
0.280 & 0.513 & 0.215 & -58.1 \\
0.300 & 0.550 & 0.257 & -53.3 \\
0.320 & 0.374 & 0.229 & -38.8 \\
0.340 & 0.496 & 0.197 & -60.3 \\
0.360 & 0.593 & 0.256 & -56.8 \\
0.380 & 0.763 & 0.379 & -50.3 \\
0.400 & 0.950 & 0.537 & -43.4 \\
0.420 & 0.974 & 0.777 & -20.2 \\
0.440 & 1.000 & 1.000 & 0.00 \\
0.460 & 0.743 & 0.781 & -5.10 \\
0.480 & 0.477 & 0.233 & -51.2 \\
0.500 & 0.280 & 0.011 & -96.1 \\
\hline
\end{tabular}

$\uparrow$ (Numerical glow curve-Experimental glow curve)/Experimental glow curve x 100 (\%) Data collected from the TLD mounted on $0.0125 \mathrm{~cm}$ thick glass substrate.

I Data collected from the TLD without the substrate. 
Table 14a. Least-squares fitting values of EGS4 depth-dose from $\mathrm{Sr} / \mathrm{Y}-90$ beta-particles in shallow dose region and related errors. $\left(\mathrm{Chi}^{\wedge} 2=2.33\right)$

\begin{tabular}{clcc}
\hline $\begin{array}{c}\text { Depth } \\
(\mathrm{cm})\end{array}$ & $\begin{array}{l}\text { EGS4 } \\
\text { data }(\mathrm{MeV})\end{array}$ & $\begin{array}{l}\text { Fitted } \\
\text { data }(\mathrm{MeV})\end{array}$ & $\begin{array}{l}\text { Fitting } \\
\text { error }(\%)\end{array}$ \\
\hline 0.000 & 385.088 & 381.024 & 1.0 \\
0.004 & 269.952 & 277.829 & -2.9 \\
0.008 & 206.184 & 207.186 & -0.5 \\
0.012 & 159.639 & 158.551 & 0.7 \\
0.016 & 127.371 & 124.809 & 2.0 \\
0.020 & 105.691 & 101.159 & 4.3 \\
0.024 & 88.937 & 84.361 & 5.1 \\
0.028 & 73.013 & 72.225 & 1.1 \\
0.032 & 61.202 & 63.275 & -3.4 \\
0.036 & 54.552 & 56.510 & -3.6 \\
0.040 & 47.145 & 51.256 & -8.7 \\
\hline † (EGS4 data-Fitted data)/EGS4 data x 100(\%) \\
\hline
\end{tabular}

Table 14b. Least-squares fitting values of EGS4 depth-dose from $\mathrm{Sr} / \mathrm{Y}-90$ beta-particles in shallow dose region and related errors. $\left(\mathrm{Chi}^{\wedge} 2=2.30\right)$

\begin{tabular}{clcc}
\hline $\begin{array}{c}\text { Depth } \\
(\mathrm{cm})\end{array}$ & $\begin{array}{l}\text { EGS4 } \\
\text { data }(\mathrm{MeV})\end{array}$ & $\begin{array}{l}\text { Fitted } \\
\text { data (MeV) }\end{array}$ & $\begin{array}{l}\text { Fitting } \\
\text { error (\%) }\end{array}$ \\
\hline 0.000 & 385.088 & 380.791 & 1.1 \\
0.004 & 269.952 & 277.925 & -2.9 \\
0.008 & 206.184 & 207.382 & -0.6 \\
0.012 & 159.639 & 158.736 & 0.6 \\
0.016 & 127.371 & 124.939 & 1.9 \\
0.020 & 105.691 & 101.224 & 4.2 \\
0.024 & 88.937 & 84.365 & 5.1 \\
0.028 & 73.013 & 72.182 & 1.1 \\
0.032 & 61.202 & 63.197 & -3.3 \\
0.036 & 54.552 & 56.411 & -3.4 \\
0.040 & 47.145 & 51.146 & -8.5 \\
\hline \$(EGS4 data-Fitted data)/EGS4 data x 100(\%) & \\
\hline
\end{tabular}


Table 15a. Least-squares fitting values of EGS4 depth-dose from $\mathrm{Sr} / \mathrm{Y}-90$ beta-particles in deep dose region and related errors. $\left(\mathrm{Chi}^{\wedge}{ }^{2}=2.33\right)$

\begin{tabular}{clcc}
\hline $\begin{array}{c}\text { Depth } \\
(\mathrm{cm})\end{array}$ & $\begin{array}{l}\text { EGS4 } \\
\text { data }(\mathrm{MeV})\end{array}$ & $\begin{array}{l}\text { Fitted } \\
\text { data }(\mathrm{MeV})\end{array}$ & $\begin{array}{l}\text { Fitting } \\
\text { error }(\%)\end{array}$ \\
\hline 0.356 & 0.270 & 0.256 & 5.1 \\
0.360 & 0.159 & 0.214 & -34.7 \\
0.364 & 0.130 & 0.175 & -34.1 \\
0.368 & 0.175 & 0.137 & 21.7 \\
0.372 & 0.035 & 0.101 & -189.5 \\
0.376 & 0.084 & 0.067 & 20.1 \\
\hline \$ (EGS4 data-Fitted data)/EGS4 data x 100(\%) & \\
\hline
\end{tabular}

Table 15b. Least-squares fitting values of EGS4 depth-dose from $\mathrm{Sr} / \mathrm{Y}-90$ beta-particles in deep dose region and related errors. $\left(\mathrm{Chi}^{\wedge} 2=2.30\right)$

\begin{tabular}{cllc}
\hline $\begin{array}{c}\text { Depth } \\
\text { (cm) }\end{array}$ & $\begin{array}{l}\text { EGS4 } \\
\text { data (MeV) }\end{array}$ & $\begin{array}{l}\text { Fitted } \\
\text { data (MeV) }\end{array}$ & $\begin{array}{l}\text { Fitting } \\
\text { error (\%) }\end{array}$ \\
\hline 0.356 & 0.270 & 0.207 & 23.3 \\
0.360 & 0.159 & 0.164 & -3.0 \\
0.364 & 0.130 & 0.123 & 5.6 \\
0.368 & 0.175 & 0.084 & 51.9 \\
0.372 & 0.035 & 0.047 & -35.2 \\
0.376 & 0.084 & 0.012 & 85.4 \\
\hline \%(EGS4 data-Fitted data)/EGS4 data x 100(\%) & \\
\hline
\end{tabular}


Table 16a. Least-squares fitting values of EGS4 depth-dose from Cs-137 gamma-rays in shallow dose region and related errors. $\left(\mathrm{Chi}^{\wedge} 2=0.24\right)$

\begin{tabular}{clcc}
\hline $\begin{array}{c}\text { Depth } \\
(\mathrm{cm})\end{array}$ & $\begin{array}{l}\text { EGS4 } \\
\text { data }(\mathrm{MeV})\end{array}$ & $\begin{array}{l}\text { Fitted } \\
\text { data }(\mathrm{MeV})\end{array}$ & $\begin{array}{l}\text { Fitting } \\
\text { error }(\%) \%\end{array}$ \\
\hline 0.000 & 0.252 & 0.397 & -57.5 \\
0.004 & 1.134 & 1.069 & 5.8 \\
0.008 & 1.417 & 1.673 & -18.0 \\
0.012 & 1.824 & 2.214 & -21.4 \\
0.016 & 2.200 & 2.697 & -22.6 \\
0.020 & 2.852 & 3.125 & -9.6 \\
0.024 & 3.526 & 3.502 & 0.7 \\
0.028 & 3.974 & 3.833 & 3.5 \\
0.032 & 4.558 & 4.120 & 9.6 \\
0.036 & 4.877 & 4.368 & 10.4 \\
0.040 & 4.932 & 4.757 & 3.5 \\
\hline † (EGS4 data-Fitted data)/EGS4 data x 100(\%) & \\
\hline
\end{tabular}

Table 16b. Least-squares fitting values of EGS4 depth-dose from Cs-137 gamma-rays in deep dose region and related errors. $\left(\mathrm{Chi}^{\wedge}{ }^{2}=0.24\right)$

\begin{tabular}{clcc}
\hline $\begin{array}{c}\text { Depth } \\
(\mathrm{cm})\end{array}$ & $\begin{array}{l}\text { EGS4 } \\
\text { data }(\mathrm{MeV})\end{array}$ & $\begin{array}{l}\text { Fitted } \\
\text { data }(\mathrm{MeV})\end{array}$ & $\begin{array}{l}\text { Fitting } \\
\text { error }(\%)\end{array}$ \\
\hline 0.356 & 5.089 & 4.908 & 3.6 \\
0.358 & 4.606 & 4.916 & -6.7 \\
0.360 & 5.203 & 4.926 & 5.3 \\
0.362 & 4.465 & 4.940 & -10.6 \\
0.364 & 4.459 & 4.957 & -11.1 \\
0.366 & 3.772 & 4.977 & -31.9 \\
0.368 & 4.369 & 5.001 & -14.5 \\
0.370 & 3.915 & 5.029 & -28.5 \\
0.372 & 4.327 & 5.062 & -17.0 \\
0.374 & 4.624 & 5.098 & -10.3 \\
0.376 & 4.434 & 5.140 & -15.9 \\
0.378 & 3.518 & 5.186 & -47.4 \\
\hline (EGS4 data-Fitted data)/EGS4 data x 100(\%) & \\
\hline
\end{tabular}


Table 17. Unfolded results of shallow doses from pulsed heating scheme of focused laser beam on the thick laser TLD exposed by single or mixed beta/gamma radiation. The radiation exposure was simulated by the EGS4 Monte Carlo code with 50,000 histories for each radiation field.

\begin{tabular}{|c|c|c|c|}
\hline $\begin{array}{l}\text { Source } \\
\text { type }\end{array}$ & $\begin{array}{l}\text { Unfolded } \\
\text { shallow dose }\end{array}$ & $\begin{array}{l}\text { EGS4 } \\
\text { shallow dose }\end{array}$ & $\begin{array}{l}\text { Relative } \\
\text { error (\%) }\end{array}$ \\
\hline 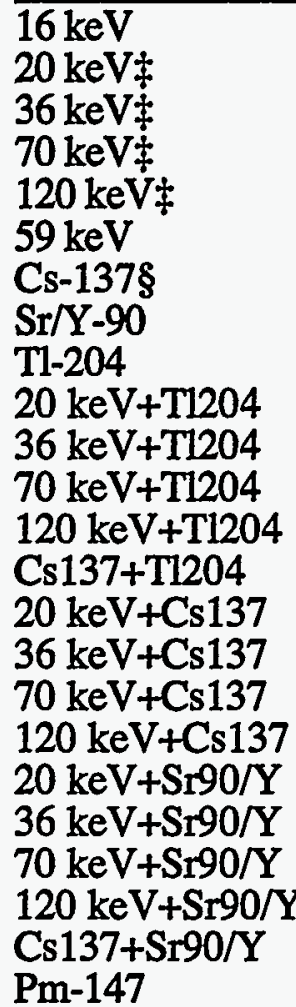 & $\begin{array}{l}2.82 \dagger(2.82 \pm 0.01) \rrbracket \\
1.76(1.76 \pm 0.01) \\
0.53(0.53 \pm 0.01) \\
0.28(0.28 \pm 0.00) \\
0.38(0.38 \pm 0.00) \\
0.26(0.26 \pm 0.00) \\
0.98(0.99 \pm 0.05) \\
305.0(303.2 \pm 15.6) \\
440.2(437.0 \pm 19.7) \\
441.9(438.7 \pm 19.6) \\
440.8(437.6 \pm 19.6) \\
440.5(437.4 \pm 19.6) \\
440.6(437.4 \pm 19.7) \\
440.6(437.4 \pm 19.6) \\
2.13(2.14 \pm 0.04) \\
0.91(0.92 \pm 0.03) \\
0.67(0.68 \pm 0.03) \\
0.76(0.77 \pm 0.03) \\
307.5(305.0 \pm 15.6) \\
306.3(303.8 \pm 15.6) \\
306.1(303.5 \pm 15.7) \\
306.2(303.7 \pm 15.6) \\
306.0(303.5 \pm 15.5) \\
363.5(358.9 \pm 125.8)\end{array}$ & $\begin{array}{l}2.73 \dagger(2.86 \pm 0.35) \mathbb{J} \\
2.07(1.78 \pm 0.21) \\
0.57(0.51 \pm 0.08) \\
0.25(0.30 \pm 0.08) \\
0.44(0.38 \pm 0.08) \\
0.27(0.24 \pm 0.04) \\
0.79(1.04 \pm 0.33) \\
314.1(291.9 \pm 31.0) \\
442.2(425.0 \pm 34.7) \\
444.3(426.8 \pm 34.7) \\
442.8(425.3 \pm 34.7) \\
442.4(425.3 \pm 34.7) \\
442.6(425.4 \pm 34.7) \\
442.5(425.4 \pm 34.7) \\
2.42(2.19 \pm 0.24) \\
0.92(0.93 \pm 0.09) \\
0.60(0.71 \pm 0.18) \\
0.79(0.79 \pm 0.15) \\
316.1(293.7 \pm 31.0) \\
314.6(292.4 \pm 31.1) \\
314.3(292.2 \pm 30.9) \\
314.5(292.3 \pm 31.0) \\
314.4(292.3 \pm 30.9) \\
352.2(284.3 \pm 138.9)\end{array}$ & $\begin{array}{l}1.5 \# \\
1.2 \\
2.7 \\
6.0 \\
0.8 \\
7.9 \\
5.3 \\
3.9 \\
2.8 \\
2.8 \\
2.9 \\
2.8 \\
2.8 \\
2.8 \\
2.4 \\
0.9 \\
4.6 \\
2.4 \\
3.8 \\
3.9 \\
3.9 \\
3.9 \\
3.8 \\
26.2\end{array}$ \\
\hline
\end{tabular}

$\dagger$ Reading of a single value

I Average value of 5 data points

\# (Averaged EGS4 data - Averaged Unfolded data)/(Averaged EGS4 data) $\times 100(\%)$

$\$$ Monoenergetic $x$-rays

$\$ 100,000$ histories 
Table 18. Unfolded results of deep doses from pulsed heating scheme of focused laser beam on the thick laser TLD exposed by single or mixed beta/gamma radiation. The radiation exposure was simulated by the EGS4 Monte Carlo code with 50,000 histories for each radiation field.

\begin{tabular}{|c|c|c|c|}
\hline $\begin{array}{l}\text { Source } \\
\text { type }\end{array}$ & $\begin{array}{l}\text { Unfolded } \\
\text { deep dose }\end{array}$ & $\begin{array}{l}\text { EGS4 } \\
\text { deep dose }\end{array}$ & $\begin{array}{l}\text { Relative } \\
\text { error (\%) }\end{array}$ \\
\hline $\begin{array}{l}16 \mathrm{keV} \\
20 \mathrm{keV}+ \\
36 \mathrm{keV}+ \\
70 \mathrm{keV} \\
120 \mathrm{keV} \$ \\
59 \mathrm{keV} \\
\mathrm{Cs}-137 \S \\
\mathrm{Sr} / \mathrm{Y}-90 \\
20 \mathrm{keV}+\mathrm{T} 1204 \\
36 \mathrm{keV}+\mathrm{T} 1204 \\
70 \mathrm{keV}+\mathrm{T} 1204 \\
120 \mathrm{keV}+\mathrm{T1204} \\
\mathrm{Cs} 137+\mathrm{T} 1204 \\
20 \mathrm{keV}+\mathrm{Cs} 137 \\
36 \mathrm{keV}+\mathrm{Cs} 137 \\
70 \mathrm{keV}+\mathrm{Cs} 137 \\
120 \mathrm{keV}+\mathrm{Cs} 137 \\
20 \mathrm{keV}+\mathrm{Sr} 90 / \mathrm{Y} \\
36 \mathrm{keV}+\mathrm{Sr} 90 / \mathrm{Y} \\
70 \mathrm{keV}+\mathrm{Sr} 90 / \mathrm{Y} \\
120 \mathrm{keV}+\mathrm{Sr} 90 / \mathrm{Y} \\
\mathrm{Cs} 137+\mathrm{Sr} 90 / \mathrm{Y}\end{array}$ & $\begin{array}{l}0.52 \dagger(0.53 \pm 0.01) J \\
0.59(0.60 \pm 0.01) \\
0.32(0.33 \pm 0.01) \\
0.18(0.18 \pm 0.00) \\
0.26(0.26 \pm 0.00) \\
0.25(0.24 \pm 0.00) \\
2.92(3.00 \pm 0.05) \\
0.06(0.06 \pm 0.00) \\
0.59(0.60 \pm 0.00) \\
0.32(0.33 \pm 0.01) \\
0.18(0.19 \pm 0.00) \\
0.26(0.26 \pm 0.00) \\
1.49(1.51 \pm 0.03) \\
2.08(2.11 \pm 0.04) \\
1.81(1.84 \pm 0.03) \\
1.67(1.70 \pm 0.03) \\
1.75(1.77 \pm 0.03) \\
0.65(0.66 \pm 0.01) \\
0.38(0.39 \pm 0.01) \\
0.24(0.25 \pm 0.01) \\
0.32(0.33 \pm 0.01) \\
1.55(1.57 \pm 0.03)\end{array}$ & $\begin{array}{l}0.64 \uparrow(0.76 \pm 0.13) \mathrm{J} \\
0.69(0.81 \pm 0.11) \\
0.25(0.42 \pm 0.23) \\
0.24(0.22 \pm 0.02) \\
0.49(0.37 \pm 0.11) \\
0.24(0.36 \pm 0.13) \\
3.03(3.87 \pm 0.58) \\
0.11(0.09 \pm 0.04) \\
0.69(0.81 \pm 0.11) \\
0.25(0.42 \pm 0.23) \\
0.24(0.22 \pm 0.02) \\
0.49(0.37 \pm 0.11) \\
1.65(1.93 \pm 0.17) \\
2.34(2.74 \pm 0.23) \\
1.89(2.36 \pm 0.31) \\
1.89(2.15 \pm 0.15) \\
2.14(2.30 \pm 0.15) \\
0.81(0.90 \pm 0.08) \\
0.36(0.52 \pm 0.18) \\
0.35(0.31 \pm 0.04) \\
0.61(0.46 \pm 0.15) \\
1.76(2.03 \pm 0.17)\end{array}$ & $\begin{array}{l}29.9 \# \\
25.7 \\
22.5 \\
15.2 \\
28.2 \\
33.2 \\
22.5 \\
35.5 \\
25.6 \\
22.0 \\
14.3 \\
27.9 \\
21.8 \\
23.0 \\
22.0 \\
21.2 \\
22.8 \\
26.4 \\
24.2 \\
20.0 \\
28.9 \\
22.3\end{array}$ \\
\hline
\end{tabular}

\footnotetext{
$\dagger$ Reading of a single value

I Average value of 5 data points

\# (Averaged EGS4 data - Averaged Unfolded data)/(Averaged EGS4 data) X $100(\%)$

$\$$ Monoenergetic $x$-rays

$\S 100,000$ histories
} 
Table 19. Unfolded results of shallow doses from pulsed heating scheme of uniform laser beam on the thick laser TLD exposed by single or mixed beta/gamma radiation. The radiation exposure was simulated by the EGS4 Monte Carlo code with 50,000 histories for each radiation field.

\begin{tabular}{|c|c|c|c|}
\hline $\begin{array}{l}\text { Source } \\
\text { type }\end{array}$ & $\begin{array}{l}\text { Unfolded } \\
\text { shallow dose }\end{array}$ & $\begin{array}{l}\text { EGS4 } \\
\text { shallow dose }\end{array}$ & $\begin{array}{l}\text { Relative } \\
\text { error (\%) }\end{array}$ \\
\hline $\begin{array}{l}16 \mathrm{keV} \\
20 \mathrm{keV}+ \\
36 \mathrm{keV}+ \\
70 \mathrm{keV}+ \\
120 \mathrm{keV}+ \\
59 \mathrm{keV} \\
\mathrm{Cs}-137 \\
\text { Sr/Y-90 } \\
\text { Tl-204 } \\
20 \mathrm{keV}+\mathrm{Tl} 1204 \\
36 \mathrm{keV}+\mathrm{Tl} 204 \\
70 \mathrm{keV}+\mathrm{T} 1204 \\
120 \mathrm{keV}+\mathrm{Tl} 1204 \\
\mathrm{Cs} 137+\mathrm{T} 1204 \\
20 \mathrm{keV}+\mathrm{Cs} 137 \\
36 \mathrm{keV}+\mathrm{Cs} 137 \\
70 \mathrm{keV}+\mathrm{Cs} 137 \\
120 \mathrm{keV}+\mathrm{Cs} 137 \\
20 \mathrm{keV}+\mathrm{Sr} 90 / \mathrm{Y} \\
36 \mathrm{keV}+\mathrm{Sr} 90 / \mathrm{Y} \\
70 \mathrm{keV}+\mathrm{Sr} 90 / \mathrm{Y} \\
120 \mathrm{keV}+\mathrm{Sr} 90 / \mathrm{Y} \\
\mathrm{Cs} 137+\mathrm{Sr} 90 / \mathrm{Y}\end{array}$ & $\begin{array}{l}2.77 \dagger(2.77 \pm 0.00) \rrbracket \\
1.84(1.84 \pm 0.00) \\
0.55(0.55 \pm 0.00) \\
0.32(0.32 \pm 0.00) \\
0.37(0.37 \pm 0.00) \\
0.25(0.29 \pm 0.00) \\
0.67(0.67 \pm 0.01) \\
251.1(247.4 \pm 5.3) \\
370.2(365.9 \pm 6.2) \\
368.7(364.8 \pm 5.5) \\
368.7(364.6 \pm 5.8) \\
369.0(364.9 \pm 5.9) \\
368.5(364.5 \pm 5.7) \\
368.2(364.2 \pm 5.7) \\
2.44(2.45 \pm 0.01) \\
1.19(1.20 \pm 0.01) \\
0.97(0.98 \pm 0.01) \\
1.01(1.02 \pm 0.01) \\
252.7(249.0 \pm 5.3) \\
251.3(247.6 \pm 5.2) \\
251.0(247.3 \pm 5.2) \\
251.0(247.3 \pm 5.2) \\
251.6(247.9 \pm 5.3)\end{array}$ & $\begin{array}{l}2.73 \dagger(2.84 \pm 0.27) J \\
2.07(1.86 \pm 0.19) \\
0.57(0.50 \pm 0.08) \\
0.25(0.27 \pm 0.03) \\
0.44(0.40 \pm 0.07) \\
0.27(0.22 \pm 0.04) \\
0.35(0.38 \pm 0.03) \\
314.1(291.0 \pm 21.3) \\
442.2(422.9 \pm 18.6) \\
444.3(424.7 \pm 18.7) \\
442.8(423.4 \pm 18.6) \\
442.5(423.2 \pm 18.6) \\
442.7(423.3 \pm 18.6) \\
442.6(423.3 \pm 18.5) \\
2.42(2.23 \pm 0.17) \\
0.92(0.87 \pm 0.05) \\
0.60(0.65 \pm 0.06) \\
0.79(0.78 \pm 0.05) \\
316.2(292.9 \pm 21.4) \\
314.7(291.5 \pm 21.3) \\
314.4(291.3 \pm 21.3) \\
314.6(291.4 \pm 21.3) \\
314.5(291.4 \pm 21.3)\end{array}$ & $\begin{array}{l}2.5 \# \\
0.8 \\
10.5 \\
18.7 \\
9.2 \\
28.6 \\
78.0 \\
15.0 \\
13.5 \\
14.1 \\
13.9 \\
13.8 \\
13.9 \\
14.0 \\
9.7 \\
37.0 \\
50.2 \\
30.4 \\
15.0 \\
15.1 \\
15.1 \\
15.1 \\
14.9\end{array}$ \\
\hline
\end{tabular}

$\uparrow$ Reading of a single value 9 Average value of 5 data points \# (Averaged EGS4 data - Averaged Unfolded data)/(Averaged EGS4 data) $\mathrm{x} 100(\%)$

$\$$ Monoenergetic $\mathrm{x}$-rays 
Table 20. Unfolded results of deep doses from pulsed heating scheme of uniform laser beam on the thick laser TLD exposed by single or mixed beta/gamma radiation. The radiation exposure was simulated by the EGS4 Monte Carlo code with 50,000 histories for each radiation field.

\begin{tabular}{|c|c|c|c|}
\hline $\begin{array}{l}\text { Source } \\
\text { type }\end{array}$ & $\begin{array}{l}\text { Unfolded } \\
\text { deep dose }\end{array}$ & $\begin{array}{l}\text { EGS4 } \\
\text { deep dose }\end{array}$ & $\begin{array}{l}\text { Relative } \\
\text { error (\%) }\end{array}$ \\
\hline $\begin{array}{l}16 \mathrm{keV} \\
20 \mathrm{keV} \ddagger \\
36 \mathrm{keV} \ddagger \\
70 \mathrm{keV} \ddagger \\
120 \mathrm{keV} \ddagger \\
59 \mathrm{keV} \\
\mathrm{Cs}-137 \\
\text { Sr/Y-90 } \\
20 \mathrm{keV}+\mathrm{Tl} 204 \\
36 \mathrm{keV}+\mathrm{Tl} 204 \\
70 \mathrm{keV+T1204} \\
120 \mathrm{keV}+\mathrm{Tl} 204 \\
\mathrm{Cs} 137+\mathrm{Tl} 204 \\
20 \mathrm{keV}+\mathrm{Cs} 137 \\
36 \mathrm{keV}+\mathrm{Cs} 137 \\
70 \mathrm{keV}+\mathrm{Cs} 137 \\
120 \mathrm{keV}+\mathrm{Cs} 137 \\
20 \mathrm{keV}+\mathrm{Sr} 90 / \mathrm{Y} \\
36 \mathrm{keV}+\mathrm{Sr} 90 / \mathrm{Y} \\
70 \mathrm{keV}+\mathrm{Sr} 90 / \mathrm{Y} \\
120 \mathrm{keV}+\mathrm{Sr} 90 / \mathrm{Y} \\
\mathrm{Cs} 137+\mathrm{Sr} 90 / \mathrm{Y}\end{array}$ & $\begin{array}{l}0.69 \dagger(0.69 \pm 0.00)] \\
0.82(0.82 \pm 0.00) \\
0.42(0.43 \pm 0.00) \\
0.25(0.25 \pm 0.00) \\
0.33(0.33 \pm 0.00) \\
0.29(0.26 \pm 0.00) \\
1.99(1.99 \pm 0.00) \\
0.10(0.10 \pm 0.00) \\
0.79(0.79 \pm 0.00) \\
0.41(0.41 \pm 0.00) \\
0.25(0.25 \pm 0.00) \\
0.31(0.32 \pm 0.00) \\
1.94(1.94 \pm 0.00) \\
2.81(2.81 \pm 0.00) \\
2.41(2.41 \pm 0.00) \\
2.24(2.24 \pm 0.00) \\
2.31(2.31 \pm 0.00) \\
0.83(0.83 \pm 0.00) \\
0.46(0.46 \pm 0.00) \\
0.29(0.30 \pm 0.00) \\
0.36(0.36 \pm 0.00) \\
1.99(2.00 \pm 0.00)\end{array}$ & $\begin{array}{l}0.64 \uparrow(0.82 \pm 0.12) J \\
0.69(0.80 \pm 0.10) \\
0.25(0.37 \pm 0.17) \\
0.24(0.21 \pm 0.03) \\
0.49(0.37 \pm 0.08) \\
0.24(0.45 \pm 0.08) \\
1.65(2.01 \pm 0.06) \\
0.11(0.10 \pm 0.04) \\
0.69(0.80 \pm 0.10) \\
0.24(0.37 \pm 0.17) \\
0.24(0.21 \pm 0.03) \\
0.49(0.37 \pm 0.08) \\
1.65(2.01 \pm 0.06) \\
2.34(2.81 \pm 0.04) \\
1.90(2.39 \pm 0.13) \\
1.89(2.23 \pm 0.04) \\
2.14(2.38 \pm 0.14) \\
0.81(0.91 \pm 0.08) \\
0.36(0.48 \pm 0.13) \\
0.36(0.32 \pm 0.02) \\
0.61(0.48 \pm 0.12) \\
1.77(2.12 \pm 0.10)\end{array}$ & $\begin{array}{l}16.6 \# \\
2.0 \\
13.6 \\
19.2 \\
12.1 \\
43.5 \\
1.19 \\
7.7 \\
1.0 \\
10.4 \\
15.5 \\
15.1 \\
3.7 \\
0.2 \\
0.9 \\
0.7 \\
3.0 \\
8.1 \\
3.8 \\
6.9 \\
23.6 \\
5.7\end{array}$ \\
\hline
\end{tabular}

$\dagger$ Reading of a single value

I Average value of 5 data points

\# (Averaged EGS4 data - Averaged Unfolded data)/(Averaged EGS4 data) $\mathrm{x} 100(\%)$

$\$$ Monoenergetic $x$-rays 
Table 21. Variations of TL glow curves for a thick laser TLD with systematic noises of various thermal parameters following pulsed heating with a $0.032 \mathrm{~cm}$ diameter focused 4 W laser beam. TL dosimeter exposed to Tl-204 beta particles was used for this calculations.

\begin{tabular}{|c|c|c|c|c|c|c|c|c|}
\hline \multirow[b]{3}{*}{ Beam intensity } & \multicolumn{8}{|c|}{$\%$ error in individual parameters } \\
\hline & \multicolumn{2}{|c|}{$-1 \%$} & \multicolumn{2}{|c|}{$-2 \%$} & \multicolumn{2}{|c|}{$-3 \%$} & \multicolumn{2}{|c|}{$-5 \%$} \\
\hline & $-22.1 \dagger$ & $-1.9 \ddagger$ & -31.5 & -3.8 & -42.8 & -5.8 & -67.1 & -9.8 \\
\hline $\begin{array}{l}\text { Absorption } \\
\text { coefficient }\end{array}$ & -17.8 & -1.0 & -28.7 & -1.9 . & -38.1 & -2.9 & -60.2 & -5.0 \\
\hline $\begin{array}{l}\text { Thermal } \\
\text { conductivity }\end{array}$ & 0.7 & 6.1 & 1.5 & 7.1 & 2.2 & 13.3 & 3.8 & 24.3 \\
\hline Specific heat & 1.0 & 11.8 & 2.0 & 31.0 & 3.0 & 53.2 & 5.1 & 92.3 \\
\hline
\end{tabular}

\begin{tabular}{|c|c|c|c|c|c|c|c|c|}
\hline \multirow[b]{3}{*}{ Beam intensity } & \multicolumn{8}{|c|}{$\%$ errors in individual parameters } \\
\hline & \multicolumn{2}{|c|}{$1 \%$} & \multicolumn{2}{|c|}{$2 \%$} & \multicolumn{2}{|c|}{$3 \%$} & \multicolumn{2}{|c|}{$5 \%$} \\
\hline & $1.7 \dagger$ & 12.8 & 3.5 & 33.1 & 5.2 & 56.6 & 8.5 & * \\
\hline $\begin{array}{l}\text { Absorption } \\
\text { coefficient }\end{array}$ & 0.8 & 11.7 & 1.6 & 30.4 & 2.4 & 49.8 & 3.9 & 84.8 \\
\hline $\begin{array}{l}\text { Thermal } \\
\text { conductivity }\end{array}$ & -4.5 & -0.9 & -8.2 & -1.6 & -12.1 & -2.4 & -19.2 & -4.0 \\
\hline Specific heat & -17.8 & -1.1 & -28.5 & -2.1 & -37.7 & -3.1 & -59.5 & -5.2 \\
\hline
\end{tabular}


Table 22. Variations of deep and shallow doses for a thick laser TLD with systematic noises of various thermal parameters following pulsed heating with a $0.032 \mathrm{~cm}$ diameter focused $4 \mathrm{~W}$ laser beam. TL dosimeter exposed to Tl-204 beta particles was used for this calculations.

\begin{tabular}{|c|c|c|c|c|c|c|c|c|}
\hline \multirow[b]{3}{*}{ Beam intensity } & \multicolumn{8}{|c|}{$\%$ errors in individual parameters } \\
\hline & \multicolumn{2}{|c|}{$-1 \%$} & \multicolumn{2}{|c|}{$-2 \%$} & \multicolumn{2}{|c|}{$-3 \%$} & \multicolumn{2}{|c|}{$-5 \%$} \\
\hline & $-10.0 \dagger$ & $-1.9 \div$ & -18.6 & -20.6 & -26.2 & -28.7 & -39.0 & -50.0 \\
\hline $\begin{array}{l}\text { Absorption } \\
\text { coefficient }\end{array}$ & -8.0 & -1.0 & -15.0 & -16.9 & -21.4 & -23.9 & -32.6 & -35.6 \\
\hline $\begin{array}{l}\text { Thermal } \\
\text { conductivity }\end{array}$ & 2.6 & 2.9 & 5.7 & 6.2 & 8.8 & 9.8 & 15.8 & 17.8 \\
\hline Specific heat & 8.0 & 9.5 & 17.4 & 21.1 & 27.6 & 34.6 & 53.0 & 73.5 \\
\hline
\end{tabular}

\begin{tabular}{|c|c|c|c|c|c|c|c|c|}
\hline \multirow[b]{3}{*}{ Beam intensity } & \multicolumn{8}{|c|}{$\%$ errors in individual parameters } \\
\hline & \multicolumn{2}{|c|}{$1 \%$} & \multicolumn{2}{|c|}{$2 \%$} & \multicolumn{2}{|c|}{$3 \%$} & \multicolumn{2}{|c|}{$5 \%$} \\
\hline & $11.1 \dagger$ & $13.0+$ & 24.1 & 29.2 & 39.5 & 50.1 & 77.3 & * \\
\hline $\begin{array}{l}\text { Absorption } \\
\text { coefficient }\end{array}$ & 8.5 & 10.1 & 18.3 & 22.4 & 29.2 & 36.8 & 55.5 & 78.3 \\
\hline $\begin{array}{l}\text { Thermal } \\
\text { conductivity }\end{array}$ & -2.9 & -3.1 & -5.4 & -5.9 & -7.9 & -8.5 & -12.4 & -13.3 \\
\hline Specific heat & -7.5 & -8.6 & -14.0 & -15.9 & -19.9 & -22.4 & -30.1 & -33.2 \\
\hline
\end{tabular}


Table 23. Variations of TL glow curves for a thick laser TLD with systematic noises of various thermal parameters following pulsed heating with a $0.3 \mathrm{~cm}$ square uniform laser beam. TL dosimeter exposed to $20 \mathrm{keV}$ x-rays was used for this calculations.

\begin{tabular}{|c|c|c|c|c|c|c|c|c|}
\hline \multirow[b]{3}{*}{ Beam intensity } & \multicolumn{8}{|c|}{$\%$ errors in individual parameters } \\
\hline & \multicolumn{2}{|c|}{$-2 \%$} & \multicolumn{2}{|c|}{$-3 \%$} & \multicolumn{2}{|c|}{$-4 \%$} & \multicolumn{2}{|c|}{$-5 \%$} \\
\hline & -68.51 & -2.3 & -74.0 & -3.5 & -89.0 & -5.2 & -83.1 & -5.8 \\
\hline $\begin{array}{l}\text { Absorption } \\
\text { coefficient }\end{array}$ & -15.8 & 17.0 & -15.7 & 24.5 & -51.0 & 22.6 & -51.0 & 20.8 \\
\hline $\begin{array}{l}\text { Thermal } \\
\text { conductivity }\end{array}$ & 0.4 & 72.6 & -0.1 & * & -1.6 & * & 0.3 & * \\
\hline Specific heat & 1.7 & * & 2.9 & * & 3.5 & * & 4.7 & $*$ \\
\hline
\end{tabular}

\begin{tabular}{|c|c|c|c|c|c|c|c|c|}
\hline \multirow[b]{3}{*}{ Beam intensity } & \multicolumn{8}{|c|}{$\%$ errors in individual parameters } \\
\hline & \multicolumn{2}{|c|}{$2 \%$} & \multicolumn{2}{|l|}{$3 \%$} & \multicolumn{2}{|c|}{$4 \%$} & \multicolumn{2}{|c|}{$5 \%$} \\
\hline & $1.7 \dagger$ & $* \ddagger$ & 2.9 & * & 4.7 & * & 5.8 & * \\
\hline $\begin{array}{l}\text { Absorption } \\
\text { coefficient }\end{array}$ & -23.0 & 67.7 & -22.2 & 67.7 & -7.1 & 68.0 & -6.3 & 78.3 \\
\hline $\begin{array}{l}\text { Thermal } \\
\text { conductivity }\end{array}$ & -33.2 & 1.4 & -36.0 & 2.1 & -68.5 & 2.1 & -59.6 & -13.3 \\
\hline Specific heat & -67.1 & -1.7 & -60.0 & -2.9 & -84.2 & -3.5 & -74.2 & -4.7 \\
\hline
\end{tabular}


Table 24. Variations of shallow and deep doses for a thick laser TLD with systematic noises of various thermal parameters following pulsed heating with a $0.3 \mathrm{~cm}$ square uniform laser beam. TL dosimeter exposed to $20 \mathrm{keV} \mathrm{x}$-rays was used for this calculations.

\begin{tabular}{|c|c|c|c|c|c|c|c|}
\hline \multirow[b]{3}{*}{ Beam intensity } & \multicolumn{7}{|c|}{$\%$ errors in individual parameters } \\
\hline & $-2 \%$ & \multicolumn{2}{|c|}{$-3 \%$} & \multicolumn{2}{|c|}{$-4 \%$} & \multicolumn{2}{|c|}{$-5 \%$} \\
\hline & $-19.3 \dagger-7.7+$ & -27.6 & -11.3 & -35.2 & -14.9 & -42.2 & -18.4 \\
\hline $\begin{array}{l}\text { Absorption } \\
\text { coefficient }\end{array}$ & $-8.2-2.4$ & -12.3 & -3.7 & -16.4 & -5.0 & -20.3 & -6.2 \\
\hline $\begin{array}{l}\text { Thermal } \\
\text { conductivity }\end{array}$ & $5.7 \quad 1.4$ & -8.5 & 1.9 & 11.3 & 2.4 & 14.1 & 2.9 \\
\hline Specific heat & 18.17 .1 & 28.0 & 10.9 & 38.9 & 15.0 & 50.9 & 19.1 \\
\hline
\end{tabular}

\begin{tabular}{|c|c|c|c|c|c|c|c|c|}
\hline \multirow[b]{3}{*}{ Beam intensity } & \multicolumn{8}{|c|}{$\%$ errors in individual parameters } \\
\hline & \multicolumn{2}{|l|}{$2 \%$} & \multicolumn{2}{|c|}{$3 \%$} & \multicolumn{2}{|c|}{$4 \%$} & \multicolumn{2}{|c|}{$5 \%$} \\
\hline & $22.9 \dagger$ & $7.9 \%$ & 36.0 & 12.3 & $\overline{50.4}$ & 16.8 & 66.2 & 21.6 \\
\hline $\begin{array}{l}\text { Absorption } \\
\text { coefficient }\end{array}$ & 9.8 & 2.8 & 14.5 & 4.2 & 19.5 & 5.5 & 24.5 & 6.8 \\
\hline $\begin{array}{l}\text { Thermal } \\
\text { conductivity }\end{array}$ & -4.3 & -0.9 & -6.6 & -1.5 & -8.9 & -2.1 & -11.1 & -2.7 \\
\hline Specific heat & -14.2 & -6.3 & -20.7 & -9.3 & -26.6 & -12.3 & -32.1 & -15.1 \\
\hline
\end{tabular}


Table 25. Thermoluminescence light attenuation data for TL dosimeters manufactured by Harshaw/Filtrol*.

\begin{tabular}{llc}
\hline Dosimeter & \multicolumn{1}{c}{$\mu, \mathrm{mm}^{-1}$} & $1 / \mu, \mathrm{cm}^{-1}$ \\
\hline TLD 100 & $0.088 \pm 0.036$ & 114 \\
TLD 600 & $0.066 \pm 0.045$ & 151 \\
TLD 700 & $<0.072$ & 139 \\
$\operatorname{LiF}(\mathrm{Cu}, \mathrm{Mg}, \mathrm{P})$ & $1.16 \pm 0.06$ & 8.6
\end{tabular}

*Harshaw Chemical Co., 6801 Cochran Road, Solon, OH 44139 


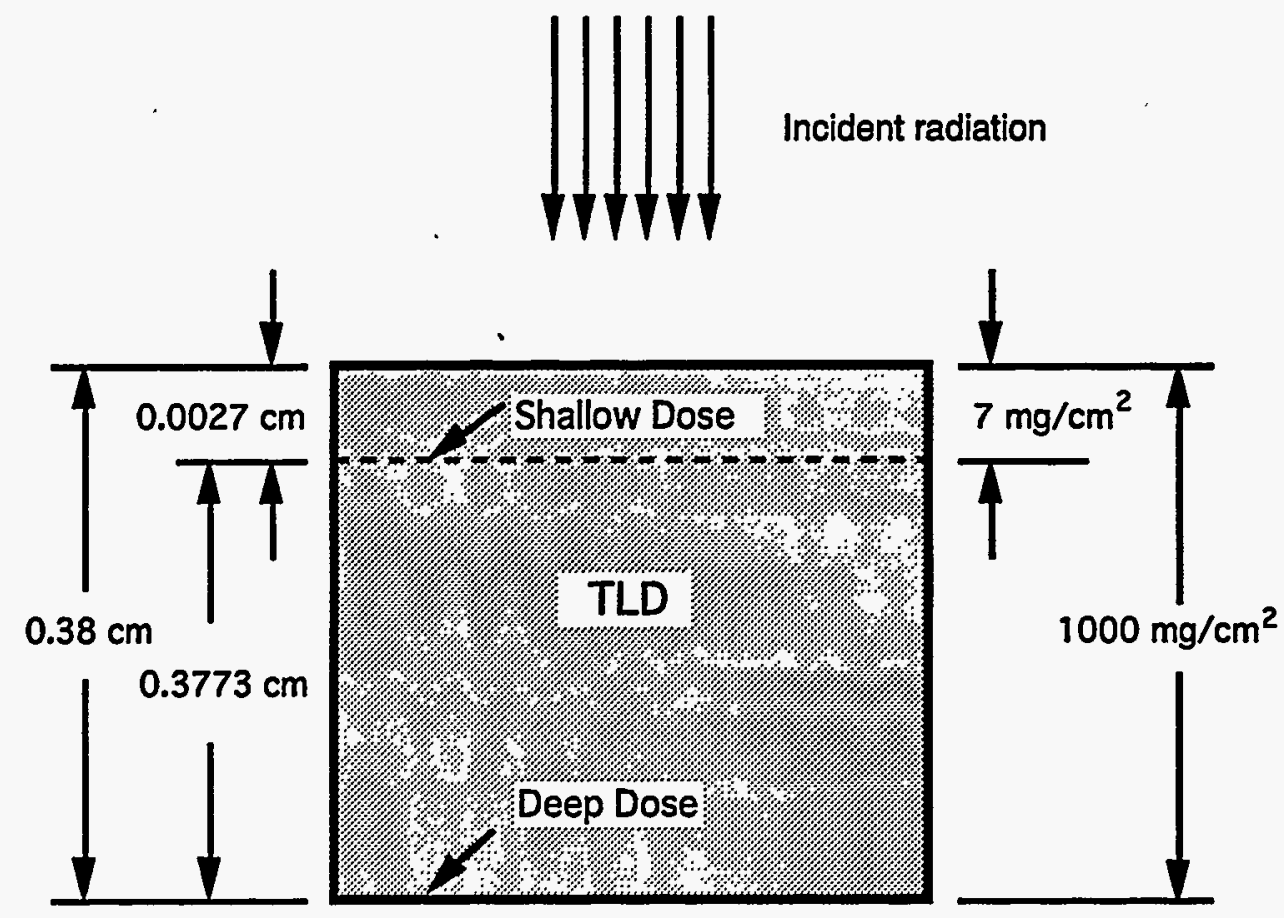

Figure 1. Schematic diagram of thick LiF laser TL dosimeter. Actual size is $0.3 \times 0.3 \times$ $0.38 \mathrm{~cm}^{3}$. The shallow dose and deep doses are determined at $0.0027 \mathrm{~cm}\left(7 \mathrm{mg} / \mathrm{cm}^{2}\right)$ and $0.38 \mathrm{~cm}\left(1000 \mathrm{mg} / \mathrm{cm}^{2}\right)$, respectively. $\mathrm{LiF}$ is a semi-opaque material with the absorption coefficient of $40 \mathrm{~cm}^{-1}$ for $10.6 \mathrm{~mm}$ infrared laser photons. 


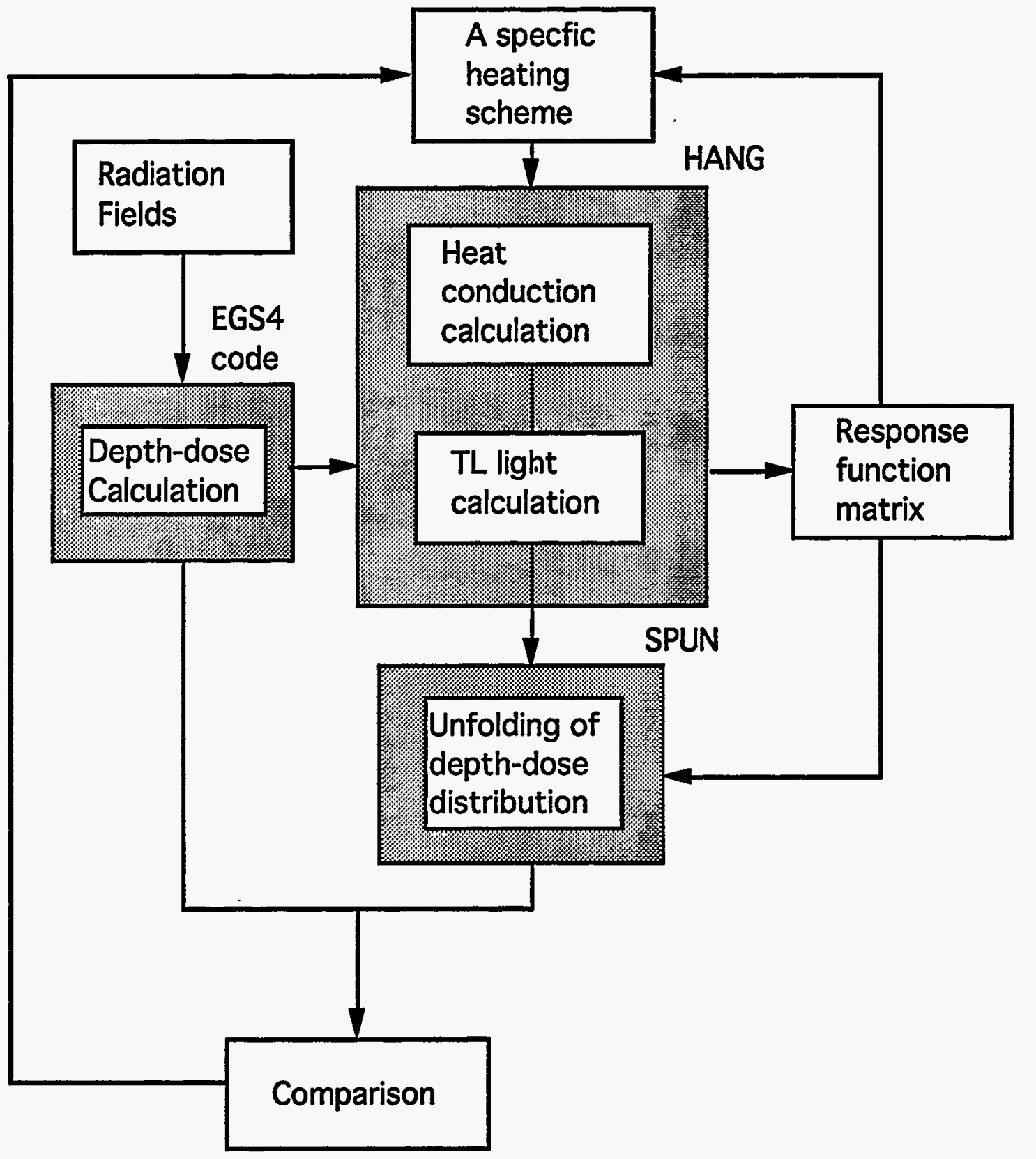

Figure 2. Schematic diagram of the logistics of the computational study. 


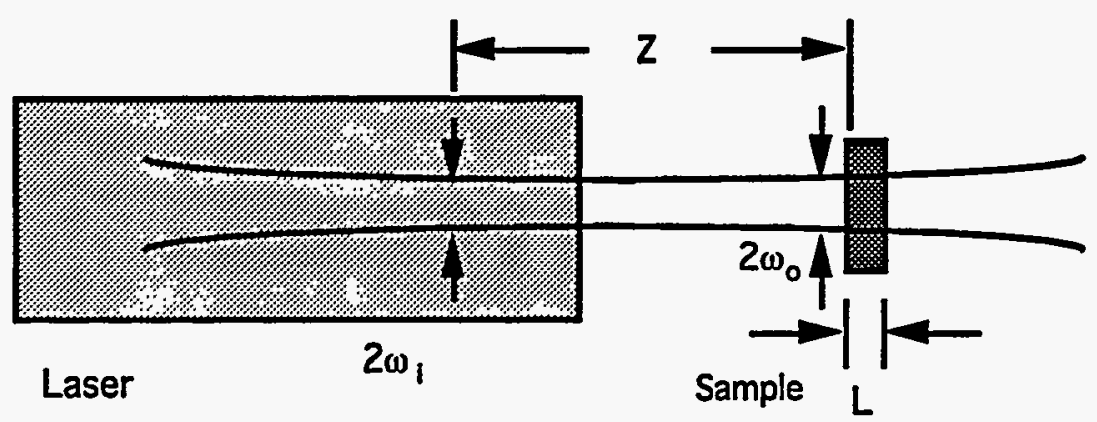

(a) Unfocused laser beam

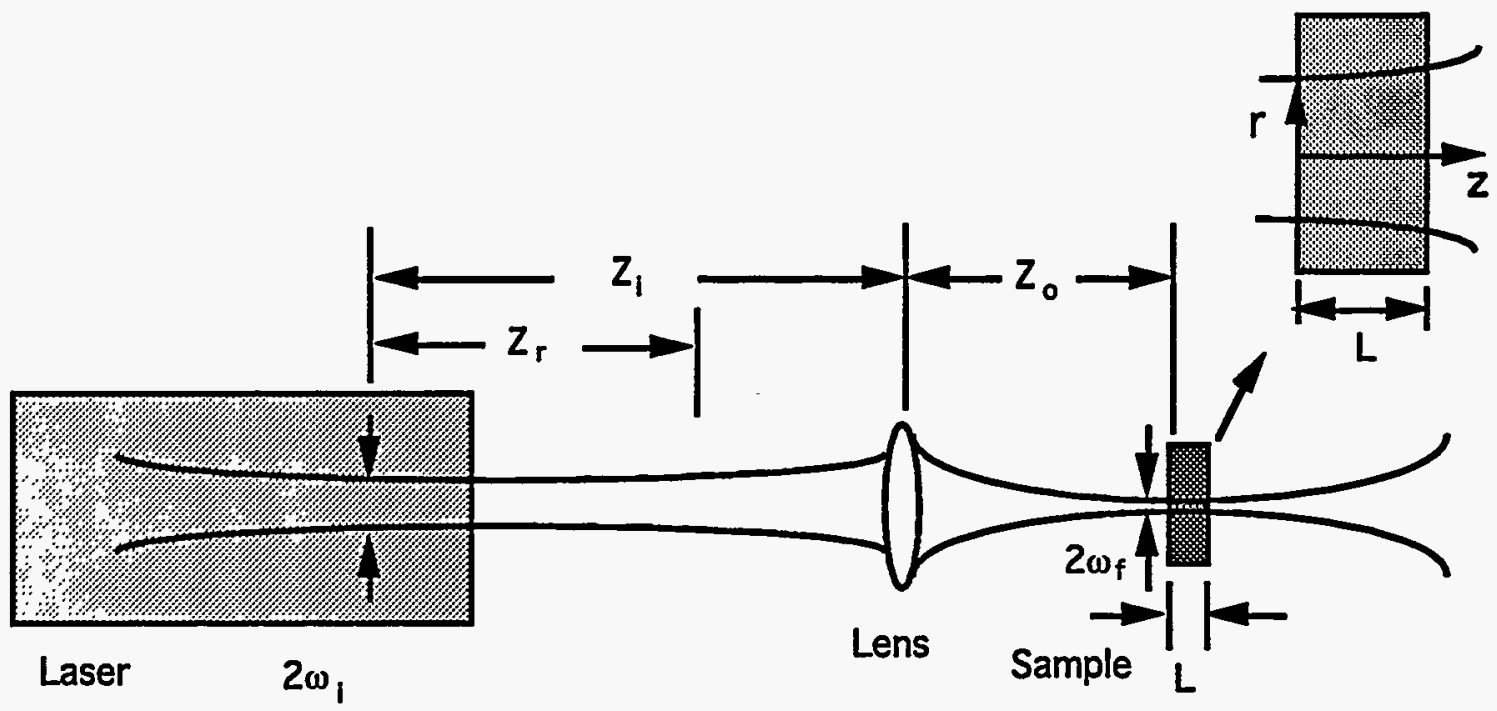

(b) Focused laser beam

Figure 3. A schematic diagram of unfocused and focused laser heating of a TLD sample; (a) In unfocused laser heating of a TL dosimeter of thickness $L$, the laser waist, or $2 \omega_{0}$, is positioned at the surface of the TL dosimeter; (b) In focused laser heating of a TL dosimeter of thickness $\mathrm{L}$, the focused waist, or $2 \omega_{\mathrm{f}}$, is positioned at the surface of the TL dosimeter. 


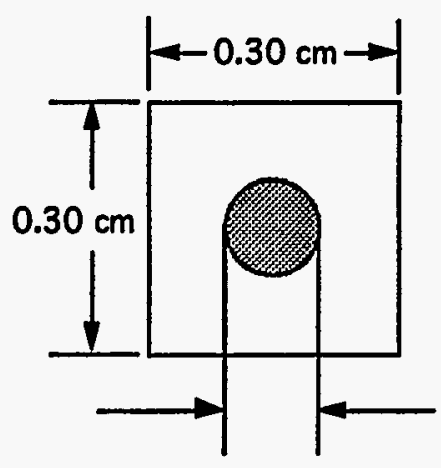

$0.170 \mathrm{~cm}$

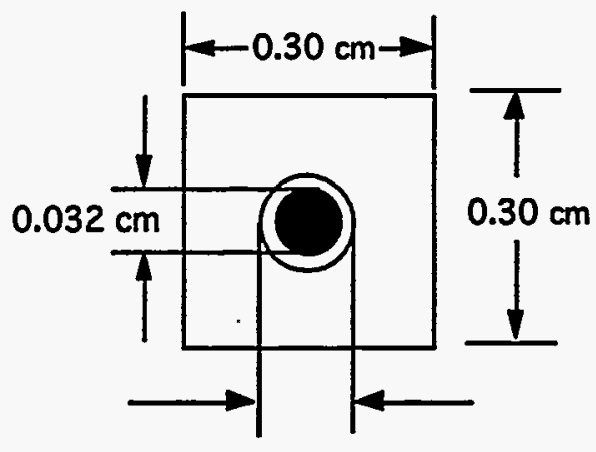

$0.170 \mathrm{~cm}$

(a) Unfocused Beam

(b) Focused Beam

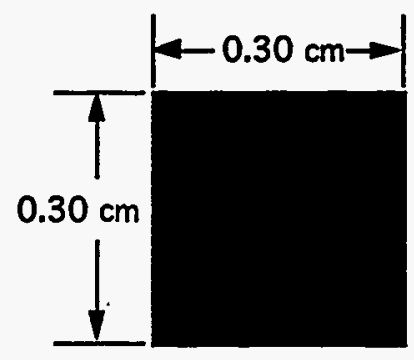

(c) Square Beam

Figure 4. Cross-sectional views of three different laser beams: (a) Unfocused Gaussian beam $(0.170 \mathrm{~cm}$ diameter); (b) Focused Gaussian beam $(0.032 \mathrm{~cm}$ diameter); and (c) Uniform square beam $\left(0.3 \times 0.3 \mathrm{~cm}^{2}\right.$ in size), which is exactly the same as the dosimeter cross-section. Focused and unfocused beam originates from a 4 Watt continuous wave (CW) laser beam. The uniform beam originates from $10 \mathrm{~W}$ continuous wave (CW) laser beam with beam shaping optics. 


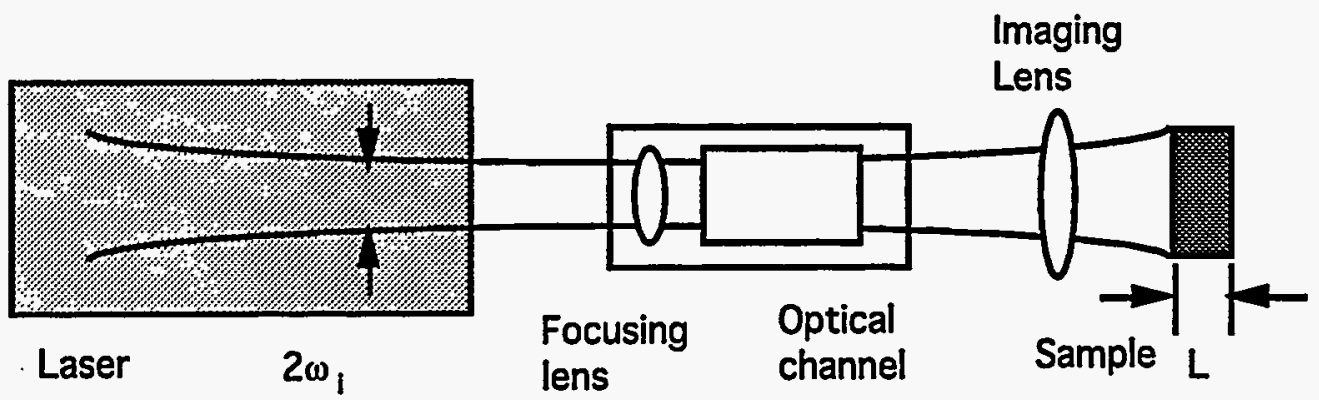

Uniform square laser beam

Figure 5. A schematic diagram of uniform laser beam heating of a sample; This uses the IST (International Sensor Technologies) TLD reader [Braunlich, 1990] which features an $\mathrm{RF}$-excited $\mathrm{CO}_{2}$ waveguide laser capable of delivering a uniform beam of around $0.3 \times 0.3$ $\mathrm{cm}^{2}$ spot size (adjustable) to discrete a TL sample of thickness $L$. 


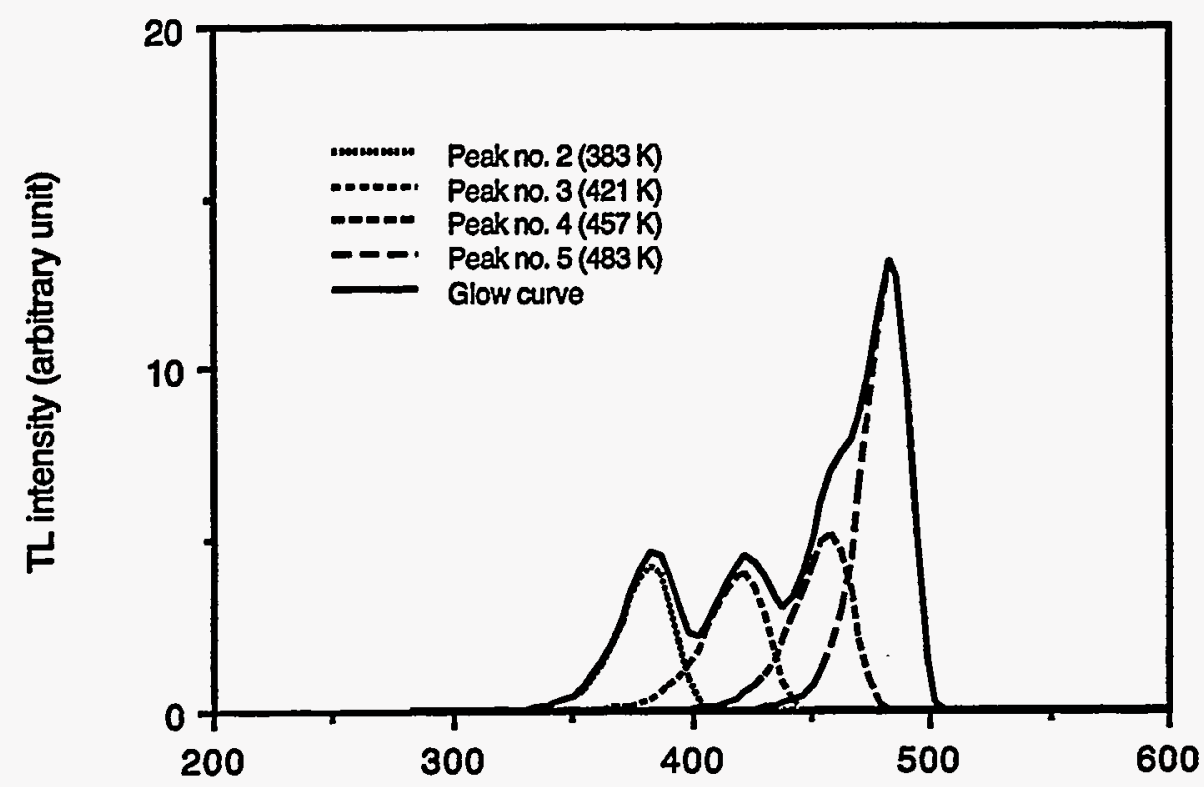

Temperature (K)

Figure 6. Thermoluminescence TL glow curves of LiF (TLD-100) analyzed by McKeever [McKeever, 1980]. These are the numerical fitting parameters obtained by experimental fits of LiF glow peaks (peaks 2-5), notably the trap depths of $1.13,1.23,1.54$, and $2.17 \mathrm{eV}$ and frequency factors of $6.1 \times 10^{13}, 4.0 \times 10^{13}, 7.3 \times 10^{15}$, and $4.0 \times 10^{21} / \mathrm{sec}$. 


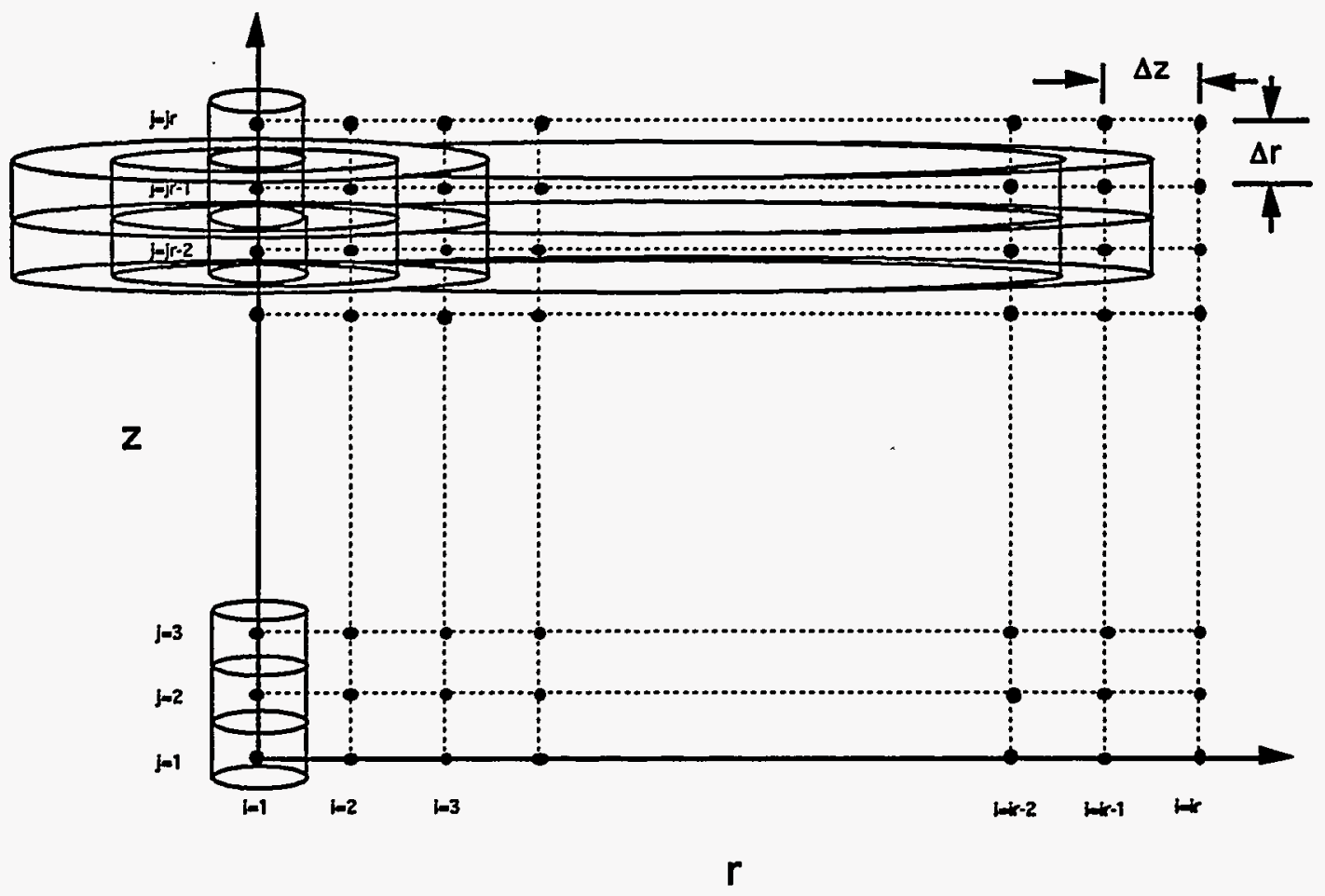

Figure 7. Schematic diagram of nodal points and meshes for TL glow curve calculation. TL intensity was generated at every nodal point (closed circles), then integrated with the surrounding cylindrical volume (annular ring) elements; For nodes inside and at the center: volume element, $\Delta \mathrm{V}=\pi(\Delta \mathrm{r} / 2)^{2} \Delta \mathrm{z}$ and $\Delta \mathrm{V}=\pi / 2(\Delta \mathrm{r} / 2)^{2}(\Delta \mathrm{z} / 2)$, respectively; For nodes at edge: $\Delta V=2 \pi r(\Delta r / 2)^{2} \Delta z$; and For other nodes: $\Delta V=2 \pi r \Delta r \Delta z$. 


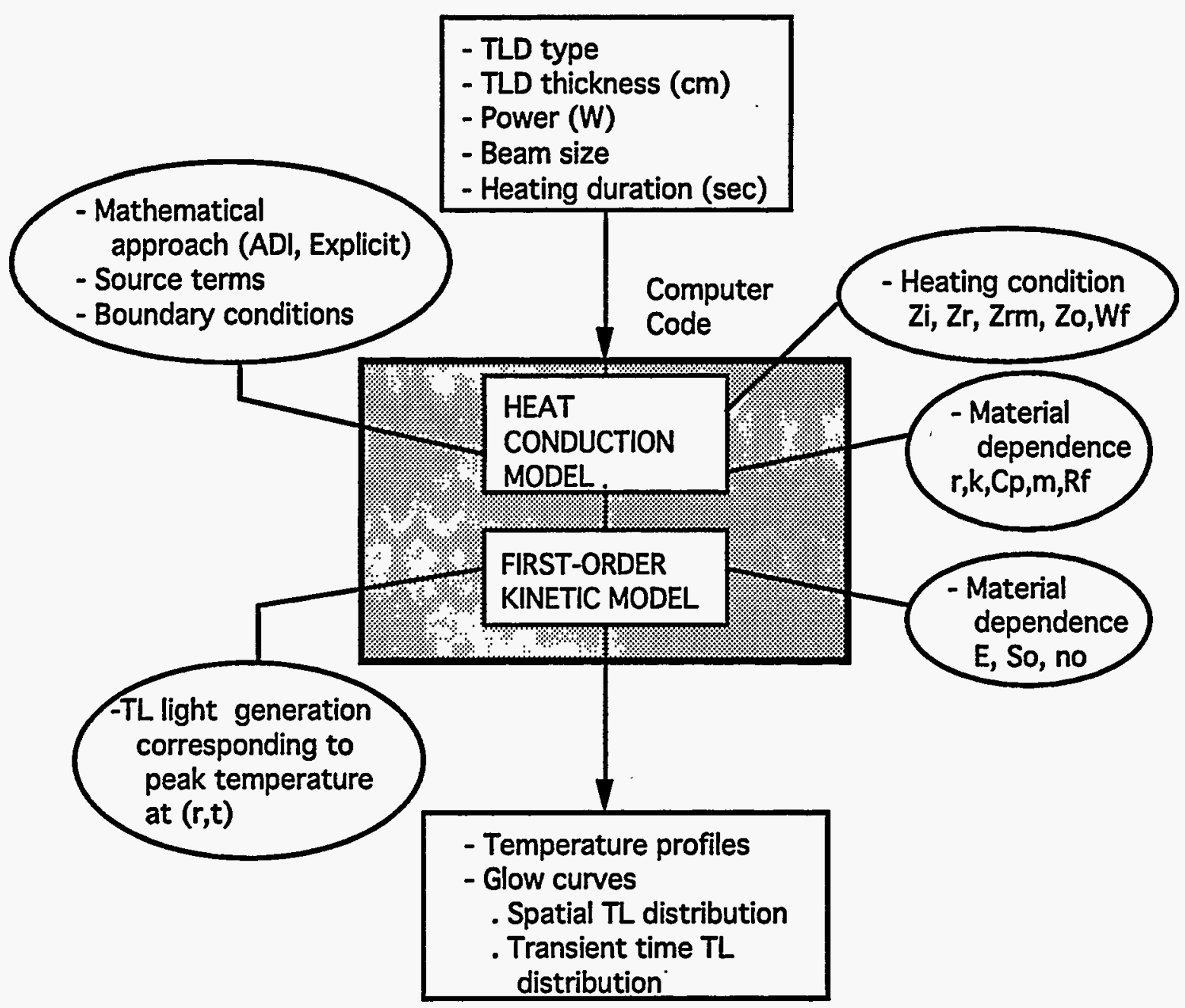

Figure 8. Schematic representation of computer code structure for heat conduction and TL glow curve calculation for laser heating system. 


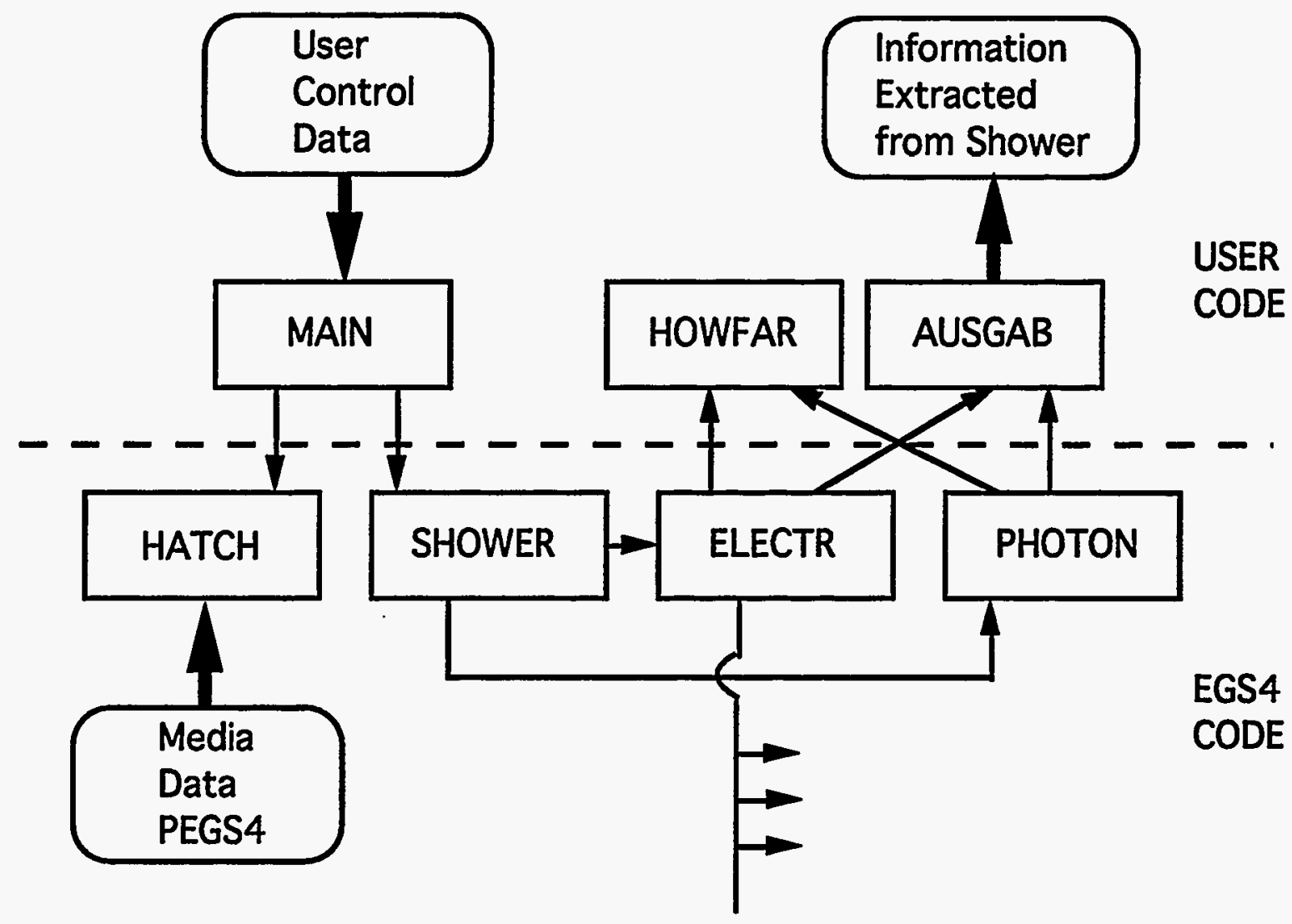

Figure 9. Schematic diagram of subroutines (user codes) of EGS4 code. 


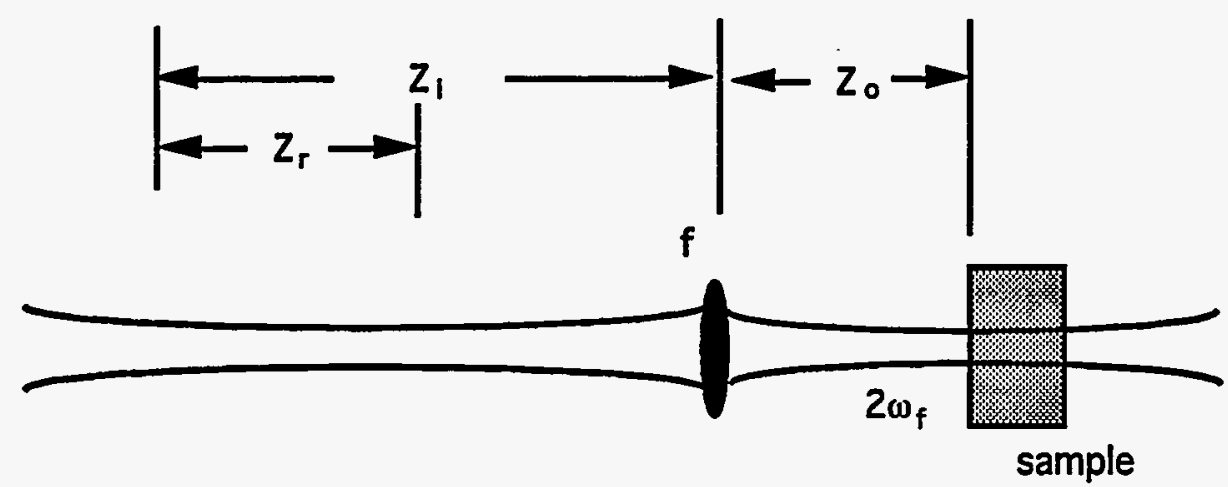

(a) Long focal length

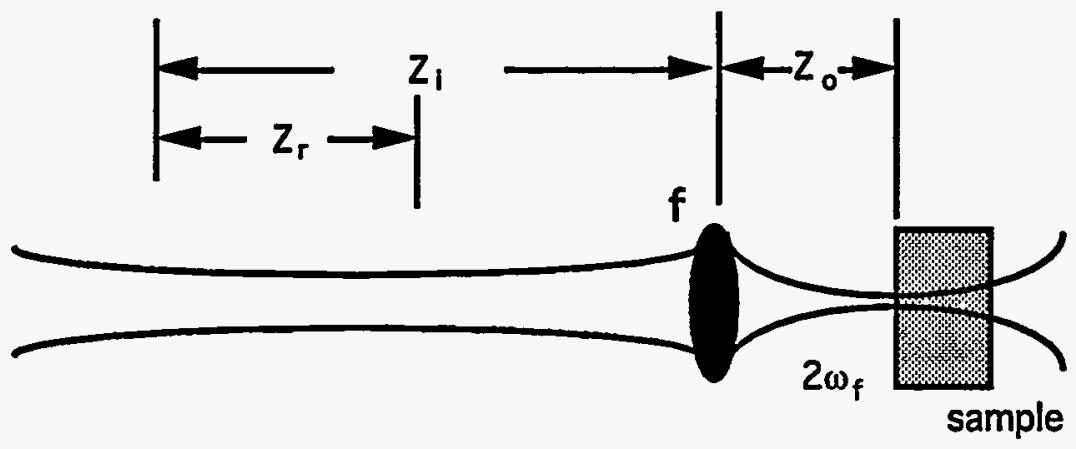

(b) Short focal length

Figure 10. A schematic diagram of geometry of focused laser heating of a TLD sample; (a) Long focal length, and (b) Short focal length; These view the dependence of beam diameter varying with the depth within the thick TL dosimeter. The focused beam with a typical focal length $10 \mathrm{~cm}$ lens was used in this study. 


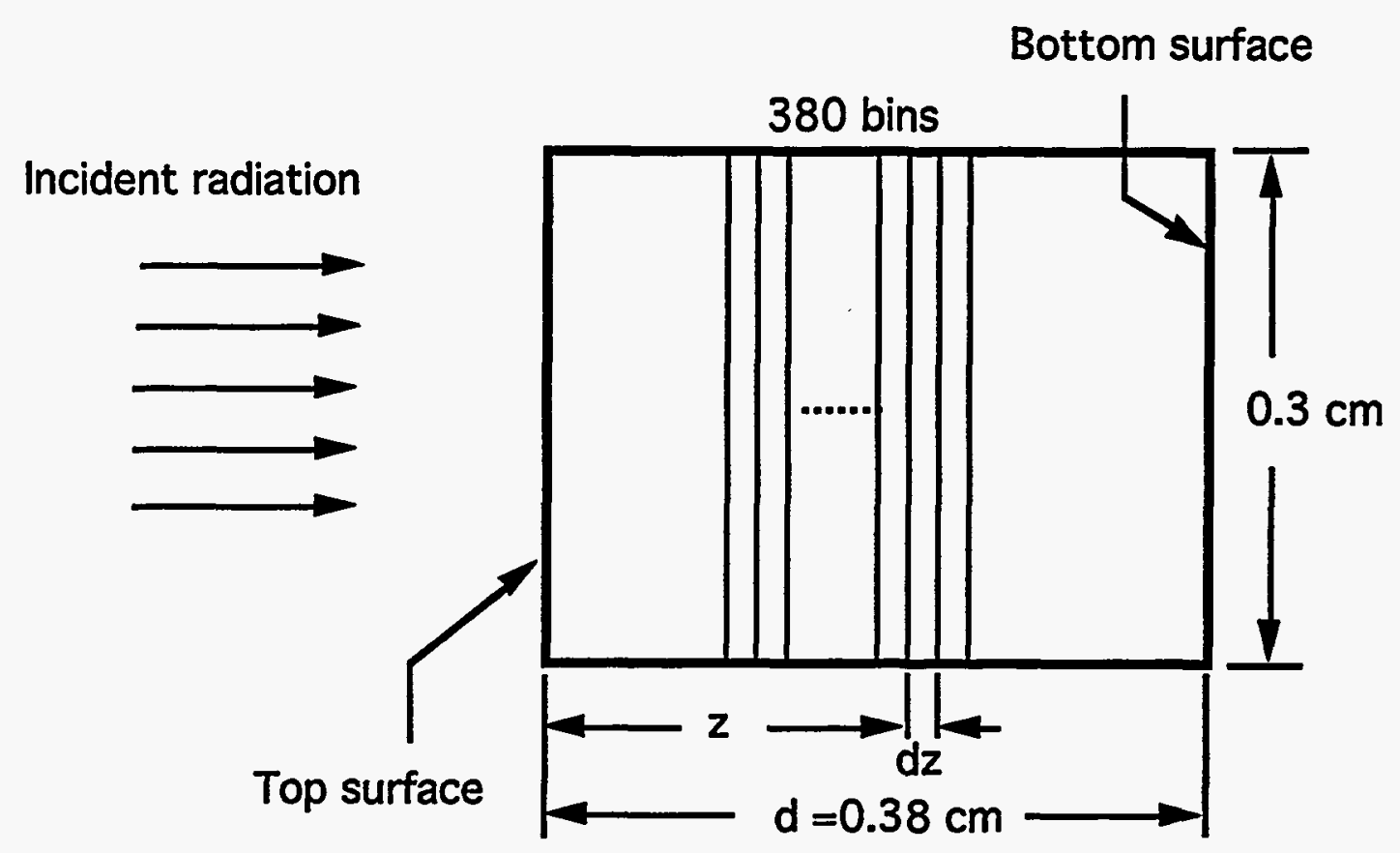

Figure 11. Calculation method of depth-dose distribution within TL dosimeter. The depthdoses were calculated as total energy deposited in $\mathrm{a}$ bin, $\mathrm{dz}$ at $\mathrm{z}$ and for all 380 bins. 


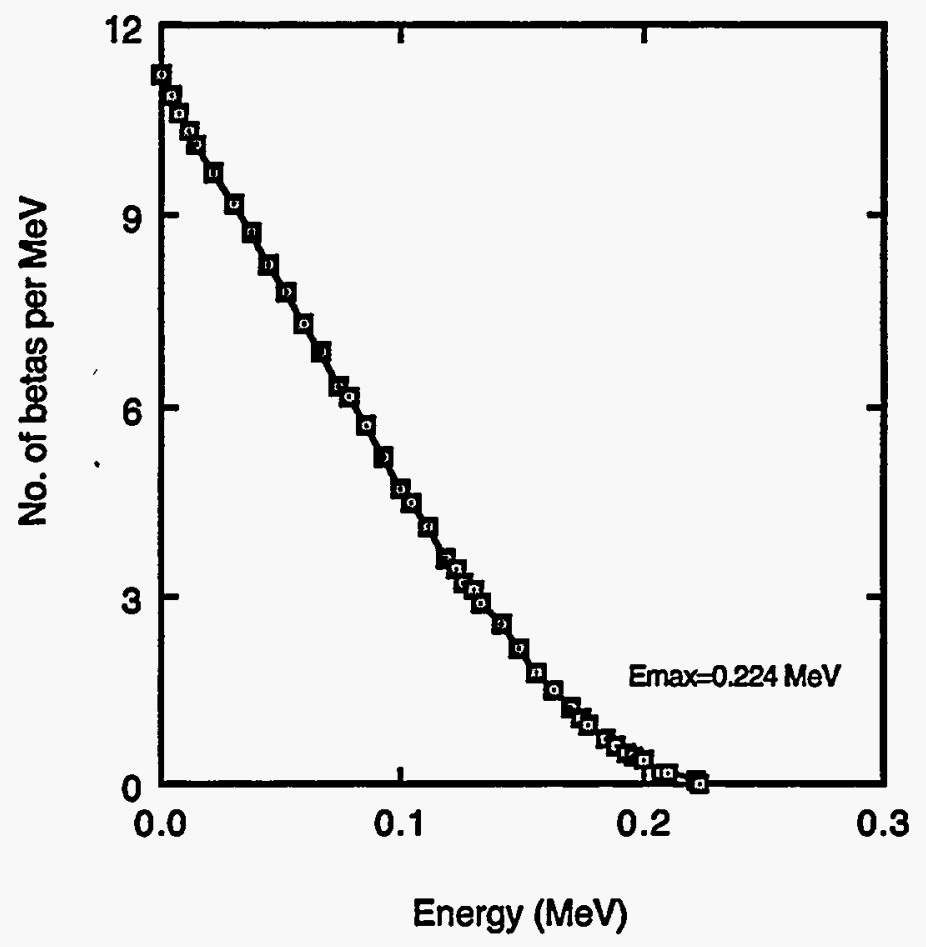

Figure 12. Beta-ray spectra from Pm-147 $\left(E_{\max }=0.224 \mathrm{MeV}\right)$ source [Cross, 1983]. The beta particles from this source were used as input data to EGS4 Monte Carlo simulation. The continuous slowing down approximation (CSDA) range corresponding to the maximum beta energy within LiF TL dosimeter is $0.025 \mathrm{~cm}$ [Berger et al., 1982]. 


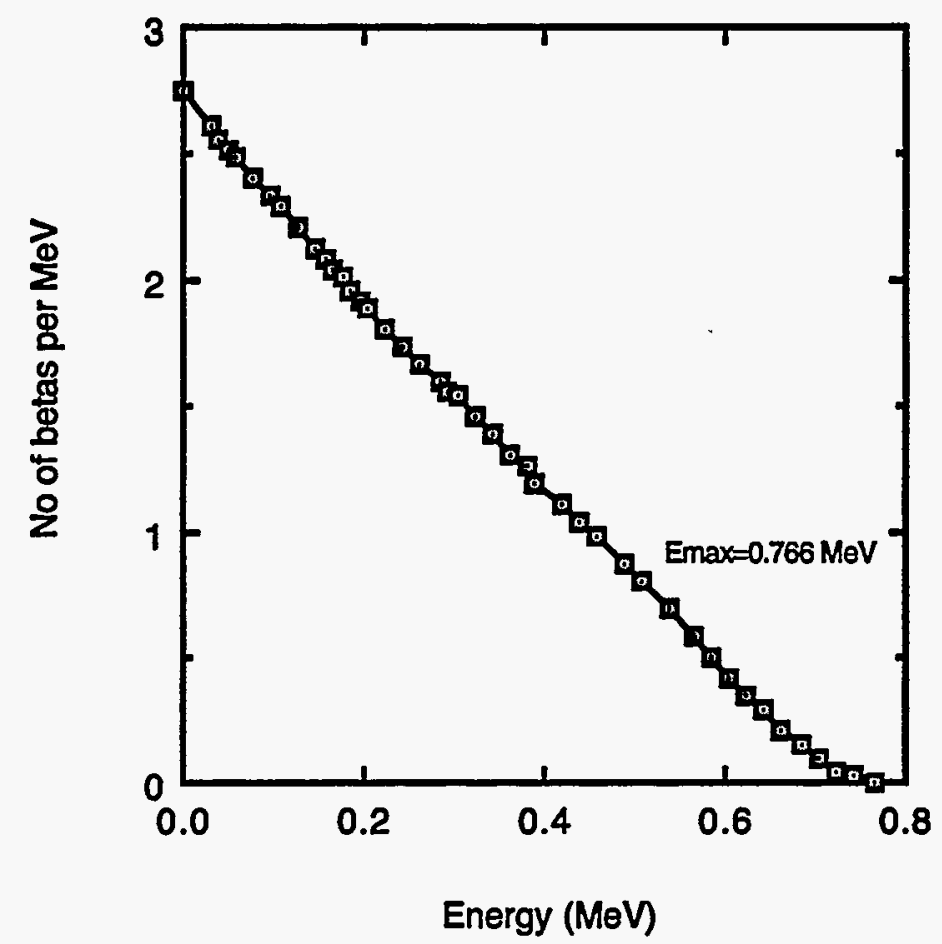

Figure 13. Beta-ray spectra from $\mathrm{Tl}-204\left(\mathrm{E}_{\max }=0.766 \mathrm{MeV}\right)$ source [Cross, 1983]. The beta particles from this source were used as input data to EGS4 Monte Carlo simulation. The continuous slowing down approximation (CSDA) range corresponding to the maximum beta energy within LiF TL dosimeter is $0.147 \mathrm{~cm}$ [Berger et al., 1982]. 


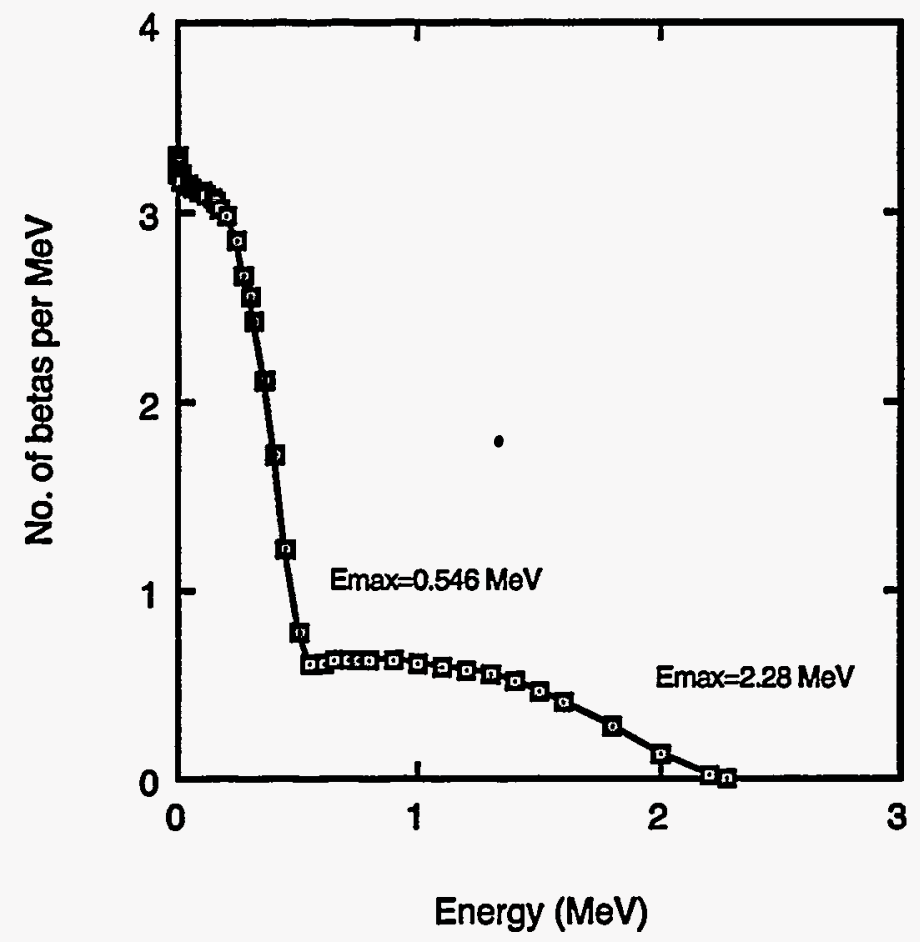

Figure 14. Beta-ray spectra from $\mathrm{Sr} / \mathrm{Y}-90\left(\mathrm{E}_{\max }=2.28 \mathrm{MeV}\right)$ source [Cross, 1983]. The beta particles from this source were used as input data to EGS4 Monte Carlo simulation. The continuous slowing down approximation (CSDA) range corresponding to the maximum beta energy within LiF TL dosimeter is $0.53 \mathrm{~cm}$ [Berger et al., 1982]. 


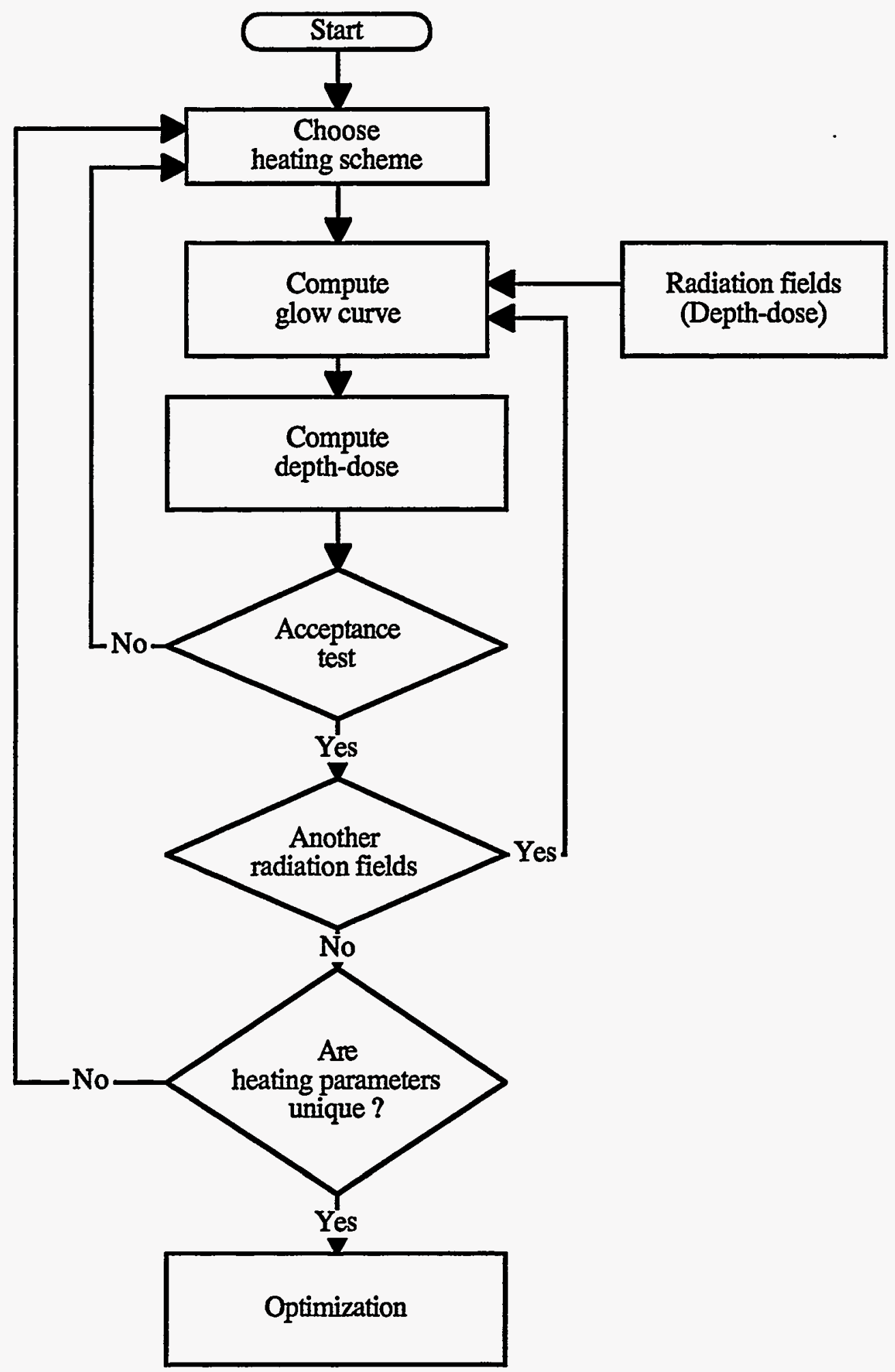

Figure 15. Optimization procedure of pulsed heating scheme for the single and mixed radiation fields. The optimization was performed to determine a unique top-and-bottom pulsed heating scheme by doing depth-dose unfolding and comparison with actual dose for 23 DOELAP radiation fields. 

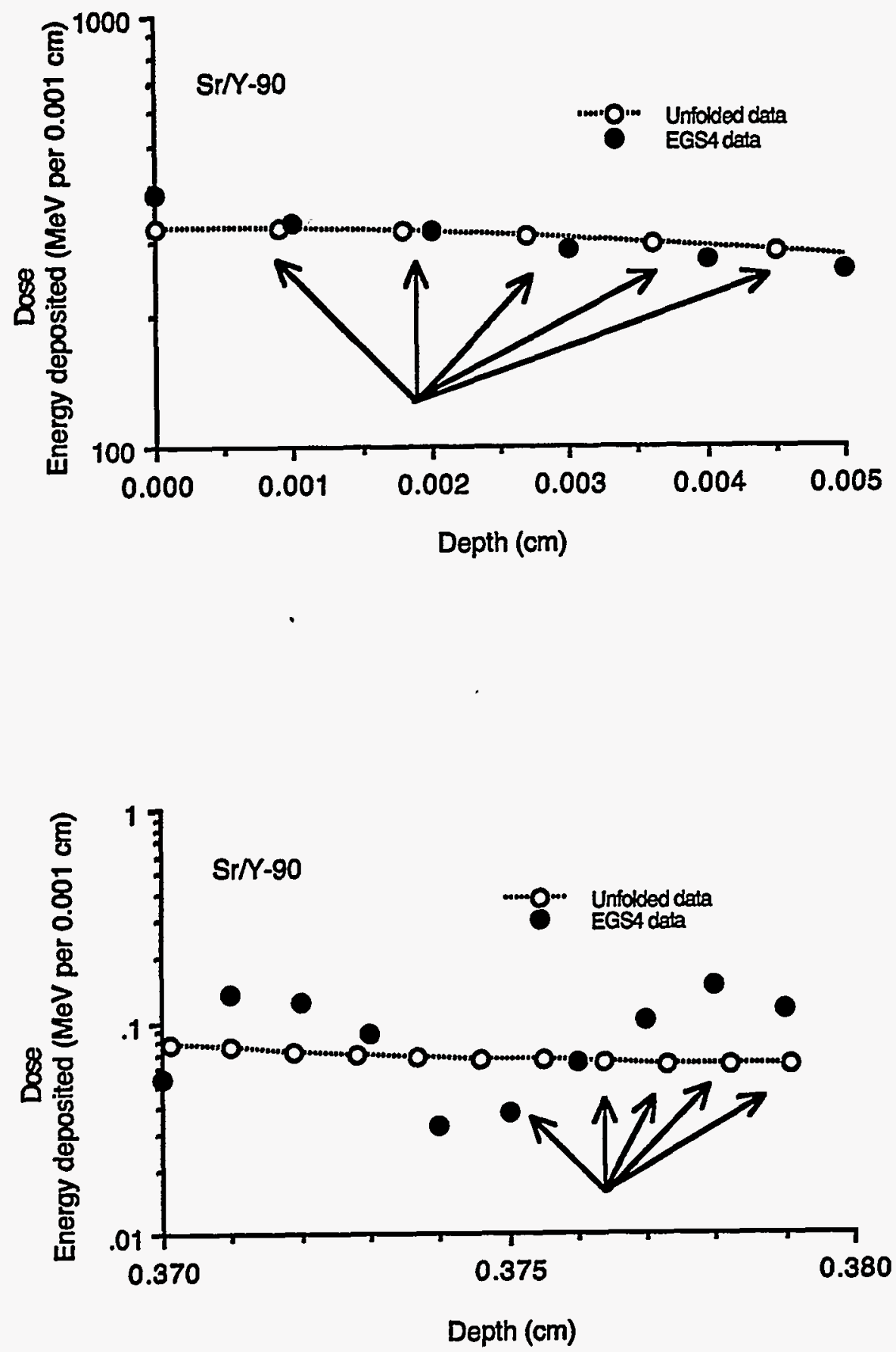

Figure 16. Descriptions of the data points averaged to determine the shallow and deep doses following the focused laser heating: (a) data points at the shallow dose region (above) and (b) data points at deep dose region. 

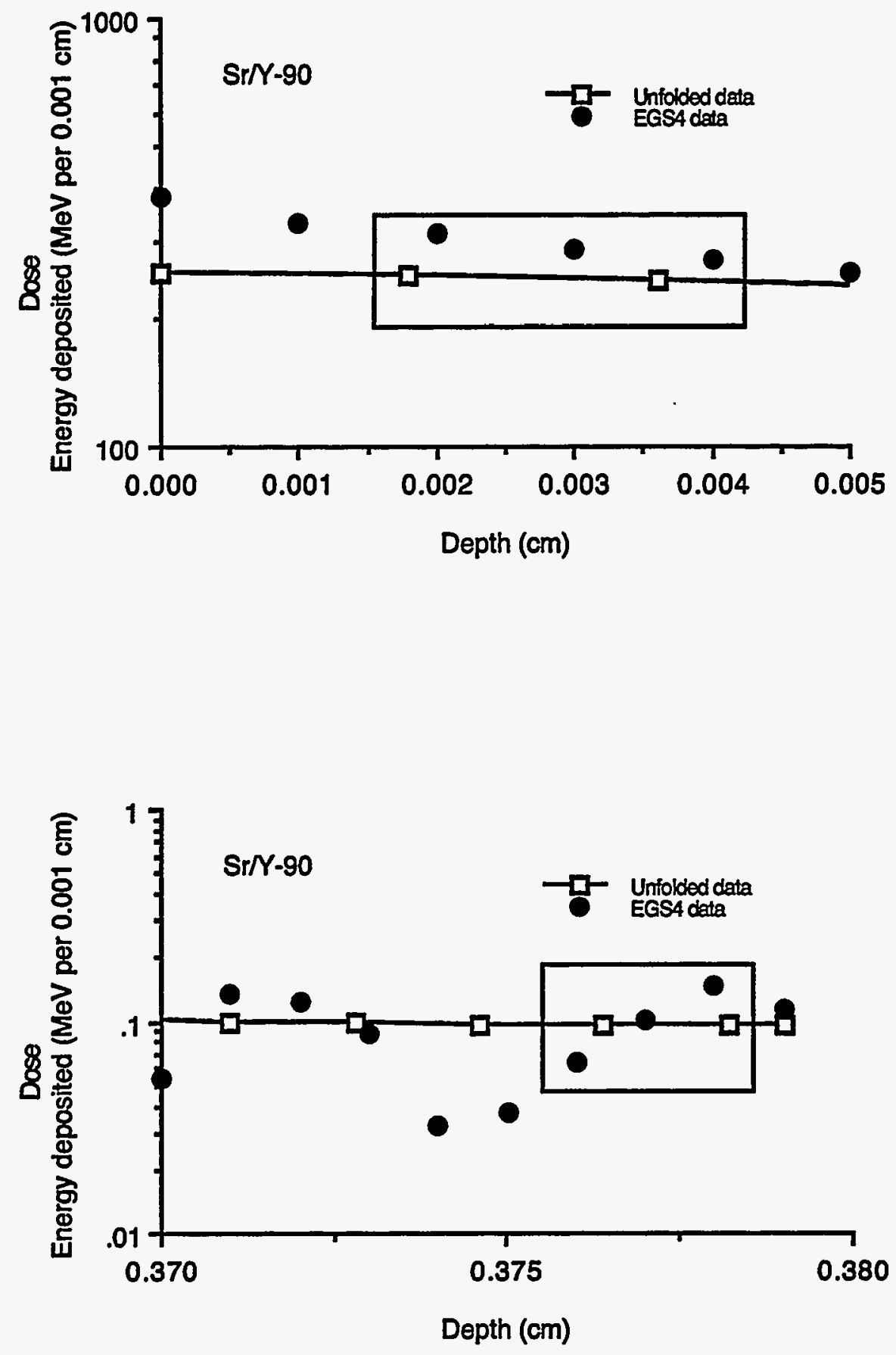

Figure 17. Descriptions of the data points averaged to determine the shallow and deep doses following the uniform laser heating: (a) data points in the box at the shallow dose region (above) and (b) data points in the box at deep dose region. 


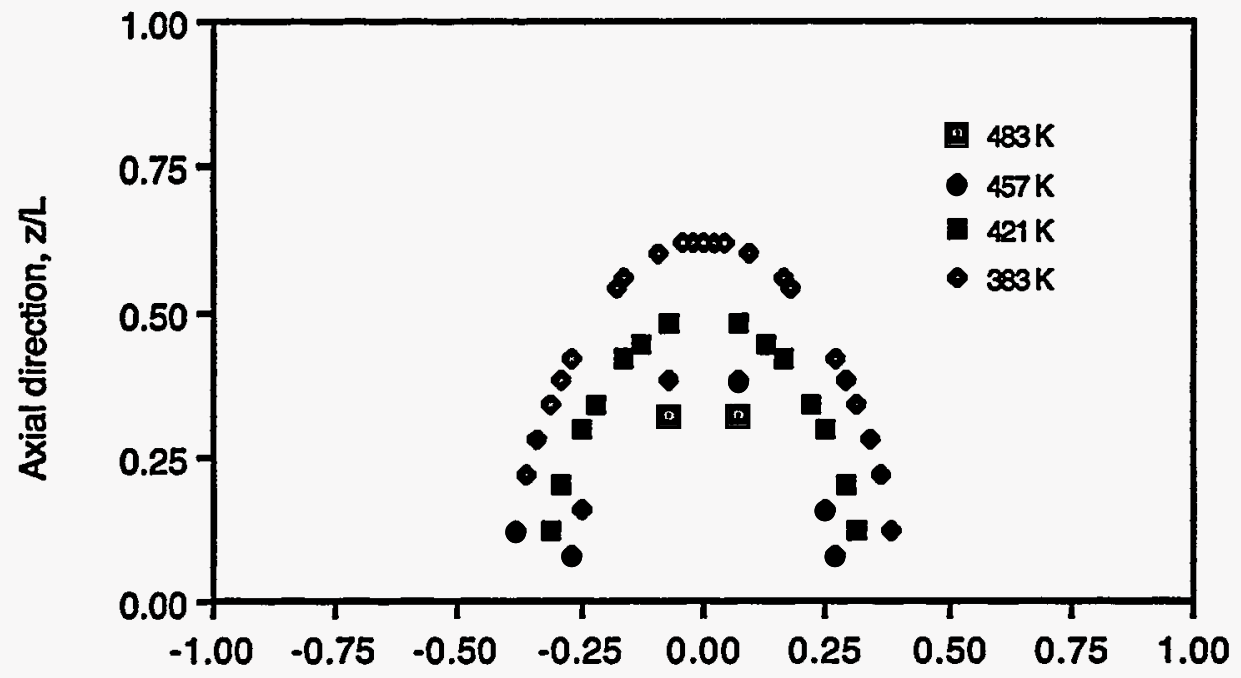

Radial direction, $\mathrm{r} / \mathbf{R}$

Figure 18. Temperature distribution in radial and axial directions in a $0.09 \mathrm{~cm}$ thick $\mathrm{LiF}$ TLD, predicted using an analytical solution, following heating with a $0.084 \mathrm{~cm}$ diameter focused $4.93 \mathrm{~W}$ continuous wave (CW) laser beam for $100 \mathrm{msec}$. Axial and radial distances were normalized for $\mathrm{L}=0.09 \mathrm{~cm}$ and $\mathrm{R}=0.15 \mathrm{~cm}$, respectively. 


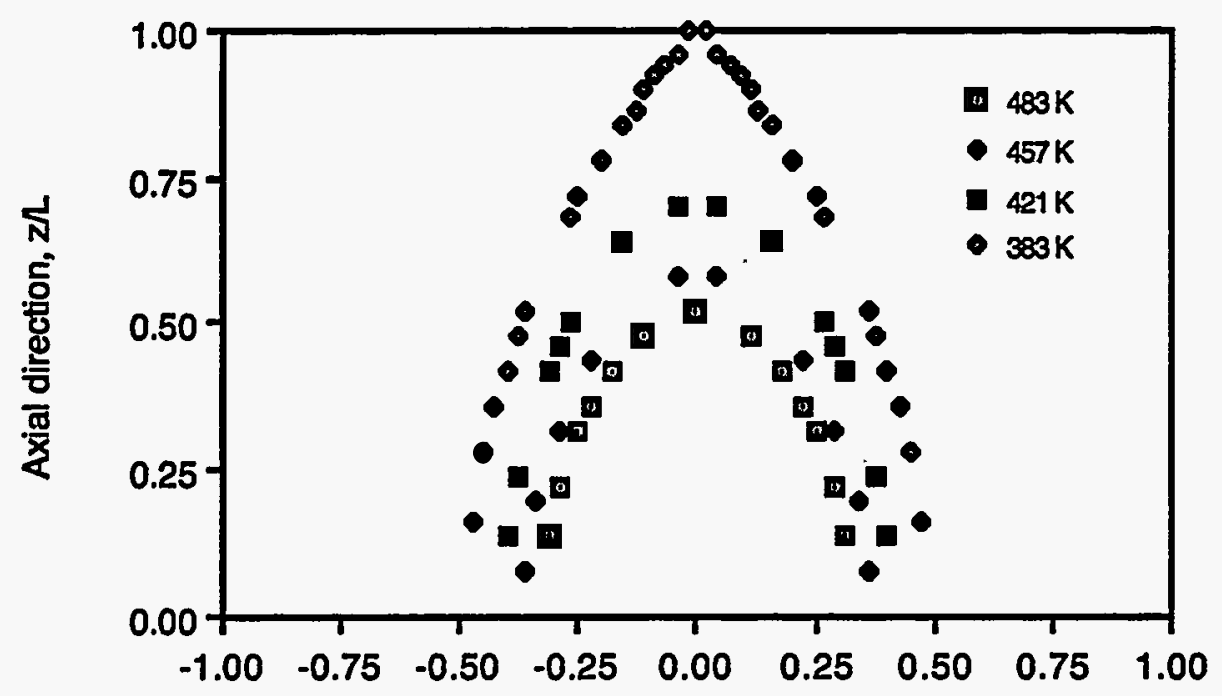

Radial direction, $r / R$

Figure 19. Temperature distribution in radial and axial directions in a $0.09 \mathrm{~cm}$ thick $\mathrm{LiF}$ TLD, predicted using an analytical solution, following heating with a $0.084 \mathrm{~cm}$ diameter focused $4.93 \mathrm{~W}$ continuous wave (CW) laser beam for $200 \mathrm{msec}$. Axial and radial distances were normalized for $L=0.09 \mathrm{~cm}$ and $R=0.15 \mathrm{~cm}$, respectively. 


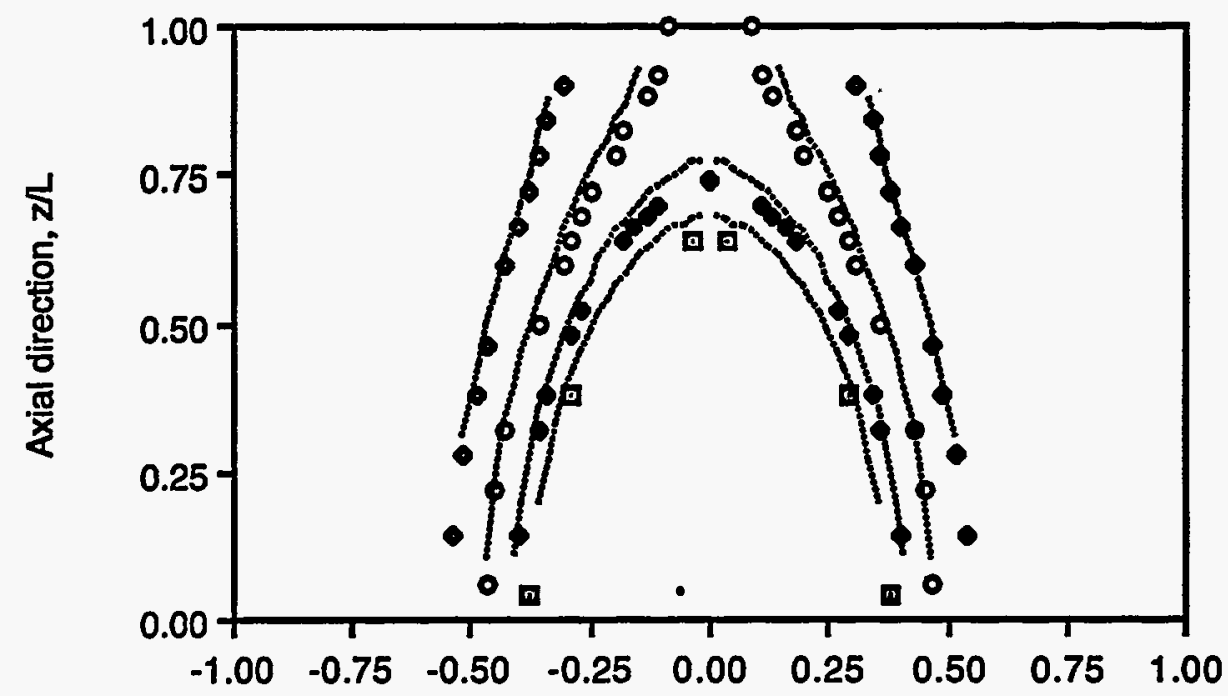

Radial direction, $\mathrm{r} / \mathbf{R}$

Figure 20. Temperature distribution in radial and axial directions in a $0.09 \mathrm{~cm}$ thick $\mathrm{LiF}$ TLD, predicted using an analytical solution, following heating with a $0.084 \mathrm{~cm}$ diameter focused $4.93 \mathrm{~W}$ continuous wave (CW) laser beam for $300 \mathrm{msec}$. Axial and radial. distances were normalized for $L=0.09 \mathrm{~cm}$ and $R=0.15 \mathrm{~cm}$, respectively. The dotted lines superimposed are the simulated data published by Abtahi [Abtahi et. al, 1985]. 


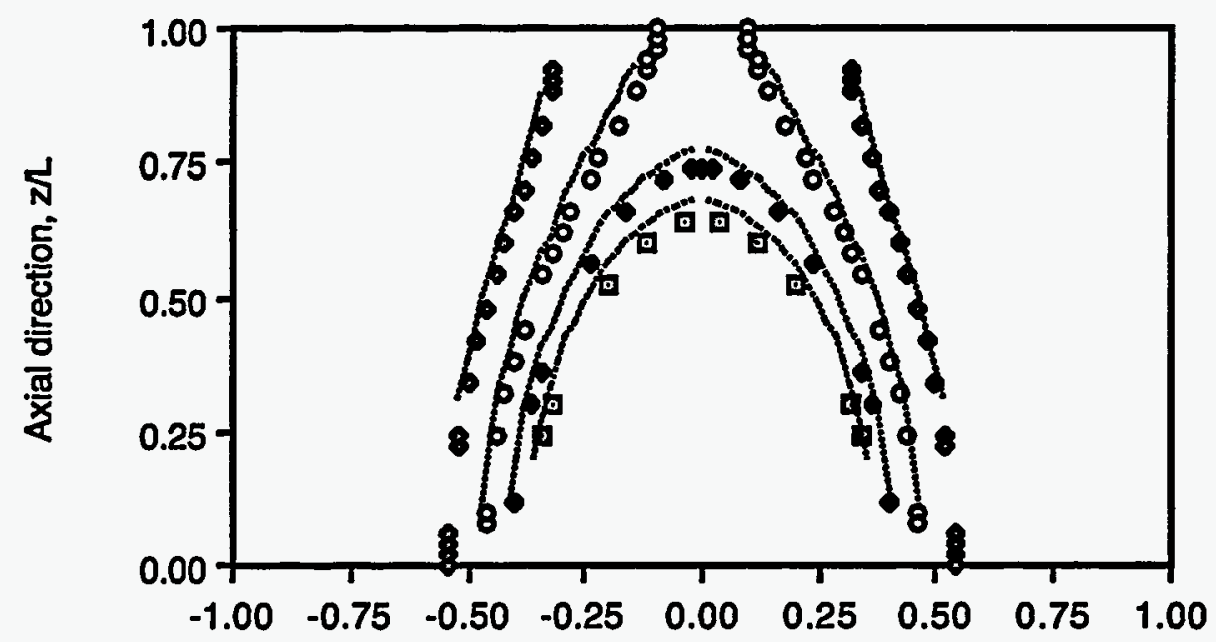

Radial direction, $\mathrm{r} / \mathbf{R}$

Figure 21. Temperature distribution in radial and axial directions in a $0.09 \mathrm{~cm}$ thick $\mathrm{LiF}$ TLD, predicted using an numerical solution, following heating with a $0.084 \mathrm{~cm}$ diameter focused $4.93 \mathrm{~W}$ continuous wave (CW) laser beam for $300 \mathrm{msec}$. Axial and radial distances were normalized for $L=0.09 \mathrm{~cm}$ and $R=0.15 \mathrm{~cm}$, respectively. The dotted lines superimposed are the simulated data published by Abtahi [Abtahi et. al, 1985]. 


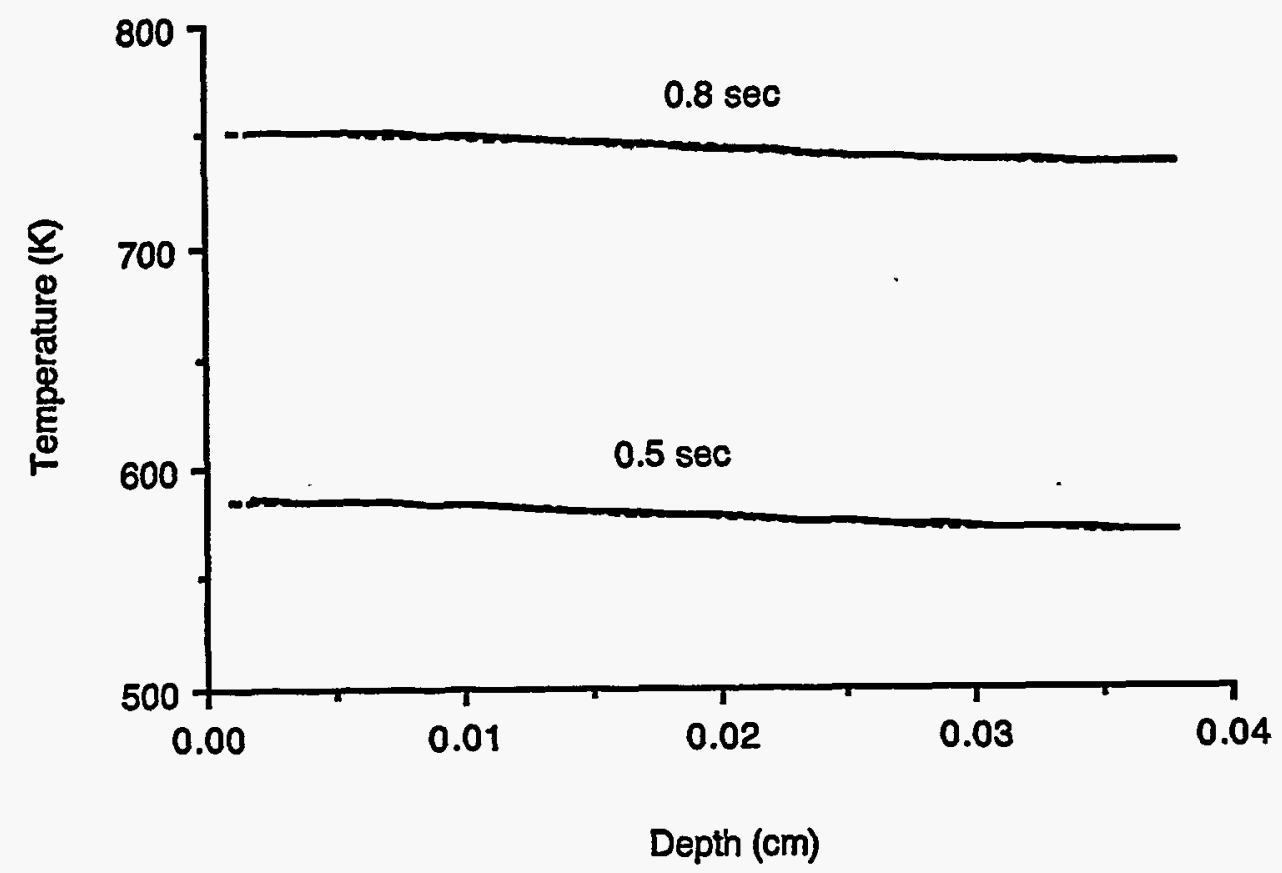

Figure 22. Temperature profiles in axial direction in a $0.038 \mathrm{~cm}$ thick LiF TLD, predicted using analytical solution (solid line) and numerical solution (dotted line), following heating with a $0.3 \times 0.3 \mathrm{~cm}$ square $10 \mathrm{~W}$ uniform laser beam for $0.5 \mathrm{sec}$ and $0.8 \mathrm{sec}$, respectively. 


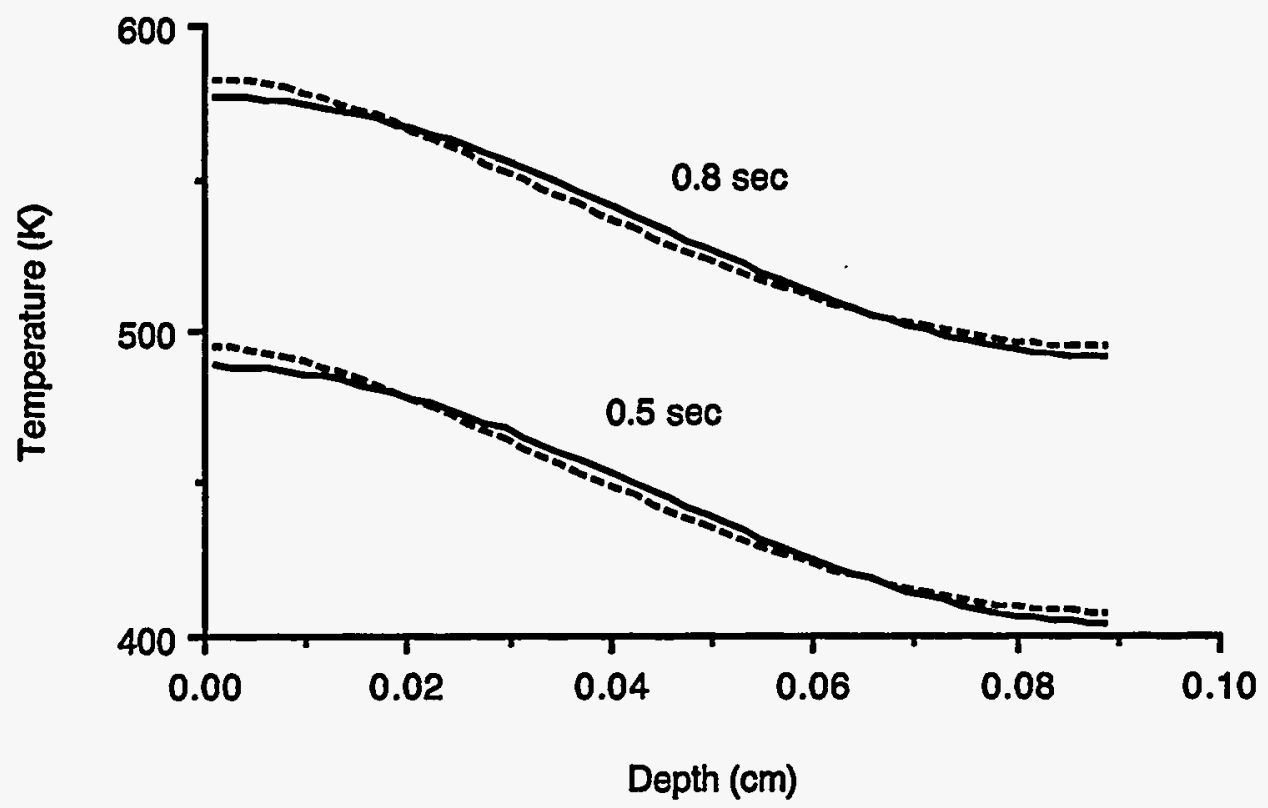

Figure 23. Temperature profiles in axial directions in a $0.09 \mathrm{~cm}$ thick LiF TLD, predicted using analytical solution (solid line) and numerical solution (dotted line), following heating with a $0.3 \times 0.3 \mathrm{~cm}$ square $10 \mathrm{~W}$ uniform laser beam for $0.5 \mathrm{sec}$ and $0.8 \mathrm{sec}$, respectively. 


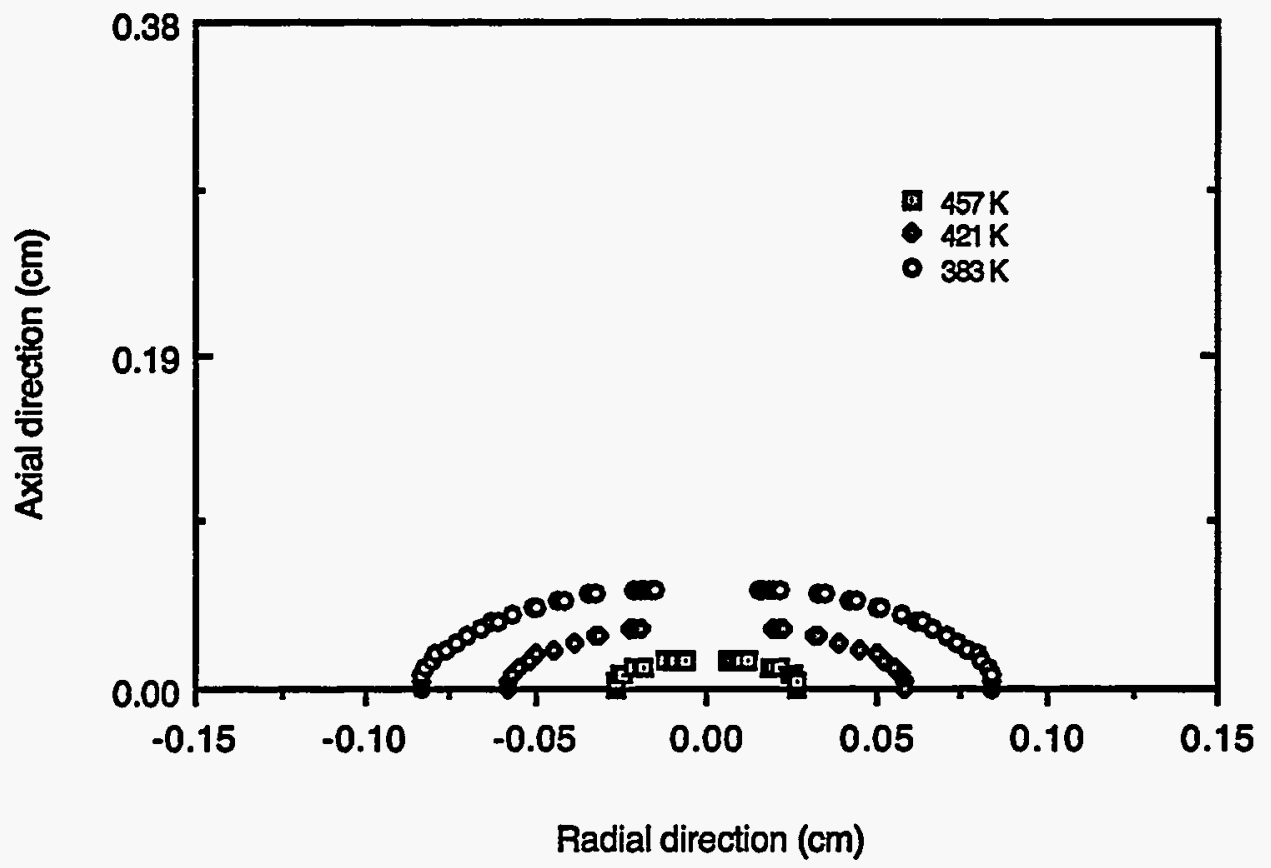

Figure 24. Temperature profiles in a thick laser TL dosimeter after $0.5 \mathrm{sec}$ of heating by a $0.170 \mathrm{~cm}, 4 \mathrm{~W}$ unfocused laser. 


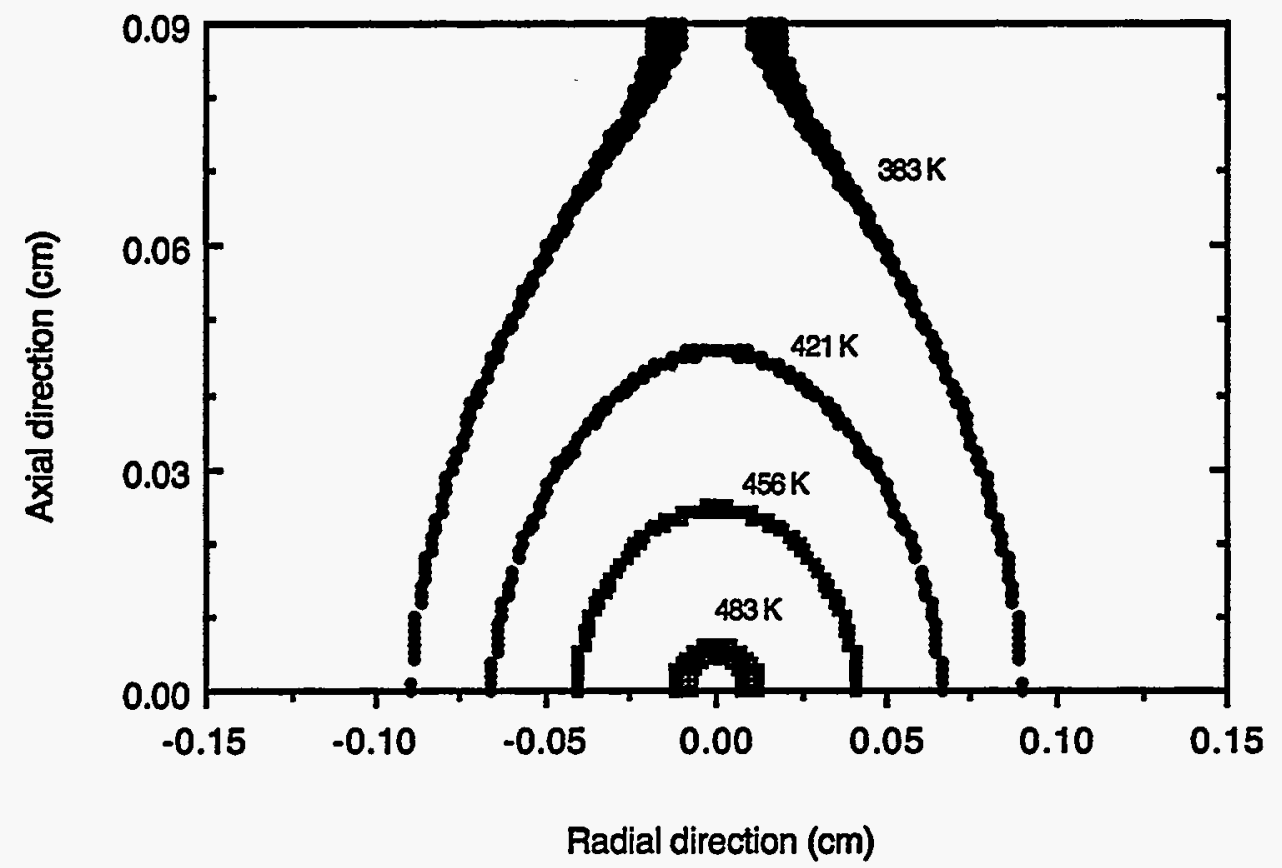

Figure 25. Temperature profiles of a $0.09 \mathrm{~cm}$ thick laser TL dosimeter after $0.5 \mathrm{sec}$ heating by $a .170 \mathrm{~cm}, 4 \mathrm{~W}$ unfocused laser beam. 


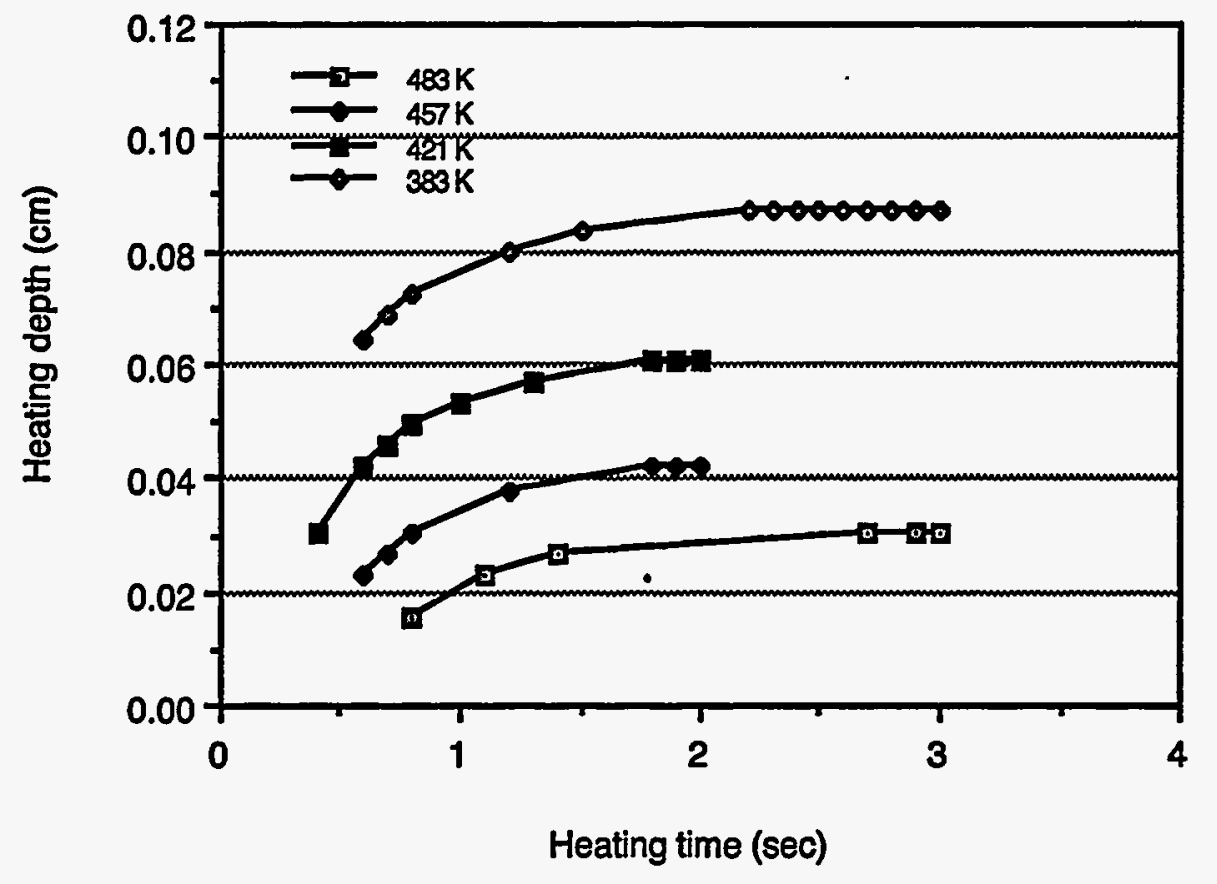

Figure 26. Variations of heating depths corresponding to the peak temperatures of peaks 25 with various unfocused beam heating durations. These simulated results were obtained for a thick laser TL dosimeter heated with $0.170 \mathrm{~cm}$ diameter $4 \mathrm{~W}$ unfocused laser beam. 


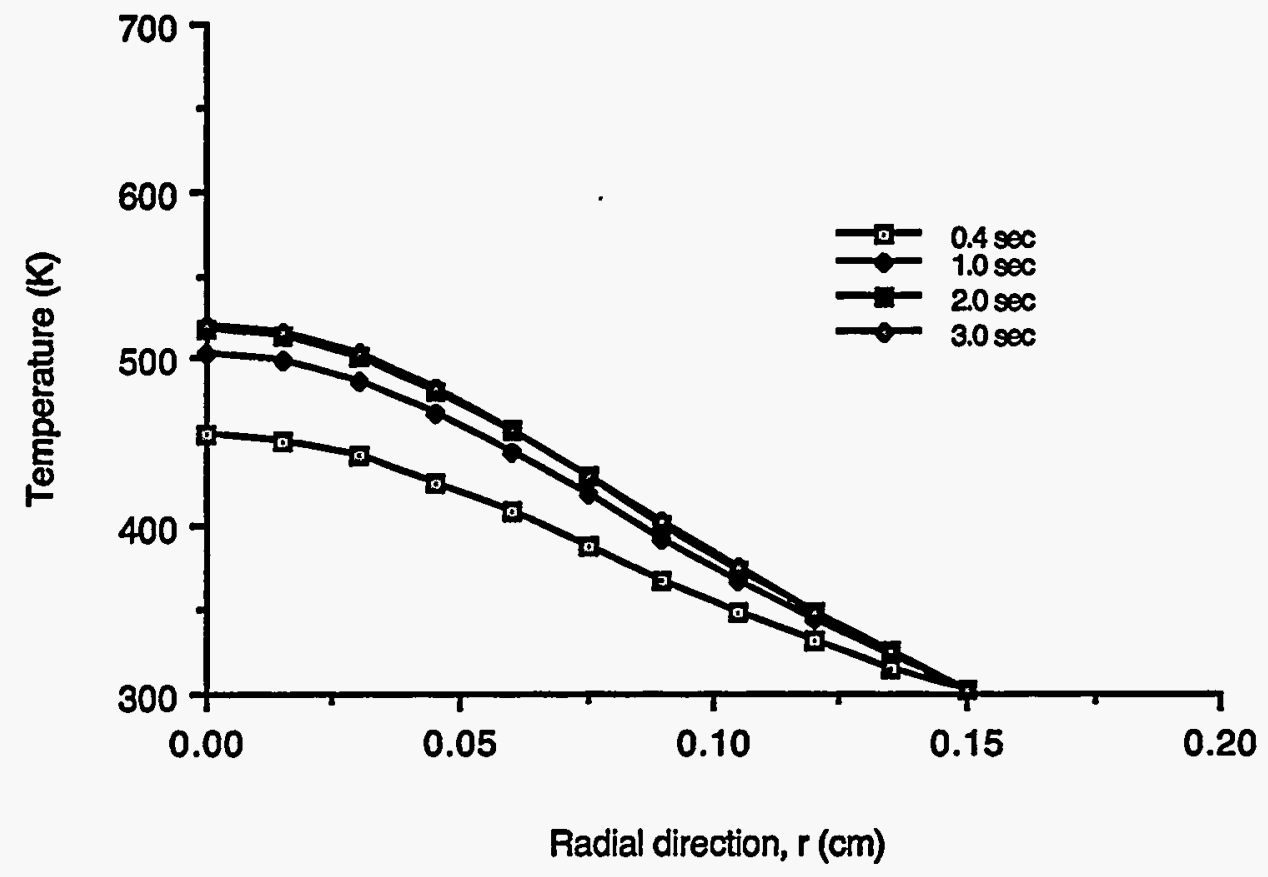

Figure 27. Radial temperature profiles at the surface of a thick laser TL dosimeter. The TLD is heated with a $0.170 \mathrm{~cm}$ diameter, $4 \mathrm{~W}$ unfocused laser beam for $0.4 \mathrm{sec}$, $1.0 \mathrm{sec}, 2.0 \mathrm{sec}$, and $3.0 \mathrm{sec}$. 


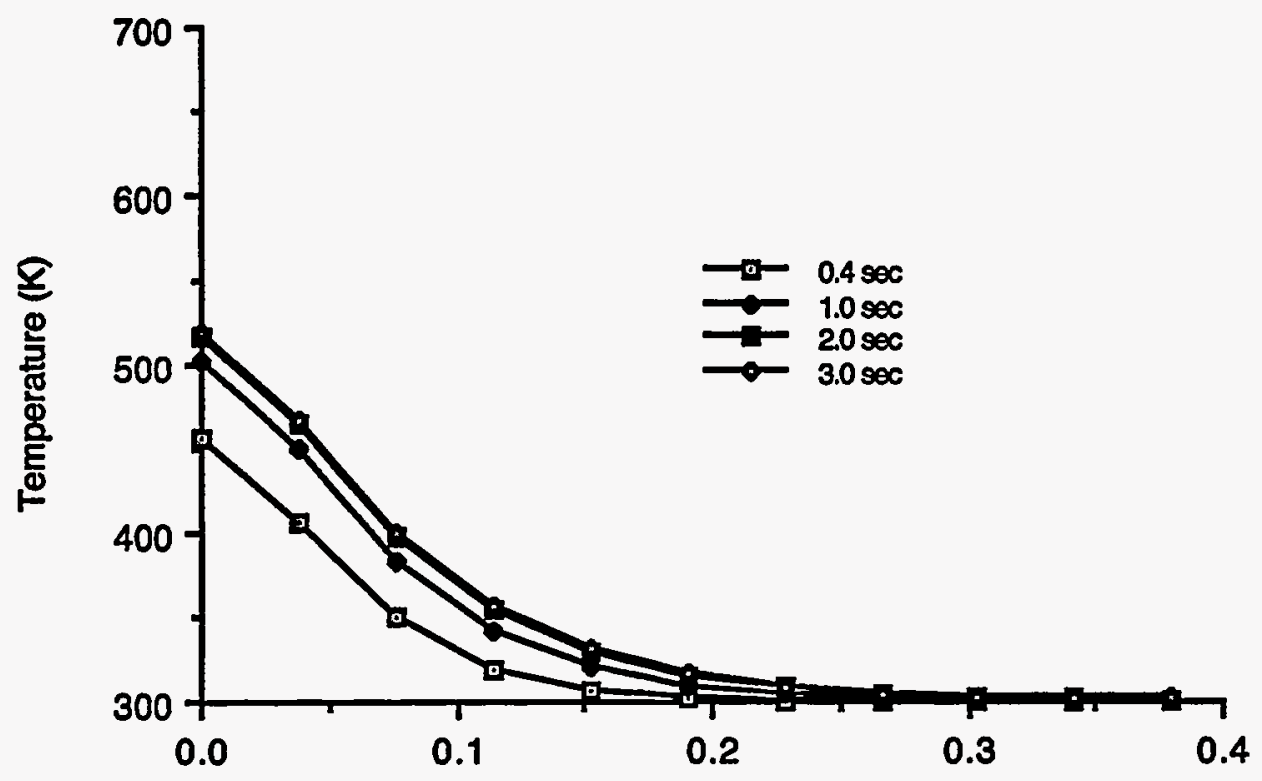

Axial direction, $\mathbf{z}(\mathrm{cm})$

Figure 28. Axial temperature profiles at the center line of a thick laser TL dosimeter. The TLD is heated with a $0.170 \mathrm{~cm}$ diameter, $4 \mathrm{~W}$ unfocused laser beam for $0.4 \mathrm{sec}$, $1.0 \mathrm{sec}, 2.0 \mathrm{sec}$, and $3.0 \mathrm{sec}$. 


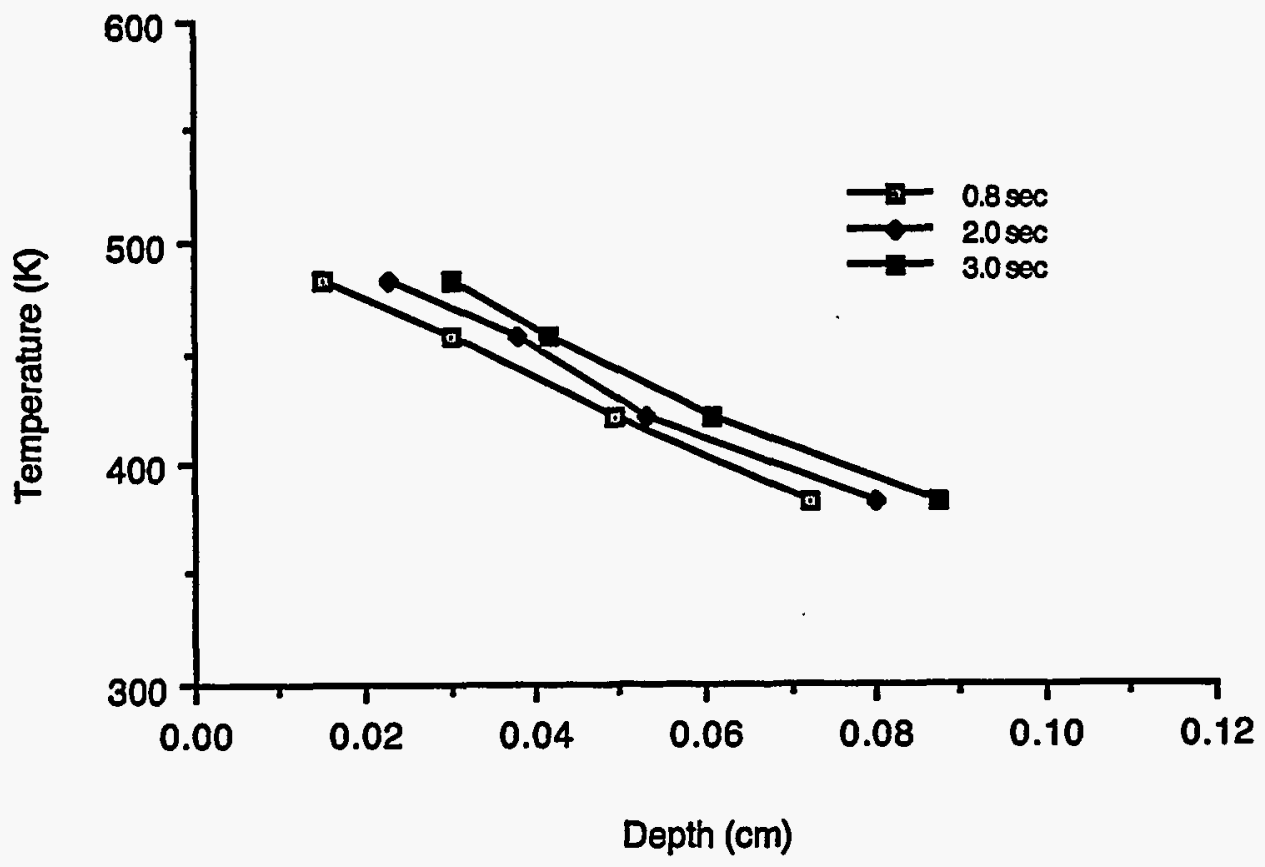

Figure 29. Axial temperature gradients at the center of a thick laser TL dosimeter with various unfocused laser heating times. The data were obtained for $a 0.170 \mathrm{~cm}$ diameter, $4 \mathrm{~W}$ unfocused Gaussian laser beam. 


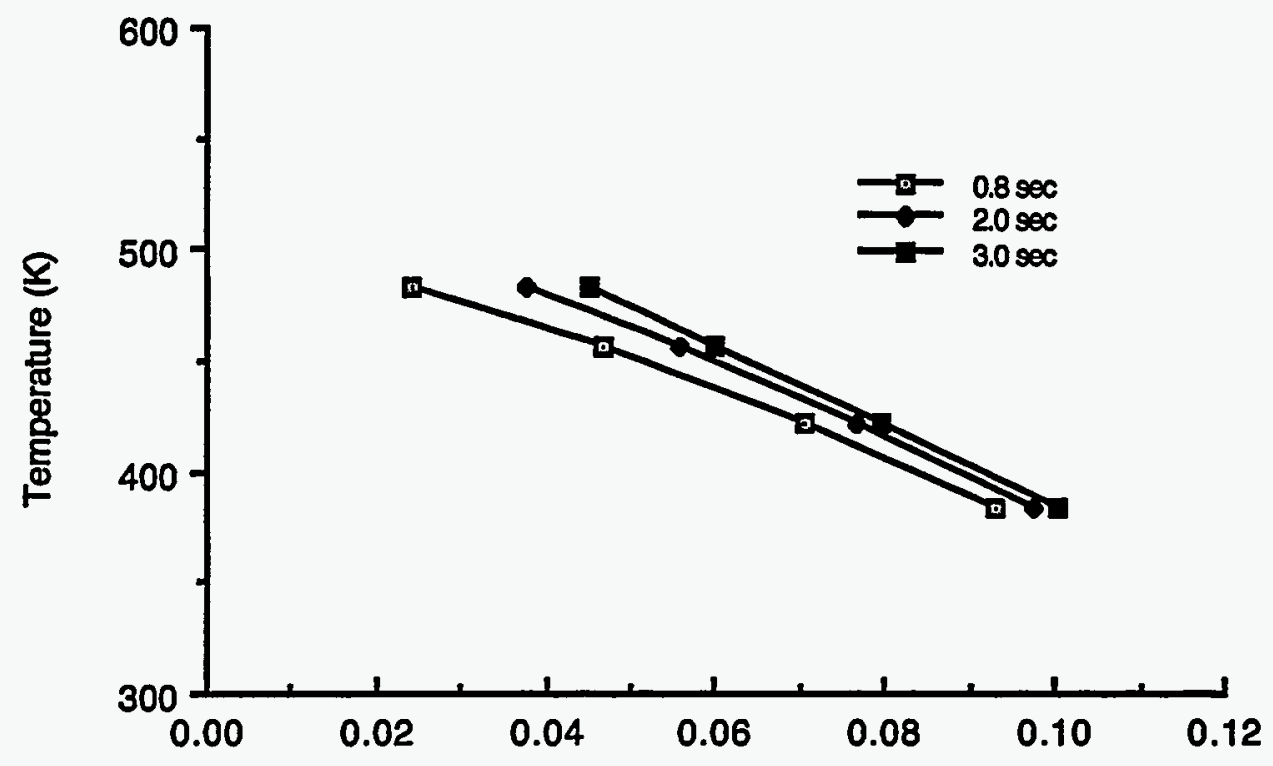

Radial distance (cm)

Figure 30. Radial temperature gradients at the surface of a thick laser TL dosimeter with various unfocused laser heating times. The data were obtained for a $0.170 \mathrm{~cm}$ diameter, 4 W unfocused Gaussian laser beam. 


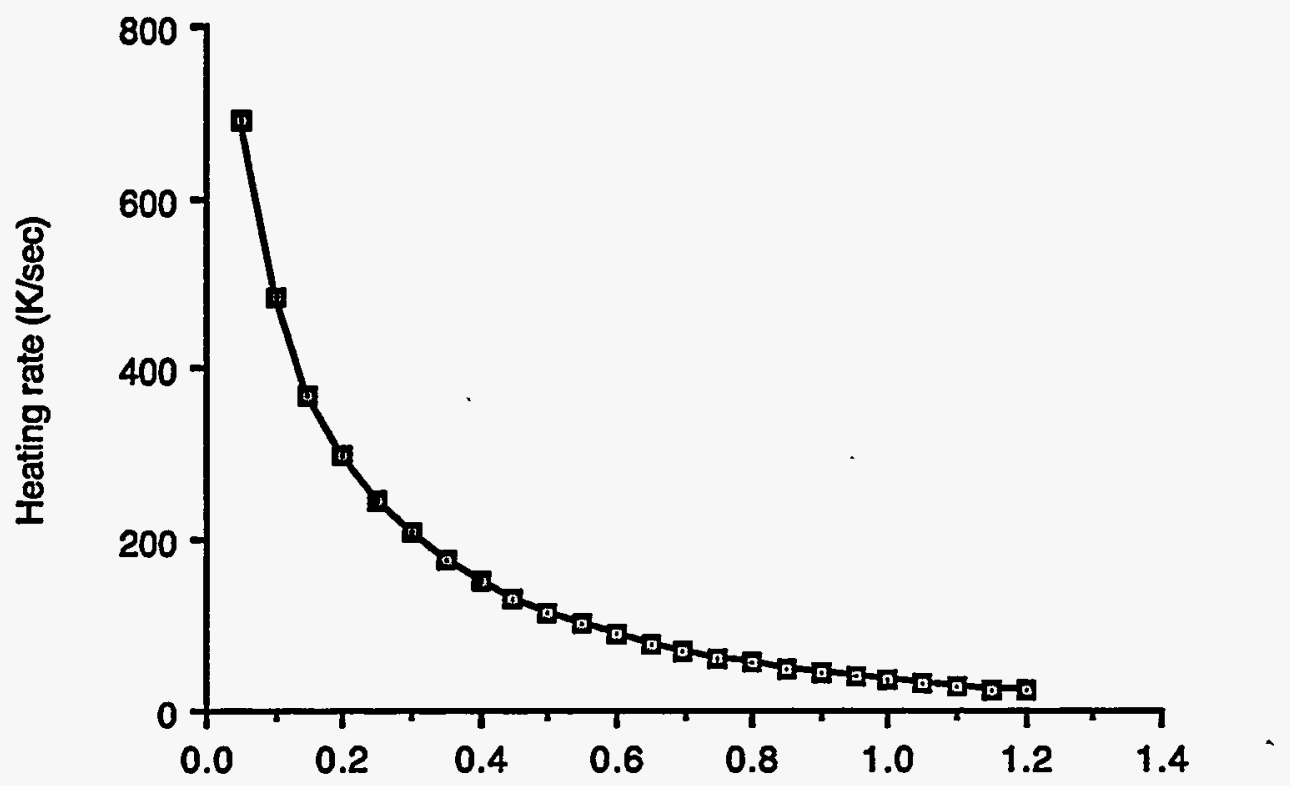

Heating time (seconds)

Figure 31. Calculated heating rate at center of the surface of a thick laser TL dosimeter by unfocused laser beam heating with a $0.170 \mathrm{~cm}$ diameter, $4 \mathrm{~W}$ unfocused laser beam. 


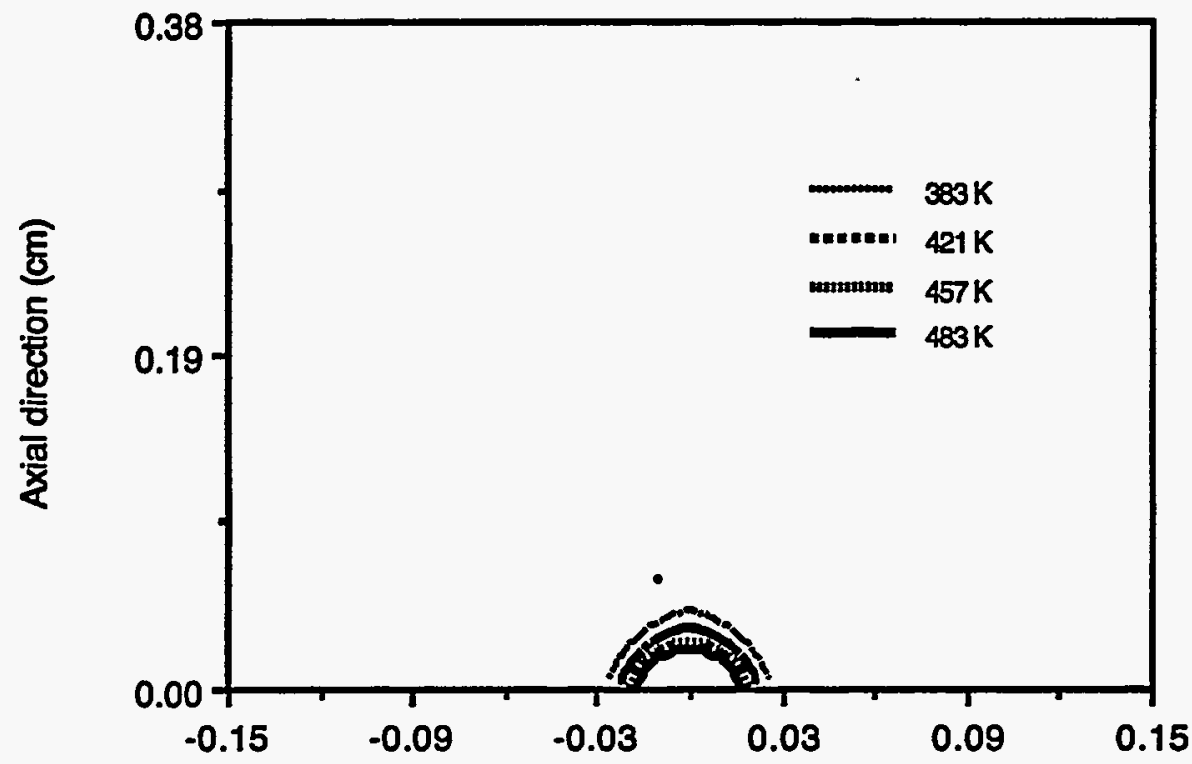

Radial direction (cm)

Figure 32. Two-dimensional temperature distributions in a thick laser TL dosimeter following heating with a $0.032 \mathrm{~cm}$ diameter focused $4 \mathrm{~W}$ continuous wave (CW) laser beam for $18 \mathrm{msec}$. 


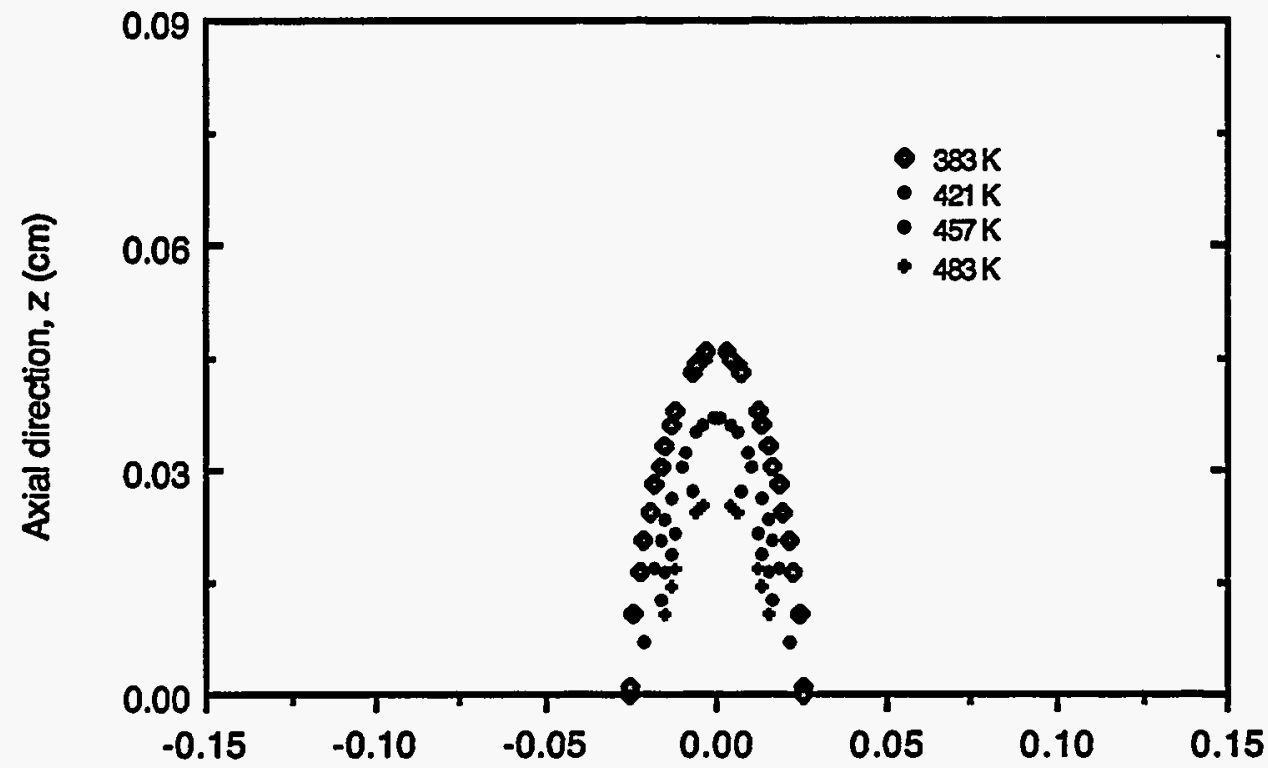

Radial direction, $\mathrm{r}(\mathrm{cm})$

Figure 33. Temperature profiles in a $0.09 \mathrm{~cm}$ thick LiF TL dosimeter after $18 \mathrm{msec}$ of heating by $a .032 \mathrm{~cm}, 4 \mathrm{~W}$ focused laser. 


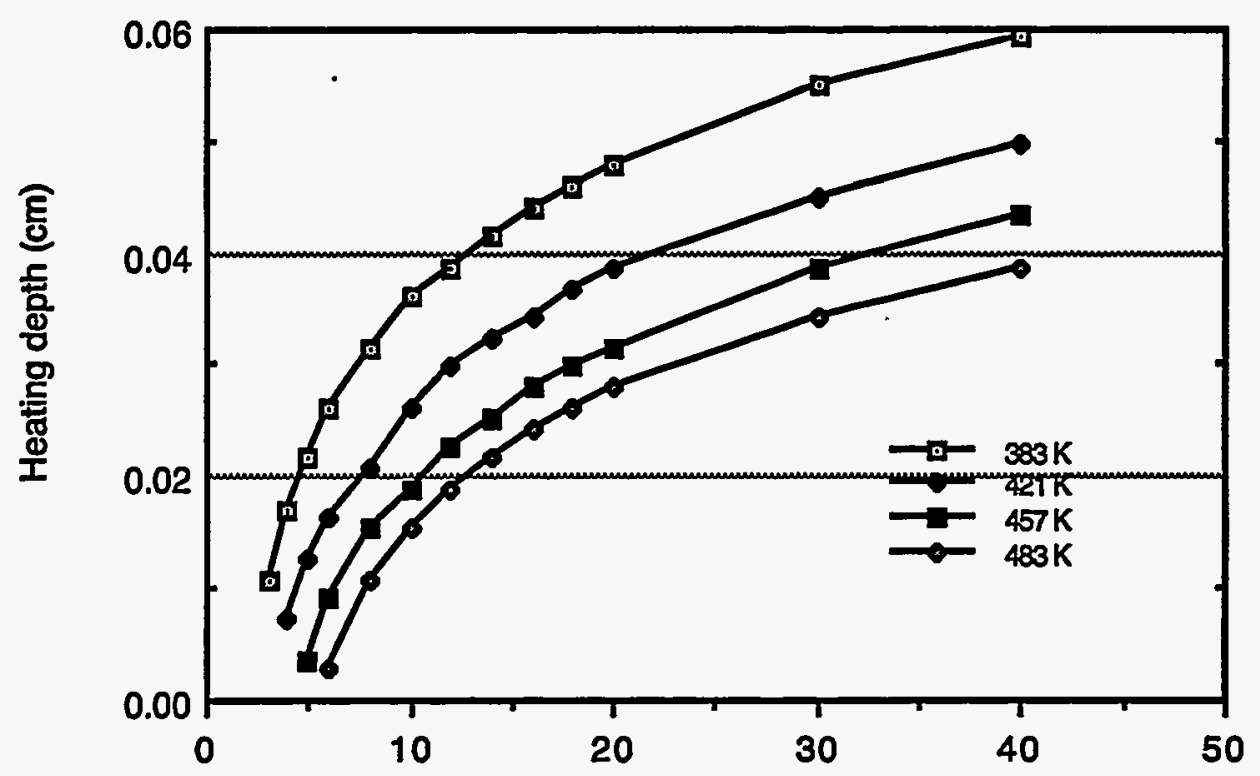

Heating time (msec)

Figure 34. Variations of heating depths corresponding to the peak temperatures of peaks 2$5(383 \mathrm{~K}, 421 \mathrm{~K}, 457 \mathrm{~K}$, and $483 \mathrm{~K}$ ) with various focused beam heating duration. These results were obtained for a $0.09 \mathrm{~cm}$ TL dosimeter heated with a $0.032 \mathrm{~cm}$ diameter, $4 \mathrm{~W}$ focused laser beam. 


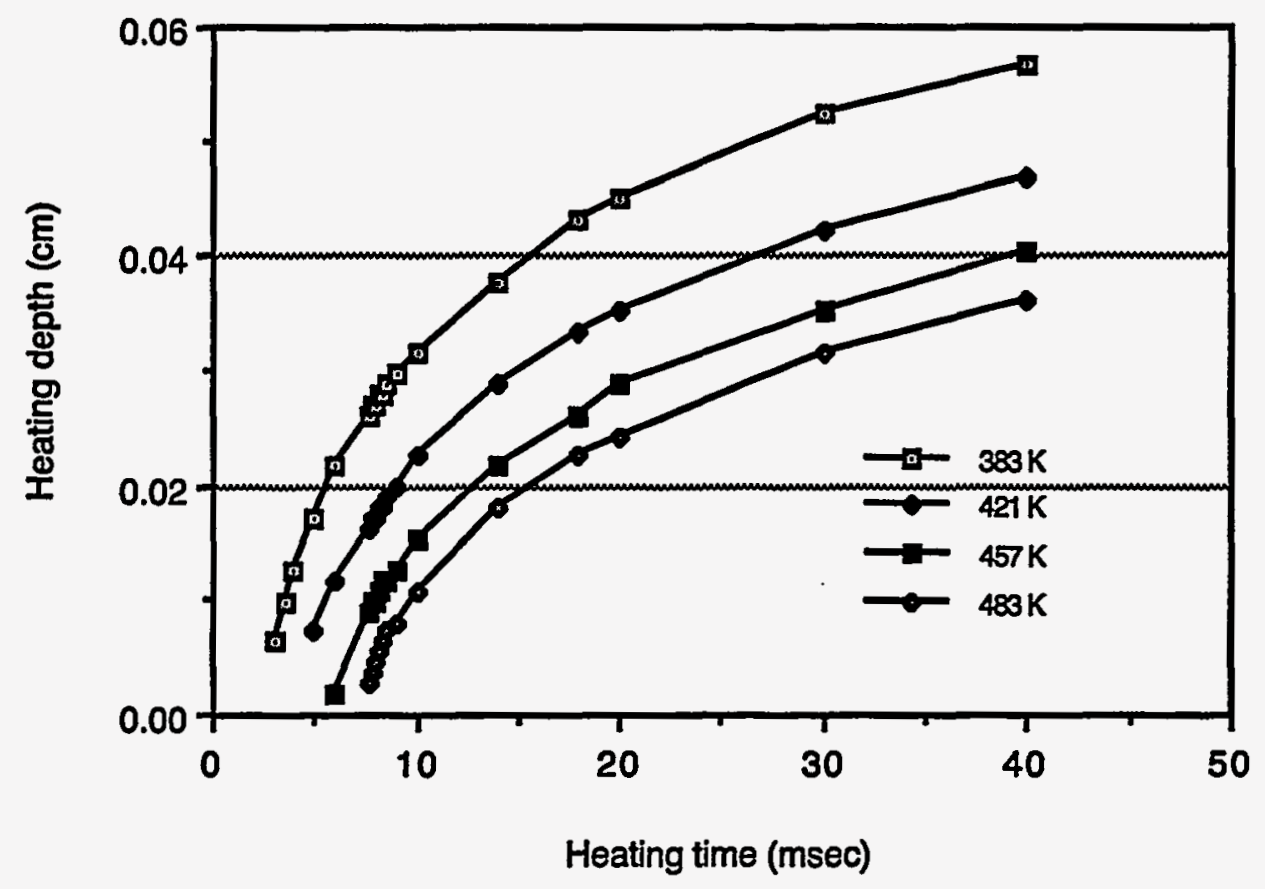

Figure 35. Variations of heating depths corresponding to the peak temperatures of peaks 2$5(383 \mathrm{~K}, 421 \mathrm{~K}, 457 \mathrm{~K}$, and $483 \mathrm{~K})$ with various focused beam heating duration. These results were obtained for a thick laser TL dosimeter heated with a $0.032 \mathrm{~cm}$ diameter, $4 \mathrm{~W}$ focused laser beam. 


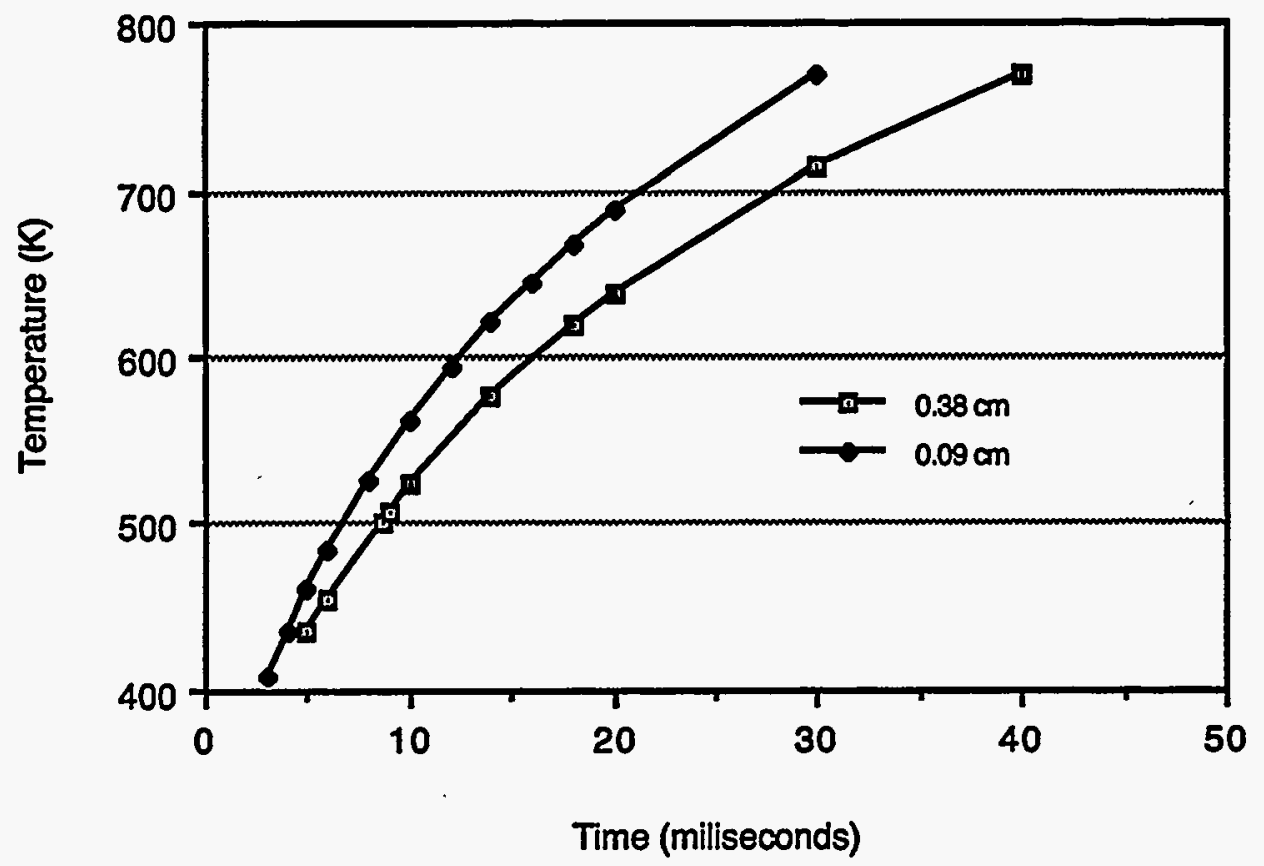

Figure 36. Surface temperatures as a function of heat duration for a $0.09 \mathrm{~cm}$ thick TLD and a thick laser TL dosimeter. These results were obtained with a $0.032 \mathrm{~cm}$ diameter, $4 \mathrm{~W}$ focused Gaussian laser beam. 


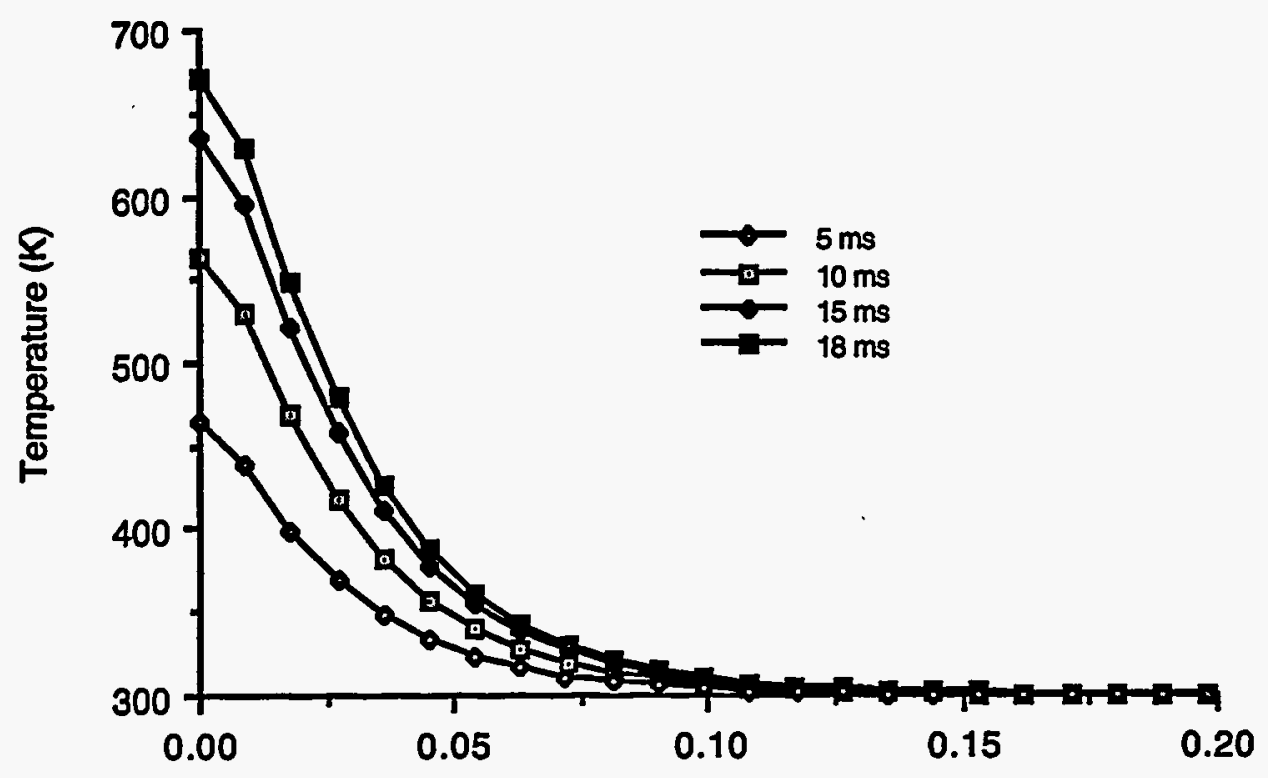

Axial direction, $\mathbf{z}(\mathrm{cm})$

Figure 37. Axial temperature profiles at the center line of a thick laser TL dosimeter heated with various focused laser beam heating durations: $5 \mathrm{~ms}, 10 \mathrm{~ms}, 15 \mathrm{~ms}$, and $18 \mathrm{~ms}$. The focused beam is $0.032 \mathrm{~cm}$ in diameter and $4 \mathrm{~W}$ in power. 


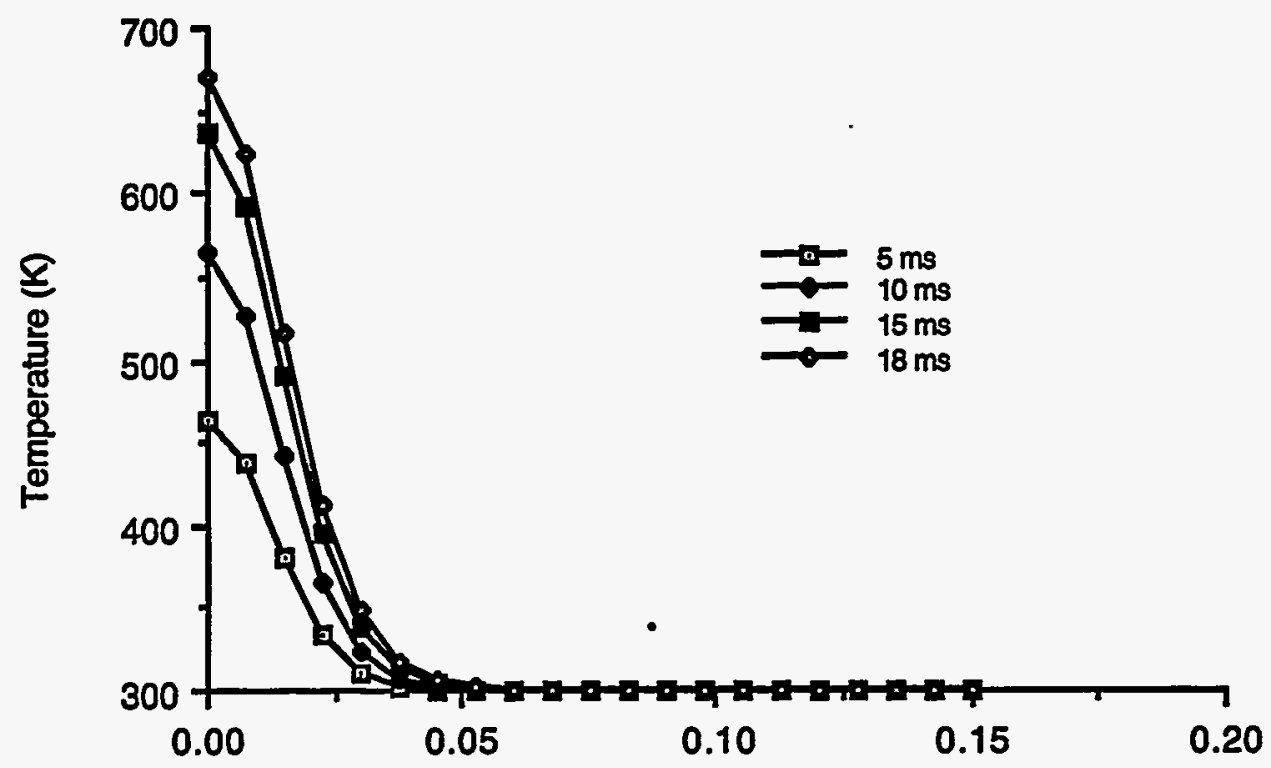

Radial direction, $\mathrm{r}(\mathrm{cm})$

Figure 38. Radial temperature profiles at the surface of a thick laser TL dosimeter arising from various focused laser beam durations: $5 \mathrm{~ms}, 10 \mathrm{~ms}, 15 \mathrm{~ms}$, and $18 \mathrm{~ms}$. The focused beam is $0.032 \mathrm{~cm}$ in diameter and $4 \mathrm{~W}$ in power. 


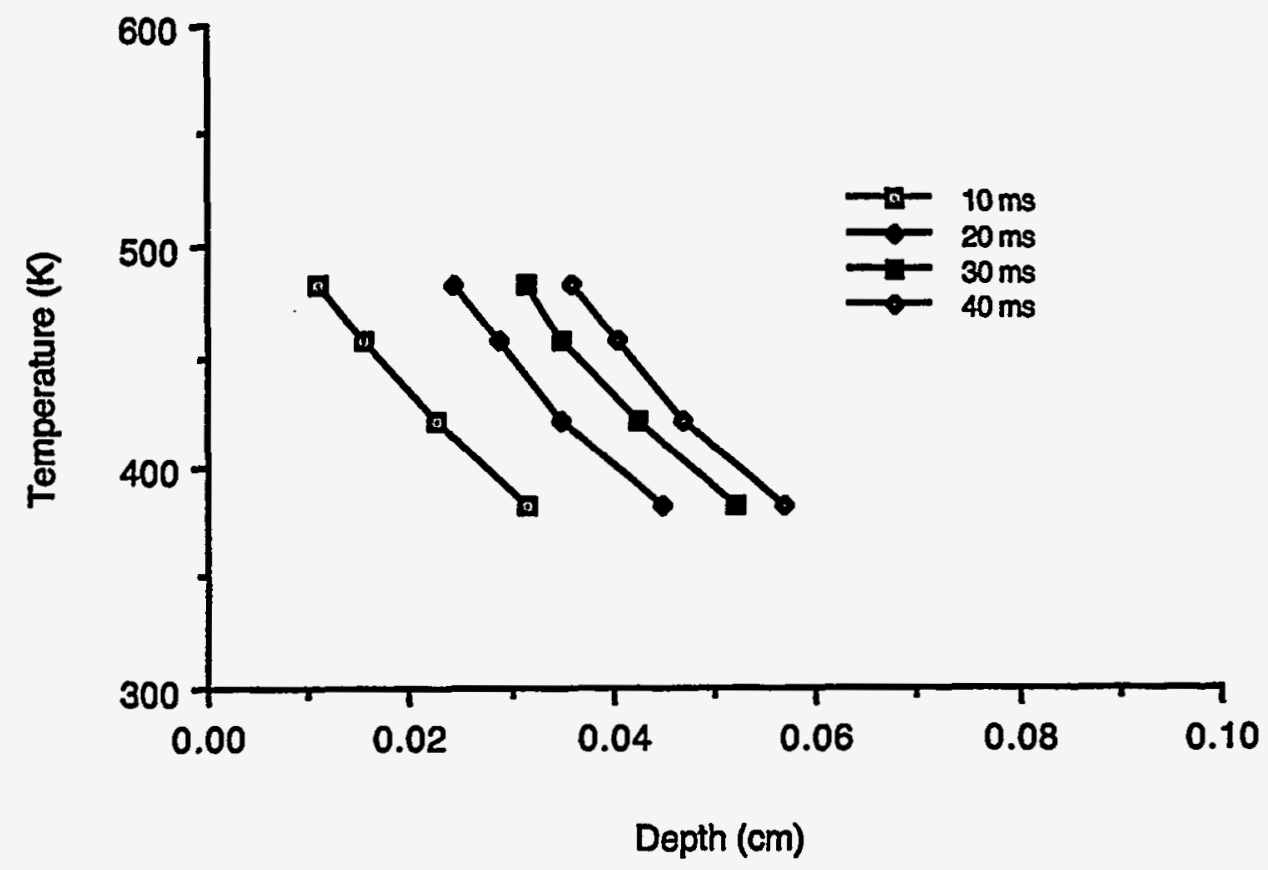

Figure 39. Axial temperature profiles at the center line of a thick laser TL dosimeter with various focused laser heating durations. The focused beam is $0.032 \mathrm{~cm}$ in diameter and 4 $\mathrm{W}$ in power. 


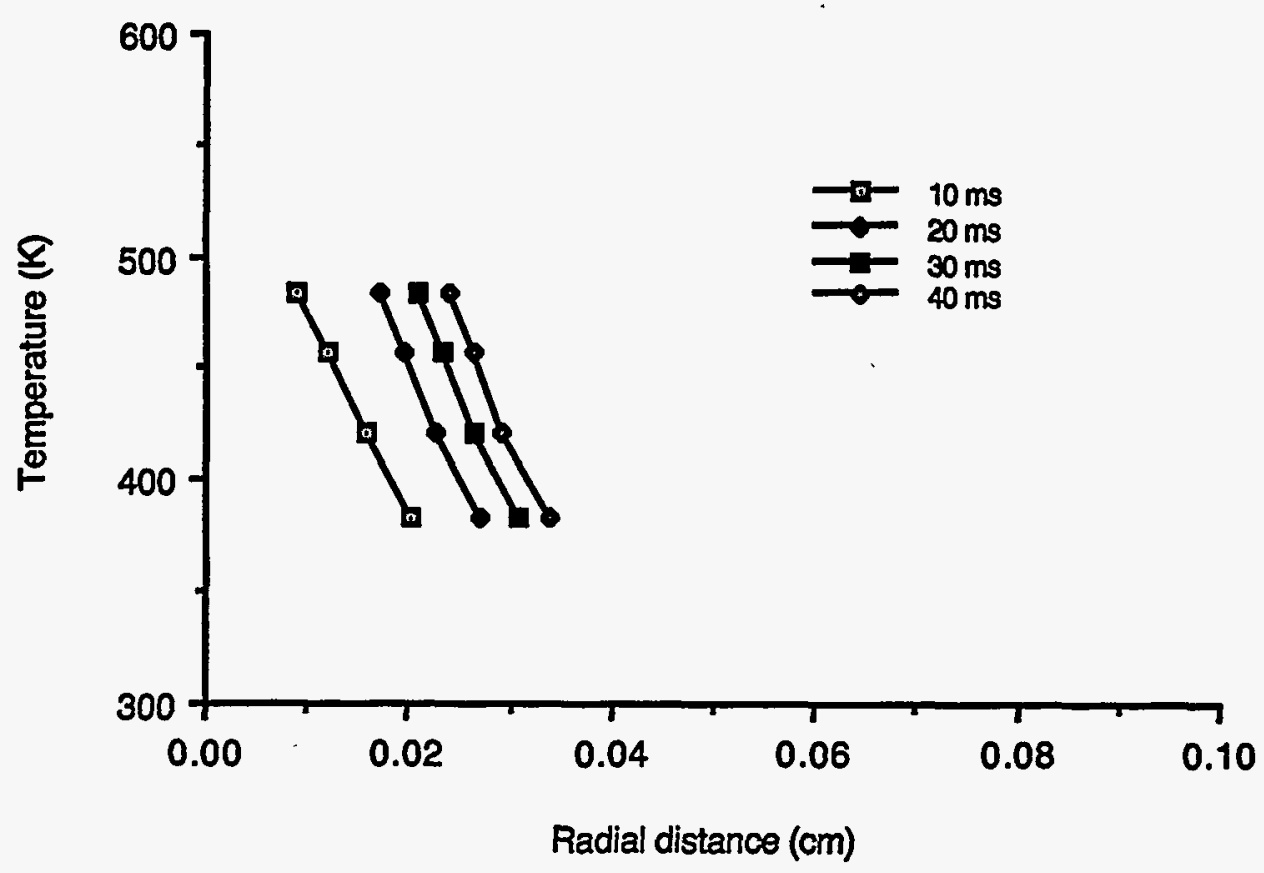

Figure 40. Radial temperature profiles at the surface of a thick laser TL dosimeter with various focused laser heating durations. The focused beam is $0.032 \mathrm{~cm}$ in diameter and 4 $\mathrm{W}$ in power. 


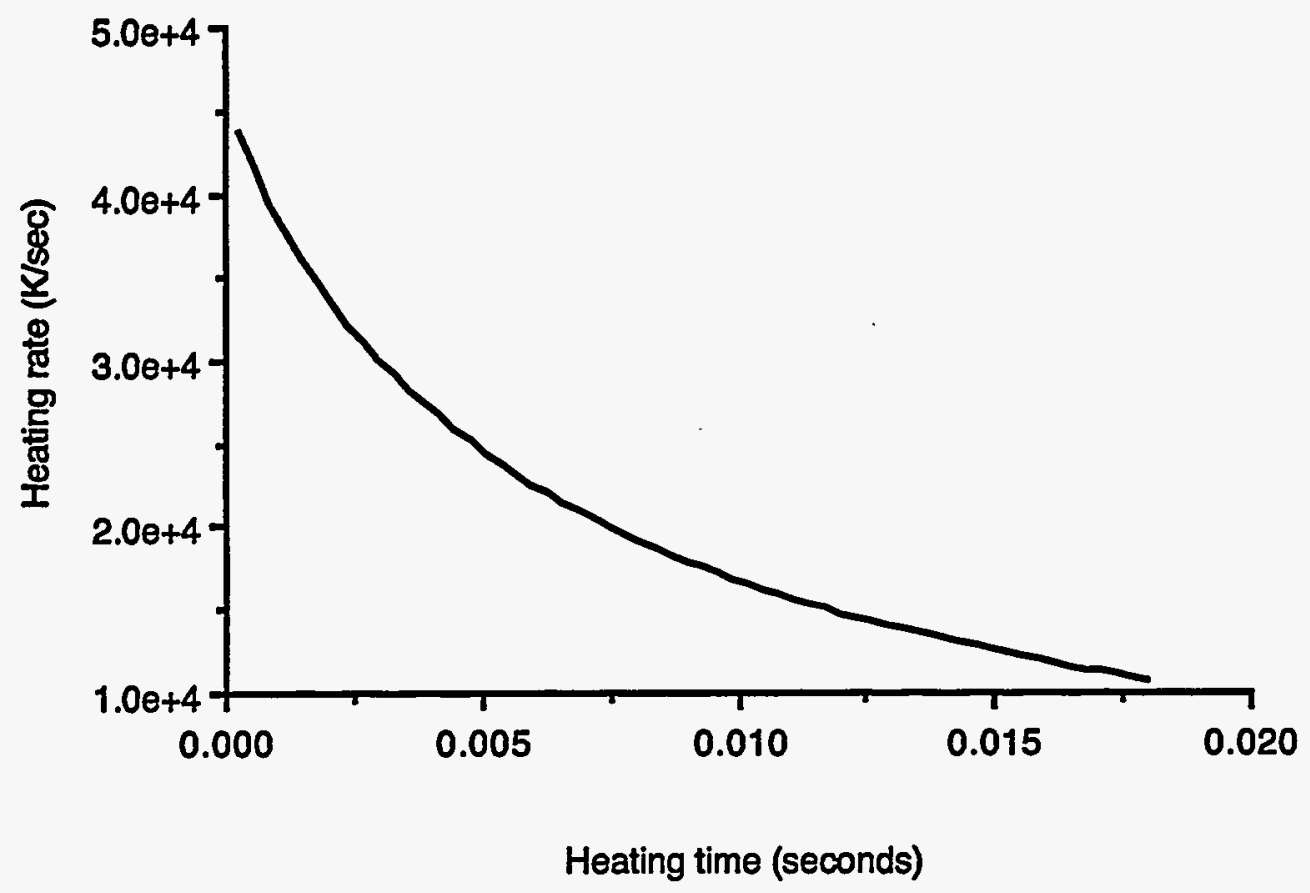

Figure 41. Calculated heating rates at the center of surface of a thick laser TL dosimeter. The results were obtained for a focused laser beam heating with a 0.032 $\mathrm{cm}$ diameter and $4 \mathrm{~W}$ in power. 


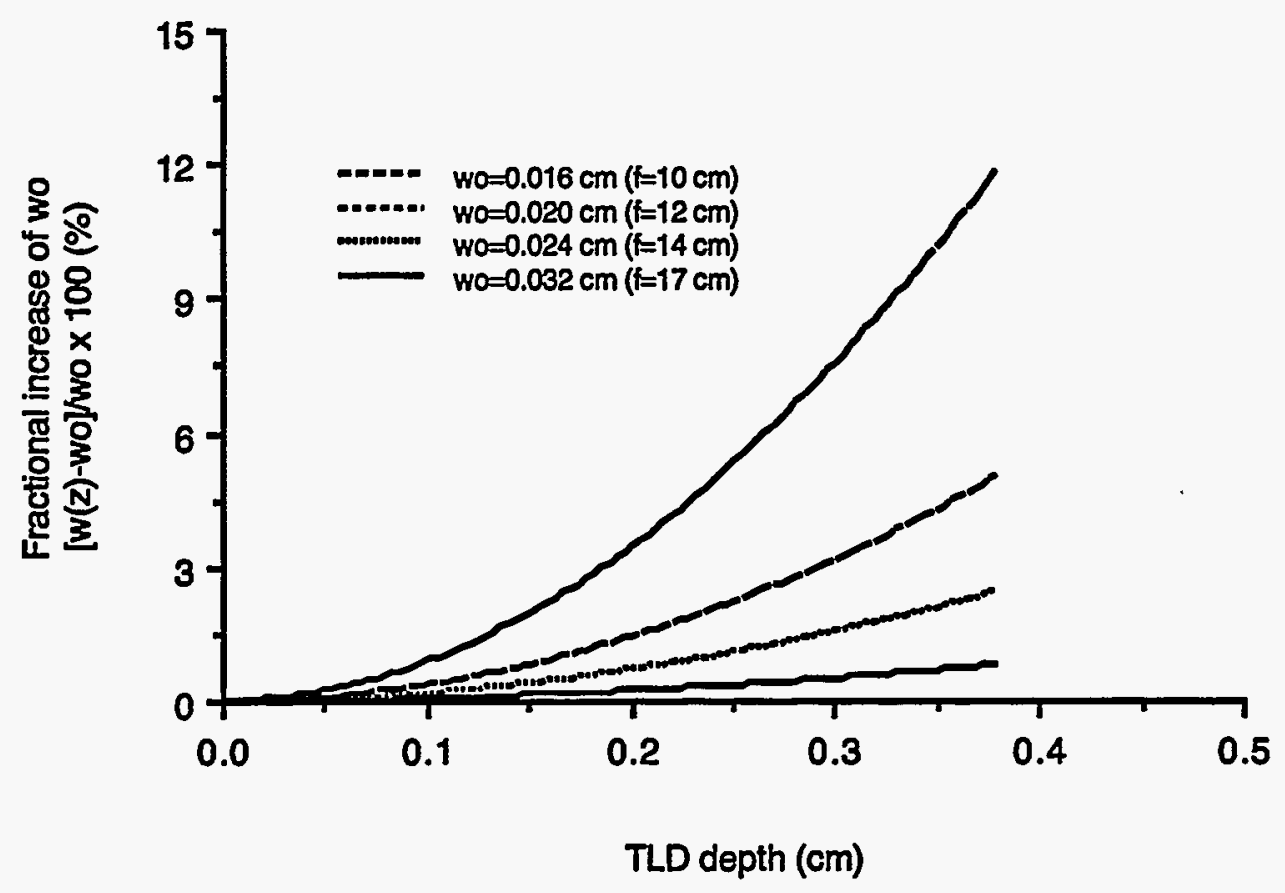

Figure 42. The variations of focused beam diameter as a function of depth in a thick TL dosimeter. These results were obtained with various focal length, $f$ for $4 \mathrm{~W}$ focused laser beam heating. 


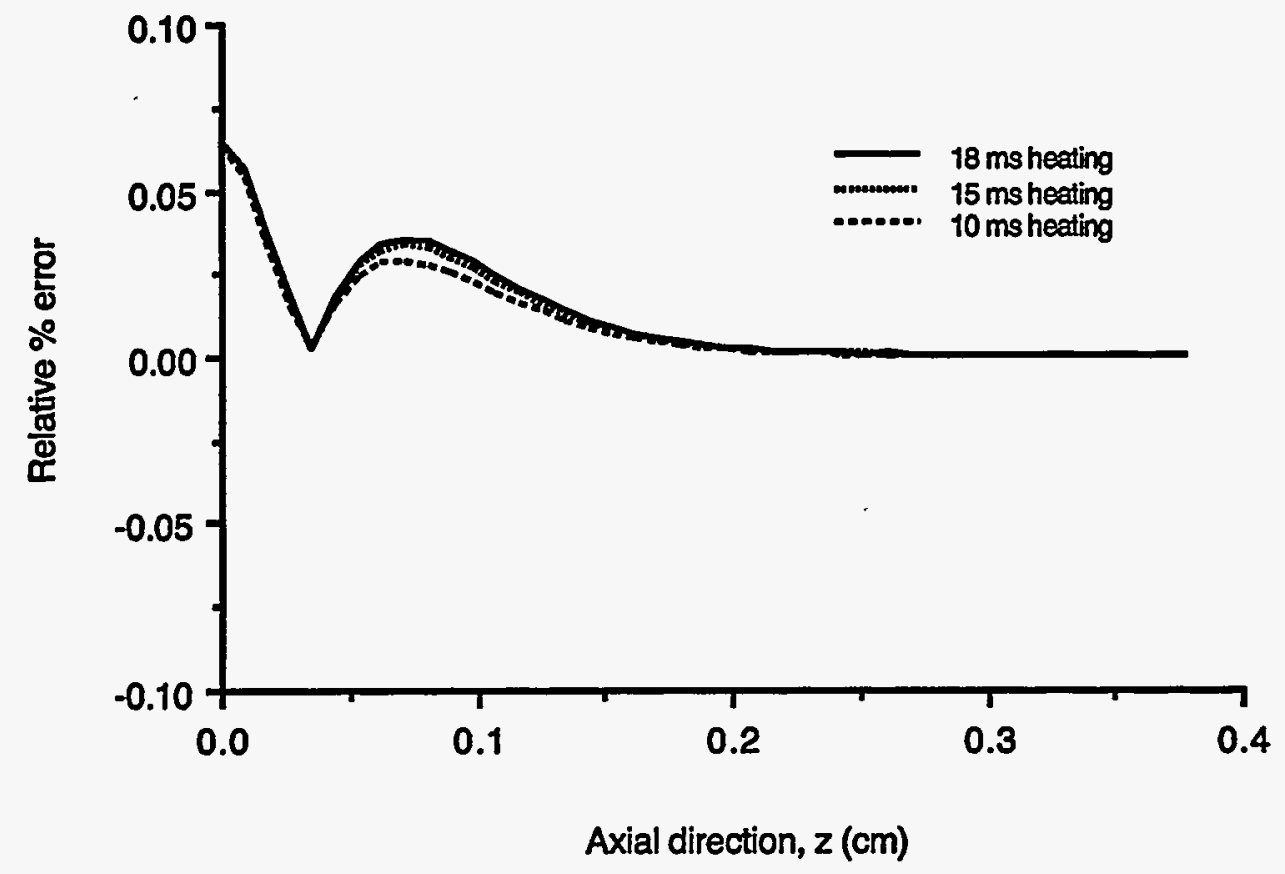

Figure 43. Relative differences of temperatures calculated with $\omega_{\mathrm{o}}$ and $\omega(\mathrm{z})$, respectively, at the center $(r=0)$ of a thick laser TL dosimeter with various heating durations. These results were obtained for $0.032 \mathrm{~cm}$ diameter $4 \mathrm{~W}$ focused laser beam and heating durations of 10 $\mathrm{ms}, 15 \mathrm{~ms}$, and $18 \mathrm{~ms}$. 


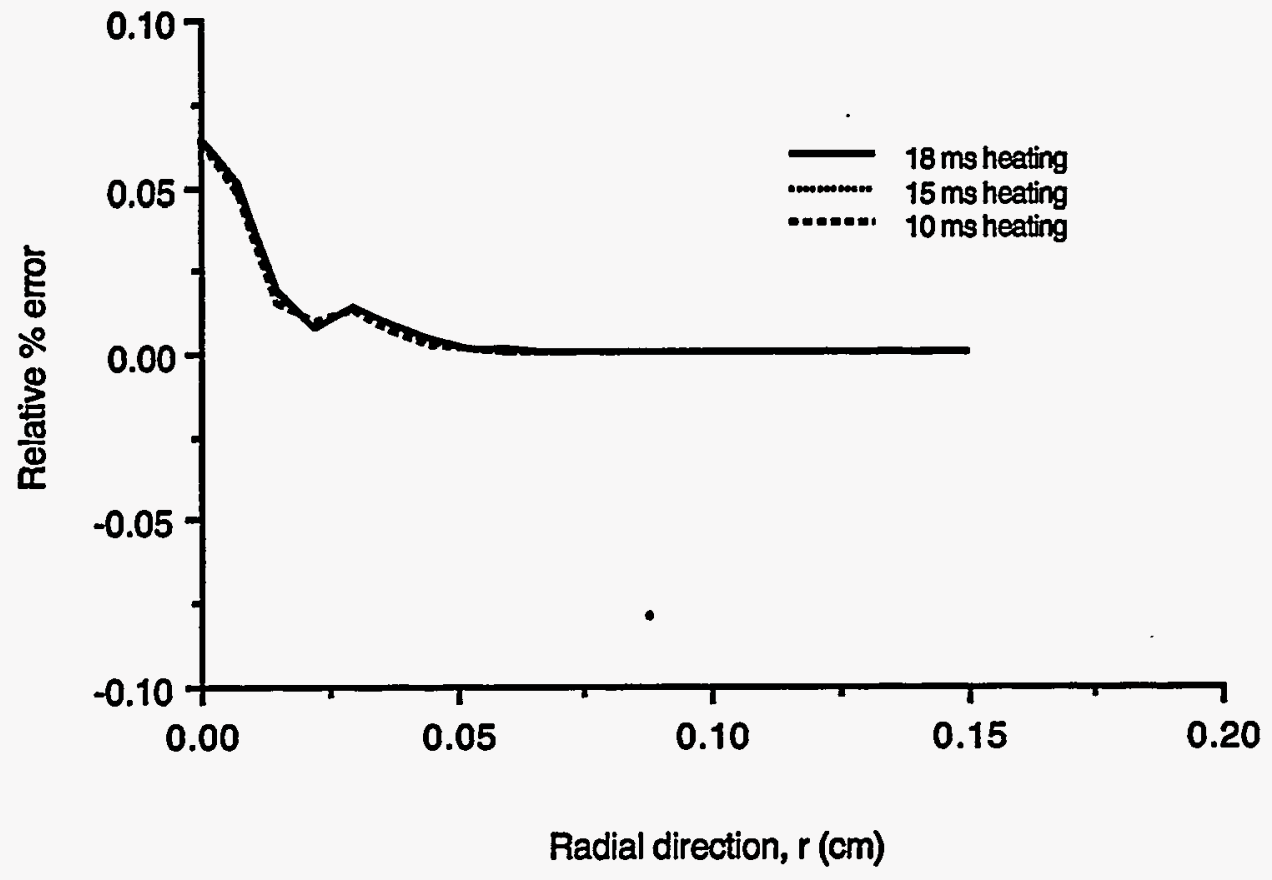

Figure 44. Relative differences of temperatures calculated with $\omega_{0}$ and $\omega(\mathrm{z})$, respectively, at the surface $(z=0)$ of a thick laser TL dosimeter with various heating durations. These results were obtained for $0.032 \mathrm{~cm}$ diameter $4 \mathrm{~W}$ focused laser beam and heating durations for $10 \mathrm{~ms}, 15 \mathrm{~ms}$, and $18 \mathrm{~ms}$. 


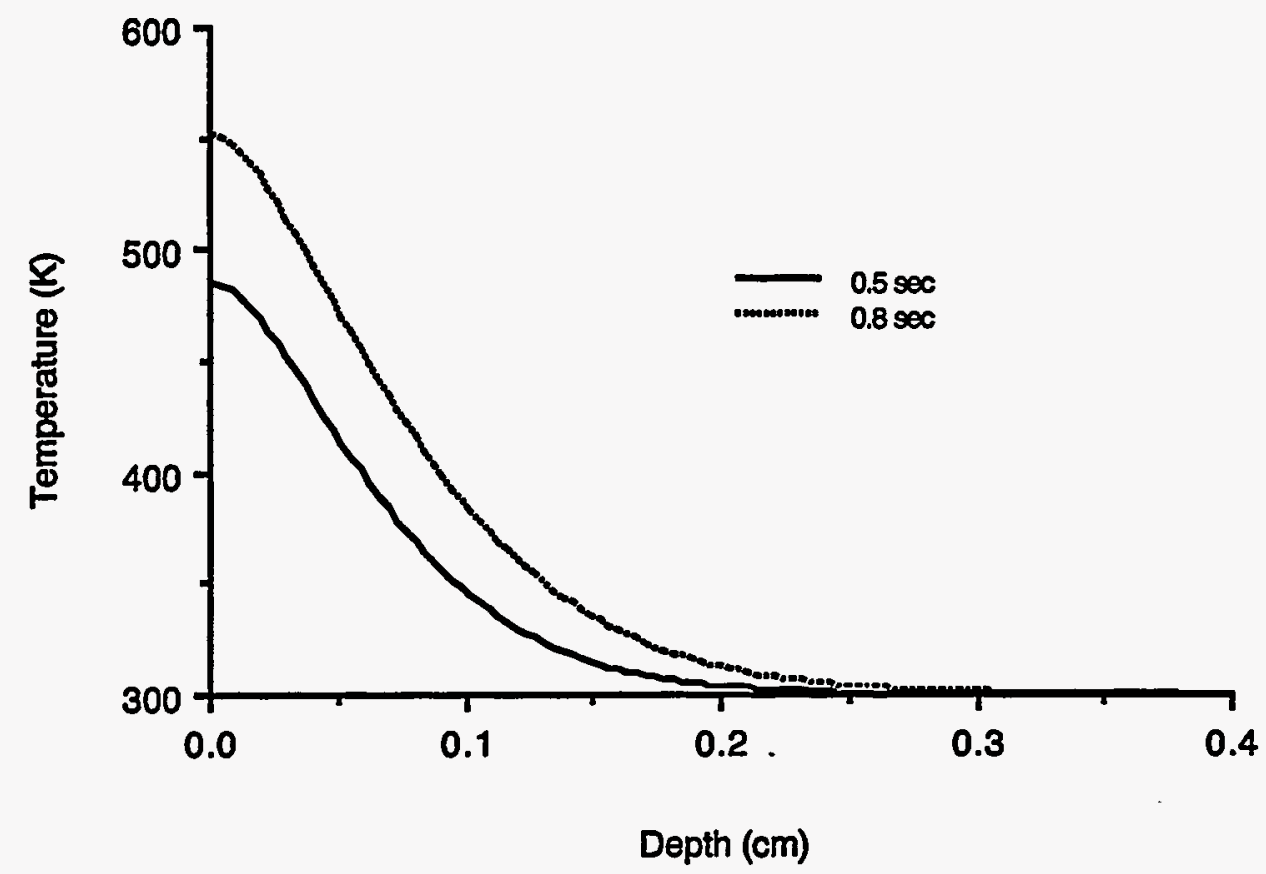

Figure 45. Temperature distributions in axial direction of a thick LiF TLD, following heating with a $0.3 \mathrm{~cm} \times 0.3 \mathrm{~cm} 10 \mathrm{~W}$ uniform laser beam for $0.5 \mathrm{sec}$ and $0.8 \mathrm{sec}$, respectively. The results were obtained using the numerical solution assuming that no heat transfer is allowed on the TLD boundaries. 


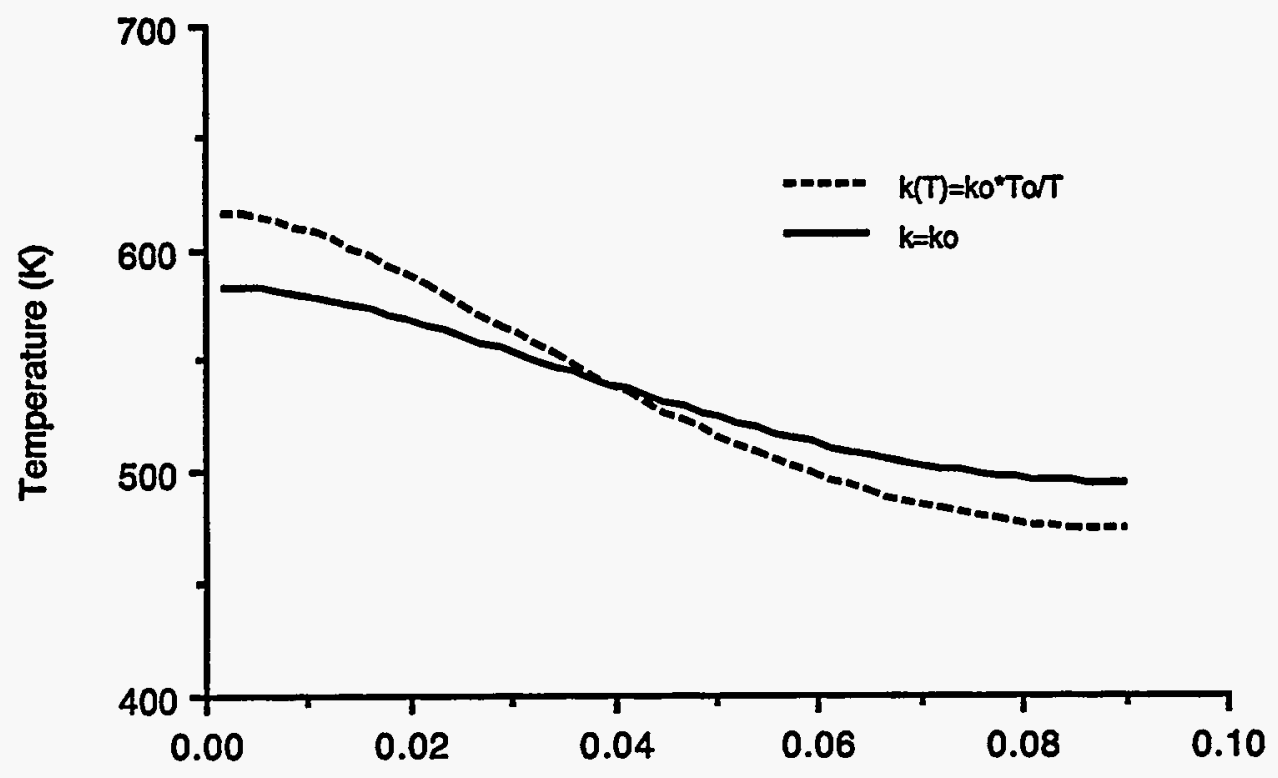

Depth (cm)

Figure 46. Temperature profiles in $0.09 \mathrm{~cm}$ thick TL dosimeter using an average thermal conductivity $\mathrm{k}_{\mathrm{o}}$ (solid line) and a temperature-dependent thermal conductivity $\mathrm{k}(\mathrm{T})$ (dashed line) for a $0.3 \mathrm{~cm} \times 0.3 \mathrm{~cm} 10 \mathrm{~W}$ uniform laser beam. 


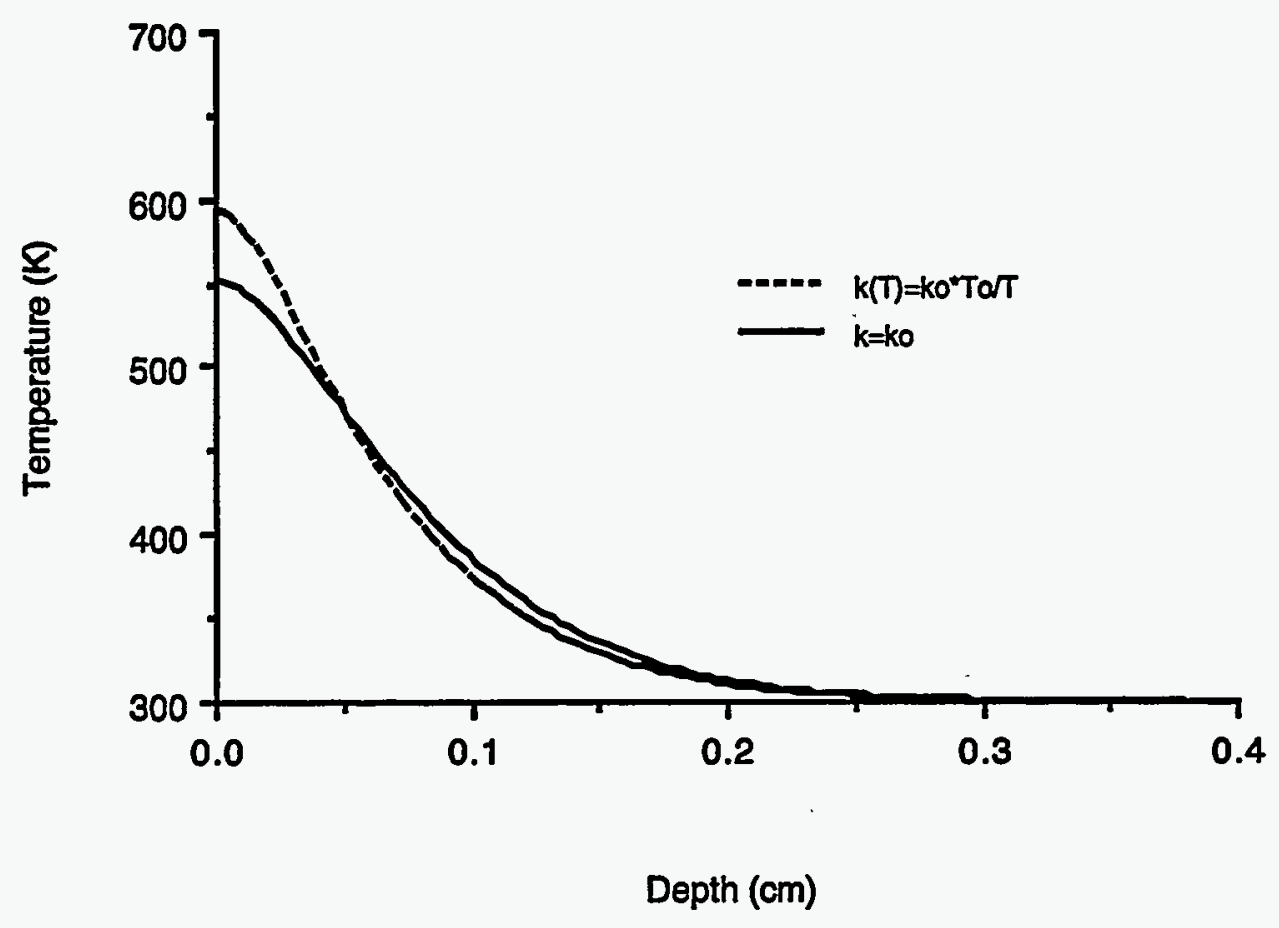

Figure 47. Temperature profiles at $0.8 \mathrm{sec}$ in a thick laser TL dosimeter using an average thermal conductivity, $k_{o}$ (solid line) and a temperature-dependent thermal conductivity, $k(T)$ (dashed line) for a $0.3 \mathrm{~cm} \times 0.3 \mathrm{~cm} 10 \mathrm{~W}$ uniform laser beam. 


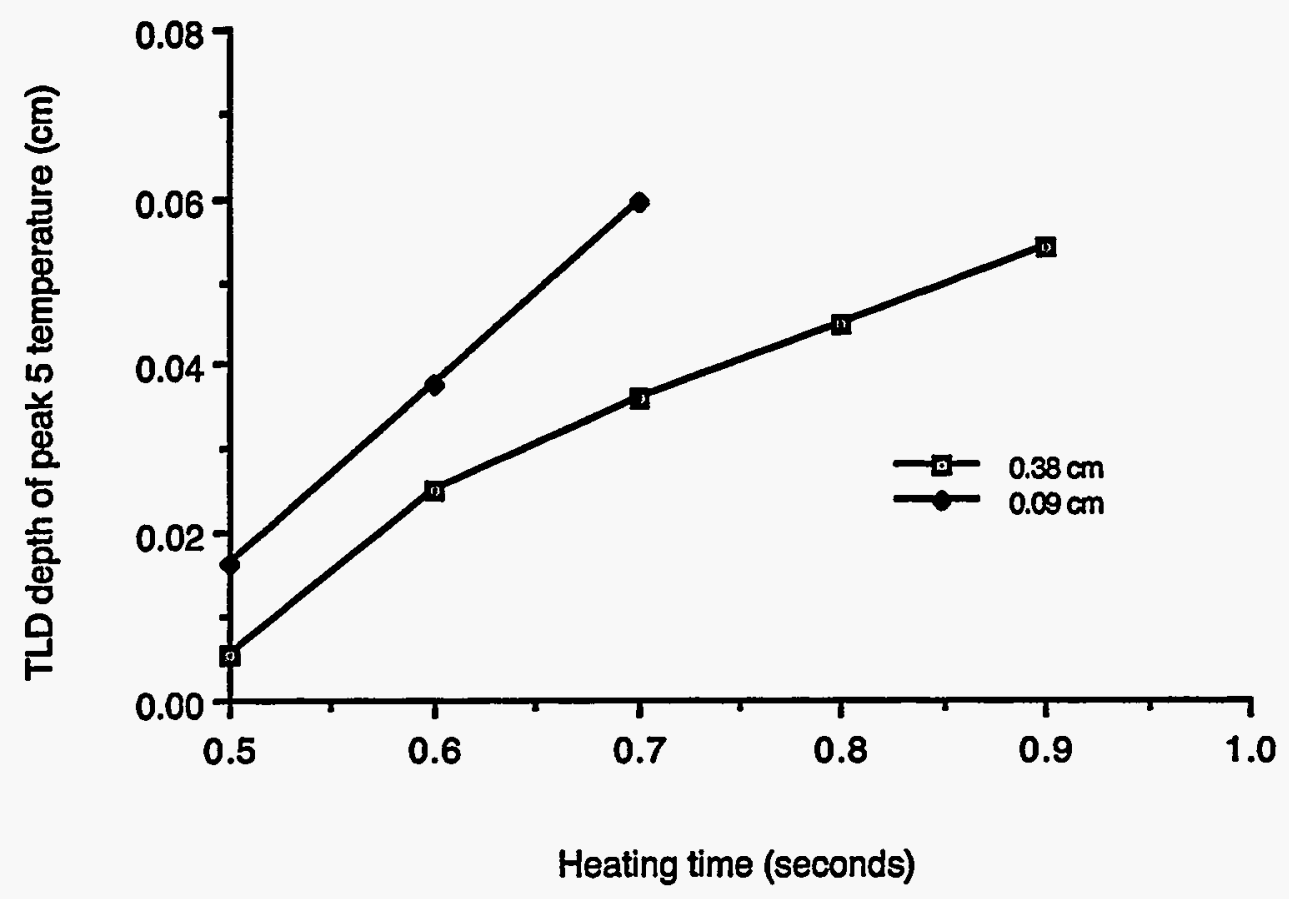

Figure 48. Variations of TL dosimeter depths corresponding to peak 5 temperature ( $483 \mathrm{~K}$ ) as a function of heating duration. These results were obtained for a $0.09 \mathrm{~cm}$ thick TLD and a thick laser TLD heated with $0.3 \mathrm{~cm} \times 0.3 \mathrm{~cm}, 10 \mathrm{~W}$ uniform beam. 


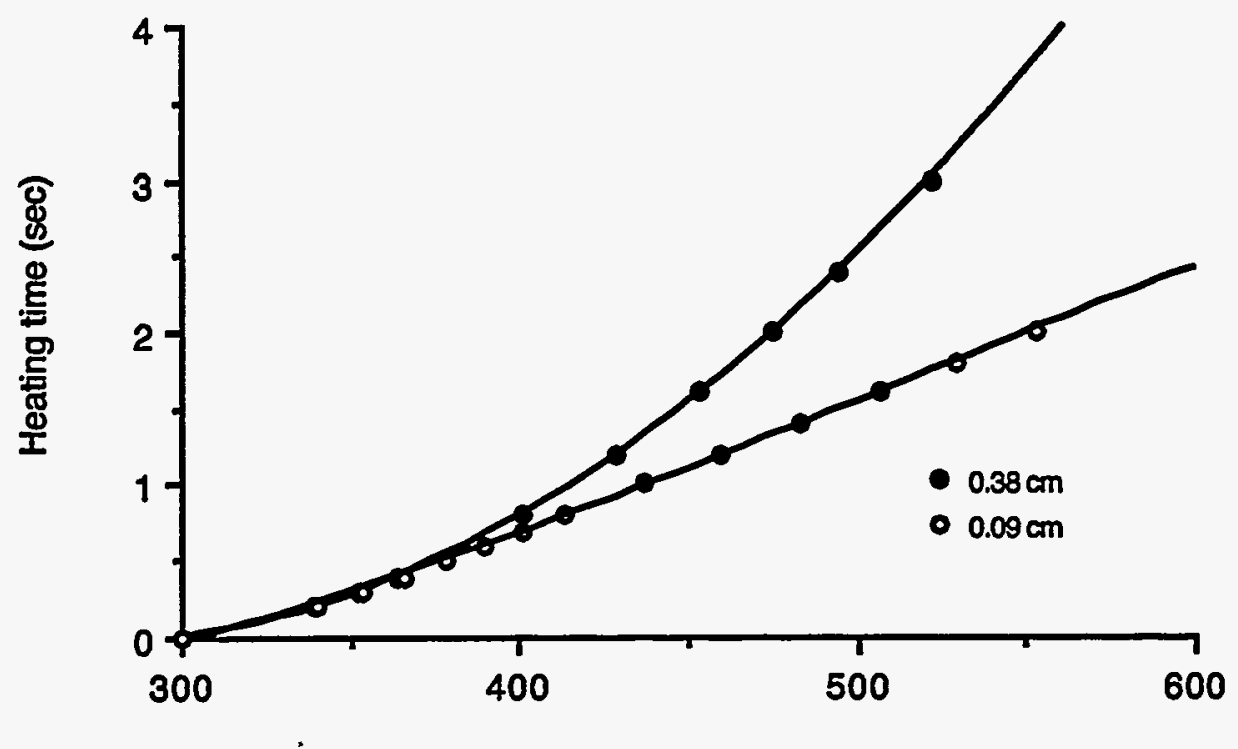

Surface temperature (K)

Fitted Data (Filled circle; $y=3.5439-2.7475 e-2 x+5.4249 e-5 x^{\wedge} 2-6.8343 e-9 x^{\wedge} \beta \quad R^{\wedge} 2=1.000$; Open circle; $y=-9.9145 e-2-7.3616 e-3 x+3.1323 e-5 x^{\wedge} 2-2.0072 e-8 x^{\wedge} 3 R^{\wedge} 2=1.000$ )

Figure 49. Surface temperature as a function of heating duration for a $0.09 \mathrm{~cm}$ thick TLD and a thick laser TL dosimeters. The results were obtained for a $0.3 \mathrm{~cm} \times 0.3 \mathrm{~cm}$ $4 \mathrm{~W}$ uniform laser beam. 


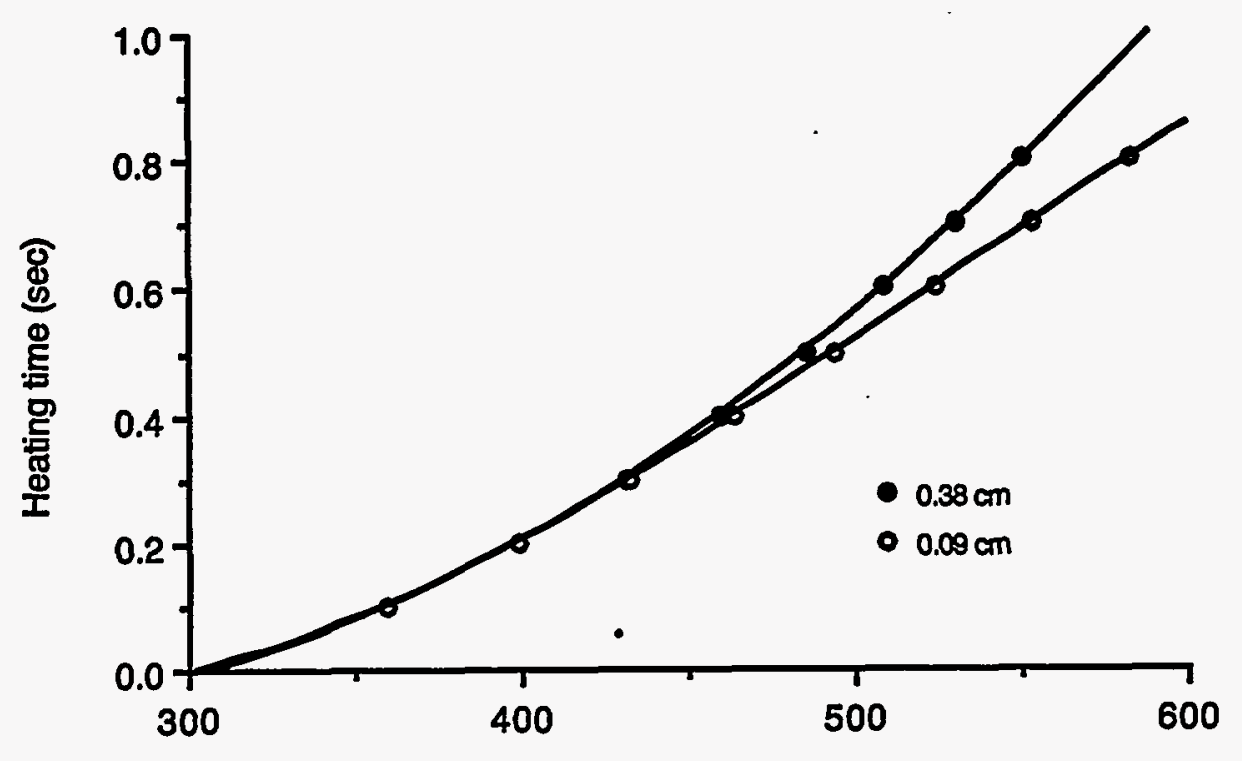

Surface temperature $(K)$

Fitted Data(Filled hole; $y=0.32199-3.5666 e-3 x+8.50300-6 x^{\wedge} 2-8.1067 e-10 x^{\wedge} 3 \quad R^{\wedge} 2=1.000$; Open hole; $y=0.74612-7.5958 e-3 x+2.0863 e-5 x^{\wedge} 2-1.3155 e-8 x^{\wedge} 3 \quad R^{\wedge} 2=1.000$ )

Figure 50. Surface temperature as a function of heating duration for a $0.09 \mathrm{~cm}$ thick TLD and a thick laser TL dosimeters. The results were obtained for a $0.3 \mathrm{~cm} \times 0.3 \mathrm{~cm}$ $10 \mathrm{~W}$ uniform laser beam. 


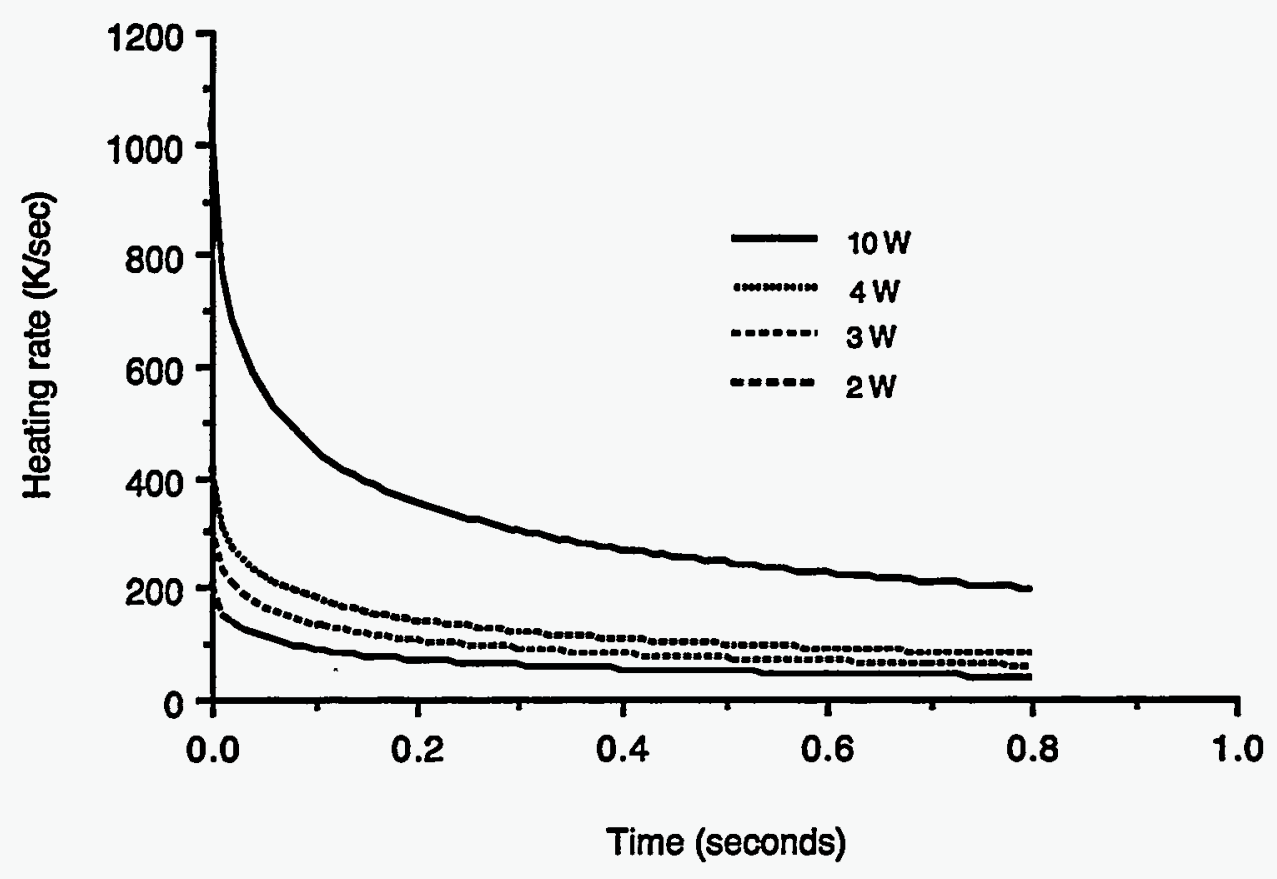

Figure 51. Calculated heating rates at the surface of a thick laser TL dosimeter by $0.3 \times 0.3$ cm square laser beam with various powers: (a) $2 \mathrm{~W}$; (b) $3 \mathrm{~W}$; (c) $4 \mathrm{~W}$; (d) $10 \mathrm{~W}$. 


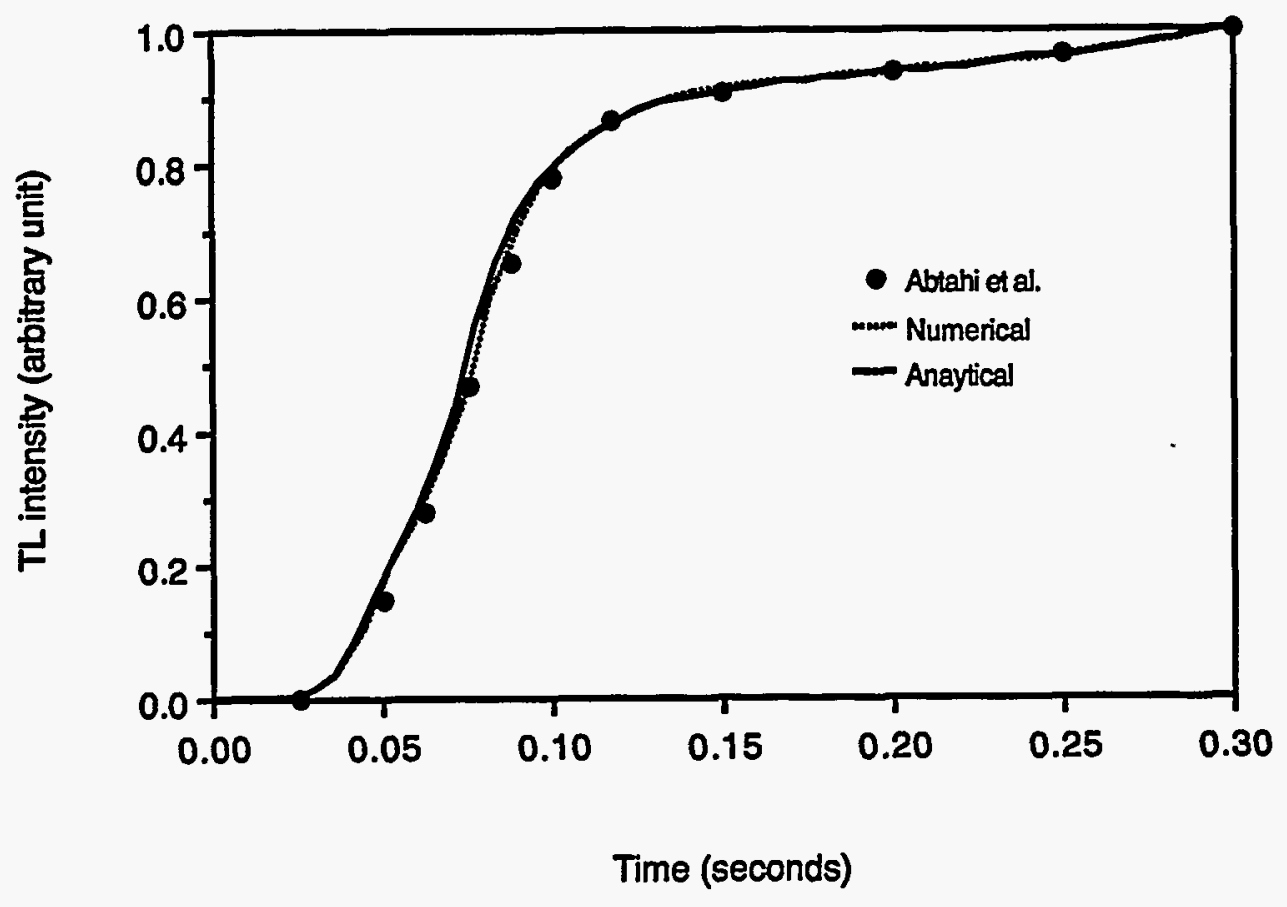

Figure 52. Glow curves for a $0.09 \mathrm{~cm}$ thick $\operatorname{LiF}$ TLD, predicted both by analytical solution (solid line) and by numerical solution (dotted line). The TLD was heated with a $0.084 \mathrm{~cm}$ diameter focused $4.93 \mathrm{~W}$ continuous wave (CW) laser beam. The published results [Abtahi et al., 1985] is also included (in dots) for comparison. 


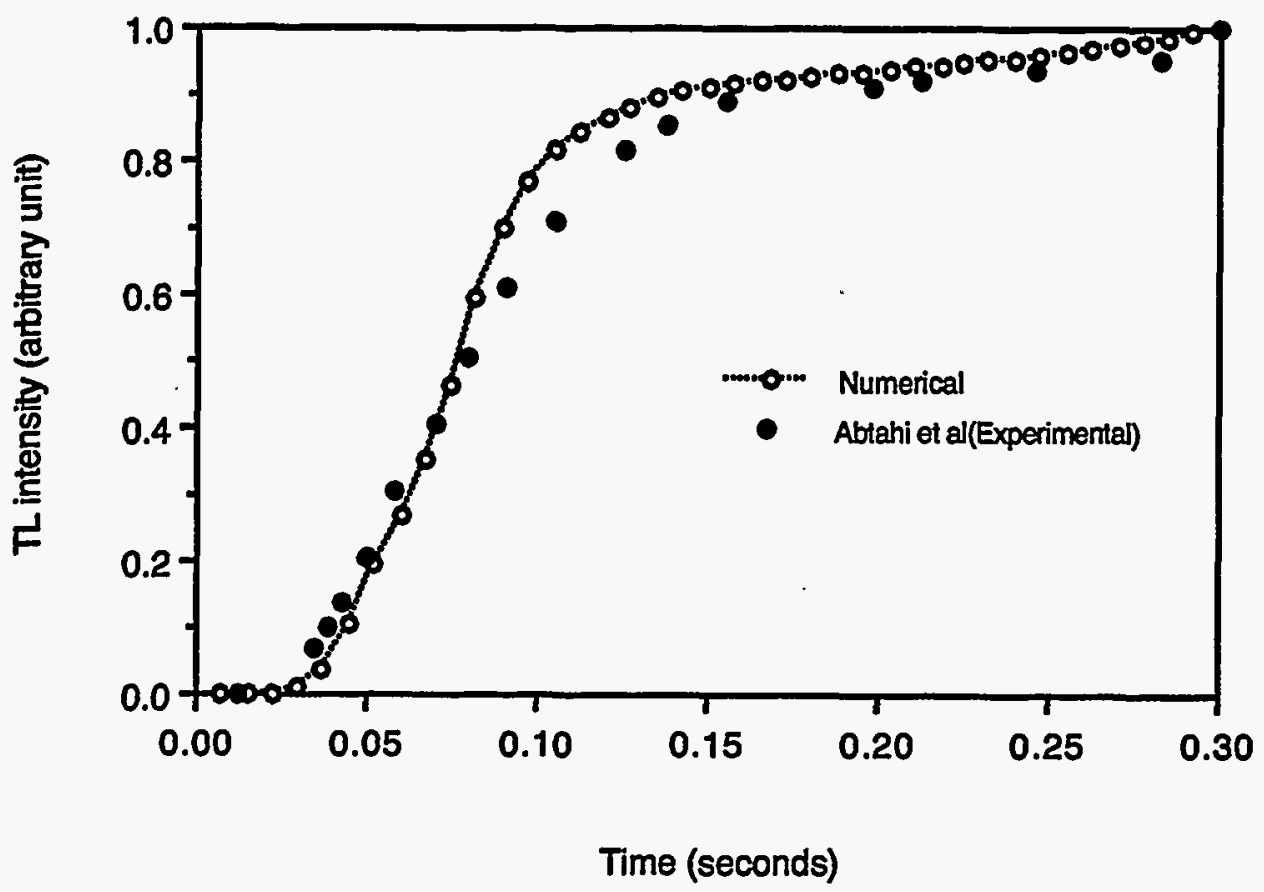

Figure 53. Glow curves calculated using a numerical solution (dotted line) for a $0.09 \mathrm{~cm}$ thick LiF TLD, following heating with a $0.084 \mathrm{~cm}$ diameter focused 4.93 W continuous wave (CW) laser beam. The experimental result (dots) published by Abtahi [Abtahi et al., 1985] is also shown for comparison. 


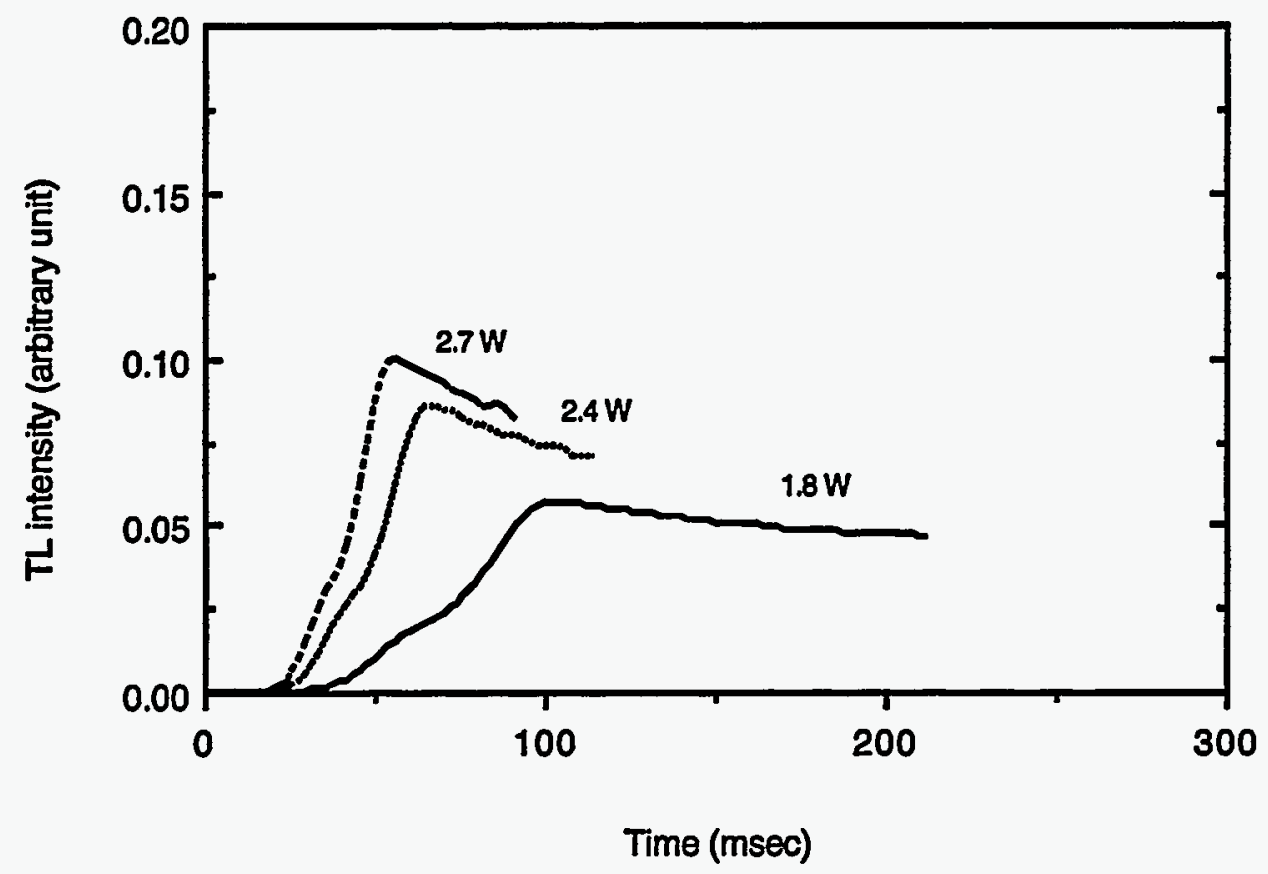

Figure 54 . Theoretical TL glow curves obtained from a $7 \mathrm{mg} / \mathrm{cm}^{2}(0.0027 \mathrm{~cm}) \mathrm{LiF}$ (TLD100) with a focused Gaussian laser beam of fixed $0.059 \mathrm{~cm}$ diameter and various laser powers. 


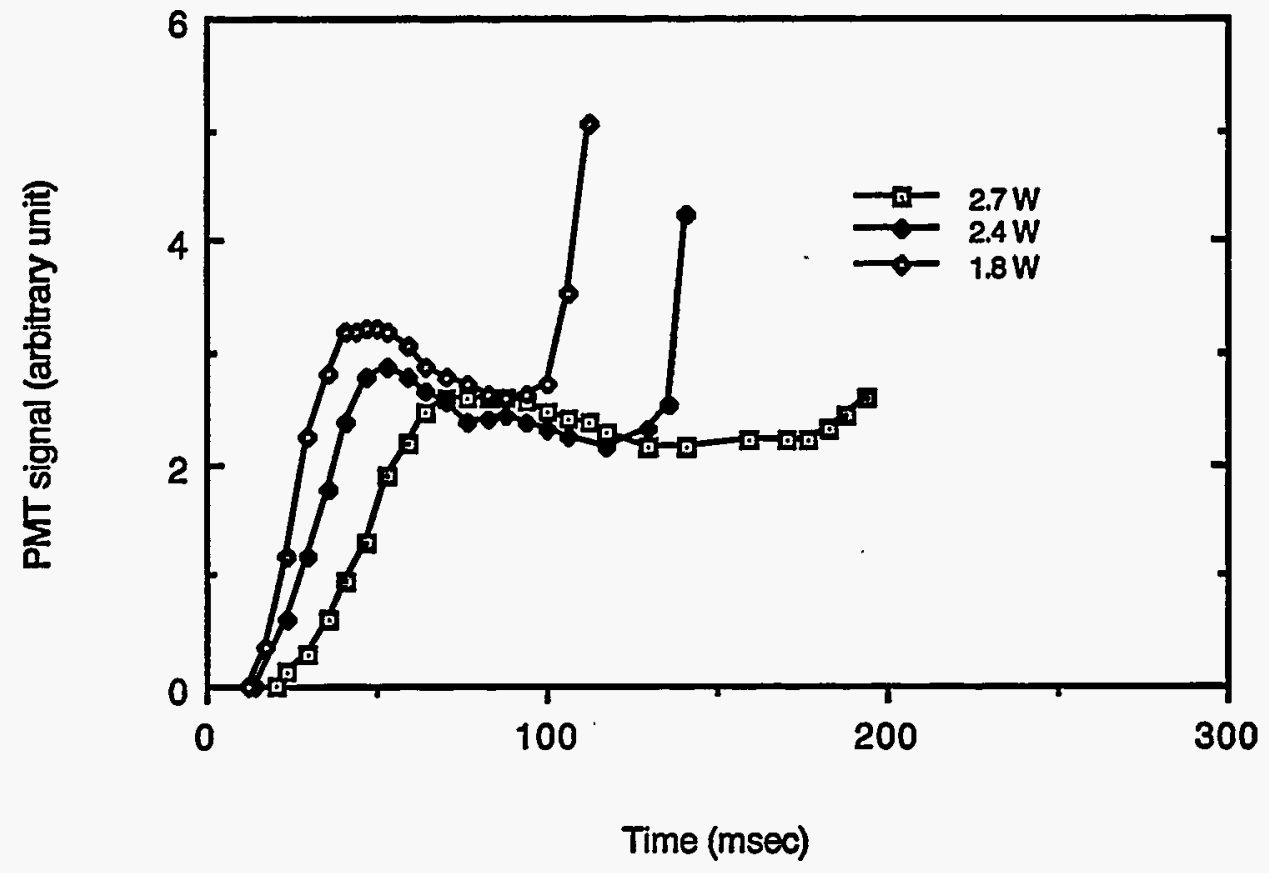

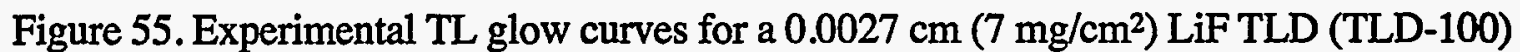
with a focused Gaussian laser beam of fixed $0.059 \mathrm{~cm}$ diameter and various laser powers [Braunlich, 1990]. 


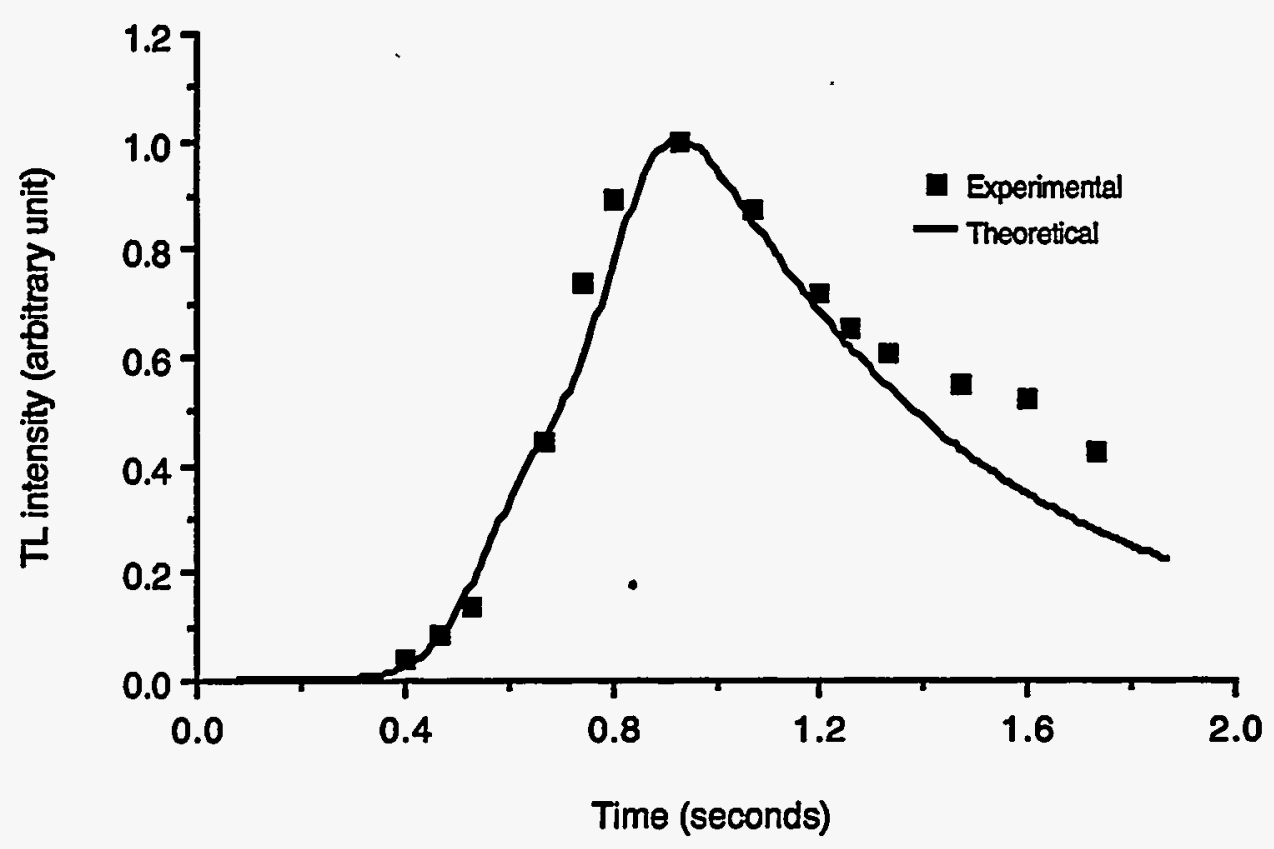

Figure 56. Theoretical and experimental glow curves for a $0.038 \mathrm{~cm}$ thick LiF TLD following heating with a $0.170 \mathrm{~cm}$ diameter unfocused $4 \mathrm{~W}$ continuous wave $(\mathrm{CW})$ laser beam; (a) glow curve calculated with frequency factors reduced by two orders of magnitude. and (b) the experimental glow curve published by Grupen-Shemansky [GrupenShemansky, 1989]. Both curves were normalized to the peak values. 


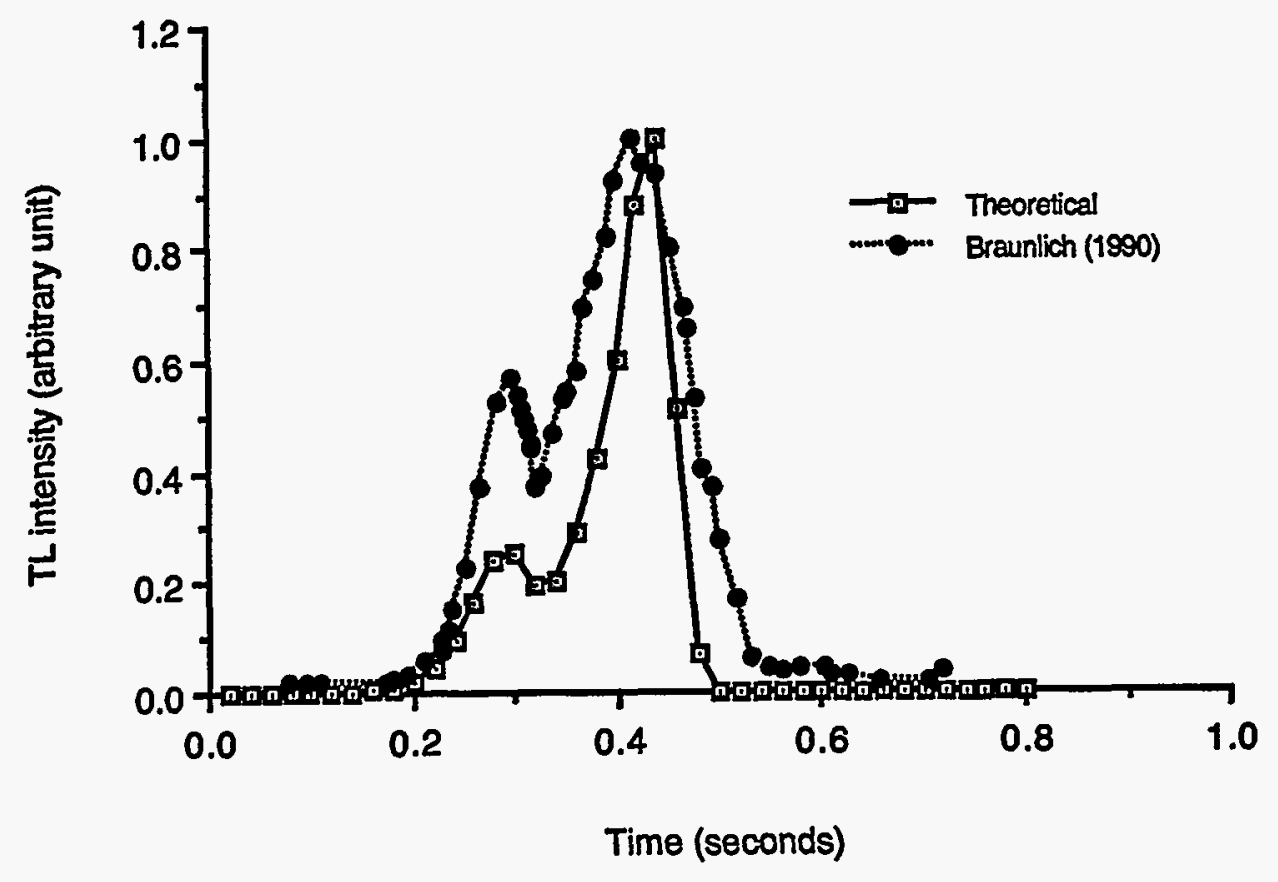

Figure 57. TL glow curves obtained from a $0.038 \mathrm{~cm}$ thick LiF (TLD-100) Harshaw chip with a uniform laser beam heating; (a) Experimental glow curve (dotted line); (b) Theoretical glow curve (solid line). These data were obtained for $0.3 \mathrm{~cm} \times 0.3 \mathrm{~cm}, 10 \mathrm{~W}$ uniform laser beam. Both curves were normalized to their major peak values. 


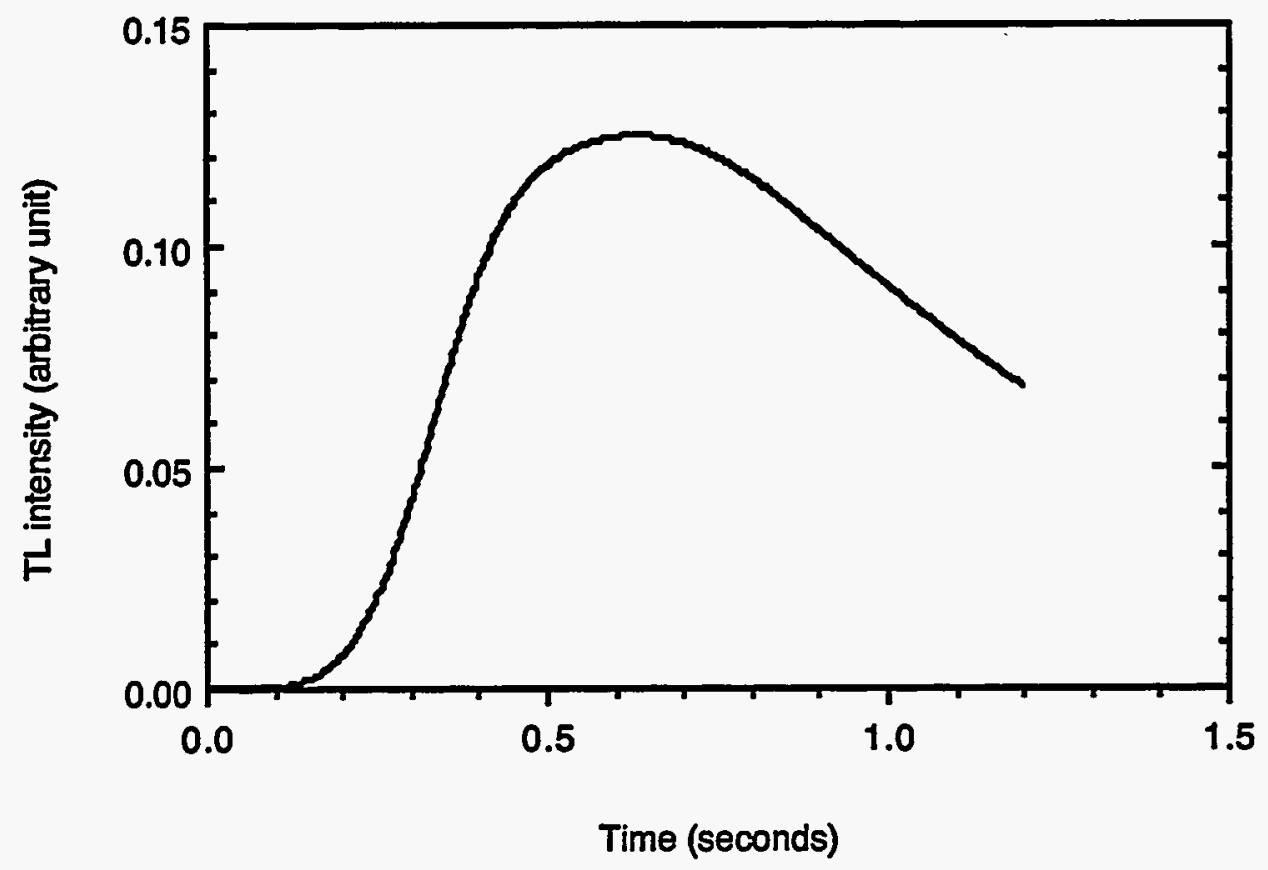

Figure 58. Glow curve for a thick laser TL dosimeter, predicted using numerical solution with assumed uniform dose, following heating with a $0.170 \mathrm{~cm}$ diameter, $4 \mathrm{~W}$ unfocused laser beam for $1.2 \mathrm{sec}$. 


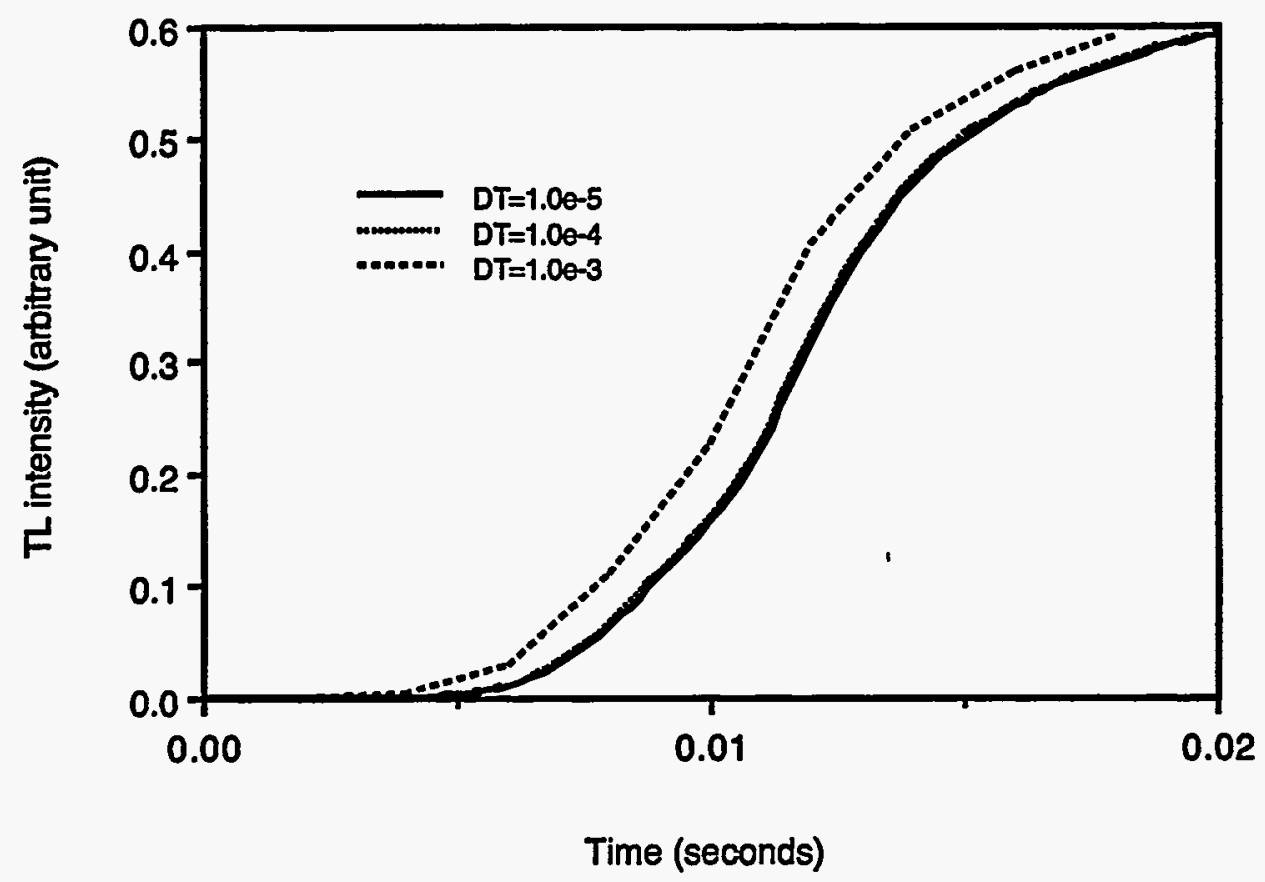

Figure 59. Variations of glow curve response for focused laser beam heating for time mesh sizes of (a) $1.0 \times 10^{-5} \mathrm{sec}$ (Solid line), (b) $1.0 \times 10^{-4} \mathrm{sec}$ (Dotted line), and (c) $1.0 \times 10^{-3} \mathrm{sec}$ (Dashed line). Radial mesh size of $7.5 \times 10^{-4} \mathrm{~cm}(\mathrm{R} / 200)$ and axial mesh size of $9.0 \times 10^{-4} \mathrm{~cm}(\mathrm{~L} / 422)$ were used. Calculation were performed for a $0.032 \mathrm{~cm}, 4 \mathrm{~W}$ focused Gaussian laser beam. 


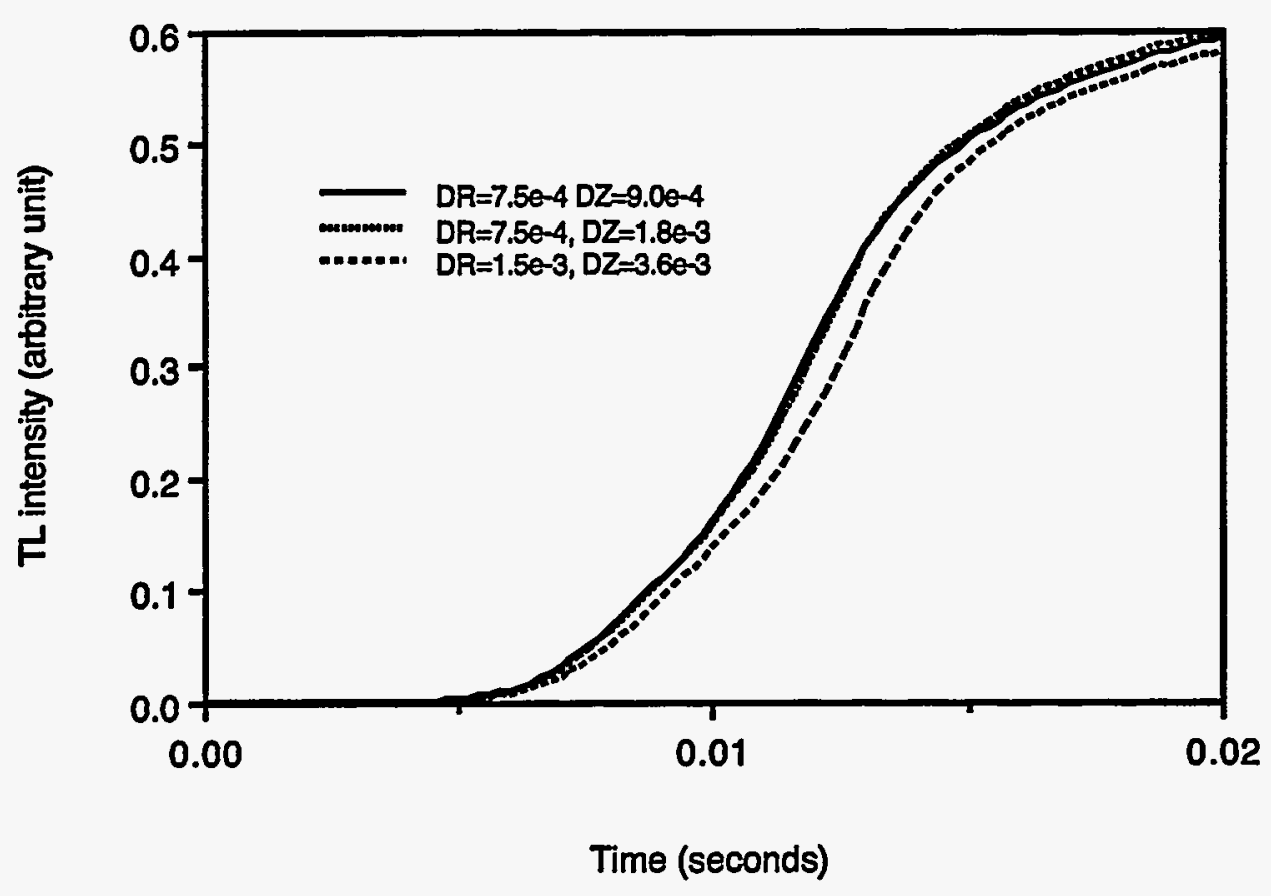

Figure 60. Variations of glow curve response for focused laser beam heating for time mesh sizes of (a) R/200; L/422 (Solid line), (b) R/200; L/211 (Dotted line), and (c) R/100; L/106 (Dashed line). Time mesh size of $1.0 \times 10^{-4} \mathrm{sec}$ was used. Calculation were performed for a $0.032 \mathrm{~cm}, 4 \mathrm{~W}$ focused Gaussian laser beam. 


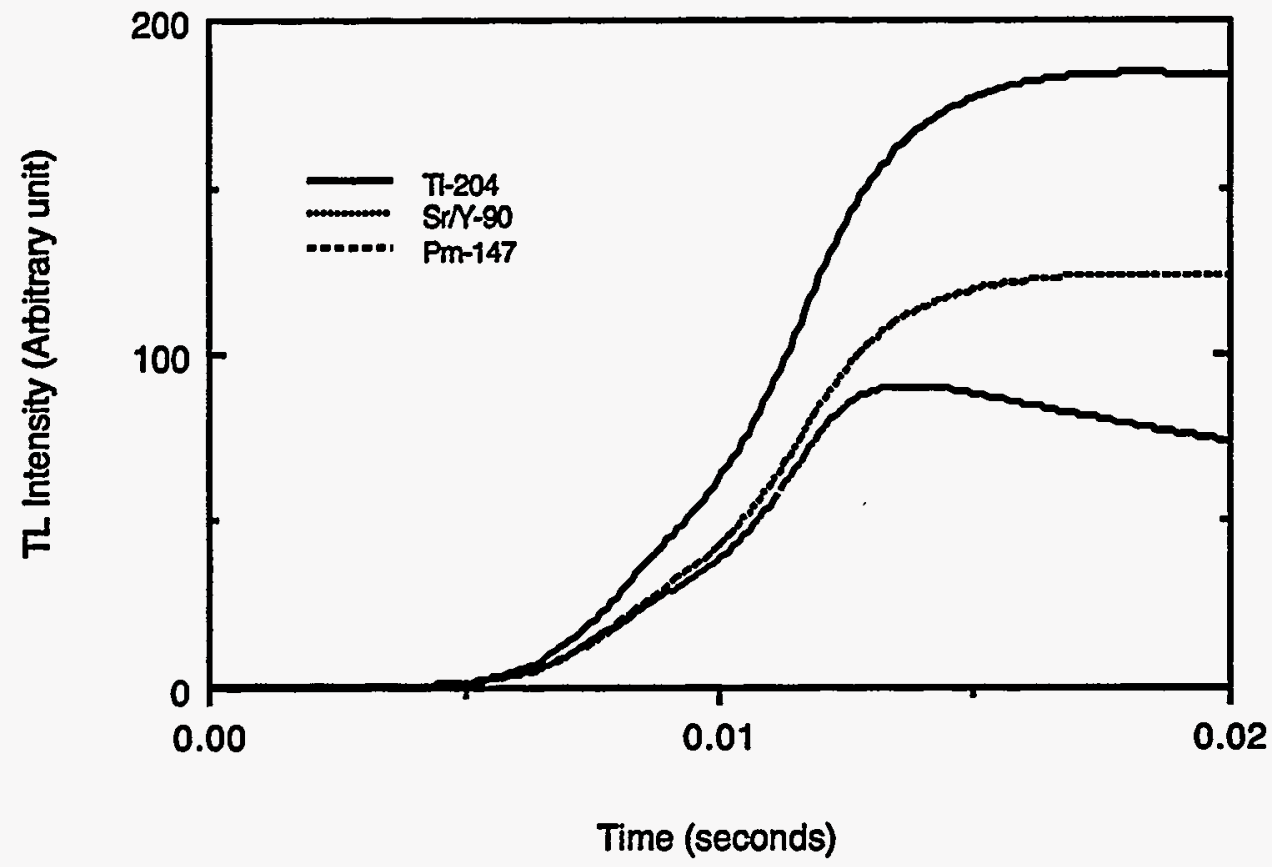

Figure 61. Theoretical glow curves of a thick laser TL dosimeter exposed to various beta sources. The depth-dose profile used for glow curve calculation were obtained from EGS4 Monte Carlo calculations with 50,000 electron histories for each beta source. TL light intensity were calculated for the TLD heated with a $0.032 \mathrm{~cm}$ diameter, $4 \mathrm{~W}$ focused Gaussian laser beam. 

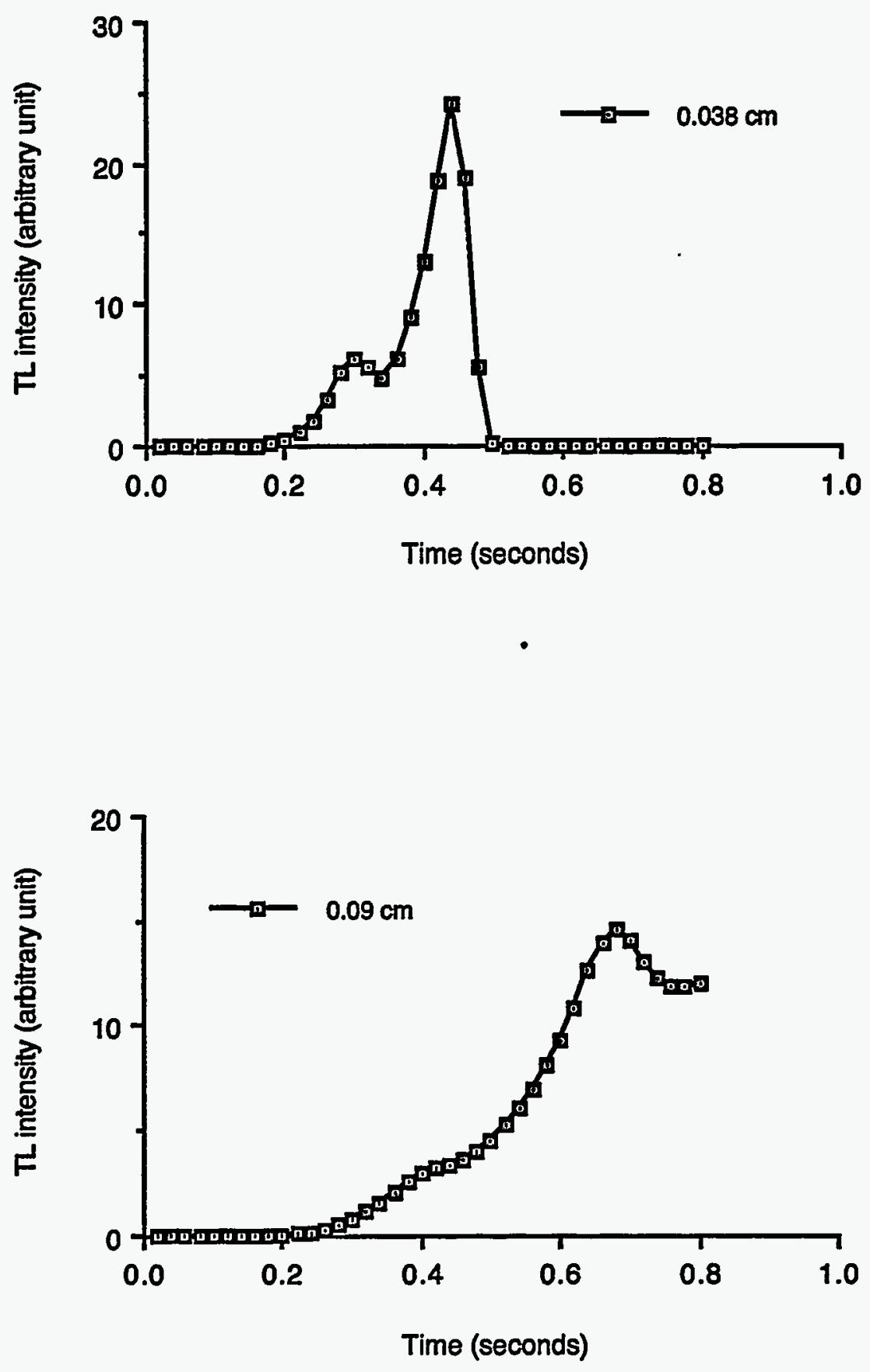

Figure 62. Glow curves based on a numerical solution for a $0.038 \mathrm{~cm}$ thick and a $0.09 \mathrm{~cm}$ thick LiF TL dosimeters, following heating with $0.3 \mathrm{~cm} \times 0.3 \mathrm{~cm} 10 \mathrm{~W}$ uniform laser beam for $0.8 \mathrm{sec}$. 


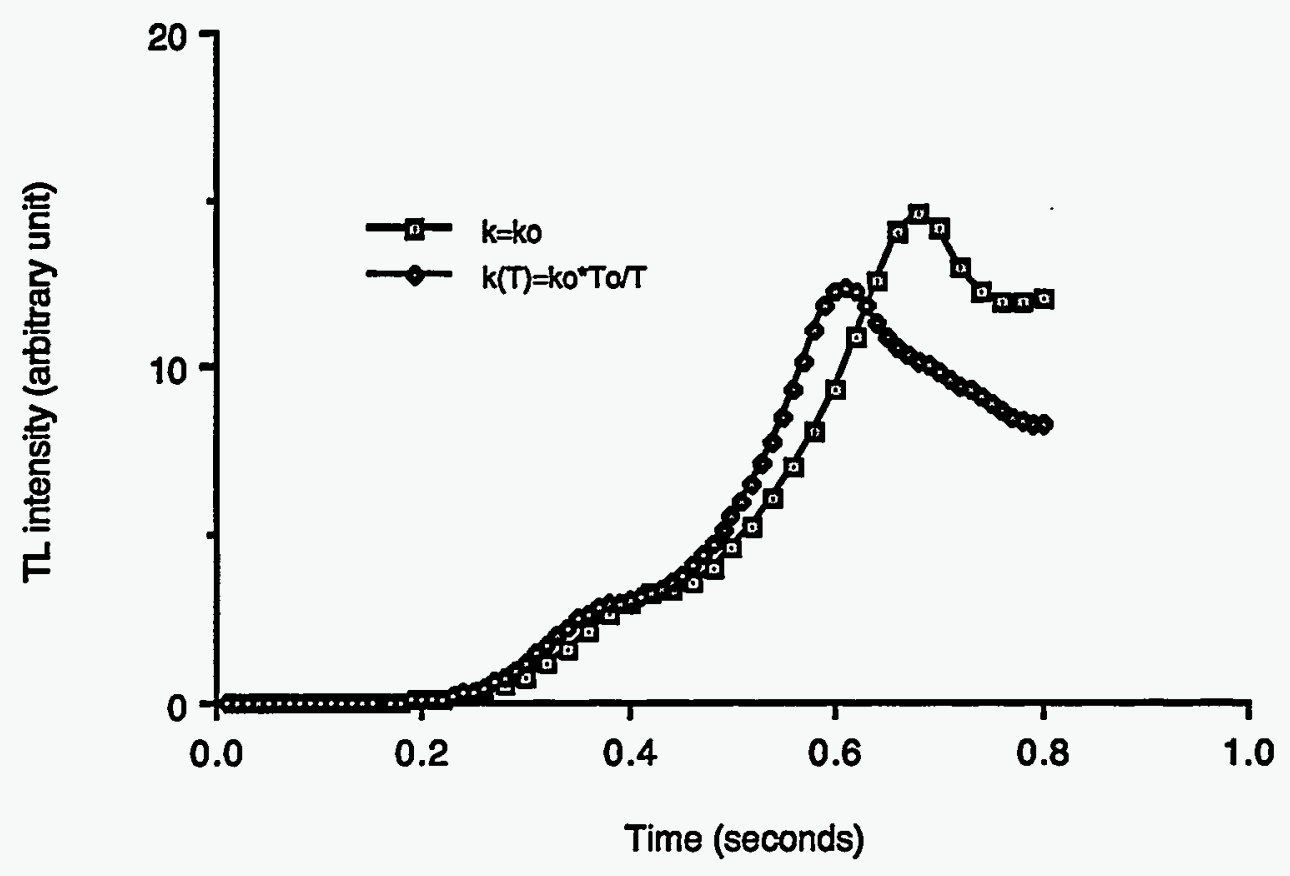

Figure 63. Variations of glow curves of a $0.09 \mathrm{~cm}$ thick TL dosimeter using temperature-dependent thermal conductivity, $k(T)$ for a $0.3 \mathrm{~cm} \mathrm{x} 0.3 \mathrm{~cm} 10 \mathrm{~W}$ uniform laser beam. 


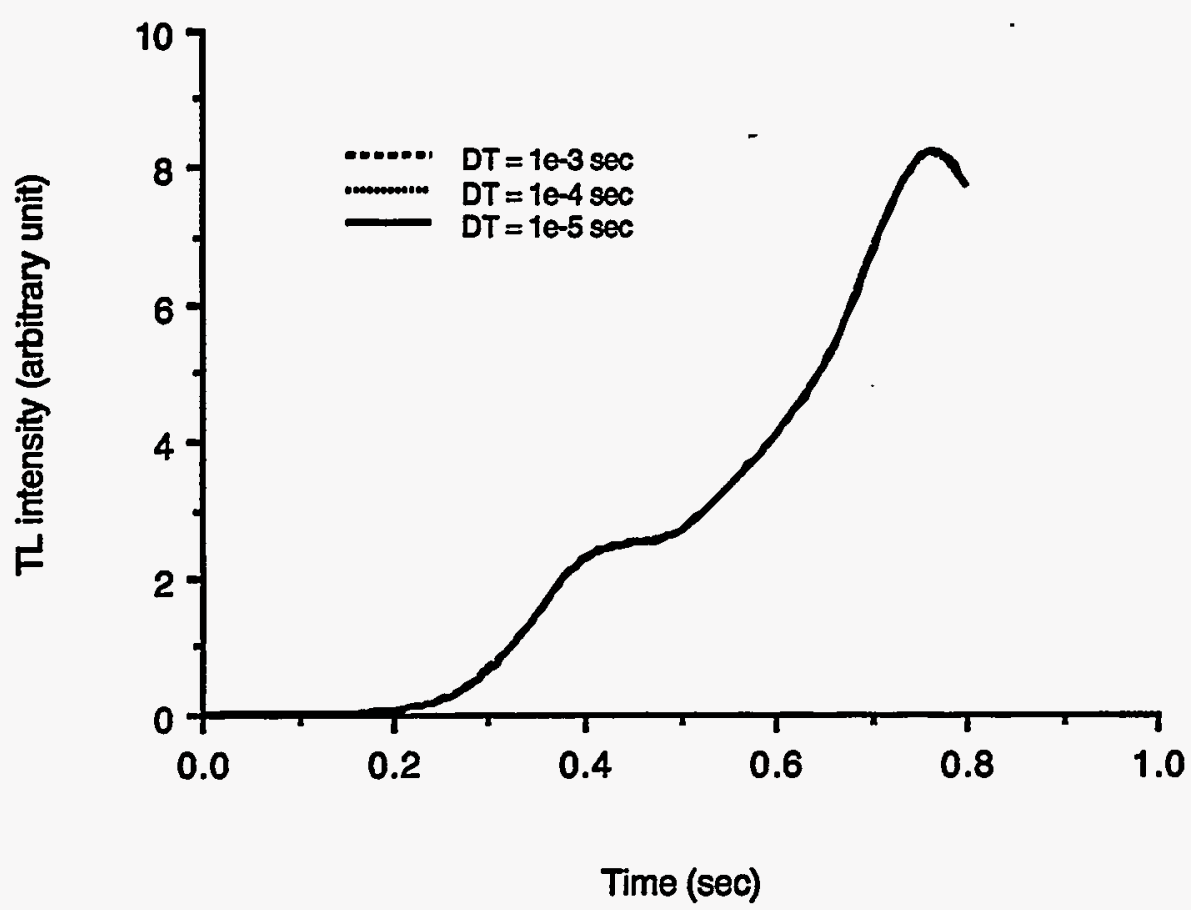

Figure 64. Variations of glow curves for uniform laser beam heating for time mesh sizes: $1.0 \times 10^{-3}$ (dashed line), $1.0 \times 10^{-4}$ (dotted line), and $1.0 \times 10^{-5}$ (dashed line). Calculations were performed for a $0.3 \mathrm{~cm} \times 0.3 \mathrm{~cm} 10 \mathrm{~W}$ uniform laser beam, and the axial mesh size used was $1.8 \times 10^{-3} \mathrm{~cm}$. 


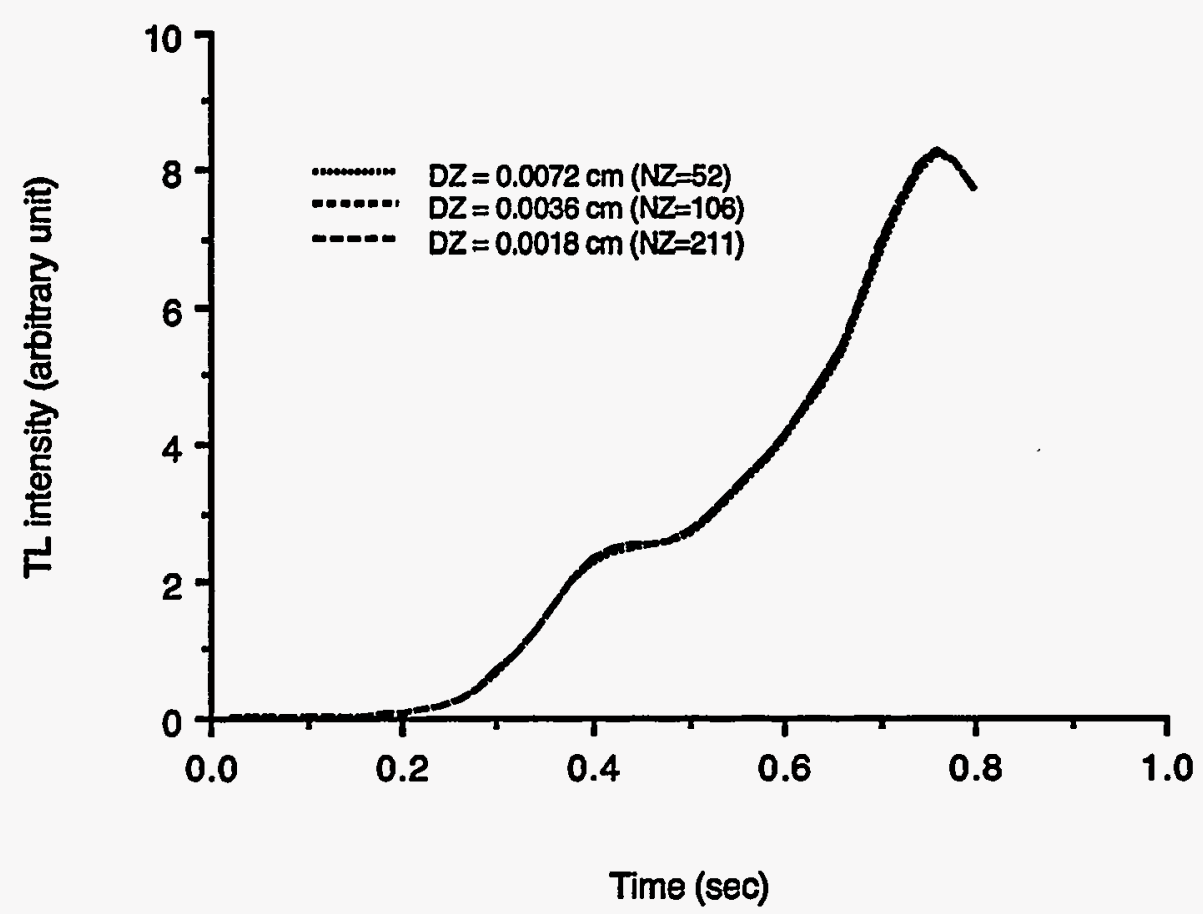

Figure 65. Variations of glow curves for uniform laser beam heating with axial mesh sizes: $7.2 \times 10^{-3} \mathrm{~cm}$ (dotted line), $3.6 \times 10^{-3} \mathrm{~cm}$ (broken line), and $1.8 \times 10^{-3}$ (dashed line). Time mesh size of $1.0 \times 10^{-4} \mathrm{sec}$ was used. Calculations were performed for a $0.3 \mathrm{~cm} \mathrm{x} 0.3 \mathrm{~cm} 10$ $\mathrm{W}$ uniform laser beam, and the time mesh size used was $1.0 \times 10^{-4} \mathrm{sec}$. 


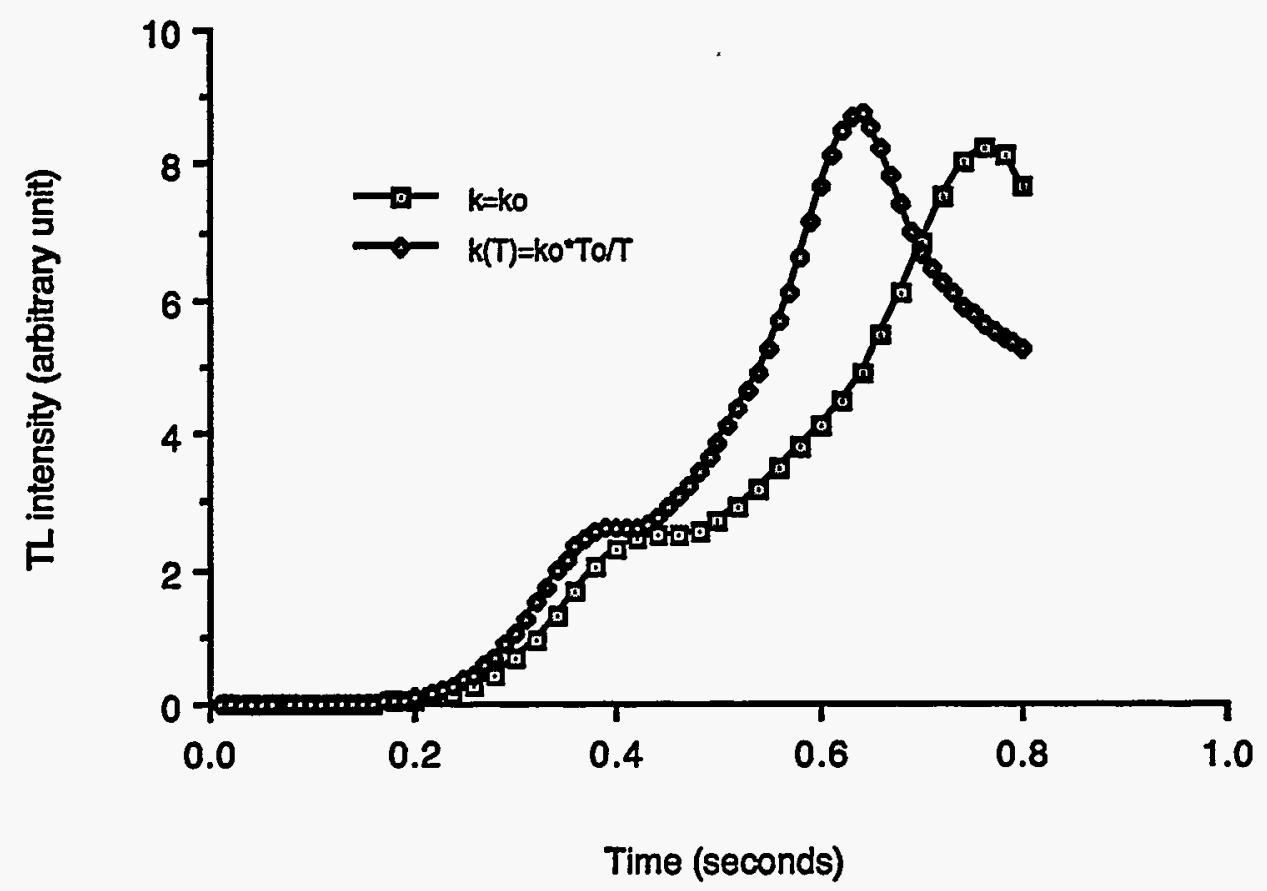

Figure 66. Variations of glows of a thick laser TL dosimeter using a temperaturedependent thermal conductivity, $\mathrm{k}(\mathrm{T})$ for a $0.3 \mathrm{~cm} \times 0.3 \mathrm{~cm} 10 \mathrm{~W}$ uniform laser heating. 


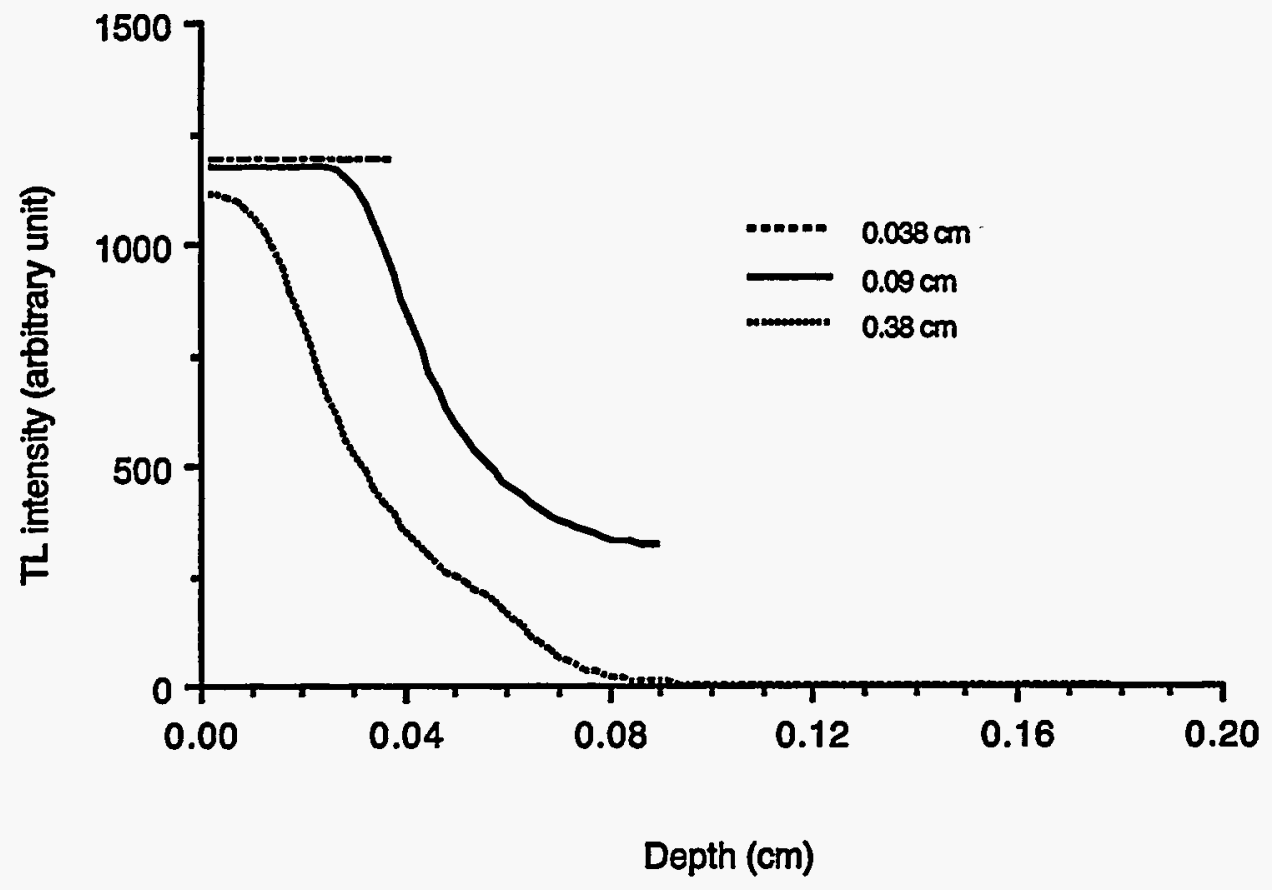

Figure 67. Numerically calculated TL light gradients as function of dosimeter depth for various TL dosimeters following heating with a $0.3 \mathrm{~cm} \mathrm{x} 0.3 \mathrm{~cm} 10 \mathrm{~W}$ laser beam for 0.8 sec: (a) $0.038 \mathrm{~cm}$ TLD (dashed line); (b) $0.09 \mathrm{~cm}$ TLD (solid line); (c) $0.38 \mathrm{~cm}$ TLD (dotted line). 


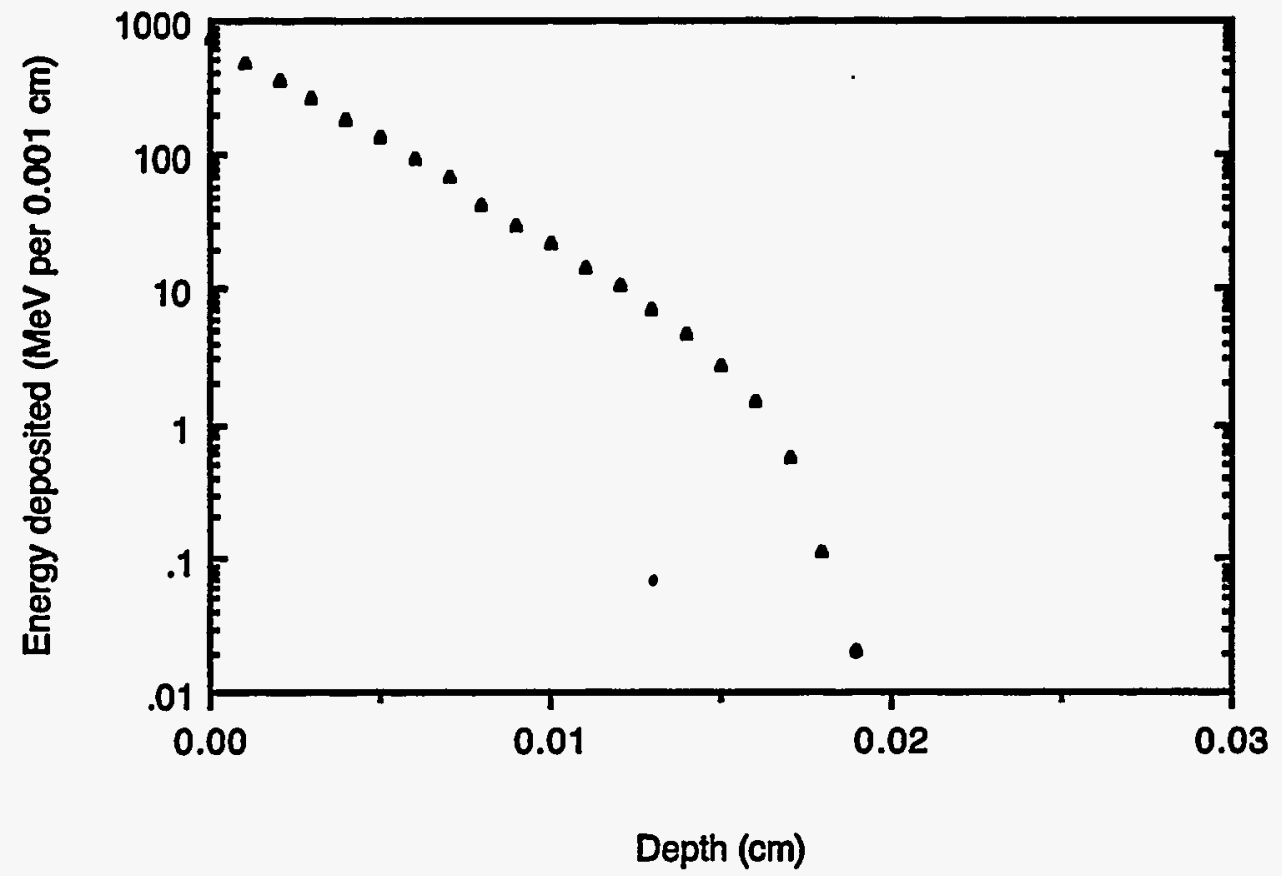

Figure 68. Calculated depth-dose curves of a thick laser TL dosimeter with Pm-147 source. These data were obtained from 50,000 history particle transport simulation by the EGS 4 Monte Carlo code. 


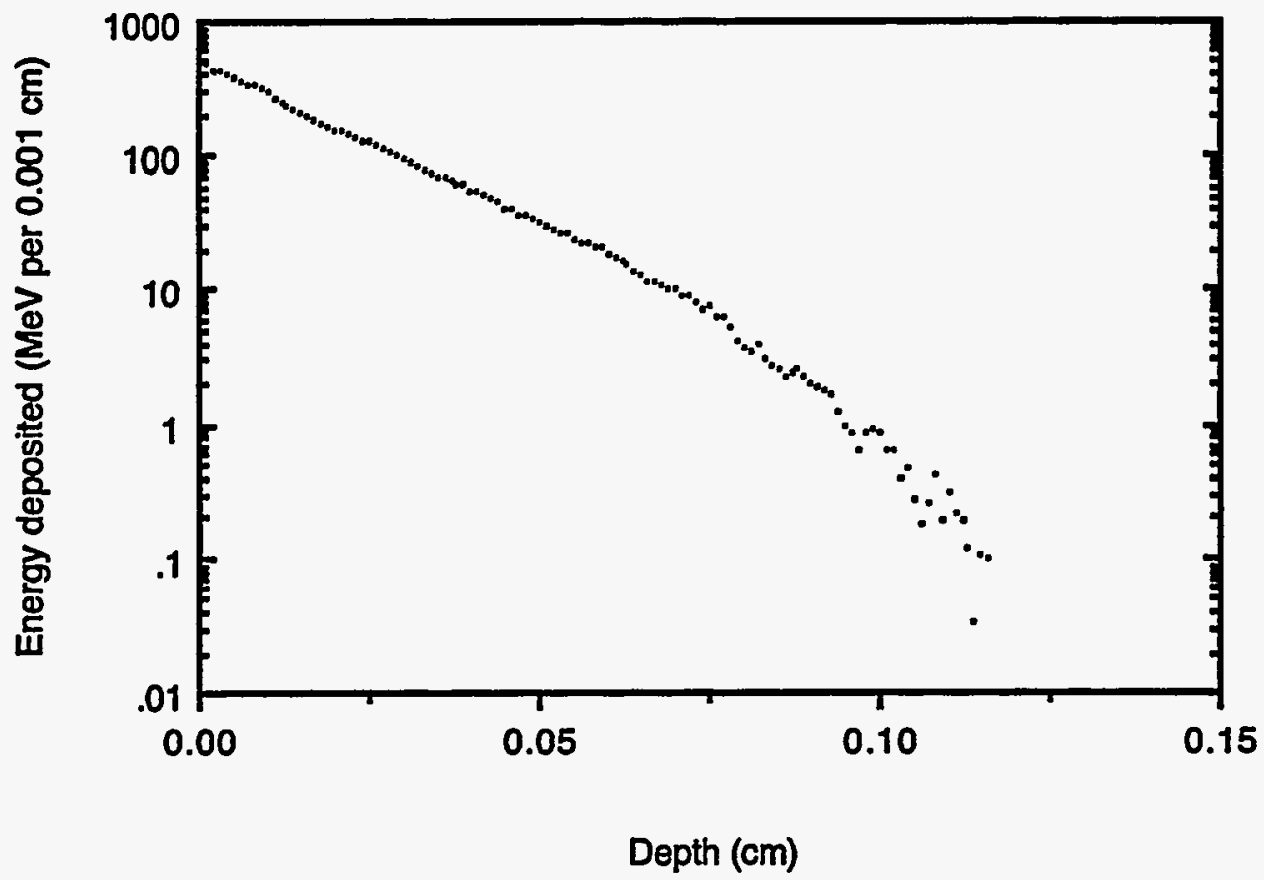

Figure 69. Calculated depth-dose curves of a thick laser TL dosimeter with Tl-204 source. These data were obtained from 50,000 history particle transport simulation by the EGS 4 Monte Carlo code. 


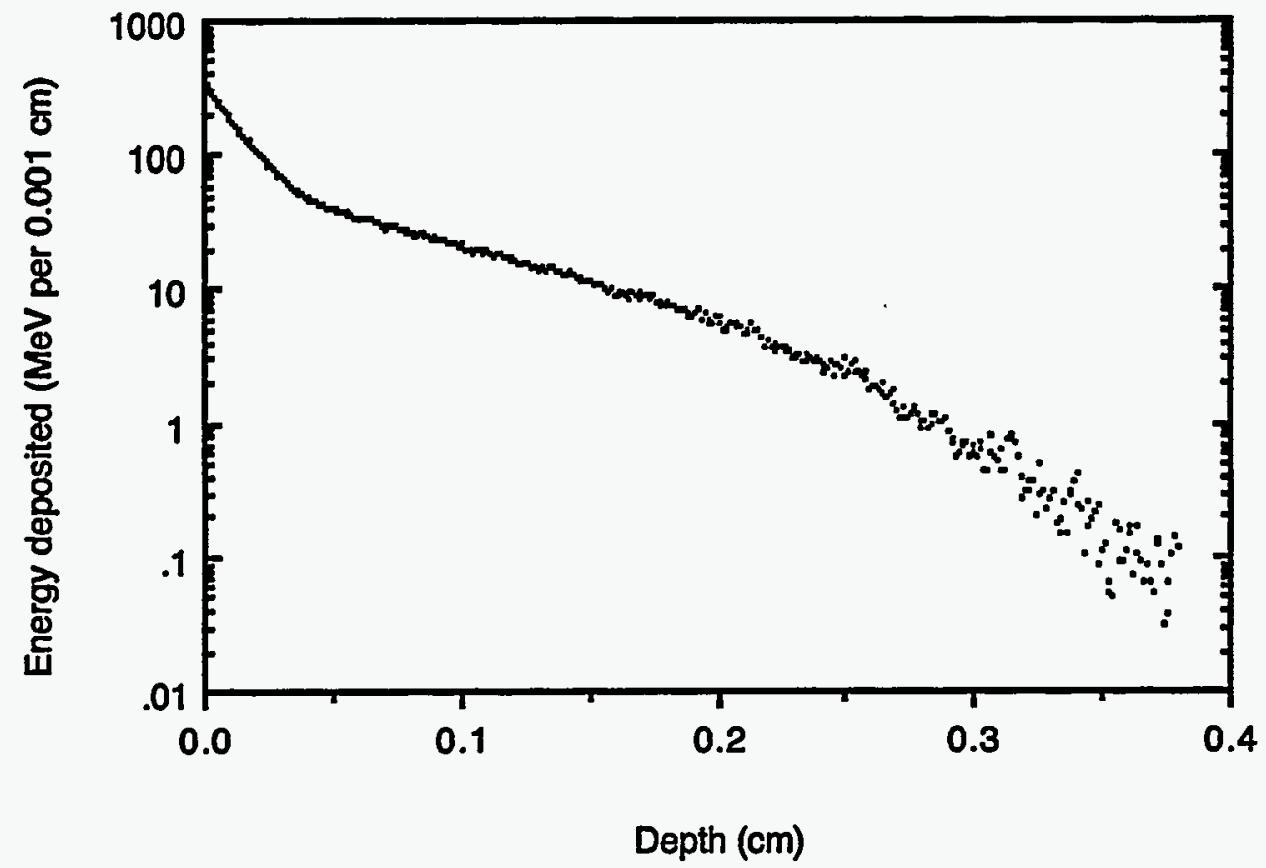

Figure 70. Calculated depth-dose curves of a thick laser TL dosimeter with Sr/Y-90 source. These data were obtained from 50,000 history particle transport simulation by the EGS4 Monte Carlo code. 


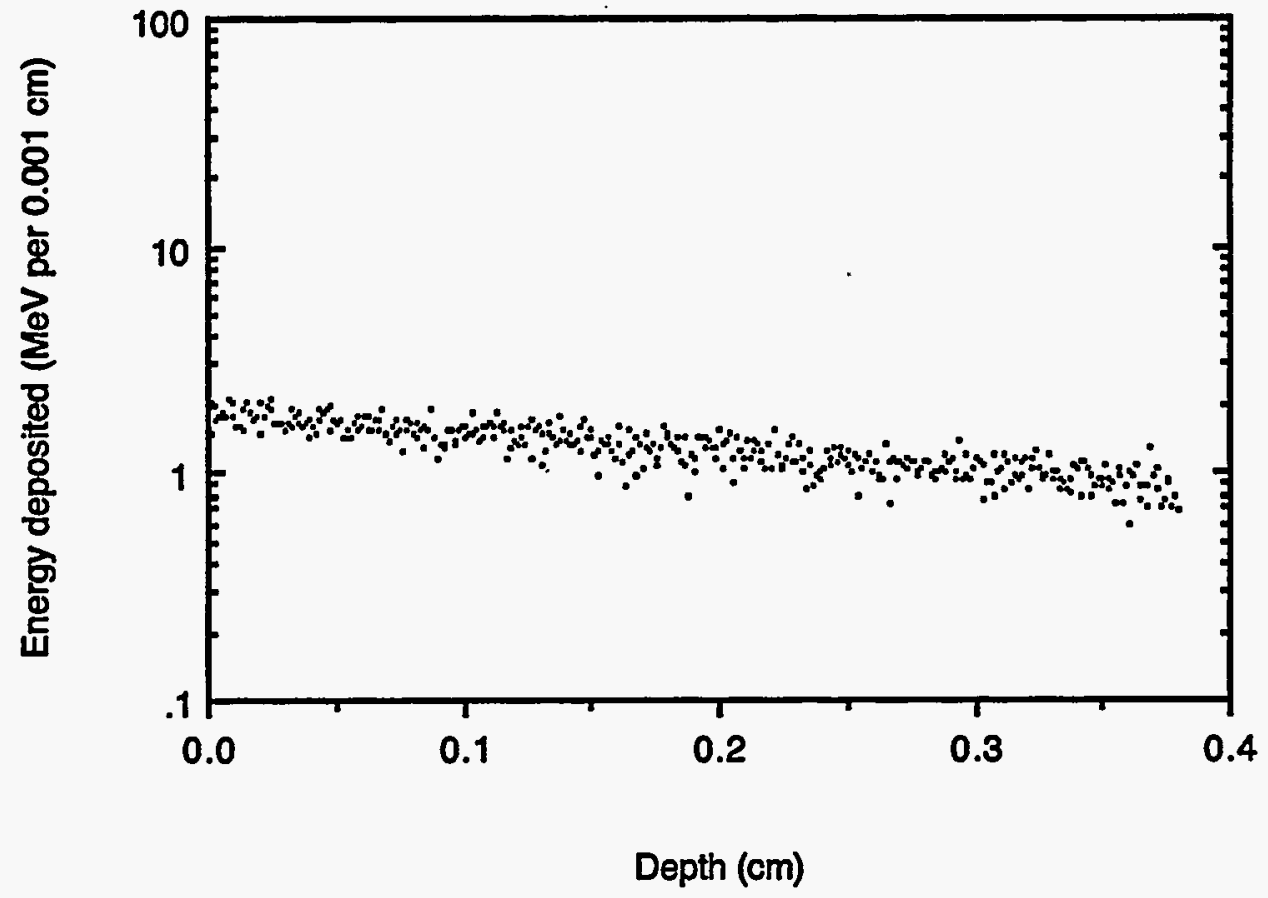

Figure 71. Calculated depth-dose curves of a thick laser TL dosimeter with $20 \mathrm{keV}$ NBS filtered x-ray source. These data were obtained from 50,000 history particle transport simulation by the EGS4 Monte Carlo code. 


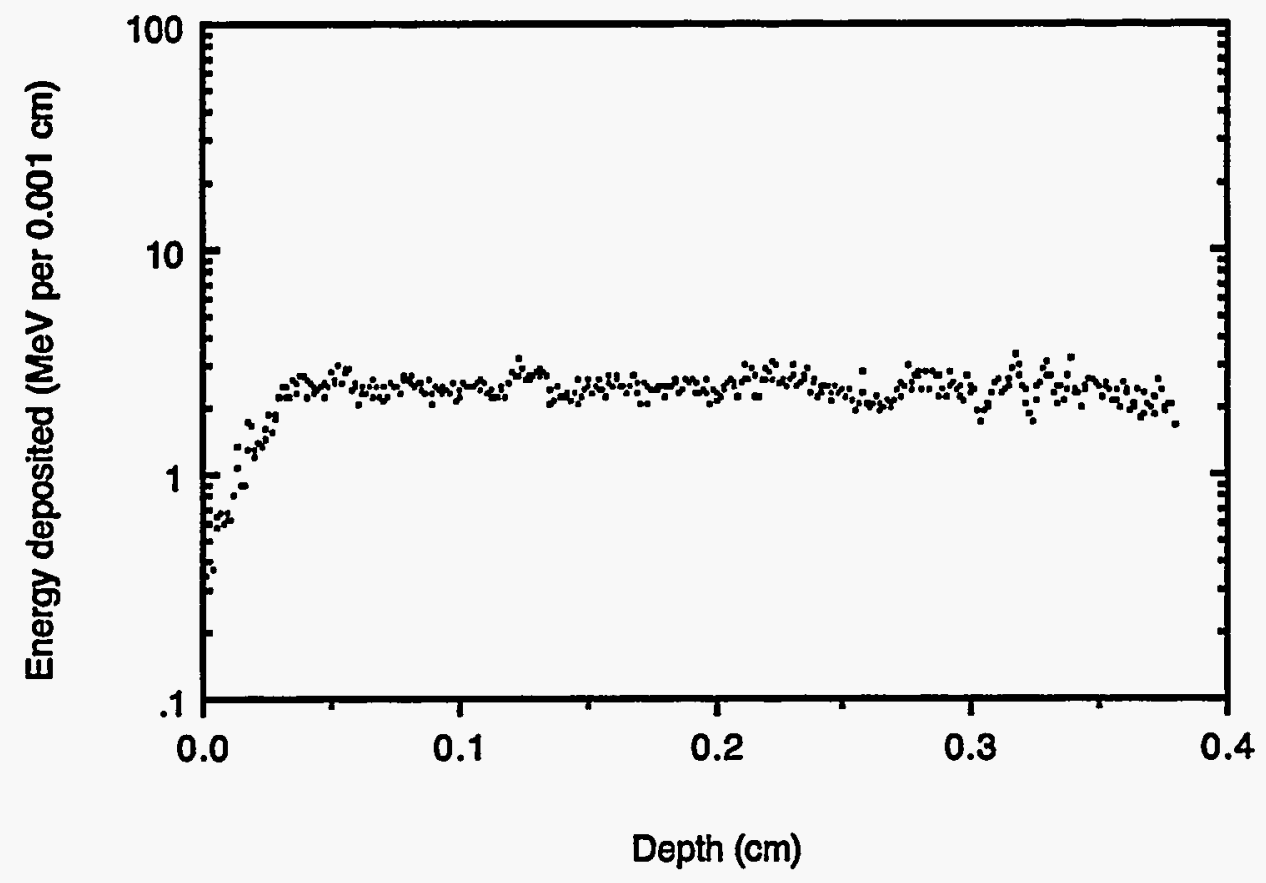

Figure 72. Calculated depth-dose curves of a thick laser TL dosimeter with Cs-137 (E = $662 \mathrm{keV}$ ) gamma-ray source. These data were obtained from 50,000 history particle transport simulation by the EGS4 Monte Carlo code. 


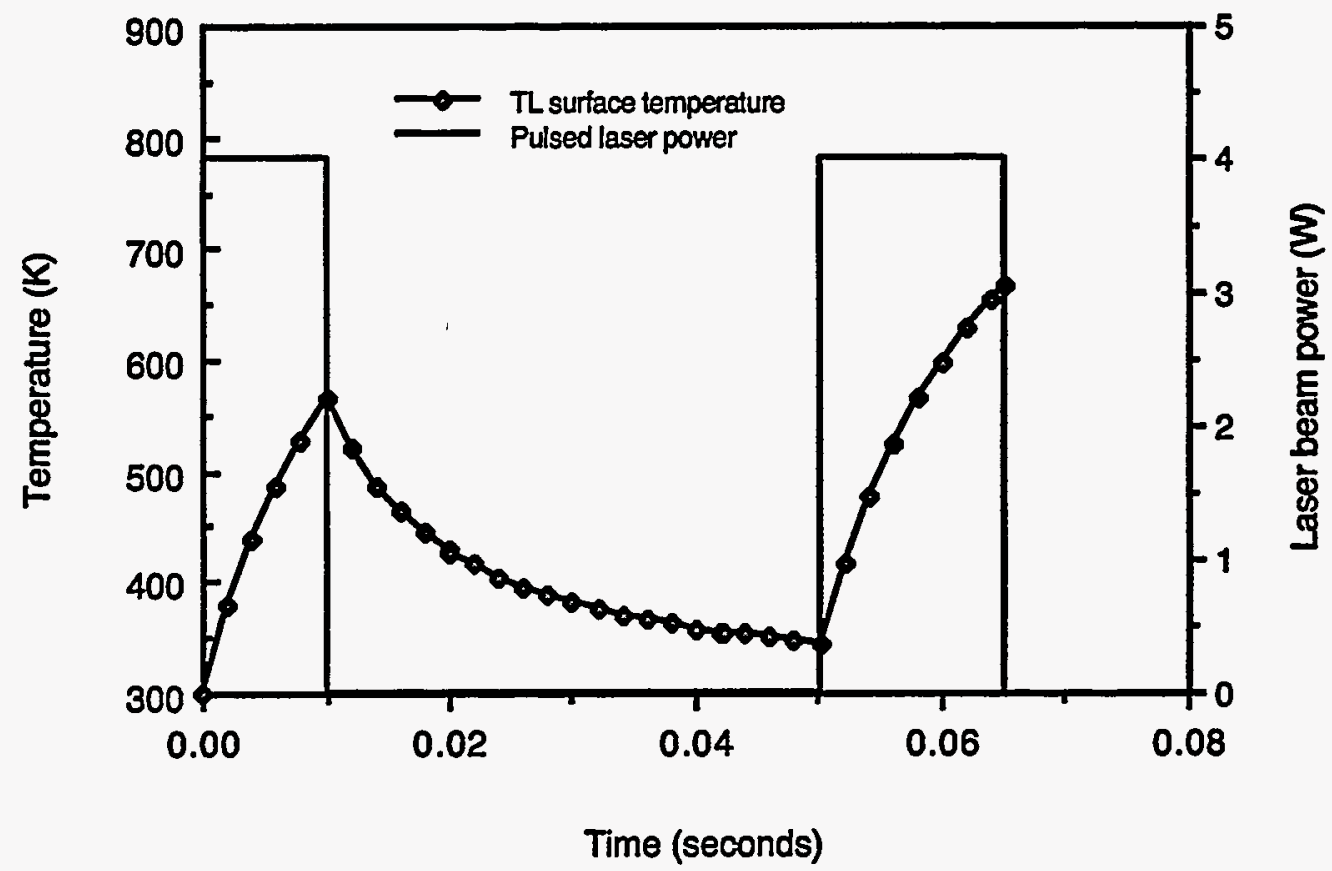

Figure 73. Surface temperature of a thick laser TL dosimeter as a function of time for the following heating scheme: $10 \mathrm{~ms}$ heating (first pulse), $40 \mathrm{~ms}$ cooling, and $15 \mathrm{~ms}$ heating (second pulse). The data were obtained for a $0.032 \mathrm{~cm}$ diameter, $4 \mathrm{~W}$ focused Gaussian laser beam. 


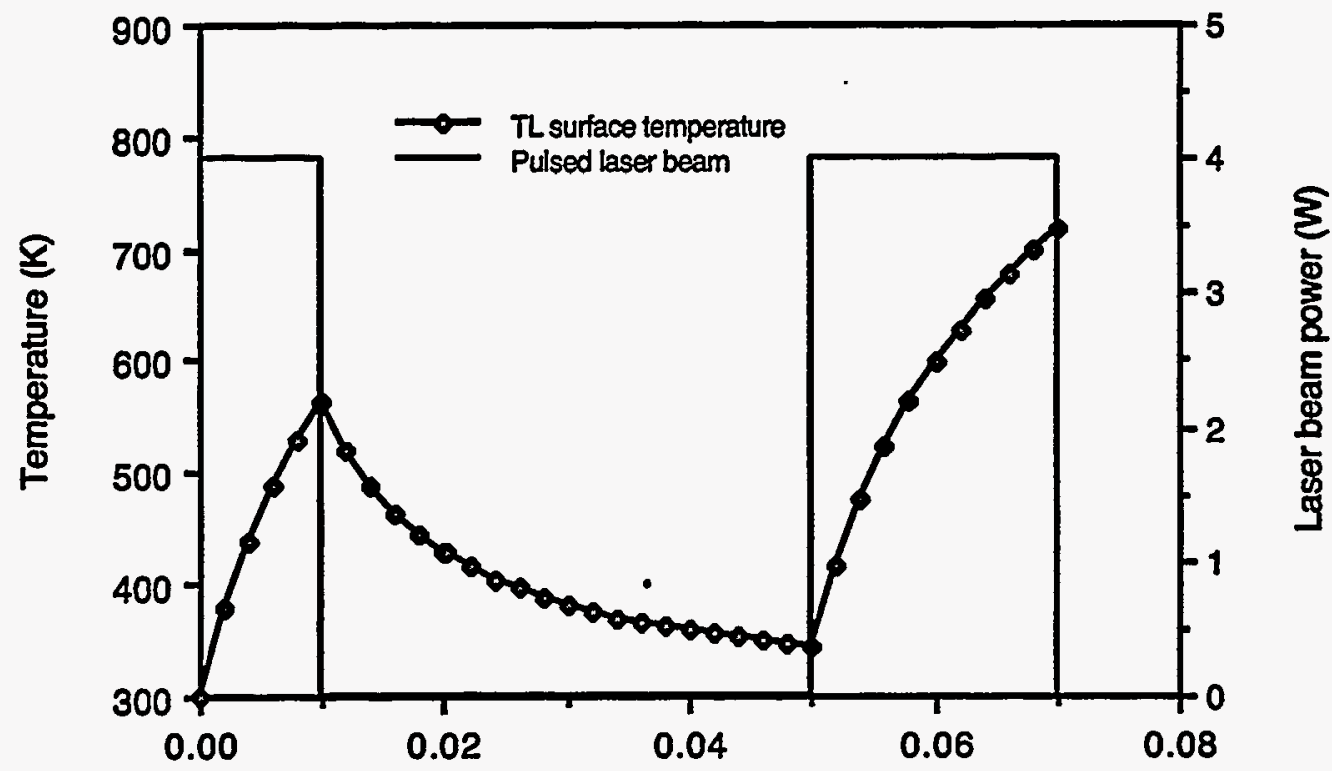

Time (seconds)

Figure 74. Surface temperature of a thick laser TL dosimeter as a function of time for the following heating scheme: $10 \mathrm{~ms}$ heating (first pulse), $40 \mathrm{~ms}$ cooling, and $20 \mathrm{~ms}$ heating (second pulse). The data were obtained for a $0.032 \mathrm{~cm}$ diameter, $4 \mathrm{~W}$ focused Gaussian. laser beam. 


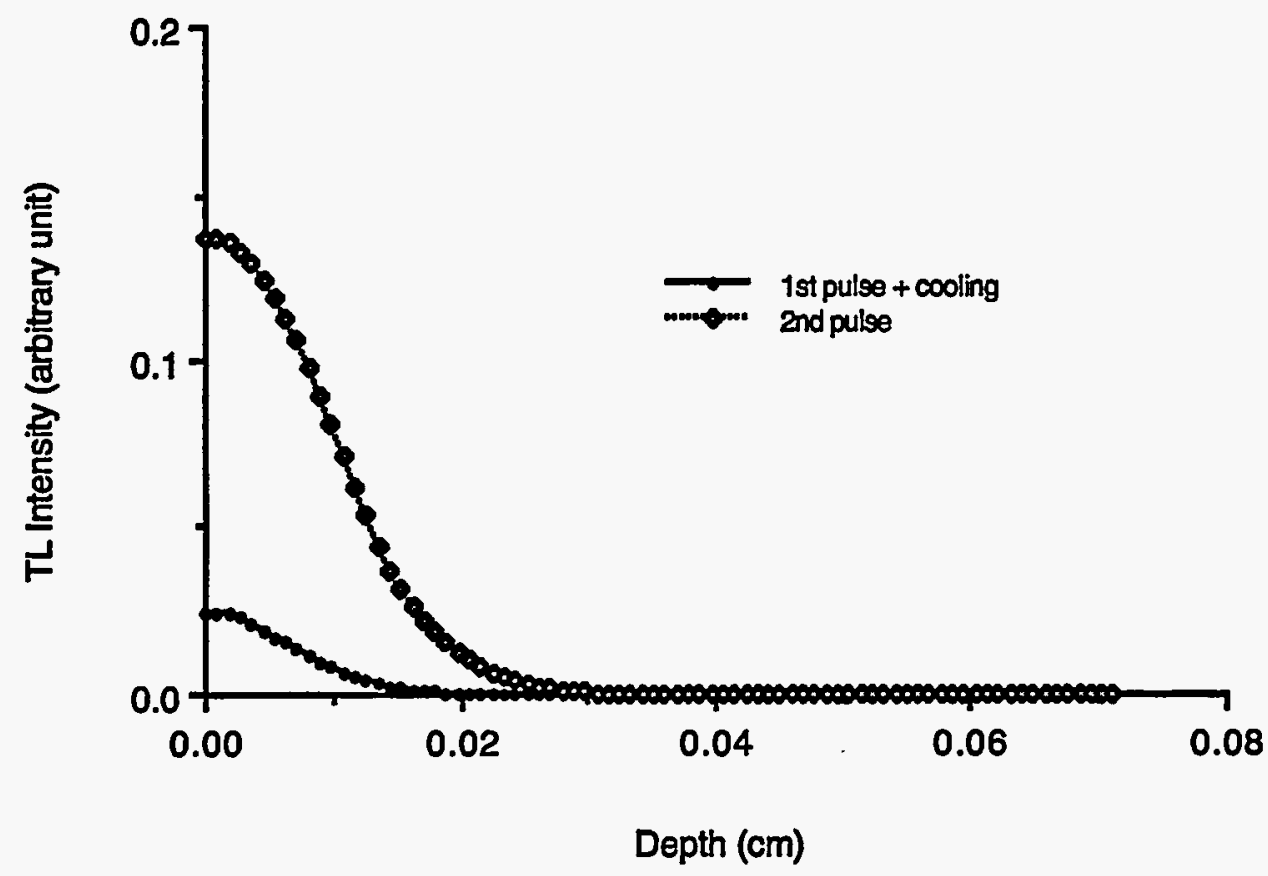

Figure 75. TL light vs. depth of a thick laser TL dosimeter heated with the following pulsed heating scheme: $10 \mathrm{msec}$ of first pulse, and $40 \mathrm{msec}$ of cooling, and then followed by $15 \mathrm{msec}$ of second pulse. The data were obtained for $0.032 \mathrm{~cm}$ diameter, $4 \mathrm{~W}$ focused Gaussian laser beam. 


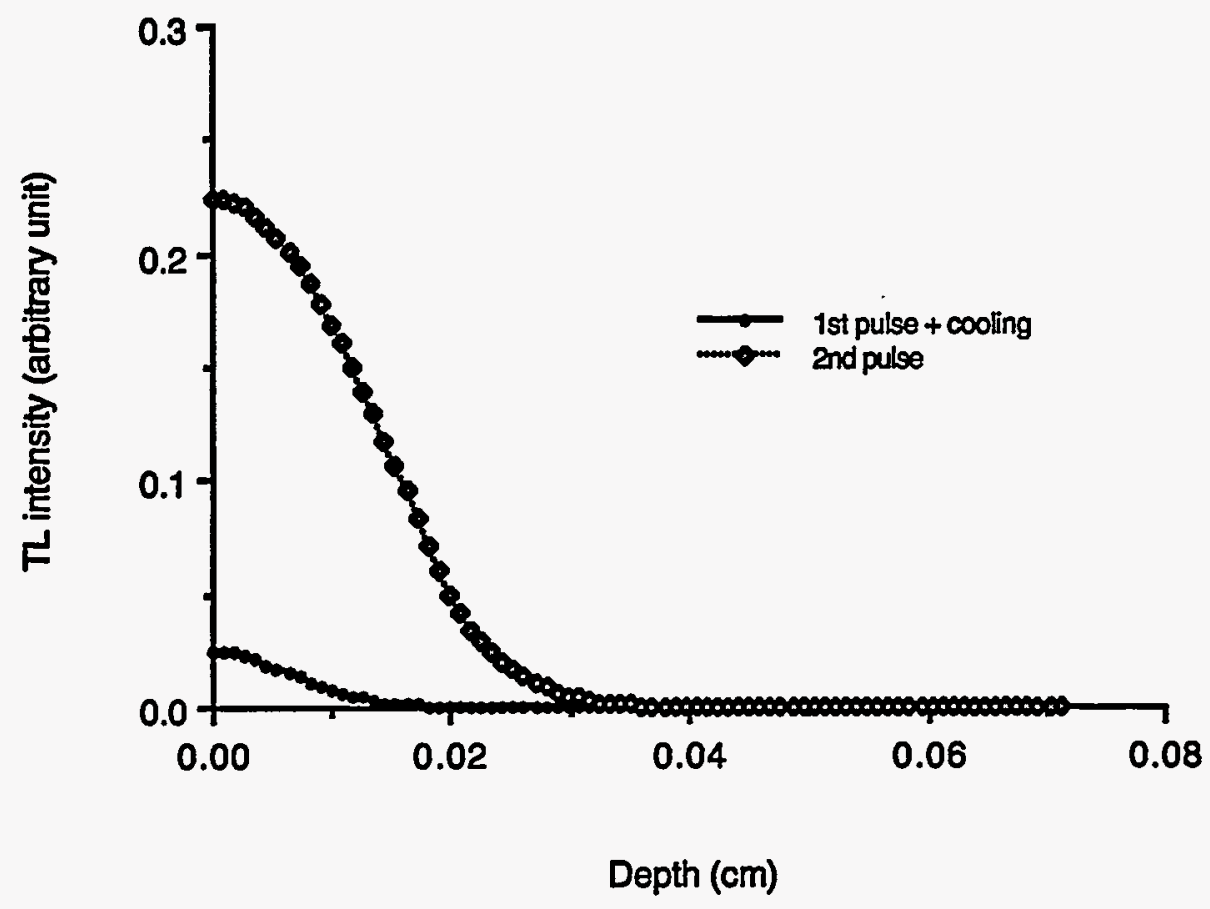

Figure 76. TL light vs. depth of a thick laser TL dosimeter heated with the following pulsed heating scheme: $10 \mathrm{msec}$ of first pulse, and $40 \mathrm{msec}$ of cooling, and then followed by $20 \mathrm{msec}$ of second pulse. The data were obtained for $0.032 \mathrm{~cm}$ diameter, $4 \mathrm{~W}$ focused Gaussian laser beam. 


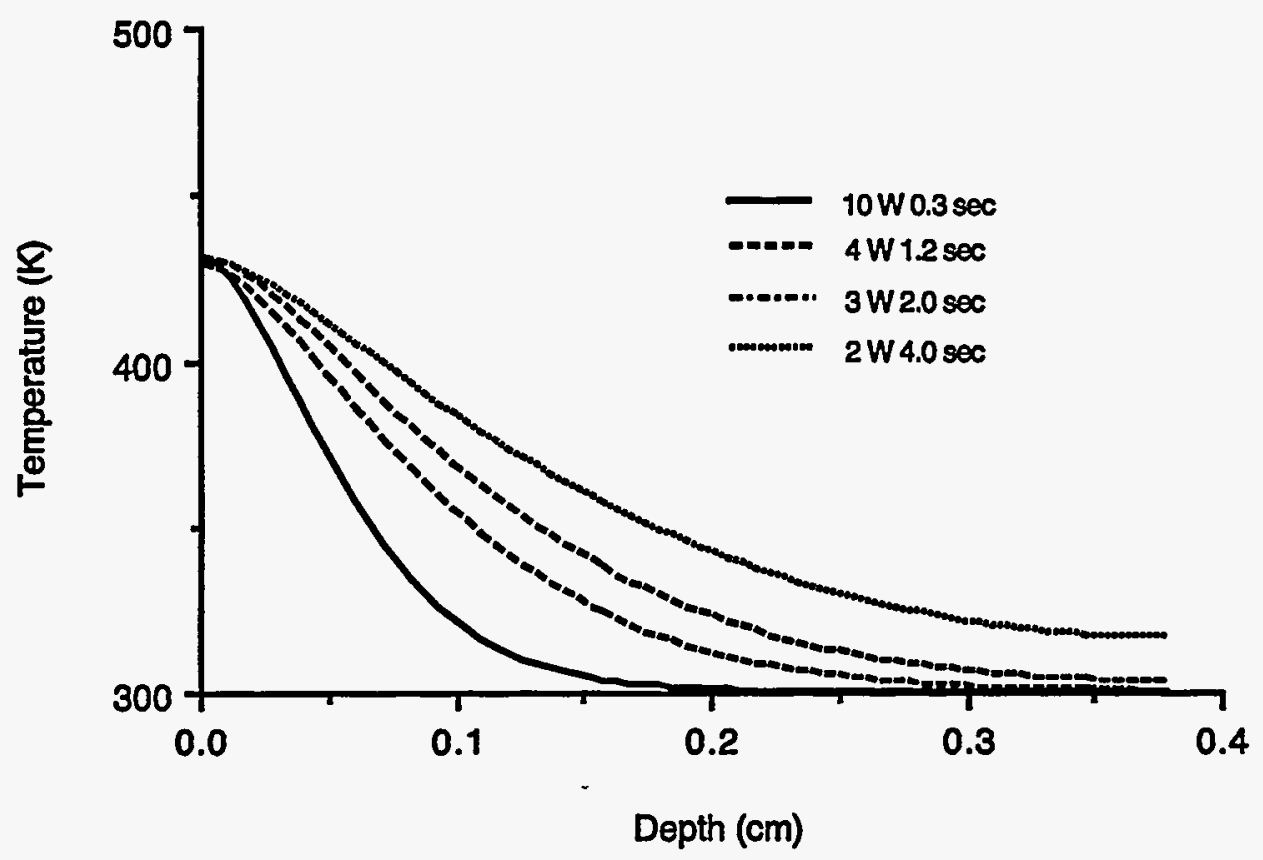

Figure 77. Temperature vs. depth of a thick laser TL dosimeter for various combinations of laser powers and heating durations: $10 \mathrm{~W}, 0.3 \mathrm{sec} ; 4 \mathrm{~W}, 1.2 \mathrm{sec} ; 3 \mathrm{~W}, 2.0 \mathrm{sec} ; 2 \mathrm{~W}, 4.0$ sec. 


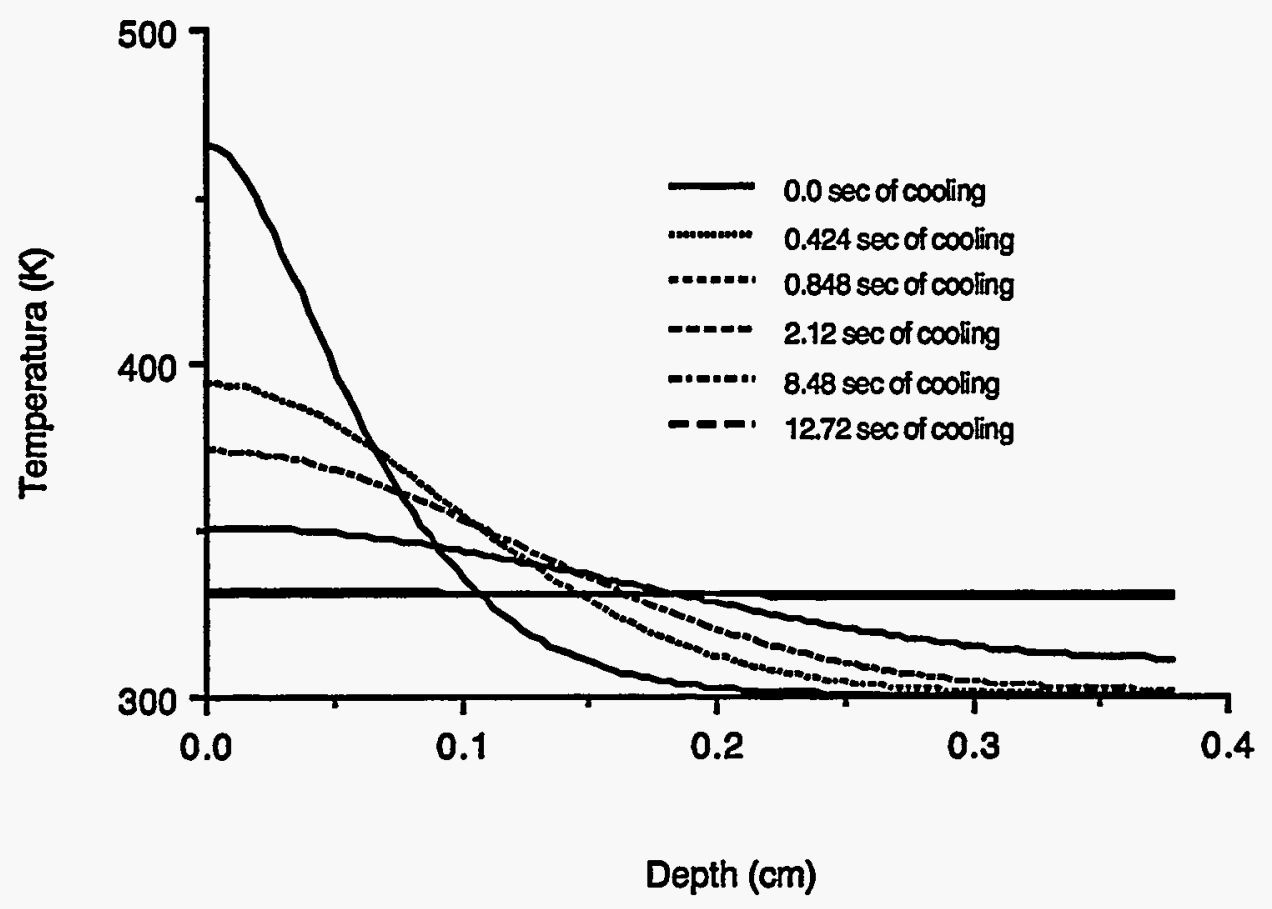

Figure 78. Variations of temperature-depth distributions of a thick laser TL dosimeter during various cooling periods (a time duration after laser is turned off): $0.424 \mathrm{sec}, 0.848$ $\mathrm{sec}, 2.12 \mathrm{sec}, 8.48 \mathrm{sec}$, and $12.72 \mathrm{sec}$. The data were obtained for a $0.3 \times 0.3 \mathrm{~cm}$ square $10 \mathrm{~W}$ uniform laser beam. 

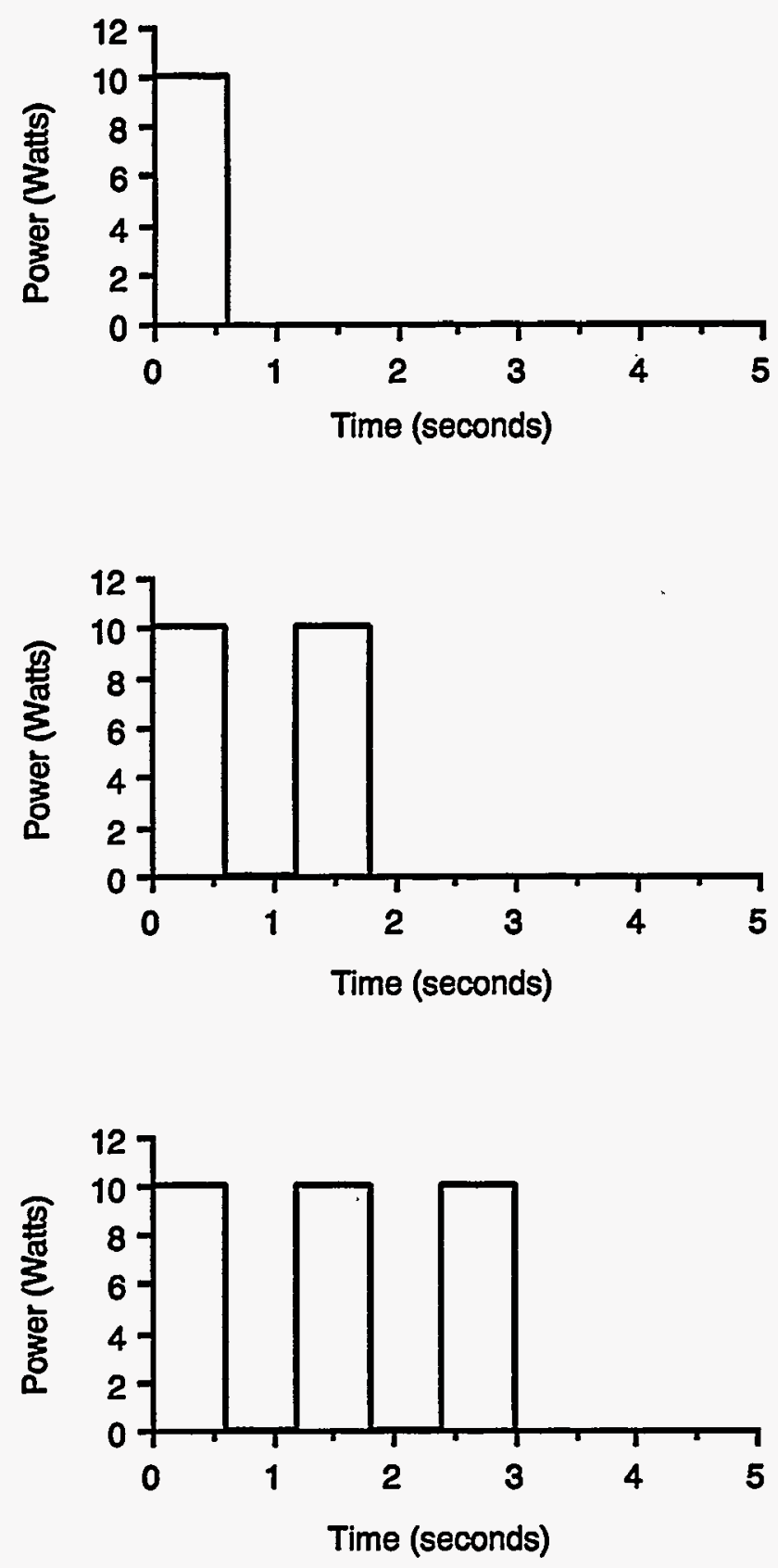

Figure 79. Pulsed heating schemes selected for use in this study. (a) Single pulse of 0.6 sec duration; (b) two $0.6 \mathrm{sec}$ pulses separated with $0.6 \mathrm{sec}$ of cooling, and (c) three $0.6 \mathrm{sec}$ pulses with $0.6 \mathrm{sec}$ of cooling between pulses. All three schemes employ the same laser power of $10 \mathrm{~W}$. 


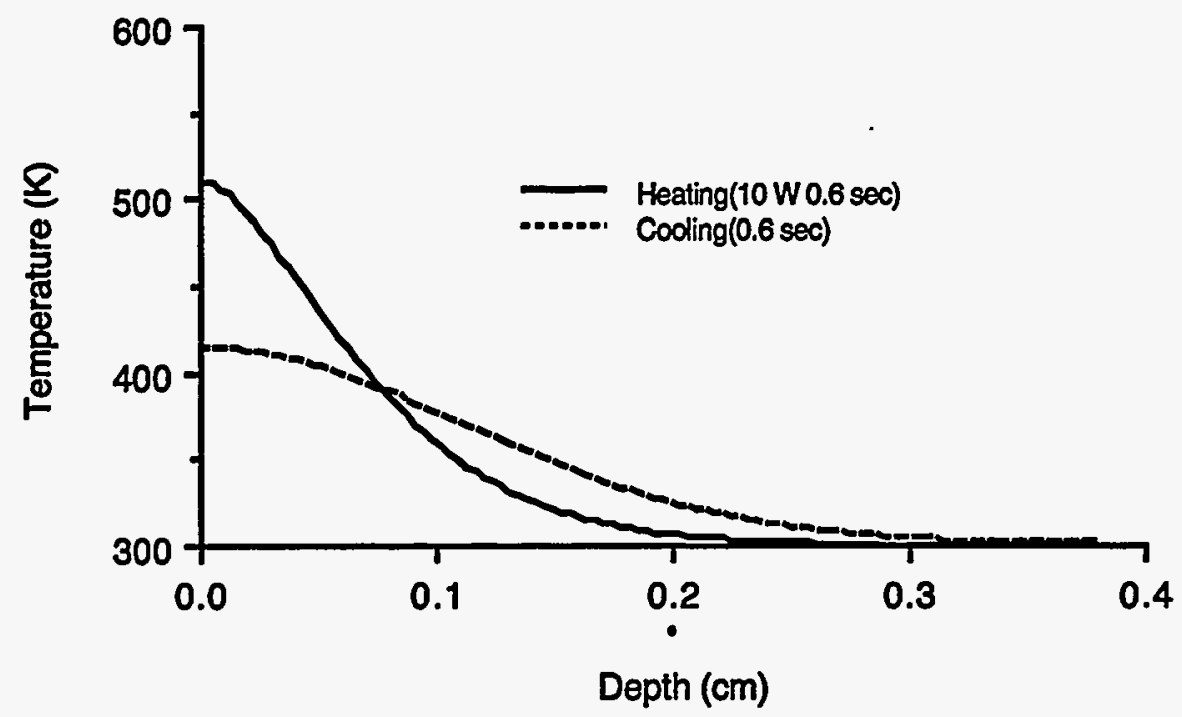

(a)

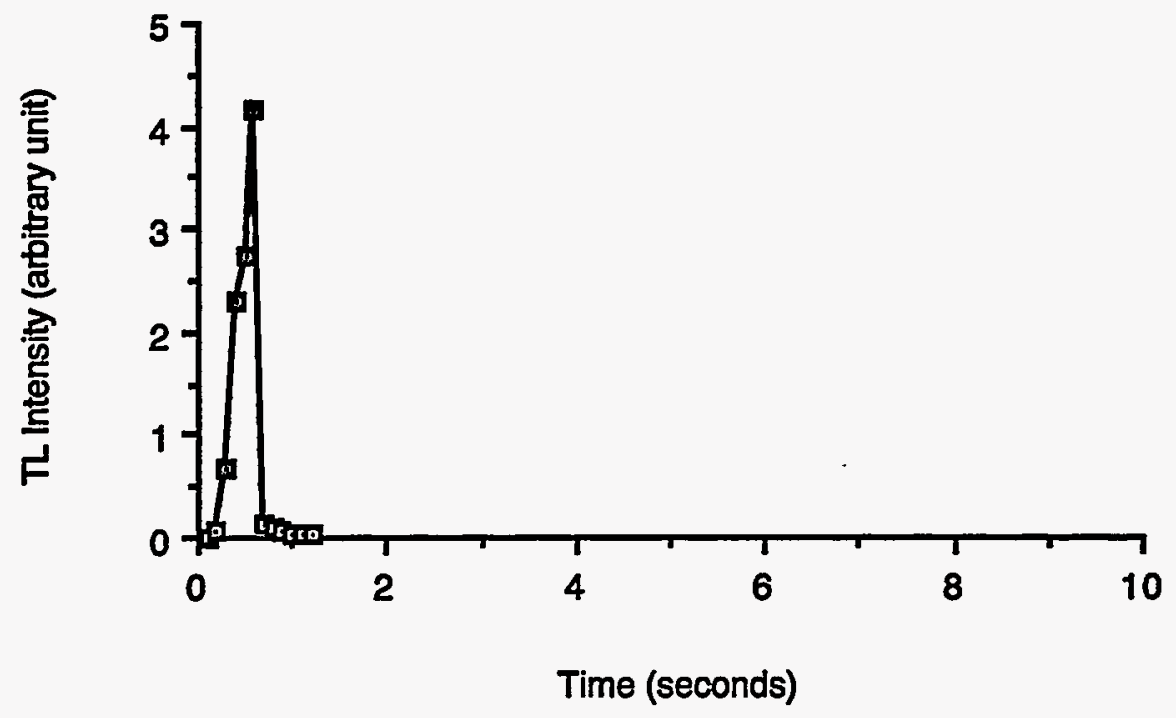

(b)

Figure 80. (a) Temperature profiles of $10 \mathrm{~W} 0.6 \mathrm{sec}$ heating and $0.6 \mathrm{sec}$ cooling and (b) the corresponding TL response of a thick laser TL dosimeter for $0.3 \mathrm{~cm} \times 0.3 \mathrm{~cm}$ uniform laser beam. 


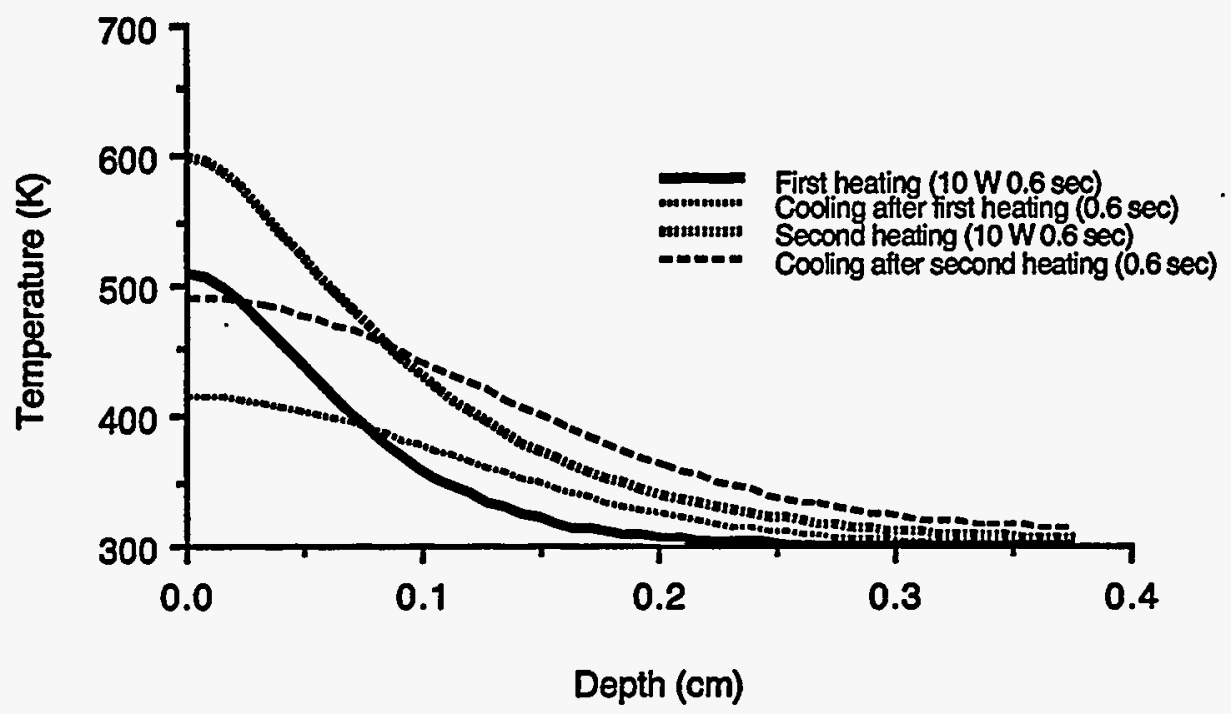

(a)

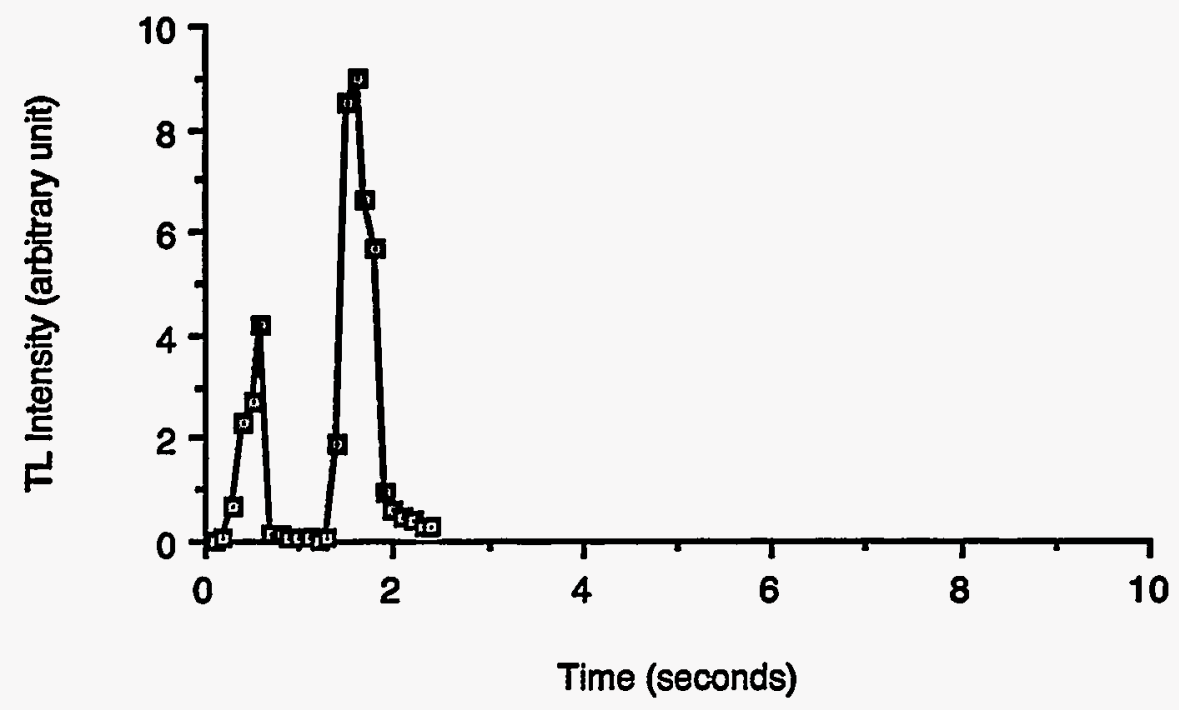

(b)

Figure 81. (a) Calculated temperature profiles for a thick laser TL dosimeter following two $10 \mathrm{~W} 0.6 \mathrm{sec}$ pulses separated with $0.6 \mathrm{sec}$ of cooling. (b) The corresponding TL response. The pulses heating were based on a $0.3 \mathrm{~cm} \times 0.3 \mathrm{~cm}$ uniform laser beam. 


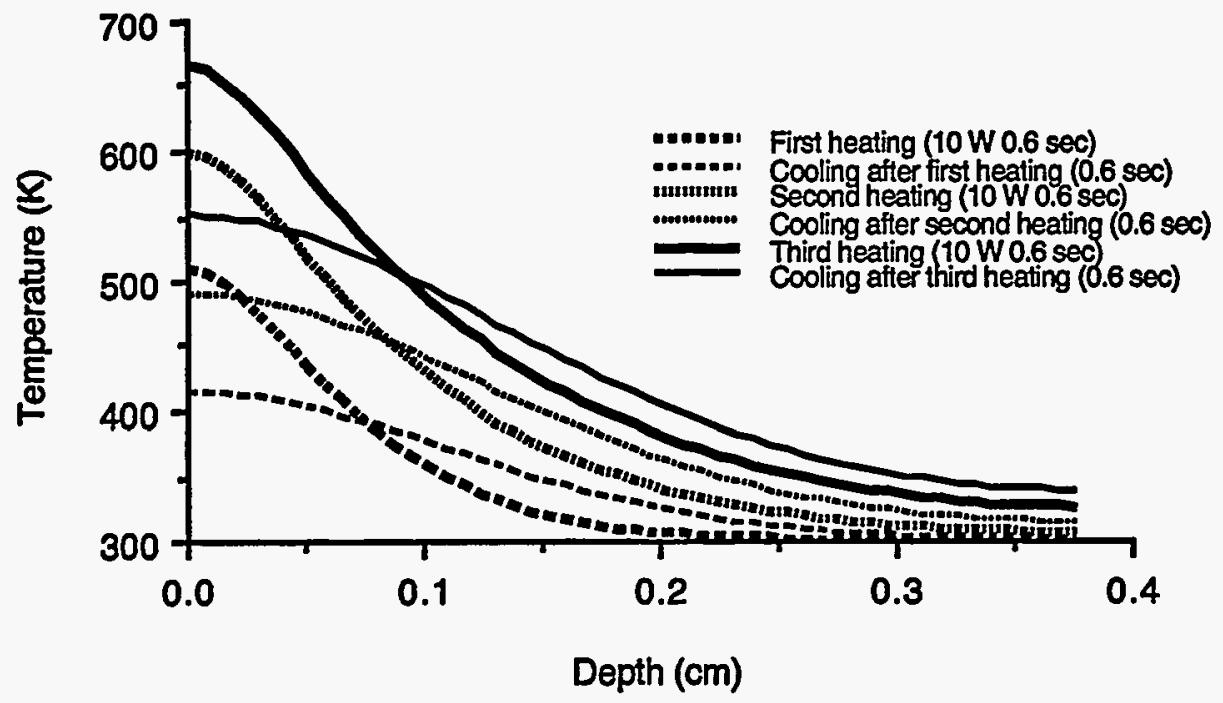

(a)

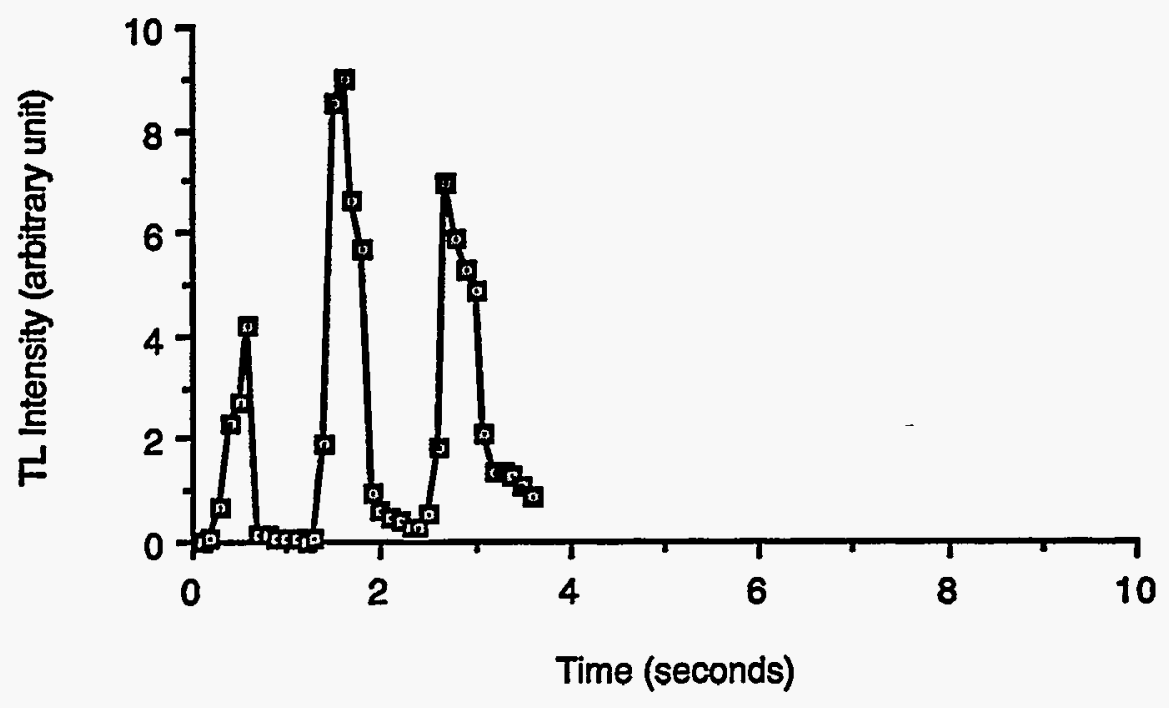

(b)

Figure 82. (a) Calculated temperature profiles for a thick laser TL dosimeter following three $10 \mathrm{~W}, 0.6 \mathrm{sec}$ pulses with $0.6 \mathrm{sec}$ of cooling between pulses. (b) The corresponding TL responses. The pulsed heating was based on a $0.3 \mathrm{~cm} \times 0.3 \mathrm{~cm}$ uniform laser beam. 


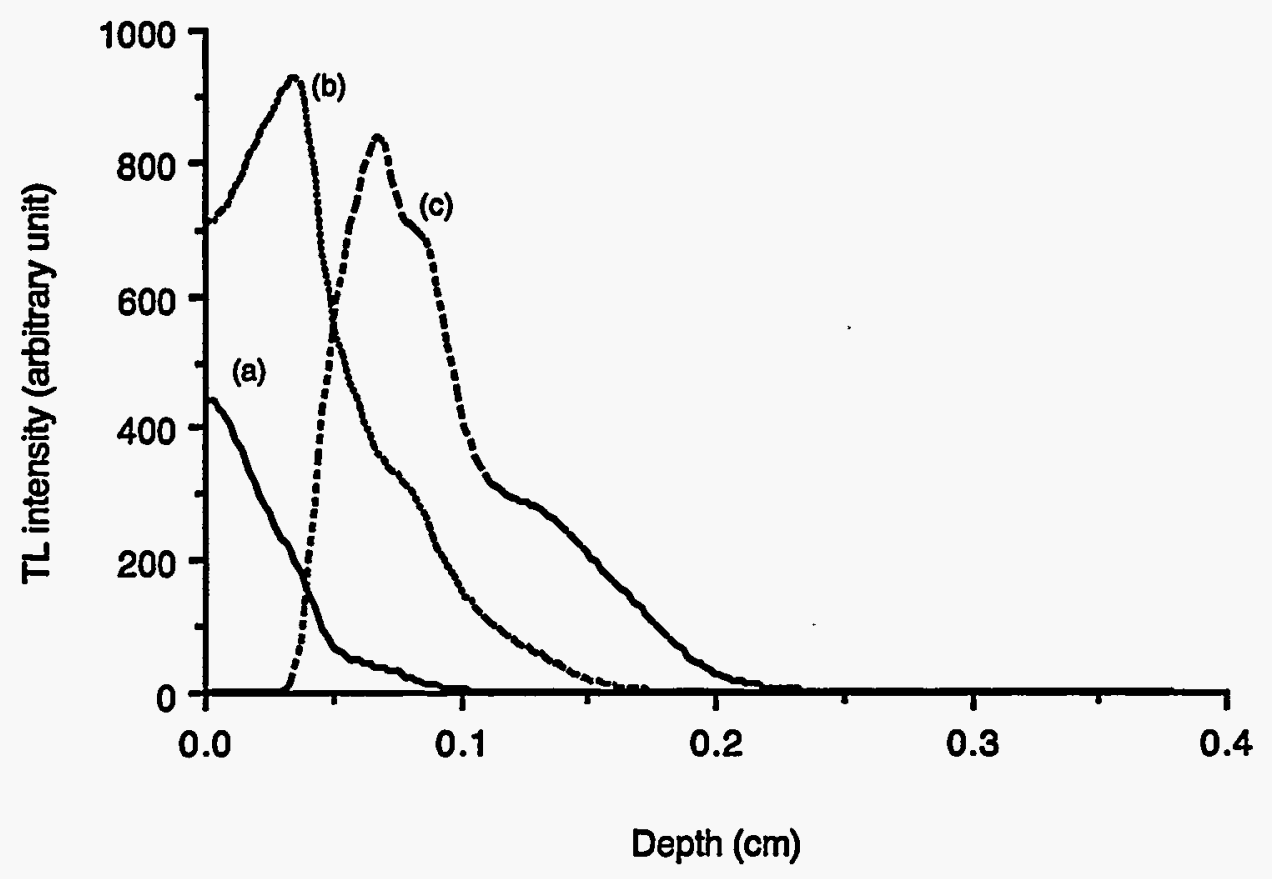

Figure 83. The calculated TL light intensity vs. depth for a thick laser TL dosimeter following three $10 \mathrm{~W}, 0.6 \mathrm{sec}$ pulses with $0.6 \mathrm{sec}$ of cooling between pulses. Three curves correspond to the TL light generated in the three durations: (a) beginning of the first pulse to $0.6 \mathrm{sec}$ of cooling after the first pulse, (b) beginning of the second pulse to $0.6 \mathrm{sec}$ of cooling after the second pulse, and (c) beginning of the third pulse to $0.6 \mathrm{sec}$ of cooling after the third pulse. The pulsed heating was based on a $0.3 \mathrm{~cm} \mathrm{x} 0.3 \mathrm{~cm}$ uniform laser beam. 


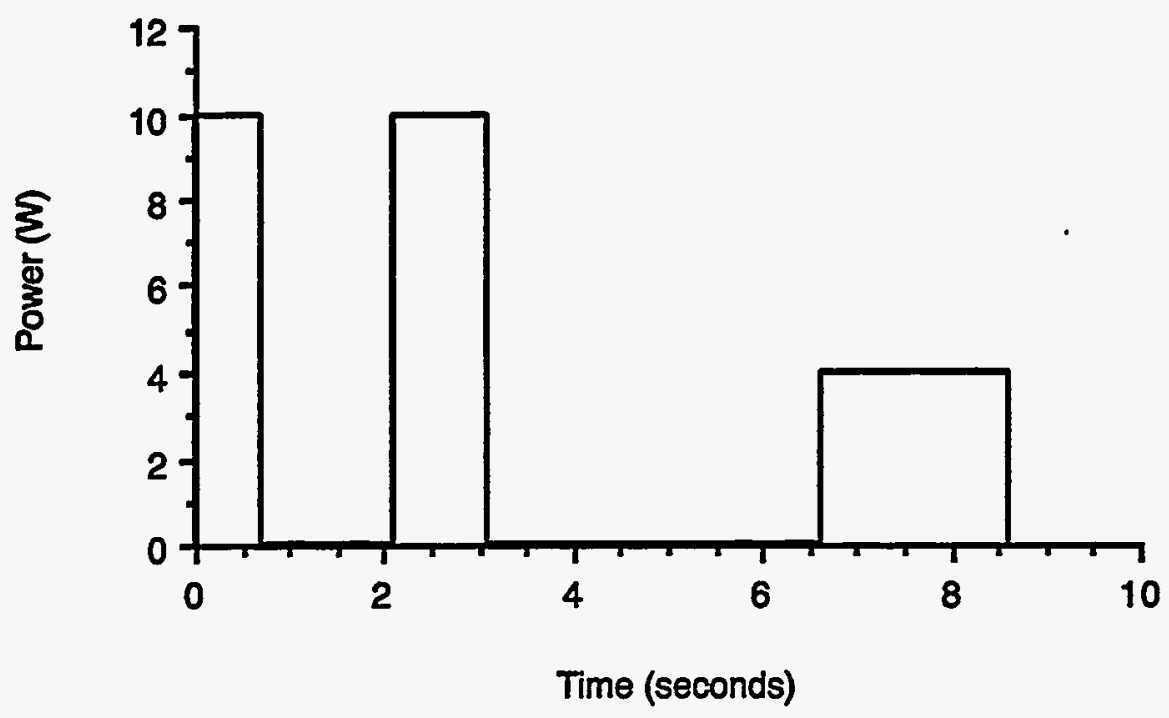

(a)

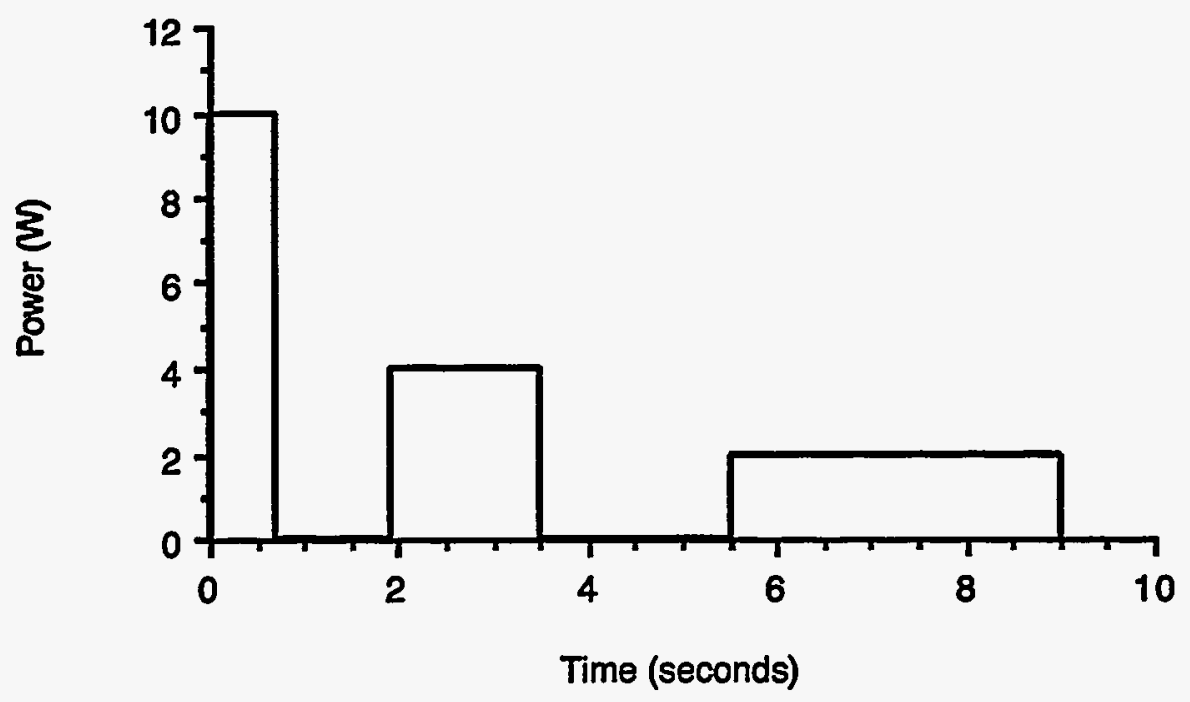

(b)

Figure 84. The heating schemes based on three consecutive pulses with varied powers, duration, and cooling time between pulses: (a) 10W-10W-4W heating scheme: first pulse $(10 \mathrm{~W} 0.7 \mathrm{sec})$ and cooling (1.4 sec); second pulse (4 W $1.0 \mathrm{sec})$ and cooling $(3.5 \mathrm{sec})$; third pulse $(2.0 \mathrm{sec})$.(b) $10 \mathrm{~W}-4 \mathrm{~W}-2 \mathrm{~W}$ heating scheme: first pulse $(10 \mathrm{~W} 0.7 \mathrm{sec})$ and cooling (1.2 sec); second pulse ( $4 \mathrm{~W} 1.6 \mathrm{sec})$ and cooling ( $2.0 \mathrm{sec})$; third pulse ( $3.5 \mathrm{sec})$. 


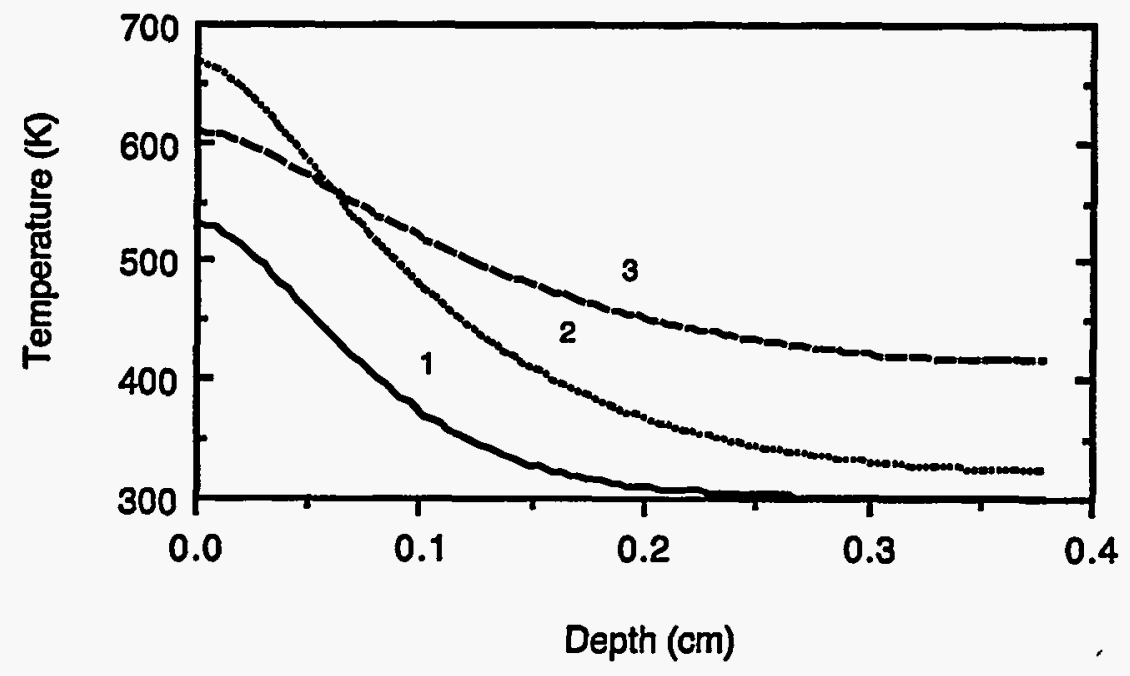

(a)

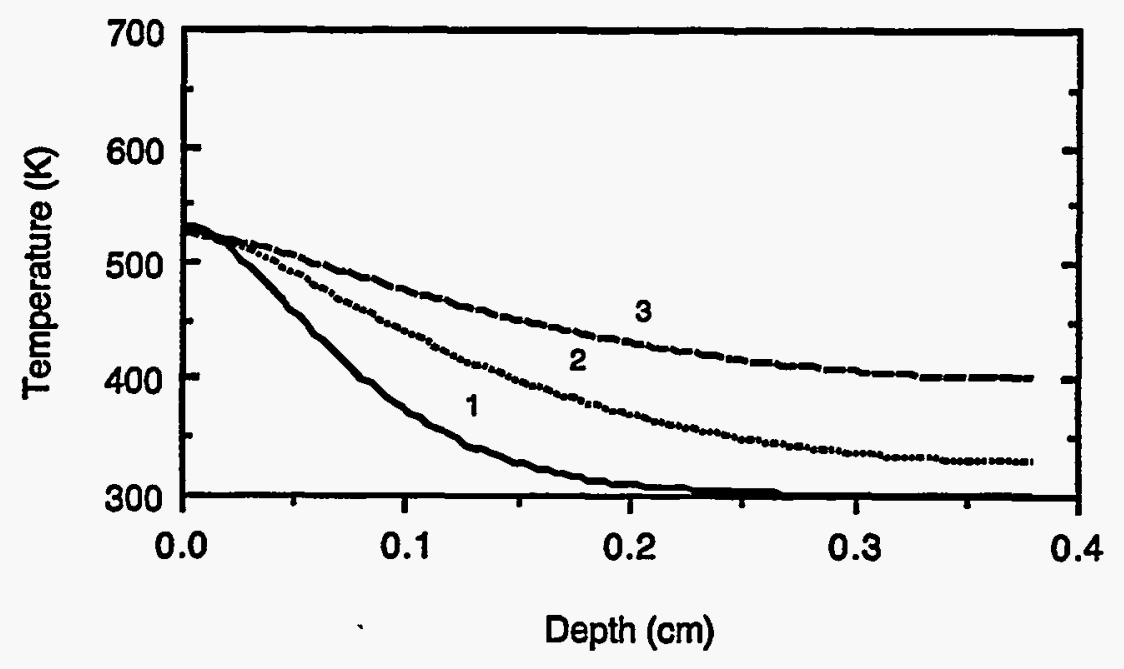

(b)

Figure 85. The calculated temperature profiles of a thick laser TL dosimeter for two pulsed heating based on three consecutive pulses with various powers, durations and cooling time between pulses: (a) $10 \mathrm{~W}-10 \mathrm{~W}-4 \mathrm{~W}$ heating scheme: $0.7 \mathrm{sec} 10 \mathrm{~W}$ first pulse, followed by $1.4 \mathrm{sec}$ of cooling time, which is then followed by a $1.0 \mathrm{sec} 10 \mathrm{~W}$ second pulse with 3.5 sec cooling, and then another $2.0 \mathrm{sec} 4 \mathrm{~W}$ third pulse; (b) $10 \mathrm{~W}-4 \mathrm{~W}-2 \mathrm{~W}$ heating scheme: $0.7 \mathrm{sec} 10 \mathrm{~W}$ first pulse, followed by $1.2 \mathrm{sec}$ of cooling time, which is then followed by a $1.6 \sec 4 \mathrm{~W}$ second pulse with $2.0 \mathrm{sec}$ of cooling, and then another $3.5 \sec 2 \mathrm{~W}$ third pulse. The three curves (i.e., 1,2 and 3) of each figure correspond to temperatures at the end of cooling period following each pulse. 


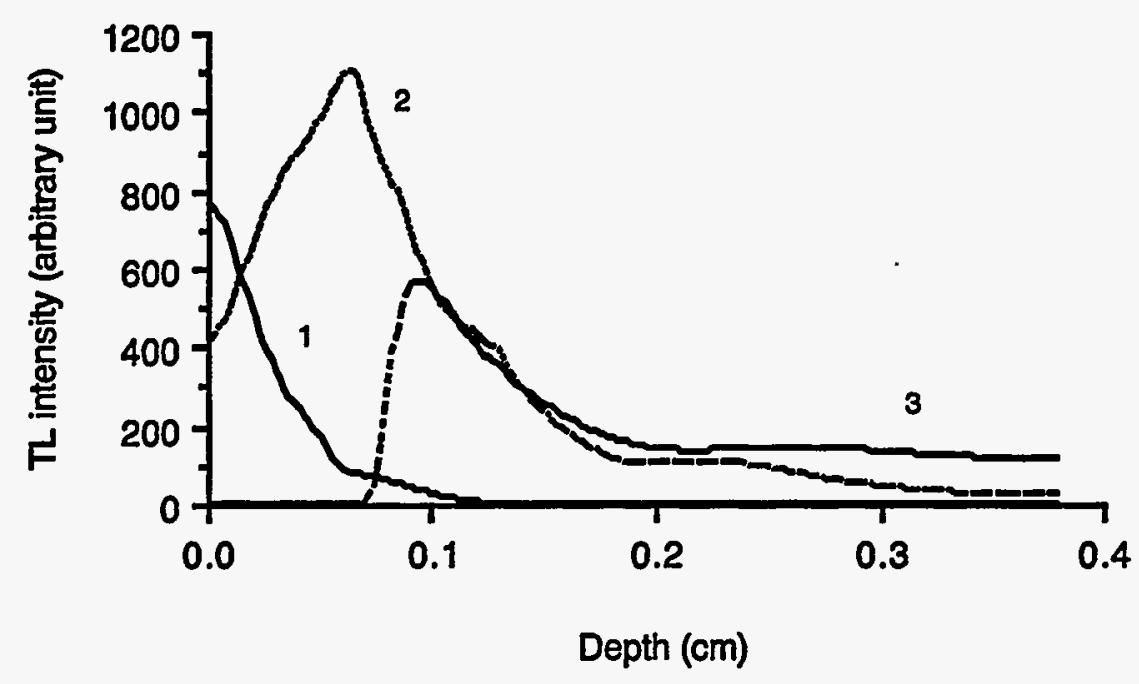

(a)

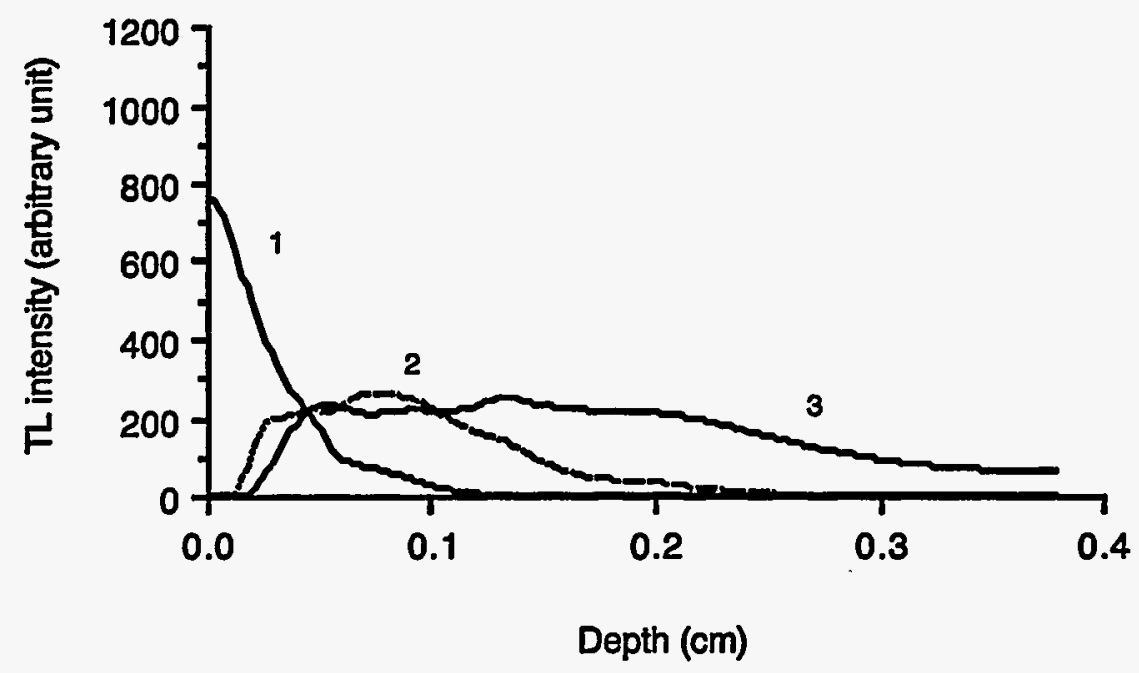

(b)

Figure 86. The calculated TL light intensity vs. TLD depth of three pulsed heating schemes based on three consecutive pulses with various powers, duration and cooling time between powers: (a) $10 \mathrm{~W}-10 \mathrm{~W}-4 \mathrm{~W}$ heating scheme: $0.7 \mathrm{sec} 10 \mathrm{~W}$ first pulse, followed by $1.4 \mathrm{sec}$ of cooling, which is then followed by a $1.0 \mathrm{sec} 10 \mathrm{~W}$ second pulse with $3.5 \mathrm{sec}$ cooling, and then another $2.0 \sec 4 \mathrm{~W}$ third pulse; (b) $10 \mathrm{~W}-4 \mathrm{~W}-2 \mathrm{~W}$ heating scheme: $0.7 \sec 10 \mathrm{~W}$ first pulse, followed by $1.2 \mathrm{sec}$ of cooling time, which is then followed by a $1.6 \sec 4 \mathrm{~W}$ second pulse with $2.0 \mathrm{sec}$ of cooling, and then another $3.5 \mathrm{sec} 2 \mathrm{~W}$ third pulse. The three curves (i.e., 1,2 and 3) of each figure correspond to temperatures at the end of cooling period following each pulse. 


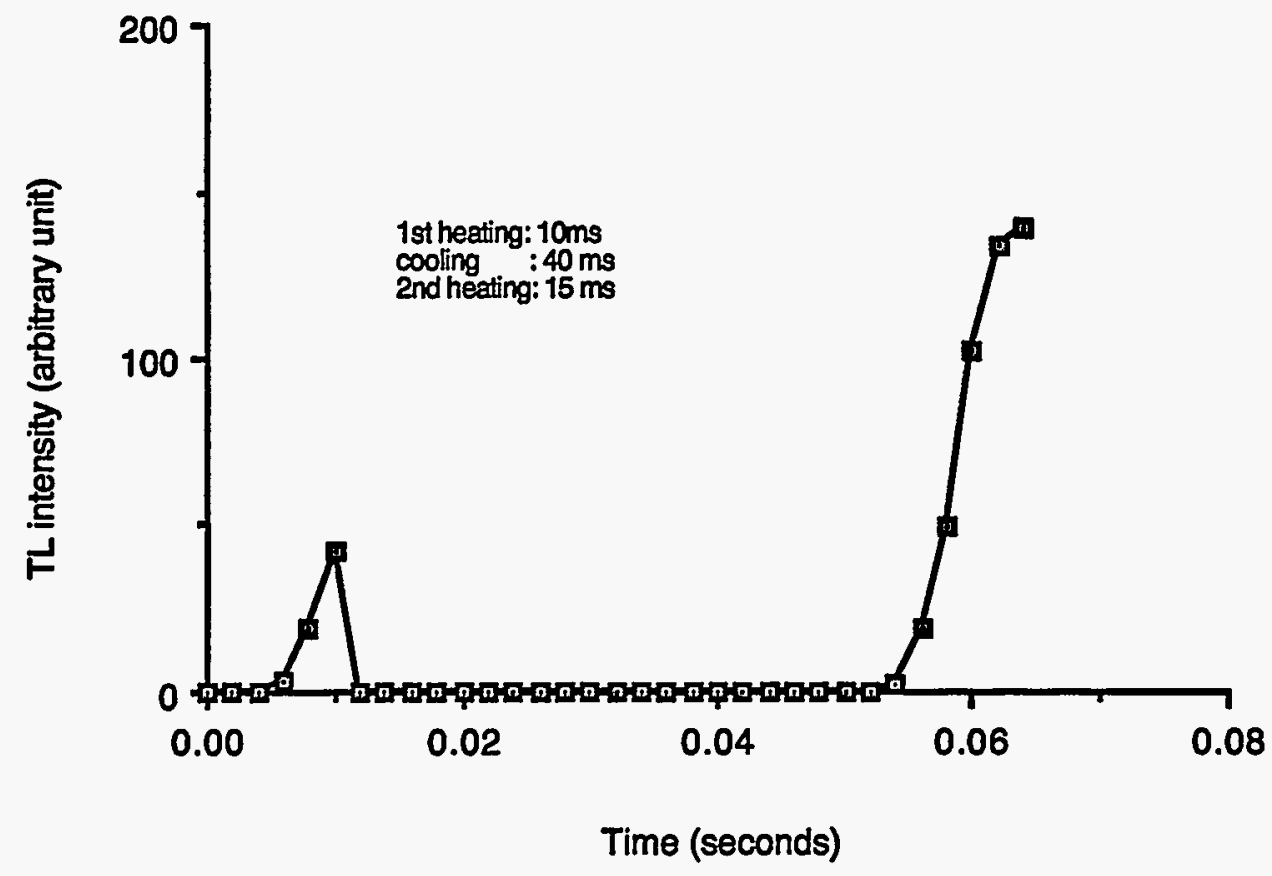

Figure 87. Calculated glow curves produced by pulsed heating using a $0.032 \mathrm{~cm}$ diameter $4 \mathrm{~W}$ focused laser beam for a thick laser TL dosimeter exposed to a broad perpendicular beam of Sr/Y-90 beta-particles. The pulsed heating scheme includes a $10 \mathrm{msec}$ first pulse followed with a $40 \mathrm{msec}$ of cooling, and then a $15 \mathrm{msec}$ second pulse. 


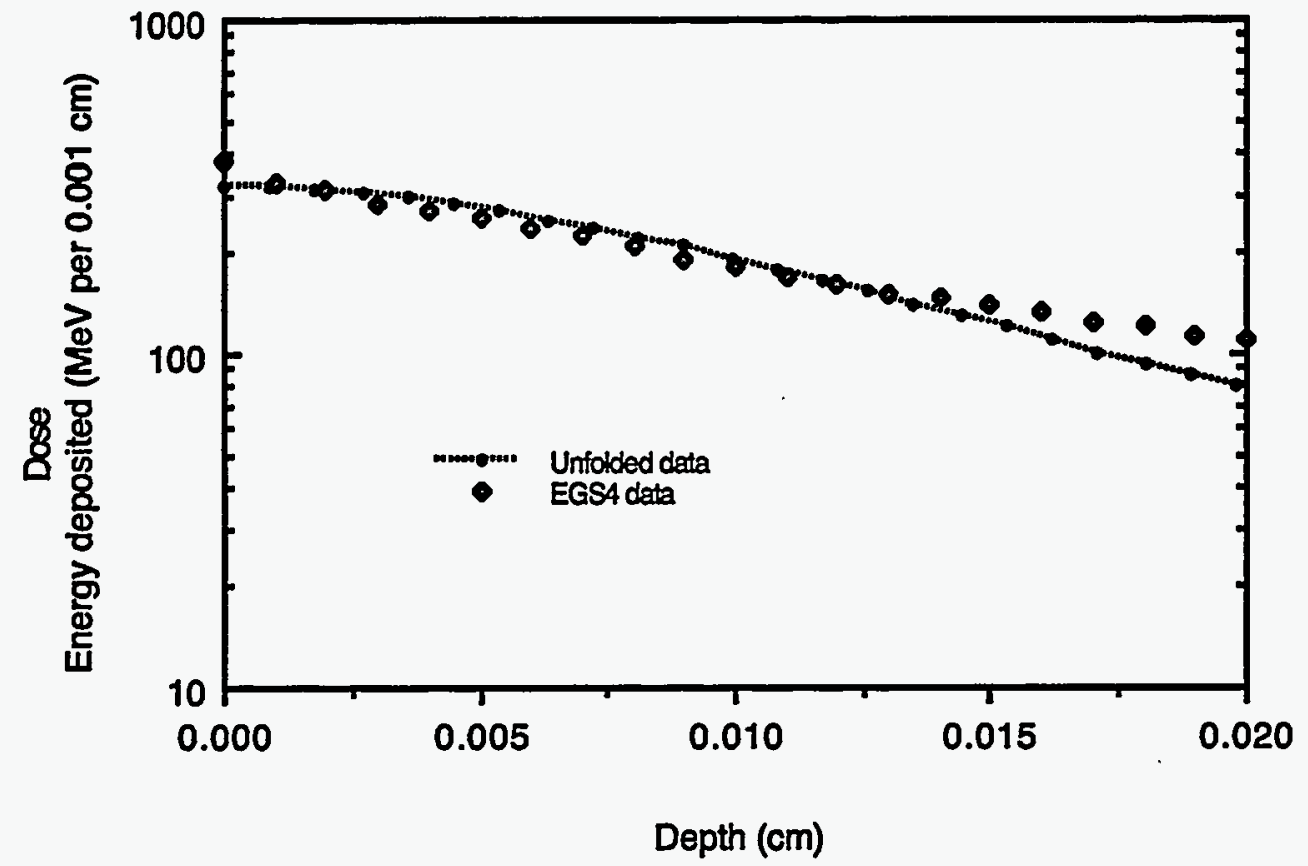

Figure 88. Unfolded depth-dose for a thick laser TL dosimeter exposed to Sr/Y-90 betaparticles. The pulsed heating scheme includes a $10 \mathrm{msec}$ first pulse followed with a 40 $\mathrm{msec}$ of cooling, and then a $15 \mathrm{msec}$ second pulse. 


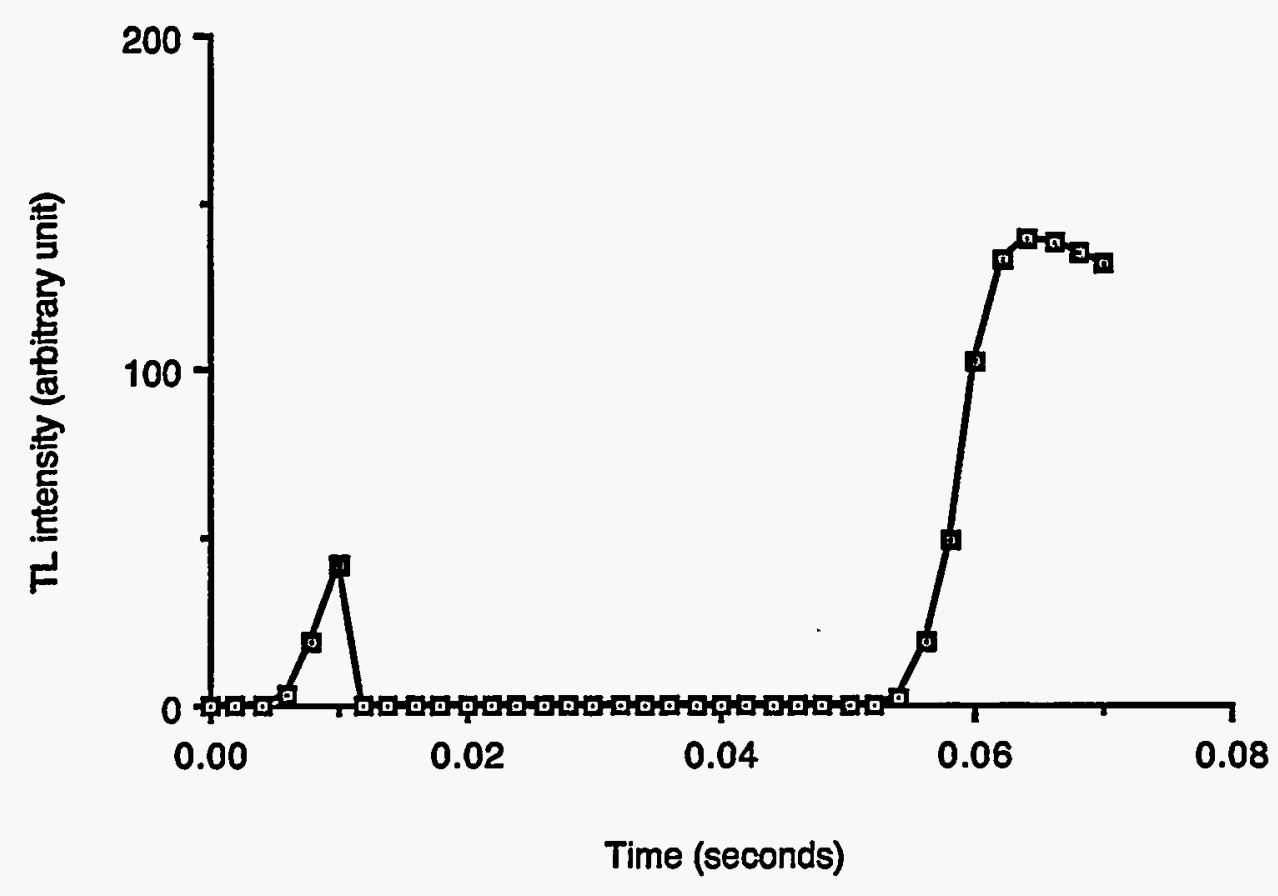

Figure 89. Calculated glow curves produced by pulsed heating using a $0.032 \mathrm{~cm}$ diameter $4 \mathrm{~W}$ focused laser beam for a thick laser TL dosimeter exposed to a broad perpendicular beam of Sr/Y-90 beta-particles. The pulsed heating scheme includes a $10 \mathrm{msec}$ first pulse followed with a $40 \mathrm{msec}$ of cooling, and then a $20 \mathrm{msec}$ second pulse. 


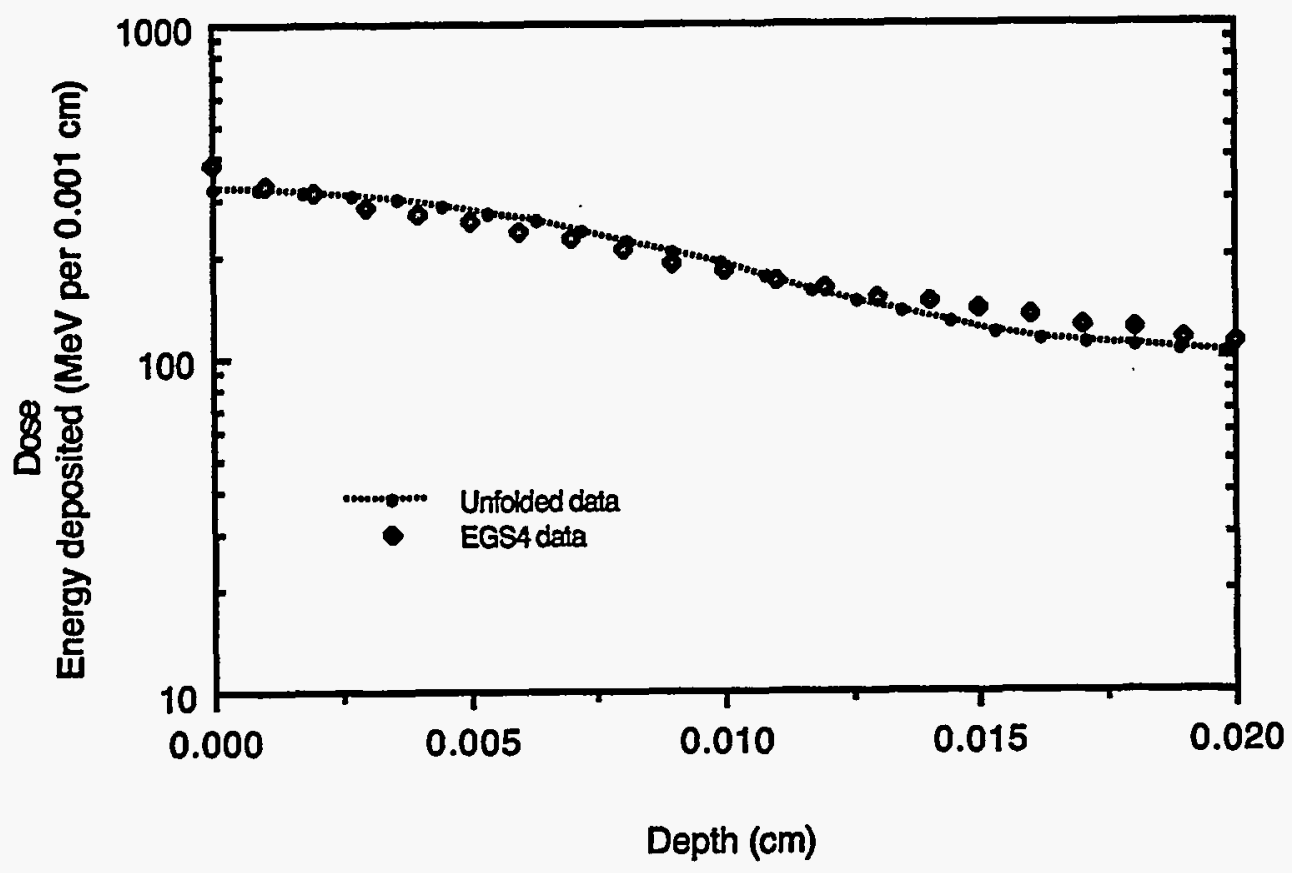

Figure 90. Unfolded depth-dose for a thick laser TL dosimeter exposed to $\mathrm{Sr} / \mathrm{Y}-90$ betaparticles. The pulsed heating scheme includes a $10 \mathrm{msec}$ first pulse followed with a 40 msec of cooling, and then a $20 \mathrm{msec}$ second pulse. 


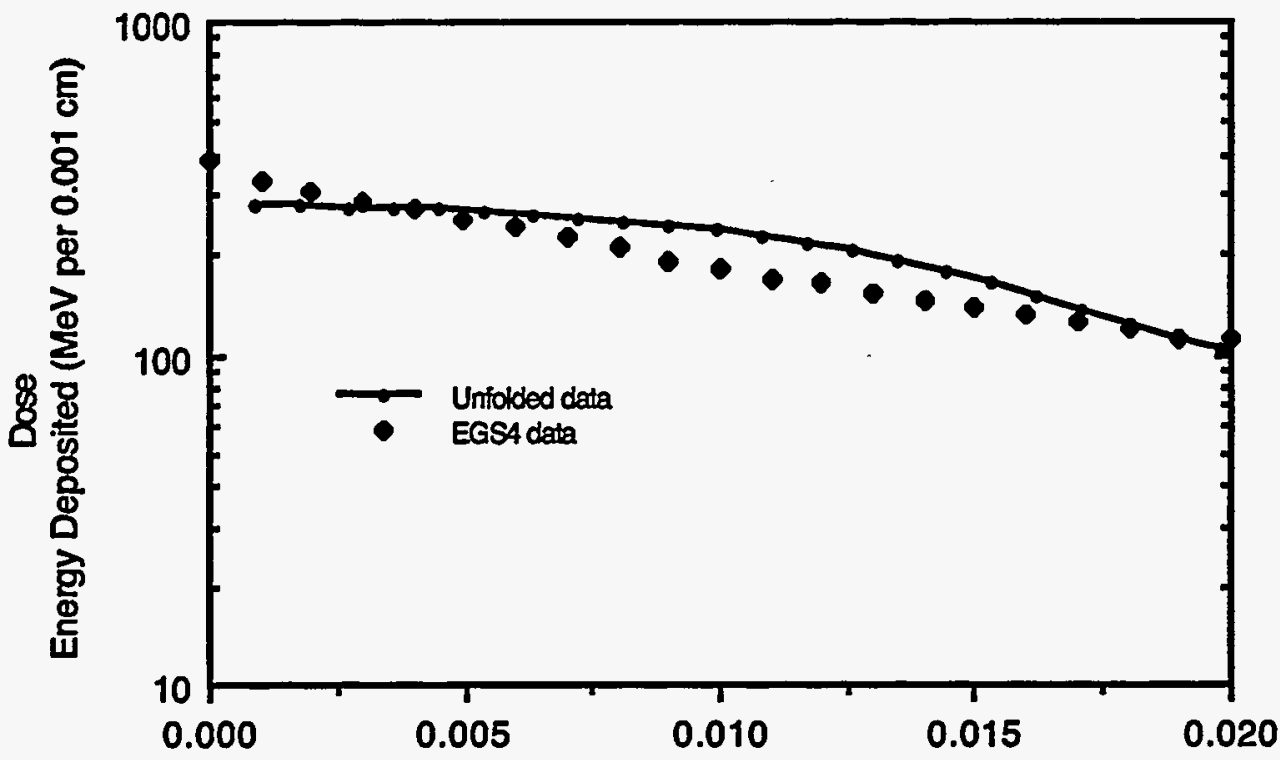

Depth (cm)

Figure 91. Unfolded depth-dose for a thick laser TL dosimeter exposed Sr/Y-90 betaparticles. The pulsed heating scheme includes a $20 \mathrm{msec}$ first pulse followed with a 40 msec of cooling, and then a $72 \mathrm{msec}$ second pulse. 

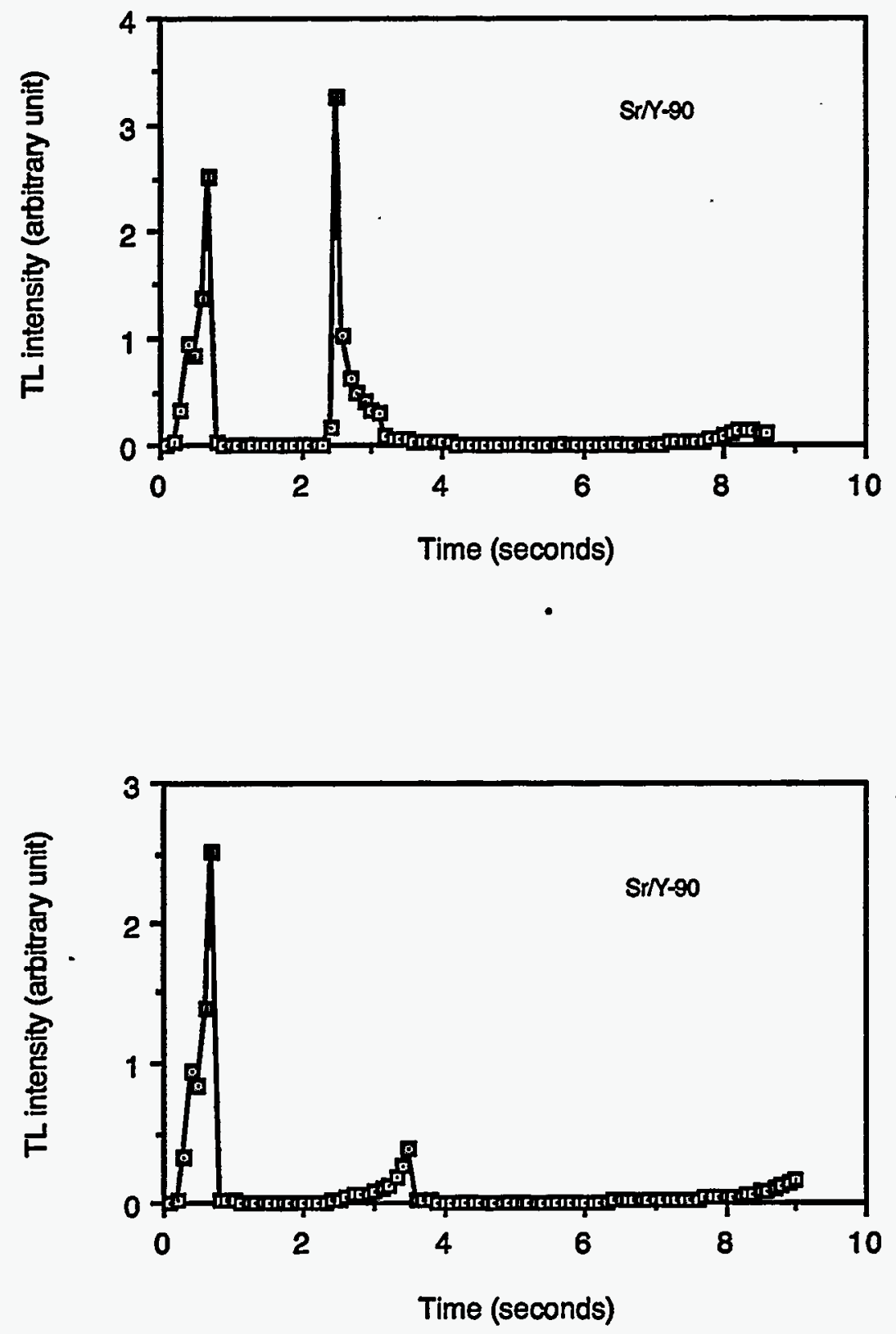

Figure 92. Calculated glow curves of a thick laser TL dosimeter exposed to $\mathrm{Sr} / \mathrm{Y}-90$ betaparticles using pulsed heating of uniform laser beam: (a) 10W-10W-4W heating scheme: $0.7 \mathrm{sec} 10 \mathrm{~W}$ first pulse, followed by $1.4 \mathrm{sec}$ of cooling, which is then followed by a 1.0 $\sec 10 \mathrm{~W}$ second pulse with $3.5 \mathrm{sec}$ of cooling, and then another $2.0 \mathrm{sec} 2 \mathrm{~W}$ third pulse; (b) $10 \mathrm{~W}-4 \mathrm{~W}-2 \mathrm{~W}$ heating scheme: $0.7 \mathrm{sec} 10 \mathrm{~W}$ first pulse, followed by $1.2 \mathrm{sec}$ of cooling, which is then followed by a $1.6 \sec 4 \mathrm{~W}$ second pulse with $2.0 \mathrm{sec}$ of cooling, and then another $3.5 \sec 2 \mathrm{~W}$ third pulse. 


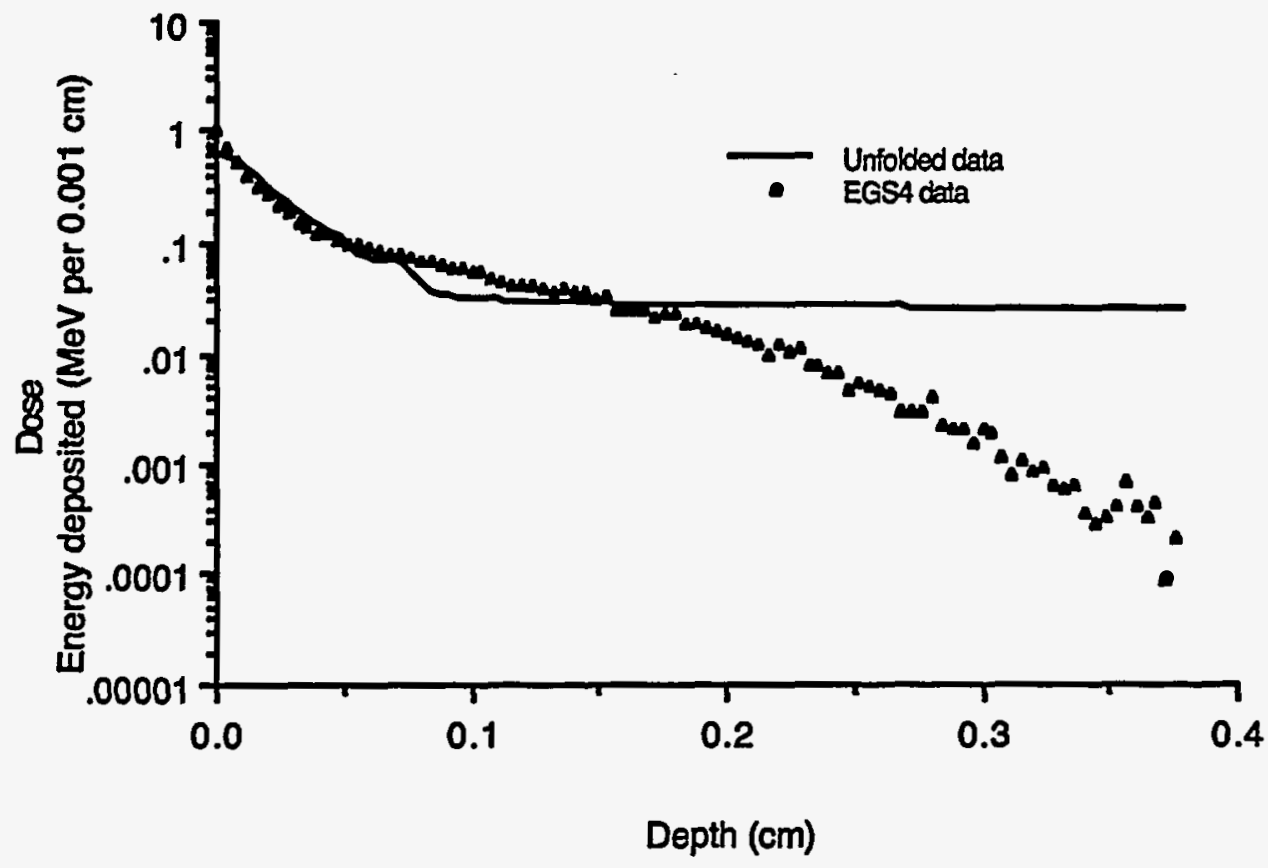

Figure 93. Unfolded depth-dose of a thick laser TL dosimeter exposed to $\mathrm{Sr} / \mathrm{Y}-90$ betaparticles. $0.7 \mathrm{sec} 10 \mathrm{~W}$ first pulse, followed by $1.4 \mathrm{sec}$ of cooling, which is then followed by a $1.0 \mathrm{sec} 10 \mathrm{~W}$ second pulse with $3.5 \mathrm{sec}$ of cooling, and then another $2.0 \sec 2 \mathrm{~W}$ third pulse. 


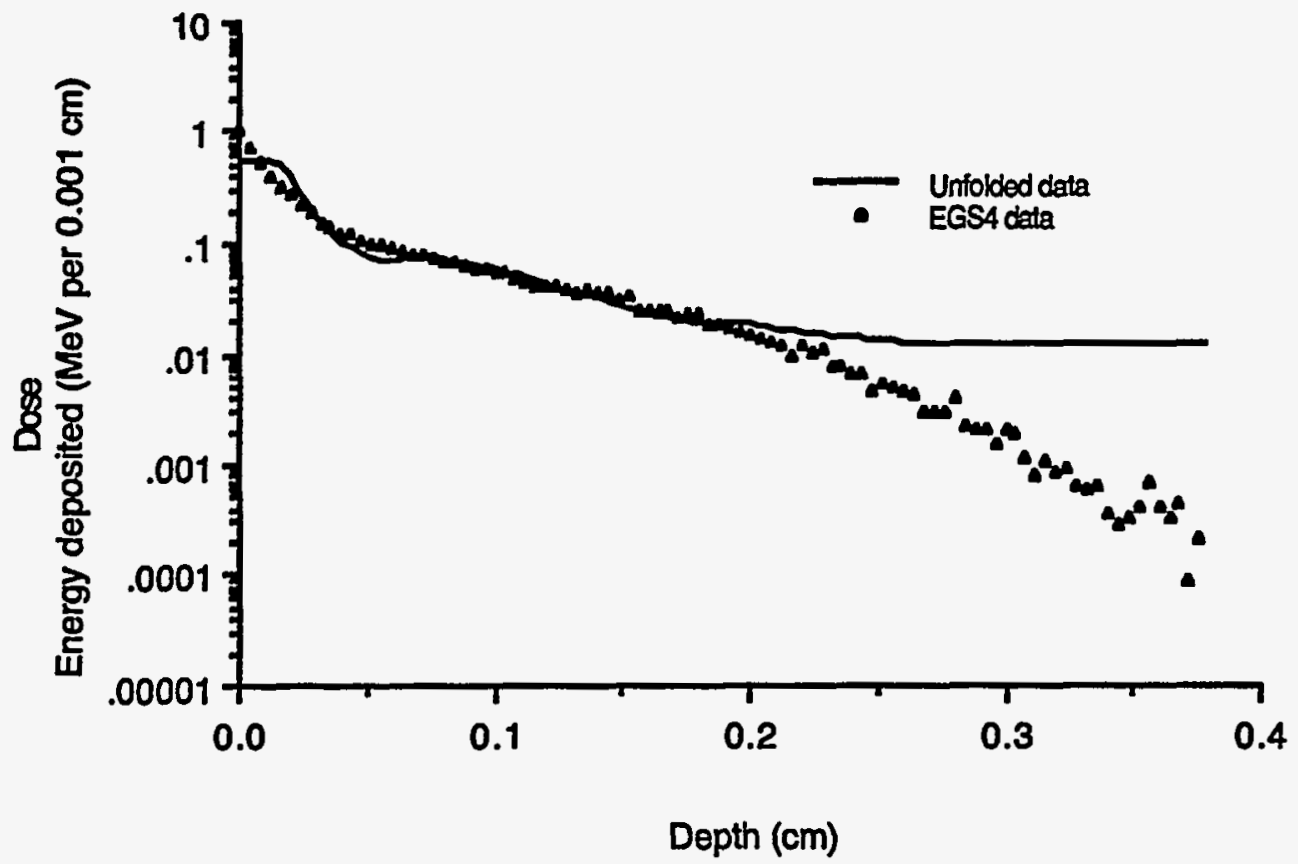

Figure 94. Unfolded depth-dose of a thick laser TL dosimeter exposed by $\mathrm{Sr} / \mathrm{Y}-90$ betaparticles. $0.7 \mathrm{sec} 10 \mathrm{~W}$ first pulse, followed by $1.2 \mathrm{sec}$ of cooling, which is then followed by a $1.6 \mathrm{sec} 4 \mathrm{~W}$ second pulse with $2.0 \mathrm{sec}$ of cooling, and then another $3.5 \mathrm{sec} 2 \mathrm{~W}$ third pulse. 


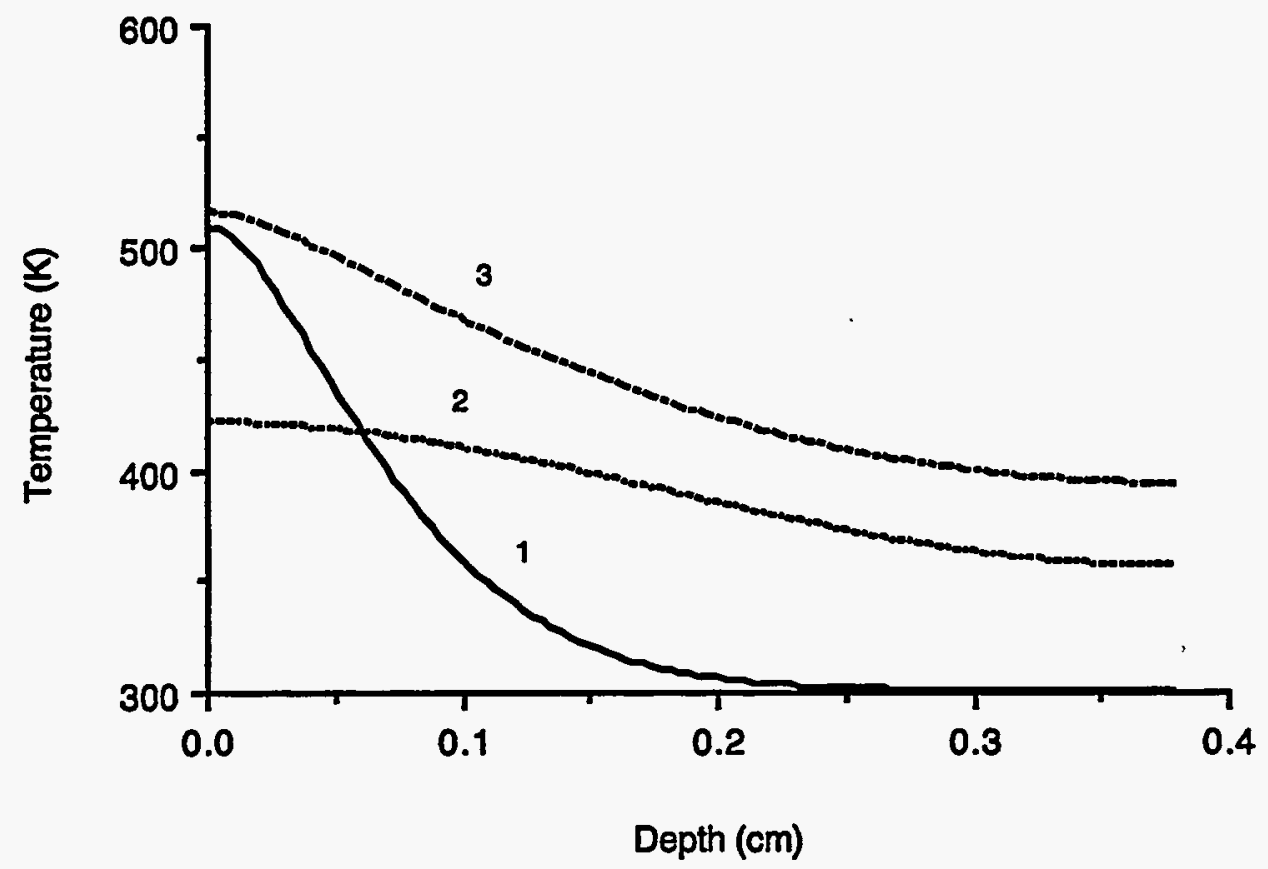

Figure 95. The calculated temperature profiles of a thick laser TL dosimeter for pulsed heating of uniform laser beam; $0.6 \mathrm{sec} 10 \mathrm{~W}$ first pulse, followed by $1.3 \mathrm{sec}$ of cooling time, which is then followed by a $1.6 \sec 4 \mathrm{~W}$ second pulse with $2.0 \mathrm{sec}$ of cooling, and then another $3.5 \sec 2 \mathrm{~W}$ third pulse. 


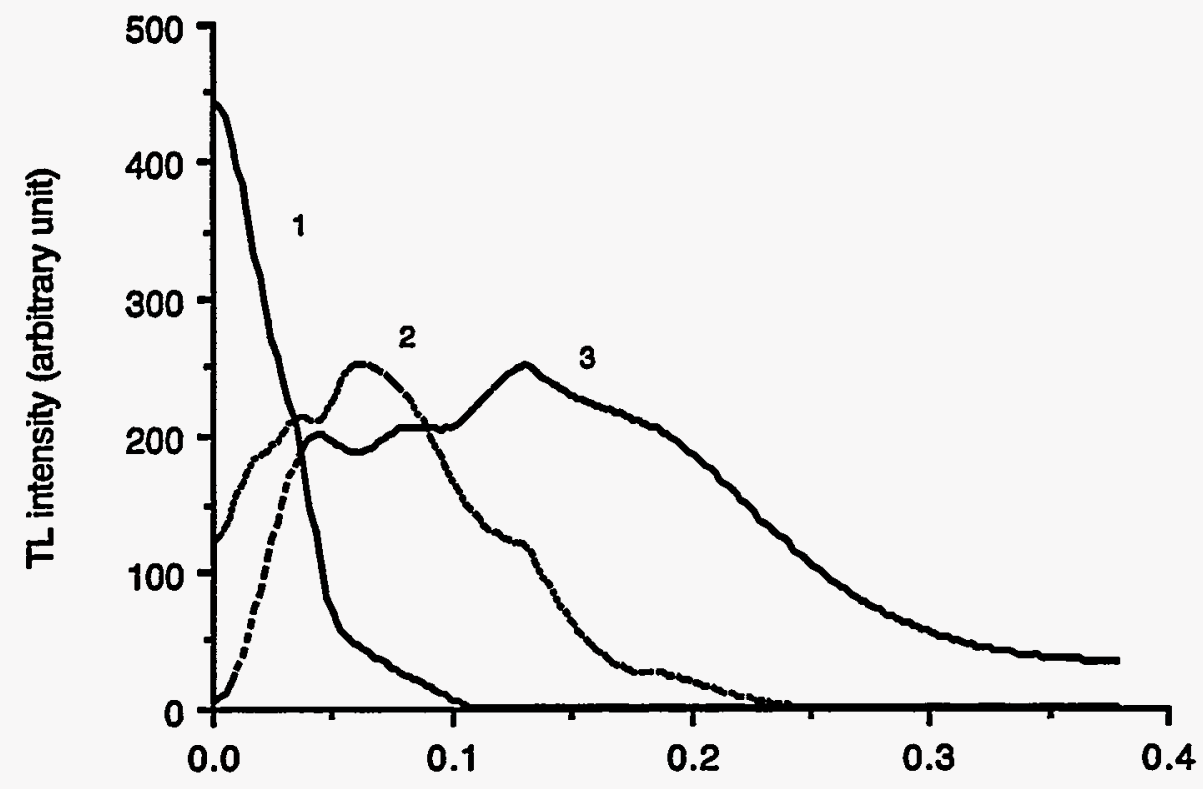

Depth (cm)

Figure 96. The calculated TL light intensity vs. TLD depth of three-pulse heating schemes based on three consecutive pulses with various powers, duration and cooling time between powers: $0.6 \mathrm{sec} 10 \mathrm{~W}$ first pulse, followed by $1.3 \mathrm{sec}$ of cooling, which is then followed by a $1.6 \mathrm{sec} 4 \mathrm{~W}$ second pulse with $2.0 \mathrm{sec}$ of cooling, and then another $3.5 \mathrm{sec} 2 \mathrm{~W}$ third pulse. 


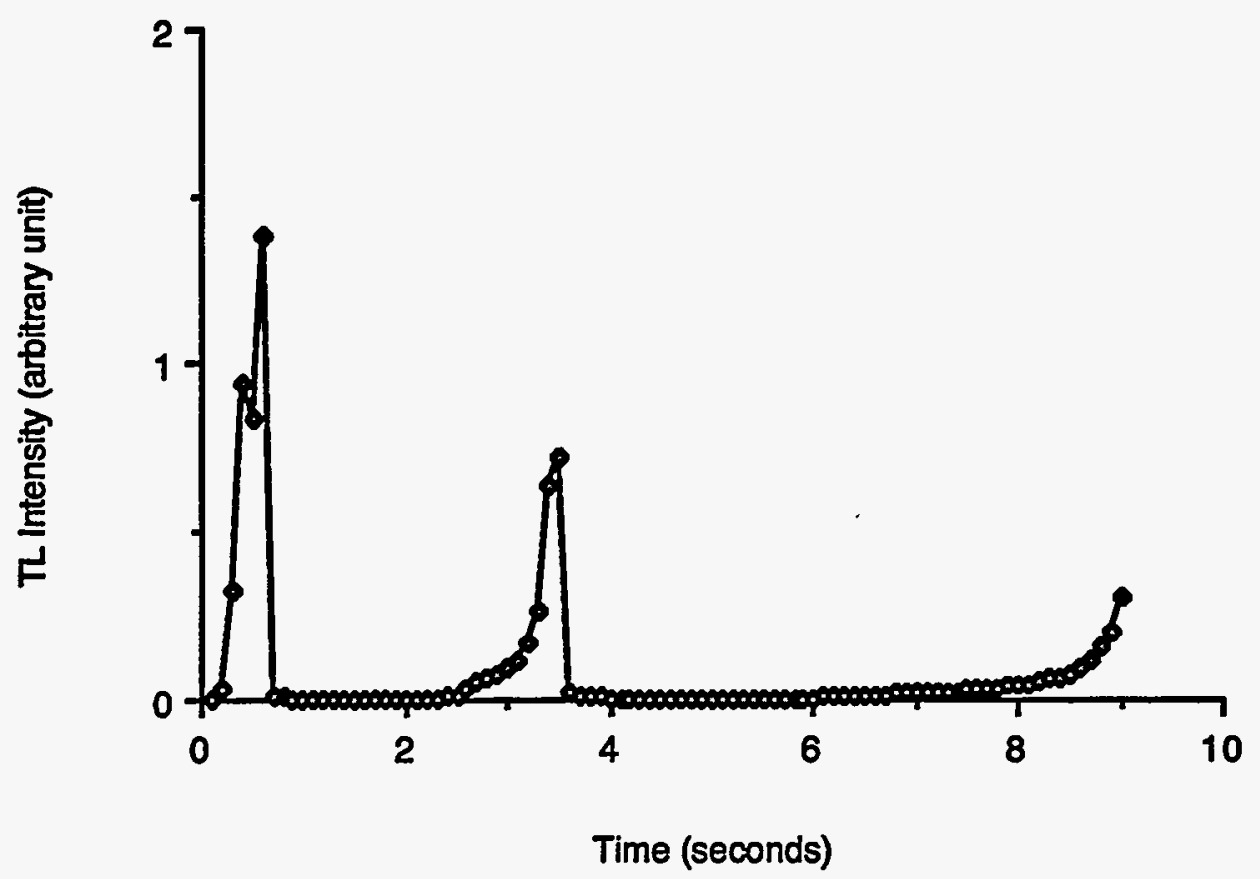

Figure 97. Calculated glow curves produced by pulsed heating using uniform laser beam for a thick laser TL dosimeter exposed to a broad perpendicular beam of Sr/Y-90 betaparticles. The pulsed heating scheme includes a $0.6 \mathrm{sec} 10 \mathrm{~W}$ first pulse, followed by 1.3 sec of cooling, which is then followed by a $1.6 \mathrm{sec} 4 \mathrm{~W}$ second pulse with $2.0 \mathrm{sec}$ of cooling, and then another $3.5 \sec 2 \mathrm{~W}$ third pulse. 


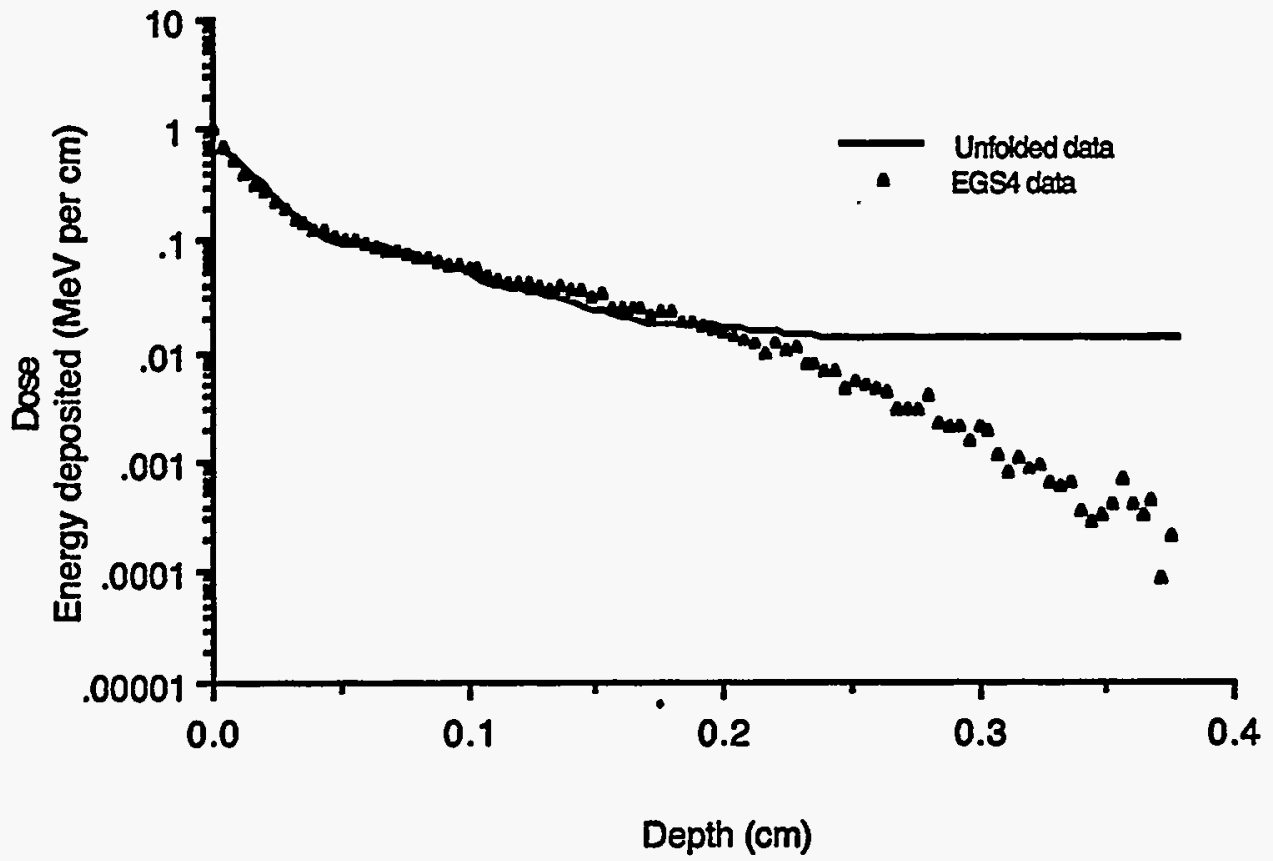

Figure 98. Unfolded depth-dose of a thick laser TL dosimeter exposed to Sr/Y-90 betaparticles. The pulsed heating scheme includes a $0.6 \mathrm{sec} 10 \mathrm{~W}$ first pulse, followed by 1.3 sec of cooling, which is then followed by a $1.6 \mathrm{sec} 4 \mathrm{~W}$ second pulse with $2.0 \mathrm{sec}$ of cooling, and then another $3.5 \sec 2 \mathrm{~W}$ third pulse. 


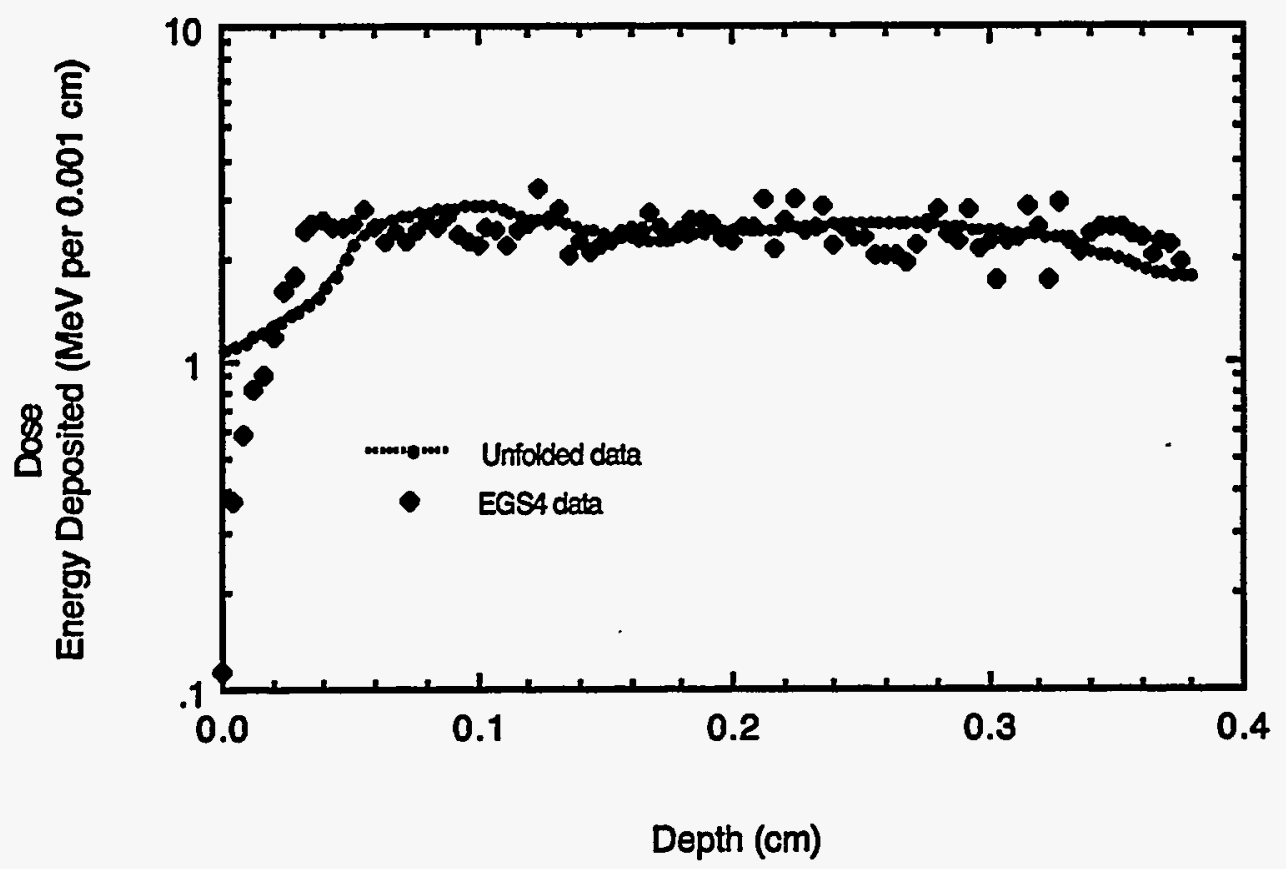

Figure 99. Unfolded depth-dose for a thick laser TL dosimeter exposed to Cs-137 gammarays. The pulsed heating scheme includes a $0.6 \mathrm{sec} 10 \mathrm{~W}$ first pulse, followed by $1.3 \mathrm{sec}$ of cooling, which is then followed by a $1.6 \mathrm{sec} 4 \mathrm{~W}$ second pulse with $2.0 \mathrm{sec}$ of cooling, and then another $3.5 \sec 2 \mathrm{~W}$ third pulse. 


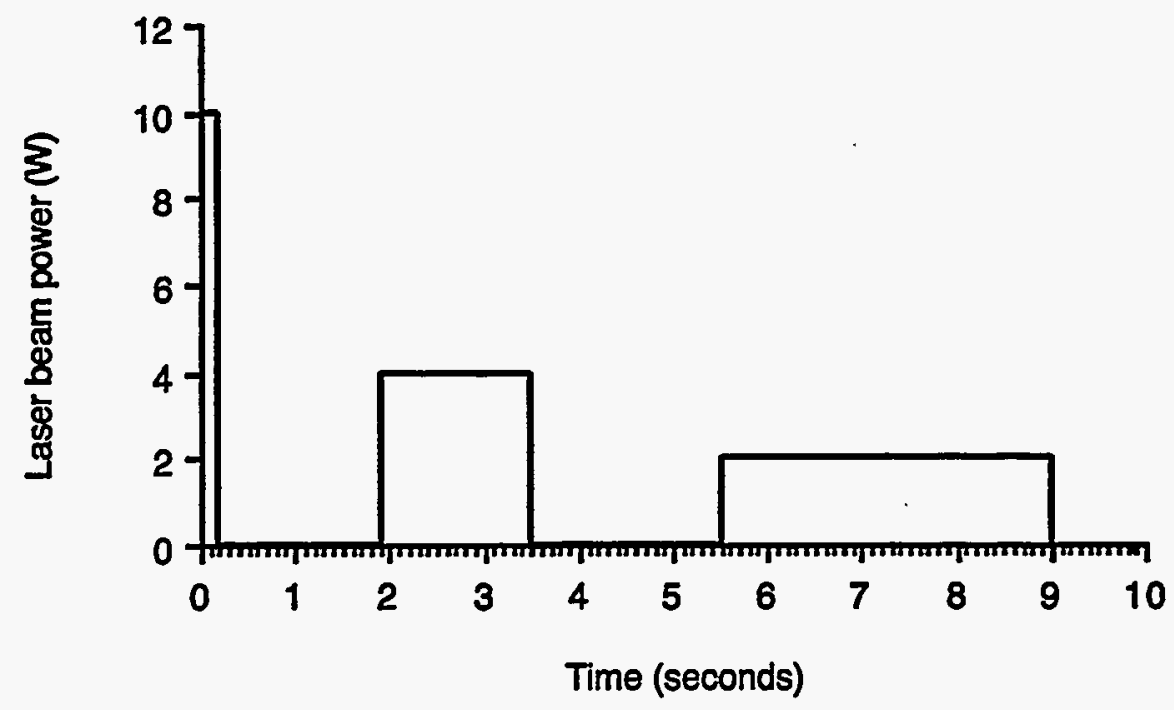

(a)

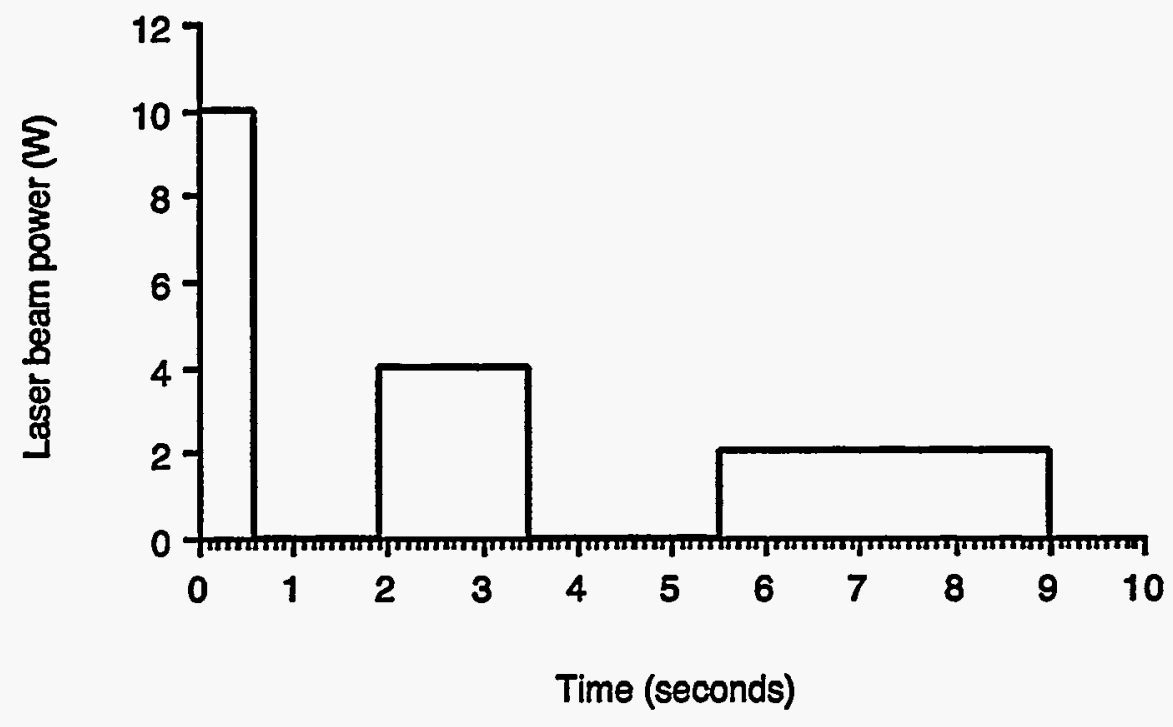

(b)

Figure 100. Optimum pulsed heating schemes for uniform laser beam on the top and bottom surface of the thick laser TL dosimeter. The pulsed heating scheme includes (a) Top heating (above): a $0.2 \mathrm{sec} 10 \mathrm{~W}$ first pulse, followed by $1.7 \mathrm{sec}$ of cooling, which is then followed by a $1.8 \mathrm{sec} 4 \mathrm{~W}$ second pulse with $2.0 \mathrm{sec}$ of cooling, and then another $3.5 \mathrm{sec} 2$ W third pulse; (a) Bottom heating (below): a $0.6 \mathrm{sec} 10 \mathrm{~W}$ first pulse, followed by $1.3 \mathrm{sec}$ of cooling, which is then followed by a $1.6 \sec 4 \mathrm{~W}$ second pulse with $2.0 \mathrm{sec}$ of cooling, and then another $3.5 \sec 2 \mathrm{~W}$ third pulse. 

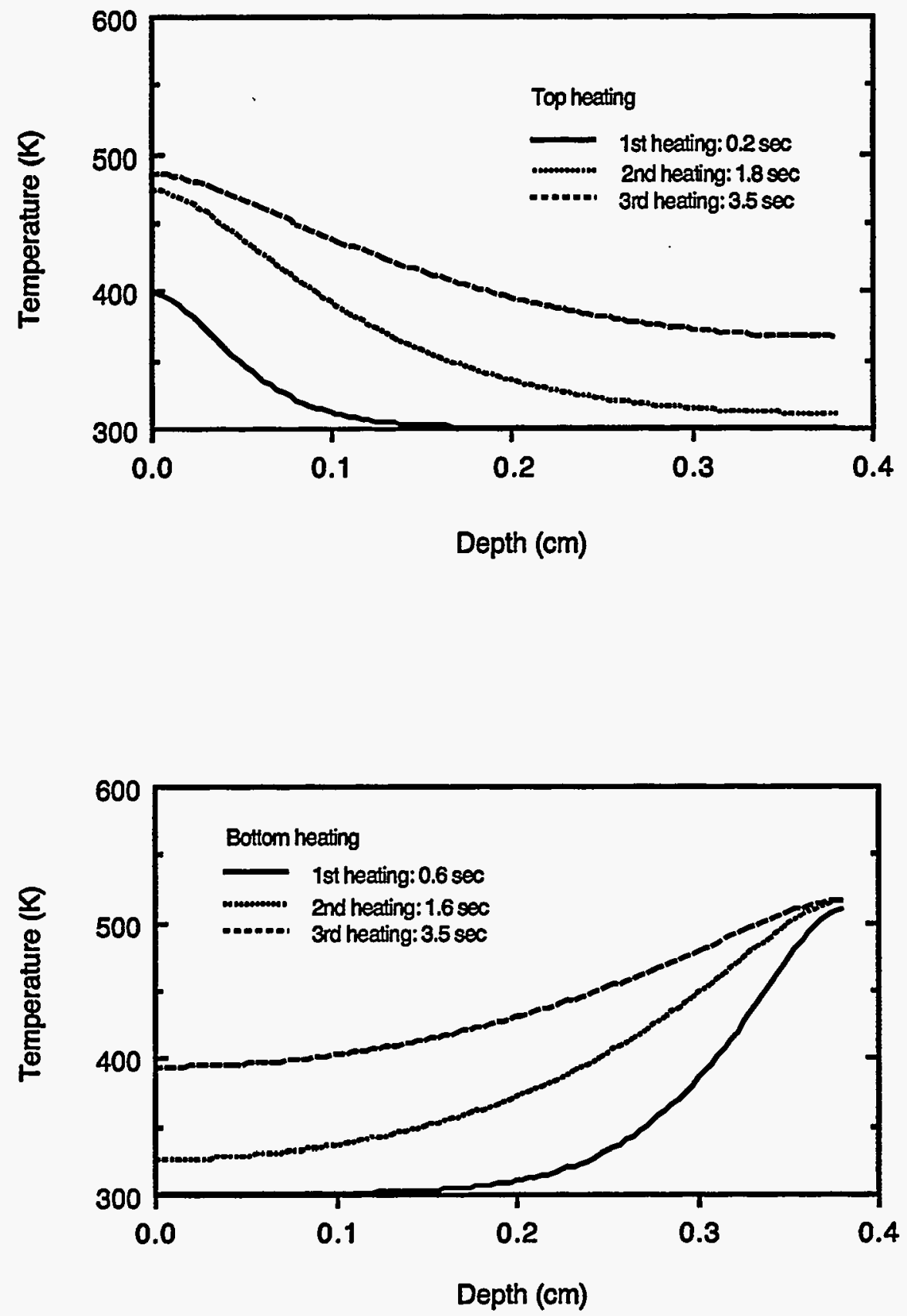

Figure 101. The calculated temperature profiles of a thick laser TL dosimeter for pulsed heating of uniform laser beam. The pulsed heating scheme includes (a) Top heating (above): a $0.2 \mathrm{sec} 10 \mathrm{~W}$ first pulse, followed by $1.7 \mathrm{sec}$ of cooling, which is then followed by a $1.8 \mathrm{sec} 4 \mathrm{~W}$ second pulse with $2.0 \mathrm{sec}$ of cooling, and then another $3.5 \sec 2 \mathrm{~W}$ third pulse; (a) Bottom heating (below): a $0.6 \mathrm{sec} 10 \mathrm{~W}$ first pulse, followed by $1.3 \mathrm{sec}$ of cooling, which is then followed by a $1.6 \sec 4 \mathrm{~W}$ second pulse with $2.0 \mathrm{sec}$ of cooling, and then another $3.5 \sec 2 \mathrm{~W}$ third pulse. 

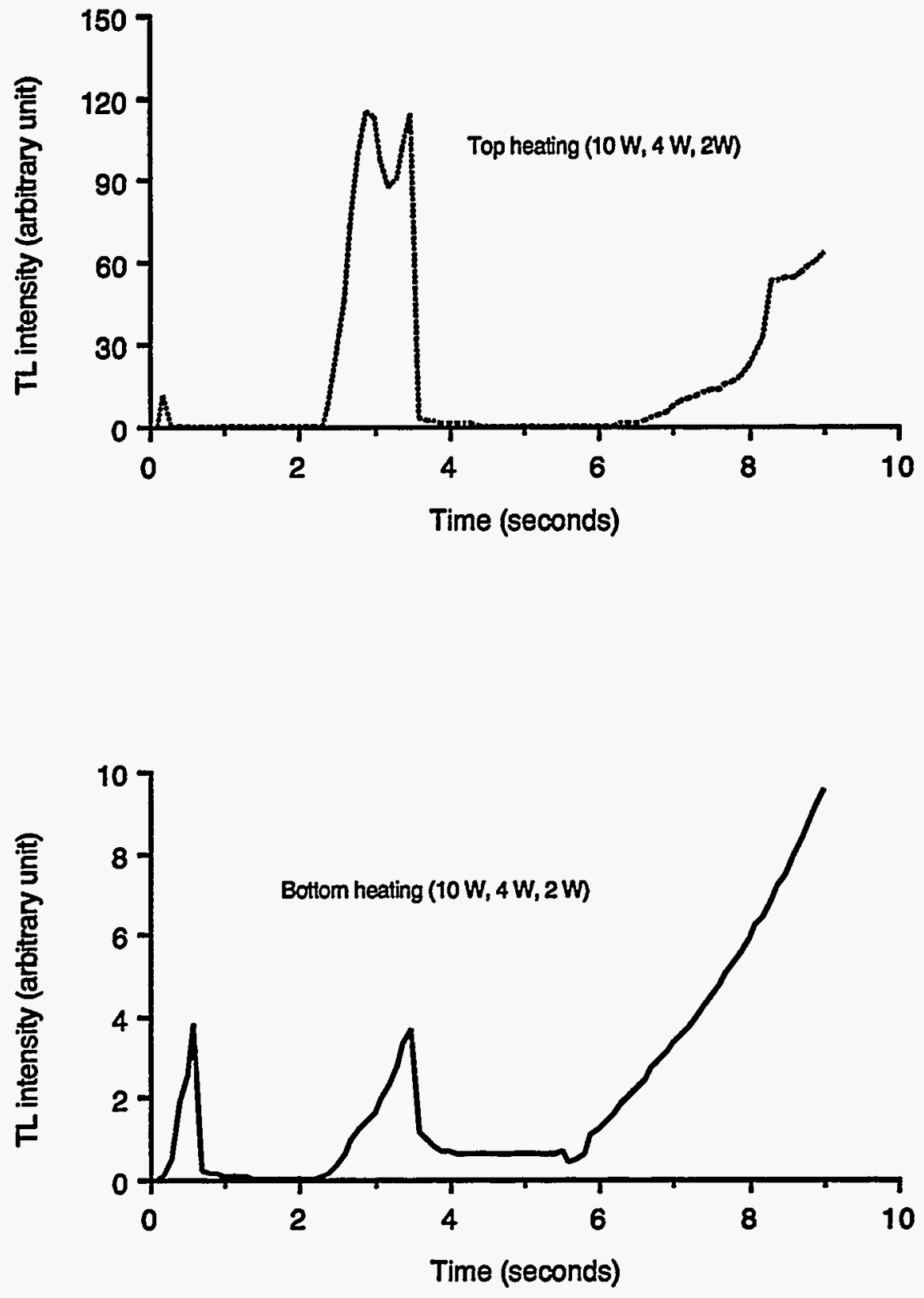

Figure 102. Calculated glow curves of a thick TL dosimeter exposed by Sr/Y-90 betaparticles using pulsed heating of uniform laser beam. The pulsed heating scheme includes (a) Top heating (above): a $0.2 \mathrm{sec} 10 \mathrm{~W}$ first pulse, followed by $1.7 \mathrm{sec}$ of cooling, which is then followed by a $1.8 \sec 4 \mathrm{~W}$ second pulse with $2.0 \mathrm{sec}$ of cooling, and then another $3.5 \sec 2 \mathrm{~W}$ third pulse; (a) Bottom heating (below): a $0.6 \mathrm{sec} 10 \mathrm{~W}$ first pulse, followed by $1.3 \mathrm{sec}$ of cooling, which is then followed by a $1.6 \sec 4 \mathrm{~W}$ second pulse with $2.0 \mathrm{sec}$ of cooling, and then another $3.5 \sec 2 \mathrm{~W}$ third pulse. 


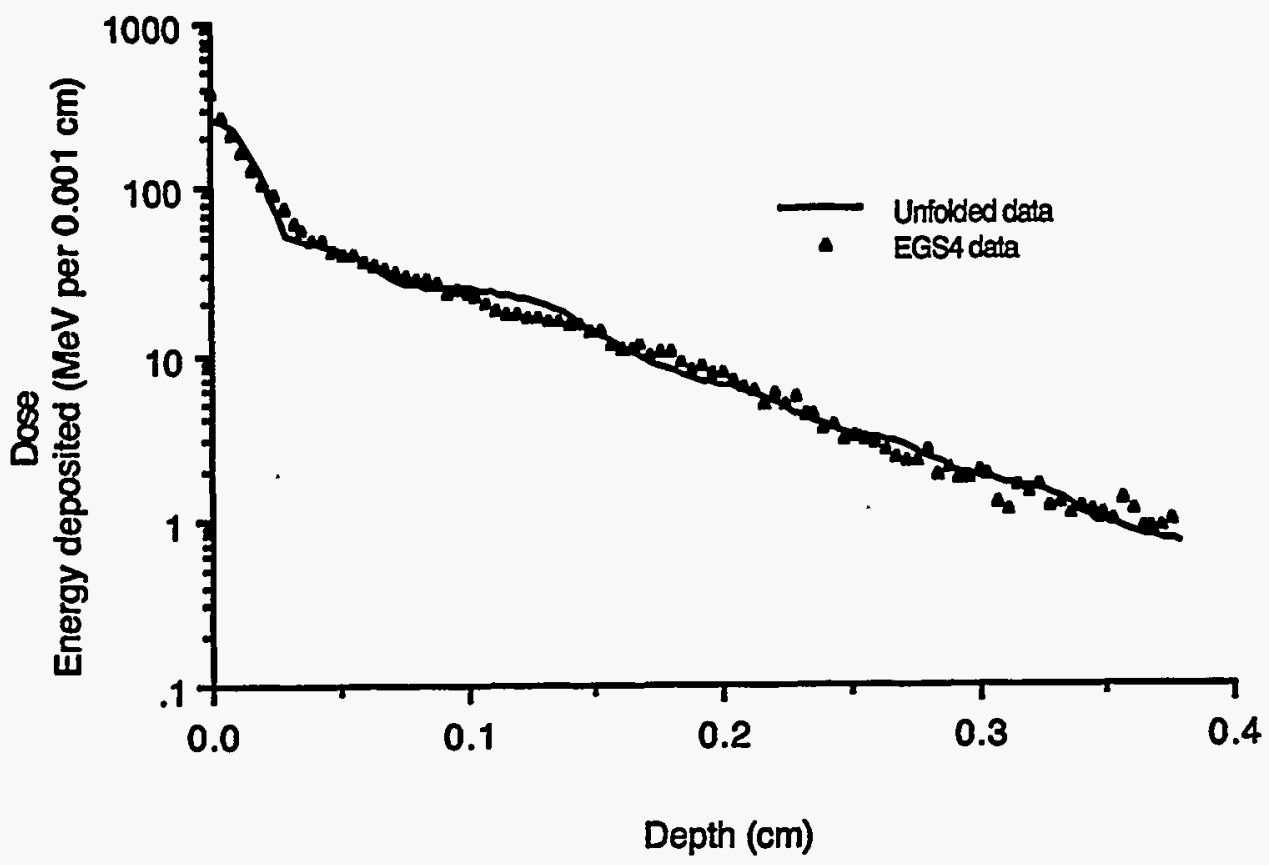

Figure 103. Unfolded depth-dose of a thick laser TL dosimeter exposed to Sr/Y-90 betaparticles using pulsed heating of uniform laser beam. The pulsed heating scheme includes (a) Top heating: a $0.2 \mathrm{sec} 10 \mathrm{~W}$ first pulse, followed by $1.7 \mathrm{sec}$ of cooling, which is then followed by a $1.8 \mathrm{sec} 4 \mathrm{~W}$ second pulse with $2.0 \mathrm{sec}$ of cooling, and then another $3.5 \mathrm{sec} 2$ W third pulse; (a) Bottom heating: a $0.6 \mathrm{sec} 10 \mathrm{~W}$ first pulse, followed by $1.3 \mathrm{sec}$ of cooling, which is then followed by a $1.6 \sec 4 \mathrm{~W}$ second pulse with $2.0 \mathrm{sec}$ of cooling, and then another $3.5 \sec 2 \mathrm{~W}$ third pulse. 


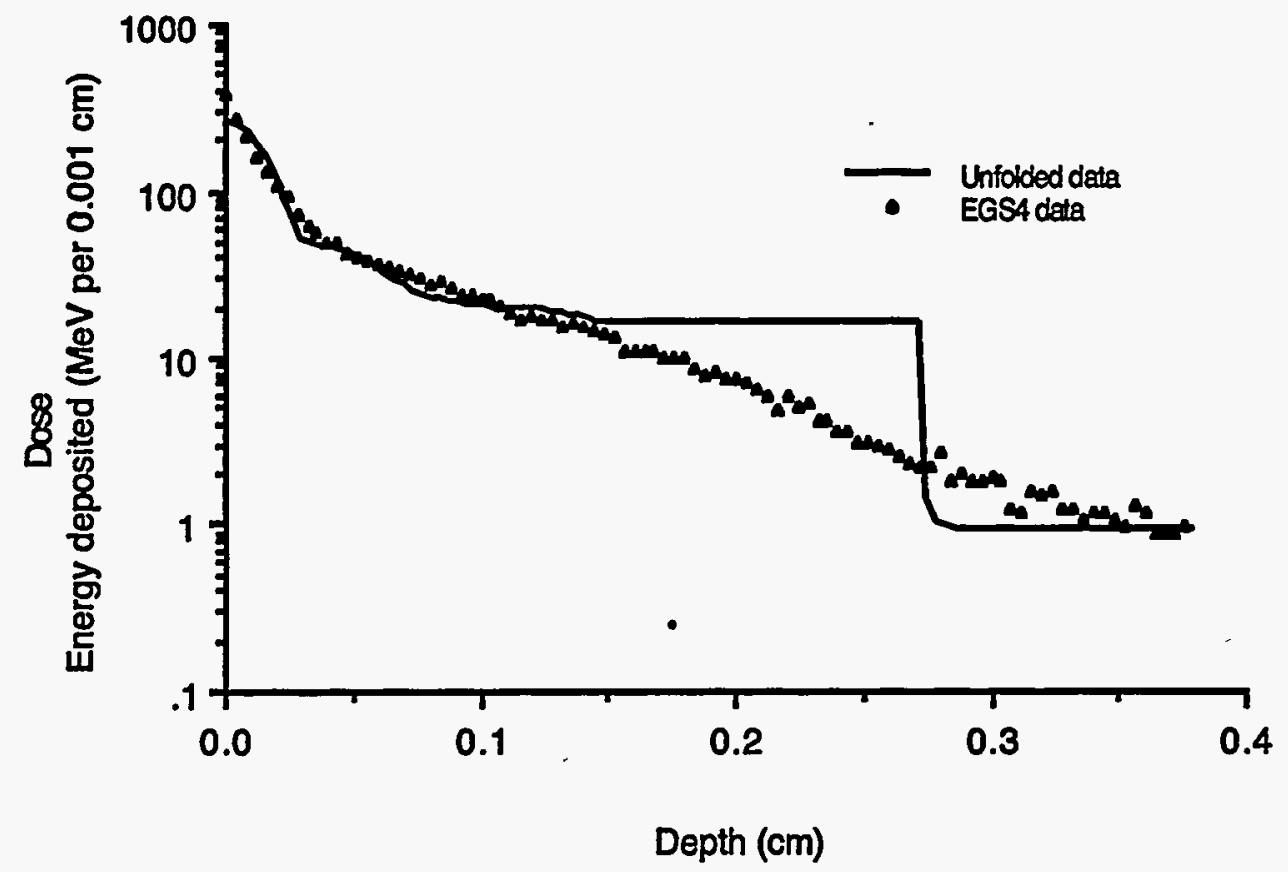

Figure 104. Unfolded depth-dose of a thick laser TL dosimeter exposed to Sr/Y-90 betaparticles using pulsed heating of uniform laser beam ( 3 pulsed heating on the top and 1 pulsed heating on the bottom); (a) Top; first heating ( $10 \mathrm{~W}, 0.2 \mathrm{sec})$ and cooling $(1.7 \mathrm{sec})$; second heating ( $4 \mathrm{~W}, 1.8 \mathrm{sec})$ and cooling $(2.0 \mathrm{sec})$; third heating ( $2 \mathrm{~W}, 3.5 \mathrm{sec}) ;(\mathrm{b})$. Bottom; first heating $(10 \mathrm{~W}, 0.6 \mathrm{sec})$. 


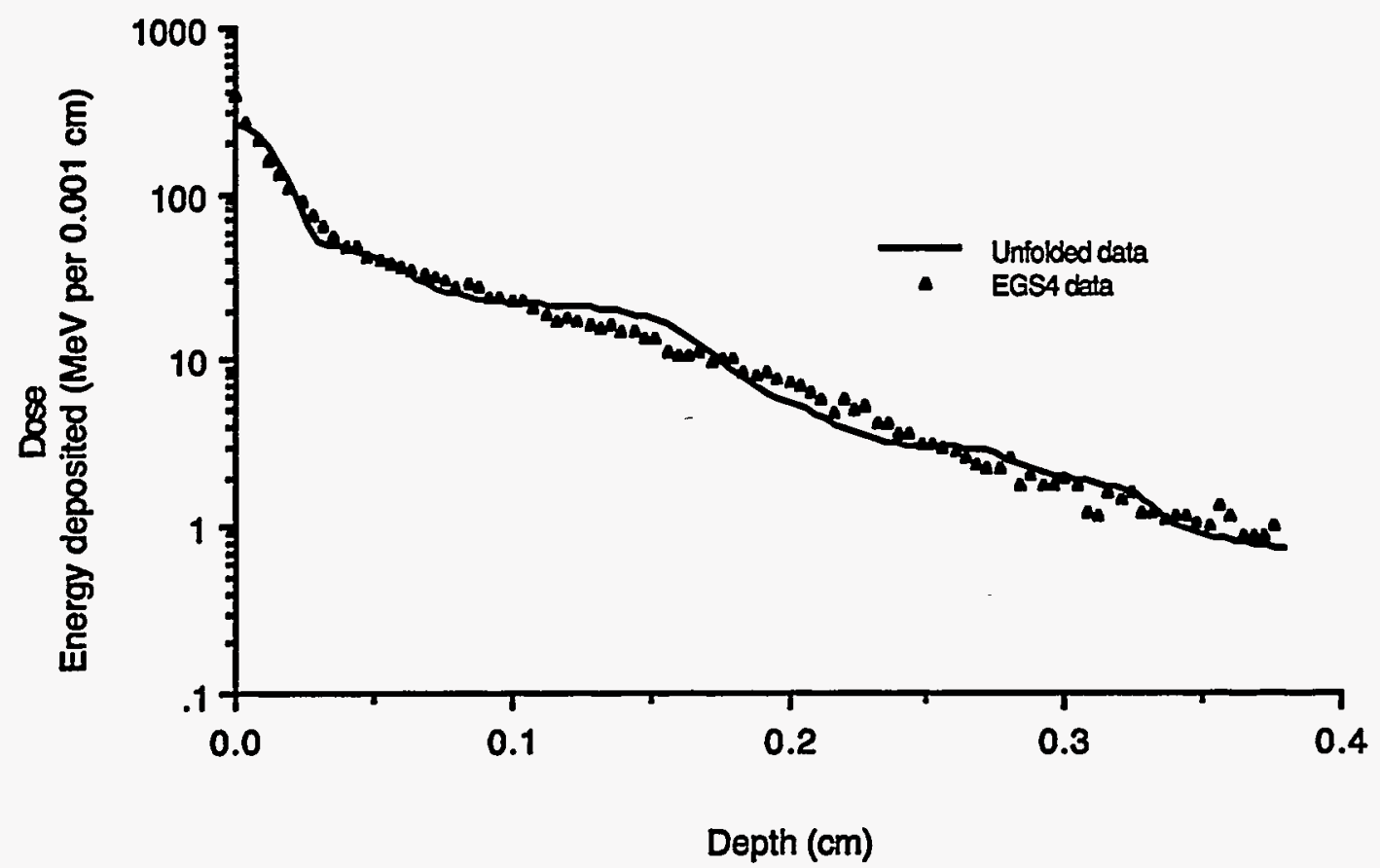

Figure 105. Unfolded depth-dose of a thick laser TL dosimeter exposed to $\mathrm{Sr} / \mathrm{Y}-90$ betaparticles using pulsed heating of uniform laser beam ( 3 pulsed heating on the top and 2 pulsed heating on the bottom); (a) Top; first heating ( $10 \mathrm{~W}, 0.2 \mathrm{sec})$ and cooling (1.7 sec); second heating ( $4 \mathrm{~W}, 1.8 \mathrm{sec})$ and cooling $(2.0 \mathrm{sec})$; third heating $(2 \mathrm{~W}, 3.5 \mathrm{sec}) ;(\mathrm{b})$ Bottom; first heating $(10 \mathrm{~W}, 0.6 \mathrm{sec})$ and cooling $(1.3 \mathrm{sec})$; second heating $(4 \mathrm{~W}, 1.6 \mathrm{sec})$. 


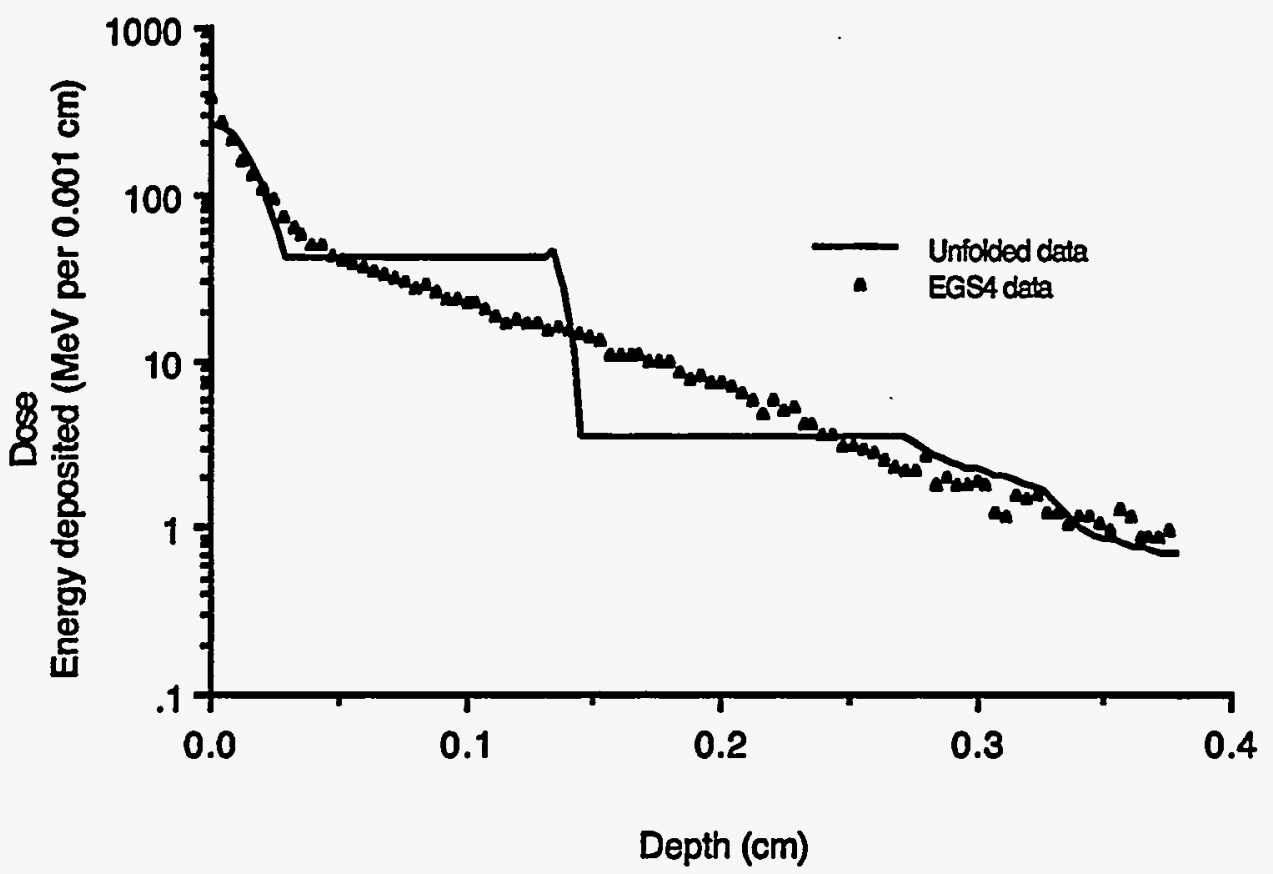

Figure 106. Unfolded depth-dose of a thick laser TL dosimeter exposed to $\mathrm{Sr} / \mathrm{Y}-90$ betaparticles using pulsed heating of uniform laser beam ( 2 pulsed heating on the top and 2 pulsed heating on the bottom); (a) Top; first heating (10 W, $0.2 \mathrm{sec})$ and cooling (1.7 sec); second heating ( $4 \mathrm{~W}, 1.8 \mathrm{sec})$; (b) Bottom; first heating (10 W, $0.6 \mathrm{sec})$ and cooling (1.3 $\mathrm{sec})$; second heating $(4 \mathrm{~W}, 1.6 \mathrm{sec})$. 


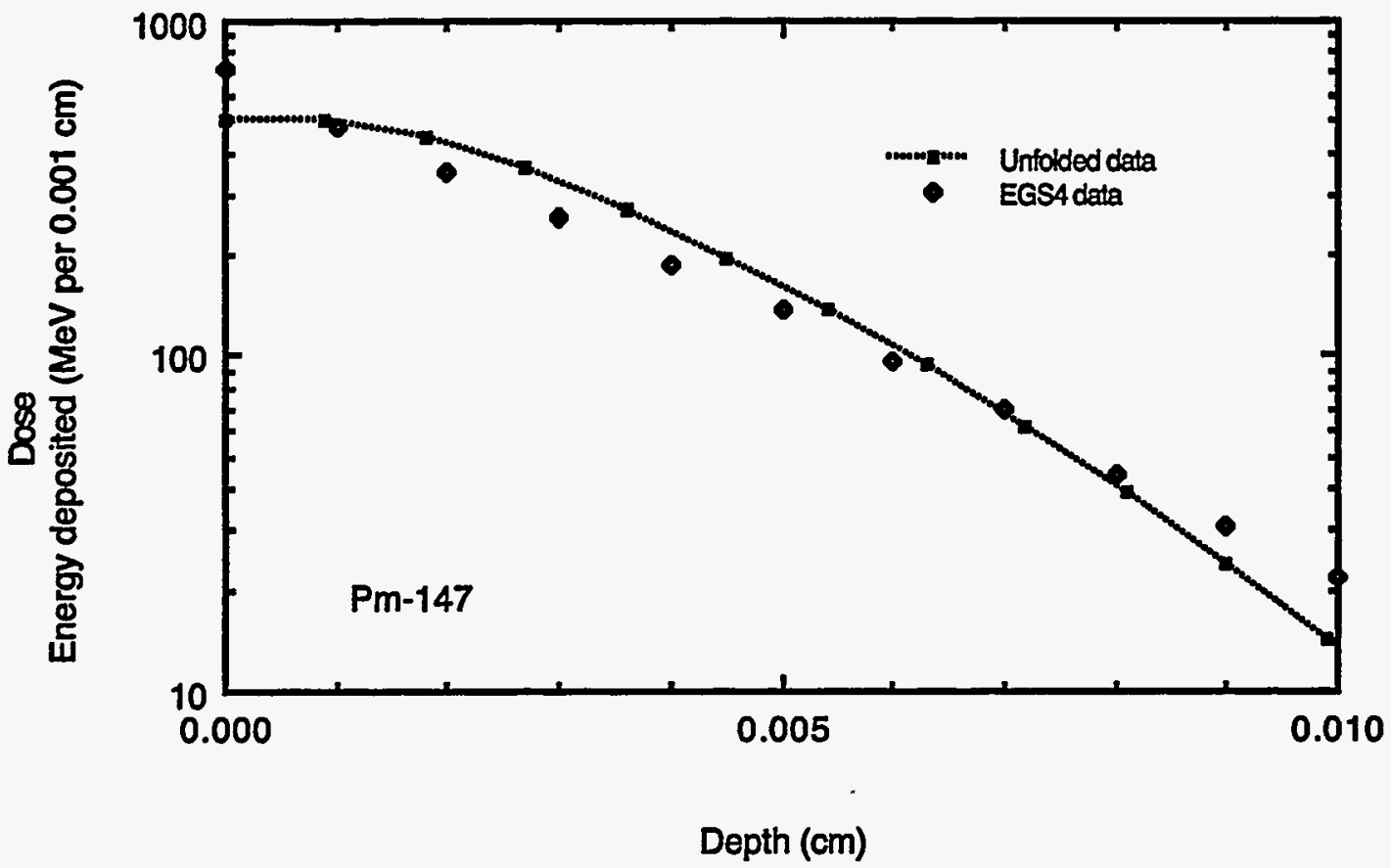

Figure 107. Unfolded shallow depth-dose of a thick laser TL dosimeter exposed to Pm-147 beta-particles following pulsed heating of $0.032 \mathrm{~cm}$ diameter $4 \mathrm{~W}$ laser beam. 


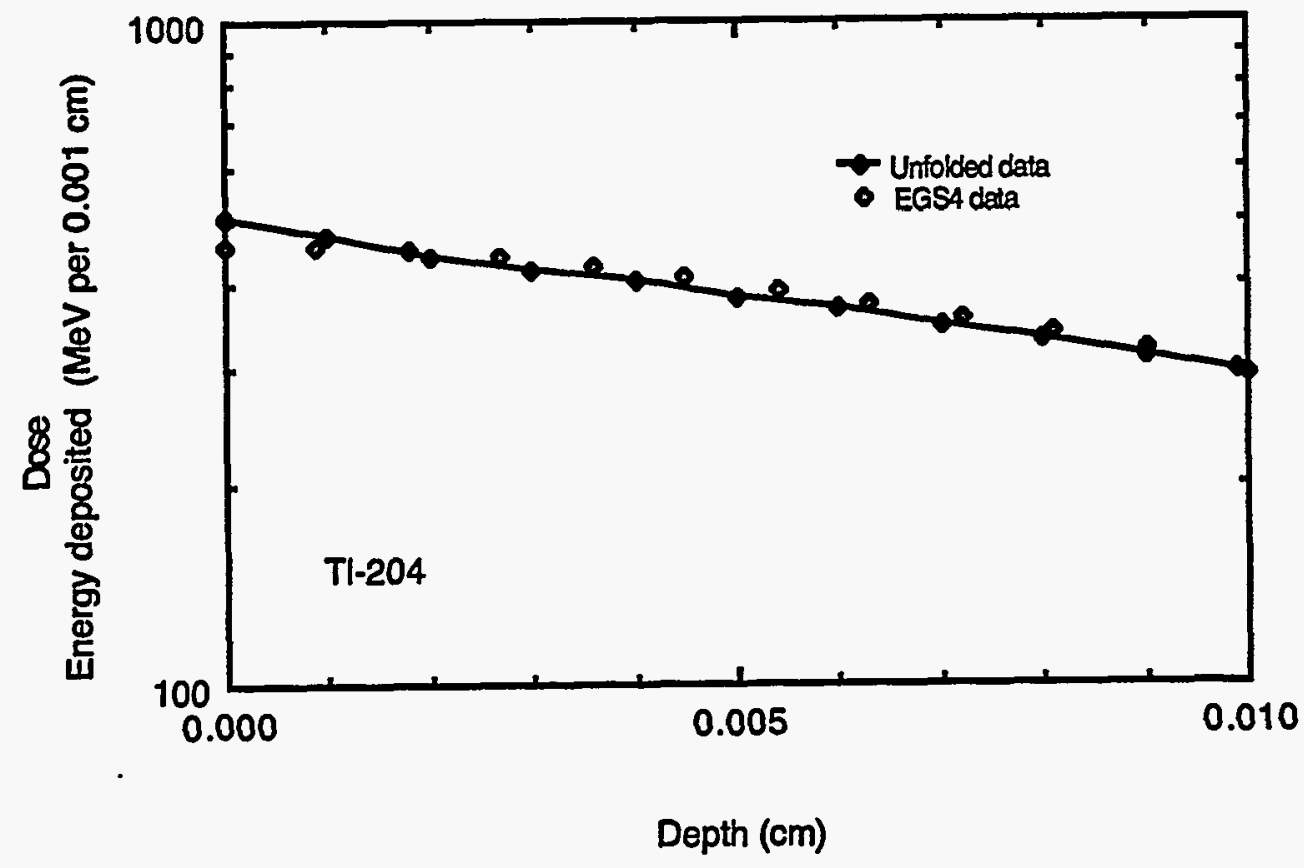

Figure 108. Unfolded shallow depth-dose of a thick laser TL dosimeter exposed to T1-204 beta-particles following pulsed heating of $0.032 \mathrm{~cm}$ diameter $4 \mathrm{~W}$ laser beam. 


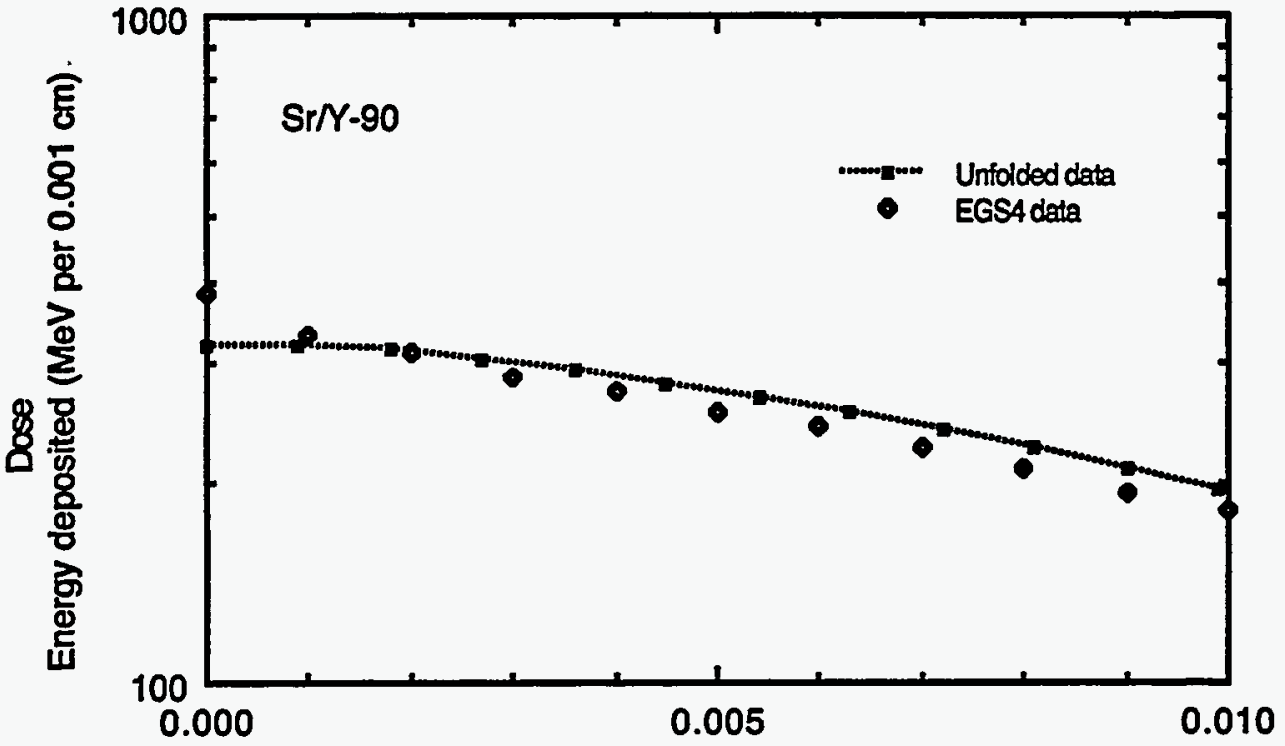

Depth (cm)

Figure 109. Unfolded shallow depth-dose of a thick laser TL dosimeter exposed to Sr/Y-90 beta-particles following pulsed heating of $0.032 \mathrm{~cm}$ diameter $4 \mathrm{~W}$ laser beam. 


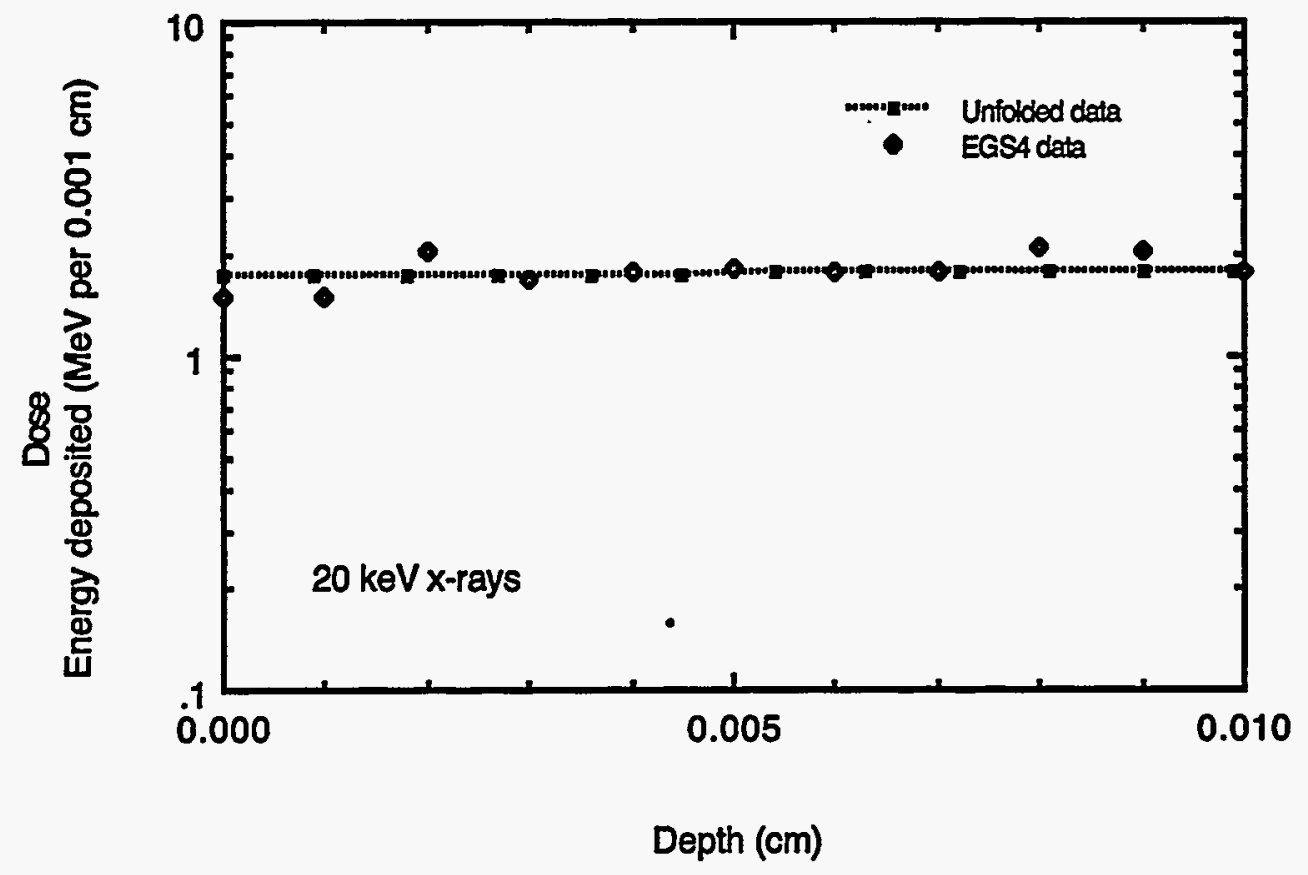

Figure 110. Unfolded shallow depth-dose of a thick laser TL dosimeter exposed to $20 \mathrm{keV}$ $\mathrm{x}$-rays following pulsed heating of $0.032 \mathrm{~cm}$ diameter $4 \mathrm{~W}$ laser beam. 


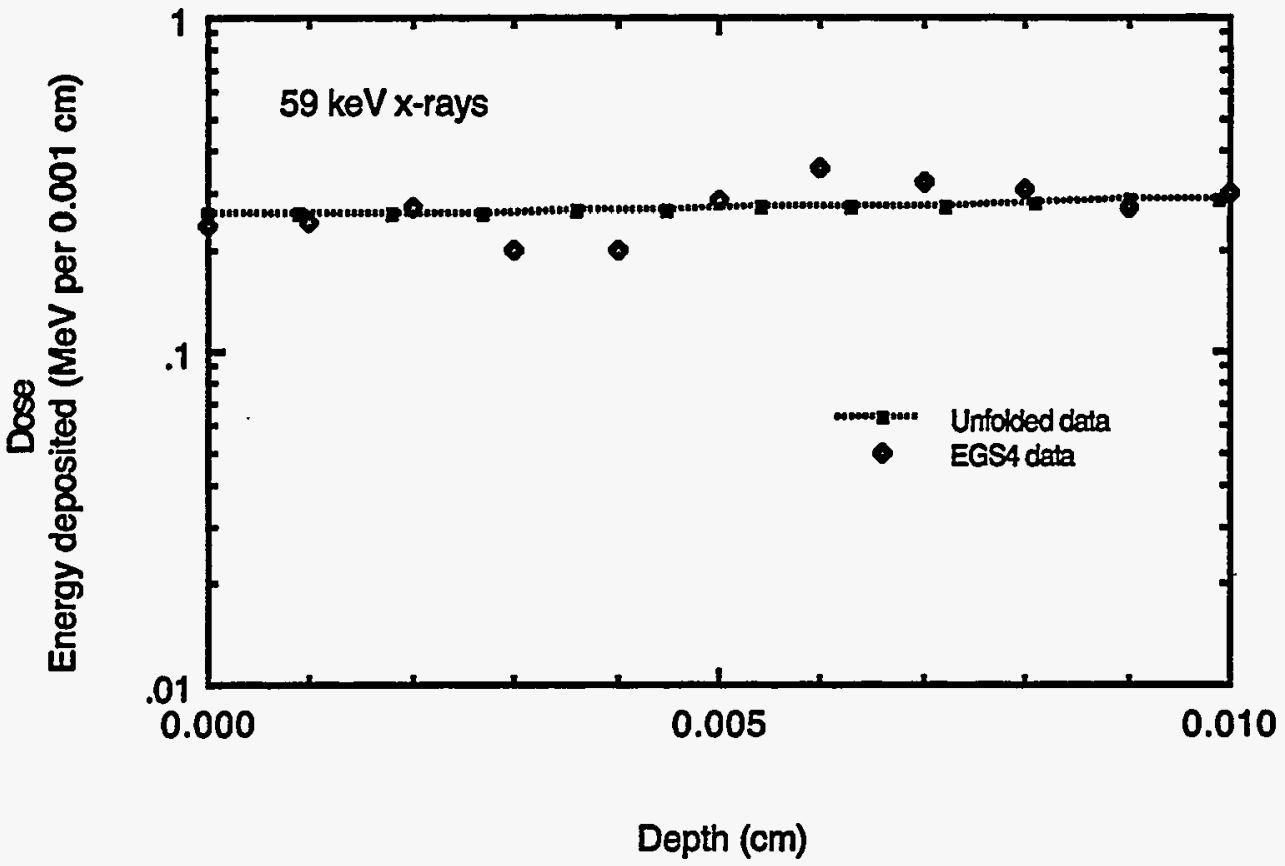

Figure 111. Unfolded shallow depth-dose of a thick laser TL dosimeter exposed to $59 \mathrm{keV}$ $\mathrm{x}$-rays following pulsed heating of $0.032 \mathrm{~cm}$ diameter $4 \mathrm{~W}$ laser beam. 


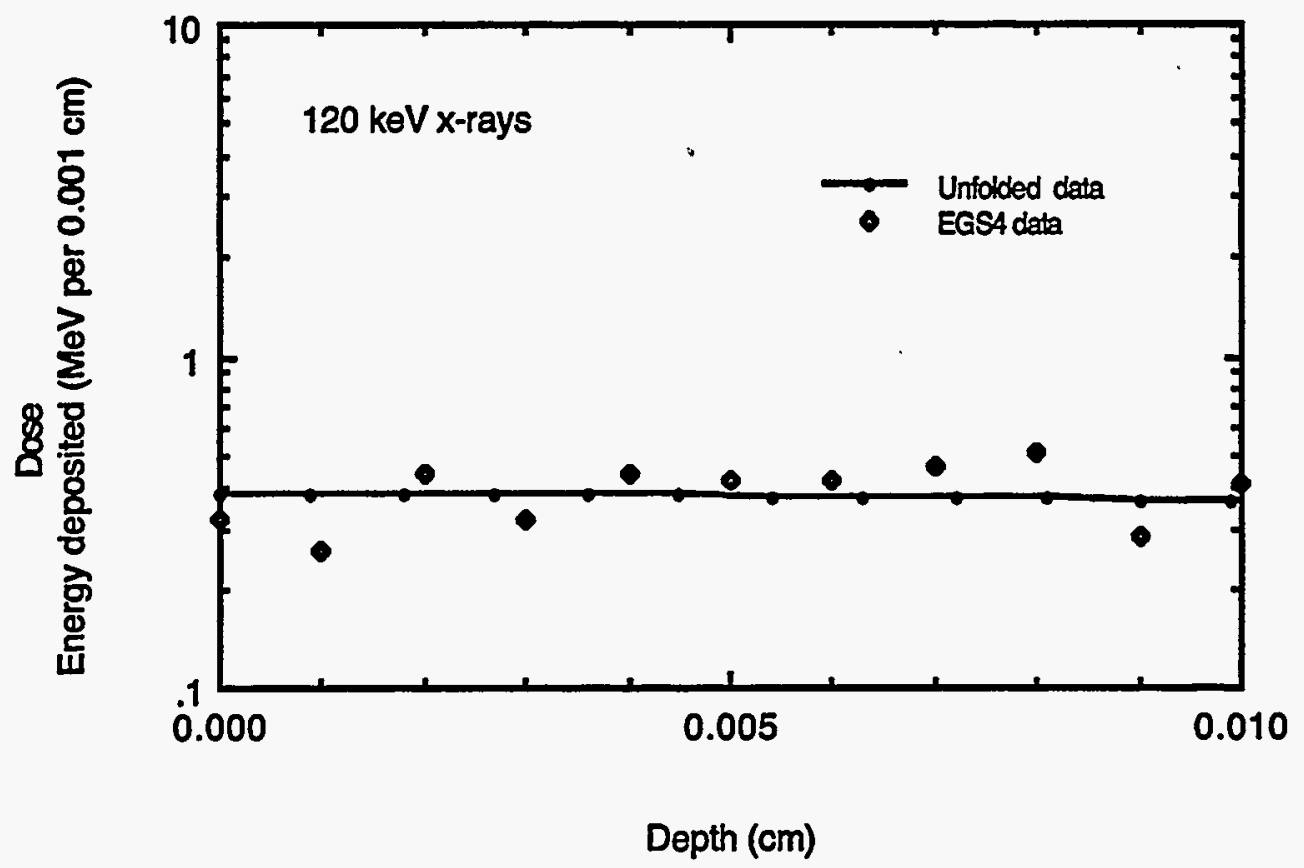

Figure 112. Unfolded shallow depth-dose of a thick laser TL dosimeter exposed to 120 $\mathrm{keV} \mathrm{x}$-rays following pulsed heating of $0.032 \mathrm{~cm}$ diameter $4 \mathrm{~W}$ laser beam. 


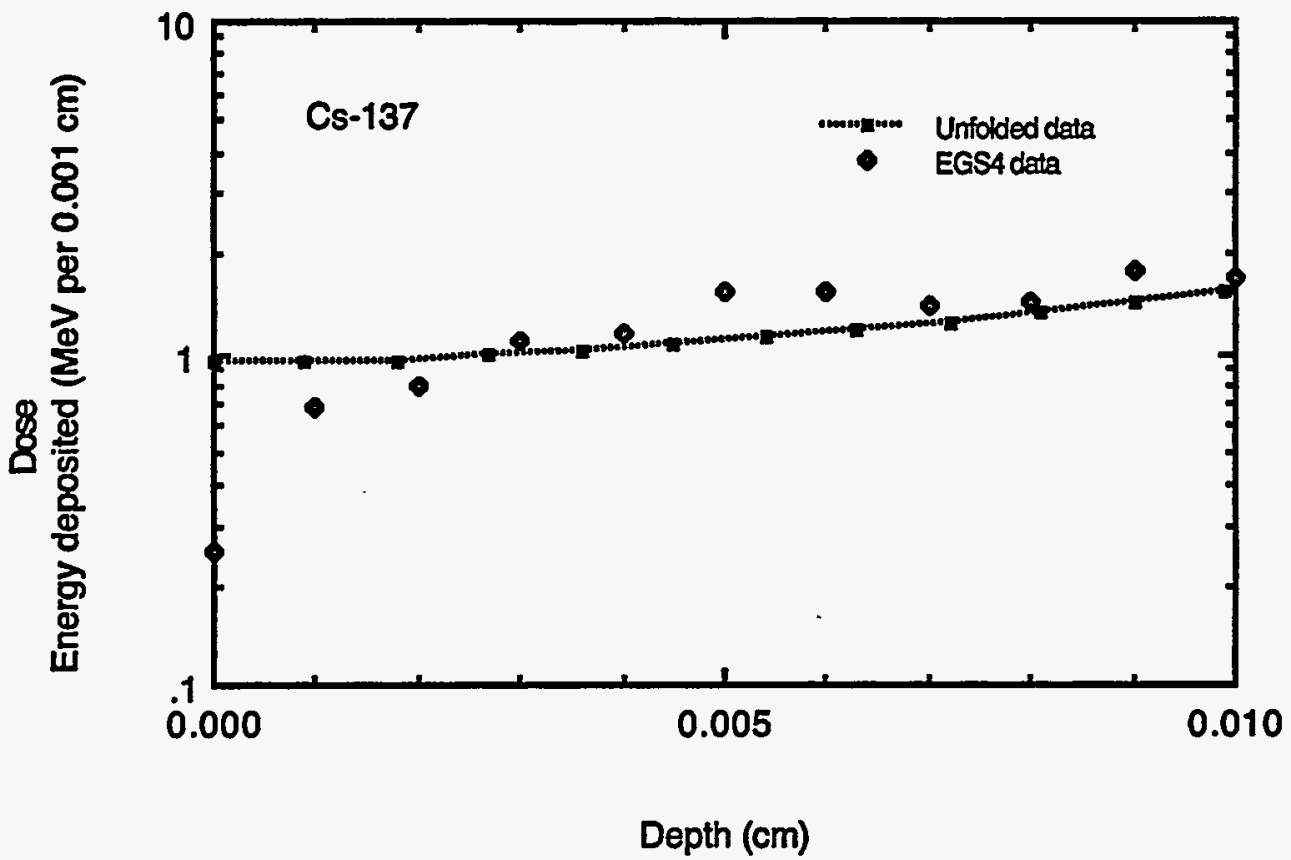

Figure 113. Unfolded shallow depth-dose of a thick laser TL dosimeter exposed to Cs-137 gamma-rays following pulsed heating of $0.032 \mathrm{~cm}$ diameter $4 \mathrm{~W}$ laser beam. 


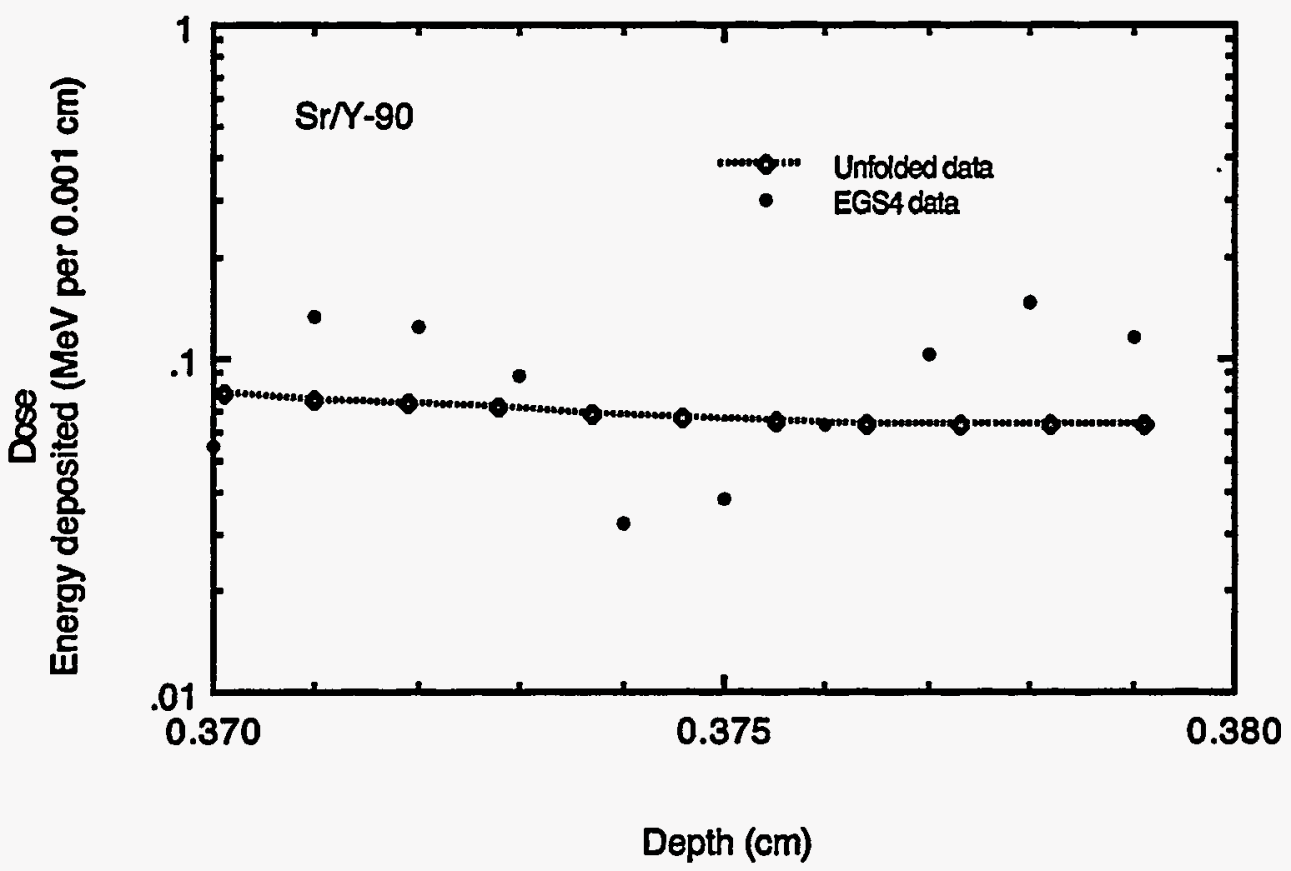

Figure 114. Unfolded deep depth-dose of a thick laser TL dosimeter exposed to Sr/Y-90 beta-particles following pulsed heating of $0.032 \mathrm{~cm}$ diameter $4 \mathrm{~W}$ laser beam. 


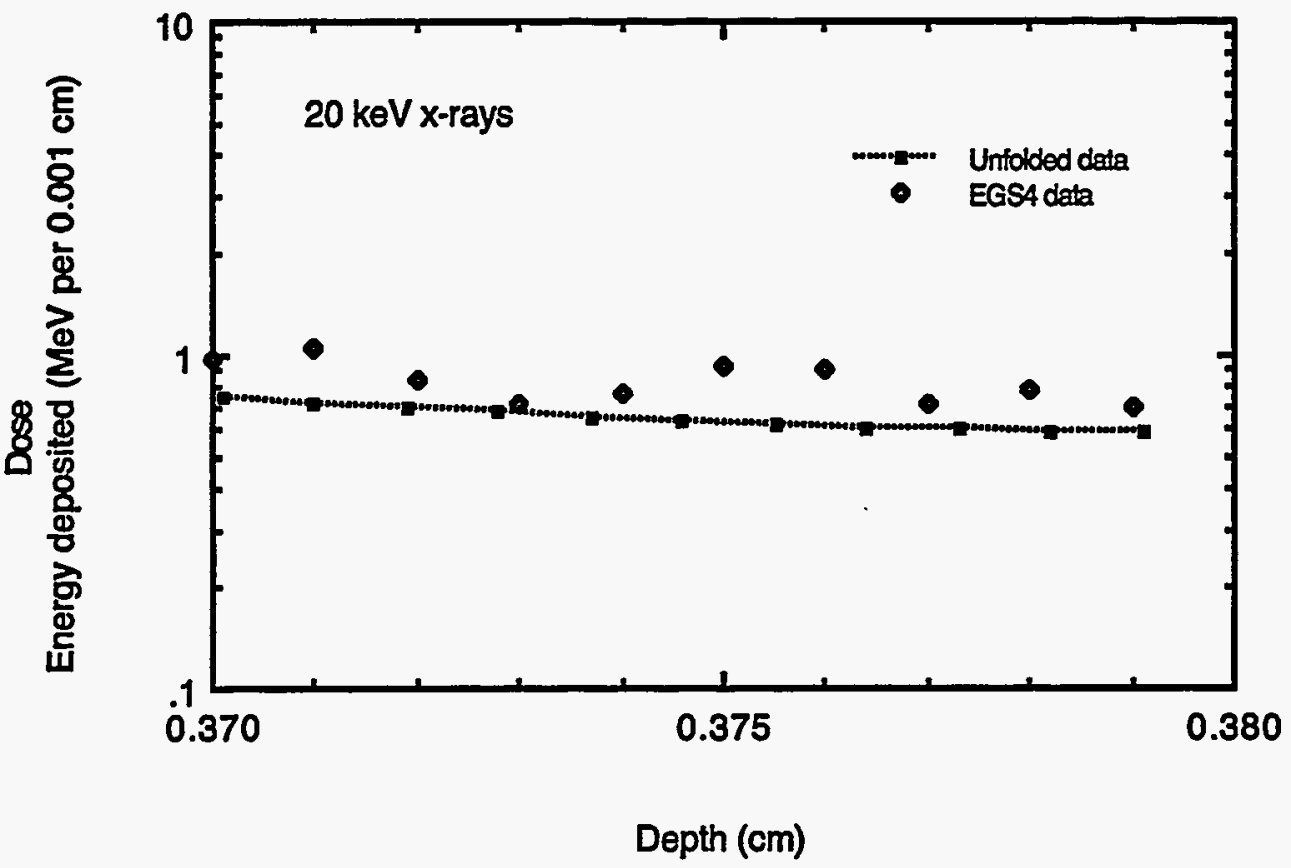

Figure 115. Unfolded deep depth-dose of a thick laser TL dosimeter exposed to $20 \mathrm{keV} \mathrm{x}$ rays following pulsed heating of $0.032 \mathrm{~cm}$ diameter $4 \mathrm{~W}$ laser beam. 


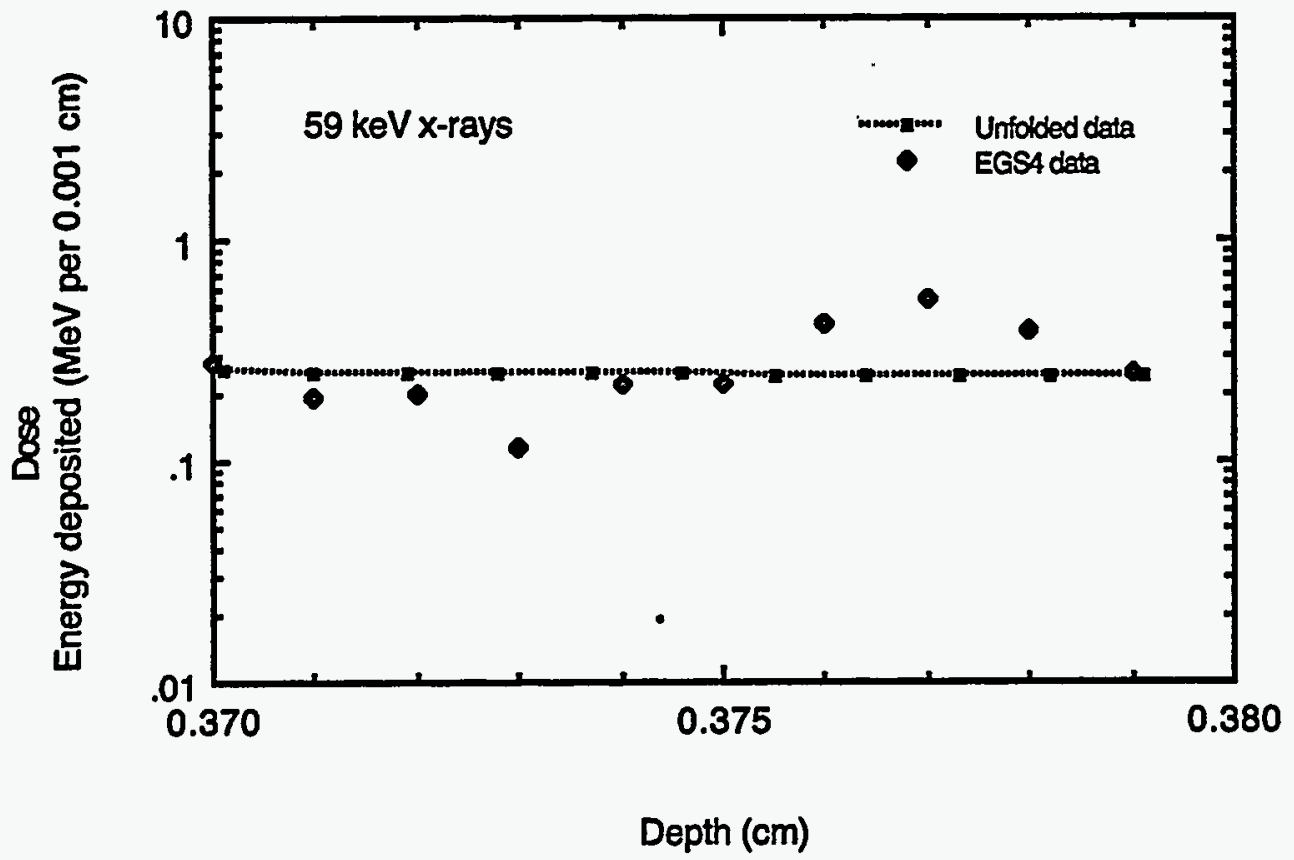

Figure 116. Unfolded deep depth-dose of a thick laser TL dosimeter exposed to $59 \mathrm{keV} \mathrm{x}$ rays following pulsed heating of $0.032 \mathrm{~cm}$ diameter $4 \mathrm{~W}$ laser beam. 


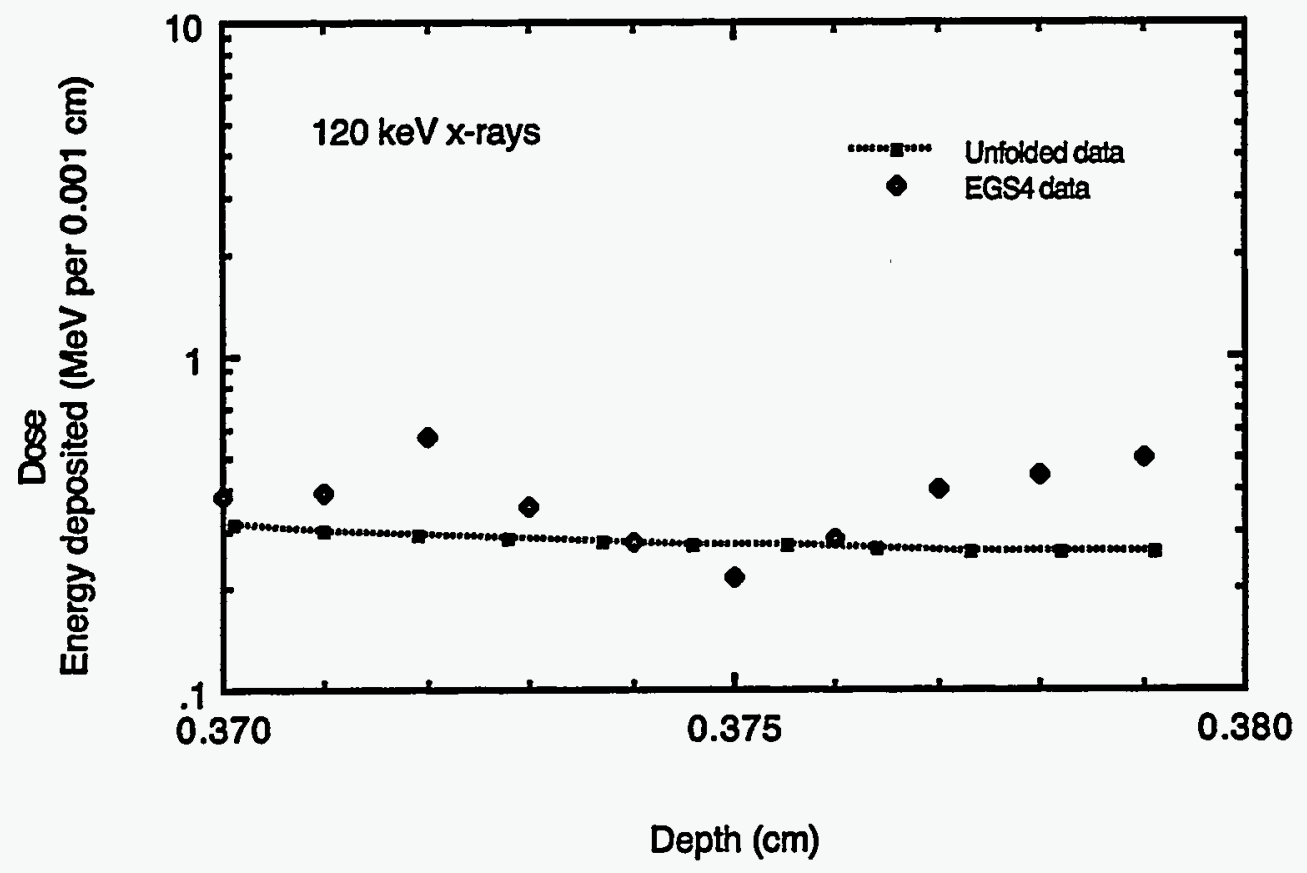

Figure 117. Unfolded deep depth-dose of a thick laser TL dosimeter exposed to $120 \mathrm{keV} \mathrm{x}$ rays following pulsed heating of $0.032 \mathrm{~cm}$ diameter $4 \mathrm{~W}$ laser beam. 


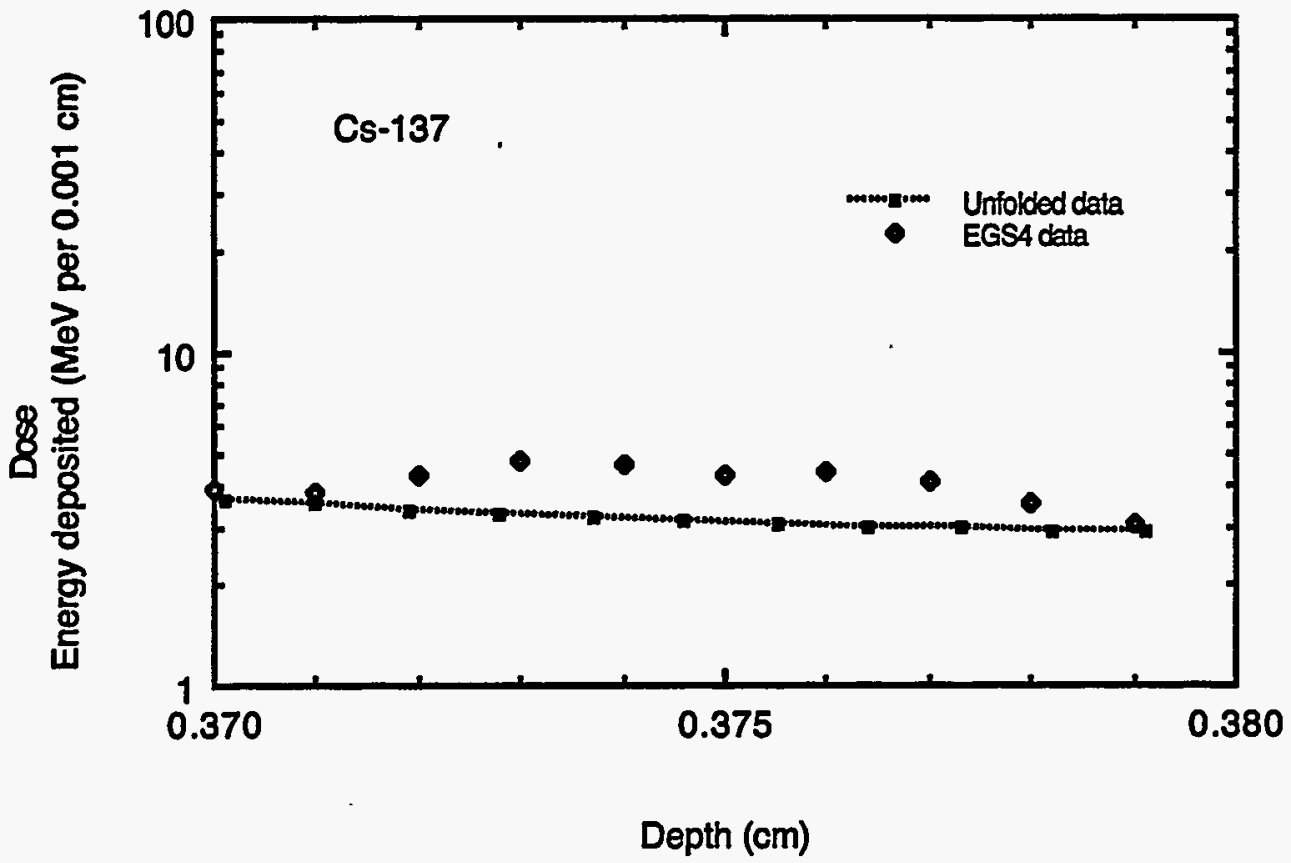

Figure 118. Unfolded deep depth-dose of a thick laser TL dosimeter exposed to Cs-137 gamma-rays following pulsed heating of $0.032 \mathrm{~cm}$ diameter $4 \mathrm{~W}$ laser beam. 


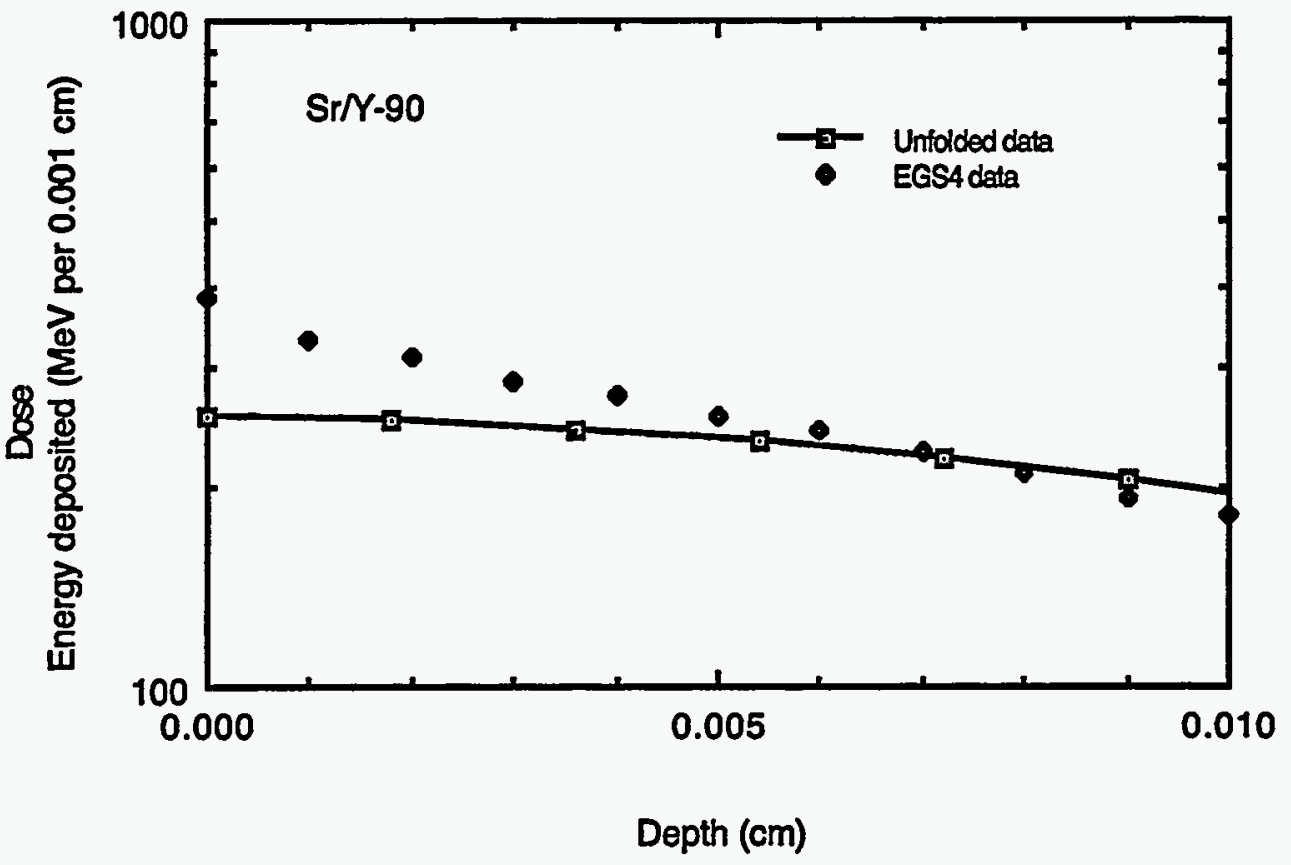

Figure 119. Unfolded shallow depth-dose of a thick laser TL dosimeter exposed to Sr/Y-90 beta-particles following pulsed heating of uniform laser beam. 


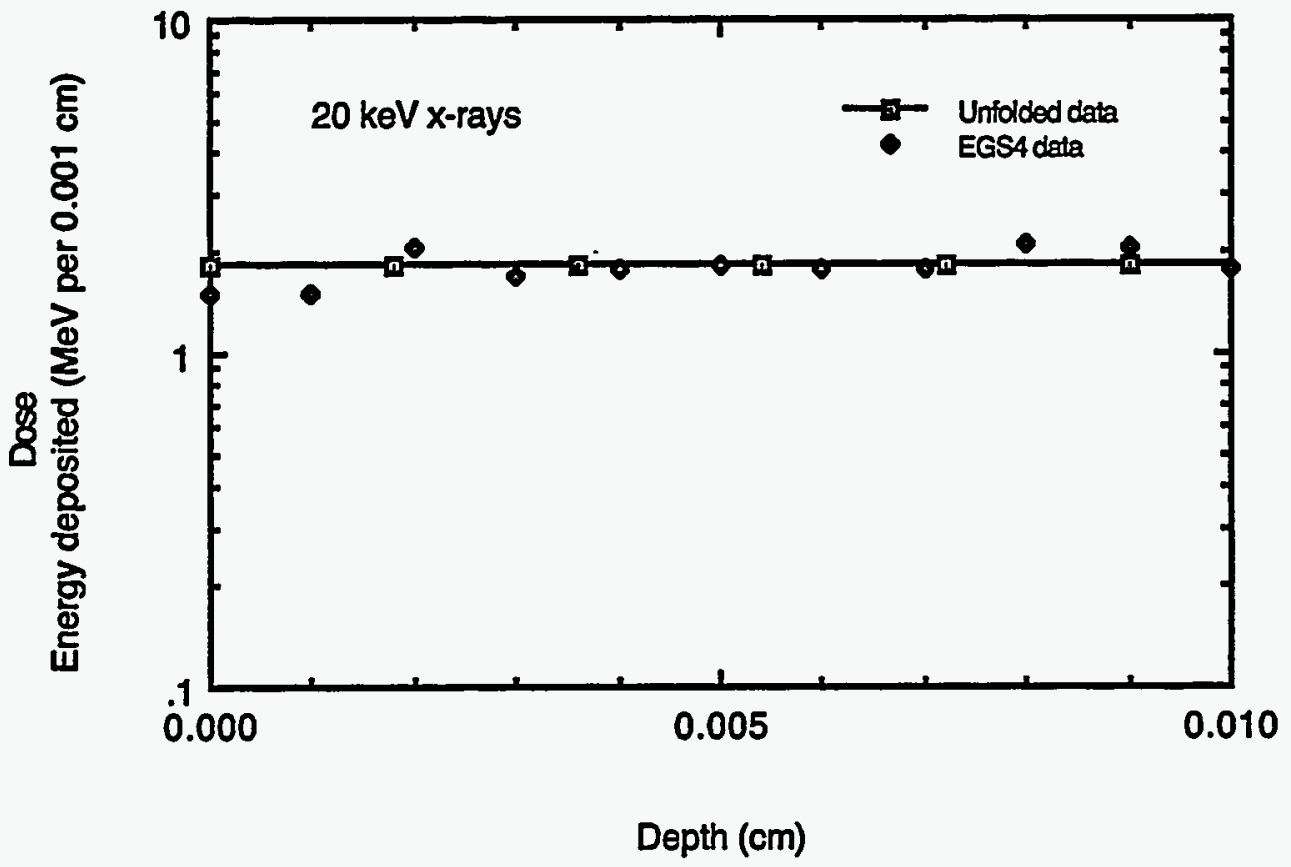

Figure 120. Unfolded shallow depth-dose of a thick laser TL dosimeter exposed to $20 \mathrm{keV}$ $\mathrm{X}$-rays following pulsed heating of uniform laser beam. 


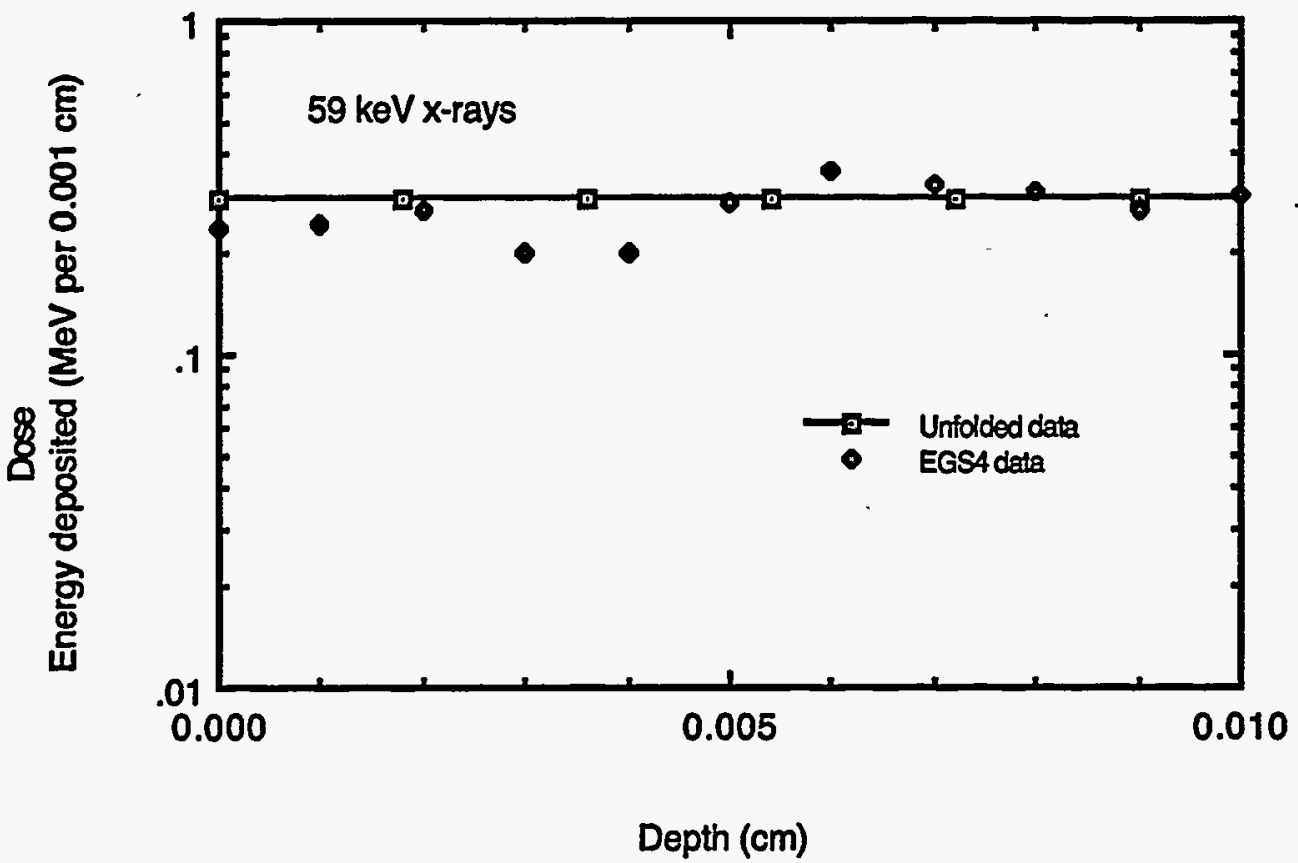

Figure 121. Unfolded shallow depth-dose of a thick laser TL dosimeter exposed to $59 \mathrm{keV}$ $\mathrm{X}$-rays following pulsed heating of uniform laser beam. 


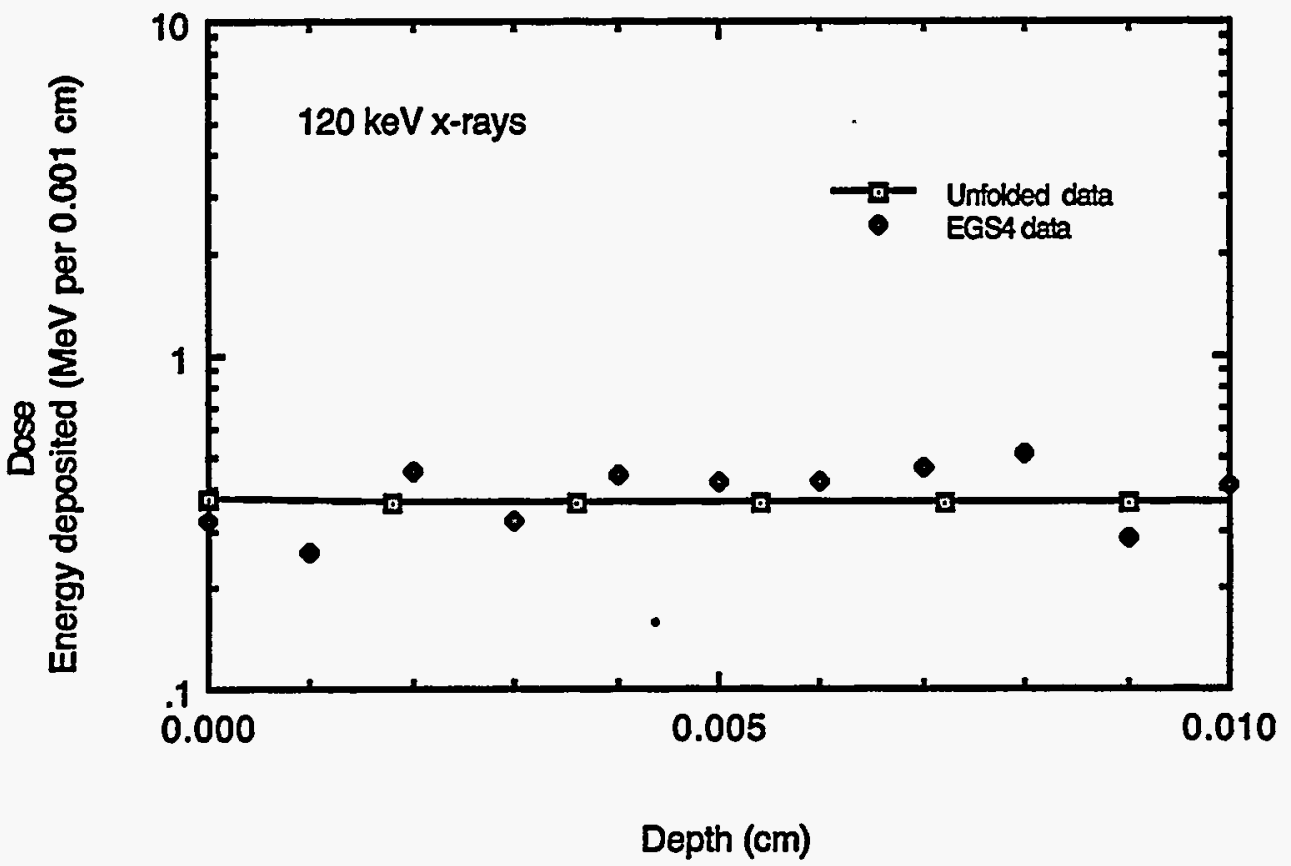

Figure 122. Unfolded shallow depth-dose of a thick laser TL dosimeter exposed to 120 $\mathrm{keV} \mathrm{x}$-rays following pulsed heating of uniform laser beam. 


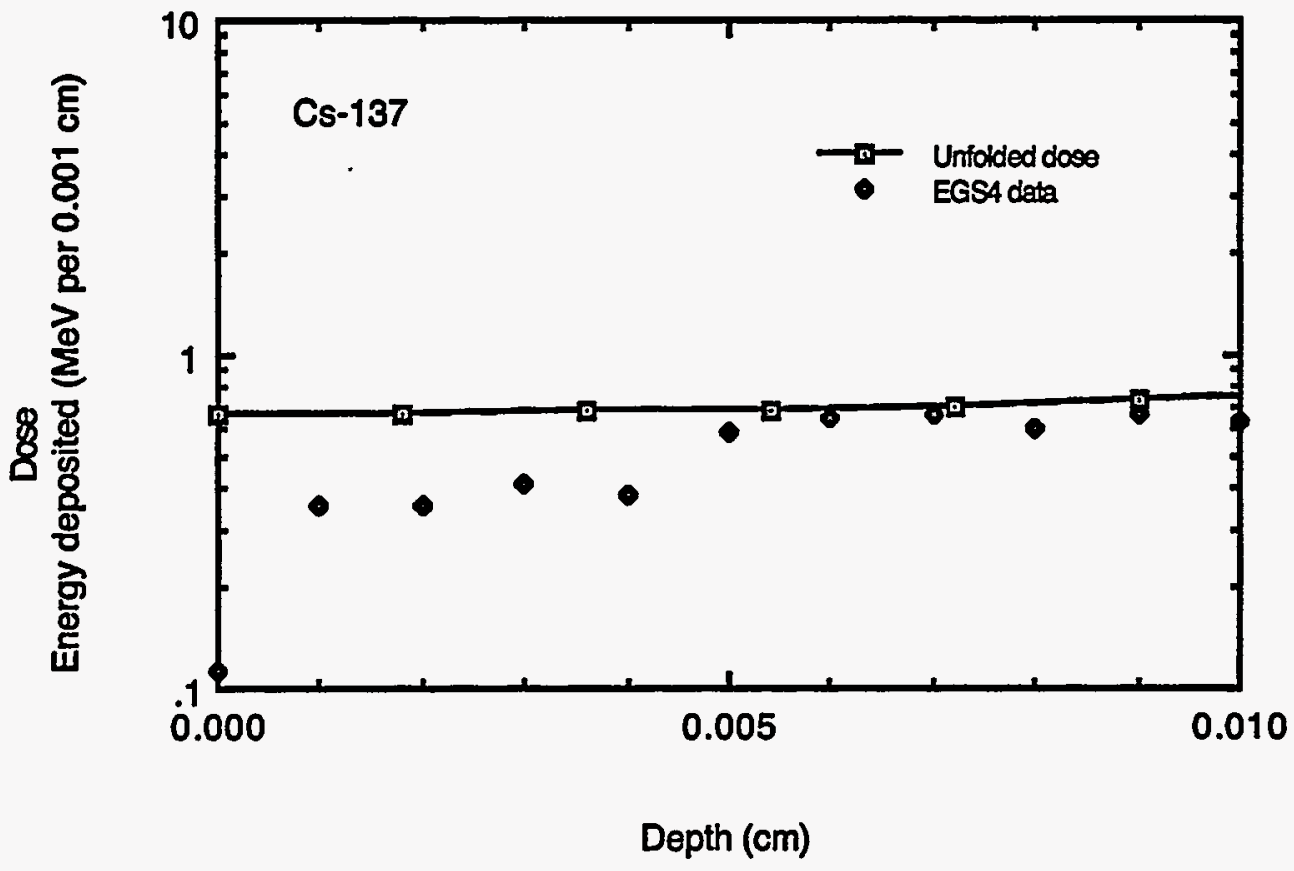

Figure 123. Unfolded shallow depth-dose of thick laser TL dosimeter exposed to Cs-137 gamma-rays following pulsed heating of uniform laser beam . 


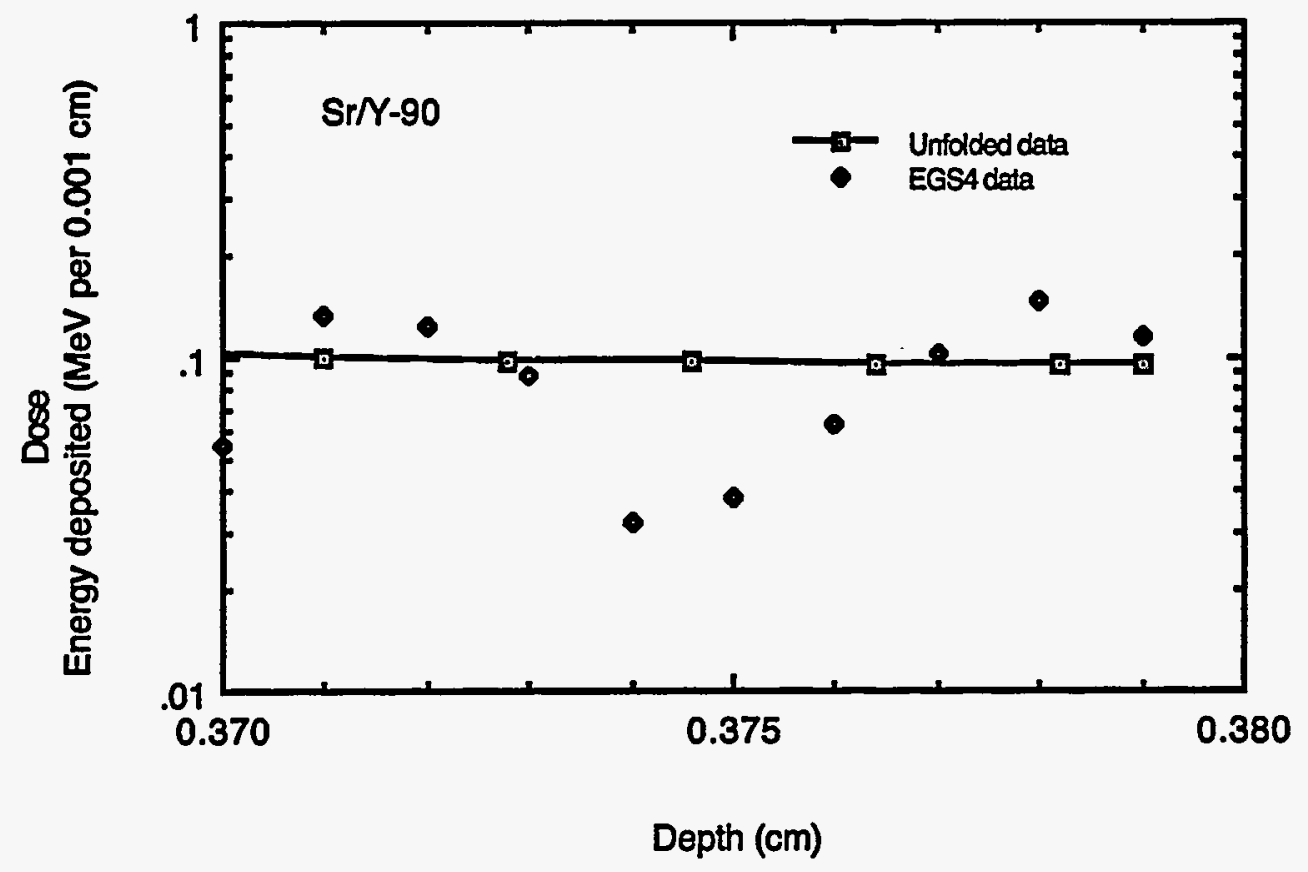

Figure 124. Unfolded deep depth-dose of a thick laser TL dosimeter exposed to Sr/Y-90 beta-particles following pulsed heating of uniform laser beam . 


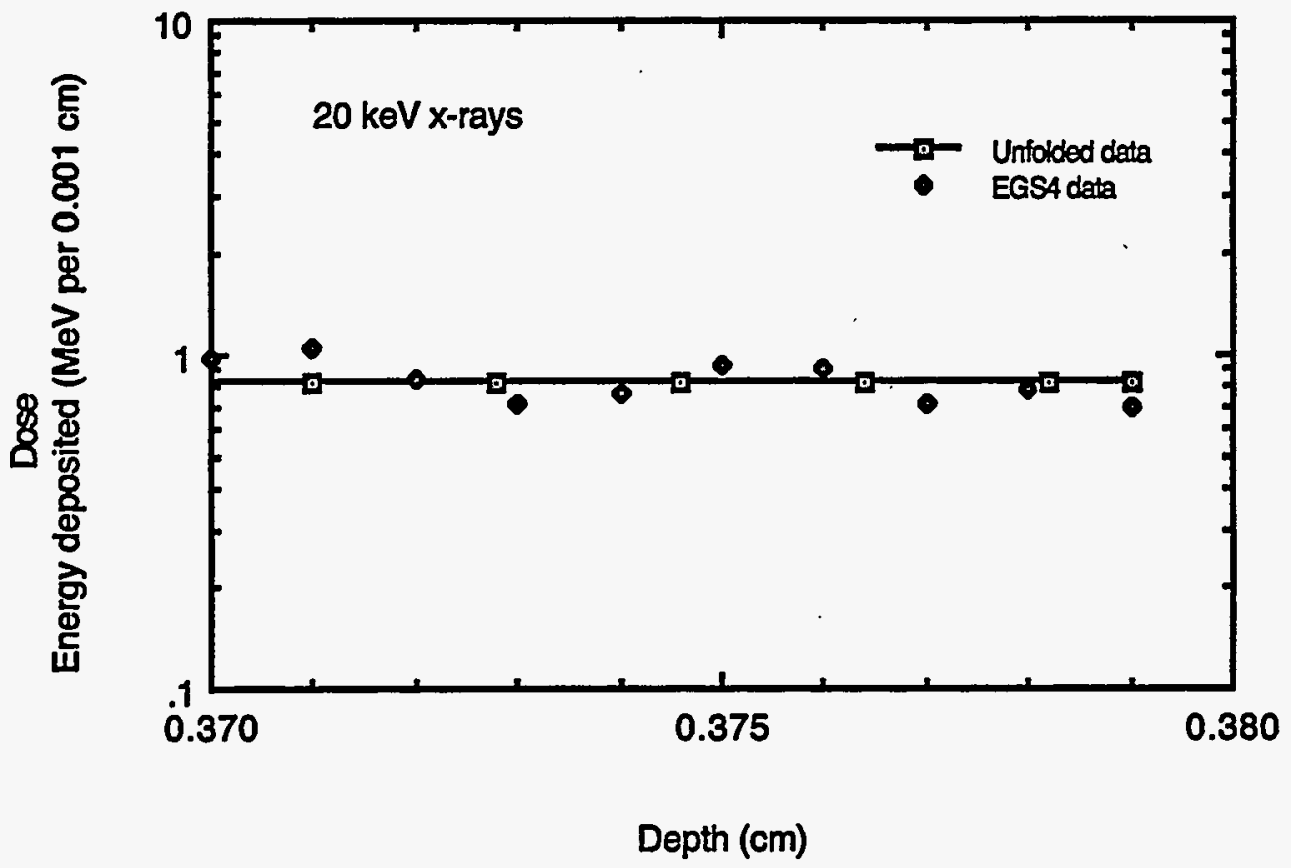

Figure 125. Unfolded deep depth-dose of a thick laser TL dosimeter exposed to $20 \mathrm{keV} \mathrm{X}$ rays following pulsed heating of uniform laser beam. 


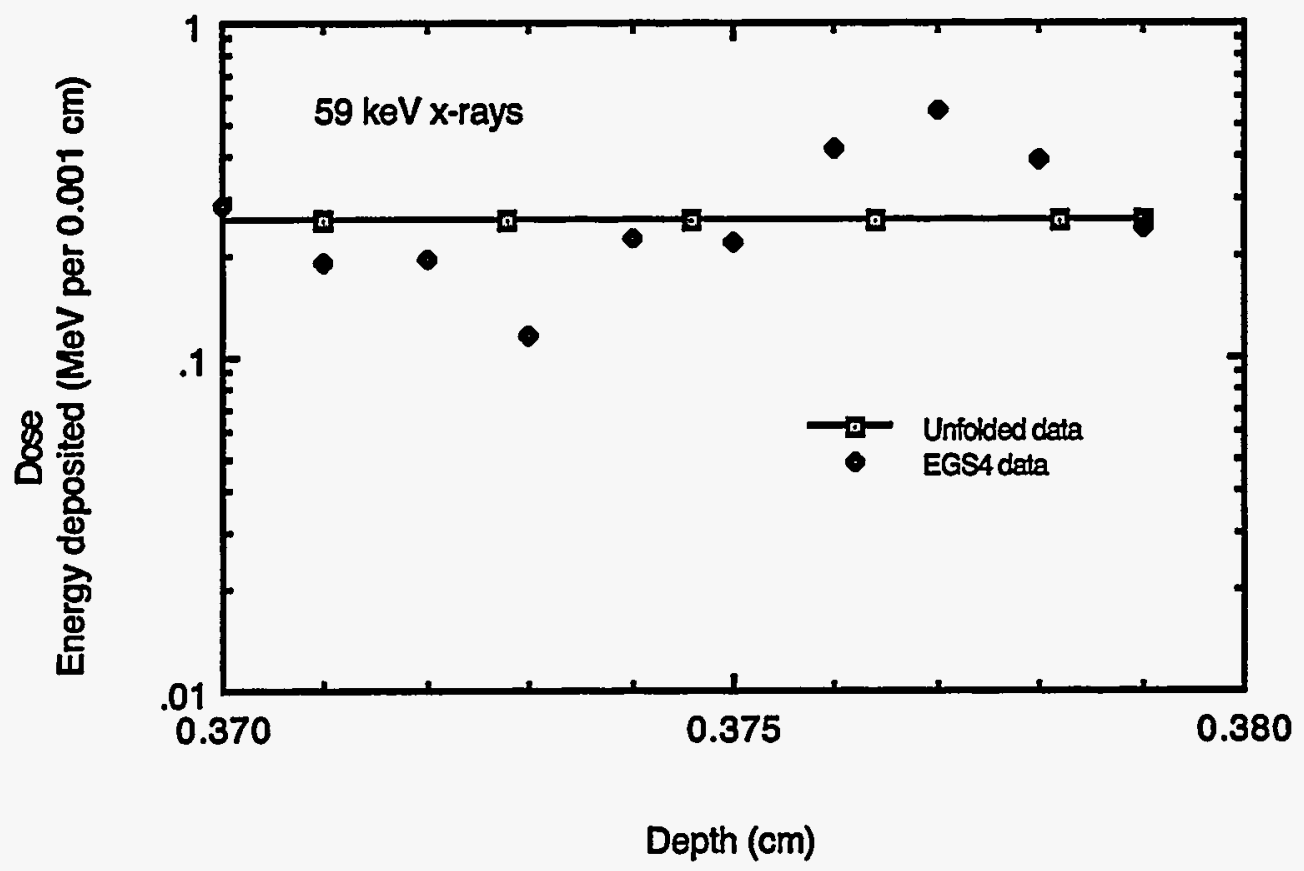

Figure 126. Unfolded deep depth-dose of a thick laser TL dosimeter exposed to $59 \mathrm{keV} \mathrm{X}$ rays following pulsed heating of uniform laser beam. 


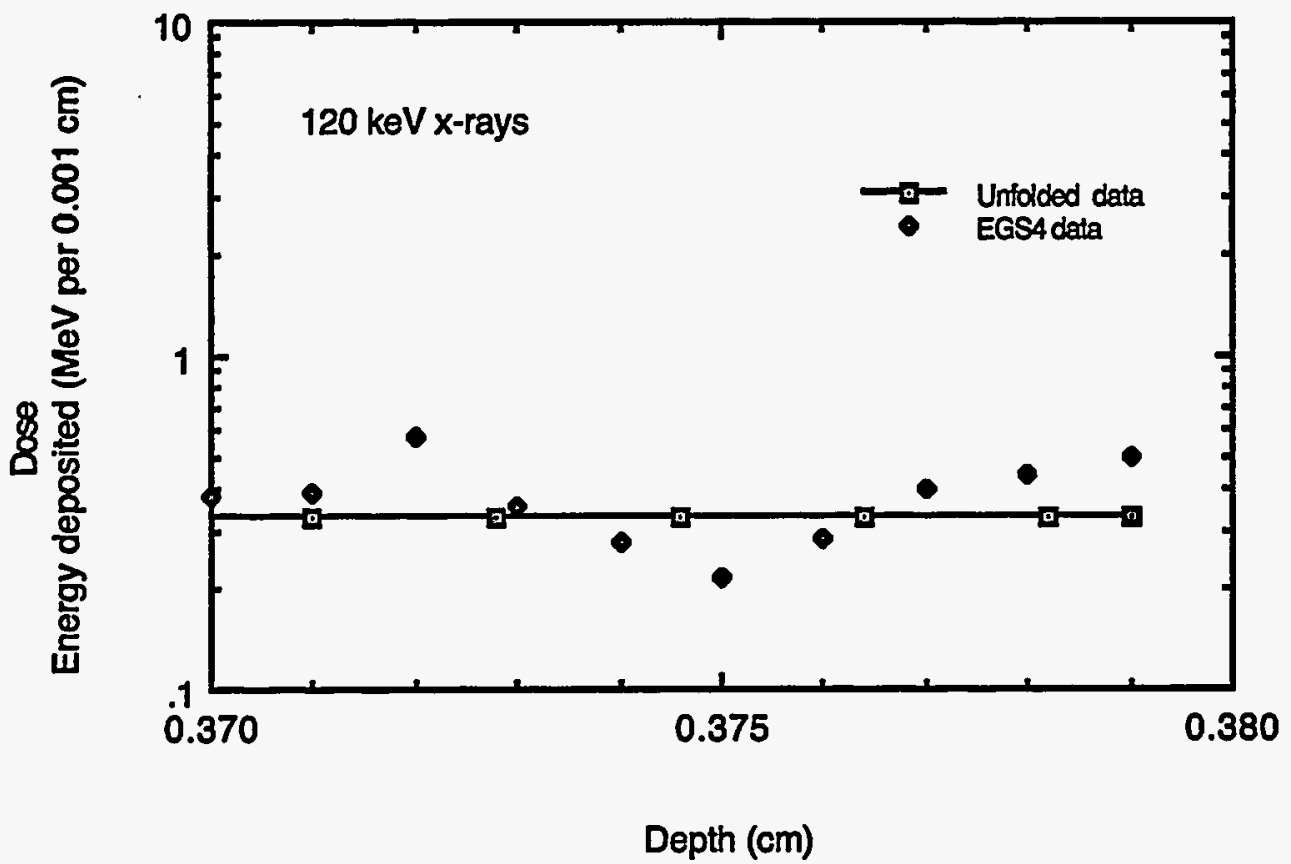

Figure 127. Unfolded deep depth-dose of a thick laser TL dosimeter exposed to $120 \mathrm{keV} \mathrm{x}$ rays following pulsed heating of uniform laser beam. 


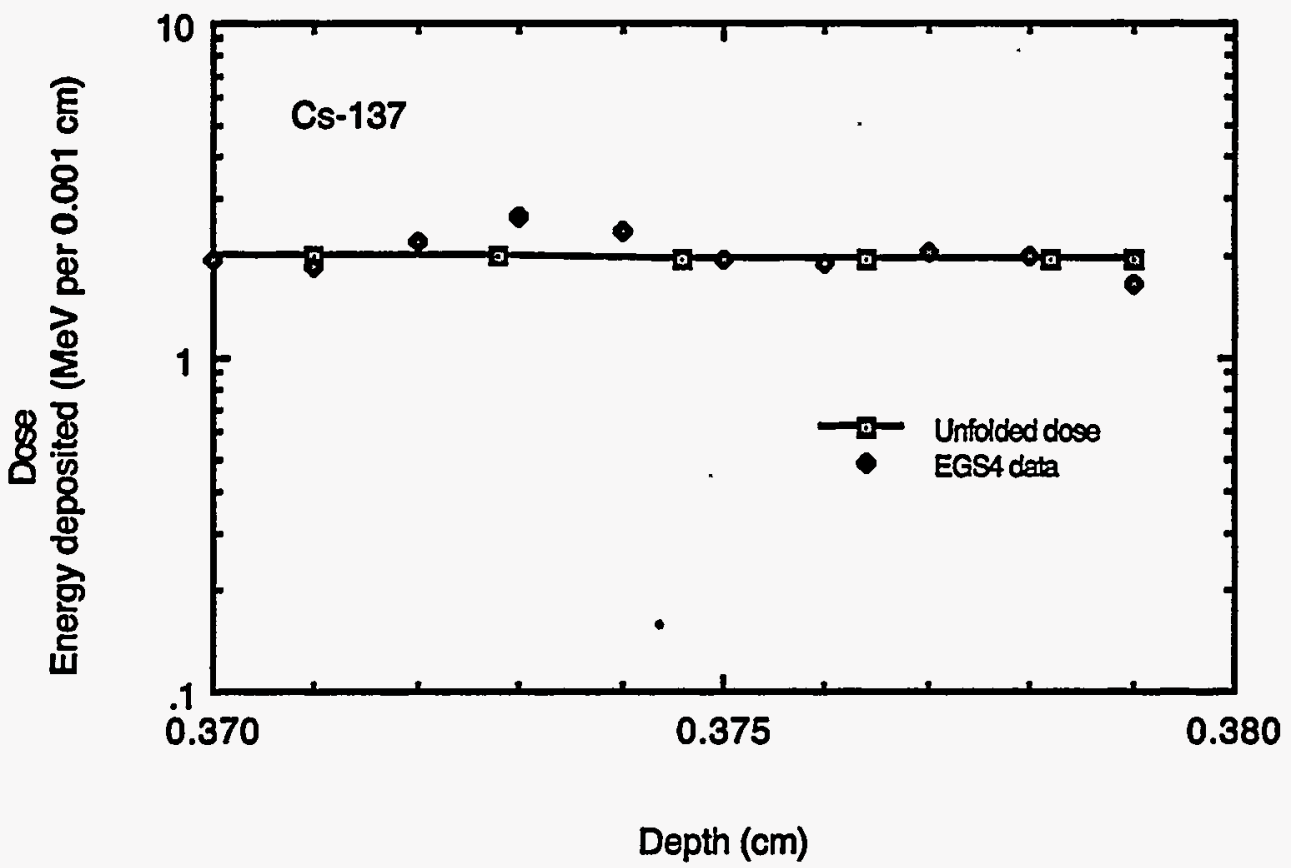

Figure 128. Unfolded deep depth-dose of a thick laser TL dosimeter exposed to Cs-137 gamma-rays following pulsed heating of uniform laser beam. 

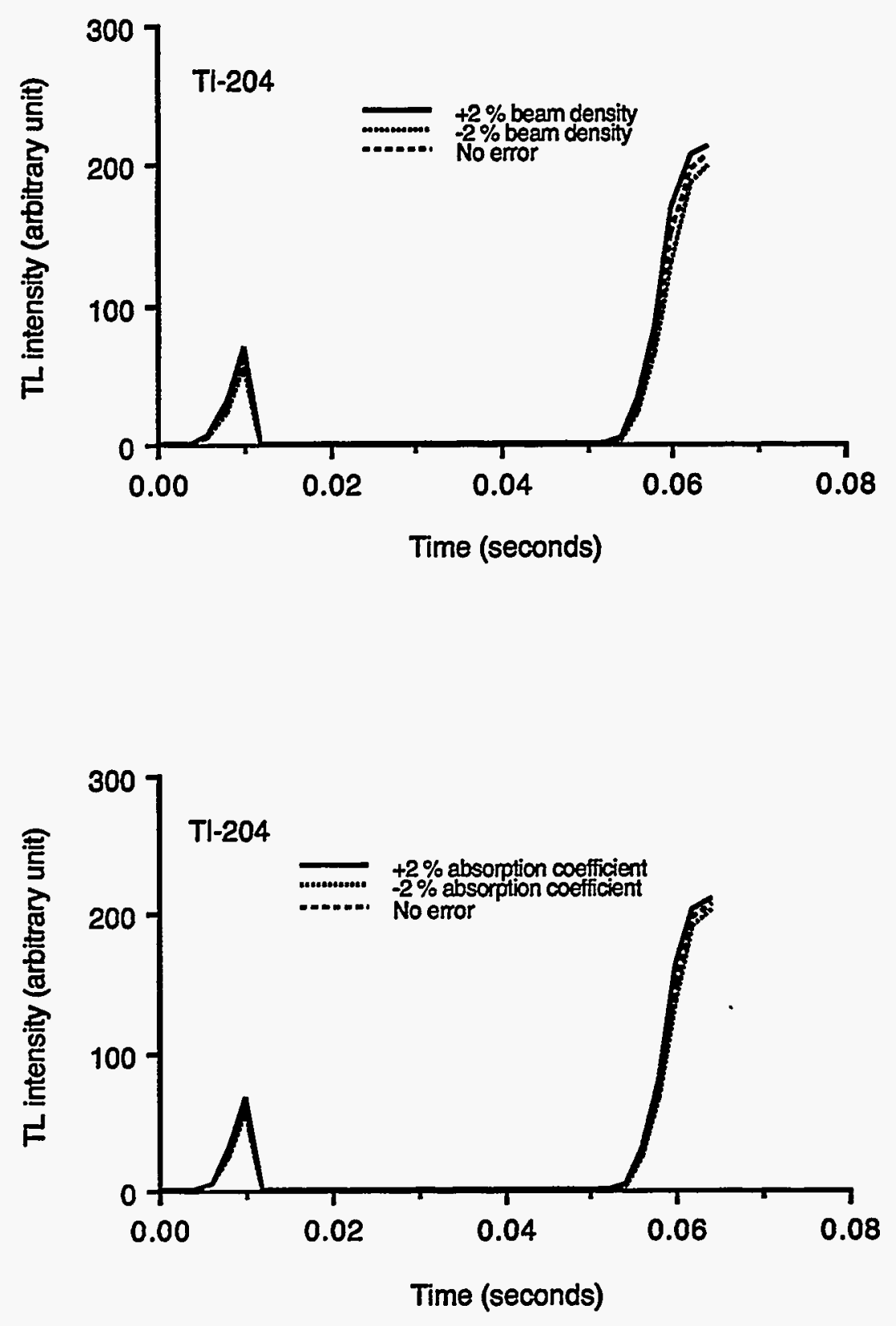

Figure 129. (1) Variations of TL glow curve with (a) $\pm 2 \%$ changes of beam density (above) and (2) $\pm 2 \%$ changes of absorption coefficient (below) of a thick laser TL dosimeter exposed to Tl-204 beta particles following pulsed heating of focused laser beam. 

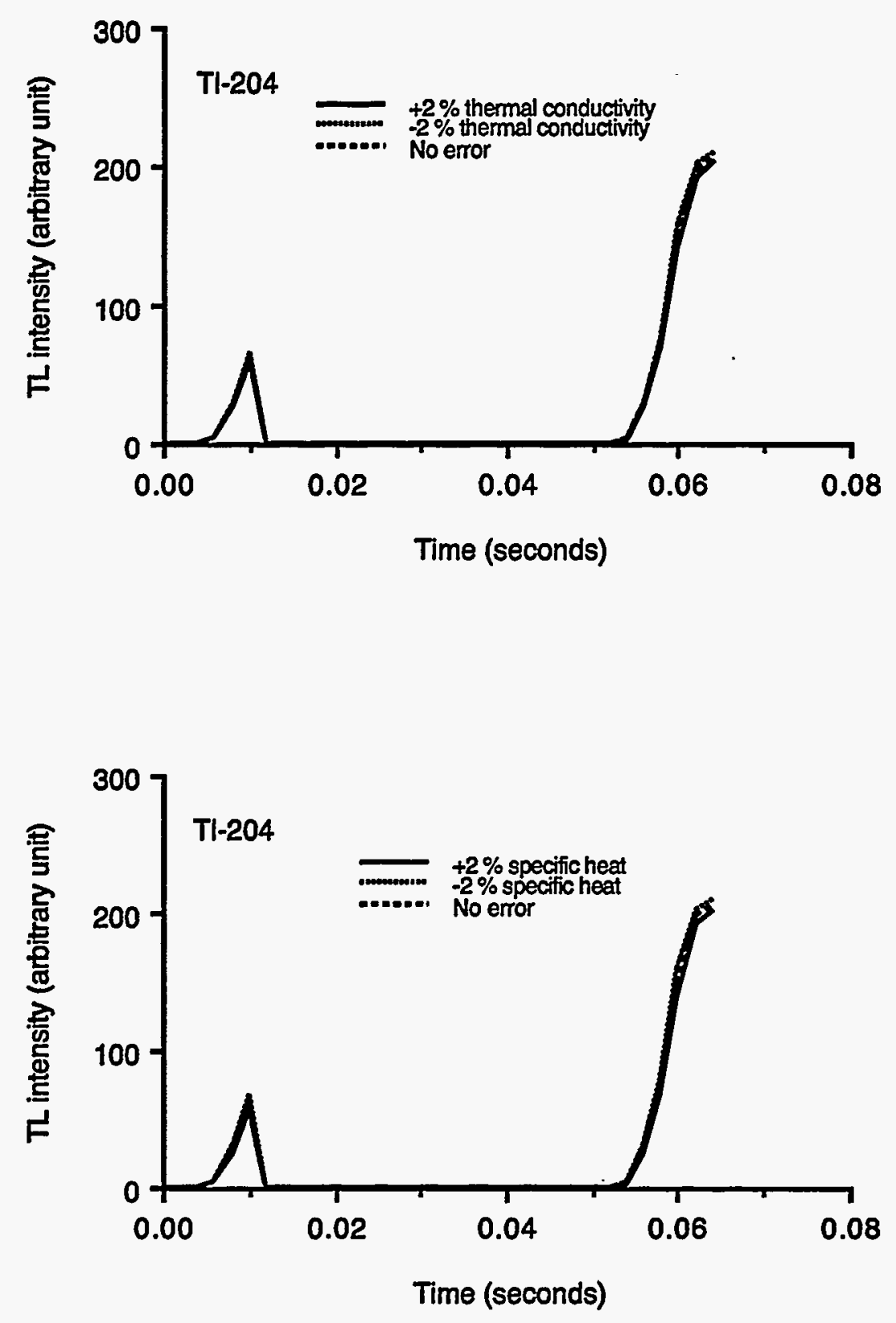

Figure 130. Variations of glow curve with (1) $\pm 2 \%$ changes of thermal conductivity (above) and (2) $\pm 2 \%$ changes of specific heat (below) of a thick laser TL dosimeter exposed to Tl-204 beta particles following pulsed heating of focused laser beam. 


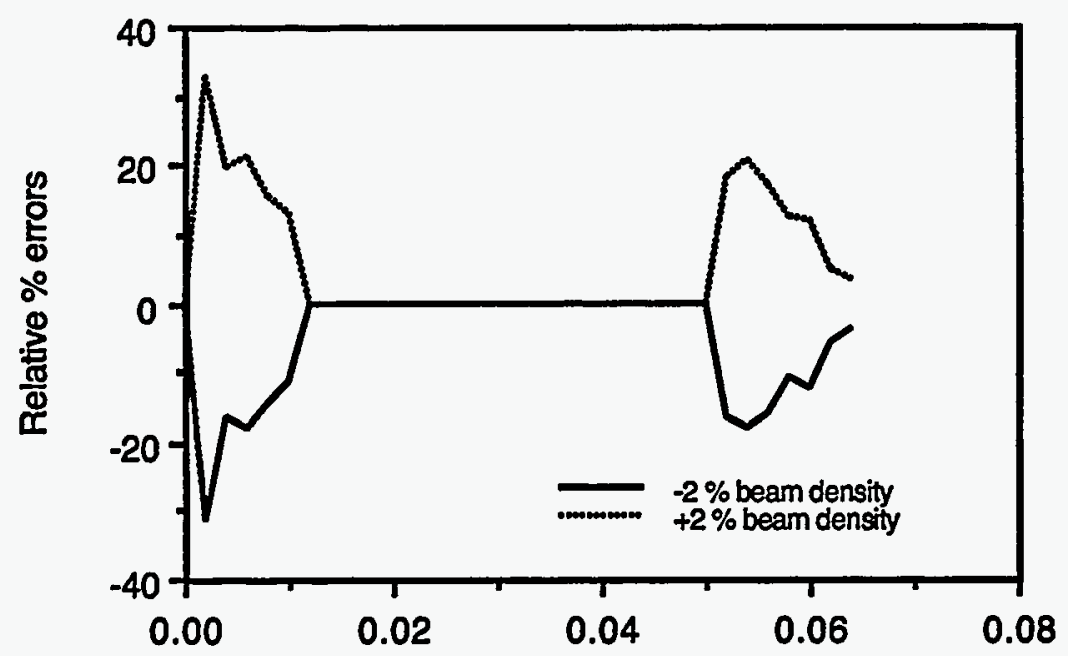

Heating time (seconds)

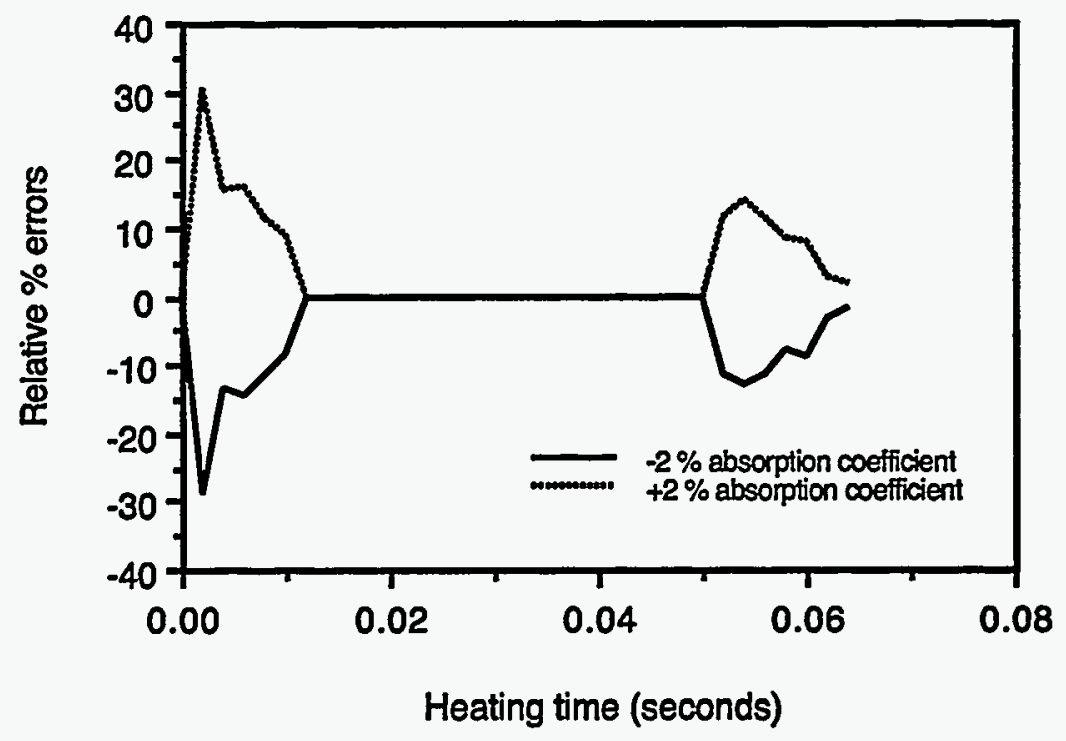

Figure 131. Variations of TL glow curves with (a) $\pm 2 \%$ changes of beam density (above) and (b) $\pm 2 \%$ changes of absorption coefficient (below) of a thick laser TL dosimeter exposed to Tl-204 beta particles following pulsed heating of focused laser beam. 

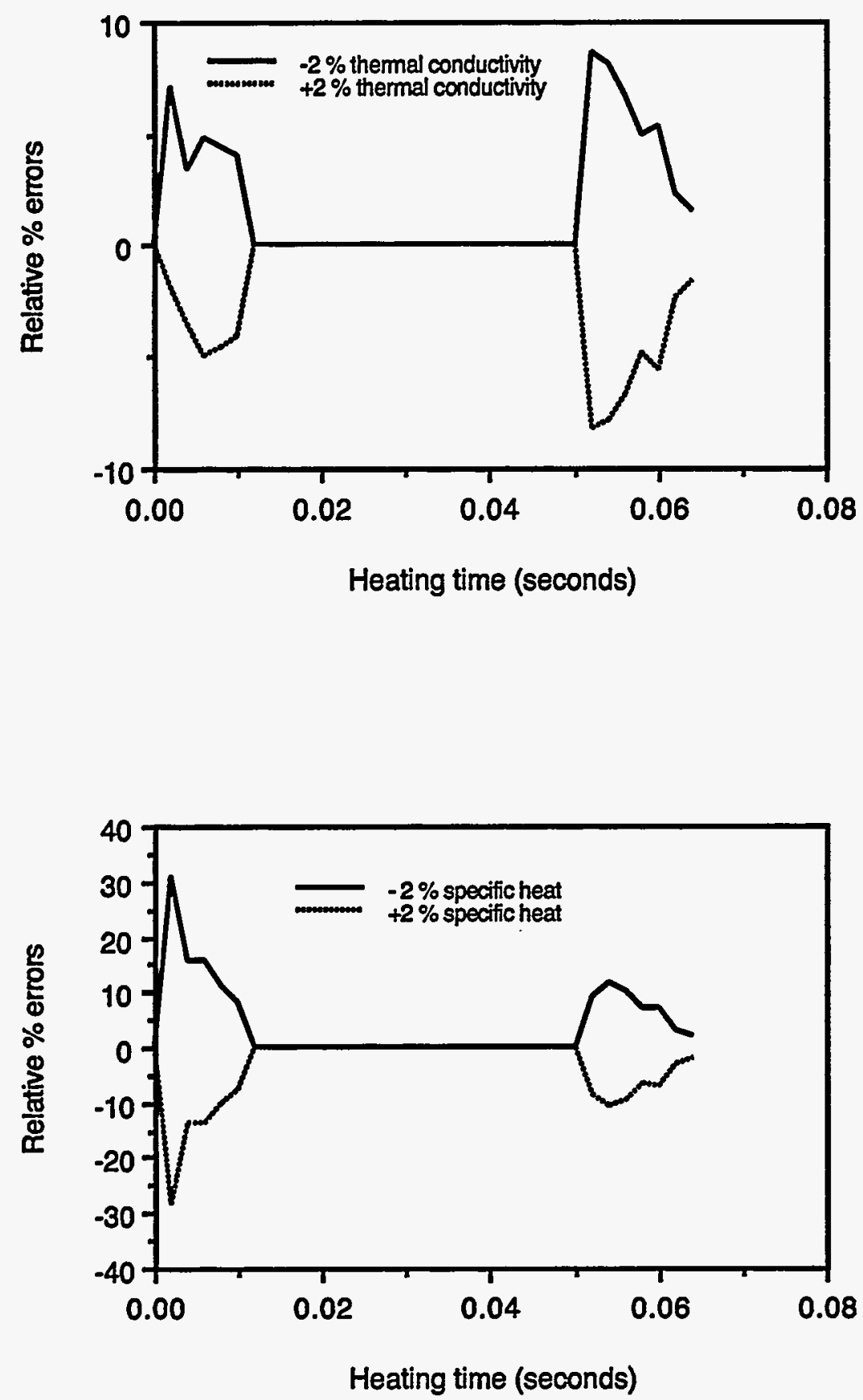

Figure 132. Variations of TL glow curves with (a) $\pm 2 \%$ changes of thermal conductivity (above) and (b) $\pm 2 \%$ changes of specific heat (below) of a thick laser TL dosimeter exposed to Tl-204 beta particles following pulsed heating of focused laser beam. 

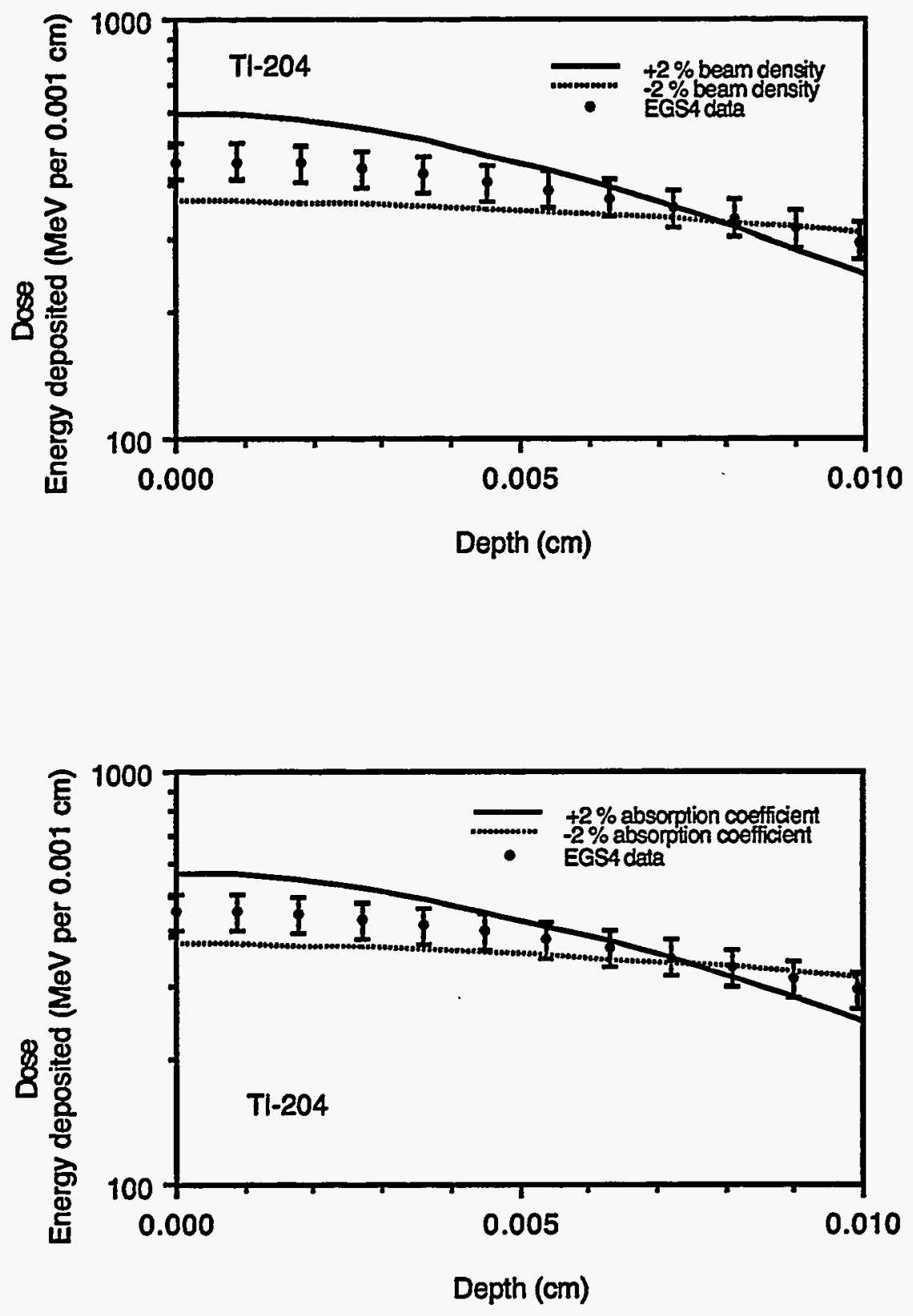

Figure 133. Variations of the depth-doses with $(1) \pm 2 \%$ changes of beam density (above) and (2) $\pm 2 \%$ changes of absorption coefficient (below) for a thick laser TL dosimeter exposed to Tl-204 beta particles following pulsed heating of focused laser beam. 

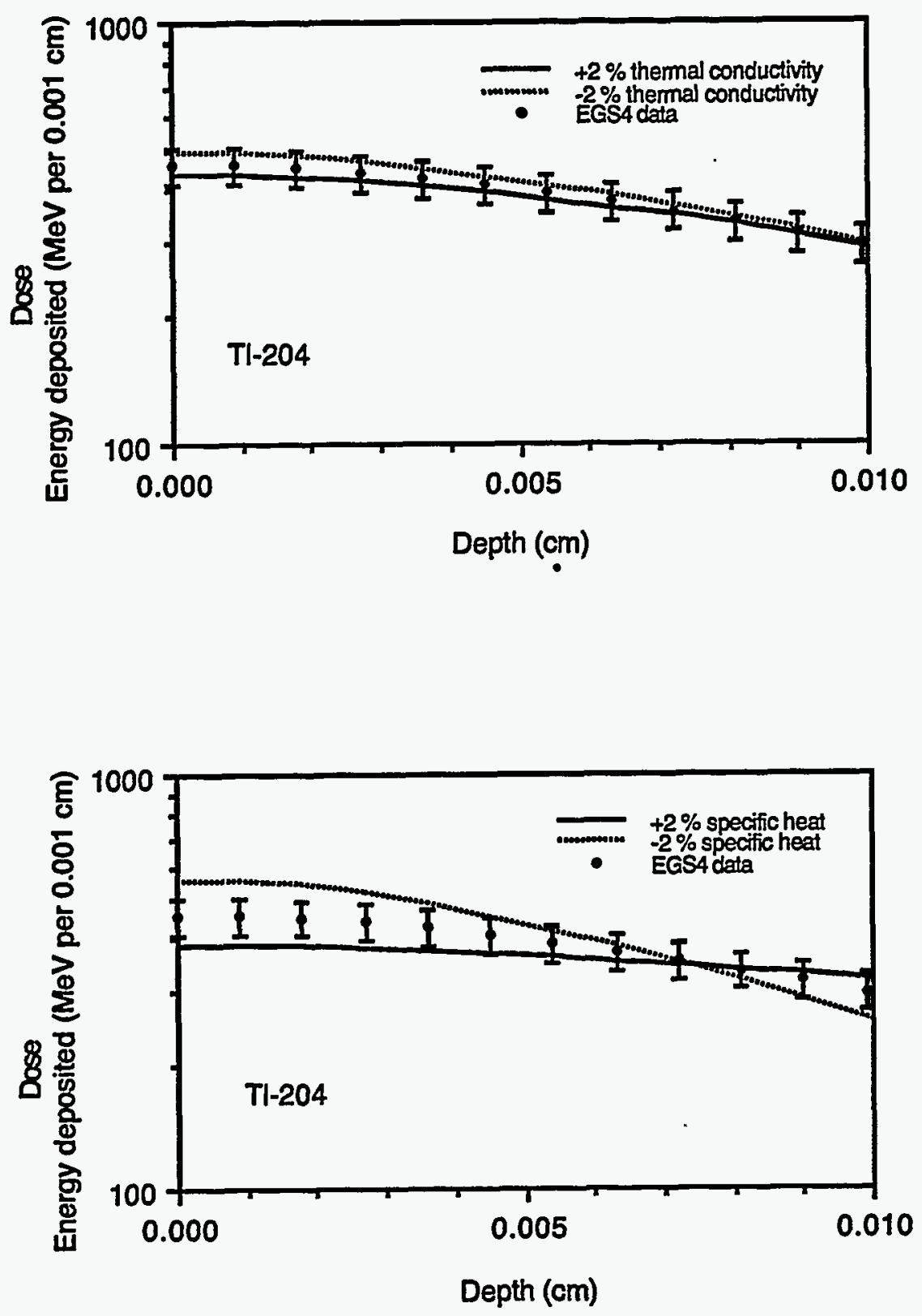

Figure 134. Variations of the depth-doses with $(1) \pm 2 \%$ changes of thermal conductivity (above) and (2) $\pm 2 \%$ changes of specific heat (below) of a thick laser TL dosimeter exposed to $\mathrm{Tl}-204$ beta particles following pulsed heating of focused laser beam. 

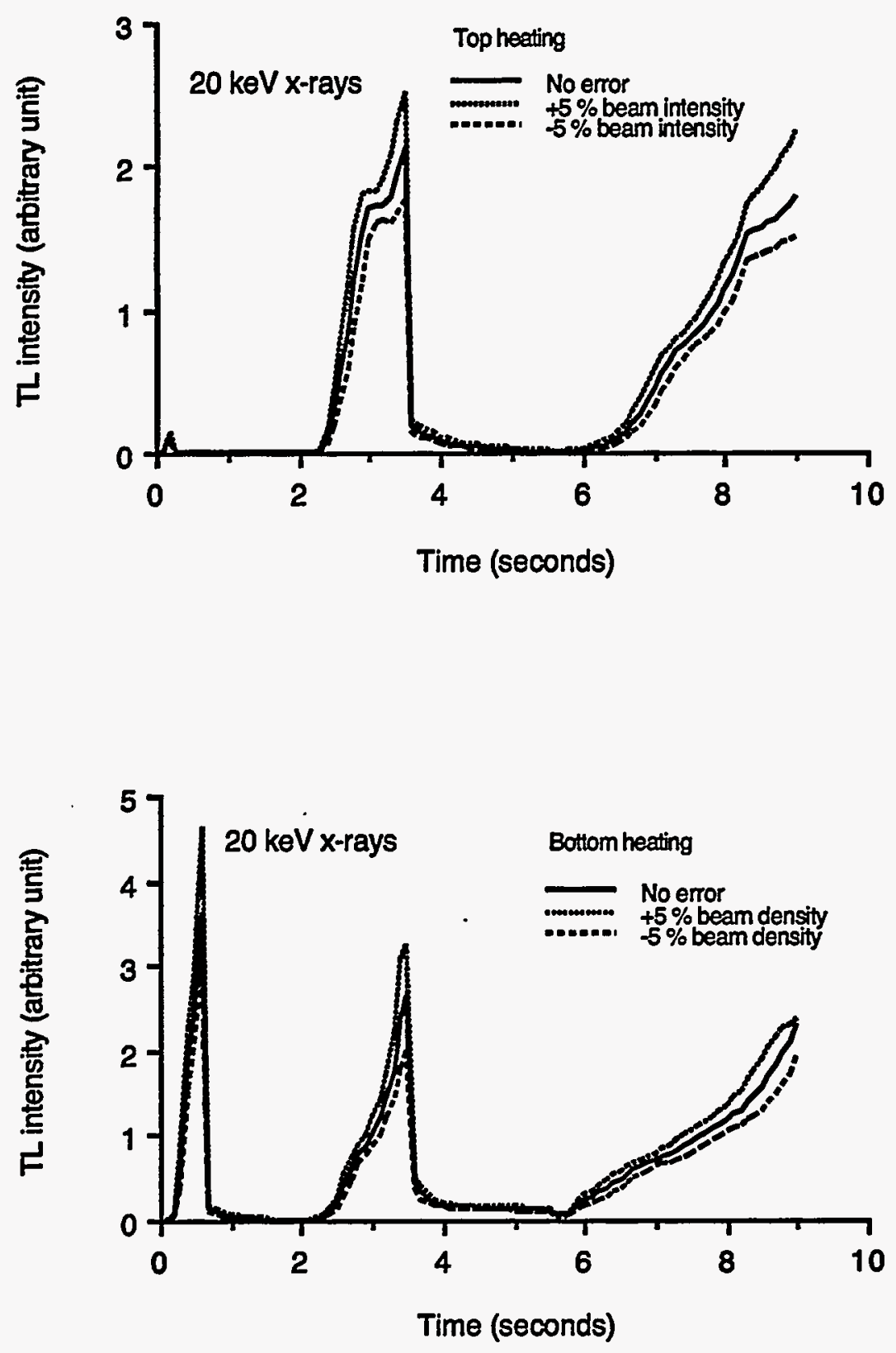

Figure 135. Variations of TL glow curves with $\pm 5 \%$ changes of beam density of a thick laser TL dosimeter exposed to $20 \mathrm{keV}$ x-rays following pulsed heating of uniform laser beam. 

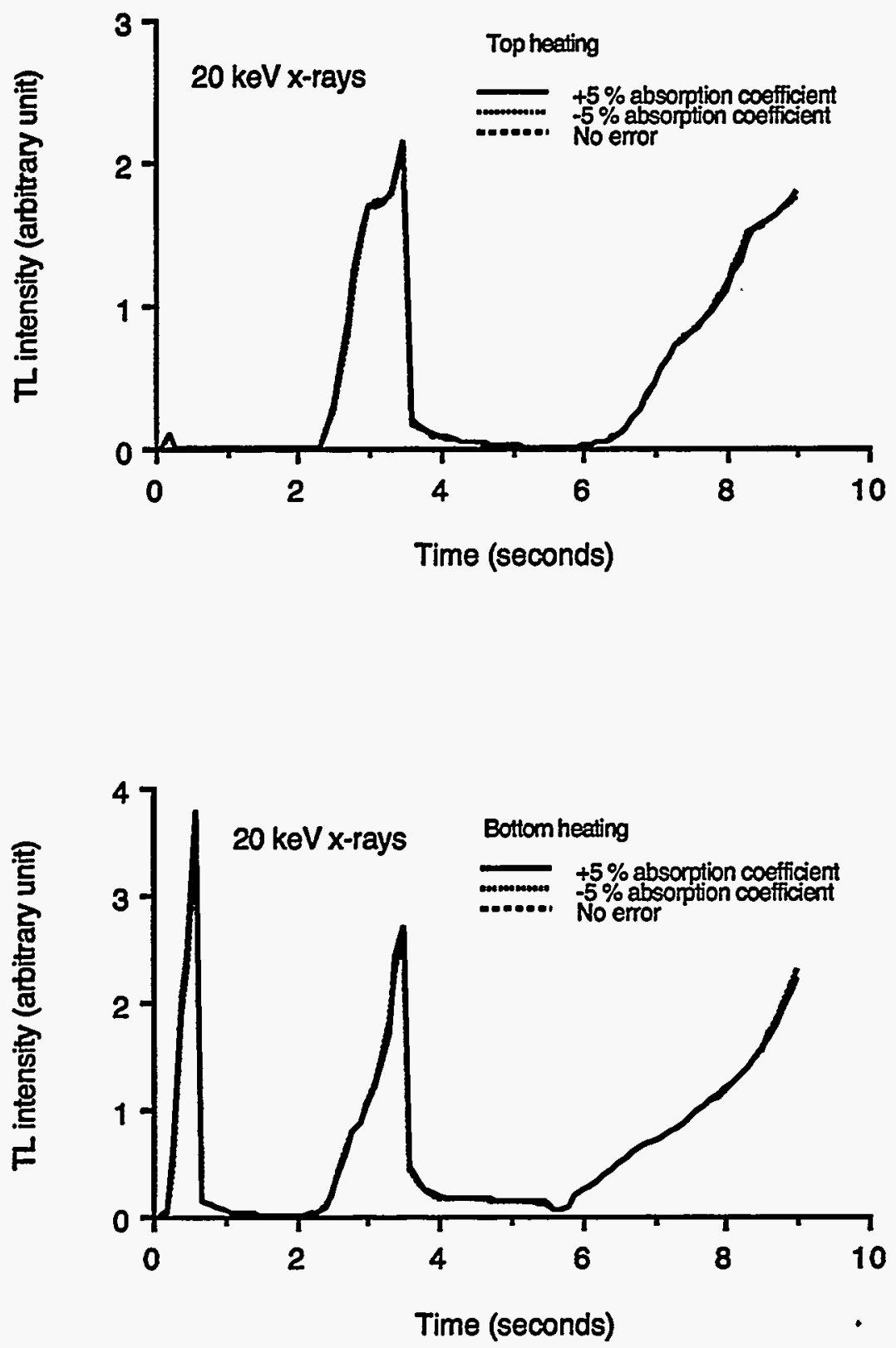

Figure 136. Variations of glow curve with $\pm 5 \%$ changes of absorption coefficient of a thick laser TL dosimeter exposed to $20 \mathrm{keV}$ x-rays following pulsed heating of uniform laser beam. 

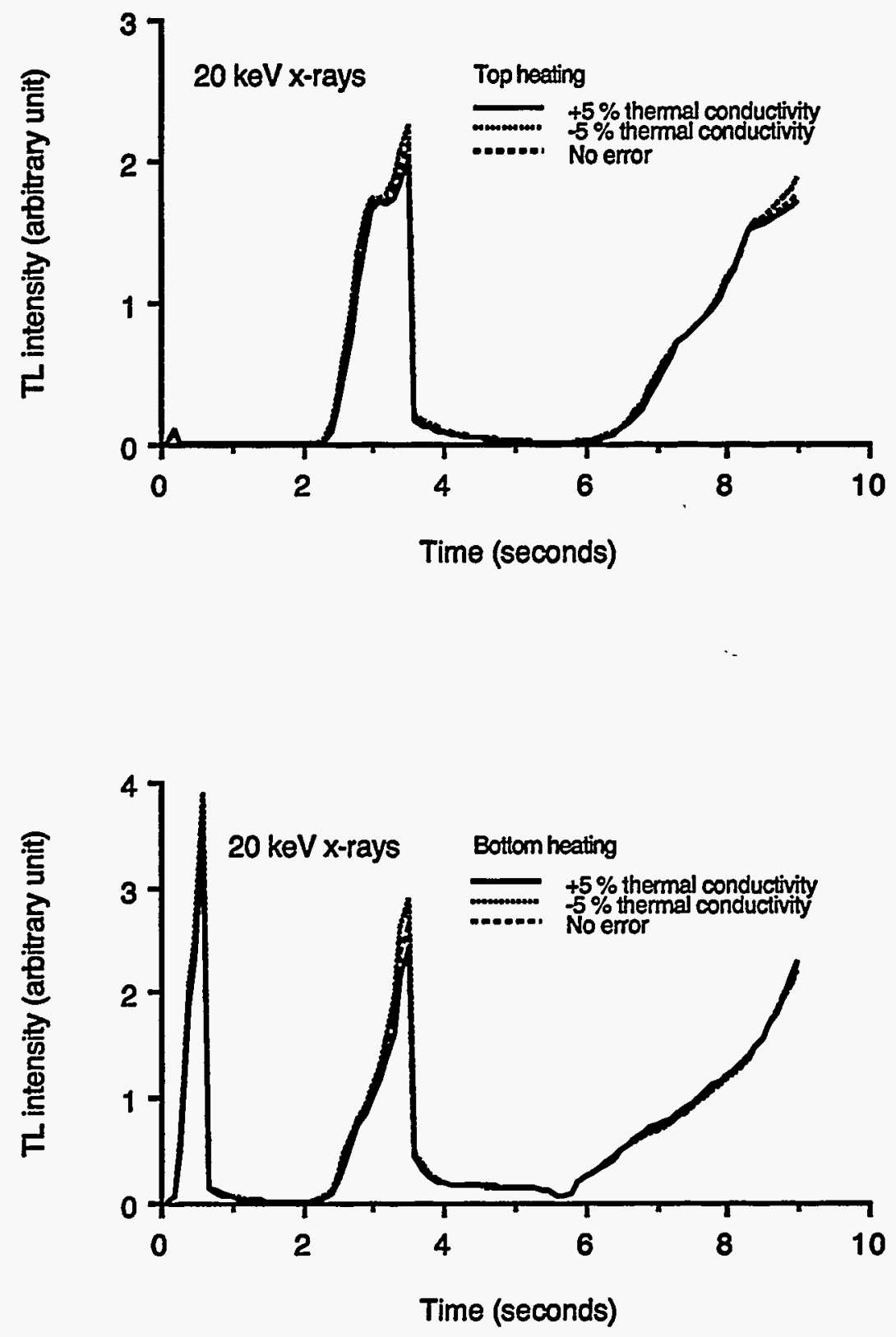

Figure 137. Variations of glow curve with $\pm 5 \%$ changes of thermal conductivity of a thick laser TL dosimeter exposed to $20 \mathrm{keV}$ x-rays following pulsed heating of uniform laser beam. 

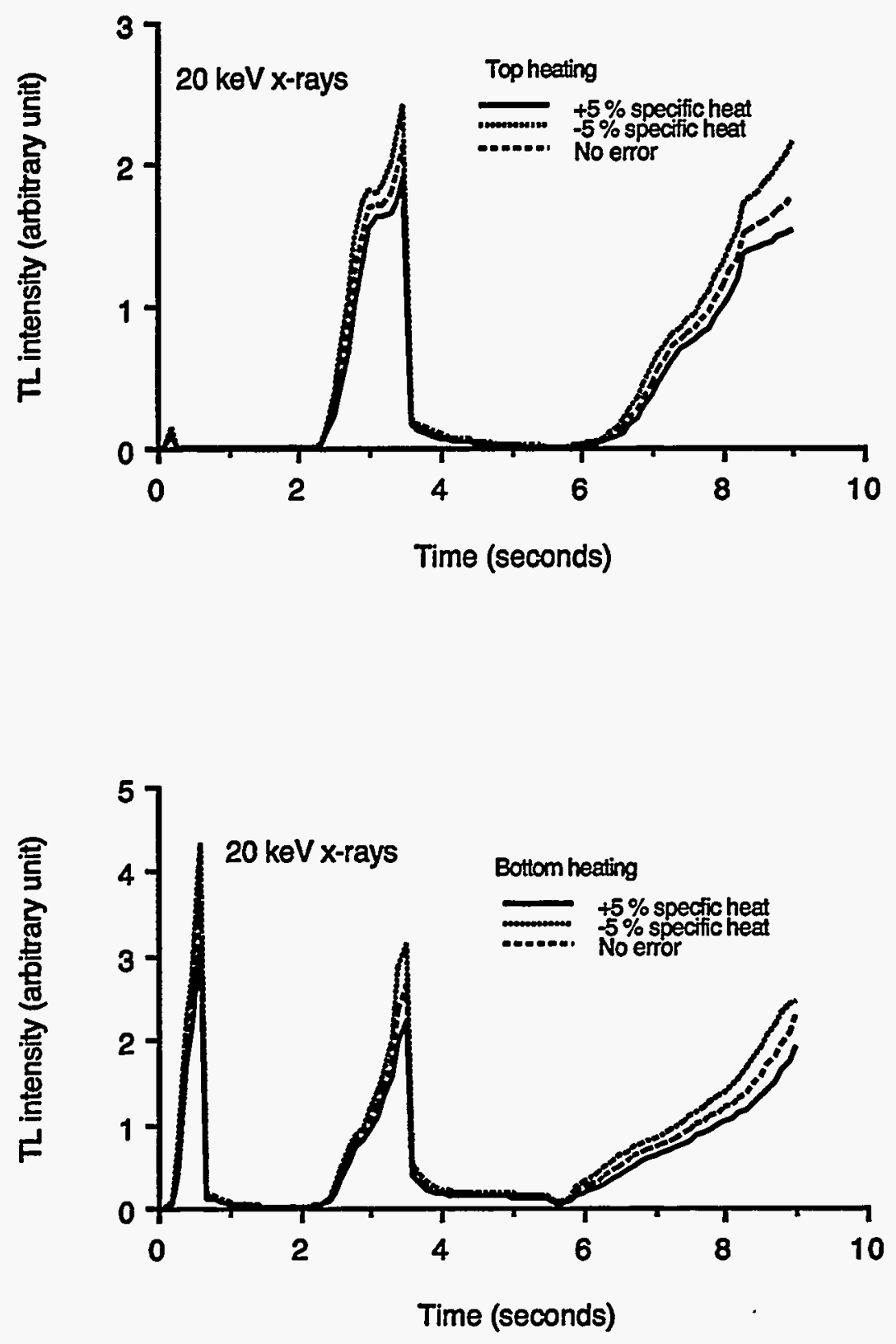

Figure 138. Variations of glow curve with $\pm 5 \%$ changes of specific heat of a thick laser TL dosimeter exposed to $20 \mathrm{keV} x$-rays following pulsed heating of uniform laser beam. 

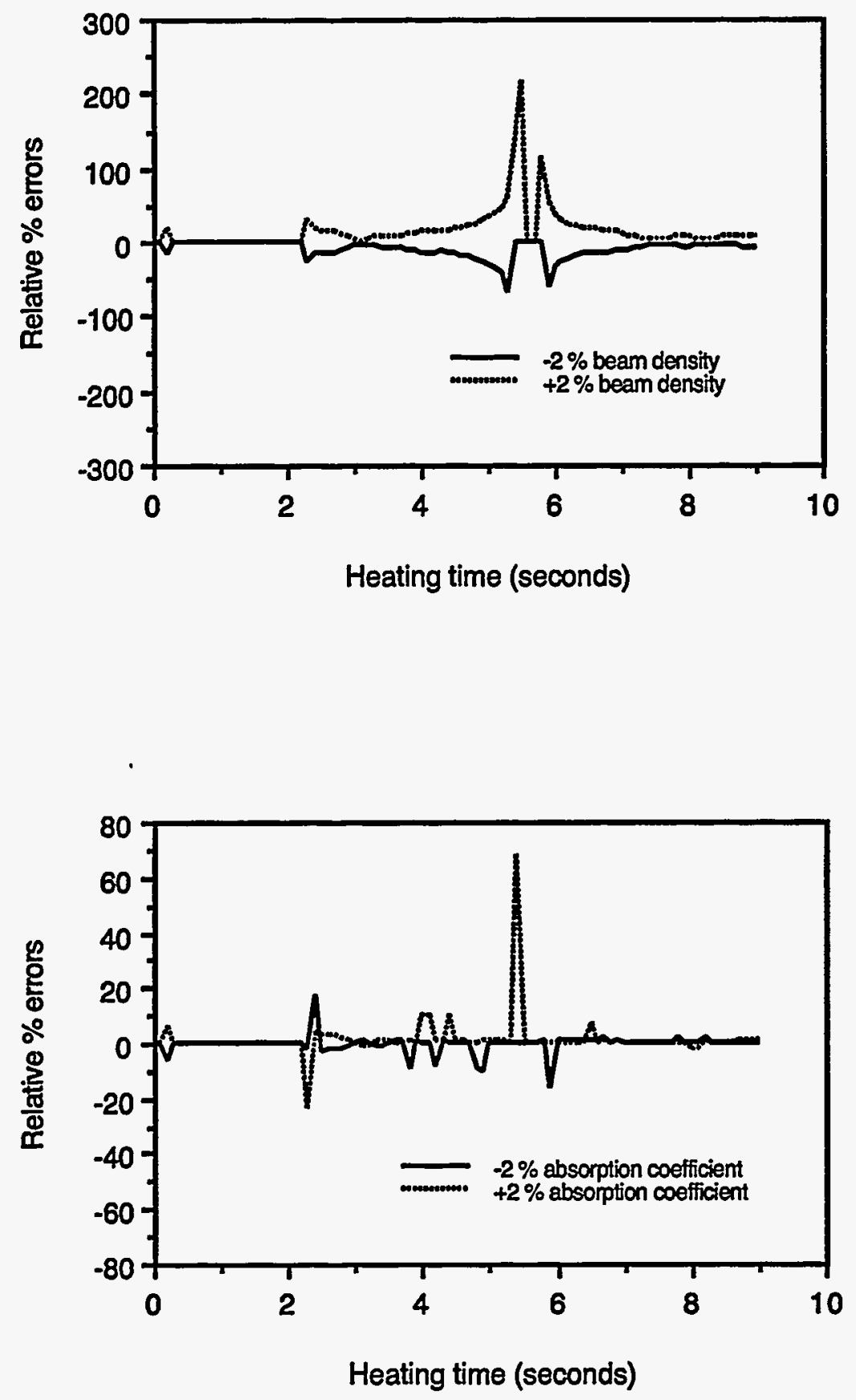

Figure 139. Variations of TL glow curves with (a) $\pm 2 \%$ changes of beam density (above) and (b) $\pm 2 \%$ changes of absorption coefficient (below) of a thick laser TL dosimeter exposed to $20 \mathrm{keV} \mathrm{X}$-rays following pulsed heating of uniform laser beam. 

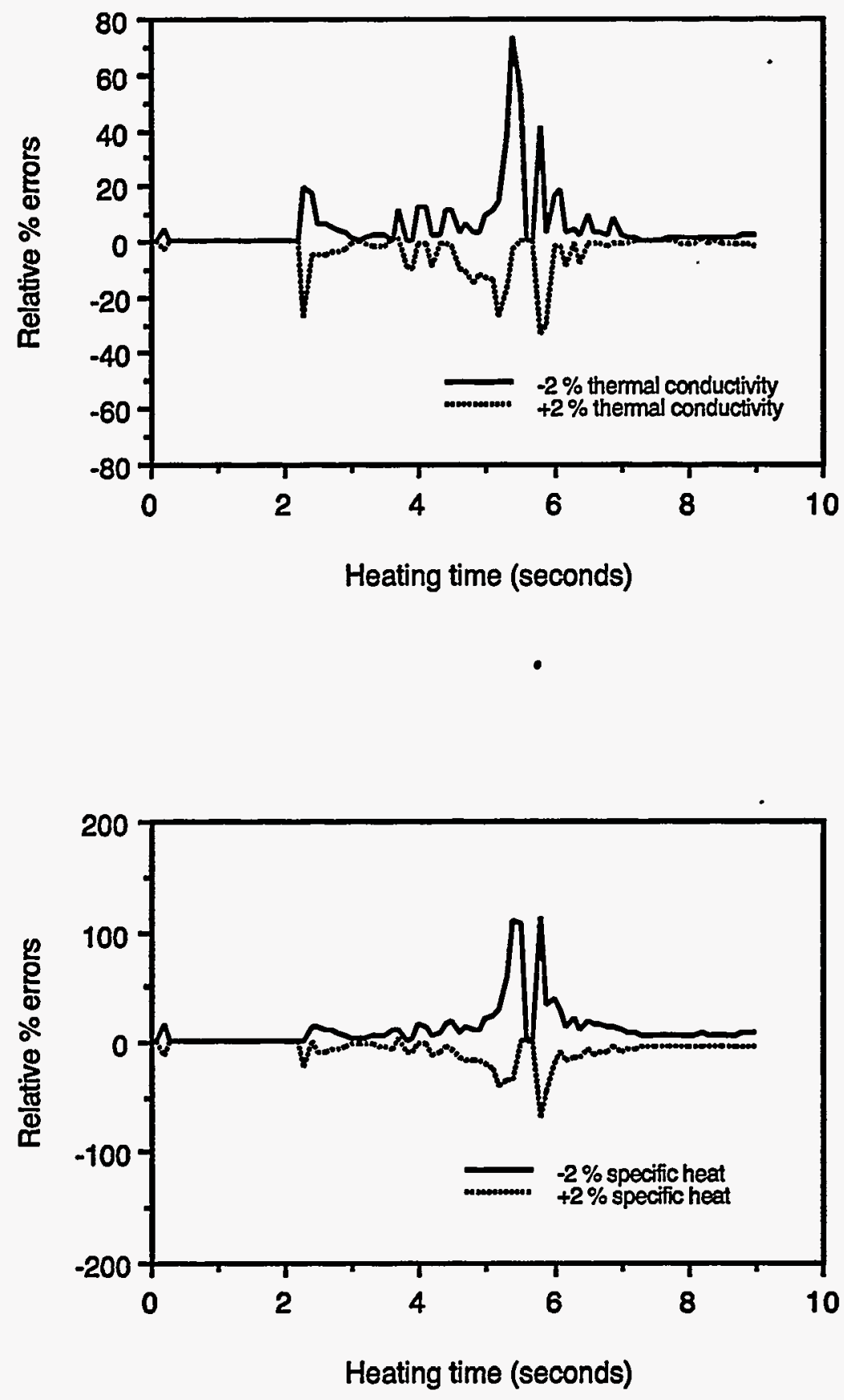

Figure 140. Variations of TL glow curves with (a) $\pm 2 \%$ changes of thermal conductivity (above) and (b) $\pm 2 \%$ changes of specific heat (below) of a thick laser TL dosimeter exposed to $20 \mathrm{keV} x$-rays following pulsed heating of uniform laser beam. 

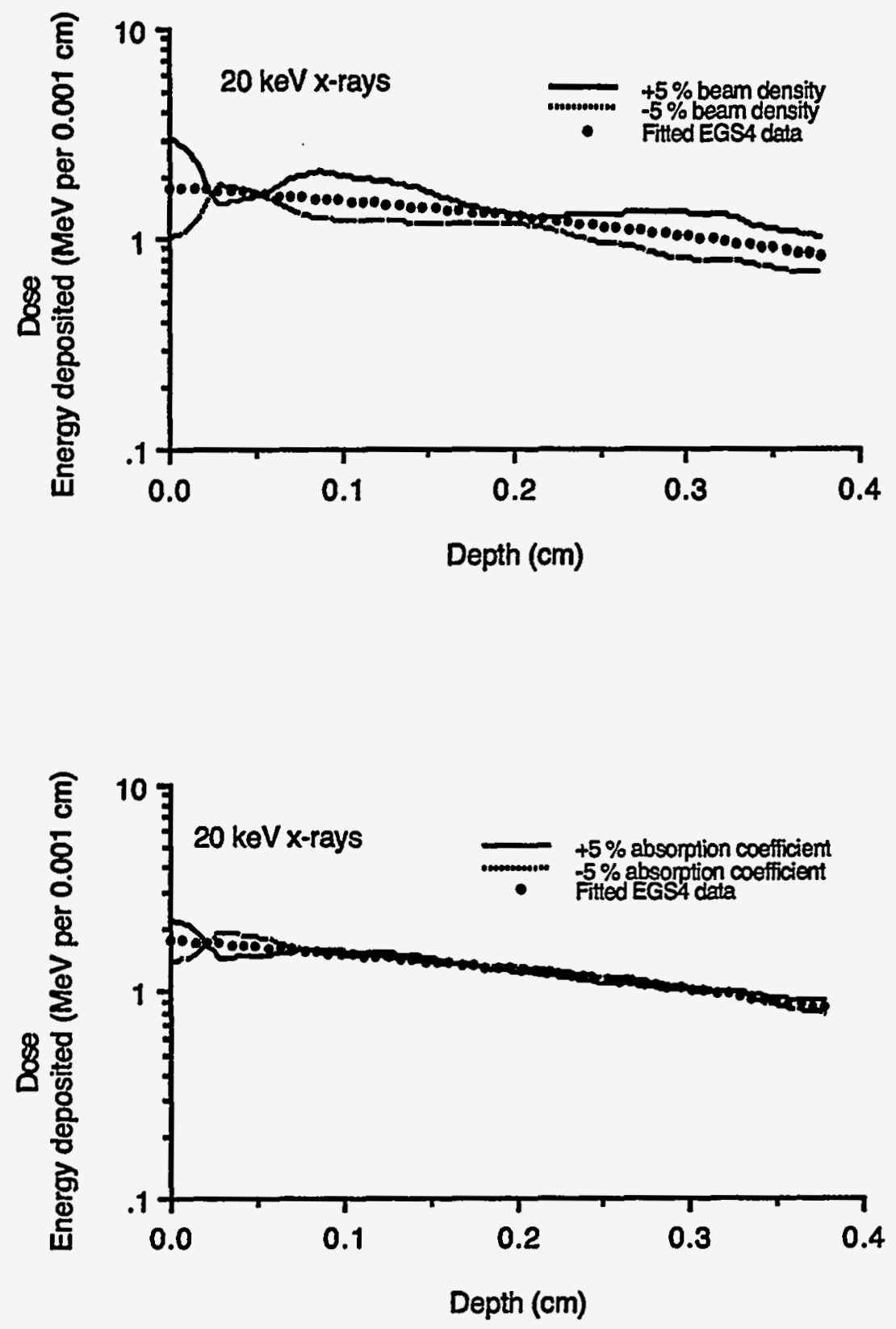

Figure 141. Variations of the depth-doses with (1) $\pm 5 \%$ changes of beam density (above) and (2) $\pm 5 \%$ changes of absorption coefficient (below) of a thick laser TL dosimeter exposed to $20 \mathrm{keV}$ x-rays following pulsed heating of uniform laser beam. 

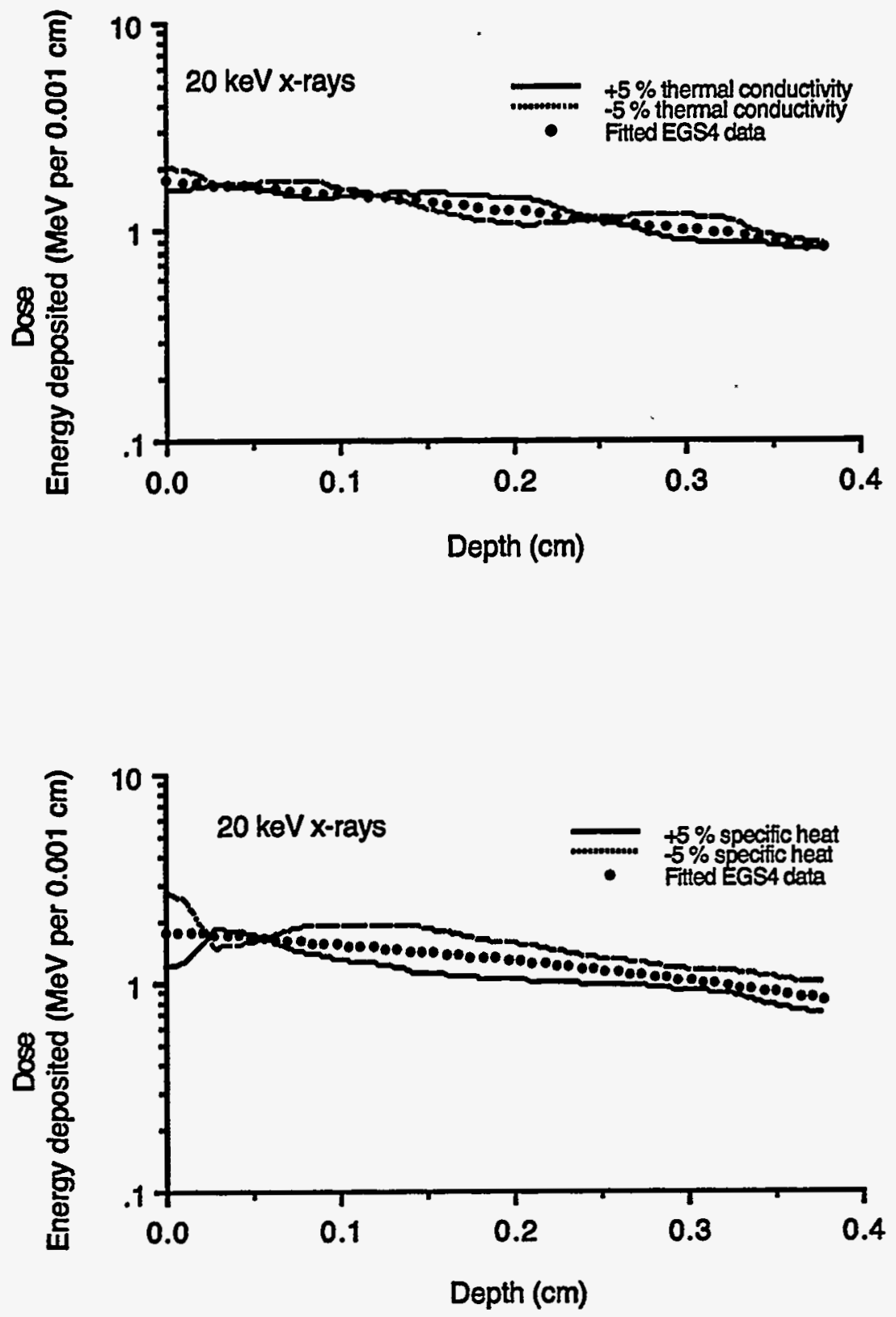

Figure 142. Variations of the depth-doses with (1) $\pm 5 \%$ changes of thermal conductivity (above) and (2) with $\pm 5 \%$ changes of specific heat (below) of a thick laser TL dosimeter exposed to $20 \mathrm{keV} x$-rays following pulsed heating of uniform laser beam. 


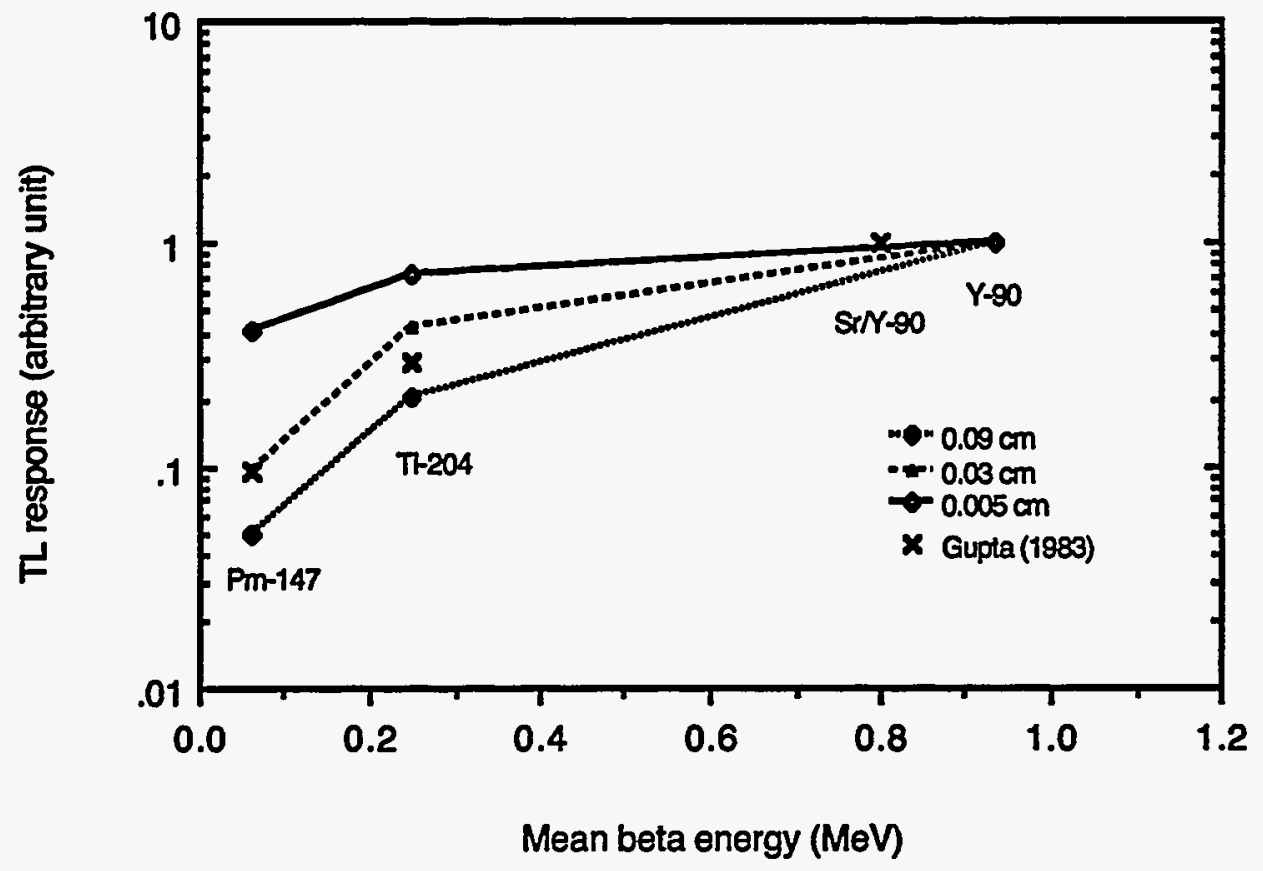

Figure 143. Theoretical beta-ray energy responses at the depth of $7 \mathrm{mg} / \mathrm{cm}^{2}$ of various $\mathrm{LiF}$ TL dosimeters as a function of mean beta energy. The data were obtained for conventional heating (planchet heating) at heating rate of $4.3 \mathrm{~K} / \mathrm{s}$ during $60 \mathrm{sec}$. The depth-doses from each beta source were calculated by EGS4 Monte Carlo transport code and based on the same dose rates. The symbols $(x)$ superimposed are the experimental data for a $0.09 \mathrm{~cm}$ thick LiF TLD published by Gupta [Gupta, 1983]. 


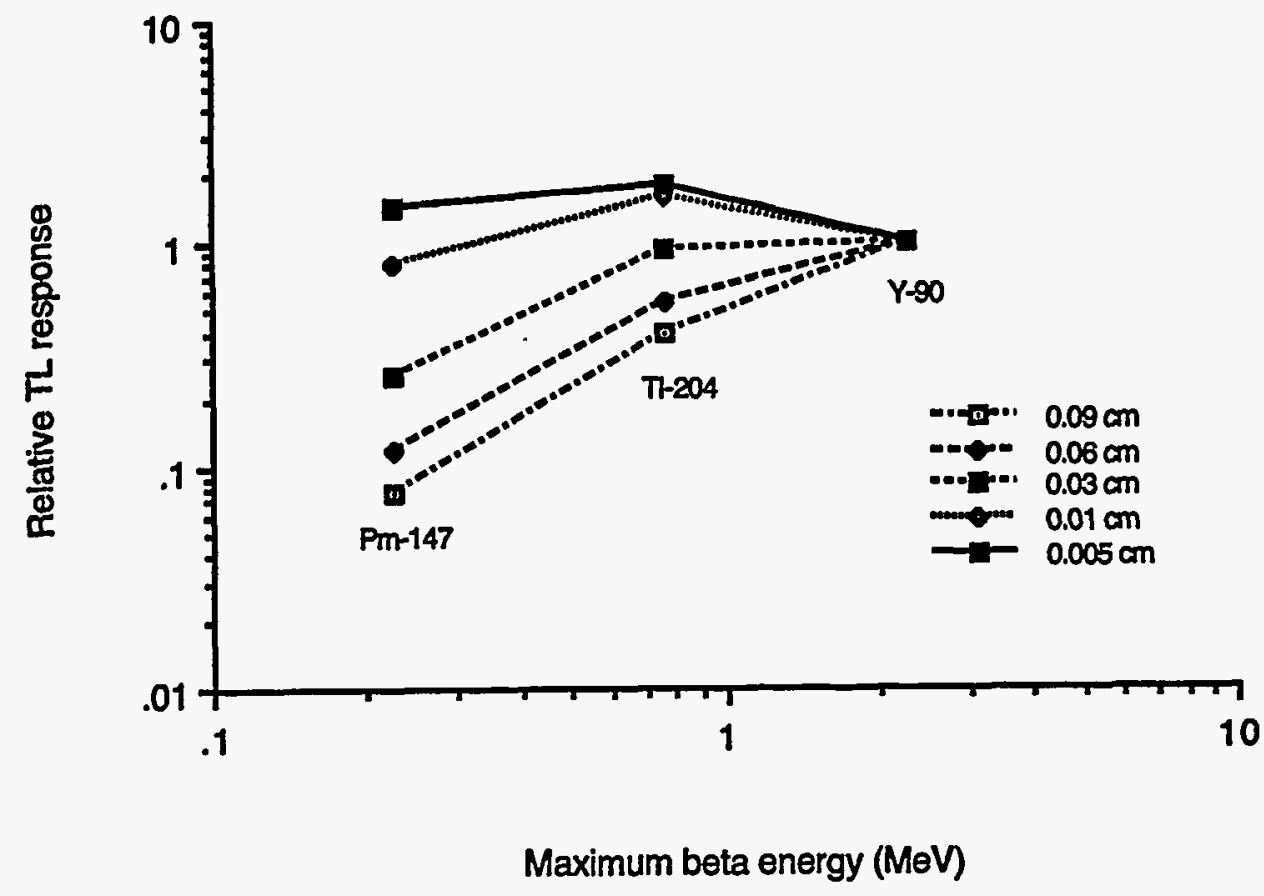

Figure 144. Theoretical beta-ray energy responses of various thick LiF TL dosimeters as a function of maximum beta energy. The data were obtained for conventional heating (planchet heating) on TLDs at heating rate of $4.3 \mathrm{~K} / \mathrm{s}$ during $60 \mathrm{sec}$. The depth-doses from each beta source were calculated by EGS4 Monte Carlo transport code and based on the same dose rates. 


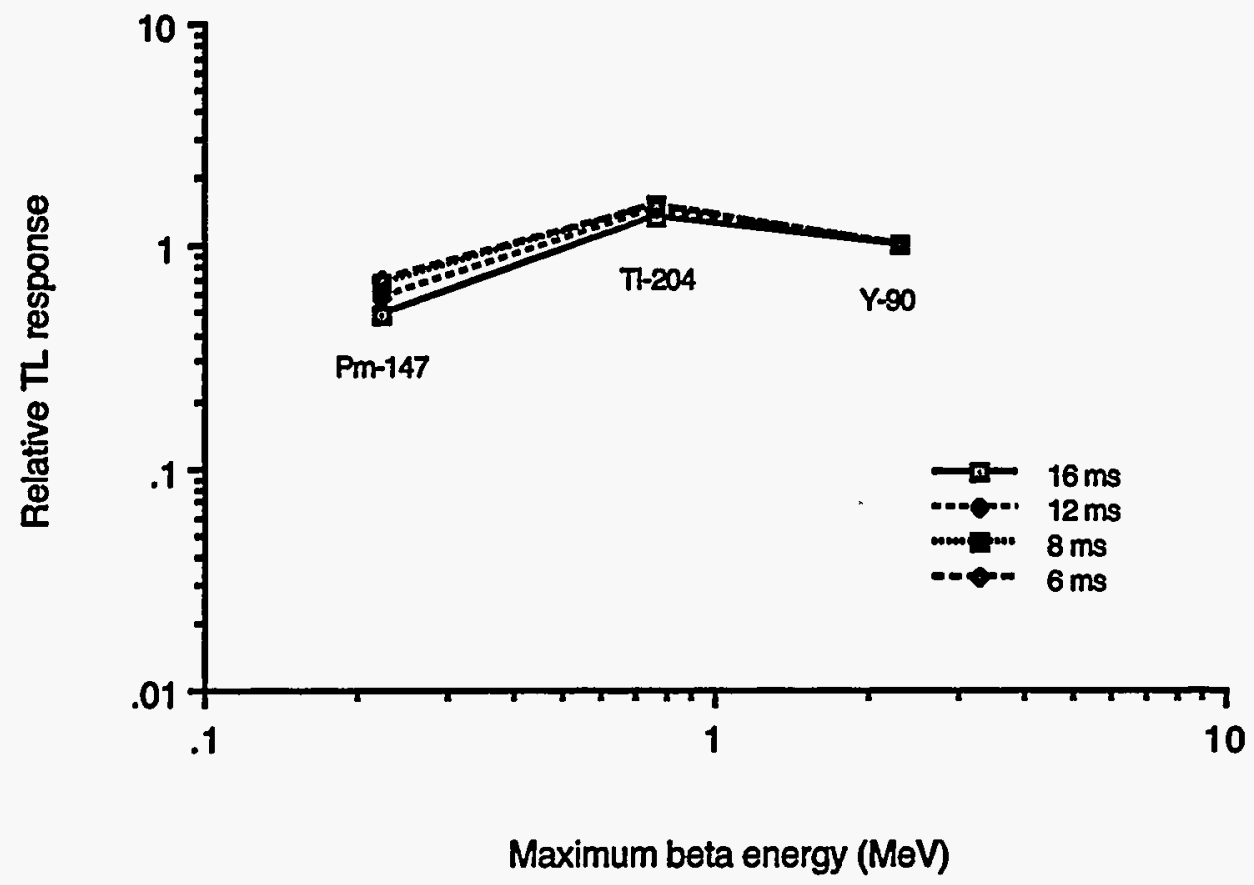

Figure 145. Theoretical beta-ray energy responses of $\mathrm{LiF} \mathrm{TL}$ dosimeter. Heating times correspond to a given depth of maximum thermoluminescence emission; $6 \mathrm{~ms}(0.0025$ $\mathrm{cm}), 8 \mathrm{~ms}(0.01 \mathrm{~cm}), 12 \mathrm{~ms}(0.018 \mathrm{~cm})$, and $16 \mathrm{~ms}(0.024 \mathrm{~cm})$. The data were obtained for $0.032 \mathrm{~cm}$ diameter, $4 \mathrm{~W}$ focused laser beam heating on $0.09 \mathrm{~cm}$ thick LiF TLD. The depth-doses from each beta source were calculated by EGS4 Monte Carlo transport code and based on the same dose rates. 


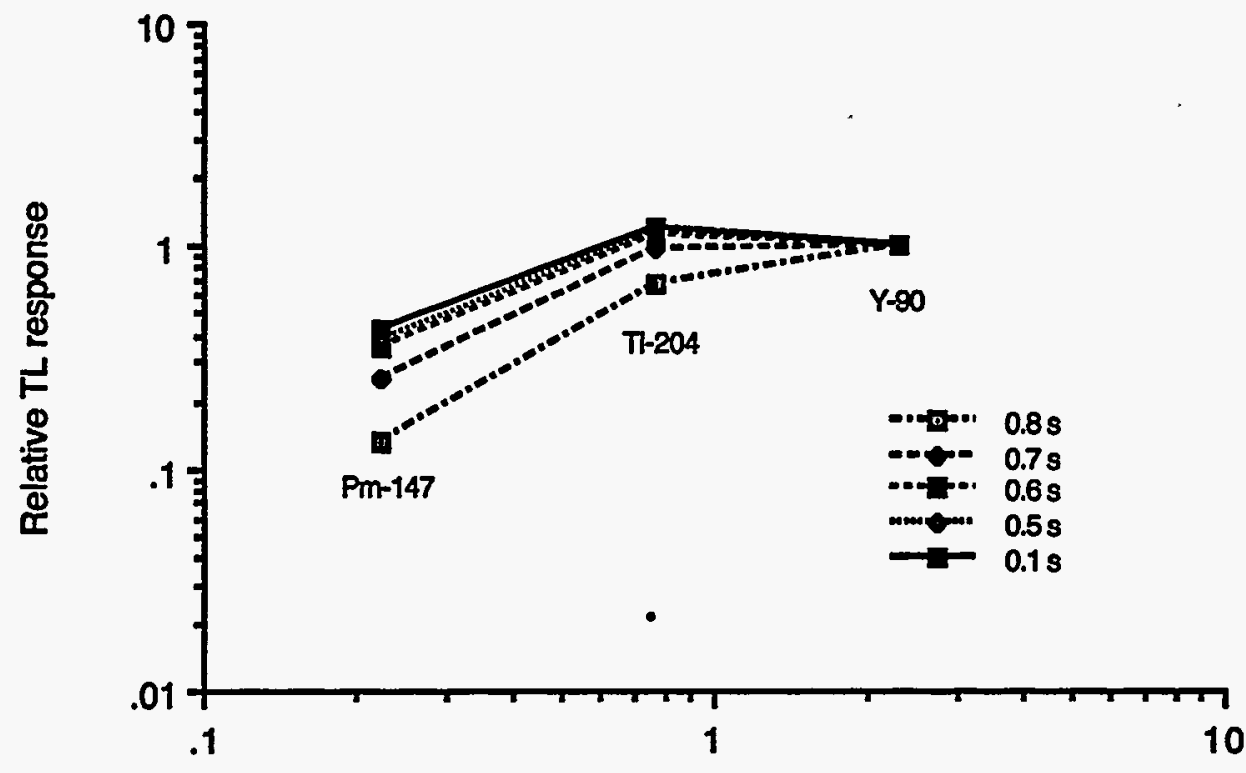

Maximum beta energy (MeV)

Figure 146. Theoretical beta-ray energy responses of LiF TL dosimeter. Heating times correspond to a given depth of maximum thermoluminescence emission; $0.1 \mathrm{~s}(0.0025$ $\mathrm{cm}), 0.5 \mathrm{~s}(0.0050 \mathrm{~cm}), 0.6 \mathrm{~s}(0.024 \mathrm{~cm}), 0.7 \mathrm{~s}(0.035 \mathrm{~cm})$, and $0.8 \mathrm{~s}(0.045 \mathrm{~cm})$.The data were obtained for $0.3 \mathrm{~cm} \times 0.3 \mathrm{~cm}, 10 \mathrm{~W}$ uniform laser beam heating on $0.09 \mathrm{~cm}$ thick. LiF TLD. The depth-doses from each beta source were calculated by EGS4 Monte Carlo transport code and based on the same dose rates. 


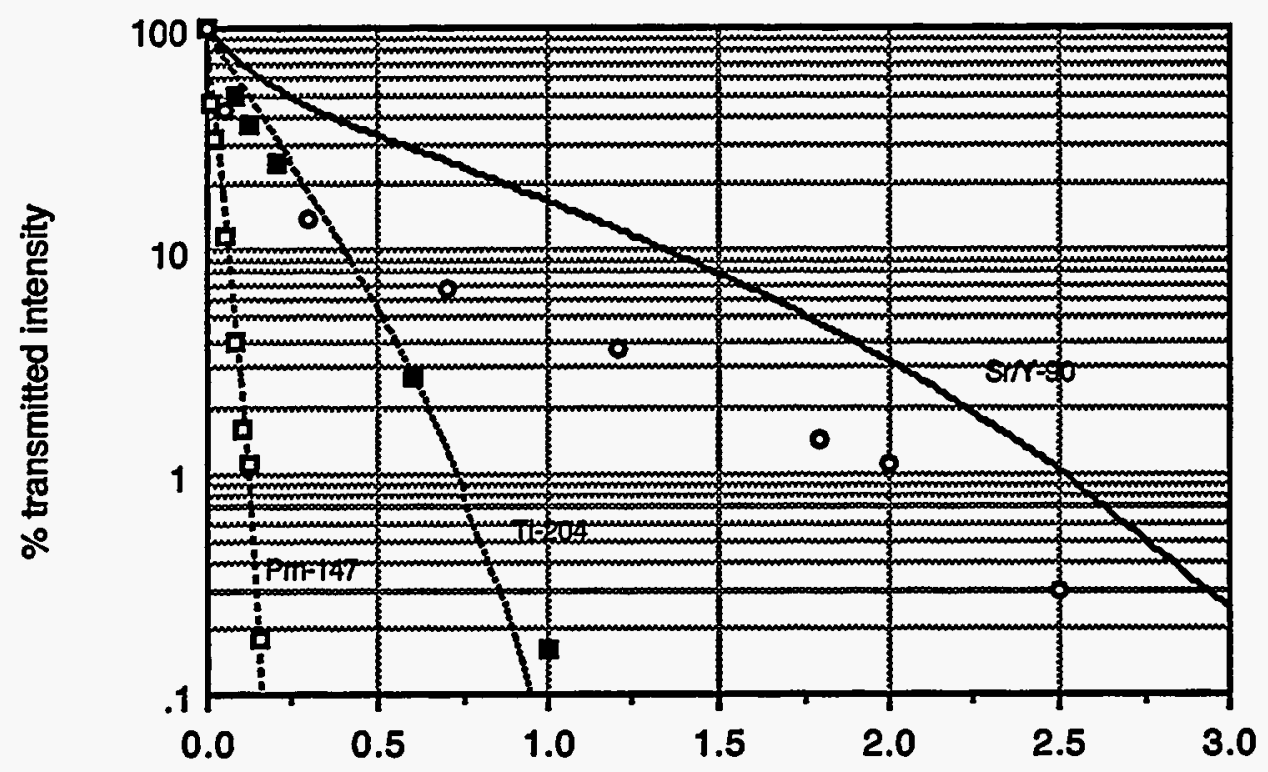

Absorber thickness (LiF), mm

Figure 147. A semilog scale plot of per cent transmitted intensity LiF absorber thickness $(\mathrm{mm})$ for pure beta emitters obtained for 50,000 history particle transport simulation by the EGS4 Monte Carlo code (lines). Experimental values superimposed: Pm-147 (open square), T1-204 (closed square), and Sr/Y-90 (open hole)[Paliwal et al., 1976]. 


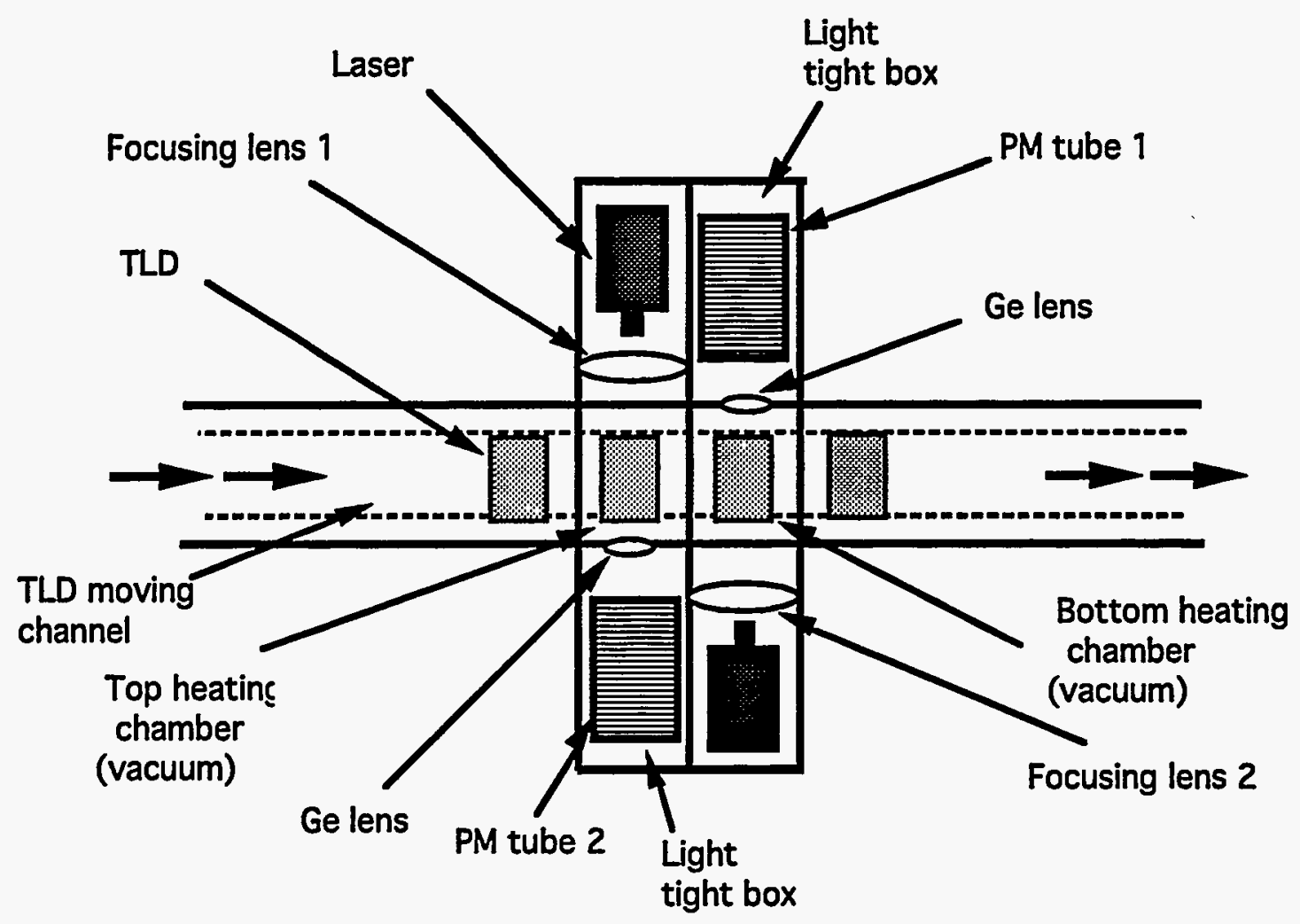

Figure 148. Conceptual diagram of automatic TL reading system. Laser heating is performed in chambers 1 and 2 . In differential heating mode, laser 1 is used heat top of TLD, collecting TL light by PM tube 1; laser 2 is used to heat bottom of TLD collecting TL light by PM tube 2. Focusing lenses 1 and 2 are used to focused the beam while Ge lenses 1 and 2 to prevent the laser beam from passing to the PM tube. 


\section{BIBLIOGRAPHY}

Abtahi, A., Braunlich, P., Kelly, P., Gasiot, J. Laser Stimulated Thermoluminescence, J. Appl. Phys. 58: 1626-1639, 1985.

Abtahi, A., Braunlich, P., Haugan, T., Kelly, P. Investigation of Thermoluminescence Efficiencies at High Laser Heating Rates, Rad. Prot. Dos. 17: 313-316, 1986 a.

Abtahi, A., Braunlich, P., Kelly, P. Theory of Transient Temperature Response of a TwoLayer System Heated with a Localized Laser Beam, J. Appl. Phys. 6: 3417-3421, 1986 b.

Abtahi, A., Haugan, T., Kelly, P. Investigation of the Dosimetric Properties of MgB407:Dy under Laser Heat, Rad. Prot. Dos. 21: 211-217, 1987.

Alexander, R.E. Regulatory Considerations in Beta Measurements, In Proc. Int. Beta Dos. Symp. Washington, D.C.: U.S. Nuclear Regulatory Commission, NUREG/CP0050, p 421-424, 1983.

Becker, K. Photographic Film Dosimetry, New York: Focal Press, 1966.

Berger, M.J., Seltzer, S.M. Stopping Powers and Ranges of Electrons and Positrons, National Bureau of Standards, NBSIR 82-2550, 1982.

Birkhoff, G., Varga, R.S., Young, D. Alternation Direction Implicit Methods, in Advances in Computers, F.L. Alt and M. Rubinoff, Eds. (Academic, New York), 1962.

Braunlich, P., Jones, S. C., Abtahi, A., DeMurcia, M. Heating of Continuous Thermoluminescent Layers with Localised Laser Beams, Rad. Prot. Dos. 6: 8386, 1984.

Braunlich, P.F., Tetzlaff, W. An Automated Laser Heated Thermoluminescence Dosimetry System, Rad. Prot. Dos. 17:321-324, 1986.

Braunlich, P. Present State and Future of TLD Laser Heating, Rad. Prot. Dos. 34:345351, 1990.

Brodsky, A. Accuracy and Sensitivity of Film Measurements of Gamma Radiation-Part II: Limits of Sensitivity and Precision, Health Phys. 9: 463-471, 1963.

CFR. Code of Federal Regulations, Title 10, Chapter 20, the Office of the Federal Register, U.S. Government Printing Office, Washington, D.C. $20402,1992$.

Charles, M. The Development of a Practical $5 \mathrm{mg} / \mathrm{cm}-2$ Skin Dosimeter, Proc. 5th Int. Conf. on Luminescence Dosimetry, Sao Paulo ed., Brazil, pp.313-323, 1977.

Christensen, P. Review of Personal Monitoring Techniques for the Measurement of Absorbed Dose from External Beta and Low Energy Photon Radiation, Rad. Prot. Dos. 2: 127-135, 1986 
Cross, W. G., Ing, H., Freedman, N. A Short Atlas of Beta-Ray Spectra, Phys. Med. Biol. 28:1251-1260, 1983.

Devine, R.T., Moscovitch, M., Blake, P.K. The US Naval Dosimetry Center Thermoluminescence Dosimetry System, Rad. Prot. Dos. 30: 231-236, 1990.

DOE/DH-0026, Handbook for the Department of Energy Laboratory Accreditation Program for Personnel Dosimetry Systems, DOE/EH-0026, 1986.

Doroshenko, J.J., Kraitor, S.N., Kuznetsova, T.V, Kushnereva, K.K, Leonov, E.S. New Methods for Measuring Neutron Spectra with Energy from $0.4 \mathrm{eV}$ to $10 \mathrm{MeV}$ by Track and Activation Detectors, Nucl. Technol. 33 : 296-304, 1977.

Driscoll, C. M. H., Francis, T. M., Richards, D. J. The Response of Thermoluminescent Materials to Beta Radiation, Rad. Prot. Dos. 9: 295-298, 1984.

Francis, T. M., O'Hagan, J. B., Richards, D. J., Driscoll, C. M. H. Responses of Thermoluminescent Materials to Beta Radiation and Low Energy Photons, Rad. Prot. Dos. 17: 89-92, 1986.

Francis, T. M., O'Hagan, J. B., Williams, S. M., Driscoll, C. M. H., Bartlett, D.T. Responses Characteristics of Carbon-Loaded TL Detectors to Beta Radiation, Rad. Prot. Dos. 28: 201-205, 1989.

Gasiot, J., Braunlich, P. Laser Heating in Thermoluminescence Dosimetry, J. Appl. Physics, 53(7): 5200-5209, 1982.

Gesell, T. F., Jones, D. E., Gupta, V. P., Kalbeizer, F. L., Cusimano, J. P. A Personnel Beta-Dosimetry Method for Reducing Energy Dependence, Idaho National Engineering Laboratory, Idaho Falls, ID 83401, IDO-12090, 1979.

Gotlib, V. I., Grebenshicov, V. L., Kantorovich, L. N., Sahre, P. Thermoluminescence Dosimetry of Beta Radiation, Rad. Prot. Dos. 22: 13-17, 1988.

Gotlib, V. I., Kantorovich, L. N., Grebenshicov, V. L., Bichev, V. R., Nemiro, E. A. The Study of Thermoluminescence Using the Contact Method of Sample Heating, J. Phys. D: Appl. Phys. 17, 2097-2114,1984.

Grupen-Shemansky, M.E. Three-Dimensional Spatial and Dosimetric Characterization of Radiotherapy Beams Using Laser Read-Out of Thermoluminescent Dosimeters, Ph.D. Dissertation, Arizona State University, 1989.

Gupta, V.P. Limitations of Commonly used Thick Element Personnel Dosimeters, In Proc. Int. Beta Dos. Symp. Washington, D.C.: U.S. Nuclear Regulatory Commission, NUREG/CP-0050, p 99-104, 1983.

Harshaw Filtrol, Crystal and Electronics Products, 6801 Cochran Rd, Solon, Ohio 44139.

Heinzelman, M., Schuren, H., Keller, M. Dosimeter for Determining Skin Dose, Rad. Prot. Dos. 2: 115-118, 1982. 
Hildebrand, J. D., Schmidt, J. W. Development of a Multi-Element Thermoluminescent Dosimeter at the Three Mile Island Nuclear Station, In Proc. Int. Beta Dos. Symp. Washington, D.C.: U.S. Nuclear Regulatory Commission, NUREG/CP-0050, p 387-400, 1983.

IAEA. Summary Report on the Post-Accident Review Meeting on the Chernobyl Accident, IAEA Safety Series 75-INSAG-1, STI/PUB/740 (Vienna: IAEA), 1986.

ICRP. International Commission on Radiological Protection, Oxford: Pergamon Press; ICRP Publication 26, 1977.

ICRU. International Commission on Radiation Units and Measurements, Determination of Dose Equivalents Resulting from External Radiation Sources, Bethesda MD: ICRU; ICRU Report 39, 1985.

Ishiguro, H., Fukuda, S. Personnel Dosimetry System Based on TLD at PNC Toki Works, In Proc. 5th Cong. IRPA. Oxford: Pergammon Press, pp. 76-79, 1980.

Kartha, M. Some Intrinsic Inaccuracies of Thermoluminescent Dosimetry, Health Phys. 20: 431-435,1971.

Kathren, R. Thermal Fogging of Personnel Monitoring Film, Health Phys. 12: 61-63, 1966.

Kathren, R. External Beta-Photon Dosimetry for Radiation Protection, In: The Dosimetry of Ionizing Radiation (Academic Press, Inc.): 321-370, 1987.

Kearfott, K. J., Nabelssi, B. K., Rucker, R. H., Klingler, G. W. Evaluation of Two Thermoluminescent Detection Systems for Medical Imaging Environments, Health Phys. 59: 827-836, 1990 a.

Kearfott, K. J., Grupen-Shemansky, M.E. Design of a Positionally Sensitive LaserHeated Thermoluminescent Detector System, Health Phys. 59: 421-431, 1990 b.

Kearfott, K. J., Grupen-Shemansky, M.E. Positional Radiotherapy Beam Dosimetry Using a Laser Heated Thermoluminescent Plate, Med. Phys. 17: 429-435, $1990 \mathrm{c}$.

Kearfott, K. J., Chesser, J. M., Mitchell, H. E., Coombs, M. A. Apparent Dose Equivalents Resulting from Severe Heating of Film Dosimeters, Health Phys. 60: 597-601, 1991 a.

Kearfott, K.J., Han, S., McMahan, K.L. Sensitivity of a Mixed Field Dosimetry Algorithm to Uncertainties in Thermoluminescent Element Readings, Health Phys., 1991 b (Unpublished).

Kelly, P., Abtahi, A., Braunlich, P. F. Laser-Stimulated Thermoluminescence. II, J. Appl. Phys. 61(2): 738-747, 1987.

Laakmann Electro-Optics, 33051 Calle Aviador, San Juan Capistrano, CA 92675

Landauer Tech Ops, 2 Science Road, Glenwood, Ill 60425.

Mansuripur, M., Connell, N., Goodman, J. W. Laser-Induced Local Heating of Multilayers, Applied Optics, 21:1106-1114, 1982. 
Marshall, M., Docherty, J. Measurement of Skin Dose from Low Energy Beta and Gamma Radiation Using Thermoluminescent Disc, Phys. Med. Biol. 16: 503-510, 1971.

McKeever, S. W. S. Thermoluminescence of in LiF: Analysis of the Glow-Curves, Nucl. Instr. and Meth. 19: 19-20, 1980.

McKeever, S. W. S. Thermoluminescence of Solids, New York: Cambridge University Press, 1985.

Moreau, Y., Gasiot, J., Marah, M., Zangaro, R. Laser Thermoluminescence: Thermal and Optical Aspects, Rad. Prot. Dos. 17: 317-320, 1986.

Moscovitch, M., Chamberlain, Velbeck, K. J. Dose Determination Algorithms for a Nearly Tissue Equivalent Multi-Element Thermoluminescent Dosimeter, ORNL/TM10971, p 48-59, 1988.

NCRP, National Council on Radiation Protection and Measurement, Basic Radiation Protection Criteria, Report 39, NCRP, B̧ethesda, MD, 1971.

Nelson, W.R., Hirayama, H., Rogers, D. W. O. The EGS4 Code System, Stanford Linear Accelerator Center Report SLAC-365, 1985.

Ozisik, M.N. Heat Conduction, John Wiley and Sons, Inc., 1980.

Paliwal, B. R., Almond, P. R. Electron Attenuation Characteristics of LiF, Health Phys. 31: $151-154,1976$.

Panasonic, Matsushita Electric Trading Co., Ltd., P.O. Box 288, Central Osaka, Japan.

Petrov, A.V., Tsypkina, N.S., Seleznev, V.E. High Temperatures-High Pressures 8, 537, 1976.

Quam, W. Beta Dosimetry Using Laser Heating of Hot-Pressed TLD, Health Phys. 44: 75-76, 1983 a.

Quam, W. Beta Dosimetry by Using Pulsed Laser Heating of TL Materials, In Proc. Int. Beta Dosimetry Symp. Washington, D.C.: U.S. Nuclear Regulatory Commission, NUREG/CP-0050, pp. 307-318, 1983 b.

Randall, J. T., Wilkins, M. H. F. Proc. Royal Soc. A184, 347, 366, 390, 1945.

Rayes, I.M., Stoebe, T.G. Personnel Dosimetry Badge System for Mixed Radiation Fields Based on Teflon-Embedded CaSO4:Dy TLD Material, Rad. Prot. Dos. 32: 5-13, 1990.

Sahre, P. A New Method for Discriminating Beta and Gamma Doses in Thick TLDosimeters, Nucl. Instr. and Meth. 217: 525-526, 1983.

Scherpelz, R. I., Endres, G. W. R., Roberson, P. L. The Application of Multi-Element Dosimeter, In Proc. Int. Beta Dos. Symp. Washington, D.C.: U.S. Nuclear Regulatory Commission, NUREG/CP-0050: 209-220 1983. 
Shen, L., Catchen, G. L., Levine, S. H. Experimental and Computational Techniques for Beta-Particle Dosimetry, Health Phys. 53: 37-47, 1987.

Sherbini, S., Sykes , J., Lodd, G., Porter , S.W The Use of Multi-Element TL Dosimeters for Beta and Mixed Beta/Gamma Personnel Monitoring, In Proc. Int. Beta Dosimetry Symp. Washington, D.C.: U.S. Nuclear Regulatory Commission, NUREG/CP-0050, pp. 569-575, 1983.

Sherbini, S., Sykes III, J. , G., Porter Jr., S. Experimental Evaluation of a Method for Performing Personnel $\beta$ dosimetry using Multi-Element Thermoluminescent Dosimeters, Health Phys. 49: 55-64, 1985.

Siemens Gammasonics, Inc., Health Physics Services, 2000 Nuclear Drive, Des Plaines, Illinois 60018-5991.

Stanford, N., McCurdy, D. E. A Single TLD Dose Algorithm to Satisfy Federal Standards and Typical Field Conditions, Health Phys. 58: 691-704, 1990.

SUN, SUN@ SPARC Station 4/370, SUN Microsystems, Inc., 2550 Garcia Ave., Mountain View, California 94043.

Uchrin, G. Beta Dosimetry, Different Solutions, Rad. Prot. Dos. 17: 99-104, 1986.

Young, J. F., Kelly, P., Abtahi, A., Braunlich, P. F. Transient Solution of the Diffusion Equation for a Composite System Heated with a Laser Beam, J. Appl. Phys. 66: 5627-5629, 1989.

Wang, C. K., Blue, T. E. A Neutron Spectrometer for Neutrons with Energies Between 1 $\mathrm{eV}$ and $10 \mathrm{keV}$, Nucl. Instr. and Meth. A290: 237-241, 1990.

Zangaro, R., Gasiot, J., Moreau, Y. CaSO4:Mn PVF2 as a Thermoluminescent Material for Laser Heating, Rad. Prot. Dos. 30: 111-116, 1990. 
APPENDIX A
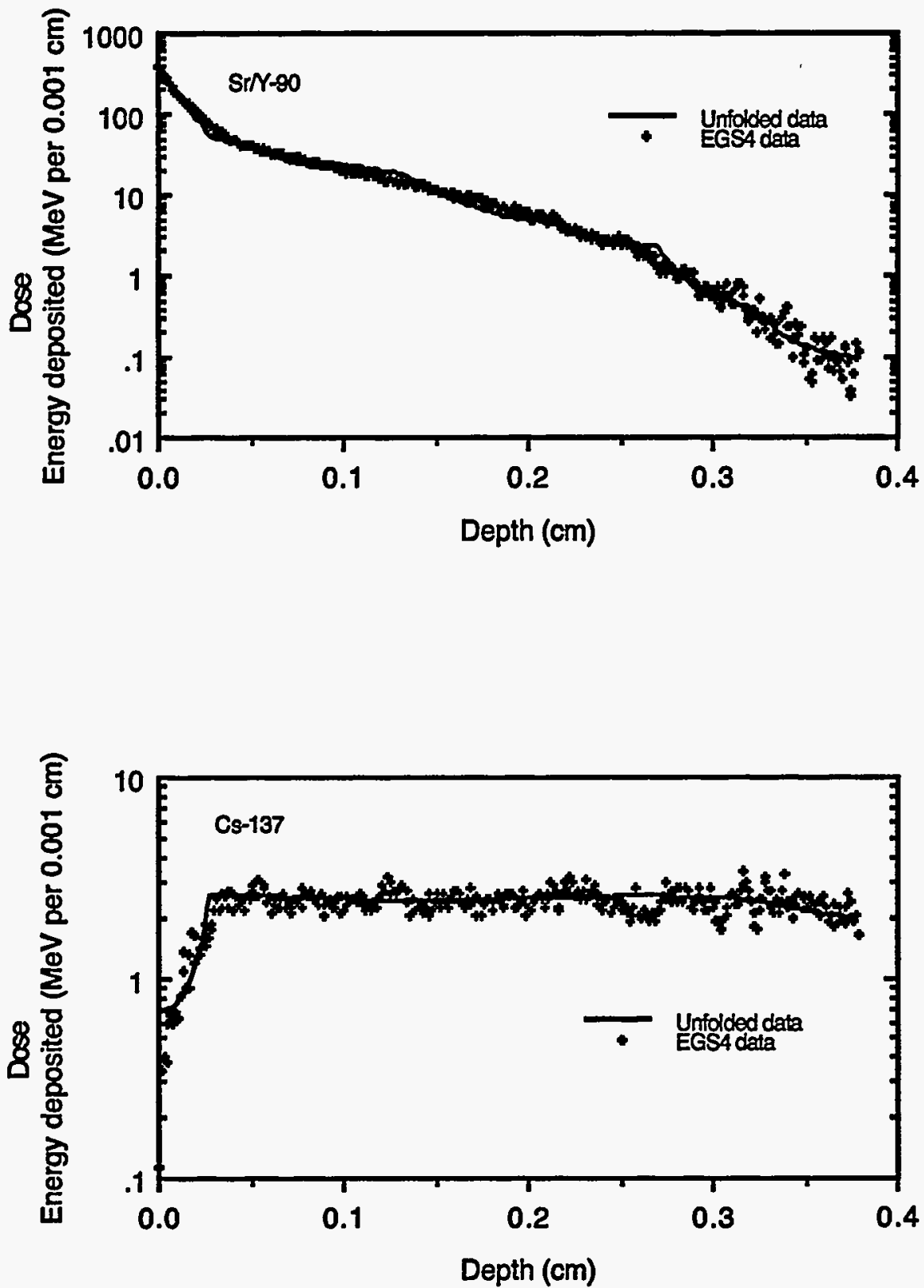

Figure A-1. Unfolded depth-dose of a thick laser TL dosimeter exposed to $\mathrm{Sr} / \mathrm{Y}-90$ beta particles (above) and Cs-137 photons (below) using pulsed heating of uniform laser beam. The pulsed heating scheme includes (a) Top heating (above): a $0.2 \mathrm{sec} 10 \mathrm{~W}$ first pulse, followed by $1.7 \mathrm{sec}$ of cooling, which is then followed by a $1.8 \mathrm{sec} 4 \mathrm{~W}$ second pulse with $2.0 \mathrm{sec}$ of cooling, and then another $3.5 \mathrm{sec} 2 \mathrm{~W}$ third pulse; (a) Bottom heating (below): a $0.6 \mathrm{sec} 10 \mathrm{~W}$ first pulse, followed by $1.3 \mathrm{sec}$ of cooling, which is then followed by a 1.6 $\sec 4 \mathrm{~W}$ second pulse with $2.0 \mathrm{sec}$ of cooling, and then another $3.5 \sec 2 \mathrm{~W}$ third pulse. 

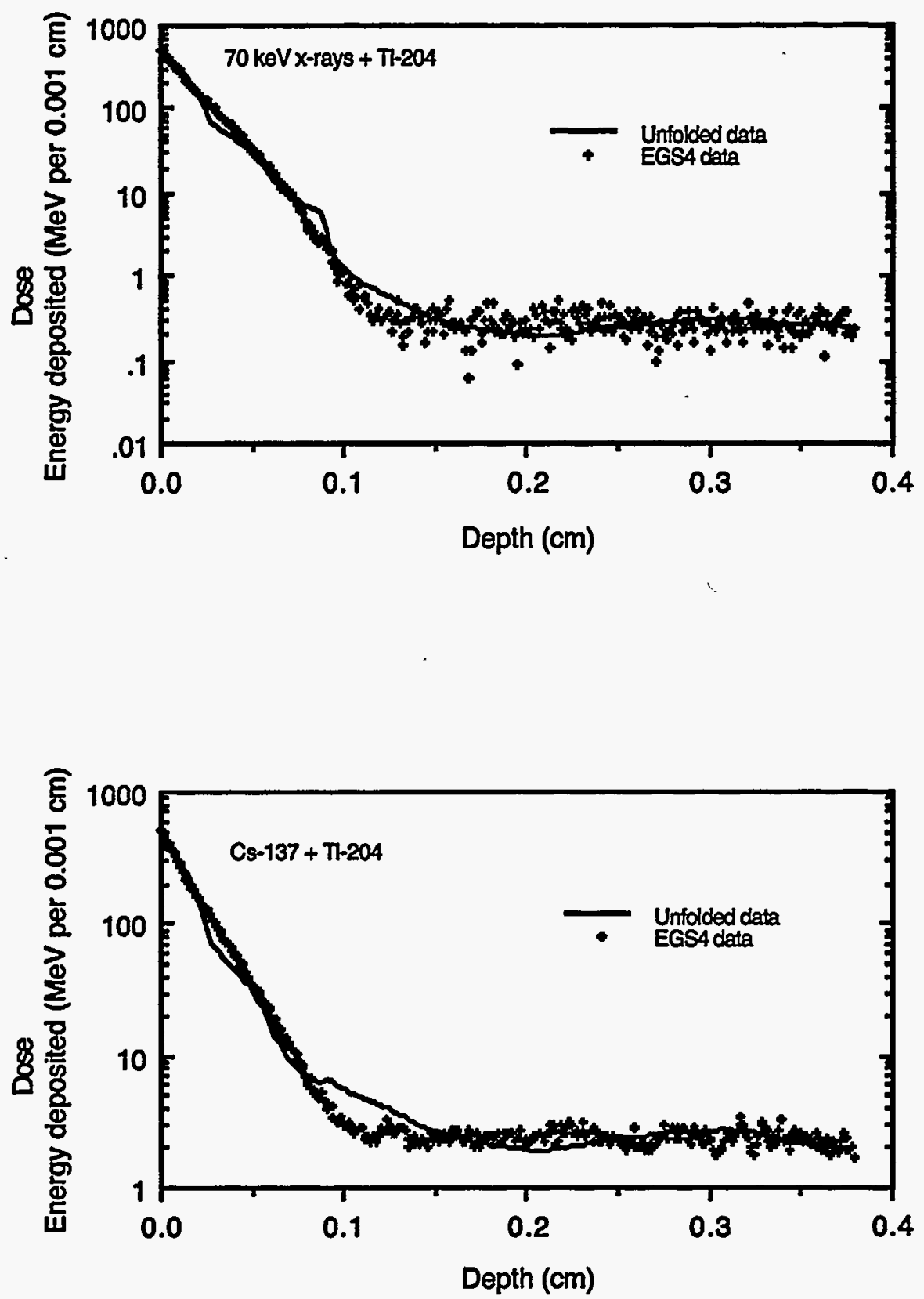

Figure A-2. Unfolded depth-dose of a thick laser TL dosimeter exposed to mixed $70 \mathrm{keV}$ $\mathrm{X}$-rays+T1-204 (above) and Cs-137+T1-204 (below) using pulsed heating of uniform laser beam. The pulsed heating scheme includes (a) Top heating: a $0.2 \mathrm{sec} 10 \mathrm{~W}$ first pulse, followed by $1.7 \mathrm{sec}$ of cooling, which is then followed by a $1.8 \sec 4 \mathrm{~W}$ second pulse with $2.0 \mathrm{sec}$ of cooling, and then another $3.5 \mathrm{sec} 2 \mathrm{~W}$ third pulse; (a) Bottom heating: a $0.6 \mathrm{sec}$ $10 \mathrm{~W}$ first pulse, followed by $1.3 \mathrm{sec}$ of cooling, which is then followed by a $1.6 \sec 4 \mathrm{~W}$ second pulse with $2.0 \mathrm{sec}$ of cooling, and then another $3.5 \sec 2 \mathrm{~W}$ third pulse. 

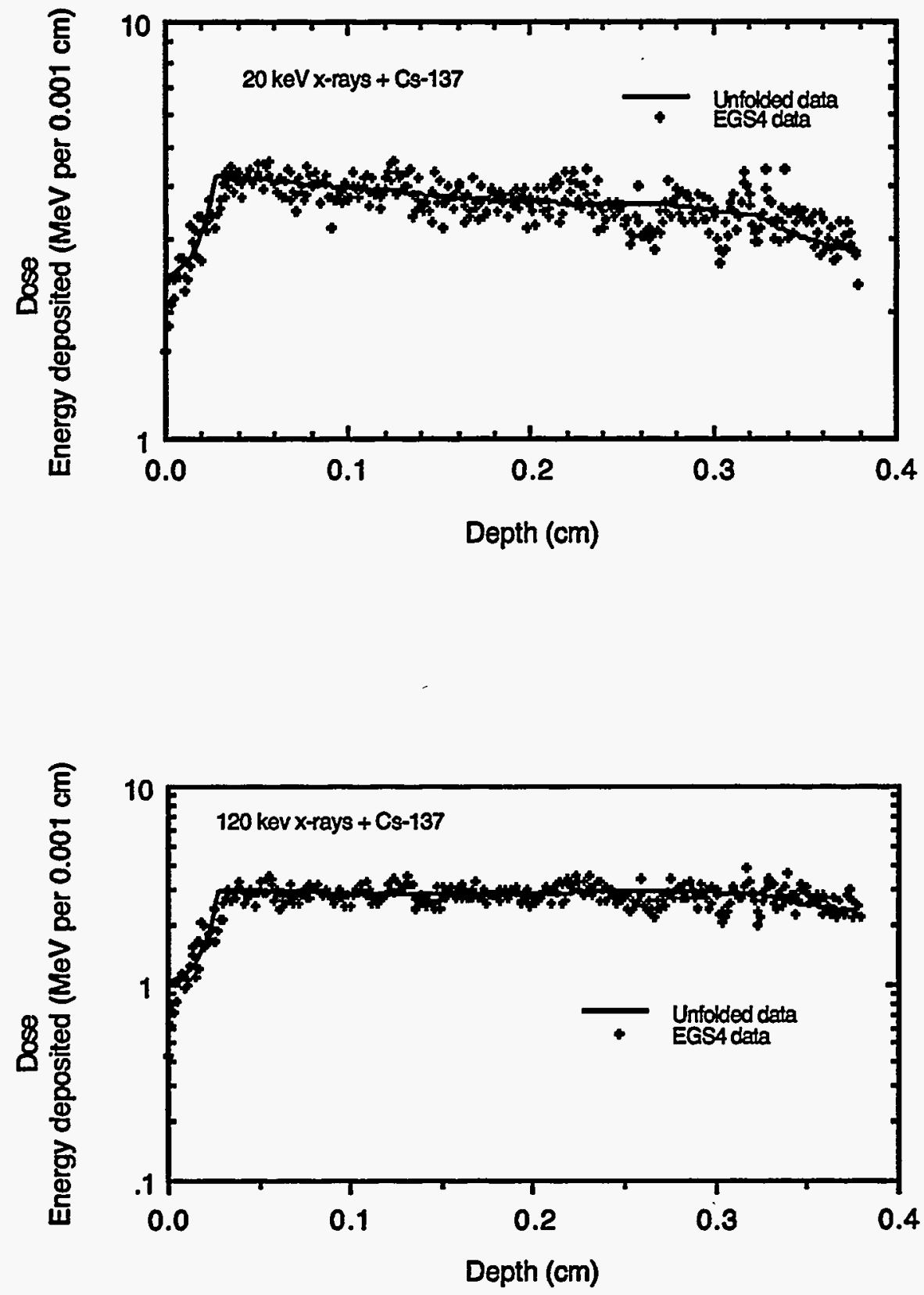

Figure A-3. Unfolded depth-dose of a thick laser TL dosimeter exposed to mixed $20 \mathrm{keV}$ $\mathrm{x}$-rays+Cs-137 (above) and mixed $120 \mathrm{keV} \mathrm{x}$-rays+Cs-137 (below) using pulsed heating of uniform laser beam. The pulsed heating scheme includes (a) Top heating: a $0.2 \mathrm{sec} 10 \mathrm{~W}$ first pulse, followed by $1.7 \mathrm{sec}$ of cooling, which is then followed by a $1.8 \sec 4 \mathrm{~W}$ second pulse with $2.0 \mathrm{sec}$ of cooling, and then another $3.5 \mathrm{sec} 2 \mathrm{~W}$ third pulse; (a) Bottom heating: a $0.6 \mathrm{sec} 10 \mathrm{~W}$ first pulse, followed by $1.3 \mathrm{sec}$ of cooling, which is then followed by a $1.6 \sec 4 \mathrm{~W}$ second pulse with $2.0 \mathrm{sec}$ of cooling, and then another $3.5 \sec 2 \mathrm{~W}$ third pulse. 

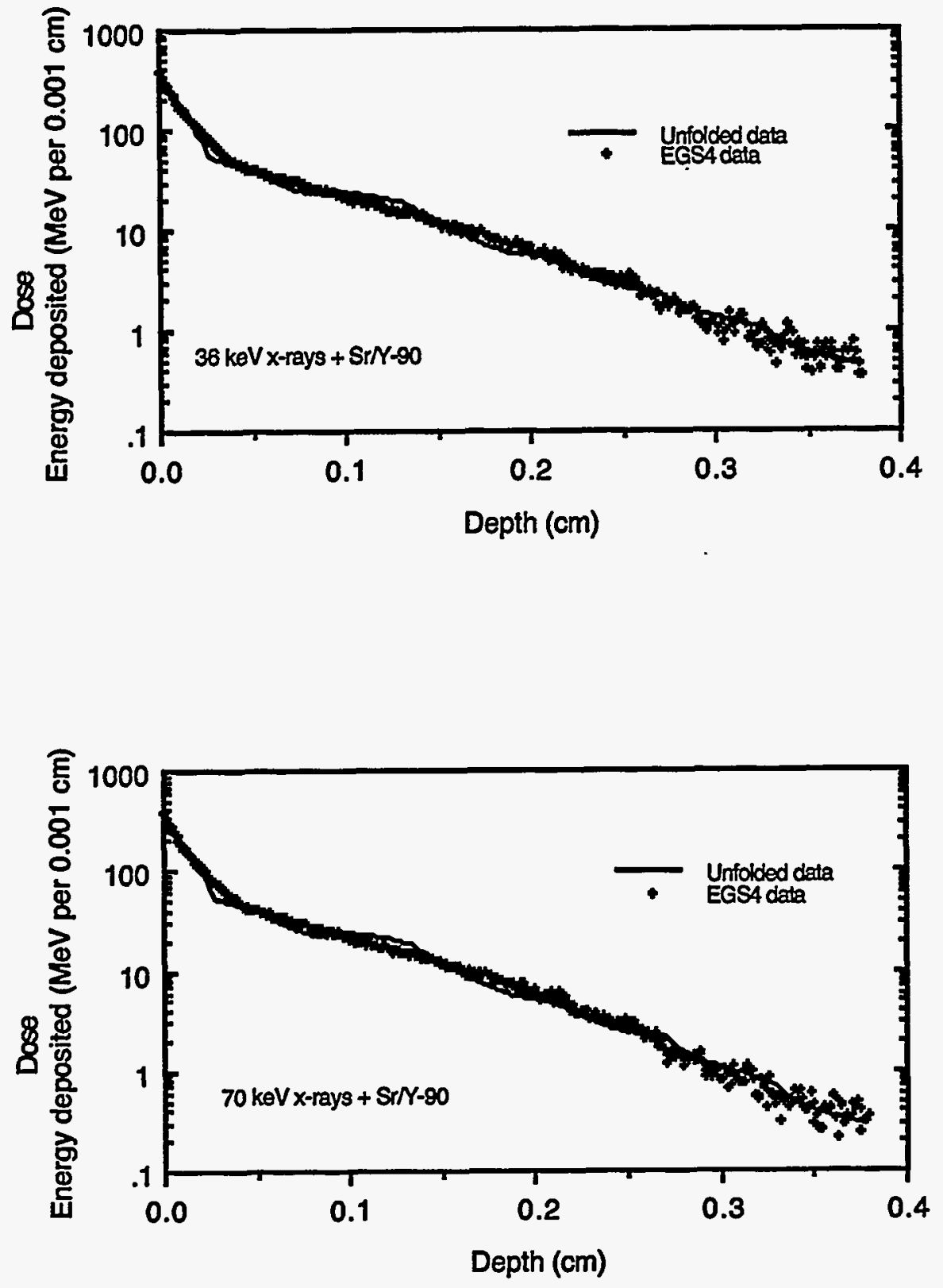

Figure A-4. Unfolded depth-dose of a thick laser TL dosimeter exposed to mixed $36 \mathrm{keV}$ $\mathrm{X}$-rays $+\mathrm{Sr} / \mathrm{Y}-90$ (above) and mixed $70 \mathrm{keV} \mathrm{x}$-rays $+\mathrm{Sr} / \mathrm{Y}-90$ (below) using pulsed heating of uniform laser beam. The pulsed heating scheme includes (a) Top heating: a $0.2 \mathrm{sec} 10 \mathrm{~W}$ first pulse, followed by $1.7 \mathrm{sec}$ of cooling, which is then followed by a $1.8 \mathrm{sec} 4 \mathrm{~W}$ second pulse with $2.0 \mathrm{sec}$ of cooling, and then another $3.5 \sec 2 \mathrm{~W}$ third pulse; (a) Bottom heating: a $0.6 \mathrm{sec} 10 \mathrm{~W}$ first pulse, followed by $1.3 \mathrm{sec}$ of cooling, which is then followed by a $1.6 \sec 4 \mathrm{~W}$ second pulse with $2.0 \mathrm{sec}$ of cooling, and then another $3.5 \sec 2 \mathrm{~W}$ third pulse. 


\section{APPENDIX B}

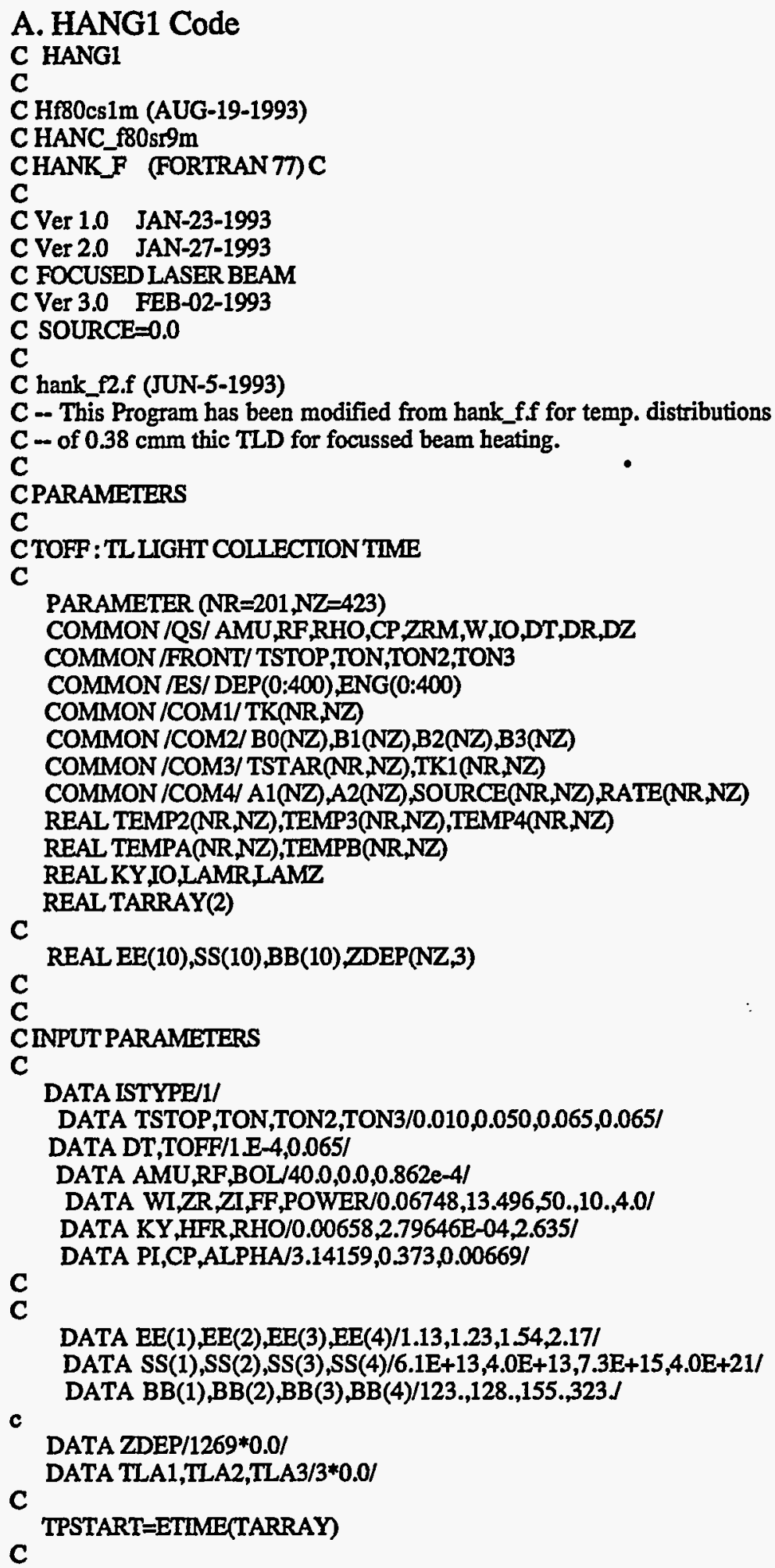




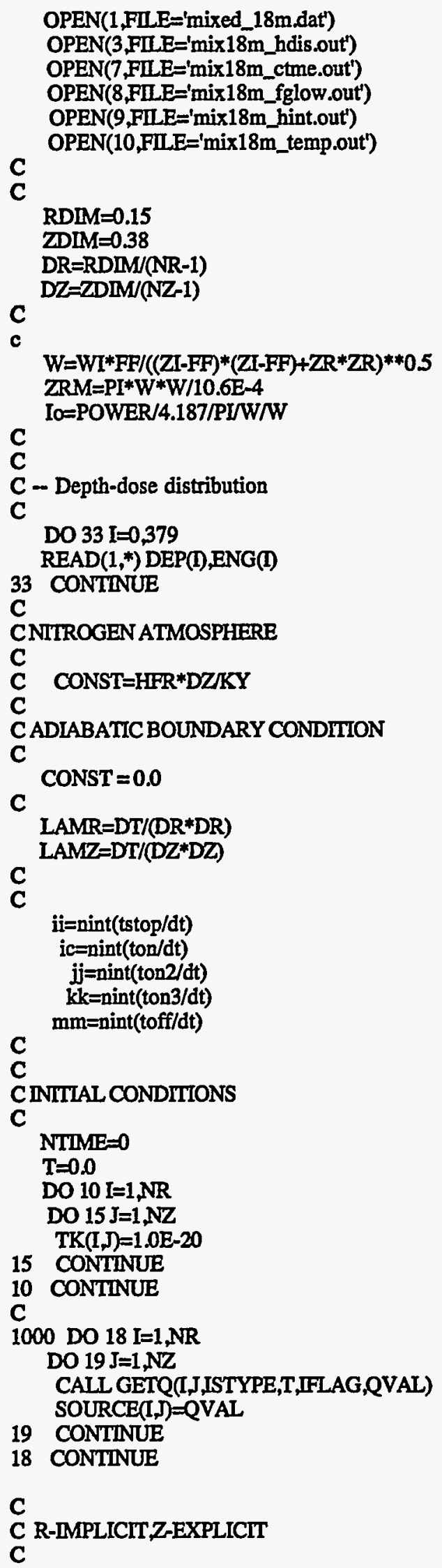


C

$$
\mathrm{BO}(2)=0.0
$$

B1(2) $=2.0 *$ ALPHA $*$ LAMR $/ 3.0+1.0$ B2(2) $=-2.0 *$ ALPHA $*$ LAMR/3.0

$\mathrm{M}=\mathrm{NR}-1$

DO $20 \mathrm{I}=3, \mathrm{M}$

BO(I) $=-L A M R * A L P H A / 2.0+L A M R * A L P H A /(4.0 *(I-1))$

$B 1(\mathrm{I})=\mathrm{LAMR} * A L P H A+1.0$

B2(I) $=-L A M R * A L P H A /(4.0 *(I-1))-L A M R * A L P H A / 2.0$

20 CONTINUE

C

C

$\mathrm{B} 2(\mathrm{NR}-1)=0.0$

CTRIDIA,R-DIRECTION

C

$N=N Z-1$

M=NR-1

$\mathrm{L}=2$

$M A X=N R-1$

$M \mathrm{MIN}=\mathrm{L}+1$

C

LAST $=$ MAX -1

DO $30 \mathrm{~J}=2, \mathrm{~N}$

DO $35 \mathrm{I}=2, \mathrm{M}$

$\mathrm{B} 3(\mathrm{I})=\mathrm{TK}(\mathrm{I}, \mathrm{J})+(\mathrm{ALPHA} * \mathrm{LAMZ} / 2.0) *(\mathrm{TK}(\mathrm{I}, \mathrm{J}+1)-2.0 * \mathrm{TK}(\mathrm{I}, \mathrm{J})$

$1+$ TK $(I, J-1))+$ SOURCE(I,J)

35 CONTINUE

c

C

$A 1(L)=-B 2(L) / B 1(L)$

DO 36 II=MIN,MAX

$\mathrm{A} 1$ (II) $=\mathrm{B} 2$ (II) $/(\mathrm{B} 1$ (II) $+\mathrm{B} 0(\mathrm{II}) * \mathrm{~A} 1$ (II-1))

$\mathrm{A} 2$ (II) $=(\mathrm{B} 3$ (II) $-\mathrm{B} 0(\mathrm{II}) * \mathrm{~A} 2(\mathrm{II}-1)) /(\mathrm{B} 1$ (II) $+\mathrm{B} 0(\mathrm{II}) * \mathrm{~A} 1(\mathrm{II}-1))$

36 CONTINUE

C

C

$\operatorname{TSTAR}(\mathrm{MAX}, \mathrm{J})=\mathbf{A} 2(\mathrm{MAX})$

DO $37 \mathrm{KK}=1, \mathrm{LAST}$

$\mathrm{I}=\mathrm{MAX}-\mathrm{KK}$

$\operatorname{TSTAR}(\mathrm{I}, \mathrm{J})=\mathrm{A} 2(\mathrm{I})+\mathrm{A} 1(\mathrm{I}) * \operatorname{TSTAR}(\mathrm{I}+1, \mathrm{~J})$

37 CONTINUE

30 CONTINUE

C

CBOUNDARY CONDITIONS

C

DO $45 \mathrm{I}=1, \mathrm{NR}$

TSTAR $(I, N Z)=T S T A R(I, N Z-1) /(1.0+$ CONST $)$

$\operatorname{TSTAR}(1,1)=\operatorname{TSTAR}(1,2) /(1.0-\operatorname{CONST})$

45 CONIINUE

C

$\mathrm{M}=\mathrm{NZ}-1$

C

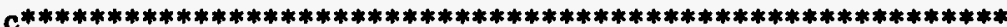

DO $50 \mathrm{~J}=2, \mathrm{M}$

TSTAR(NR, $)=0.0$

$\operatorname{TSTAR}(1, \mathrm{~J})=4.0 * \operatorname{TSTAR}(2, \mathrm{~J}) / 3.0-\operatorname{TSTAR}(3, \mathrm{~J}) / 3.0$

50 CONIINUE

C

CZ-IMPLICIT,R-EXPLICIT

C

$\mathrm{BO}(2)=0.0$

B1(2) $=($ ALPHA $*$ LAMZ) $/(2.0 *(1.0-$ CONST $))-A L P H A * L A M Z-1.0$

B2(2)=ALPHA*LAMZ/2.0 


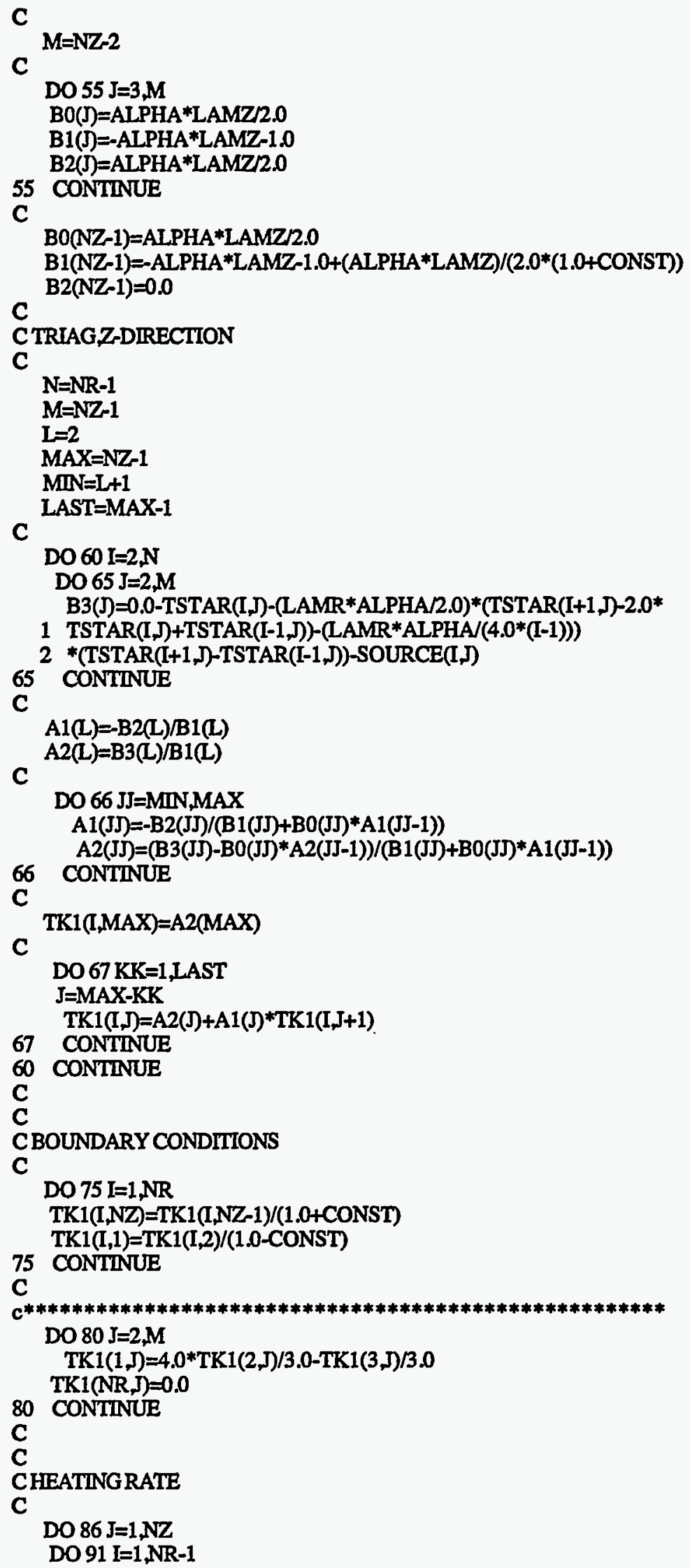


RATE $(1, J)=(\operatorname{TK} 1(I, J)-T K(I, J)) / D T$

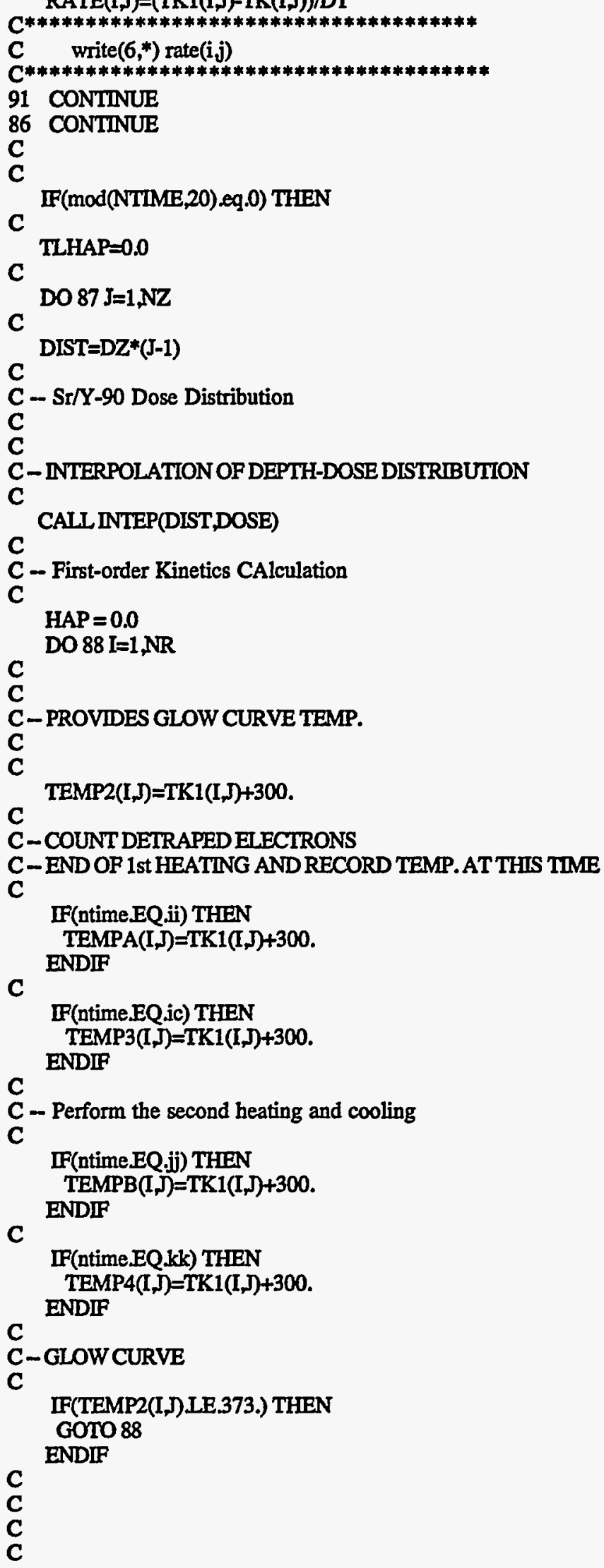


C-TL MUST NOT GENERATE WHENLESS THAN TEMP3 WHIIE C-THE SECOND HEATING IS PERFORMED.

C

C

IF(IFLAG.EQ.1 AND. TEMP2(I,J)LE.TEMPA(I,J)) then GOTO 88

C ENDIF

C-TL MUST NOT GENERATE WHEN LESS THAN TEMP3 WHIIE C-THE SECOND HEATING IS PERFORMED.

C

IF(IFLAG.EQ 2 AND. TEMP2(I,J)ILE.TEMP3(I,J).OR.

1 IFLAG.EQ2 AND. TEMP2(I,J).LE.TEMPA(IJ)) THEN GOTO 88

C ENDIF

C-TL MUST NOT GENERATE WHEN LESS THAN TEMP3 WHIIL C - THE SECOND HEATING IS PERFORMED.

C

IF(IFLAG.EQ.3 .AND. TEMP2(I,J)LE.TEMPB(I,J).OR.

1 IFLAG.EQ.3 AND. TEMP2(I).LE.TEMP3(I).OR.

2 IFLAG.EQ.3 AND. TEMP2(I,) ILE.TEMPA(I)) THEN GOTO 88

C ENDIF

C

C-TLMUST NOT GENERATE WHEN LESS THAN TEMP4 WHILE C-THE THIRD HEATING IS PERFORMED.

C

IF(IFLAG.EQ.4 AND. TEMP2(I)ILE.TEMP4(I,).OR.

1 IFLAG.EQ.4 AND. TEMP2(IJ).LE.TEMPB(I).OR.

2 IFLAG.EQ.4 AND. TEMP2(I,J) IE.TEMP3(I,J).OR.

3 IFLAG.EQ.4 AND. TEMP2(I,J)LE.TEMPA(I,)) THEN GOTO 88

C

ENDIF

C

C

C - WILKINS-RANDALL FIRST KINETICMODEL

DELTI $=0.0$

DO $115 \mathrm{M}=1,4$

$\mathrm{DD}=\mathrm{EE}(\mathrm{M}) /(\mathrm{BOL} * \mathrm{TEMP} 2(\mathrm{I}, \mathrm{J}))$

IF(RATE(I,J).LE.0.0) GOTO 115

$\mathrm{FAC}=1.0-2 / \mathrm{DD}+6 /(\mathrm{DD} * \mathrm{DD})-24 /(\mathrm{DD} * \mathrm{DD} * \mathrm{DD})$

HTEM=SS(M)*TEMP2(I,J)/DD*EXP(-DD)*FAC/RATE(I,J)

DELTI=DEITI+SS(M)*BB(M)*EXP(-DD)*EXP(-HTEM)

115 CONTINUE

C

IF(i.EQ.1) THEN

$\mathrm{TL}=\mathrm{PI} * \mathrm{DR} * \mathrm{DR} / 4 . * \mathrm{DZ} * \mathrm{DELTI}$

ELSEIF(i.EQ.1.AND.jEQ.1) THEN

$T L=P I * D R * D R / 8 . * D Z * D E L T I$

C

ELSEIF(j.EQNZ.OR.iEQNR) THEN

$\mathrm{TL}=2.0 * \mathrm{PI} *(\mathrm{DR} / 2 .+(\mathrm{i}-2) * \mathrm{DR}) * \mathrm{DR} * \mathrm{DZ} * 0.5 * \mathrm{DELTI}$

ELSE

c 
c

$\mathrm{TL}=2.0 * \mathrm{PI} *(\mathrm{DR} / 2 .+(\mathrm{i}-2) * \mathrm{DR}) * \mathrm{DR} * \mathrm{DZ} * \mathrm{DELTI}$

ENDIF

c

C

HAP=HAP+TL*DOSE

C

CGLOW CURVEDISTRIBUTION

C $\quad G L(I, J, K)=T L$

c

88 CONTINUE

C

C

IF(IFLAG.EQ.0.OR.IFLAG.EQ.1) THEN

ZDEP(J,1)=ZDEP(J,1)+HAP

TLA1=TLA1+HAP

ENDIF

C

IF(IFLAGEQ.2.OR.IFLAG.EQ.3) THEN

ZDEP(J, 2) $=$ ZDEP(J,2)+HAP

TLA2 $=$ TLA2+HAP

ENDIF

C

IF(IFLAGEQ.4) THEN

$\mathrm{ZDEP}(\mathrm{J}, 3)=\mathrm{ZDEP}(\mathrm{J}, 3)+\mathrm{HAP}$

TLA3=TLA3+HAP

ENDIF

C

TLHAP $=$ TLHAP+HAP

C

87 CONTINUE

C

CDIFFERENTIAL GLOW CURVE WITH TIME

C

WRITE $(8,180)$ T,TLHAP

180 FORMAT(5X,F10.5,' ',1PE12.3)

C

C

ENDIF

C SET PRESENT TO PAST TEMPS. FOR NEXT TIME STEP

C

DO $85 \mathrm{~J}=1, \mathrm{NZ}$

DO $90 \mathrm{I}=1, \mathrm{NR}$

$\operatorname{TK}(I, J)=$ TK1 $(I$,

90 CONTINUE

85 CONTINUE

C

COUTPUT

C

NTIME=NTIME+1

T=NTIME*DT

IF(T LE.TOFF) GOTO 1000

c

DO $16 \mathrm{~J}=1,80$

WRITE(3,102) (J-1)*DZZDEP(J,1),ZDEP(J,2),ZDEP(J,3)

16 CONTINUE

C

WRITE $(9,101)$ TLA1,TLA2,TLA3

C WRITE(10,*) TK1(1,1)+300.

101 format(10x,3(/,1PE12.3)) 


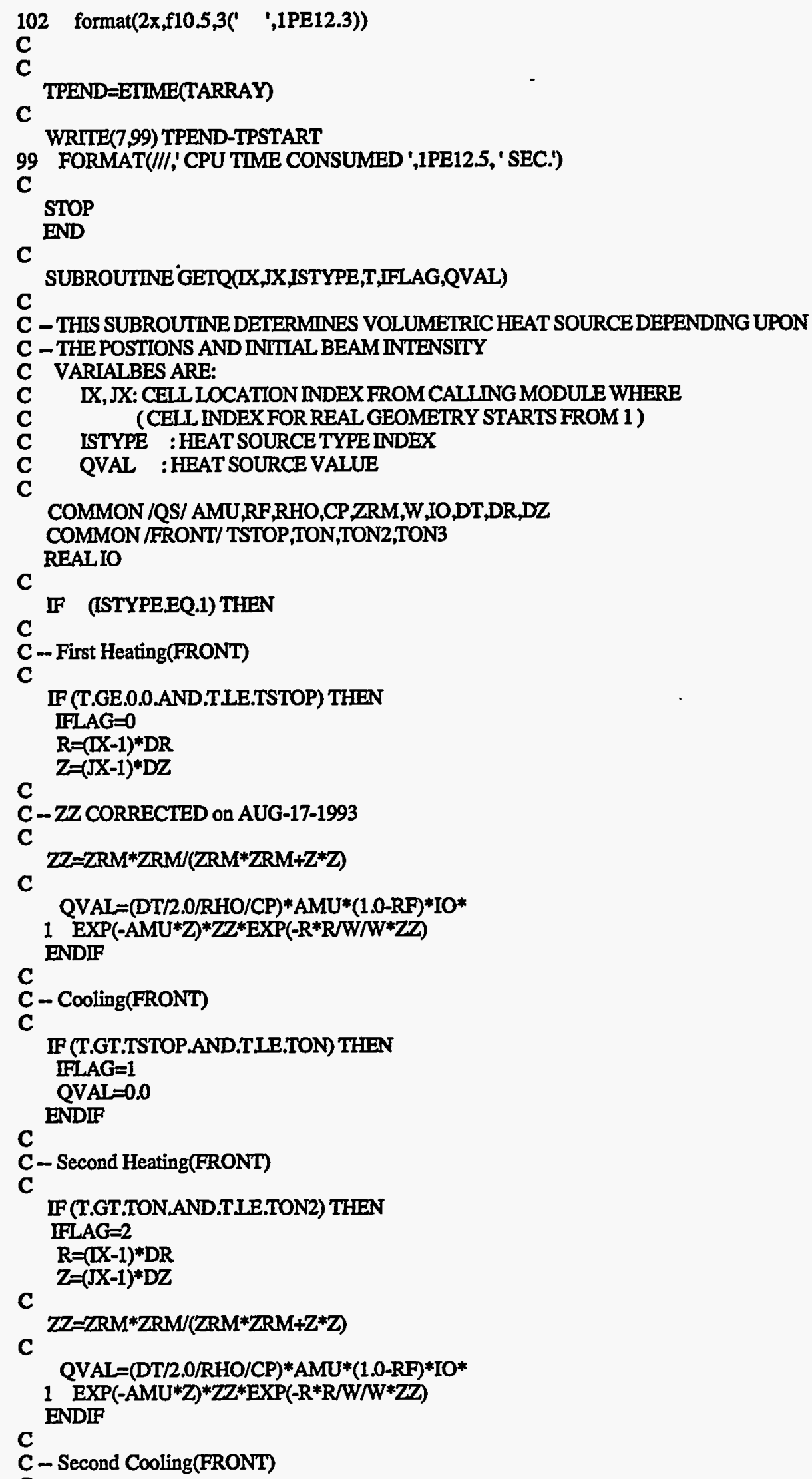




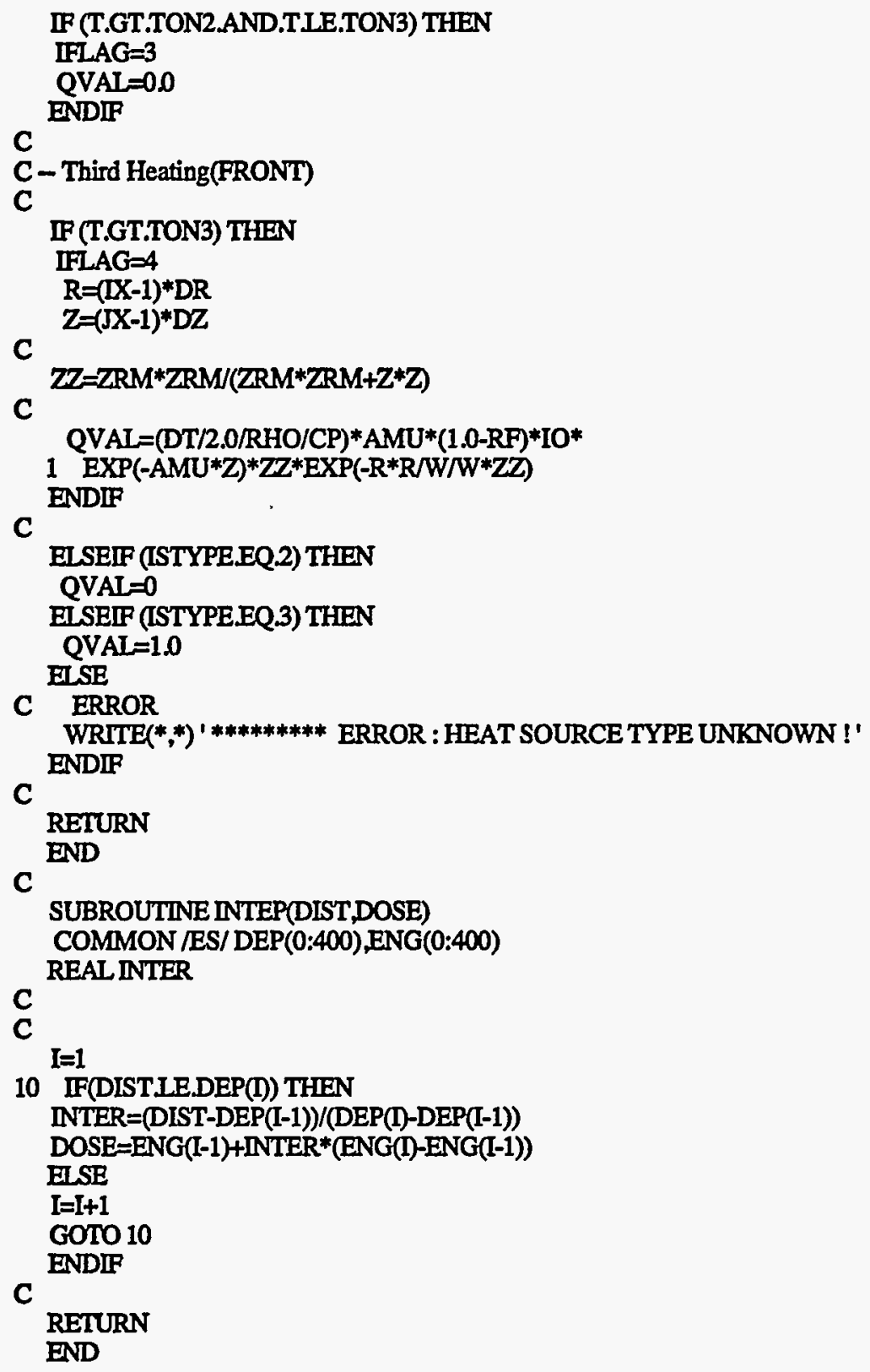




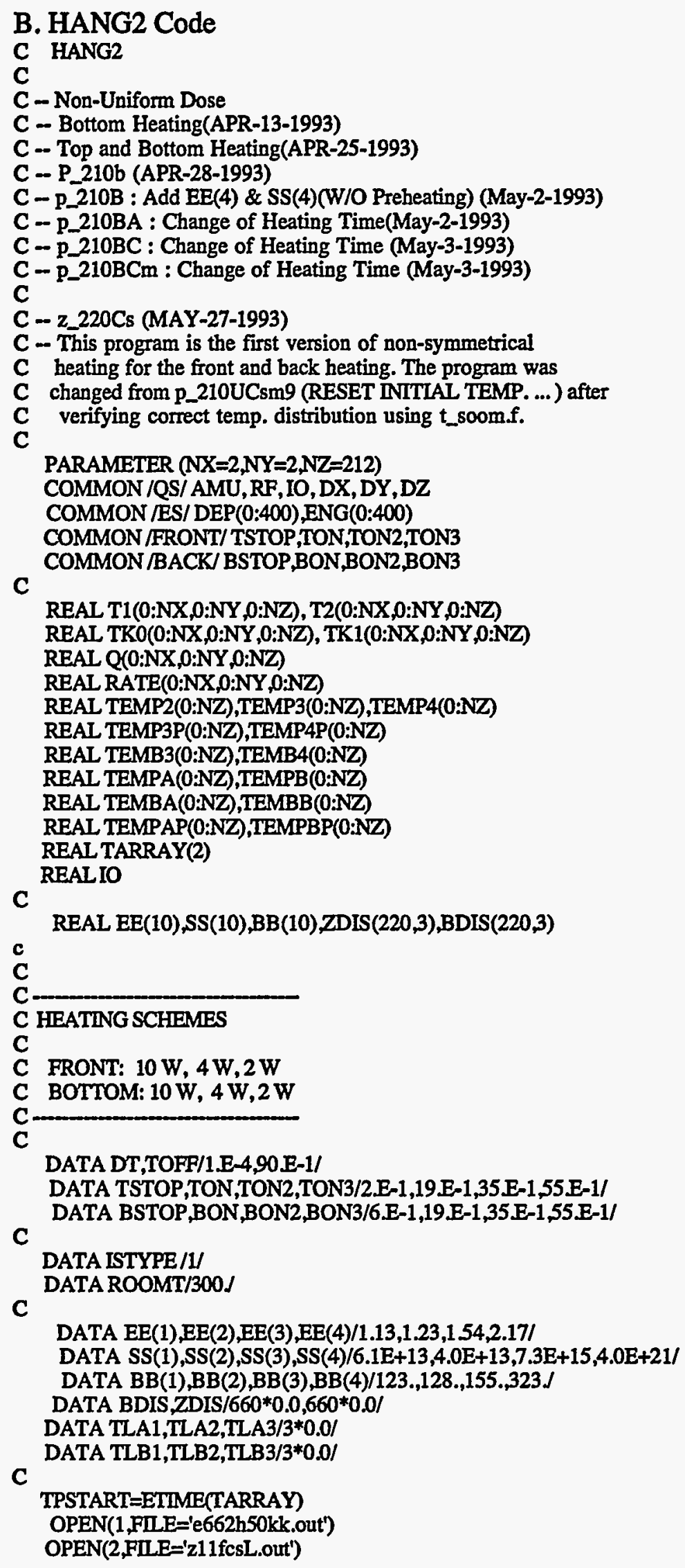




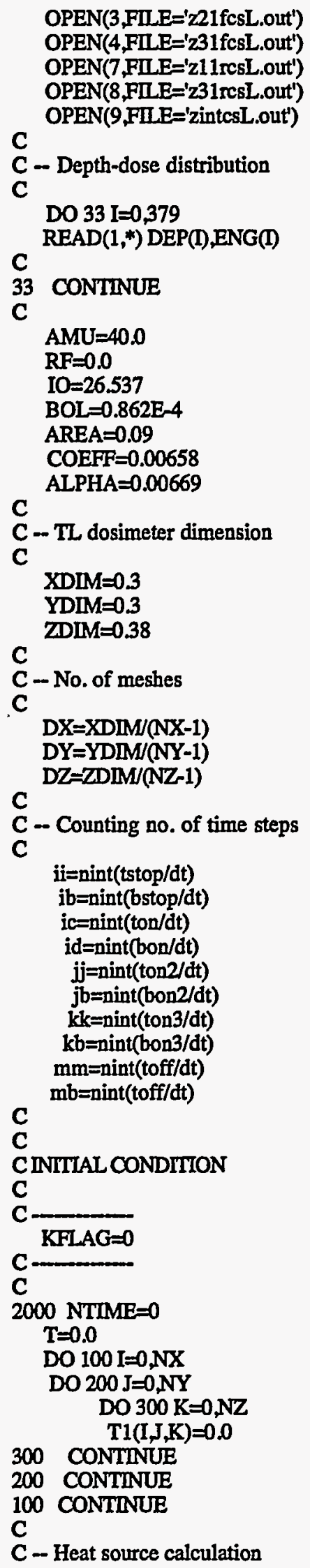


C

1000 DO $110 \mathrm{I}=0, \mathrm{NX}$

DO $210 \mathrm{~J}=0, \mathrm{NY}$

DO $310 \mathrm{~K}=0, \mathrm{NZ}$

CALL GETQ(I,J,K,ISTYPE,T,KFLAG,IFLAG,QVAL)

310 CONTINUE

210 CONTINUE

110 CONTINUE

C

C - Explicit technique

C

DO $10 \mathrm{I}=1, \mathrm{NX}-1$

DO $20 \mathrm{~J}=1, \mathrm{NY}-1$

DO $30 \mathrm{~K}=1, \mathrm{NZ}-1$

$\mathrm{T} 2(\mathrm{I}, \mathrm{J}, \mathrm{K})=\left(\left(\mathrm{T} 1(\mathrm{I}+1, \mathrm{~J}, \mathrm{~K})+\mathrm{T} 1(\mathrm{I}-1, \mathrm{~J}, \mathrm{~K})-2.0^{*}\right.\right.$

$1 \mathrm{~T} 1(\mathrm{I} J, \mathrm{~K}))(\mathrm{DX} * \mathrm{DX})+(\mathrm{T} 1(\mathrm{I}, \mathrm{J}+1, \mathrm{~K})+\mathrm{T} 1(\mathrm{I}, \mathrm{J}-1, \mathrm{~K})$

$2 \quad-2 . * \mathrm{~T} 1(\mathrm{I}, \mathrm{J}, \mathrm{K})) /(\mathrm{DY} * \mathrm{DY})+(\mathrm{T} 1(\mathrm{I}, \mathrm{J}, \mathrm{K}+1)+\mathrm{T} 1(\mathrm{I}, \mathrm{J}, \mathrm{K}-1)$

$3-2 . * \mathrm{~T} 1(\mathrm{I}, J, \mathrm{~K}))(\mathrm{DZ} * \mathrm{DZ})+1, J \mathrm{COEFF} * \mathrm{Q}(\mathrm{I}, J, \mathrm{~K})) * A L P H A$

$4 \quad$ *DT+T1 $(I, J, K)$

$\operatorname{RATE}(\mathrm{I}, \mathrm{J}, \mathrm{K})=\mathrm{ABS}((\mathrm{T} 2(\mathrm{I}, \mathrm{J}, \mathrm{K})-\mathrm{T} 1(\mathrm{I}, \mathrm{J}, \mathrm{K})) / \mathrm{DT})$

30 CONTINUE

20 CONTINUE

10 CONTINUE

C

C-WRITE TEMPERATURE PROFILE

C

IF(KFLAG.eq.0) then

IF(ntime.eq.ii.or.ntime.eq.j.j.or.ntime.eq.mm) then

WRITE(2,'(3X,E12,2,A)') T 'SEC'

DO $7 \mathrm{~K}=1, \mathrm{NZ}-1,2$

DO $5 \mathrm{I}=1, \mathrm{NX}-1,20$

C

DO $6 \mathrm{~J}=1, \mathrm{NY}-1,10$

C - Front

C

TEMP $=$ T1 $(\mathrm{I}, \mathrm{J}, \mathrm{K})+\mathrm{ROOMT}$

write $(2,102) \mathrm{k}^{*} \mathrm{dz}, \mathrm{TEMP}$

6 CONTINUE

5 CONTINUE

7 CONTINUE

ENDIF

C

ENDIF

IF(KFLAG.EQ.1) THEN

IF(ntime.eq.ib.or.ntime.eq.jb.or.ntime.eq.mb) then

WRITE(7,'(3X,E12.2,A)') T 'SEC'

DO $81 \mathrm{~K}=1, \mathrm{NZ}-1,2$

DO $82 \mathrm{I}=1, \mathrm{NX}-1,20$

C

DO $83 \mathrm{~J}=1, \mathrm{NY}-1,10$

C.-Back

C

$m=n z-k$

TEMP $=T 1(I, J, m)+R O O M T$

write $(7,102) k^{*} d z$, TEMP

C

83 CONTINUE

82 CONTINUE

81 CONTINUE

ENDIF

ENDIF

C 
C-DONEXT TIME STEP

C

NTIME $=$ NTIME+1

C $T=N T I M E * D T$

C-ASSIGNE CALCULATED TEMPERATURE VALUES

C - AS THOSE FOR PREVIOUS TIME STEP

C

DO $15 \mathrm{I}=1, \mathrm{NX}-1$

DO $25 \mathrm{~J}=1, \mathrm{NY}-1$

DO $35 \mathrm{~K}=1, \mathrm{NZ}-1$

C-ASSIGN

C

C

$T 1(I, J, K)=T 2(I, J, K)$

35 CONTINUE

25 CONTINUE

15 CONTINUE

C

C -- Boundary conditions

C

DO $40 \mathrm{~K}=0, \mathrm{NZ}, \mathrm{NZ}$

DO $45 \mathrm{I}=0, \mathrm{NX}$

DO $50 \mathrm{~J}=0, \mathrm{NY}$

IF(KEQ.0) THEN

$\mathrm{T} 1(\mathrm{I}, J, \mathrm{~K})=\mathrm{T} 1(\mathrm{I}, \mathrm{J}, \mathrm{K}+1)$

ELSE

$\mathrm{T} 1(\mathrm{I}, \mathrm{J}, \mathrm{K})=\mathrm{T} 1(\mathrm{I}, \mathrm{J}, \mathrm{K}-1)$

ENDIF

50 CONTINUE

45 CONTINUE

40 CONTINUE

C

DO $60 \mathrm{~J}=0, N Y, N Y$

DO $65 \mathrm{I}=0, \mathrm{NX}$

DO $70 \mathrm{~K}=0, \mathrm{NZ}$

IF(J.EQ.0) THEN

$\mathrm{T} 1(\mathrm{I}, \mathrm{J}, \mathrm{K})=\mathrm{T} 1(\mathrm{I}, \mathrm{J}+1, \mathrm{~K})$

EISE

$T 1(I, J, K)=T 1(I, J-1, K)$

ENDIP

70 CONTINUE

65 CONTINUE

60 CONTINUE

C

DO $75 \mathrm{I}=0, \mathrm{NX}, \mathrm{NX}$

DO $80 \mathrm{~J}=0, \mathrm{NY}$

DO $85 \mathrm{~K}=0, \mathrm{NZ}$

IF(I.EQ.0) THEN

$\mathrm{T} 1(\mathrm{I}, J, \mathrm{~K})=\mathrm{T} 1(\mathrm{I}+1, \mathrm{~J}, \mathrm{~K})$

ELSE

$T 1(I, J, K)=T 1(I-1, J, K)$

85 CONTINUE

80 CONTINUE

75 CONTINUE

C

C-END OF ASSIGNMENT

C

C-RESET INITLAL TEMP. FOR BOTTOM and TOP

C

DO $19 \mathrm{I}=0, \mathrm{NX}$

DO $24 \mathrm{~J}=0, \mathrm{NY}$ 


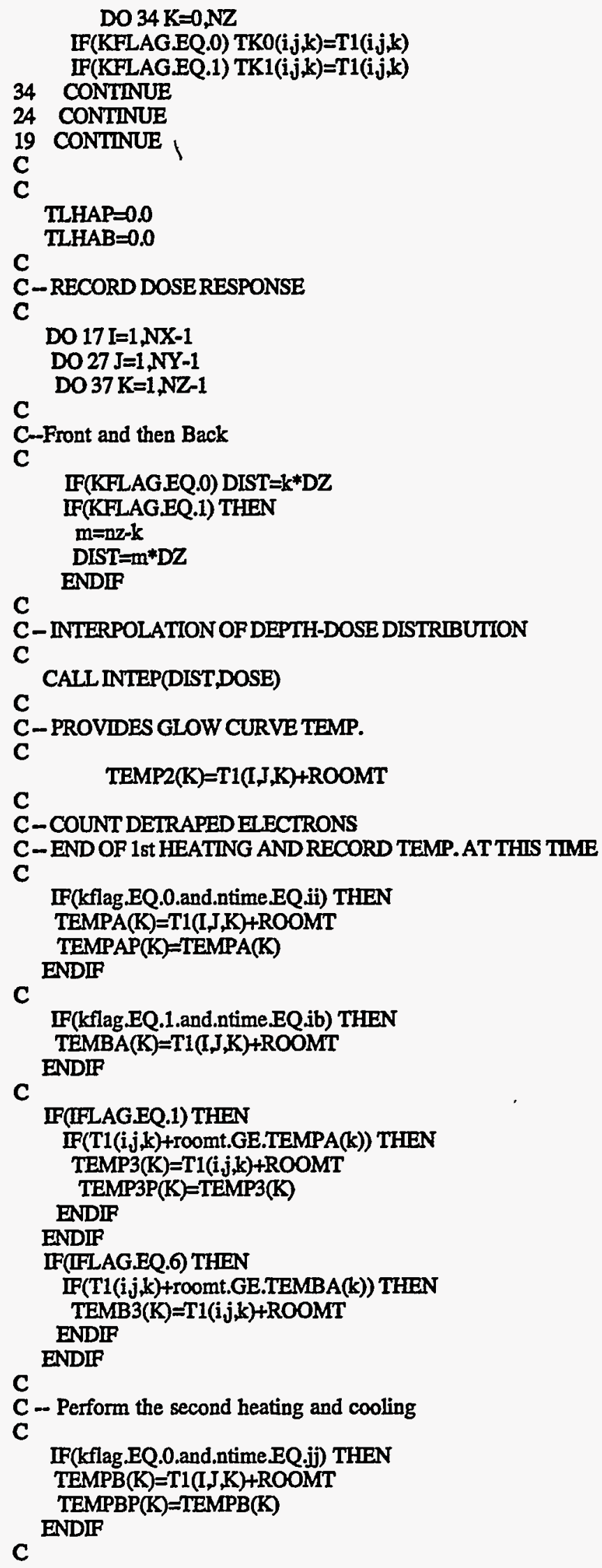

DO $34 \mathrm{~K}=0, \mathrm{NZ}$ 
C

IF(kflag.EQ.1.and.ntime.EQ.jb) THEN

$\operatorname{TEMBB}(\mathrm{K})=\mathrm{T} 1(\mathrm{I}, \mathrm{J}, \mathrm{K})+\mathrm{ROOMT}$

ENDIF

IF(IFLAGEQ3) THEN

IF(T1(i,j,k)+roomt.GE.TEMPB(k)) THEN

TEMP4(K) $=\mathrm{T} 1(\mathrm{i}, \mathrm{j}, \mathrm{k})+\mathrm{ROOMT}$

TEMP4P(K)=TEMP4(K)

ENDIF

ENDIF

IF(IFLAGEQ.8) THEN

IF(T1 $(\mathrm{i}, \mathrm{j}, \mathrm{k})+$ +roomt.GE.TEMBB(k)) THEN

TEMB4(K)=T1(ij,k)+ROOMT

ENDIF

ENDIF

C

C-GLOWCURVE

C

IF(TEMP2(K)LE.373.) THEN GOTO 37

C ENDIF

C

IF(KFLAGEQ.1) GOTO 38

C-TL MUST NOT GENERATE WHEN LESS THAN TEMP3 WHIIE

C-COOLING.

C

C

IF(IFLAG.EQ.1 AND. TEMP2(K).LE.TEMPA(K)) THEN GOTO 37

C ENDIF

C-TL MUST NOT GENERATE WHEN LESS THAN TEMP3 WHILE

C-THE SECOND HEATING IS PERFORMED.

C

IF(IFLAG.EQ.2 AND. TEMP2(K).LE.TEMP3(K).OR.

1 IFLAG.EQ.2 .AND. TEMP2(K)LE.TEMPA(k)) THEN GOTO 37

C ENDIF

C-TL MUSTNOT GENERATE WHENLESS THAN TEMP4 WHIIE

C-THE SECOND COOLING IS PERFORMED.

C

IF(IFLAG.EQ.3 AND. TEMP2(K)LE.TEMPB(K).OR.

1 IFLAGEQ.3 AND. TEMP2(K) LE.TEMP3(K).OR.

2 IFLAG.EQ3 .AND.TEMP2(K)LE.TEMPA(K)) THEN GOTO 37

C

ENDIF

C-TL MUST NOT GENERATE WHENLESS THAN TEMP4 WHIIE

C-THE THIRD HEATING IS PERFORMED.

C

IF(IFLAG.EQ.4 .AND. TEMP2(K).LE.TEMP4(K).OR.

1 IFLAG.EQ.4 AND. TEMP2(K).LE.TEMPB(K).OR.

2 IFLAG.EQ.4 AND. TEMP2(K)ILE.TEMP3(K).OR.

3 IFLAG.EQ.4 .AND. TEMP2(K).LE.TEMPA(K)) THEN GOTO 37

C ENDIF

C

GOTO 55

C -- Bottom heating

C

$38 m=n z-k$ 


\section{C}

IF(IFLAG.EQ.6 .AND. TEMP2(K).LE.TEMBA(K).OR.

1 IFLAG.EQ.6 AND. TEMP2(K) LE.TEMPAP(m)) THEN GOTO 37

C

ENDIF

IF(IFLAG.EQ.7 AND. TEMP2(K)LE.TEMB3(K).OR.

1 IFLAG.EQ.7 .AND. TEMP2(K) LE.TEMBA(K).OR.

2 IFLAG.EQ.7 AND. TEMP2(K).LE.TEMP3P(m).OR.

3 IFLAGEQ.7 AND. TEMP2(K) LE.TEMPAP(m)) THEN GOTO 37

C ENDIF

IF(IFLAG.EQ.8 .AND. TEMP2(K).LE.TEMBB(K).OR.

1 IFLAG.EQ.8 AND. TEMP2(K).LE.TEMB3(K).OR.

2 IFLAG.EQ.8 AND. TEMP2(K).LE.TEMBA(K).OR.

3 IFLAGEQ.8 AND. TEMP2(K).LE.TEMPBP(m).OR.

4 IFLAGEQ.8 AND. TEMP2(K) LE.TEMP3P(m).OR.

5 IFLAG.EQ.8 AND. TEMP2(K) LE.TEMPAP(m)) THEN GOTO 37

C ENDIF

IF(IFLAG.EQ.9 AND. TEMP2(K)LE.TEMB4(K).OR. 1 IFLAG.EQ.9 AND. TEMP2(K) LE.TEMBB(K).OR.

2 IFLAG.EQ.9 AND. TEMP2(K) LE.TEMB3(K).OR.

3 IFLAG.EQ.9 AND. TEMP2(K).LE.TEMBA(K).OR.

4 IFLAG.EQ.9 .AND. TEMP2(K)LE.TEMP4P(m).OR.

5 IFLAG.EQ.9 AND. TEMP2(K) LE.TEMPBP(m).OR.

6 IFLAG.EQ.9 AND. TEMP2(K)LE.TEMP3P(m).OR.

7 IFLAGEQ.9 AND. TEMP2(K) LE.TEMPAP(m)) THEN GOTO 37

C

ENDIF

C - WILKINS-RANDALL FIRST KINETICMODEI

C

55 DELTI $=0.0$

DO $14 M=14$

$\mathrm{DD}=\mathrm{EE}(\mathrm{M}) /(\mathrm{BOL} * \mathrm{TEMP} 2(\mathrm{~K}))$

$\mathrm{FAC}=1.0-2 / \mathrm{DD}+6 /(\mathrm{DD} * \mathrm{DD})-24 /(\mathrm{DD} * \mathrm{DD} * \mathrm{DD})$

HH=SS(M)*TEMP2(K)/DD*EXP(-DD)*FACRATE(I, J,K)

DELTI $=D E L T I+E X P(-H H) * S S(M) * B B(M) * E X P(-D D)$

14 CONTINUE

C

C

$\mathrm{TL}=\mathrm{DZ} * A R E A * D E L T$

c

IF(iflag.eq.0.or.iflag.eq.1) then ZDIS(K, 1)=ZDIS $(K, 1)+T L * D O S E$

TLA1=TLA1+TL*DOSE

ENDIF

IF(iflag.eq.5.or.iflag.eq.6) then

BDIS $(K, 1)=B D I S(K, 1)+T L * D O S E$

TLB1=TLB1+TL*DOSE

ENDIP

IF(iflag.eq.2.or.iflag.eq.3) then $\mathrm{ZDIS}(\mathrm{K}, 2)=\mathrm{ZDIS}(\mathrm{K}, 2)+\mathrm{TL} * \mathrm{DOSE}$

TLA2 $=$ TLA2+TL*DOSE

ENDIF

IF(iflag.eq.7.or.iflag.eq.8) then BDIS $(K, 2)=B D I S(K, 2)+T L * D O S E$ TLB2 $=$ TLB2+TL*DOSE

ENDIF

IF(iflag.eq.4) then $\mathrm{ZDIS}(\mathrm{K}, 3)=\mathrm{ZDIS}(\mathrm{K}, 3)+\mathrm{TL} * \mathrm{DOSE}$ 
$T L A 3=T L A 3+T L * D O S E$

ENDIF

IF(iflag.eq.9) then

BDIS $(K, 3)=B D I S(K, 3)+T L * D O S E$

$T L B 3=T L B 3+T L * D O S E$

C

ENDIF

\section{IF(KFLAG.EQ.0)then}

TLHAP=TL HAP+TL*DOSE

ENDIF

IF(KFLAG.EQ.1)then

TLHAB $=T L H A B+T L * D O S E$

ENDIF

C

37 CONTINUE

27 CONTINUE

17 CONTINUE

C

C - WRITE TIME vs. TL INTENSITY

C

if $(\bmod (n t i m e, 1000) \cdot e q .0)$ then

if(kflag.eq.0) write $(4,140)$ T,TLHAP

if(kflag.eq.1) write( 8,140$)$ T,TLHAB

140 FORMAT(2x,19.3;' ',1PE12.2)

C

ENDIF

IF(KFLAG.EQ.1) GOTO 43

c

KFLAG=1

IF(ntime.eq.1)then

GOTO 2000

ELSE

ntime=ntime-1

C

C- INTLALIZE BOTTOM TEMP.

C

DO $76 \mathrm{I}=0, \mathrm{NX}$

DO $86 \mathrm{~J}=0, N Y$

DO $96 \mathrm{~K}=0, \mathrm{NZ}$

$\mathrm{T} 1(\mathrm{I}, J, \mathrm{~K})=\mathrm{TK} 1(\mathrm{I}, \mathrm{J}, \mathrm{K})$

96 CONTINUE

86 CONTINUE

76 CONTINUE

C

GOTO 1000

C

ENDIF

101 format(10x,6(/,1pe12.4))

102 format(10x,f10.4,' ',f10.4)

103 format(2x,f10.4,6(',f10.4))

C

C- INTTLAIIZE TOP TEMP.

C

$43 \mathrm{KFLAG}=0$

DO $77 \mathrm{I}=0, \mathrm{NX}$

DO $87 \mathrm{~J}=0, \mathrm{NY}$

DO $97 \mathrm{~K}=0, \mathrm{NZ}$

T1 $(I, J, K)=T K O(I, J, K)$

97 CONTINUE

87 CONTINUE

77 CONTINUE

C 


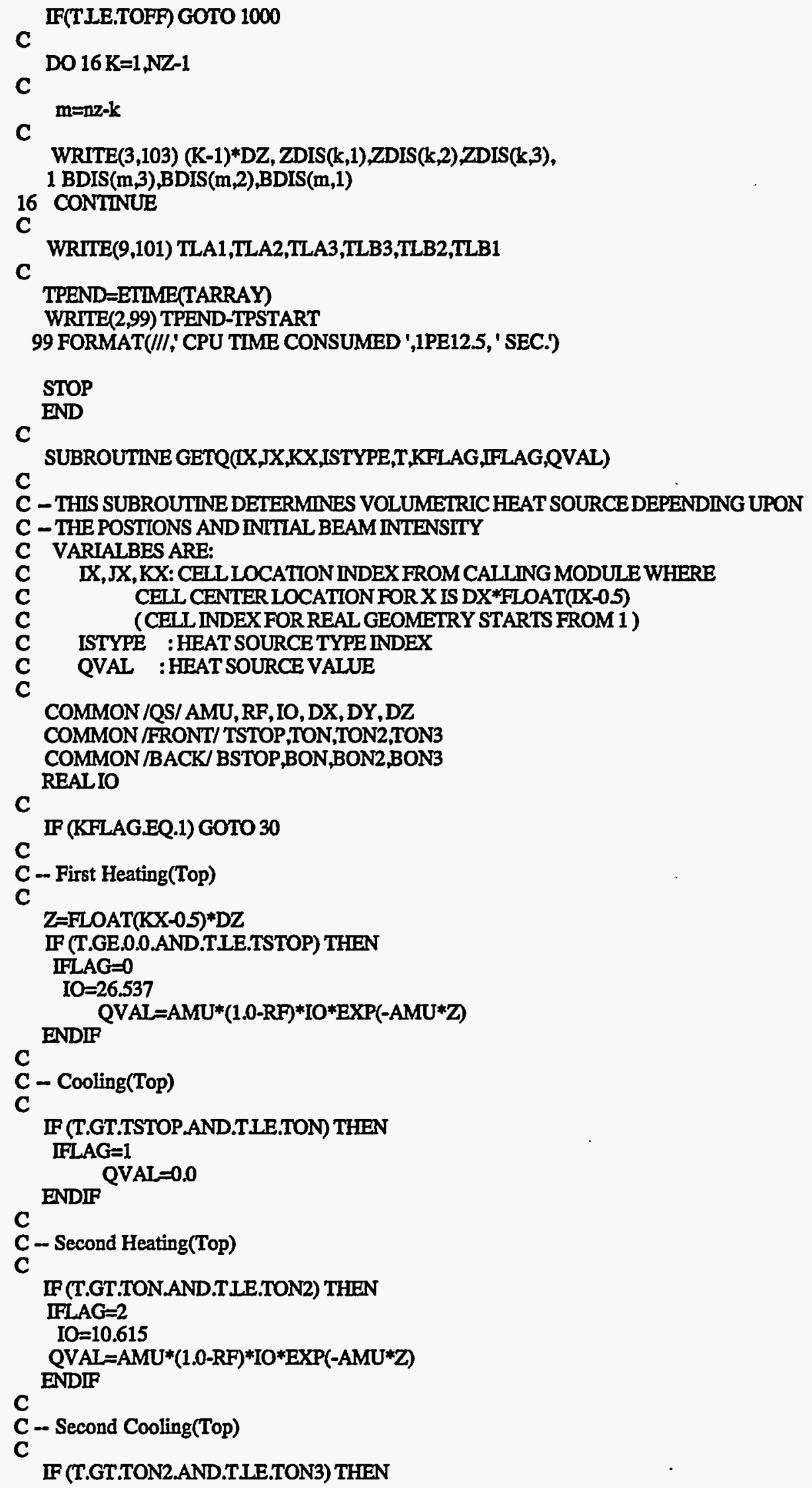




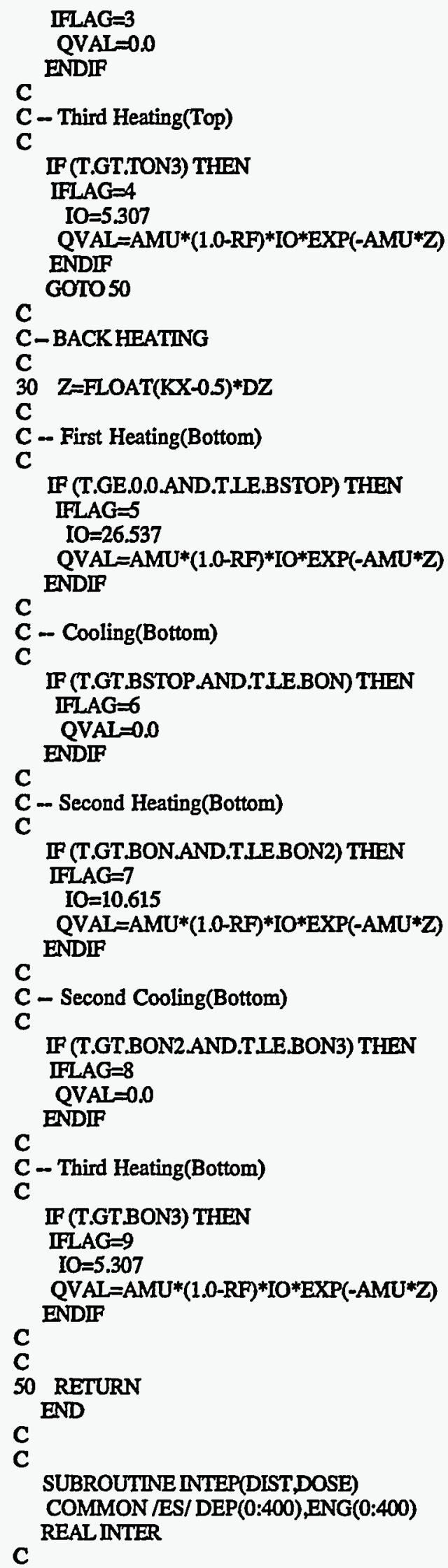


C $\mathrm{I}=1$

10 IF(DIST.LE.DEP(I)) THEN

INTER=(DIST-DEP(I-1))/(DEP(I)-DEP(I-1))

DOSE=ENG(I-1)+INTER*(ENG(I)-ENG(I-1))

ELSE

I $=I+1$

GOTO 10

ENDIF

C

RETURN

END 